\title{
Asymmetric Total Synthesis and Biological Evaluation of the Natural PDE4 Inhibitor Toddacoumalone
}

\author{
Guangdong Key Laboratory of Chiral Molecule and Drug Discovery, \\ School of Pharmaceutical Sciences, \\ Sun Yat-sen University, \\ Guangzhou, Guangdong 510006 (China). \\ E-mail: xiongxf7@mail.sysu.edu.cn
}

Ke-Qiang Hou, Xue-Ping Chen, Yiyou Huang, Albert S. C. Chan, Hai-Bin Luo, and Xiao-Feng Xiong*

Table of Contents

\begin{tabular}{|l|l|}
\hline 1. General information & $\mathrm{S} 2$ \\
\hline 2. Preparation of catalysts & $\mathrm{S} 3$ \\
\hline 3. Preparation of quinolinones (Figure S1) & $\mathrm{S} 7$ \\
\hline 4. Preparation of pyranoquinolinones (Figure S2) & $\mathrm{S} 8$ \\
\hline 5. Optimization of the reaction conditions (Table S1) & $\mathrm{S} 13$ \\
\hline 6. General procedures for the [4+2] cycloaddition reaction & $\mathrm{S} 15$ \\
\hline 7. Synthesis of acid 9 from $4 \mathbf{4 a a}$, & $\mathrm{S} 41$ \\
\hline 8. Total synthesis of toddacoumalone & $\mathrm{S} 42$ \\
\hline 9. PDE4 inhibition potency in enzymatic assays & $\mathrm{S} 46$ \\
\hline 10. Transition state model for the reaction (Figure S4) & $\mathrm{S} 47$ \\
\hline 11. References & $\mathrm{S} 47$ \\
\hline 12. Crystal data for $6 a$ acid 9 and toddacoumalone 1a & $\mathrm{S} 48$ \\
\hline 13. NMR spectra & $\mathrm{S} 51$ \\
\hline
\end{tabular}




\section{General information}

All reagents used in the synthesis were obtained commercially and used without further purification unless otherwise specified. Proton nuclear magnetic resonance ( ${ }^{1} \mathrm{H}$ NMR) spectra were recorded on a Bruker ( 400 or $\left.500 \mathrm{MHz}\right)$ spectrometer. Carbon nuclear magnetic resonance $\left({ }^{13} \mathrm{C}\right.$ NMR) spectra were recorded on a Bruker $[(400 \mathrm{MHz})(100 \mathrm{MHz})$ or $(500 \mathrm{MHz})$ $(125 \mathrm{MHz})]$ spectrometer. Chemical shifts of protons are reported in parts per million downfield from tetramethylsilane and are referenced to residual protium in the NMR solvent $\left(\mathrm{CDCl}_{3}: \delta 7.26\right.$; DMSO- $\left.d_{6}: \delta 2.50\right)$. Chemical shifts of carbon are referenced to the carbon resonances of the solvent ( $\mathrm{CDCl}_{3}: \delta 77.0$; DMSO- $\left.d_{6}: \delta 39.5\right) .{ }^{1} \mathrm{H}$ NMR splitting patterns are designated as singlet (s), doublet (d), triplet ( $\mathrm{t}$ ), quartet (q), dd (doublet of doublets); m (multiplets), and etc. All first-order splitting patterns were assigned on the basis of the appearance of the multiplet. Splitting patterns that could not be easily interpreted are designated as multiplet (m) or broad (br). The high-resolution mass spectra (HRMS) were obtained using a Shimadzu LCMS-ITTOF mass spectrometer. The determination of ee was performed via chiral HPLC analysis using Shimadzu LC-20AD HPLC work station. In each case, enantiomeric ratio was determined by HPLC analysis on a chiral column in comparison with authentic racemate, using a Daicel Chiralpak AD-H Column $(250 \times 4.6 \mathrm{~mm})$, Chiralpak ODH Column $(250 \times 4.6 \mathrm{~mm})$, Chiralpak AS-H Column $(250 \times 4.6 \mathrm{~mm})$, Chiralpak IA Column $(250 \times 4.6 \mathrm{~mm})$ or Chiralpak IG Column $(250 \times 4.6 \mathrm{~mm})$. UV detection was monitored at $230 \mathrm{~nm}$. X-ray crystallography analysis was performed on Bruker X8 APEX X-ray diffractionmeter. Optical rotations were measured in $\mathrm{CHCl}_{3}$ solution at $20{ }^{\circ} \mathrm{C}$ using a $1 \mathrm{~mL}$ cell with a $1 \mathrm{dm}$ path length on a Jasco P-1030 polarimeter and are reported as follows: $[\alpha]^{20} \mathrm{D}(c$ in $\mathrm{g}$ per $100 \mathrm{~mL}$ solvent). Analytical thin-layer chromatography (TLC) was performed on glass-backed silica plates. UV light, $\mathrm{I}_{2}$, and solution of potassium permanganate were used to visualize products. Column chromatography was performed on silica gel (200-300 mesh). All chemicals were used without purification as commercially available unless otherwise noted. Petroleum ether and ethyl acetate were distilled. Unless otherwise noted, experiments involving moisture and/or air sensitive components were performed under a positive pressure of argon in oven-dried glassware equipped with a rubber septum inlet. Dried solvents and liquid reagents were transferred by oven-dried syringes. 


\section{Preparation of catalysts}

Catalysts 8a, 8b, and 8h-8r purchased from Alfa Aesar or Sigma-Aldrich were used as received. Catalysts 8c, 8d, 8e, 8f and $\mathbf{8 g}$ were prepared through a similar process according to the literature. ${ }^{[1-3]}$

\section{Preparation of catalyst 8c.}

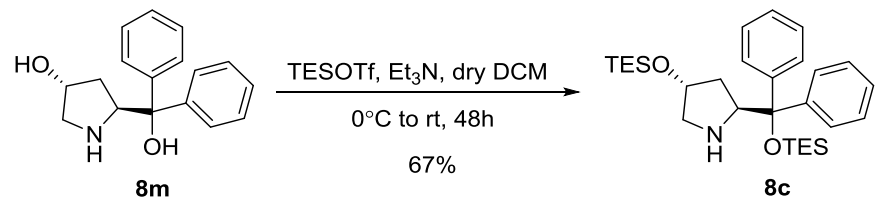

In a flame dried round bottom flask equipped with a magnetic stirring bar, (3R, 5S)-5(hydroxydiphenylmethyl)pyrrolidin-3-ol 8m (324 mg, $1.2 \mathrm{mmol}, 1 \mathrm{eq}$ ) and $\mathrm{Et}_{3} \mathrm{~N}$ (461 mg, $\left.4.6 \mathrm{mmol}, 3.8 \mathrm{eq}\right)$ were suspended in dry DCM (5 mL). The flask was filled with argon and placed in an ice bath, then TESOTf (1.10 g, $4.2 \mathrm{mmol}$, $3.5 \mathrm{eq}$ ) was slowly added over $15 \mathrm{mins}$. The reaction was then allowed to reach to room temperature and was stirred for 48 $\mathrm{h}$ until full conversion was confirmed by TLC analysis. The reaction mixture was then diluted with brine $(5 \mathrm{~mL})$ and extracted with DCM $(5 \mathrm{~mL} \times 3)$. The combined organic layers were dried over $\mathrm{Na}_{2} \mathrm{SO}_{4}$, filtered and concentrated under reduced pressure. The crude material was purified by column chromatography on silica gel, eluting with petroleum ether and ethyl acetate (100:1), to afford $\mathbf{8 c}$ in $67 \%$ yield $(400 \mathrm{mg}, 0.8 \mathrm{mmol})$ as a colorless oil. ${ }^{1} \mathbf{H} \mathbf{~ N M R}\left(500 \mathrm{MHz} \mathrm{CDCl}_{3}\right) \delta$ $=7.49-7.21(\mathrm{~m}, 10 \mathrm{H}), 4.35(\mathrm{t}, J=7.5 \mathrm{~Hz}, 1 \mathrm{H}), 3.83-3.76(\mathrm{~m}, 1 \mathrm{H}), 2.71-2.62(\mathrm{~m}, 2 \mathrm{H}), 1.73-1.58(\mathrm{~m}, 3 \mathrm{H}), 0.89(\mathrm{t}, J$ $=8.0 \mathrm{~Hz}, 9 \mathrm{H}), 0.85(\mathrm{t}, J=8.0 \mathrm{~Hz}, 9 \mathrm{H}), 0.50(\mathrm{q}, J=8.0 \mathrm{~Hz}, 6 \mathrm{H}), 0.33(\mathrm{qd}, J=8.0,3.0 \mathrm{~Hz}, 6 \mathrm{H}) .{ }^{13} \mathbf{C} \mathbf{N M R}(100 \mathrm{MHz}$, $\left.\mathrm{CDCl}_{3}\right) \delta=146.4,145.2,128.8,128.4,127.6,127.1,127.1,126.8,82.7,72.2,63.7,56.0,37.9,7.2,6.8,6.4,4.7$. HRMS (ESI) $\mathrm{m} / \mathrm{z}:[\mathrm{M}+\mathrm{H}]^{+}$calcd. for $\mathrm{C}_{29} \mathrm{H}_{48} \mathrm{NO}_{2} \mathrm{Si}_{2} 498.3218$, found 498.3219 .

\section{Preparation of catalyst 8d.}

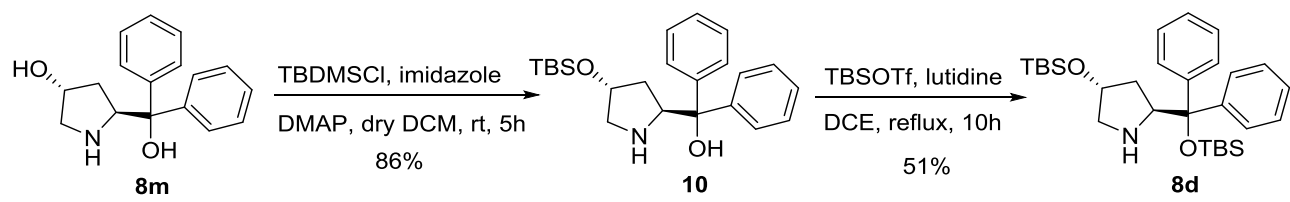

In a flame dried round bottom flask equipped with a magnetic stirring bar, a solution of (3R, 5S)-5(hydroxydiphenylmethyl)pyrrolidin-3-ol $8 \mathrm{~m}(0.807 \mathrm{~g}, 3.0 \mathrm{mmol}, 1 \mathrm{eq})$ in dry DCM (15 mL), imidazole $(0.306 \mathrm{~g}, 4.5 \mathrm{mmol}$, $1.5 \mathrm{eq})$ and DMAP (0.036 g, $0.3 \mathrm{mmol}, 0.1 \mathrm{eq})$ were added followed by TBDMSCl (0.675 g, $4.5 \mathrm{mmol}, 1.5 \mathrm{eq})$. After stirring at room temperature for $5 \mathrm{~h}$, the reaction mixture was then diluted with brine $(15 \mathrm{~mL})$, and extracted with DCM $(15 \mathrm{~mL} \times 3)$. The combined organic layers were dried over $\mathrm{Na}_{2} \mathrm{SO}_{4}$, filtered and concentrated under reduced pressure. The crude material was purified by column chromatography on silica gel, eluting with petroleum ether and ethyl acetate (2:1), to afford 10 in $86 \%$ yield $(0.99 \mathrm{~g}, 2.6 \mathrm{mmol})$ as a colorless oil. ${ }^{1} \mathbf{H} \mathbf{N M R}\left(400 \mathrm{MHz}, \mathrm{CDCl}_{3}\right) \delta=7.56(\mathrm{~d}, J=8.0 \mathrm{~Hz}, 2 \mathrm{H})$, $7.46(\mathrm{~d}, J=8.0 \mathrm{~Hz}, 2 \mathrm{H}), 7.32-7.21(\mathrm{~m}, 4 \mathrm{H}), 7.20-7.09(\mathrm{~m}, 2 \mathrm{H}), 4.56(\mathrm{dd}, J=9.2,7.2 \mathrm{~Hz}, 1 \mathrm{H}), 4.26(\mathrm{~s}, 1 \mathrm{H}), 3.08(\mathrm{dd}, J$ $=10.8,4.4 \mathrm{~Hz}, 1 \mathrm{H}), 2.86(\mathrm{dd}, J=10.8,2.8 \mathrm{~Hz}, 1 \mathrm{H}), 1.81-1.70(\mathrm{~m}, 1 \mathrm{H}), 1.44(\mathrm{dd}, J=13.2,6.8 \mathrm{~Hz}, 1 \mathrm{H}), 0.86(\mathrm{~s}, 9 \mathrm{H}), 0.00$ $(\mathrm{s}, 6 \mathrm{H}) .{ }^{13} \mathrm{C}$ NMR $\left(100 \mathrm{MHz}, \mathrm{CDCl}_{3}\right) \delta=147.9,145.1,128.3,128.0,126.6,126.4,126.0,125.5,73.0,63.4,55.8,36.4$, 25.9, 18.1, -4.7, -4.8. HRMS (ESI) m/z: $[\mathrm{M}+\mathrm{H}]^{+}$calcd. for $\mathrm{C}_{23} \mathrm{H}_{34} \mathrm{NO}_{2} \mathrm{Si} 384.2353$, found 384.2342.

In a flame dried round bottom flask equipped with a magnetic stirring bar, $10(0.199 \mathrm{~g}, 0.52 \mathrm{mmol})$ was dissolved in dry DCM (4 mL) under argon. Then, lutidine $(0.49 \mathrm{~mL}, 4.20 \mathrm{mmol})$ and TBDMSOTf $(0.48 \mathrm{~mL}, 2.1 \mathrm{mmol})$ were added to the flask and the reaction mixture was heated at reflux for $10 \mathrm{~h}$. The reaction mixture was diluted with brine $(2 \mathrm{~mL})$ and the $\mathrm{pH}$ maintained at 9-10 with $10 \%$ aq. $\mathrm{NaOH}$ solution. Layers were separated and the aqueous phase was further extracted with DCM (4 $\mathrm{mL} \times 3)$. The organic layer was dried over $\mathrm{Na}_{2} \mathrm{SO}_{4}$, filtered and concentrated under reduced pressure. The crude material was purified by column chromatography on silica gel, eluting with petroleum ether and ethyl acetate (10:1), 
to afford $\mathbf{8 d}$ in $51 \%$ yield $(0.131 \mathrm{~g}, 0.26 \mathrm{mmol})$ as a colorless oil. ${ }^{1} \mathbf{H} \mathbf{~ N M R ~}\left(500 \mathrm{MHz}, \mathrm{CDCl}_{3}\right) \delta=7.51(\mathrm{~d}, J=6.5 \mathrm{~Hz}, 2 \mathrm{H})$, $7.35(\mathrm{~d}, J=7.0 \mathrm{~Hz}, 2 \mathrm{H}), 7.30-7.21(\mathrm{~m}, 6 \mathrm{H}), 4.35(\mathrm{t}, J=8.0 \mathrm{~Hz}, 1 \mathrm{H}), 3.83-3.76(\mathrm{~m}, 1 \mathrm{H}), 2.70-2.61(\mathrm{~m}, 2 \mathrm{H}), 1.78(\mathrm{~s}$, 1H), $1.67-1.62(\mathrm{~m}, 2 \mathrm{H}), 0.94$ (s, 9H), 0.84 (s, 9H), -0.04 (s, 6H), -0.20 (s, 3H), -0.50 (s, 3H). ${ }^{13} \mathbf{C}$ NMR $\left(125 \mathrm{MHz}, \mathrm{CDCl}_{3}\right)$ $\delta=146.2,145.0,129.1,128.7,127.7,127.2,127.1,126.9,82.9,72.5,63.6,56.0,38.1,26.3,25.9,19.0,18.1,-2.7,-3.4,-$ 4.7, -4.8. HRMS (ESI) m/z: $[\mathrm{M}+\mathrm{H}]^{+}$calcd. for $\mathrm{C}_{29} \mathrm{H}_{48} \mathrm{NO}_{2} \mathrm{Si}_{2} 498.3218$, found 498.3221 .

\section{Preparation of catalyst 8e.}

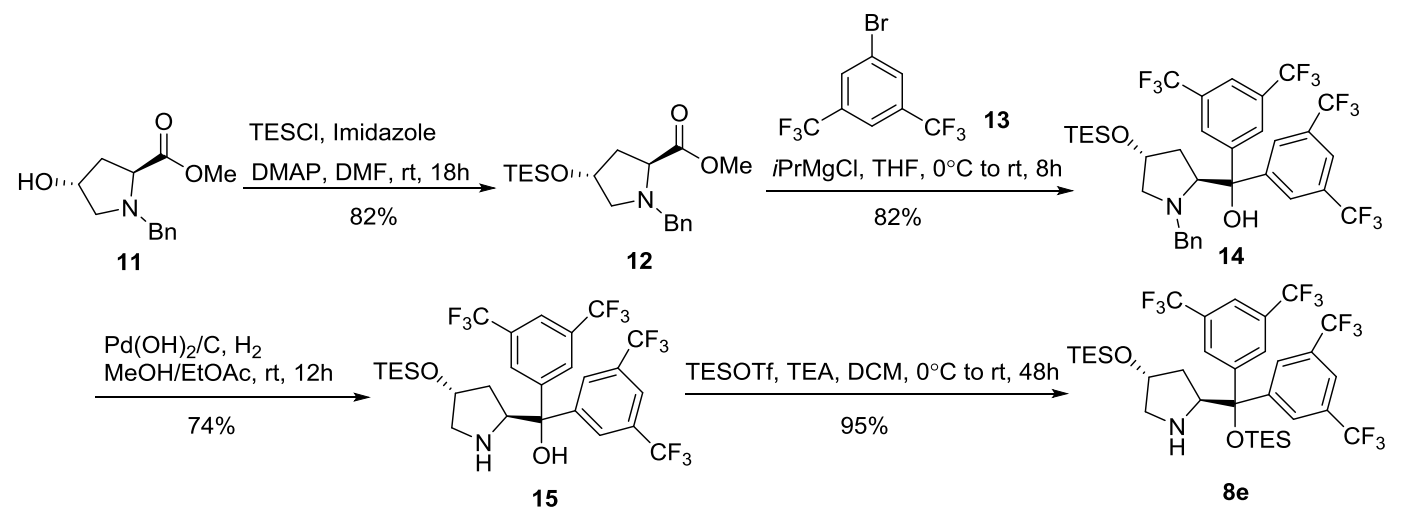

In a round bottom flask equipped with a magnetic stirring bar, $(2 S, 4 R)$-methyl 1-benzyl-4-hydroxypyrrolidine-2carboxylate 11 ( $3.5 \mathrm{~g}, 15 \mathrm{mmol}, 1 \mathrm{eq})$ was dissolved in DMF (150 mL). Subsequently imidazole (3.06 g, $45 \mathrm{mmol}, 3 \mathrm{eq})$, TESCl ( $4.5 \mathrm{~g}, 30 \mathrm{mmol}, 2 \mathrm{eq})$ and DMAP $(0.55 \mathrm{~g}, 4.5 \mathrm{mmol}, 0.3 \mathrm{eq})$ were added in this order. The reaction mixture was stirred for $18 \mathrm{~h}$ and quenched with $15 \mathrm{~mL} \mathrm{MeOH}$. Hereafter, the mixture was diluted with ethyl acetate $(150 \mathrm{~mL})$ and washed with water. The aqueous phase was extracted with ethyl acetate $(50 \mathrm{~mL} \times 3)$ and the combined organic extracts were dried over $\mathrm{Na}_{2} \mathrm{SO}_{4}$. After filtration and evaporation of the solvent, the crude material was purified by column chromatography on silica gel, eluting with petroleum ether and ethyl acetate (40:1 to 20:1), to afford $\mathbf{1 2}$ in 82\% yield (4.3 $\mathrm{g}, 12.3 \mathrm{mmol})$ as a colorless oil. ${ }^{1} \mathbf{H}$ NMR $\left(400 \mathrm{MHz}, \mathrm{CDCl}_{3}\right) \delta=7.32-7.22(\mathrm{~m}, 5 \mathrm{H}), 4.44-4.37(\mathrm{~m}, 1 \mathrm{H}), 3.89(\mathrm{~d}, J=$ $12.8 \mathrm{~Hz}, 1 \mathrm{H}), 3.64(\mathrm{~s}, 3 \mathrm{H}), 3.59(\mathrm{~d}, J=12.8 \mathrm{~Hz}, 1 \mathrm{H}), 3.52(\mathrm{t}, J=8.0 \mathrm{~Hz}, 1 \mathrm{H}), 3.26(\mathrm{dd}, J=9.6,5.9 \mathrm{~Hz}, 1 \mathrm{H}), 2.37(\mathrm{dd}, J=$ 9.6, $5.2 \mathrm{~Hz}, 1 \mathrm{H}), 2.24-2.15(\mathrm{~m}, 1 \mathrm{H}), 2.06-1.99(\mathrm{~m}, 1 \mathrm{H}), 0.92(\mathrm{t}, J=8.0 \mathrm{~Hz}, 9 \mathrm{H}), 0.55(\mathrm{q}, J=8.0 \mathrm{~Hz}, 6 \mathrm{H}) .{ }^{13} \mathbf{C} \mathbf{~ N M R}$ $\left(100 \mathrm{MHz}, \mathrm{CDCl}_{3}\right) \delta=174.3,138.1,129.3,128.2,127.2,70.1,64.3,61.8,59.3,51.8,39.6,6.7,4.7$. HRMS (ESI) m/z: $[\mathrm{M}+\mathrm{H}]^{+}$calcd. for $\mathrm{C}_{19} \mathrm{H}_{32} \mathrm{NO}_{3} \mathrm{Si} 350.2146$, found 350.2139 .

In a $100 \mathrm{~mL}$ three-neck round-bottom flask equipped with a magnetic stirring bar, 1-bromo-3,5bis(trifluoromethyl)benzene $\mathbf{1 3}(5.8 \mathrm{~g}, 19.8 \mathrm{mmol}, 3 \mathrm{eq})$ was dissolved in $15 \mathrm{~mL}$ of anhydrous THF under argon and this solution was cooled in $0{ }^{\circ} \mathrm{C}$. Then, isopropylmagnesium chloride $(10.0 \mathrm{~mL}, 2.0 \mathrm{M}$ in THF, $21.1 \mathrm{mmol}$, 3.2eq) was added to the reaction mixture. The reaction mixture, which turned pale browngreen, was stirred at $0{ }^{\circ} \mathrm{C}$ for $1 \mathrm{~h}$. In parallel, $12(2.3$ $\mathrm{g}, 6.6 \mathrm{mmol}, 1 \mathrm{eq}$ ) was dissolved in $10 \mathrm{~mL}$ of anhydrous THF, giving a clear solution. This solution was added to the reaction mixture with the aid of a cannula under argon at $0{ }^{\circ} \mathrm{C}$. The reaction mixture was then stirred at room temperature for $8 \mathrm{~h}$, and conversion was confirmed by TLC (petroleum ether and ethyl acetate $=30: 1$ ). The reaction mixture was then diluted with saturated $\mathrm{NH}_{4} \mathrm{Cl}(20 \mathrm{~mL})$ and extracted with ethyl acetate $(60 \mathrm{~mL} \times 3)$. The combined organic layers were dried over $\mathrm{Na}_{2} \mathrm{SO}_{4}$, filtered and concentrated under reduced pressure. The crude material was purified by column chromatography on silica gel, eluting with petroleum ether and ethyl acetate (100:1), to afford $\mathbf{1 4}$ in $82 \%$ yield (4.0 g, 5.3 mmol) as a colorless oil. ${ }^{1} \mathbf{H}$ NMR $\left(400 \mathrm{MHz}, \mathrm{CDCl}_{3}\right) \delta=8.21(\mathrm{~s}, 2 \mathrm{H}), 8.04(\mathrm{~s}, 2 \mathrm{H}), 7.74(\mathrm{~d}, J=16.0 \mathrm{~Hz}, 2 \mathrm{H}), 7.23-7.20$ (s, 3H), $6.97-6.90(\mathrm{~m}, 2 \mathrm{H}), 5.46(\mathrm{~s}, 1 \mathrm{H}), 4.43(\mathrm{t}, J=8.4 \mathrm{~Hz}, 1 \mathrm{H}), 4.20(\mathrm{~s}, 1 \mathrm{H}), 3.52(\mathrm{~d}, J=13.2 \mathrm{~Hz}, 1 \mathrm{H}), 3.17(\mathrm{~d}, J=13.2$ $\mathrm{Hz}, 1 \mathrm{H}), 3.05(\mathrm{dd}, J=11.6,4.0 \mathrm{~Hz}, 1 \mathrm{H}), 2.70-2.61(\mathrm{~m}, 1 \mathrm{H}), 1.81-1.71(\mathrm{~m}, 1 \mathrm{H}), 1.68-1.58(\mathrm{~m}, 1 \mathrm{H}), 0.94(\mathrm{t}, J=8.0 \mathrm{~Hz}$, 9H), $0.57(\mathrm{q}, J=8.0 \mathrm{~Hz}, 6 \mathrm{H}) .{ }^{19} \mathbf{F}$ NMR $\left(376 \mathrm{MHz}, \mathrm{CDCl}_{3}\right) \delta=-62.9,-62.9 .{ }^{13} \mathbf{C} \mathbf{N M R}\left(100 \mathrm{MHz}, \mathrm{CDCl}_{3}\right) \delta=149.5,147.2$, $138.6,132.4\left(\mathrm{q}, J_{\mathrm{C}, \mathrm{F}}=33 \mathrm{~Hz}\right), 131.7\left(\mathrm{q}, J_{\mathrm{C}, \mathrm{F}}=33 \mathrm{~Hz}\right), 128.5,128.1,127.4,126.0\left(\mathrm{~d}, J_{\mathrm{C}, \mathrm{F}}=3.2 \mathrm{~Hz}\right), 125.7,124.6\left(\mathrm{~d}, J_{\mathrm{C}, \mathrm{F}}\right.$ 
= 4.5 Hz), $121.8\left(\mathrm{~d}, J_{\mathrm{C}, \mathrm{F}}=4.5 \mathrm{~Hz}\right), 121.5(\mathrm{~m}), 121.3(\mathrm{~m}), 76.3,71.2,71.1,62.9,61.8,39.4,6.8,4.7$. HRMS (ESI) m/z: $[\mathrm{M}+\mathrm{H}]^{+}$calcd. for $\mathrm{C}_{34} \mathrm{H}_{36} \mathrm{~F}_{12} \mathrm{NO}_{2} \mathrm{Si} 746.2318$, found 746.2336.

In a flame dried round bottom flask equipped with a magnetic stirring bar, 14 (4.0 g, $5.3 \mathrm{mmol}, 1 \mathrm{eq})$ was dissolved in methanol $(30 \mathrm{~mL})$ and ethyl acetate $(10 \mathrm{~mL}) . \mathrm{Pd}(\mathrm{OH})_{2} / \mathrm{C}$ was added $(1.6 \mathrm{~g}, 40 \mathrm{wt} . \%)$. The mixture was subsequently saturated with hydrogen gas and stirred under hydrogen atmosphere for $12 \mathrm{~h}$. Hereafter, the reaction mixture was filtered through a short bed of celite and the solution was evaporated under reduced pressure. The crude material was purified by column chromatography on silica gel, eluting with petroleum ether and ethyl acetate (15:1), to afford $\mathbf{1 5}$ in $74 \%$ yield (2.57 g, 3.92mmol) as a colorless oil. ${ }^{1} \mathbf{H}$ NMR $\left(400 \mathrm{MHz}, \mathrm{CDCl}_{3}\right) \delta=8.06$ (s, 2H), 7.95 (s, 2H), 7.77 (d, J=5.2 Hz, 2H), 4.96 $(\mathrm{s}, 1 \mathrm{H}), 4.65(\mathrm{dd}, J=9.6,6.4 \mathrm{~Hz}, 1 \mathrm{H}), 4.35-4.29(\mathrm{~m}, 1 \mathrm{H}), 3.13(\mathrm{dd}, J=11.2,4.0 \mathrm{~Hz}, 1 \mathrm{H}), 3.02-2.97(\mathrm{~m}, 1 \mathrm{H}), 1.96-$ $1.77(\mathrm{br}, 1 \mathrm{H}), 1.69-1.60(\mathrm{~m}, 1 \mathrm{H}), 1.47-1.39(\mathrm{~m}, 1 \mathrm{H}), 0.94(\mathrm{t}, J=8.0 \mathrm{~Hz}, 9 \mathrm{H}), 0.59(\mathrm{q}, J=8.0 \mathrm{~Hz}, 6 \mathrm{H}) .{ }^{19} \mathbf{F ~ N M R}(376$ $\left.\mathrm{MHz}, \mathrm{CDCl}_{3}\right) \delta=-62.8,-63.0 .{ }^{13} \mathbf{C ~ N M R}\left(100 \mathrm{MHz}, \mathrm{CDCl}_{3}\right) \delta=149.2,146.2,132.4\left(\mathrm{q}, J_{\mathrm{C}, \mathrm{F}}=33 \mathrm{~Hz}\right), 131.7\left(\mathrm{q}, J_{\mathrm{C}, \mathrm{F}}=33\right.$ $\mathrm{Hz}), 126.2\left(\mathrm{~d}, J_{\mathrm{C}, \mathrm{F}}=3.2 \mathrm{~Hz}\right), 125.7\left(\mathrm{~d}, J_{\mathrm{C}, \mathrm{F}}=2.9 \mathrm{~Hz}\right), 124.6,121.9,121.6(\mathrm{~m}), 121.4(\mathrm{~m}), 76.5,72.5$, 63.4, 56.1, 36.9, 6.7, 4.7. HRMS (ESI) m/z: $[\mathrm{M}+\mathrm{H}]^{+}$calcd. for $\mathrm{C}_{27} \mathrm{H}_{30} \mathrm{~F}_{12} \mathrm{NO}_{2} \mathrm{Si} 656.1849$, found 656.1860.

In a flame dried round bottom flask equipped with a magnetic stirring bar, $15(2.57 \mathrm{~g}, 3.92 \mathrm{mmol}, 1 \mathrm{eq})$ and $\mathrm{Et}_{3} \mathrm{~N}$ $(1.58 \mathrm{~g}, 15.7 \mathrm{mmol}, 4 \mathrm{eq})$ were dissolved in dry DCM (25 mL) under argon. The solution was cooled to $0{ }^{\circ} \mathrm{C}$ and TESOTf $(3.1 \mathrm{~g}, 11.76 \mathrm{mmol}, 3 \mathrm{eq})$ was added dropwise over $15 \mathrm{mins}$. The reaction was then allowed to reach to room temperature and was stirred for $48 \mathrm{~h}$ until full conversion was confirmed by TLC analysis. The reaction mixture was then diluted with brine $(15 \mathrm{~mL})$ and extracted with DCM $(15 \mathrm{~mL} \times 3)$. The combined organic layers were dried over $\mathrm{Na}_{2} \mathrm{SO}_{4}$, filtered and concentrated under reduced pressure. The crude material was purified by column chromatography on silica gel, eluting with petroleum ether and ethyl acetate (100:1), to afford 8e in 95\% yield (2.86 g, $3.7 \mathrm{mmol})$ as a colorless oil. ${ }^{\mathbf{1}} \mathbf{H}$ NMR $\left(400 \mathrm{MHz} \mathrm{CDCl}_{3}\right) \delta=8.08(\mathrm{~s}, 2 \mathrm{H}), 7.85(\mathrm{~d}, J=5.6 \mathrm{~Hz}, 2 \mathrm{H}), 7.76(\mathrm{~s}, 2 \mathrm{H}), 4.50(\mathrm{t}, J=8.0 \mathrm{~Hz}, 1 \mathrm{H}), 3.79(\mathrm{~s}, 1 \mathrm{H}), 2.74(\mathrm{~d}, J$ $=11.6 \mathrm{~Hz}, 1 \mathrm{H}), 2.24(\mathrm{dd}, J=11.6,4.0 \mathrm{~Hz}, 1 \mathrm{H}), 1.84-1.76(\mathrm{~s}, 1 \mathrm{H}), 1.43-1.33(\mathrm{~m}, 1 \mathrm{H}), 0.91(\mathrm{t}, J=8.0 \mathrm{~Hz}, 9 \mathrm{H}), 0.84(\mathrm{t}$, $J=8.0 \mathrm{~Hz}, 9 \mathrm{H}), 0.52(\mathrm{q}, J=8.0 \mathrm{~Hz}, 6 \mathrm{H}), 0.41-0.23(\mathrm{~m}, 6 \mathrm{H}) .{ }^{19} \mathbf{F} \mathbf{N M R}\left(376 \mathrm{MHz}, \mathrm{CDCl}_{3}\right) \delta=-62.8,-62.9 .{ }^{\mathbf{1 3}} \mathbf{C} \mathbf{~ N M R}$ $\left(100 \mathrm{MHz}, \mathrm{CDCl}_{3}\right) \delta=147.9,146.1,132.6\left(\mathrm{q}, J_{\mathrm{C}, \mathrm{F}}=33 \mathrm{~Hz}\right), 130.6\left(\mathrm{q}, J_{\mathrm{C}, \mathrm{F}}=33 \mathrm{~Hz}\right), 128.8\left(\mathrm{~d}, J_{\mathrm{C}, \mathrm{F}}=2.2 \mathrm{~Hz}\right), 128.7,127.4$ $\left(\mathrm{d}, J_{\mathrm{C}, \mathrm{F}}=25 \mathrm{~Hz}\right), 124.7\left(\mathrm{~d}, J_{\mathrm{C}, \mathrm{F}}=25 \mathrm{~Hz}\right), 121.9(\mathrm{~m}), 121.5(\mathrm{~m}), 82.0,72.3,63.0,55.9,37.9,6.8,6.7,6.3$, 4.6. HRMS (ESI) $\mathrm{m} / \mathrm{z}:[\mathrm{M}+\mathrm{H}]^{+}$calcd. for $\mathrm{C}_{33} \mathrm{H}_{44} \mathrm{~F}_{12} \mathrm{NO}_{2} \mathrm{Si}_{2} 770.2714$, found 770.2726 .

\section{Preparation of catalyst 8f.}

Catalysts 8 f was prepared through a similar process according to the catalysts 8 e from $(2 R, 4 S)$-4-hydroxypyrrolidine2-carboxylic acid. Spectral data of $\mathbf{8 f}$ is in agreement with $\mathbf{8 e}$.

\section{Preparation of catalyst 8g.}

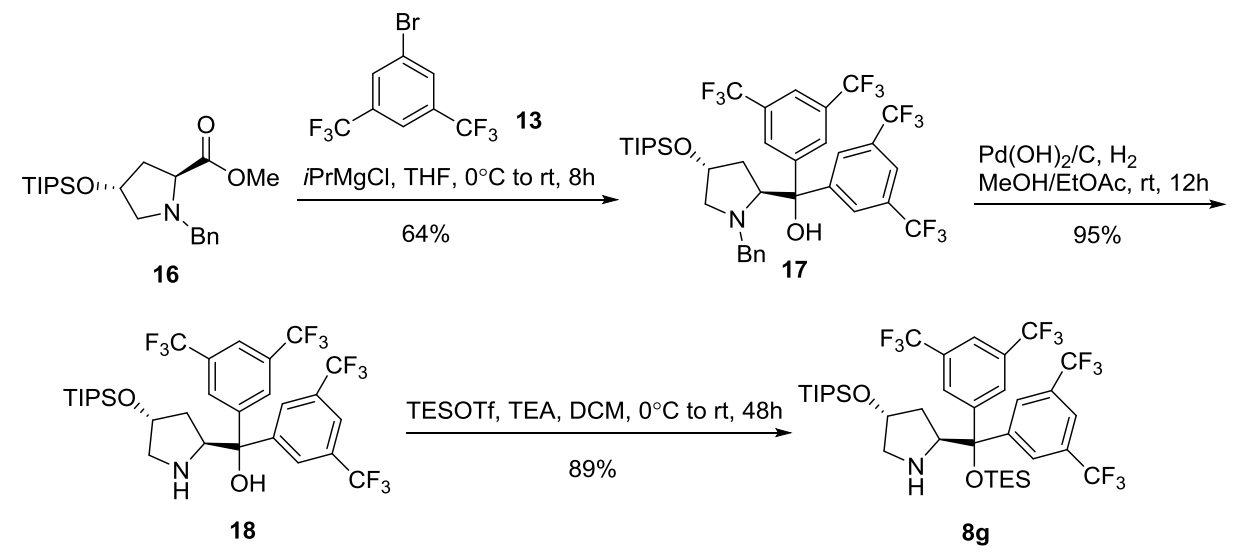


In a flame dried round bottom flask equipped with a magnetic stirring bar, 1-bromo-3,5-bis(trifluoromethyl)benzene $13(6.15 \mathrm{~g}, 21 \mathrm{mmol}, 3 \mathrm{eq})$ was dissolved in $18 \mathrm{~mL}$ of anhydrous THF under argon and this solution was cooled in $0{ }^{\circ} \mathrm{C}$. Then, isopropylmagnesium chloride $(11.0 \mathrm{ml}, 2.0 \mathrm{M}$ in THF, $23 \mathrm{mmol}, 3.2 \mathrm{eq})$ was added to the reaction mixture. The reaction mixture, which turned pale browngreen, was stirred at $0{ }^{\circ} \mathrm{C}$ for $1 \mathrm{~h}$. In parallel, $16(2.74 \mathrm{~g}, 7 \mathrm{mmol}, 1 \mathrm{eq})$ was dissolved in $10 \mathrm{~mL}$ of anhydrous THF, giving a clear solution. This solution was added to the reaction mixture with the aid of a cannula under argon at $0{ }^{\circ} \mathrm{C}$. The reaction mixture was then stirred at room temperature for $8 \mathrm{~h}$, and conversion was confirmed by TLC (petroleum ether and ethyl acetate $=50: 1$ ). The reaction mixture was then diluted with saturated $\mathrm{NH}_{4} \mathrm{Cl}(25 \mathrm{~mL})$ and extracted with ethyl acetate $(60 \mathrm{~mL} \times 3)$. The combined organic layers were dried over $\mathrm{Na}_{2} \mathrm{SO}_{4}$, filtered and concentrated under reduced pressure. The crude material was purified by column chromatography on silica gel, eluting with petroleum ether and ethyl acetate (100:1), to afford $\mathbf{1 7}$ in $64 \%$ yield $(3.5 \mathrm{~g}, 4.44 \mathrm{mmol})$ as a white solid. ${ }^{1} \mathbf{H}$ NMR $\left(400 \mathrm{MHz}, \mathrm{CDCl}_{3}\right) \delta=8.21(\mathrm{~s}, 2 \mathrm{H}), 8.04(\mathrm{~s}, 2 \mathrm{H}), 7.75(\mathrm{~d}, J=18.0 \mathrm{~Hz}, 2 \mathrm{H}), 7.26-7.19(\mathrm{~m}, 3 \mathrm{H}), 6.95(\mathrm{~d}, J=7.2 \mathrm{~Hz}, 2 \mathrm{H})$, $5.47(\mathrm{~s}, 1 \mathrm{H}), 4.46(\mathrm{t}, J=7.6 \mathrm{~Hz}, 1 \mathrm{H}), 4.31(\mathrm{~s}, 1 \mathrm{H}), 3.57(\mathrm{~d}, J=13.02 \mathrm{~Hz}, 1 \mathrm{H}), 3.16(\mathrm{~d}, J=13.2 \mathrm{~Hz}, 1 \mathrm{H}), 3.07(\mathrm{dd}, J=11.6$, $3.6 \mathrm{~Hz}, 1 \mathrm{H}), 2.72(\mathrm{~d}, J=11.6 \mathrm{~Hz}, 1 \mathrm{H}), 1.84-1.77(\mathrm{~m}, 1 \mathrm{H}), 1.67-7.59(\mathrm{~m}, 1 \mathrm{H}), 1.05(\mathrm{~s}, 21 \mathrm{H}) .{ }^{19} \mathbf{F} \mathbf{N M R}\left(376 \mathrm{MHz}, \mathrm{CDCl}_{3}\right)$ $\delta=-62.9,-63.0 .{ }^{13} \mathbf{C}$ NMR $\left(100 \mathrm{MHz}, \mathrm{CDCl}_{3}\right) \delta=149.4,147.2,138.7,132.3\left(\mathrm{q}, J_{\mathrm{C}, \mathrm{F}}=33 \mathrm{~Hz}\right), 131.7\left(\mathrm{q}, J_{\mathrm{C}, \mathrm{F}}=33 \mathrm{~Hz}\right)$, 128.5, 128.1, 127.4, $125.9\left(\mathrm{~d}, J_{\mathrm{C}, \mathrm{F}}=2.6 \mathrm{~Hz}\right), 125.6\left(\mathrm{~d}, J_{\mathrm{C}, \mathrm{F}}=2.7 \mathrm{~Hz}\right), 124.5\left(\mathrm{~d}, J_{\mathrm{C}, \mathrm{F}}=4.2 \mathrm{~Hz}\right), 121.8\left(\mathrm{~d}, J_{\mathrm{C}, \mathrm{F}}=4.4 \mathrm{~Hz}\right)$, 121.5 (m), 121.3 (m), 76.1, 71.7, 71.3, 63.0, 61.9, 39.5, 18.0, 12.0. HRMS (ESI) m/z: [M+H] $]^{+}$calcd. for $\mathrm{C}_{37} \mathrm{H}_{42} \mathrm{~F}_{12} \mathrm{NO}_{2} \mathrm{Si}$ 788.2692, found 788.2710 .

In a flame dried round bottom flask equipped with a magnetic stirring bar, $17(3.5 \mathrm{~g}, 4.44 \mathrm{mmol}, 1 \mathrm{eq})$ was dissolved in methanol $(30 \mathrm{~mL})$ and ethyl acetate $(10 \mathrm{~mL}) . \mathrm{Pd}(\mathrm{OH})_{2} / \mathrm{C}$ was added $(1.4 \mathrm{~g}, 40 \mathrm{wt} . \%)$. The mixture was subsequently saturated with hydrogen gas and stirred under hydrogen atmosphere for $12 \mathrm{~h}$. Hereafter, the reaction mixture was filtered through a short bed of celite and the solution was evaporated under reduced pressure. The crude material was purified by column chromatography on silica gel, eluting with petroleum ether and ethyl acetate (100:1), to afford $\mathbf{1 8}$ in $95 \%$ yield $(2.92 \mathrm{~g}, 4.18 \mathrm{mmol})$ as a colorless oil. ${ }^{1} \mathrm{H}$ NMR $\left(500 \mathrm{MHz}, \mathrm{CDCl}_{3}\right) \delta=8.09(\mathrm{~s}, 2 \mathrm{H}), 8.00(\mathrm{~s}, 2 \mathrm{H}), 7.76(\mathrm{~s}, 2 \mathrm{H}), 5.49(\mathrm{~s}, 1 \mathrm{H})$, $4.51(\mathrm{dd}, J=9.5,3.5 \mathrm{~Hz}, 1 \mathrm{H}), 4.41(\mathrm{~s}, 1 \mathrm{H}), 3.10(\mathrm{~d}, J=10.5 \mathrm{~Hz}, 1 \mathrm{H}), 3.03(\mathrm{dd}, J=10.5,2.5 \mathrm{~Hz}, 1 \mathrm{H}), 2.02-1.94(\mathrm{~m}, 1 \mathrm{H})$, $1.58(\mathrm{~d}, J=14.5 \mathrm{~Hz}, 1 \mathrm{H}), 1.10-1.04(\mathrm{~m}, 21 \mathrm{H}) .{ }^{19} \mathbf{F} \mathbf{~ N M R}\left(471 \mathrm{MHz}, \mathrm{CDCl}_{3}\right) \delta=-62.7,-62.9 .{ }^{13} \mathbf{C} \mathbf{N M R}\left(125 \mathrm{MHz}, \mathrm{CDCl}_{3}\right)$ $\delta=148.4,148.0,131.3\left(\mathrm{q}, J_{\mathrm{C}, \mathrm{F}}=33 \mathrm{~Hz}\right), 131.9\left(\mathrm{q}, J_{\mathrm{C}, \mathrm{F}}=33 \mathrm{~Hz}\right), 126.5,125.9\left(\mathrm{~d}, J_{\mathrm{C}, \mathrm{F}}=2.8 \mathrm{~Hz}\right), 124.4\left(\mathrm{~d}, J_{\mathrm{C}, \mathrm{F}}=6.3 \mathrm{~Hz}\right)$, $122.2\left(\mathrm{~d}, J_{\mathrm{C}, \mathrm{F}}=6.3 \mathrm{~Hz}\right), 121.4(\mathrm{~m}$, overlapped signals), 77.4, 72.2, 63.8, 56.2, 36.9, 17.9, 17.9, 12.0. HRMS (ESI) m/z: $[\mathrm{M}+\mathrm{H}]^{+}$calcd. for 698.2318 , found 698.2329 .

In a flame dried round bottom flask equipped with a magnetic stirring bar, $18(2.92 \mathrm{~g}, 4.18 \mathrm{mmol}, 1 \mathrm{eq})$ and $\mathrm{Et}_{3} \mathrm{~N}$ $(1.69 \mathrm{~g}, 16.74 \mathrm{mmol}, 4 \mathrm{eq})$ were dissolved in dry DCM $(25 \mathrm{~mL})$ under argon. The solution was cooled to $0{ }^{\circ} \mathrm{C}$ and TESOTf ( $3.32 \mathrm{~g}, 12.56 \mathrm{mmol}, 3 \mathrm{eq}$ ) was added dropwise over $15 \mathrm{mins}$. The reaction was then allowed to reach to room temperature and was stirred for $48 \mathrm{~h}$ until full conversion was confirmed by TLC analysis. The reaction mixture was then diluted with brine $(20 \mathrm{~mL})$ and extracted with $\mathrm{DCM}(20 \mathrm{~mL} \times 3)$. The combined organic layers were dried over $\mathrm{Na}_{2} \mathrm{SO}_{4}$, filtered and concentrated under reduced pressure. The crude material was purified by column chromatography on silica gel, eluting with petroleum ether and ethyl acetate (100:1), to afford $\mathbf{8 g}$ in $89 \%$ yield ( $3 \mathrm{~g}, 3.7 \mathrm{mmol}$ ) as a white solid. ${ }^{\mathbf{1}} \mathbf{H} \mathbf{~ N M R}$ (400 $\left.\mathrm{MHz}, \mathrm{CDCl}_{3}\right) \delta 8.09(\mathrm{~s}, 2 \mathrm{H}), 7.85(\mathrm{~d}, J=7.2 \mathrm{~Hz}, 2 \mathrm{H}), 7.75(\mathrm{~s}, 2 \mathrm{H}), 4.51(\mathrm{t}, J=7.6 \mathrm{~Hz}, 1 \mathrm{H}), 3.87(\mathrm{~s}, 1 \mathrm{H}), 2.79(\mathrm{~d}, J=11.6$ $\mathrm{Hz}, 1 \mathrm{H}), 2.32(\mathrm{dd}, J=11.2,3.6 \mathrm{~Hz}, 1 \mathrm{H}), 1.90-1.83(\mathrm{~m}, 1 \mathrm{H}), 1.44-1.35(\mathrm{~m}, 1 \mathrm{H}), 1.00(\mathrm{~s}, 21 \mathrm{H}), 0.84(\mathrm{t}, J=8.0 \mathrm{~Hz}, 9 \mathrm{H})$, $0.40-0.25(\mathrm{~m}, 6 \mathrm{H}) .{ }^{19} \mathbf{F}$ NMR $\left(376 \mathrm{MHz}, \mathrm{CDCl}_{3}\right) \delta=-62.8,-63.0 .{ }^{13} \mathbf{C} \mathbf{~ N M R}\left(100 \mathrm{MHz}, \mathrm{CDCl}_{3}\right) \delta=147.9,146.1,131.6$ $\left(\mathrm{q}, J_{\mathrm{C}, \mathrm{F}}=33 \mathrm{~Hz}\right), 130.6\left(\mathrm{q}, J_{\mathrm{C}, \mathrm{F}}=33 \mathrm{~Hz}\right), 128.8\left(\mathrm{~d}, J_{\mathrm{C}, \mathrm{F}}=2.7 \mathrm{~Hz}\right), 128.7\left(\mathrm{~d}, J_{\mathrm{C}, \mathrm{F}}=2.7 \mathrm{~Hz}\right), 124.7\left(\mathrm{~d}, J_{\mathrm{C}, \mathrm{F}}=25 \mathrm{~Hz}\right), 121.9$ (m, overlapped signals), $122.5(\mathrm{~m}), 81.9,77.4,77.0,76.7,72.5,63.0,56.0,38.1,17.9,12.0,6.8,6.2$. HRMS (ESI) m/z: $[\mathrm{M}+\mathrm{H}]^{+}$calcd. for $\mathrm{C}_{36} \mathrm{H}_{50} \mathrm{~F}_{12} \mathrm{NO}_{2} \mathrm{Si}_{2} 812.3183$, found 812.3198. 


\section{Preparation of the quinolinones}

Figure S1. Structure of the quinolinones.
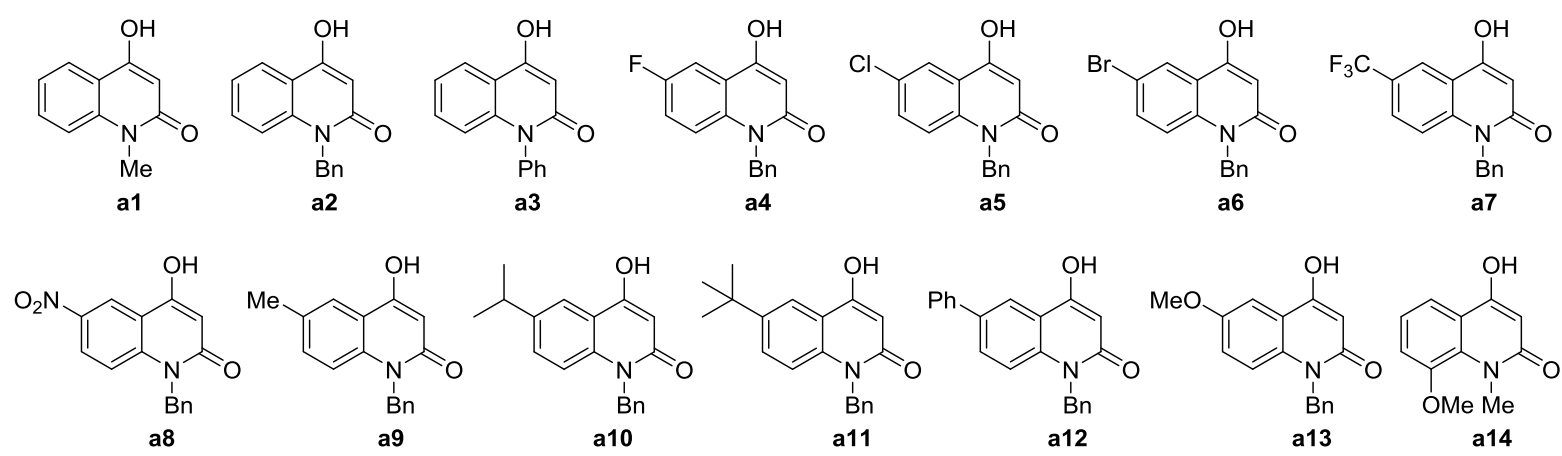<smiles>COc1cc2c(O)cc(=O)n(Cc3ccccc3)c2cc1OC</smiles><smiles>C=[10B]n1c(=O)cc(O)c2c3ccccc3ccc21</smiles><smiles>[B]n1c(=O)cc(O)c2cccnc21</smiles><smiles>[13C]=[10B]n1c(=O)cc(O)c2c3cccnc3ccc21</smiles><smiles></smiles><smiles>O=c1cc(O)c2cccc3c2n1CC[R1]3</smiles>

The quinolinone a1 purchased from Sigma-Aldrich were used as received. The quinolinones a2-a21 could be conveniently synthesized according to the literature procedures. ${ }^{[4]}$ Among them, a6, a8, $\mathbf{a 1 3}, \mathbf{a 1 7}$ and $\mathbf{a 1 9}$ are the new compounds.

\section{Characterization datas for the quinolinones:}

1-benzyl-6-bromo-4-hydroxyquinolin-2(1H)-one (a6)

$81 \%$ yield, off-white solid, ${ }^{1}$ H NMR (400 MHz, DMSO-d $\left.{ }_{6}\right) \delta=7.99(\mathrm{~s}, 1 \mathrm{H}), 7.65(\mathrm{~d}, J=8.8 \mathrm{~Hz}, 1 \mathrm{H}), 7.34-7.13(\mathrm{~m}, 7 \mathrm{H})$ $6.04(\mathrm{~s}, 1 \mathrm{H}), 5.45$ (s, 2H). ${ }^{13} \mathrm{C}$ NMR $\left(100 \mathrm{MHz}, \mathrm{DMSO}-\mathrm{d}_{6}\right) \delta=162.6,160.4,138.4,137.0,133.7,128.7,127.0,126.4$, 125.4, 118.3, 117.5, 113.6, 98.7, 44.1. HRMS (ESI) m/z: [M+H] $]^{+}$calcd. for $\mathrm{C}_{16} \mathrm{H}_{13} \mathrm{BrNO}_{2} 330.0124$, found 330.0117.

\section{1-benzyl-4-hydroxy-6-nitroquinolin-2(1H)-one (a8)}

$56 \%$ yield, off-white solid, ${ }^{1}$ H NMR (400 MHz, DMSO-d 6$) \delta=8.73(\mathrm{~d}, J=2.8 \mathrm{~Hz}, 1 \mathrm{H}), 8.37(\mathrm{dd}, J=9.2,2.8 \mathrm{~Hz}, 1 \mathrm{H})$, $7.58(\mathrm{~d}, J=9.6 \mathrm{~Hz}, 1 \mathrm{H}), 7.40-7.22(\mathrm{~m}, 5 \mathrm{H}), 6.15(\mathrm{~s}, 1 \mathrm{H}), 5.57(\mathrm{~s}, 2 \mathrm{H}) .{ }^{13} \mathbf{C}$ NMR $\left(100 \mathrm{MHz}, \mathrm{DMSO}-\mathrm{d}_{6}\right) \delta=162.8,161.1$, 143.3, 141.2, 136.5, 128.7, 127.2, 126.4, 125.7, 119.4, 116.4, 116.3, 99.0, 44.5. HRMS (ESI) m/z: [M+H] $]^{+}$calcd. for $\mathrm{C}_{16} \mathrm{H}_{13} \mathrm{~N}_{2} \mathrm{O}_{4}$ 297.0870, found 297.0879.

\section{1-benzyl-4-hydroxy-6-methoxyquinolin-2(1H)-one (a13)}

$78 \%$ yield, off-white solid, ${ }^{1}$ H NMR (400 MHz, DMSO-d $\left.{ }_{6}\right) \delta=11.53(\mathrm{~s}, 1 \mathrm{H}), 7.35-7.14(\mathrm{~m}, 7 \mathrm{H}), 6.00(\mathrm{~s}, 1 \mathrm{H}), 5.43(\mathrm{~s}$, 2H), 3.78 (s, 3H). ${ }^{13}$ C NMR (100 MHz, DMSO-d 6 ) $\delta=162.4,160.9,153.9,137.5,133.7,128.6,126.9,126.4,119.6,117.1$, 116.6, 105.2, 98.2, 55.4, 44.0. HRMS (ESI) m/z: [M+H] ${ }^{+}$calcd. for $\mathrm{C}_{17} \mathrm{H}_{16} \mathrm{NO}_{3}$ 282.1125, found 282.1135.

\section{4-benzyl-1-hydroxybenzo[f]quinolin-3(4H)-one (a17)}

$81 \%$ yield, off-white solid, ${ }^{1} \mathbf{H}$ NMR (400 MHz, DMSO-d $\left.{ }_{6}\right) \delta=11.90(\mathrm{~s}, 1 \mathrm{H}), 9.49(\mathrm{~d}, J=8.8 \mathrm{~Hz}, 1 \mathrm{H}), 8.01(\mathrm{~d}, J=9.2$ $\mathrm{Hz}, 1 \mathrm{H}), 7.91(\mathrm{~d}, J=8.0 \mathrm{~Hz}, 1 \mathrm{H}), 7.68-7.48(\mathrm{~m}, 3 \mathrm{H}), 7.33-7.16(\mathrm{~m}, 5 \mathrm{H}), 6.22(\mathrm{~s}, 1 \mathrm{H}), 5.66(\mathrm{~s}, 2 \mathrm{H}) .{ }^{13} \mathbf{C ~ N M R}(100$ MHz, DMSO-d $\left.{ }_{6}\right) \delta=165.1,162.0,140.1,137.5,132.7,129.7,128.9,128.6,128.4,127.8,126.9,126.4,126.3,124.9,116.0$, 109.0, 99.2, 44.8. HRMS (ESI) m/z: $[\mathrm{M}+\mathrm{H}]^{+}$calcd. for $\mathrm{C}_{20} \mathrm{H}_{16} \mathrm{NO}_{2} 302.1176$, found 302.1181 .

\section{4-benzyl-1-hydroxy-4,7-phenanthrolin-3(4H)-one (a19)}

$82 \%$ yield, off-white solid, ${ }^{1} \mathbf{H}$ NMR (400 MHz, DMSO-d 6$) \delta=12.12(\mathrm{~s}, 1 \mathrm{H}), 9.83(\mathrm{~d}, J=9.2 \mathrm{~Hz}, 1 \mathrm{H}), 8.87(\mathrm{~d}, J=2.8$ $\mathrm{Hz}, 1 \mathrm{H}), 8.10(\mathrm{~d}, J=9.6 \mathrm{~Hz}, 1 \mathrm{H}), 7.85(\mathrm{~d}, J=9.6 \mathrm{~Hz}, 1 \mathrm{H}), 7.66(\mathrm{dd}, J=8.8,4.0 \mathrm{~Hz}, 1 \mathrm{H}), 7.36-7.15(\mathrm{~m}, 5 \mathrm{H}), 6.25(\mathrm{~s}$, 1H), $5.68(\mathrm{~s}, 2 \mathrm{H}) .{ }^{13} \mathrm{C}$ NMR (100 MHz, DMSO-d 6$) \delta=164.8,162.1,148.5,143.5,139.8,137.2,134.8,133.0,128.7$, 127.1, 126.3, 125.3, 122.5, 119.8, 108.7, 99.8, 44.8. HRMS (ESI) m/z: [M+H] ${ }^{+}$calcd. for $\mathrm{C}_{19} \mathrm{H}_{15} \mathrm{~N}_{2} \mathrm{O}_{2} 303.1128$, found 303.1130 . 


\title{
4. Preparation of pyranoquinolinones
}

Aldehydes 7a purchased from Alfa Aesar or Sigma-Aldrich were used as received. Aldehydes 7b-7d were synthesized from the corresponding ketones by a three-step sequence: Horner-Wadsworth-Emmons olefination/DIBAL-H reduction/ $\mathrm{MnO}_{2}$ oxidation according to the literature procedures. ${ }^{[5]}$

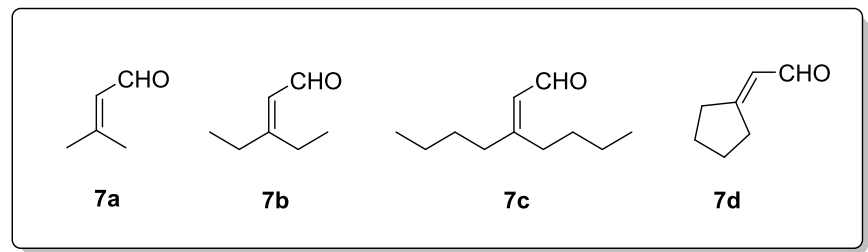

\section{General procedure for the preparation of pyranoquinolinones 6a-6x:}

The substituted pyranoquinolinones $\mathbf{6 a - 6 x}$ could be conveniently synthesized according to the similar literature procedures. ${ }^{[6]}$ Among them, $\mathbf{6} \mathbf{a}^{[6]}$ and $\mathbf{6} \mathbf{n}^{[7]}$ are the known compounds and the structure of $\mathbf{6 a}$ was further assigned by the single-crystal X-ray analysis (CCDC 1952168). The selected quinolinones a1-a21 (5.0 mmol, $1 \mathrm{eq}$ ) and selected aldehyde 7a-7d $(6.0 \mathrm{mmol}, 1.2 \mathrm{eq})$ were added to $10 \mathrm{ml}$ of pyridine and refluxed for $3 \mathrm{~h}$. The solvent removed in vacuo, and $10 \mathrm{~mL}$ of $\mathrm{H}_{2} \mathrm{O}$ was added and the solution was extracted with ethyl acetate $(3 \times 10 \mathrm{~mL})$, and the combined organic layers were collected and washed with $1 \mathrm{~N} \mathrm{HCl}$ solution. After drying over $\mathrm{Na}_{2} \mathrm{SO}_{4}$, the solvent was removed in vacuo to afford crude product. Then, the pyranoquinolinones 6a-6x were purified by flash chromatography (petroleum ether / ethyl acetate $=10: 1$ to 4:1).

Figure S2. Structure of pyranoquinolinones 6a-6x.<smiles></smiles>

$6 a$<smiles>CC1(C)C=Cc2c(c3cc(C(F)(F)F)ccc3[nH]c2=O)O1</smiles>

$\mathrm{Bn}$

$6 \mathrm{~g}$<smiles>COc1ccc2c(c1)c1c(c(=O)n2Cc2ccccc2)C=CC(C)(C)O1</smiles>

$6 m$
(CCDC 1952168)<smiles>CC1(C)C=Cc2c(c3cc([N+](=O)[O-])ccc3n(Cc3ccccc3)c2=O)O1</smiles>

$6 h$<smiles>CC1(C)C=Cc2c(c3ccccc3[nH]c2=O)O1</smiles>

$\mathrm{Ph}$

6c<smiles></smiles>

$\mathrm{Bn}$ 6d<smiles></smiles><smiles>CC1(C)C=Cc2c(c3cc(N)ccc3[nH]c2=O)O1</smiles>

$\mathrm{Bn}$<smiles>CC1(C)C=Cc2c(c3cc(C(C)(C)C)ccc3[nH]c2=O)O1</smiles>

$\mathrm{Bn}$<smiles></smiles>

$\mathrm{Bn}$
$6 \mathbf{k}$<smiles>[2H]n1c(=O)c2c(c3ccccc31)OC(CCCC)(CCCC)C=C2</smiles>

$6 u$
$6 p$<smiles>CC1(C)C=Cc2c(c3ccc4ccccc4c3n(N)c2=O)O1</smiles><smiles>O=c1c2c(c3ccccc3n1Cc1ccccc1)OC1(C=C2)CCCC1</smiles>

$6 v$

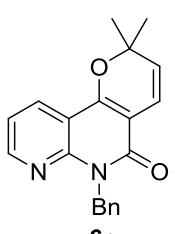<smiles>[B]n1c(=O)c2c(c3c4ccccc4ccc31)OC(C)(C)C=C2</smiles>

$6 q$<smiles></smiles><smiles>CC1(C)C=Cc2c(c3cccc4c3n(c2=O)CCC4)O1</smiles>

$6 x$

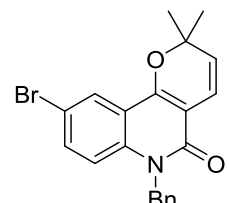<smiles>Cn1c(=O)c2c(c3cc(-c4ccccc4)ccc31)OC(C)(C)C=C2</smiles>

$\mathrm{Bn}$
$6 \mathrm{I}$<smiles>C[13CH2]n1c2c(c3c(ccc4ncccc43)c1=O)C=CC(C)(C)O2</smiles>

$6 s$<smiles>CCC1(CC)C=Cc2c(c3ccccc3n(Cc3ccccc3)c2=O)O1</smiles>

$6 t$

\section{.}


Characterization datas for the pyranoquinolinones:

\section{6-benzyl-2,2-dimethyl-2,6-dihydro-5H-pyrano[3,2-c]quinolin-5-one (6b)}

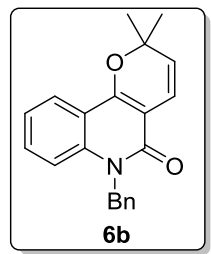

Following the general procedure, quinolinone $\mathbf{a} 2$ and $\alpha, \beta$-unsaturated aldehyde $7 \mathbf{a}$ was converted to product $\mathbf{6 b}, 79 \%$ yield, off-white solid. ${ }^{1} \mathbf{H}$ NMR $\left(400 \mathrm{MHz}, \mathrm{CDCl}_{3}\right) \delta=7.97(\mathrm{dd}, J=8.0,1.4 \mathrm{~Hz}, 1 \mathrm{H})$, $7.42-7.36(\mathrm{~m}, 1 \mathrm{H}), 7.31-7.25(\mathrm{~m}, 3 \mathrm{H}), 7.24-7.14(\mathrm{~m}, 4 \mathrm{H}), 6.81(\mathrm{~d}, J=10.0 \mathrm{~Hz}, 1 \mathrm{H}), 5.60-5.50(\mathrm{~m}$, $3 \mathrm{H}), 1.55(\mathrm{~s}, 6 \mathrm{H}) .{ }^{13} \mathbf{C} \mathbf{N M R}\left(100 \mathrm{MHz}, \mathrm{CDCl}_{3}\right) \delta=161.2,155.6,138.8,136.8,130.9,128.7,127.1,126.6$, 126.4, 123.2, 121.8, 118.0, 116.3, 114.9, 105.6, 79.0, 45.8, 28.4. HRMS (ESI) m/z: $[\mathrm{M}+\mathrm{Na}]^{+}$calcd. for

$\mathrm{C}_{21} \mathrm{H}_{19} \mathrm{NO}_{2} \mathrm{Na} 340.1308$, found 340.1303 .

\section{2,2-dimethyl-6-phenyl-2,6-dihydro-5H-pyrano[3,2-c]quinolin-5-one (6c)}

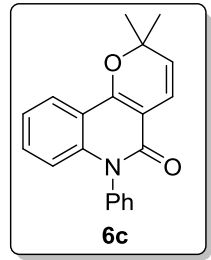

Following the general procedure, quinolinone $\mathbf{a} 3$ and $\alpha, \beta$-unsaturated aldehyde $\mathbf{7 a}$ was converted to product 6c, $85 \%$ yield, off-white solid. ${ }^{1} \mathbf{H}$ NMR $\left(400 \mathrm{MHz}, \mathrm{CDCl}_{3}\right) \delta=7.99(\mathrm{dd}, J=8.0,1.4 \mathrm{~Hz}, 1 \mathrm{H})$, $7.61-7.53(\mathrm{~m}, 2 \mathrm{H}), 7.53-7.46(\mathrm{~m}, 1 \mathrm{H}), 7.33-7.26(\mathrm{~m}, 3 \mathrm{H}), 7.22-7.15(\mathrm{~m}, 1 \mathrm{H}), 6.74(\mathrm{~d}, J=10.0 \mathrm{~Hz}$, $1 \mathrm{H}), 6.62(\mathrm{~d}, J=8.4 \mathrm{~Hz}, 1 \mathrm{H}), 5.56(\mathrm{~d}, J=10.0 \mathrm{~Hz}, 1 \mathrm{H}), 1.57(\mathrm{~s}, 6 \mathrm{H}) .{ }^{13} \mathbf{C ~ N M R}\left(100 \mathrm{MHz}, \mathrm{CDCl}_{3}\right) \delta=$ $161.2,155.9,140.4,137.9,130.4,130.1,129.2,128.7,126.3,122.8,122.0,117.7,115.8,115.8,105.9$, 79.1, 28.4. HRMS (ESI) m/z: [M+H] ${ }^{+}$calcd. for $\mathrm{C}_{20} \mathrm{H}_{18} \mathrm{NO}_{2} 304.1332$, found 304.1333.

\section{6-benzyl-9-fluoro-2,2-dimethyl-2,6-dihydro-5H-pyrano[3,2-c]quinolin-5-one (6d)}

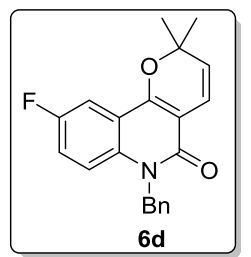

Following the general procedure, quinolinone a4 and $\alpha, \beta$-unsaturated aldehyde $7 \mathbf{a}$ was converted to product 6d, $71 \%$ yield, off-white solid. ${ }^{1} \mathbf{H} \mathbf{~ N M R}\left(400 \mathrm{MHz}, \mathrm{CDCl}_{3}\right) \delta=7.61$ (dd, $J=8.8,2.8 \mathrm{~Hz}$, $1 \mathrm{H}), 7.32-7.10(\mathrm{~m}, 7 \mathrm{H}), 6.79(\mathrm{~d}, J=10.0 \mathrm{~Hz}, 1 \mathrm{H}), 5.59$ (d, $J=10.0 \mathrm{~Hz}, 1 \mathrm{H}), 5.52(\mathrm{~s}, 2 \mathrm{H}), 1.55$ (s, $6 \mathrm{H}) .{ }^{19} \mathbf{F}$ NMR $\left(376 \mathrm{MHz}, \mathrm{CDCl}_{3}\right) \delta=-120.8 .{ }^{13} \mathbf{C ~ N M R}\left(100 \mathrm{MHz}, \mathrm{CDCl}_{3}\right) \delta=160.9,157.9\left(\mathrm{~d}, J_{\mathrm{C}, \mathrm{F}}\right.$ $=240.6 \mathrm{~Hz}), 154.7\left(\mathrm{~d}, J_{\mathrm{C}, \mathrm{F}}=3.1 \mathrm{~Hz}\right), 136.6,135.3,128.8,127.3,127.0,126.5,118.3\left(\mathrm{~d}, J_{\mathrm{C}, \mathrm{F}}=23.9\right.$ $\mathrm{Hz}), 117.8,117.4\left(\mathrm{~d}, J_{\mathrm{C}, \mathrm{F}}=8.3 \mathrm{~Hz}\right), 116.6\left(\mathrm{~d}, J_{\mathrm{C}, \mathrm{F}}=7.9 \mathrm{~Hz}\right), 108.5\left(\mathrm{~d}, J_{\mathrm{C}, \mathrm{F}}=23.9 \mathrm{~Hz}\right), 106.4,79.3$, 46.0, 28.4 HRMS (ESI) m/z: $[\mathrm{M}+\mathrm{H}]^{+}$calcd. for $\mathrm{C}_{21} \mathrm{H}_{19} \mathrm{FNO}_{2} 336.1394$, found 336.1404.

\section{6-benzyl-9-chloro-2,2-dimethyl-2,6-dihydro-5H-pyrano[3,2-c]quinolin-5-one (6e)}

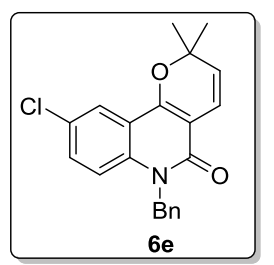

Following the general procedure, quinolinone $\mathbf{a 5}$ and $\alpha, \beta$-unsaturated aldehyde $7 \mathbf{a}$ was converted to product $\mathbf{6 e}, 70 \%$ yield, off-white solid. ${ }^{1} \mathbf{H} \mathbf{~ N M R}\left(400 \mathrm{MHz}, \mathrm{CDCl}_{3}\right) \delta=7.91(\mathrm{~s}, 1 \mathrm{H}), 7.34-7.10(\mathrm{~m}$, $7 \mathrm{H}), 6.78(\mathrm{~d}, J=10.0 \mathrm{~Hz}, 1 \mathrm{H}), 5.59(\mathrm{~d}, J=10.0 \mathrm{~Hz}, 1 \mathrm{H}), 5.50(\mathrm{~s}, 2 \mathrm{H}), 1.55(\mathrm{~s}, 6 \mathrm{H}) .{ }^{13} \mathbf{C} \mathbf{N M R}(100$ $\left.\mathrm{MHz} \mathrm{CDCl}_{3}\right) \delta=160.9,154.4,137.3,136.4,130.8,128.9,127.6,127.3,127.1,126.5,122.6,117.7$, 117.5, 116.4, 106.4, 79.4, 45.9, 28.4. HRMS (ESI) m/z: $[\mathrm{M}+\mathrm{H}]^{+}$calcd. for $\mathrm{C}_{21} \mathrm{H}_{19} \mathrm{ClNO}_{2} 352.1099$, found 352.1085 .

\section{6-benzyl-9-bromo-2,2-dimethyl-2,6-dihydro-5H-pyrano[3,2-c]quinolin-5-one (6f)}

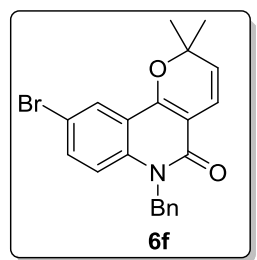

Following the general procedure, quinolinone $\mathbf{a 6}$ and $\alpha, \beta$-unsaturated aldehyde $7 \mathbf{a}$ was converted to product 6f, 76\% yield, off-white solid. ${ }^{1} \mathbf{H} \mathbf{~ N M R}\left(400 \mathrm{MHz}, \mathrm{CDCl}_{3}\right) \delta=8.05(\mathrm{~d}, J=2.0 \mathrm{~Hz}, 1 \mathrm{H}), 7.45$ $(\mathrm{dd}, J=9.2,2.4 \mathrm{~Hz}, 1 \mathrm{H}), 7.32-7.16(\mathrm{~m}, 5 \mathrm{H}), 7.07(\mathrm{~d}, J=8.8 \mathrm{~Hz}, 1 \mathrm{H}), 6.78(\mathrm{~d}, J=10.0 \mathrm{~Hz}, 1 \mathrm{H})$, $5.59(\mathrm{~d}, J=10.0 \mathrm{~Hz}, 1 \mathrm{H}), 5.50(\mathrm{~s}, 2 \mathrm{H}), 1.55(\mathrm{~s}, 6 \mathrm{H}) .{ }^{13} \mathbf{C ~ N M R}\left(100 \mathrm{MHz}, \mathrm{CDCl}_{3}\right) \delta=160.8,154.3$, 137.6, 136.4, 133.5, 128.8, 127.3, 127.1, 126.5, 125.6, 117.9, 117.7, 116.7, 115.0, 106.3, 79.4, 45.9, 28.4. HRMS (ESI) m/z: $[\mathrm{M}+\mathrm{H}]^{+}$calcd. for $\mathrm{C}_{21} \mathrm{H}_{19} \mathrm{BrNO}_{2} 396.0594$, found 396.0607. 


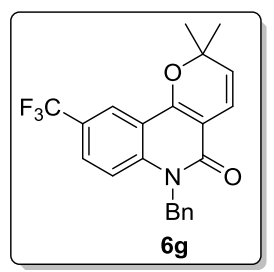

Following the general procedure, quinolinone $\mathbf{a} 7$ and $\alpha, \beta$-unsaturated aldehyde $7 \mathbf{a}$ was converted to product $\mathbf{6 g}, 72 \%$ yield, off-white solid. ${ }^{1} \mathbf{H}$ NMR $\left(400 \mathrm{MHz}, \mathrm{CDCl}_{3}\right) \delta=8.20(\mathrm{~s}, 1 \mathrm{H}), 7.60(\mathrm{~d}, J=$ $8.8 \mathrm{~Hz}, 1 \mathrm{H}), 7.35-7.17(\mathrm{~m}, 6 \mathrm{H}), 6.79(\mathrm{~d}, J=10.0 \mathrm{~Hz}, 1 \mathrm{H}), 5.62(\mathrm{~d}, J=10.0 \mathrm{~Hz}, 1 \mathrm{H}), 5.55(\mathrm{~s}, 2 \mathrm{H})$, $1.58(\mathrm{~s}, 6 \mathrm{H}) .{ }^{19} \mathbf{F}$ NMR $\left(376 \mathrm{MHz}, \mathrm{CDCl}_{3}\right) \delta=-61.9 .{ }^{13} \mathbf{C ~ N M R}\left(100 \mathrm{MHz}, \mathrm{CDCl}_{3}\right) \delta 161.1,155.0$ 140.7, 136.2, 128.9, 127.5, 127.3, $127.1\left(\mathrm{q}, J_{\mathrm{C}, \mathrm{F}}=6.3 \mathrm{~Hz}\right), 126.5,124.1\left(\mathrm{q}, J_{\mathrm{C}, \mathrm{F}}=147.1 \mathrm{~Hz}\right), 120.8$ $\left(\mathrm{q}, J_{\mathrm{C}, \mathrm{F}}=4.1 \mathrm{~Hz}\right), 117.5,116.1,115.4,106.5,79.7,46.0,28.4$. HRMS (ESI) $\mathrm{m} / \mathrm{z}:[\mathrm{M}+\mathrm{H}]^{+}$calcd. for $\mathrm{C}_{22} \mathrm{H}_{19} \mathrm{~F}_{3} \mathrm{NO}_{2} 386.1362$, found 386.1373 .

\section{6-benzyl-2,2-dimethyl-9-nitro-2,6-dihydro-5H-pyrano[3,2-c]quinolin-5-one (6h)}

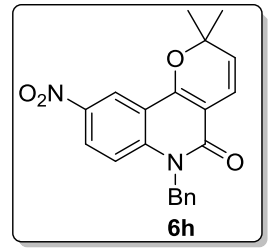

Following the general procedure, quinolinone $\mathbf{a 8}$ and $\alpha, \beta$-unsaturated aldehyde $7 \mathbf{a}$ was converted to product $\mathbf{6 h}, 70 \%$ yield, off-white solid. ${ }^{1} \mathbf{H}$ NMR $\left(400 \mathrm{MHz}, \mathrm{CDCl}_{3}\right) \delta=8.82(\mathrm{~d}, J=2.4 \mathrm{~Hz}, 1 \mathrm{H})$, $8.21(\mathrm{dd}, J=9.2,2.4 \mathrm{~Hz}, 1 \mathrm{H}), 7.34-7.27(\mathrm{~m}, 3 \mathrm{H}), 7.20(\mathrm{~d}, J=7.2 \mathrm{~Hz}, 3 \mathrm{H}), 6.77(\mathrm{~d}, J=10.0 \mathrm{~Hz}$, $1 \mathrm{H}), 5.66(\mathrm{~d}, J=10.0 \mathrm{~Hz}, 1 \mathrm{H}), 5.56(\mathrm{~s}, 2 \mathrm{H}), 1.60(\mathrm{~s}, 6 \mathrm{H}) .{ }^{13} \mathrm{C} \mathrm{NMR}\left(100 \mathrm{MHz}, \mathrm{CDCl}_{3}\right) \delta=161.0$, 154.8, 142.5, 142.1, 135.7, 129.0, 127.9, 127.7, 126.5, 125.3, 119.6, 117.1, 116.2, 115.6, 106.8, 80.2, 46.3, 28.5. HRMS (ESI) m/z: [M+Na] $]^{+}$calcd. for $\mathrm{C}_{21} \mathrm{H}_{18} \mathrm{~N}_{2} \mathrm{O}_{4} \mathrm{Na} 385.1159$, found 385.1150.

\section{6-benzyl-2,2,9-trimethyl-2,6-dihydro-5H-pyrano[3,2-c]quinolin-5-one (6i)}

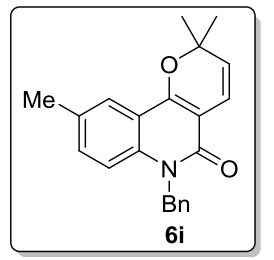

Following the general procedure, quinolinone $\mathbf{a 9}$ and $\alpha, \beta$-unsaturated aldehyde $7 \mathbf{a}$ was converted to product $\mathbf{6 i}, 75 \%$ yield, off-white solid. ${ }^{1} \mathbf{H}$ NMR $\left(400 \mathrm{MHz}, \mathrm{CDCl}_{3}\right) \delta=7.75(\mathrm{~d}, J=0.8 \mathrm{~Hz}, 1 \mathrm{H}), 7.31$ $-7.25(\mathrm{~m}, 2 \mathrm{H}), 7.24-7.15(\mathrm{~m}, 4 \mathrm{H}), 7.10(\mathrm{~d}, J=8.8 \mathrm{~Hz}, 1 \mathrm{H}), 6.81(\mathrm{~d}, J=10.0 \mathrm{~Hz}, 1 \mathrm{H}), 5.56(\mathrm{~d}, J=$ $10.0 \mathrm{~Hz}, 1 \mathrm{H}), 5.52(\mathrm{~s}, 2 \mathrm{H}), 2.38(\mathrm{~s}, 3 \mathrm{H}), 1.55(\mathrm{~s}, 6 \mathrm{H}) .{ }^{13} \mathbf{C} \mathbf{~ N M R}\left(100 \mathrm{MHz}, \mathrm{CDCl}_{3}\right) \delta=161.1,155.4$, 137.0, 136.9, 132.2, 131.4, 128.7, 127.1, 126.6, 126.2, 122.8, 118.1, 116.2, 114.9, 105.6, 78.9, 45.8, 28.3, 20.7. HRMS (ESI) m/z: $[\mathrm{M}+\mathrm{H}]^{+}$calcd. for $\mathrm{C}_{22} \mathrm{H}_{22} \mathrm{NO}_{2} 332.1645$, found 332.1645 .

\section{6-benzyl-9-isopropyl-2,2-dimethyl-2,6-dihydro-5H-pyrano[3,2-c]quinolin-5-one (6j)}

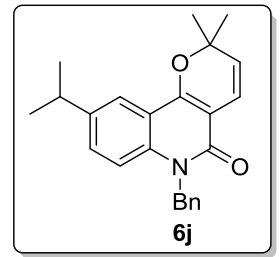

found 360.1957 .

Following the general procedure, quinolinone $\mathbf{a 1 0}$ and $\alpha, \beta$-unsaturated aldehyde 7a was converted to product $\mathbf{6 j}, 75 \%$ yield, off-white solid. ${ }^{1} \mathbf{H}$ NMR $\left(400 \mathrm{MHz}, \mathrm{CDCl}_{3}\right) \delta=7.77(\mathrm{~d}, J=2.0 \mathrm{~Hz}, 1 \mathrm{H})$, $7.31-7.20(\mathrm{~m}, 6 \mathrm{H}), 7.15(\mathrm{~d}, J=8.8 \mathrm{~Hz}, 1 \mathrm{H}), 6.81(\mathrm{~d}, J=10.0 \mathrm{~Hz}, 1 \mathrm{H}), 5.56(\mathrm{~d}, J=10.0 \mathrm{~Hz}, 1 \mathrm{H})$, $5.52(\mathrm{~s}, 2 \mathrm{H}), 3.00-2.91(\mathrm{~m}, 1 \mathrm{H}), 1.56(\mathrm{~s}, 6 \mathrm{H}), 1.27(\mathrm{~s}, 3 \mathrm{H}), 1.25(\mathrm{~s}, 3 \mathrm{H}) .{ }^{13} \mathrm{C} \mathrm{NMR}(100 \mathrm{MHz}$, $\left.\mathrm{CDCl}_{3}\right) \delta=161.2,155.7,142.5,137.2,137.0,129.5,128.7,127.1,126.6,126.2,120.3,118.1,116.2$, 115.0, 105.5, 78.9, 45.8, 33.6, 28.4, 24.0. HRMS (ESI) m/z: $[\mathrm{M}+\mathrm{H}]^{+}$calcd. for $\mathrm{C}_{24} \mathrm{H}_{26} \mathrm{NO}_{2} 360.1958$,

\section{6-benzyl-9-(tert-butyl)-2,2-dimethyl-2,6-dihydro-5H-pyrano[3,2-c]quinolin-5-one (6k)}

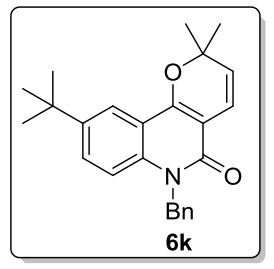

374.2117 .

Following the general procedure, quinolinone a11 and $\alpha, \beta$-unsaturated aldehyde 7a was converted to product 6k, 78\% yield, off-white solid. ${ }^{1} \mathbf{H} \mathbf{~ N M R}\left(400 \mathrm{MHz}, \mathrm{CDCl}_{3}\right) \delta=7.91(\mathrm{~d}, J=2.4 \mathrm{~Hz}, 1 \mathrm{H})$, $7.45(\mathrm{dd}, J=8.8,2.4 \mathrm{~Hz}, 1 \mathrm{H}), 7.31-7.20(\mathrm{~m}, 6 \mathrm{H}), 7.16(\mathrm{~d}, J=8.8 \mathrm{~Hz}, 1 \mathrm{H}), 6.81(\mathrm{~d}, J=10.0 \mathrm{~Hz}$, $1 \mathrm{H}), 5.56(\mathrm{~d}, J=10.0 \mathrm{~Hz}, 1 \mathrm{H}), 5.52(\mathrm{~s}, 2 \mathrm{H}), 1.56(\mathrm{~s}, 6 \mathrm{H}), 1.34(\mathrm{~s}, 9 \mathrm{H}) .{ }^{13} \mathbf{C ~ N M R}\left(100 \mathrm{MHz}, \mathrm{CDCl}_{3}\right)$ $\delta=161.2,155.8,144.8,137.1,136.9,128.8,128.7,127.1,126.7,126.2,118.9,118.1,115.9,114.8$, 105.6, 78.9, 45.8, 34.4, 31.3, 28.4. HRMS (ESI) m/z: $[\mathrm{M}+\mathrm{H}]^{+}$calcd. for $\mathrm{C}_{25} \mathrm{H}_{28} \mathrm{NO}_{2} 374.2115$, found 


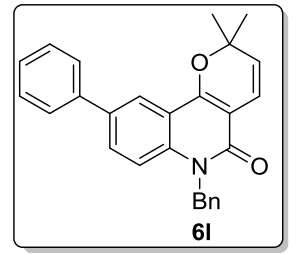

Following the general procedure, quinolinone $\mathbf{a 1 2}$ and $\alpha, \beta$-unsaturated aldehyde 7a was converted to product 61, $81 \%$ yield, off-white solid. ${ }^{1} \mathbf{H} \mathbf{N M R}\left(400 \mathrm{MHz}, \mathrm{CDCl}_{3}\right) \delta=8.16(\mathrm{~d}, J=2.0 \mathrm{~Hz}, 1 \mathrm{H})$, $7.66-7.57(\mathrm{~m}, 3 \mathrm{H}), 7.47-7.42(\mathrm{~m}, 2 \mathrm{H}), 7.38-7.22(\mathrm{~m}, 7 \mathrm{H}), 6.82(\mathrm{~d}, J=10.0 \mathrm{~Hz}, 1 \mathrm{H}), 5.59(\mathrm{~d}, J$ $=10.0 \mathrm{~Hz}, 3 \mathrm{H}), 1.57(\mathrm{~s}, 6 \mathrm{H}) .{ }^{13} \mathbf{C}$ NMR $\left(100 \mathrm{MHz}, \mathrm{CDCl}_{3}\right) \delta=161.1,155.6,140.1,138.1,136.8$, 135.0, 129.9, 128.9, 128.8, 127.4, 127.2, 127.0, 126.6, 126.6, 121.3, 118.0, 116.7, 115.5, 105.9, 79.2, 45.9, 28.4. HRMS (ESI) m/z: $[\mathrm{M}+\mathrm{H}]^{+}$calcd. for $\mathrm{C}_{27} \mathrm{H}_{24} \mathrm{NO}_{2} 394.1802$, found 394.1803.

6-benzyl-9-methoxy-2,2-dimethyl-2,6-dihydro-5H-pyrano[3,2-c]quinolin-5-one (6m)

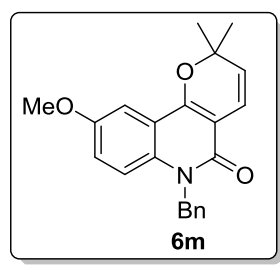

Following the general procedure, quinolinone a13 and $\alpha, \beta$-unsaturated aldehyde 7a was converted to product $6 \mathbf{m}, 77 \%$ yield, off-white solid. ${ }^{1} \mathbf{H} \mathbf{~ N M R}\left(400 \mathrm{MHz}, \mathrm{CDCl}_{3}\right) \delta=7.38(\mathrm{~d}, J=3.2 \mathrm{~Hz}, 1 \mathrm{H})$, $7.32-7.25(\mathrm{~m}, 2 \mathrm{H}), 7.21(\mathrm{t}, J=5.2 \mathrm{~Hz}, 3 \mathrm{H}), 7.13(\mathrm{~d}, J=9.2 \mathrm{~Hz}, 1 \mathrm{H}), 7.01(\mathrm{dd}, J=9.2,3.2 \mathrm{~Hz}, 1 \mathrm{H})$, $6.81(\mathrm{~d}, J=10.0 \mathrm{~Hz}, 1 \mathrm{H}), 5.57(\mathrm{~d}, J=10.0 \mathrm{~Hz}, 1 \mathrm{H}), 5.52(\mathrm{~s}, 2 \mathrm{H}), 3.85(\mathrm{~s}, 3 \mathrm{H}), 1.55(\mathrm{~s}, 6 \mathrm{H}) .{ }^{13} \mathrm{C}$ NMR $\left(100 \mathrm{MHz}, \mathrm{CDCl}_{3}\right) \delta=160.8,155.0,154.7,137.0,133.5,128.7,127.1,126.6,126.5,119.6$, 118.1, 117.0, 116.4, 106.0, 104.9, 79.0, 55.7, 45.9, 28.4. HRMS (ESI) m/z: $[\mathrm{M}+\mathrm{H}]^{+}$calcd. for $\mathrm{C}_{22} \mathrm{H}_{22} \mathrm{NO}_{3} 348.1594$, found 348.1591.

\section{6-benzyl-8,9-dimethoxy-2,2-dimethyl-2,6-dihydro-5H-pyrano[3,2-c]quinolin-5-one (6o)}

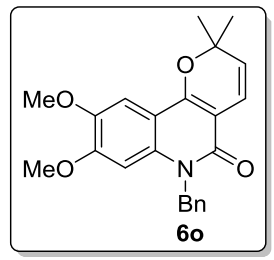

Following the general procedure, quinolinone a15 and $\alpha, \beta$-unsaturated aldehyde 7a was converted to product 6o, $78 \%$ yield, off-white solid. ${ }^{1} \mathbf{H}$ NMR $\left(400 \mathrm{MHz}, \mathrm{CDCl}_{3}\right) \delta=7.31-7.21(\mathrm{~m}, 6 \mathrm{H}), 6.81$ $(\mathrm{d}, J=10.0 \mathrm{~Hz}, 1 \mathrm{H}), 6.67(\mathrm{~s}, 1 \mathrm{H}), 5.54-5.50(\mathrm{~m}, 3 \mathrm{H}), 3.93(\mathrm{~s}, 3 \mathrm{H}), 3.74(\mathrm{~s}, 3 \mathrm{H}), 1.55(\mathrm{~s}, 6 \mathrm{H}) .{ }^{13} \mathrm{C}$ NMR $\left(100 \mathrm{MHz}, \mathrm{CDCl}_{3}\right) \delta=161.1,155.5,152.2,145.1,137.1,134.7,128.8,127.3,126.7,125.3$, 118.3, 109.1, 104.0, 103.8, 98.5, 79.0, 56.2, 55.9, 46.2, 28.4. HRMS (ESI) m/z: $[\mathrm{M}+\mathrm{H}]^{+}$calcd. for $\mathrm{C}_{23} \mathrm{H}_{24} \mathrm{NO}_{4} 378.1700$, found 378.1700.

\section{3,3,11-trimethyl-3,11-dihydro-12H-benzo[h]pyrano[3,2-c]quinolin-12-one (6p)}

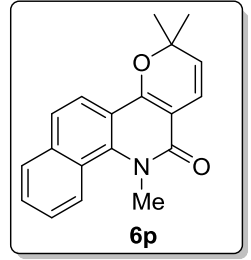

Following the general procedure, quinolinone a16 and $\alpha, \beta$-unsaturated aldehyde 7a was converted to product 6p, 77\% yield, off-white solid. ${ }^{1} \mathbf{H}$ NMR $\left(400 \mathrm{MHz}, \mathrm{CDCl}_{3}\right) \delta=8.42$ (d, $\left.J=8.4 \mathrm{~Hz}, 1 \mathrm{H}\right), 7.94$ $(\mathrm{d}, J=8.8 \mathrm{~Hz}, 1 \mathrm{H}), 7.87(\mathrm{~d}, J=7.6 \mathrm{~Hz}, 1 \mathrm{H}), 7.62-7.47(\mathrm{~m}, 3 \mathrm{H}), 6.79(\mathrm{~d}, J=10.0 \mathrm{~Hz}, 1 \mathrm{H}), 5.57(\mathrm{~d}$, $J=10.0 \mathrm{~Hz}, 1 \mathrm{H}), 4.04(\mathrm{~s}, 3 \mathrm{H}), 1.55(\mathrm{~s}, 6 \mathrm{H}) .{ }^{13} \mathbf{C ~ N M R}\left(100 \mathrm{MHz}, \mathrm{CDCl}_{3}\right) \delta=163.3,156.0,139.2$, 135.9, 128.8, 127.1, 126.5, 125.8, 125.0, 123.8, 123.4, 119.3, 117.8, 113.8, 105.7, 79.0, 40.2, 28.3. HRMS (ESI) m/z: $[\mathrm{M}+\mathrm{H}]^{+}$calcd. for $\mathrm{C}_{19} \mathrm{H}_{18} \mathrm{NO}_{2} 292.1332$, found 292.1329.

\section{6-benzyl-2,2-dimethyl-2,6-dihydro-5H-benzo[f]pyrano[3,2-c]quinolin-5-one (6q)}

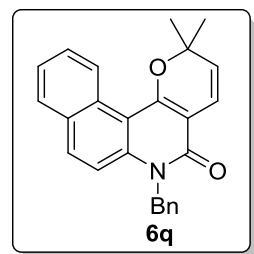

found 368.1649 .

Following the general procedure, quinolinone $\mathbf{a 1 7}$ and $\alpha, \beta$-unsaturated aldehyde 7a was converted to product 6q, 72\% yield, off-white solid. ${ }^{\mathbf{1}} \mathbf{H} \mathbf{N M R}\left(400 \mathrm{MHz}, \mathrm{CDCl}_{3}\right) \delta=9.51(\mathrm{~d}, J=8.8 \mathrm{~Hz}, 1 \mathrm{H}), 7.81$ $-7.76(\mathrm{~m}, 2 \mathrm{H}), 7.64-7.57(\mathrm{~m}, 1 \mathrm{H}), 7.51-7.41(\mathrm{~m}, 2 \mathrm{H}), 7.33-7.19(\mathrm{~m}, 5 \mathrm{H}), 6.93(\mathrm{~d}, J=10.0 \mathrm{~Hz}$, 1H), $5.72(\mathrm{~s}, 2 \mathrm{H}), 5.64(\mathrm{~d}, J=10.0 \mathrm{~Hz}, 1 \mathrm{H}), 1.69(\mathrm{~s}, 6 \mathrm{H}) .{ }^{13} \mathbf{C} \mathbf{N M R}\left(100 \mathrm{MHz}, \mathrm{CDCl}_{3}\right) \delta=160.6$, 159.2, 139.5, 136.8, 132.7, 130.0, 129.7, 128.9, 128.6, 127.8, 127.2, 126.8, 126.4, 126.0, 125.2, 118.8, 115.6, 109.9, 107.0, 79.4, 46.8, 28.3. HRMS (ESI) m/z: $[\mathrm{M}+\mathrm{H}]^{+}$calcd. for $\mathrm{C}_{25} \mathrm{H}_{22} \mathrm{NO}_{2} 368.1645$, 


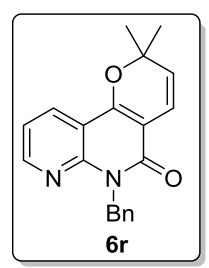

Following the general procedure, quinolinone $\mathbf{a 1 8}$ and $\alpha, \beta$-unsaturated aldehyde 7a was converted to product 6r, 75\% yield, off-white solid. ${ }^{1} \mathbf{H}$ NMR $\left(400 \mathrm{MHz}, \mathrm{CDCl}_{3}\right) \delta=8.56(\mathrm{dd}, J=4.8,2.0 \mathrm{~Hz}, 1 \mathrm{H})$, $8.19(\mathrm{dd}, J=8.0,2.0 \mathrm{~Hz}, 1 \mathrm{H}), 7.52-7.43(\mathrm{~m}, 2 \mathrm{H}), 7.28-7.12(\mathrm{~m}, 4 \mathrm{H}), 6.75(\mathrm{~d}, J=10.0 \mathrm{~Hz}, 1 \mathrm{H}), 5.72$ $(\mathrm{s}, 2 \mathrm{H}), 5.55(\mathrm{~d}, J=10.0 \mathrm{~Hz}, 1 \mathrm{H}), 1.52(\mathrm{~s}, 6 \mathrm{H}) .{ }^{13} \mathbf{C ~ N M R}\left(100 \mathrm{MHz}, \mathrm{CDCl}_{3}\right) \delta=161.4,154.2,150.1$, 149.1, 138.1, 131.3, 128.6, 128.2, 127.0, 126.9, 117.8, 117.6, 111.7, 106.6, 100.0, 79.5, 44.0, 28.4. HRMS (ESI) $\mathrm{m} / \mathrm{z}:[\mathrm{M}+\mathrm{H}]^{+}$calcd. for $\mathrm{C}_{20} \mathrm{H}_{19} \mathrm{~N}_{2} \mathrm{O}_{2} 319.1441$, found 319.1451 .

\section{6-benzyl-2,2-dimethyl-2,6-dihydro-5H-pyrano[2,3-a][4,7]phenanthrolin-5-one (6s)}

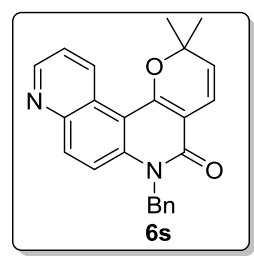

Following the general procedure, quinolinone $\mathbf{a 1 9}$ and $\alpha, \beta$-unsaturated aldehyde $7 \mathbf{a}$ was converted to product $6 \mathbf{6 s}, 71 \%$ yield, off-white solid. ${ }^{1} \mathbf{H}$ NMR $\left(400 \mathrm{MHz}, \mathrm{CDCl}_{3}\right) \delta=9.79(\mathrm{~d}, J=8.8 \mathrm{~Hz}, 1 \mathrm{H}), 8.89$ $-8.82(\mathrm{~m}, 1 \mathrm{H}), 8.07(\mathrm{~d}, J=9.6 \mathrm{~Hz}, 1 \mathrm{H}), 7.69(\mathrm{~d}, J=9.6 \mathrm{~Hz}, 1 \mathrm{H}), 7.52-7.47(\mathrm{~m}, 1 \mathrm{H}), 7.34-7.21(\mathrm{~m}$, $5 \mathrm{H}), 6.92(\mathrm{~d}, J=10.0 \mathrm{~Hz}, 1 \mathrm{H}), 5.73(\mathrm{~s}, 2 \mathrm{H}), 5.67(\mathrm{~d}, J=10.0 \mathrm{~Hz}, 1 \mathrm{H}), 1.69(\mathrm{~s}, 6 \mathrm{H}) .{ }^{13} \mathbf{C ~ N M R}(100$ $\left.\mathrm{MHz}_{2} \mathrm{CDCl}_{3}\right) \delta=160.5,158.7,148.8,144.8,139.0,136.4,134.8,133.6,128.9,127.4,126.4,126.4$, 125.6, 122.1, 119.1, 118.6, 109.4, 107.4, 79.7, 46.8, 28.4. HRMS (ESI) m/z: $[\mathrm{M}+\mathrm{H}]^{+}$calcd. for $\mathrm{C}_{24} \mathrm{H}_{21} \mathrm{~N}_{2} \mathrm{O}_{2} 369.1598$, found 369.1595 .

\section{6-benzyl-2,2-diethyl-2,6-dihydro-5H-pyrano[3,2-c]quinolin-5-one (6t)}

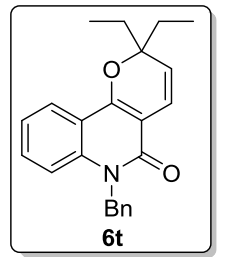

Following the general procedure, quinolinone $\mathbf{a} 2$ and $\alpha, \beta$-unsaturated aldehyde $\mathbf{7 b}$ was converted to product 6u, 71\% yield, off-white solid. ${ }^{1} \mathbf{H}$ NMR $\left(500 \mathrm{MHz}, \mathrm{CDCl}_{3}\right) \delta=7.97(\mathrm{~d}, J=8.0 \mathrm{~Hz}, 1 \mathrm{H}), 7.38$ $(\mathrm{t}, J=7.5 \mathrm{~Hz}, 1 \mathrm{H}), 7.30-7.26(\mathrm{~m}, 2 \mathrm{H}), 7.24-7.15(\mathrm{~m}, 5 \mathrm{H}), 6.91(\mathrm{~d}, J=10.0 \mathrm{~Hz}, 1 \mathrm{H}), 5.52(\mathrm{~s}, 2 \mathrm{H})$, $5.42(\mathrm{~d}, J=10.0 \mathrm{~Hz}, 1 \mathrm{H}), 1.91-1.82(\mathrm{~m}, 2 \mathrm{H}), 1.77-1.38(\mathrm{~m}, 2 \mathrm{H}), 0.99(\mathrm{t}, J=7.0 \mathrm{~Hz}, 6 \mathrm{H}) .{ }^{13} \mathbf{C} \mathbf{N M R}$ $\left(100 \mathrm{MHz} \mathrm{CDCl}_{3}\right) \delta=161.2,156.4,138.9,136.9,130.8,128.7,127.1,126.6,123.7,123.0,121.8,119.4$, 116.1, 114.9, 105.0, 85.1, 45.8, 32.9, 7.8. HRMS (ESI) m/z: $[\mathrm{M}+\mathrm{H}]^{+}$calcd. for $\mathrm{C}_{23} \mathrm{H}_{24} \mathrm{NO}_{2} 346.1802$,

found 346.1788

\section{6-benzyl-2,2-dibutyl-2,6-dihydro-5H-pyrano[3,2-c]quinolin-5-one (6u)}

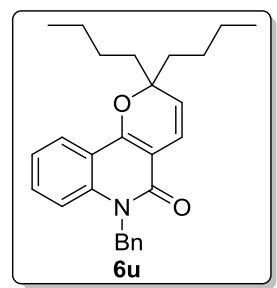

Following the general procedure, quinolinone $\mathbf{a} 2$ and $\alpha, \beta$-unsaturated aldehyde $7 \mathbf{c}$ was converted to product 6v, 72\% yield, off-white solid. ${ }^{1} \mathbf{H}$ NMR $\left(400 \mathrm{MHz}, \mathrm{CDCl}_{3}\right) \delta=7.96(\mathrm{dd}, J=8.0,1.2 \mathrm{~Hz}$, 1H), $7.41-7.35(\mathrm{~m}, 1 \mathrm{H}), 7.32-7.14(\mathrm{~m}, 7 \mathrm{H}), 6.86(\mathrm{~d}, J=10.0 \mathrm{~Hz}, 1 \mathrm{H}), 5.52(\mathrm{~s}, 2 \mathrm{H}), 5.44(\mathrm{~d}, J=$ $10.0 \mathrm{~Hz}, 1 \mathrm{H}), 1.89-1.79(\mathrm{~m}, 2 \mathrm{H}), 1.73-1.65(\mathrm{~m}, 2 \mathrm{H}), 1.48-1.38(\mathrm{~m}, 4 \mathrm{H}), 1.37-1.26(\mathrm{~m}, 4 \mathrm{H})$, $0.88(\mathrm{t}, J=7.2 \mathrm{~Hz}, 6 \mathrm{H}) .{ }^{13} \mathbf{C} \mathbf{N M R}\left(100 \mathrm{MHz}, \mathrm{CDCl}_{3}\right) \delta=161.3,156.2,138.9,136.9,130.8,128.7$, $127.1,126.6,124.5,123.1,121.8,118.8,116.2,114.9,105.0,84.6,45.8,40.5,25.6,23.0,14.0$.

HRMS (ESI) m/z: $[\mathrm{M}+\mathrm{H}]^{+}$calcd. for $\mathrm{C}_{27} \mathrm{H}_{32} \mathrm{NO}_{2} 402.2428$, found 402.2436 .

\section{6'-benzylspiro[cyclopentane-1,2'-pyrano[3,2-c]quinolin]-5'(6'H)-one (6v)}

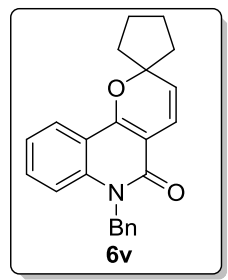

Following the general procedure, quinolinone $\mathbf{a} 2$ and $\alpha, \beta$-unsaturated aldehyde $7 \mathbf{d}$ was converted to product 6w, 72\% yield, off-white solid. ${ }^{1} \mathbf{H} \mathbf{~ N M R}\left(400 \mathrm{MHz}, \mathrm{CDCl}_{3}\right) \delta=7.90(\mathrm{dd}, J=8.0,1.6 \mathrm{~Hz}, 1 \mathrm{H})$, $7.41-7.35(\mathrm{~m}, 1 \mathrm{H}), 7.32-7.12(\mathrm{~m}, 7 \mathrm{H}), 6.83(\mathrm{~d}, J=10.0 \mathrm{~Hz}, 1 \mathrm{H}), 5.60(\mathrm{~d}, J=10.0 \mathrm{~Hz}, 1 \mathrm{H}), 5.54(\mathrm{~s}$, 2H), $2.35-2.22(\mathrm{~m}, 2 \mathrm{H}), 2.07-1.98(\mathrm{~m}, 2 \mathrm{H}), 1.87-1.65(\mathrm{~m}, 5 \mathrm{H}) .{ }^{13} \mathbf{C} \mathbf{N M R}\left(100 \mathrm{MHz}, \mathrm{CDCl}_{3}\right) \delta=$ $161.1,155.4,138.8,136.8,130.8,128.7,127.1,126.5,125.1,123.0,121.8,118.8,116.4,114.9,106.2$, 89.8, 45.7, 40.1, 23.3. HRMS (ESI) m/z: $[\mathrm{M}+\mathrm{H}]^{+}$calcd. for $\mathrm{C}_{23} \mathrm{H}_{22} \mathrm{NO}_{2} 344.1645$, found 344.1633. 
10,10-dimethyl-4,5-dihydro-7H,10H-pyrano[3,2-c]pyrrolo[3,2,1-ij]quinolin-7-one (6w)

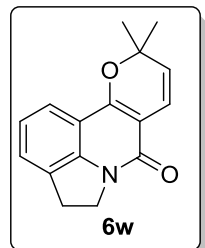

Following the general procedure, quinolinone $\mathbf{a 2 0}$ and $\alpha, \beta$-unsaturated aldehyde 7a was converted to product 6y, 78\% yield, off-white solid. ${ }^{1} \mathbf{H}$ NMR $\left(400 \mathrm{MHz}, \mathrm{CDCl}_{3}\right) \delta=7.57(\mathrm{dd}, J=8.0,0.8 \mathrm{~Hz}, 1 \mathrm{H})$, $7.30(\mathrm{dd}, J=7.2,0.8 \mathrm{~Hz}, 1 \mathrm{H}), 7.11(\mathrm{dd}, J=8.0,7.6 \mathrm{~Hz}, 1 \mathrm{H}), 6.76(\mathrm{~d}, J=10.0 \mathrm{~Hz}, 1 \mathrm{H}), 5.50(\mathrm{~d}, J=10.0$ $\mathrm{Hz}, 1 \mathrm{H}), 4.45-4.33(\mathrm{~m}, 2 \mathrm{H}), 3.39(\mathrm{t}, J=8.0 \mathrm{~Hz}, 2 \mathrm{H}), 1.51(\mathrm{~s}, 6 \mathrm{H}) .{ }^{13} \mathbf{C ~ N M R}\left(100 \mathrm{MHz}, \mathrm{CDCl}_{3}\right) \delta=$ 159.6, 156.0, 141.9, 130.4, 125.8, 125.2, 122.7, 119.3, 118.0, 113.2, 107.5, 78.7, 46.6, 28.2, 27.3. HRMS (ESI) $\mathrm{m} / \mathrm{z}:[\mathrm{M}+\mathrm{H}]^{+}$calcd. for $\mathrm{C}_{16} \mathrm{H}_{16} \mathrm{NO}_{2} 254.1176$, found 254.1167 .

\section{1,11-dimethyl-5,6-dihydro-4H,8H,11H-pyrano[3,2-c]pyrido[3,2,1-ij]quinolin-8-one (6x)}

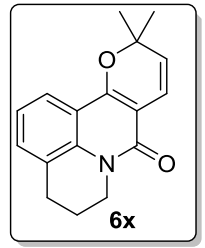

Following the general procedure, quinolinone $\mathbf{a} 21$ and $\alpha, \beta$-unsaturated aldehyde 7a was converted to product 6z, 74\% yield, off-white solid. ${ }^{1} \mathbf{H}$ NMR $\left(400 \mathrm{MHz}, \mathrm{CDCl}_{3}\right) \delta=7.79(\mathrm{~d}, J=8.0 \mathrm{~Hz}, 1 \mathrm{H}), 7.27(\mathrm{~d}$, $J=6.4 \mathrm{~Hz}, 1 \mathrm{H}), 7.10(\mathrm{t}, J=7.6 \mathrm{~Hz}, 1 \mathrm{H}), 6.76(\mathrm{~d}, J=10.0 \mathrm{~Hz}, 1 \mathrm{H}), 5.53(\mathrm{~d}, J=10.0 \mathrm{~Hz}, 1 \mathrm{H}), 4.23-4.13$ $(\mathrm{m}, 2 \mathrm{H}), 2.95(\mathrm{t}, J=6.2 \mathrm{~Hz}, 2 \mathrm{H}), 2.12-2.04(\mathrm{~m}, 2 \mathrm{H}), 1.51(\mathrm{~s}, 6 \mathrm{H}) .{ }^{13} \mathbf{C} \mathbf{N M R}\left(100 \mathrm{MHz}, \mathrm{CDCl}_{3}\right) \delta=$ 160.6, 155.3, 136.1, 130.1, 126.4, 124.7, 121.2, 121.0, 117.9, 115.8, 105.6, 78.6, 42.1, 28.2, 28.0, 20.9.

HRMS (ESI) m/z: [M+H] ${ }^{+}$calcd. for $\mathrm{C}_{17} \mathrm{H}_{18} \mathrm{NO}_{2} 268.1332$, found 268.1328.

\section{Optimization of the Reaction Conditions}

Table S1. Optimization of the asymmetric [4+2] cycloaddition reaction.

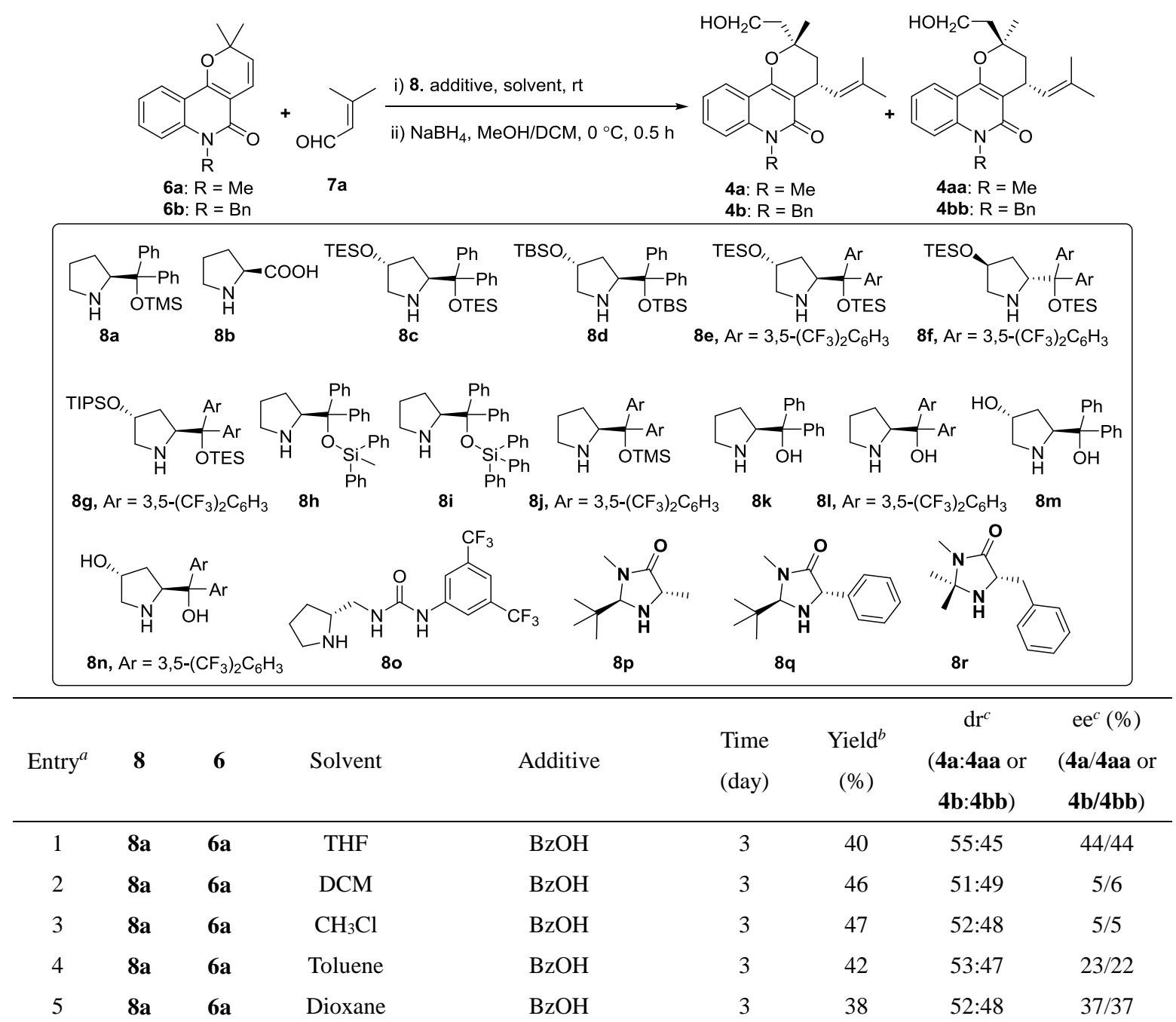




\begin{tabular}{|c|c|c|c|c|c|c|c|c|}
\hline 6 & $8 \mathbf{a}$ & $6 a$ & $\mathrm{MeCN}$ & $\mathrm{BzOH}$ & 3 & 48 & $56: 44$ & $32 / 32$ \\
\hline 7 & $\mathbf{8 a}$ & $\mathbf{6 a}$ & $\mathrm{THF}^{d}$ & $\mathrm{BzOH}$ & 3 & 38 & $52: 48$ & $44 / 43$ \\
\hline $8^{e}$ & $8 \mathbf{a}$ & $6 \mathbf{a}$ & THF & $\mathrm{BzOH}$ & 3 & Trace & - & - \\
\hline 9 & $\mathbf{8 a}$ & $\mathbf{6 a}$ & THF & $o$-nitrobenzoic acid & 3 & 31 & $54: 46$ & $45 / 44$ \\
\hline 10 & $8 \mathbf{a}$ & $\mathbf{6 a}$ & THF & Salicylic acid & 3 & 32 & $53: 47$ & $39 / 38$ \\
\hline 11 & $\mathbf{8 a}$ & $\mathbf{6 a}$ & THF & $\mathrm{AcOH}$ & 3 & 38 & $52: 48$ & $44 / 44$ \\
\hline 12 & $8 \mathbf{a}$ & $6 \mathbf{a}$ & THF & $\mathrm{TsOH}$ & 3 & N.D. & - & - \\
\hline 13 & $\mathbf{8 a}$ & $\mathbf{6 a}$ & THF & D-(-)-Tartaric acid & 3 & 19 & $56: 44$ & $40 / 42$ \\
\hline 14 & $8 \mathbf{a}$ & $\mathbf{6 a}$ & THF & L-(+)-Tartaric acid & 3 & 23 & $51: 49$ & $41 / 41$ \\
\hline 15 & $\mathbf{8 a}$ & $6 \mathbf{a}$ & THF & (S)-(+)-Mandelic acid & 3 & 31 & $55: 45$ & $41 / 41$ \\
\hline 16 & $\mathbf{8 a}$ & $\mathbf{6 a}$ & THF & (R)-(-)-Mandelic acid & 3 & 35 & $55: 45$ & $42 / 44$ \\
\hline 17 & $8 \mathbf{a}$ & $\mathbf{6 a}$ & THF & D-(+)-Phenyllactic acid & 3 & 32 & $57: 43$ & $41 / 45$ \\
\hline 18 & $\mathbf{8 a}$ & $\mathbf{6 a}$ & THF & $\mathrm{L}(+)$-Lactic acid & 3 & 35 & $53: 47$ & $33 / 32$ \\
\hline 19 & $\mathbf{8 b}$ & $\mathbf{6 a}$ & THF & $\mathrm{BzOH}$ & 3 & 35 & $51: 49$ & $1 / 0$ \\
\hline 20 & $8 c$ & $\mathbf{6 a}$ & THF & $\mathrm{BzOH}$ & 3 & 42 & $55: 45$ & $68 / 82$ \\
\hline 21 & $8 d$ & $6 \mathbf{a}$ & THF & $\mathrm{BzOH}$ & 3 & 40 & $51: 49$ & $73 / 81$ \\
\hline 22 & $8 \mathbf{e}$ & $\mathbf{6 a}$ & THF & $\mathrm{BzOH}$ & 3 & 41 & $51: 49$ & $91 / 91$ \\
\hline 23 & $8 f$ & $\mathbf{6 a}$ & THF & $\mathrm{BzOH}$ & 3 & 42 & $52: 48$ & $-91 /-91$ \\
\hline 24 & $8 g$ & $\mathbf{6 a}$ & THF & $\mathrm{BzOH}$ & 3 & 39 & $53: 47$ & $88 / 90$ \\
\hline 25 & $8 h$ & $6 a$ & THF & $\mathrm{BzOH}$ & 3 & 25 & $52: 48$ & $39 / 38$ \\
\hline 26 & $8 \mathbf{i}$ & $6 a$ & THF & $\mathrm{BzOH}$ & 3 & 28 & $52: 48$ & $36 / 36$ \\
\hline 27 & $8 \mathbf{j}$ & $6 a$ & THF & $\mathrm{BzOH}$ & 3 & 41 & $53: 47$ & $46 / 47$ \\
\hline 28 & $8 k$ & $6 a$ & THF & $\mathrm{BzOH}$ & 3 & 44 & $53: 47$ & $16 / 15$ \\
\hline 29 & 81 & $6 a$ & THF & $\mathrm{BzOH}$ & 3 & 10 & $55: 45$ & $36 / 37$ \\
\hline 30 & $8 m$ & $6 a$ & THF & $\mathrm{BzOH}$ & 3 & 5 & $47: 53$ & $46 / 45$ \\
\hline 31 & $8 n$ & $6 a$ & THF & $\mathrm{BzOH}$ & 3 & Trace & - & - \\
\hline 32 & 80 & $6 a$ & THF & $\mathrm{BzOH}$ & 3 & 46 & $56: 44$ & $-24 /-25$ \\
\hline 33 & $8 p$ & $6 a$ & THF & $\mathrm{BzOH}$ & 3 & N.D. & - & - \\
\hline 34 & $8 q$ & $6 a$ & THF & $\mathrm{BzOH}$ & 3 & N.D. & - & - \\
\hline 35 & $8 r$ & $6 a$ & THF & $\mathrm{BzOH}$ & 3 & N.D. & - & - \\
\hline 36 & $8 e$ & $6 b$ & THF & $\mathrm{BzOH}$ & 3 & 46 & $52: 48$ & $93 / 93$ \\
\hline 37 & $8 f$ & $6 b$ & THF & $\mathrm{BzOH}$ & 3 & 45 & $52: 48$ & $-93 /-93$ \\
\hline 38 & $8 e$ & $6 b$ & $\mathrm{THF}(0.5 \mathrm{ml})$ & $\mathrm{BzOH}$ & 2 & 57 & $54: 46$ & $93 / 93$ \\
\hline 39 & $8 \mathbf{e}$ & $6 b$ & THF(0.5 ml) & $\mathrm{BzOH}$ & 3 & 60 & $53: 47$ & $91 / 93$ \\
\hline $40^{f}$ & $8 e$ & $6 \mathbf{b}$ & $\mathrm{THF}(0.5 \mathrm{ml})$ & $\mathrm{BzOH}$ & 3 & 65 & $52: 48$ & $90 / 92$ \\
\hline $41^{f}$ & $8 e$ & $6 \mathbf{b}$ & THF(0.3 ml) & $\mathrm{BzOH}$ & 3 & 66 & $54: 46$ & $89 / 90$ \\
\hline $42^{g}$ & $8 e$ & $6 b$ & THF(0.5 ml) & $\mathrm{BzOH}$ & 3 & 12 & $64: 36$ & $94 / 90$ \\
\hline $43^{h}$ & $8 e$ & $6 b$ & $\mathrm{THF}(0.25 \mathrm{ml})$ & $\mathrm{BzOH}$ & 2 & 70 & $52: 48$ & $93 / 93$ \\
\hline
\end{tabular}

${ }^{a}$ Unless otherwise noted, reactions were performed with $6(0.1 \mathrm{mmol}), 7 \mathrm{a}(0.15 \mathrm{mmol})$, catalyst $8(20 \mathrm{~mol} \%)$, and additive (20 mol \%) in $1 \mathrm{~mL}$ solvent. ${ }^{b}$ Combined isolated yield of $\mathbf{4 a}$ and $\mathbf{4 a a}$ or $\mathbf{4 b}$ and $\mathbf{4 b b} .{ }^{c}$ Determined by chiral HPLC analysis of $\mathbf{4 a}$ and $\mathbf{4 a a}$ or $\mathbf{4 b}$ and 4bb. ${ }^{d}$ Adding $0.1 \mathrm{ml} \mathrm{H} 2 \mathrm{O} .{ }^{e}$ Adding $50 \mathrm{mg} 4 \AA$ A MS. ${ }^{f} 0.3 \mathrm{mmol}$ of 7a. ${ }^{g}$ under $0{ }^{\circ} \mathrm{C} .{ }^{h}$ With $\mathbf{6 b}(0.25 \mathrm{mmol}), 7 \mathbf{a}(0.375 \mathrm{mmol})$, catalyst $8 \mathbf{e}$ (20 $\mathrm{mol} \%)$, and $\mathrm{BzOH}(20 \mathrm{~mol} \%)$. 


\section{General procedure for the $[4+2]$ cycloaddition reaction}

To a solution of catalyst $8 \mathbf{e}$ or $\mathbf{8 f}(0.05 \mathrm{mmol}, 0.2$ equiv) in THF $(250 \mu \mathrm{L}), \alpha, \beta$ - unsaturated aldehyde $7 \mathbf{a}(0.375 \mathrm{mmol}$, 1.5 equiv), pyranoquinolinone 6 ( $0.25 \mathrm{mmol}, 1.0$ equiv) and $\mathrm{BzOH}(0.05 \mathrm{mmol}, 0.2$ equiv) were added at room temperature and stirred for 2 days. Then, $\mathrm{MeOH}(500 \mu \mathrm{L})$ and DCM $(500 \mu \mathrm{L})$ were added and cooled at $0{ }^{\circ} \mathrm{C}$ followed by $\mathrm{NaBH}_{4}(95$ $\mathrm{mg}, 2.5 \mathrm{mmol}, 10$ equiv) in small portions and stirred at same temperature for 30 minutes. Then, reaction was quenched with ice cold water $(5 \mathrm{~mL})$ and extracted with DCM $(3 \times 5 \mathrm{~mL})$, and organic layer was washed with brine $(10 \mathrm{~mL})$, dried over anhydrous $\mathrm{Na}_{2} \mathrm{SO}_{4}$ and concentrated under reduce pressure. The products $\mathbf{4 X}$ and $\mathbf{4 X X}$ could be separated and purified by flash column chromatography (silica gel, petroleum ether/EtOAc/DCM $=4: 1: 1$ to 2:1:1).

Figure S3. Structures of $4 X$ and $4 X X$.

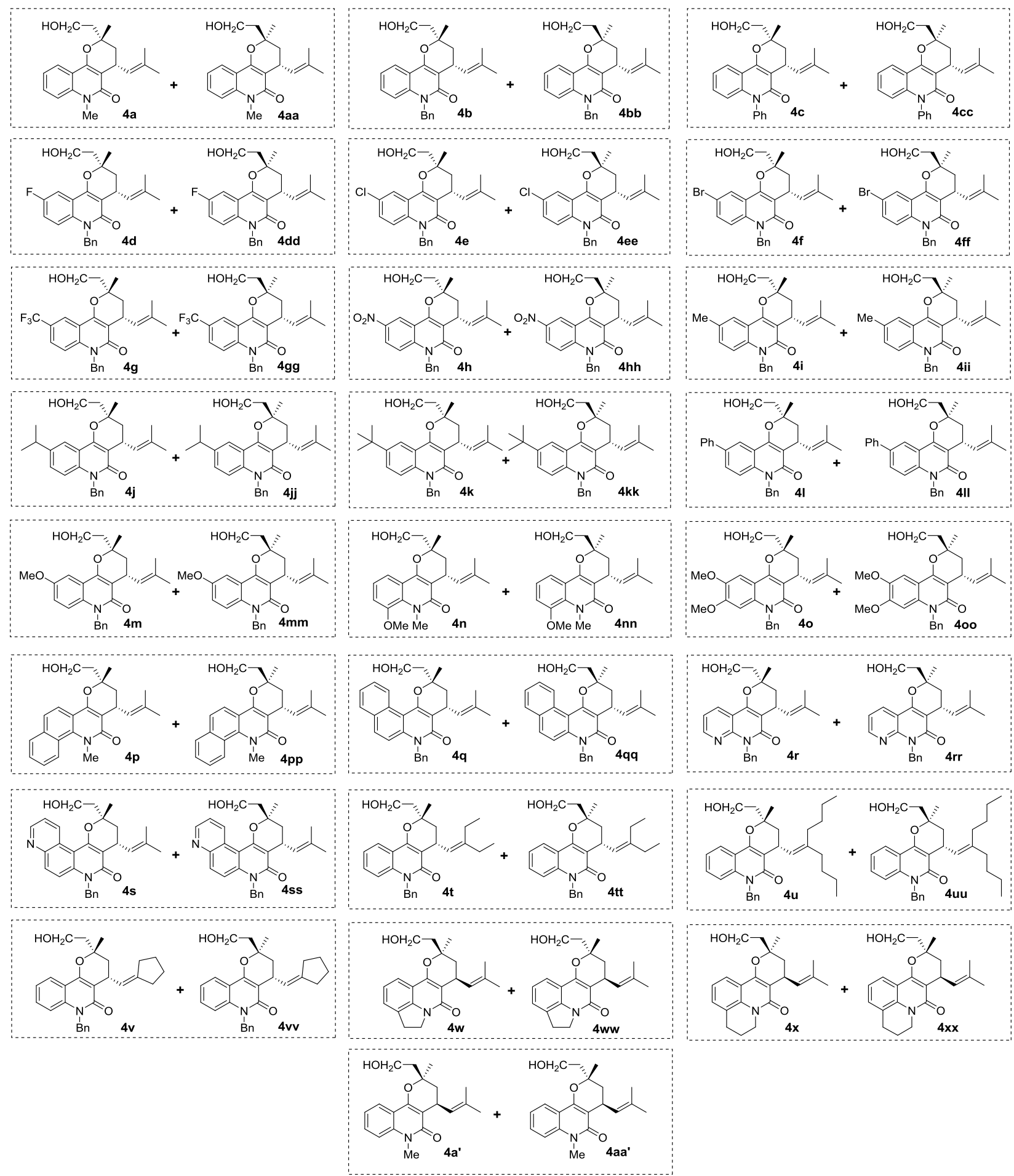




\section{Characterization datas for $4 \mathrm{X}$ and $4 \mathrm{XX}$ :}

(2S,4R)-2-(2-hydroxyethyl)-2,6-dimethyl-4-(2-methylprop-1-en-1-yl)-2,3,4,6-tetrahydro-5H-pyrano[3,2-c]quinolin-

5-one (4a):

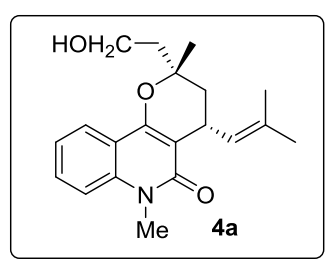

With catalyst $\mathbf{8 e}, 31 \%$ yield, $25 \mathrm{mg}$. White solid. $91 \%$ ee, determined by HPLC analysis [Chiralpak AD-H, $n$-hexane $/ i-\operatorname{PrOH}=90 / 10,1.0 \mathrm{~mL} / \mathrm{min}, \lambda=230 \mathrm{~nm}, \mathrm{t}$ (major) $=9.27 \mathrm{~min}, \mathrm{t}$ $($ minor $)=11.60 \mathrm{~min}] .{ }^{1} \mathbf{H}$ NMR $\left(400 \mathrm{MHz}, \mathrm{CDCl}_{3}\right) \delta=7.91(\mathrm{dd}, J=8.0,1.2 \mathrm{~Hz}, 1 \mathrm{H}), 7.57-$ $7.52(\mathrm{~m}, 1 \mathrm{H}), 7.33(\mathrm{~d}, J=8.4 \mathrm{~Hz}, 1 \mathrm{H}), 7.26-7.19(\mathrm{~m}, 1 \mathrm{H}), 5.09-5.01(\mathrm{~m}, 1 \mathrm{H}), 3.98(\mathrm{t}, J=6.4$ $\mathrm{Hz}, 2 \mathrm{H}), 3.74(\mathrm{dd}, J=16.4,8.8 \mathrm{~Hz}, 1 \mathrm{H}), 3.69(\mathrm{~s}, 3 \mathrm{H}), 2.11-2.05(\mathrm{~m}, 2 \mathrm{H}), 2.02-1.97(\mathrm{~m}, 1 \mathrm{H})$, $1.85-1.83(\mathrm{~m}, 1 \mathrm{H}), 1.83(\mathrm{~s}, 3 \mathrm{H}), 1.77$ (s, 3H), $1.40(\mathrm{~s}, 3 \mathrm{H}) .{ }^{13} \mathbf{C}$ NMR $\left(100 \mathrm{MHz}, \mathrm{CDCl}_{3}\right) \delta=162.6,155.0,138.8,131.4$, $130.3,127.2,122.8,121.5,116.5,113.8,109.5,78.4,58.8,43.4,39.1,29.2,28.9,25.9,22.9,18.0 .[\alpha]^{20} \mathbf{D}=+11.90^{\circ}(c=$ 1.0, $\mathrm{CHCl}_{3}, 91 \%$ ee). HRMS (ESI) m/z: $[\mathrm{M}+\mathrm{H}]^{+}$calcd. for $\mathrm{C}_{20} \mathrm{H}_{26} \mathrm{NO}_{3} 328.1907$, found 328.1899 .
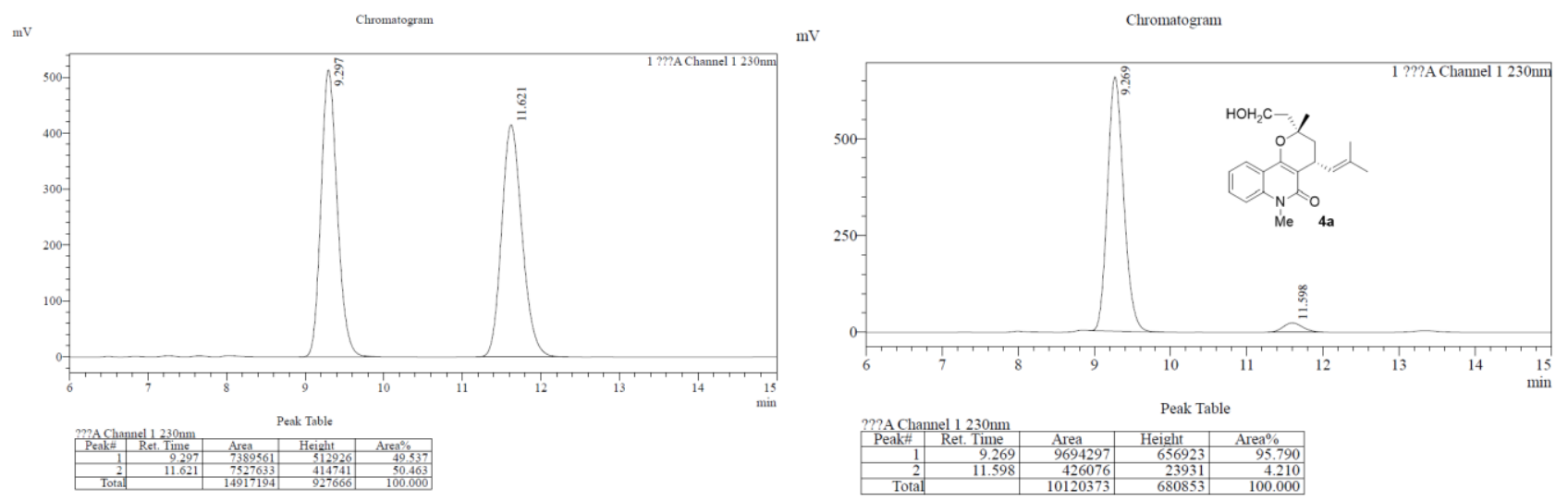

(2R,4R)-2-(2-hydroxyethyl)-2,6-dimethyl-4-(2-methylprop-1-en-1-yl)-2,3,4,6-tetrahydro-5H-pyrano[3,2-c]quinolin5-one (4aa):

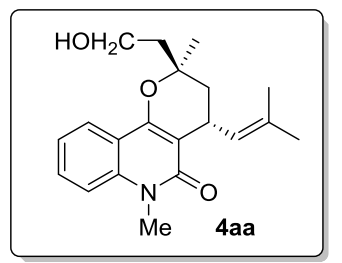

With catalyst 8e, 29\% yield, $24 \mathrm{mg}$. White solid. $91 \%$ ee, determined by HPLC analysis [Chiralpak AD-H, $n$-hexane $/ i$-PrOH $=90 / 10,1.0 \mathrm{~mL} / \mathrm{min}, \lambda=230 \mathrm{~nm}, \mathrm{t}$ (major) $=9.77 \mathrm{~min}, \mathrm{t}$ $($ minor $)=14.84 \mathrm{~min}] .{ }^{1} \mathbf{H}$ NMR $\left(400 \mathrm{MHz}, \mathrm{CDCl}_{3}\right) \delta=7.92(\mathrm{~d}, J=8.0 \mathrm{~Hz}, 1 \mathrm{H}), 7.53(\mathrm{t}, J=8.4$ $\mathrm{Hz}, 1 \mathrm{H}), 7.31(\mathrm{~d}, J=8.4 \mathrm{~Hz}, 1 \mathrm{H}), 7.21(\mathrm{t}, J=7.6 \mathrm{~Hz}, 1 \mathrm{H}), 5.08(\mathrm{~d}, J=8.4 \mathrm{~Hz}, 1 \mathrm{H}), 3.85(\mathrm{t}, J=$ $6.8 \mathrm{~Hz}, 2 \mathrm{H}), 3.75(\mathrm{dd}, J=15.2,7.6 \mathrm{~Hz}, 1 \mathrm{H}), 3.67(\mathrm{~s}, 3 \mathrm{H}), 2.16-2.03(\mathrm{~m}, 2 \mathrm{H}), 1.94-1.87(\mathrm{~m}$, $1 \mathrm{H}), 1.81(\mathrm{~s}, 3 \mathrm{H}), 1.79-1.76(\mathrm{~m}, 1 \mathrm{H}), 1.74(\mathrm{~s}, 3 \mathrm{H}), 1.50(\mathrm{~s}, 3 \mathrm{H}),{ }^{13} \mathbf{C ~ N M R}\left(100 \mathrm{MHz}, \mathrm{CDCl}_{3}\right)$ $\delta=162.7,154.9,138.8,131.4,130.3,127.5,122.8,121.5,116.5,113.8,109.5,78.1,58.8,40.6,39.6,29.2,28.9,26.0,25.8$, 18.0. $[\alpha]^{\mathbf{2 0}} \mathbf{D}=+3.60^{\circ}\left(c=1.0, \mathrm{CHCl}_{3}, 91 \%\right.$ ee $)$. HRMS (ESI) m/z: $[\mathrm{M}+\mathrm{H}]^{+}$calcd. for $\mathrm{C}_{20} \mathrm{H}_{26} \mathrm{NO}_{3} 328.1907$, found 328.1914 .
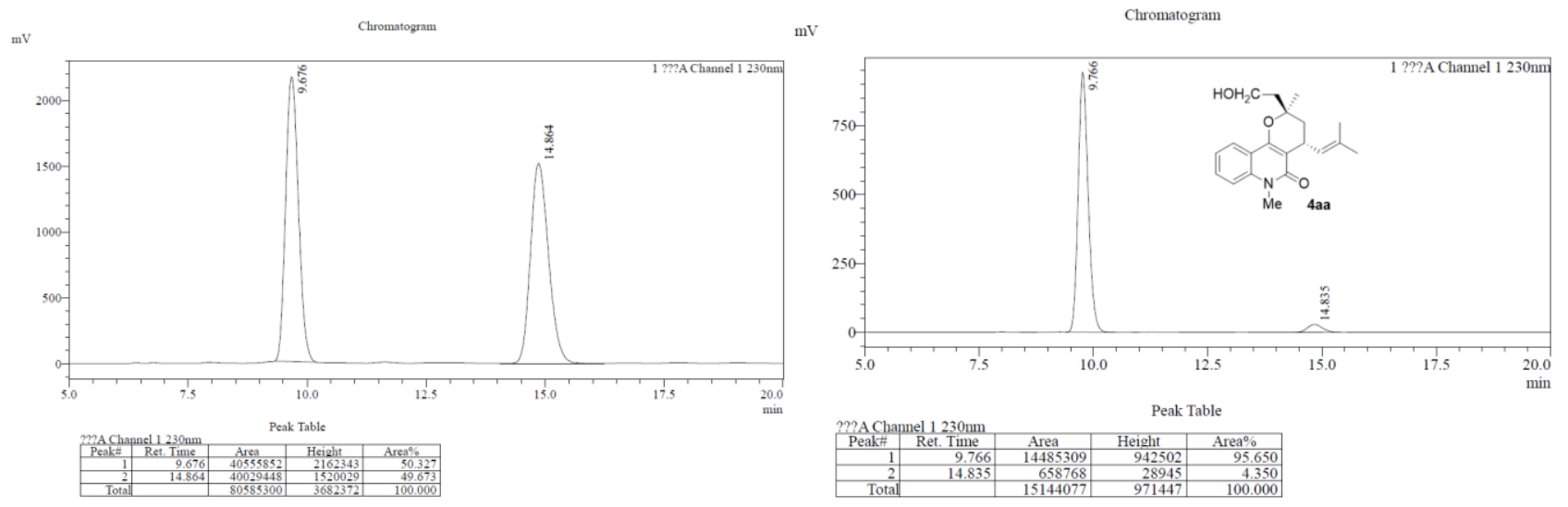
(2S,4R)-6-benzyl-2-(2-hydroxyethyl)-2-methyl-4-(2-methylprop-1-en-1-yl)-2,3,4,6-tetrahydro-5H-pyrano[3,2-c]qui nolin-5-one (4b):

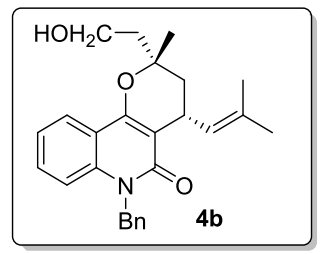

With catalyst 8e, $37 \%$ yield, $37 \mathrm{mg}$. White solid. 93\% ee, determined by HPLC analysis [Chiralpak AD-H, $n$-hexane $/ i$-PrOH $=90 / 10,1.0 \mathrm{~mL} / \mathrm{min}, \lambda=230 \mathrm{~nm}, \mathrm{t}$ (major) $=14.96 \mathrm{~min}, \mathrm{t}$ $($ minor $)=16.67 \mathrm{~min}] .{ }^{1} \mathbf{H}$ NMR $\left(400 \mathrm{MHz}, \mathrm{CDCl}_{3}\right) \delta=7.82(\mathrm{dd}, J=8.0,1.6 \mathrm{~Hz}, 1 \mathrm{H}), 7.33-7.26$ $(\mathrm{m}, 1 \mathrm{H}), 7.23-7.16(\mathrm{~m}, 2 \mathrm{H}), 7.15-7.05(\mathrm{~m}, 5 \mathrm{H}), 5.44(\mathrm{~s}, 2 \mathrm{H}), 5.02(\mathrm{~d}, J=8.4 \mathrm{~Hz}, 1 \mathrm{H}), 3.89(\mathrm{t}$, $J=6.8 \mathrm{~Hz}, 2 \mathrm{H}), 3.72(\mathrm{dd}, J=16.0,8.4 \mathrm{~Hz}, 1 \mathrm{H}), 2.04-2.00(\mathrm{~m}, 2 \mathrm{H}), 1.97-1.91(\mathrm{~m}, 1 \mathrm{H}), 1.82-$ $1.76(\mathrm{~m}, 1 \mathrm{H}), 1.73(\mathrm{~s}, 3 \mathrm{H}), 1.68(\mathrm{~s}, 3 \mathrm{H}), 1.35$ (s, 3H). ${ }^{13} \mathbf{C ~ N M R}\left(100 \mathrm{MHz}, \mathrm{CDCl}_{3}\right) \delta=162.7,155.3,138.3,137.0,131.5$, $130.3,128.7,127.3,127.0,126.5,122.8,121.5,116.7,114.7,109.3,78.5,58.8,45.6,43.4,39.1,29.0,25.9,23.2,18.0$. $[\alpha]^{20} \mathbf{D}=+16.85^{\circ}\left(c=1.0, \mathrm{CHCl}_{3}, 93 \%\right.$ ee $)$. HRMS (ESI) m/z: $[\mathrm{M}+\mathrm{H}]^{+}$calcd. for $\mathrm{C}_{26} \mathrm{H}_{30} \mathrm{NO}_{3} 404.2220$, found 404.2223 .
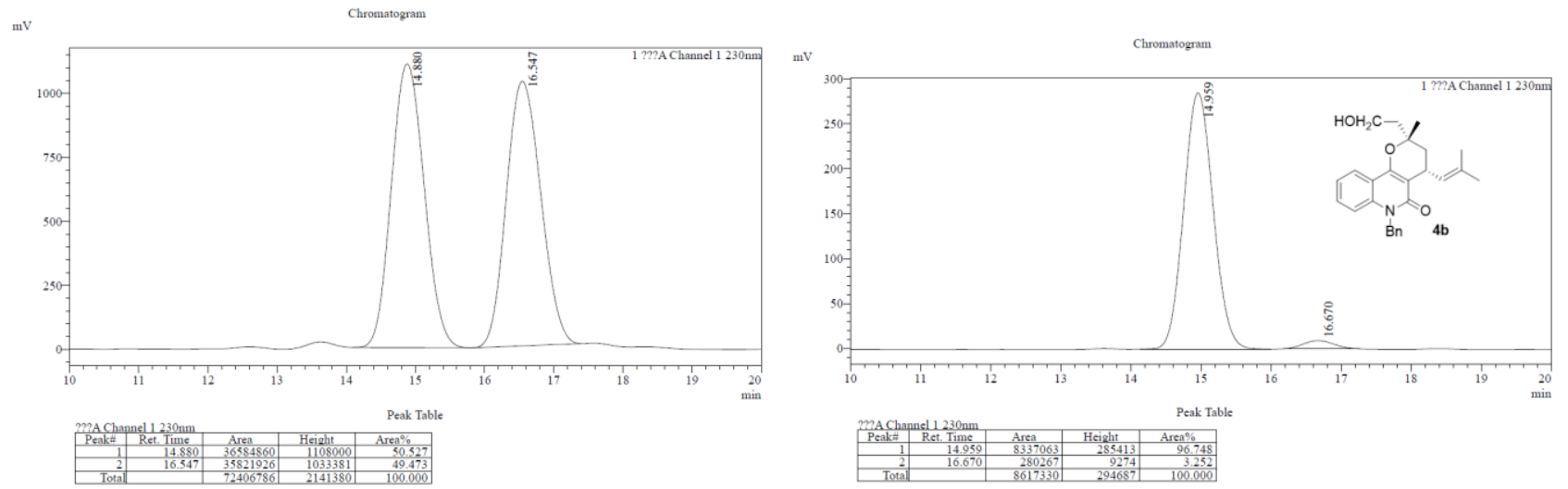

(2R,4R)-6-benzyl-2-(2-hydroxyethyl)-2-methyl-4-(2-methylprop-1-en-1-yl)-2,3,4,6-tetrahydro-5H-pyrano[3,2-c]qui nolin-5-one (4bb):

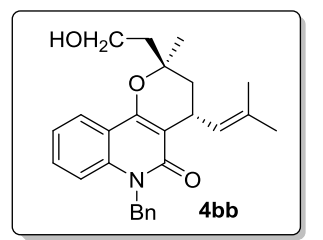

With catalyst 8e, 33\% yield, $34 \mathrm{mg}$. White solid. 93\% ee, determined by HPLC analysis [Chiralpak $\mathrm{AD}-\mathrm{H}, n$-hexane $/ \mathrm{i}-\mathrm{PrOH}=90 / 10,1.0 \mathrm{~mL} / \mathrm{min}, \lambda=230 \mathrm{~nm}, \mathrm{t}$ (major) $=13.64 \mathrm{~min}, \mathrm{t}($ minor $)=$ $17.72 \mathrm{~min}] .{ }^{1} \mathbf{H}$ NMR $\left(400 \mathrm{MHz}, \mathrm{CDCl}_{3}\right) \delta=7.92(\mathrm{~d}, J=8.0 \mathrm{~Hz}, 1 \mathrm{H}), 7.38-7.35(\mathrm{~m}, 1 \mathrm{H}), 7.28-$ $7.24(\mathrm{~m}, 3 \mathrm{H}), 7.22-7.12(\mathrm{~m}, 5 \mathrm{H}), 5.56(\mathrm{~d}, J=15.6 \mathrm{~Hz}, 1 \mathrm{H}), 5.44(\mathrm{~d}, J=14.8 \mathrm{~Hz}, 1 \mathrm{H}), 5.13(\mathrm{~d}, J$ $=8.4 \mathrm{~Hz}, 1 \mathrm{H}), 3.87(\mathrm{t}, J=6.8 \mathrm{~Hz}, 2 \mathrm{H}), 3.84-3.76(\mathrm{~m}, 1 \mathrm{H}), 2.19-2.12(\mathrm{~m}, 1 \mathrm{H}), 2.10-2.05(\mathrm{~m}$, 1H), $2.01-1.90(\mathrm{~m}, 1 \mathrm{H}), 1.84-1.76(\mathrm{~m}, 4 \mathrm{H}), 1.74(\mathrm{~s}, 3 \mathrm{H}), 1.51(\mathrm{~s}, 3 \mathrm{H}) .{ }^{13} \mathbf{C} \mathbf{N M R}\left(100 \mathrm{MHz}, \mathrm{CDCl}_{3}\right) \delta=162.8,155.2$, 138.3, 137.0, 131.5, 130.3, 128.7, 127.6, 127.0, 126.5, 122.8, 121.6, 116.7, 114.7, 109.3, 78.2, 58.8, 45.7, 40.8, 39.5, 29.0, 26.1, 25.9, 18.0. $[\boldsymbol{\alpha}]^{\mathbf{2 0}} \mathbf{D}=+13.83^{\circ}\left(c=1.0, \mathrm{CHCl}_{3}, 93 \%\right.$ ee $) . \mathbf{H R M S}(\mathrm{ESI}) \mathrm{m} / \mathrm{z}:[\mathrm{M}+\mathrm{Na}]^{+}$calcd. for $\mathrm{C}_{26} \mathrm{H}_{29} \mathrm{NO}_{3} \mathrm{Na}_{426.2040}$, found 426.2060.
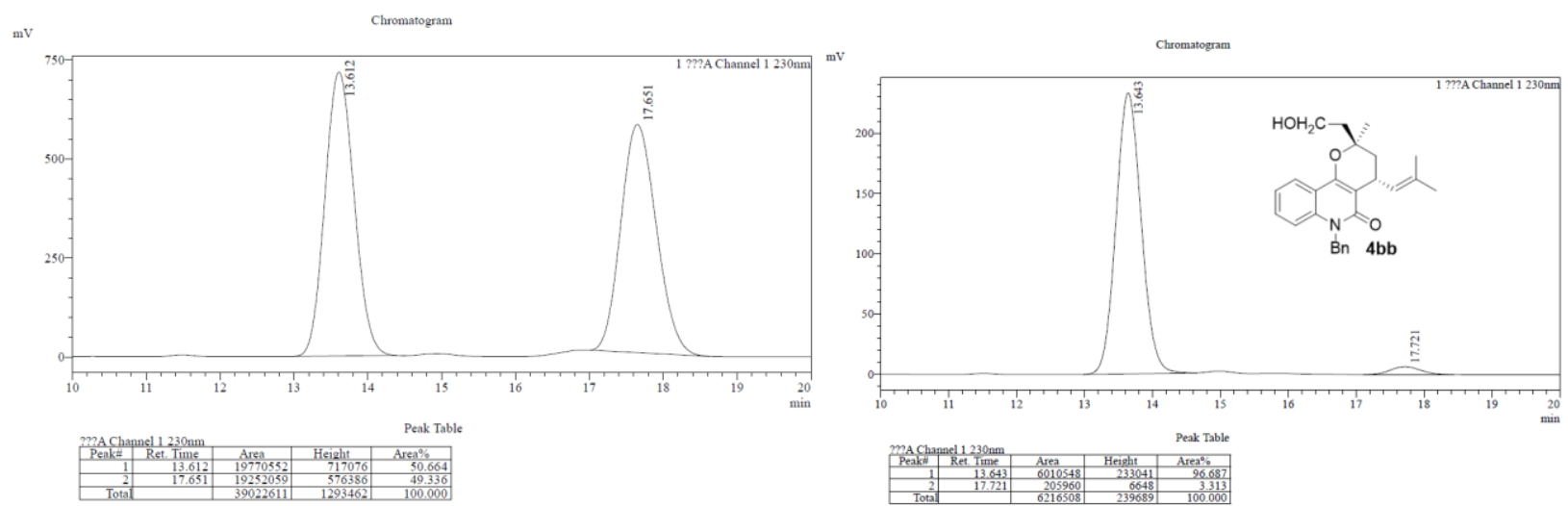
(2S,4R)-2-(2-hydroxyethyl)-2-methyl-4-(2-methylprop-1-en-1-yl)-6-phenyl-2,3,4,6-tetrahydro-5H-pyrano[3,2-c]qui nolin-5-one (4c):

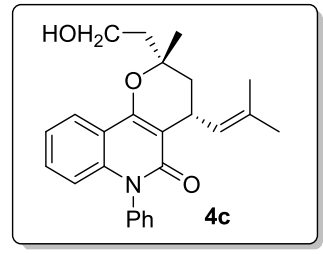

With catalyst 8e, $28 \%$ yield, $27 \mathrm{mg}$. White solid. 94\% ee, determined by HPLC analysis [Chiralpak AD-H, $n$-hexane $/ i-\mathrm{PrOH}=90 / 10,1.0 \mathrm{~mL} / \mathrm{min}, \lambda=230 \mathrm{~nm}, \mathrm{t}$ (major) $=7.58 \mathrm{~min}, \mathrm{t}$ (minor) $=5.78 \mathrm{~min}] .{ }^{1} \mathbf{H}$ NMR $\left(400 \mathrm{MHz}, \mathrm{CDCl}_{3}\right) \delta=7.90(\mathrm{~d}, J=8.0 \mathrm{~Hz}, 1 \mathrm{H}), 7.60-7.49(\mathrm{~m}$, 2H), $7.47-7.43(\mathrm{~m}, 1 \mathrm{H}), 7.32-7.20(\mathrm{~m}, 4 \mathrm{H}), 7.16(\mathrm{t}, J=7.6 \mathrm{~Hz}, 1 \mathrm{H}), 6.61(\mathrm{~d}, J=8.4 \mathrm{~Hz}, 1 \mathrm{H})$, $5.08(\mathrm{~d}, J=8.4 \mathrm{~Hz}, 1 \mathrm{H}), 3.97(\mathrm{t}, J=6.8 \mathrm{~Hz}, 2 \mathrm{H}), 3.75(\mathrm{dd}, J=16.4,8.4 \mathrm{~Hz}, 1 \mathrm{H}), 2.16-2.06(\mathrm{~m}$, $2 \mathrm{H}), 2.02-1.97(\mathrm{~m}, 1 \mathrm{H}), 1.82-1.87(\mathrm{~m}, 1 \mathrm{H}), 1.74(\mathrm{~s}, 3 \mathrm{H}), 1.69(\mathrm{~s}, 3 \mathrm{H}), 1.44(\mathrm{~s}, 3 \mathrm{H}) .{ }^{13} \mathbf{C ~ N M R}(100 \mathbf{M H z} \mathbf{C D C l}) \delta=$ 162.6, 155.6, 139.9, 138.1, 131.2, 130.1, 129.9, 129.8, 129.4, 129.3, 128.5, 127.1, 122.5, 121.6, 116.2, 115.6, 109.7, 78.6, $58.8,43.4,39.2,28.8,25.9,23.2,17.9 .[\mathbf{\alpha}]^{\mathbf{2 0}} \mathbf{D}=+30.45^{\circ}\left(c=1.0, \mathrm{CHCl}_{3}, 94 \%\right.$ ee $) . \mathbf{H R M S}(\mathrm{ESI}) \mathrm{m} / \mathrm{z}:[\mathrm{M}+\mathrm{H}]^{+}$calcd. for $\mathrm{C}_{25} \mathrm{H}_{28} \mathrm{NO}_{3} 390.2064$, found 390.2067. mV
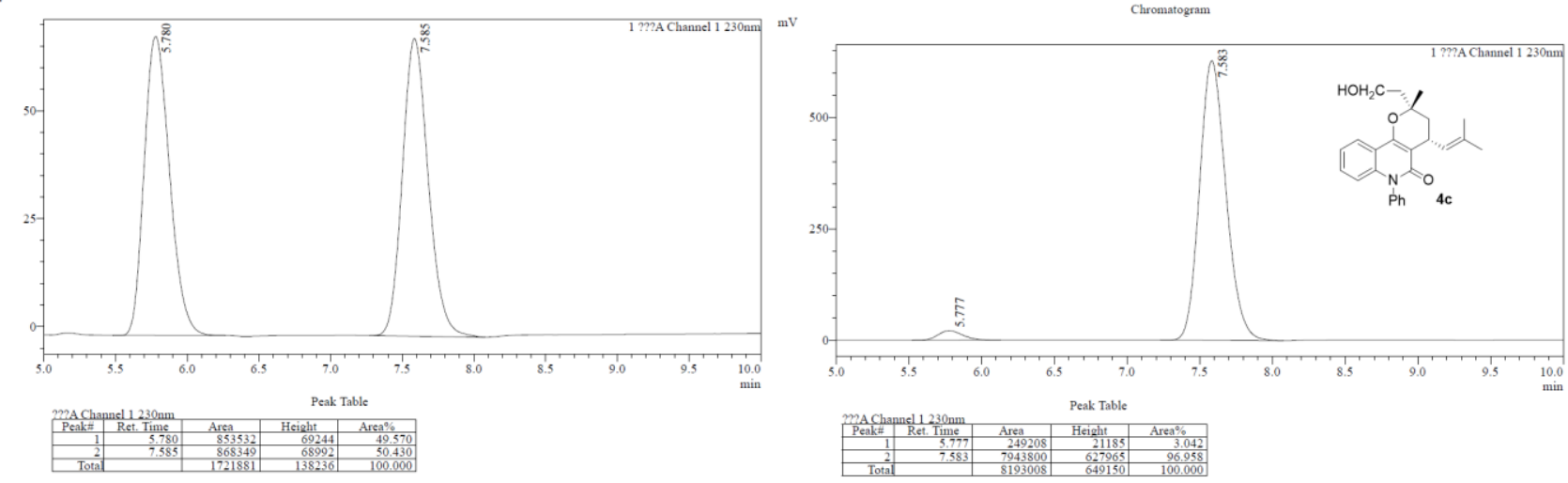

(2R,4R)-2-(2-hydroxyethyl)-2-methyl-4-(2-methylprop-1-en-1-yl)-6-phenyl-2,3,4,6-tetrahydro-5H-pyrano[3,2-c]qui nolin-5-one (4cc):

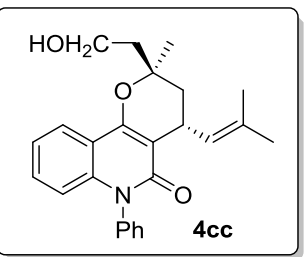

With catalyst 8e, $27 \%$ yield, $26 \mathrm{mg}$. White solid. $94 \%$ ee, determined by HPLC analysis [Chiralpak AD-H, $n$-hexane $/ i-\mathrm{PrOH}=90 / 10,1.0 \mathrm{~mL} / \mathrm{min}, \lambda=230 \mathrm{~nm}, \mathrm{t}$ (major) $=10.55 \mathrm{~min}, \mathrm{t}$ $($ minor $)=6.04 \mathrm{~min}] .{ }^{1} \mathbf{H}$ NMR $\left(400 \mathrm{MHz}, \mathrm{CDCl}_{3}\right) \delta=7.93(\mathrm{~d}, J=8.0 \mathrm{~Hz}, 1 \mathrm{H}), 7.57-7.43(\mathrm{~m}$, $3 \mathrm{H}), 7.30-7.22(\mathrm{~m}, 4 \mathrm{H}), 7.17(\mathrm{t}, J=7.6 \mathrm{~Hz}, 1 \mathrm{H}), 6.62(\mathrm{~d}, J=8.4 \mathrm{~Hz}, 1 \mathrm{H}), 5.12(\mathrm{~d}, J=8.4 \mathrm{~Hz}$, $1 \mathrm{H}), 3.89(\mathrm{t}, J=6.4 \mathrm{~Hz}, 2 \mathrm{H}), 3.77(\mathrm{dd}, J=15.2,7.6 \mathrm{~Hz}, 1 \mathrm{H}), 2.19-2.07(\mathrm{~m}, 2 \mathrm{H}), 2.00-1.90(\mathrm{~m}$, $1 \mathrm{H}), 1.80(\mathrm{dd}, J=14.0,6.8 \mathrm{~Hz}, 1 \mathrm{H}), 1.74(\mathrm{~s}, 3 \mathrm{H}), 1.69(\mathrm{~s}, 3 \mathrm{H}), 1.54(\mathrm{~s}, 3 \mathrm{H}) .{ }^{13} \mathbf{C ~ N M R}(100 \mathrm{MHz}$, $\left.\mathrm{CDCl}_{3}\right) \delta=162.7,155.5,139.9,138.1,131.3,130.1,130.0,129.8,129.4,129.3,128.5,127.4,122.5,121.7,116.2,115.6$, $109.7,78.3,58.9,40.8,39.7,28.8,26.1,25.9,17.9 .[\alpha]^{\mathbf{2 0}} \mathbf{D}=+28.57^{\circ}\left(c=1.0, \mathrm{CHCl}_{3}, 94 \%\right.$ ee $) . \mathbf{H R M S}(\mathrm{ESI}) \mathrm{m} / \mathrm{z}:[\mathrm{M}+\mathrm{H}]^{+}$ calcd. for $\mathrm{C}_{25} \mathrm{H}_{28} \mathrm{NO}_{3} 390.2064$, found 390.2079.
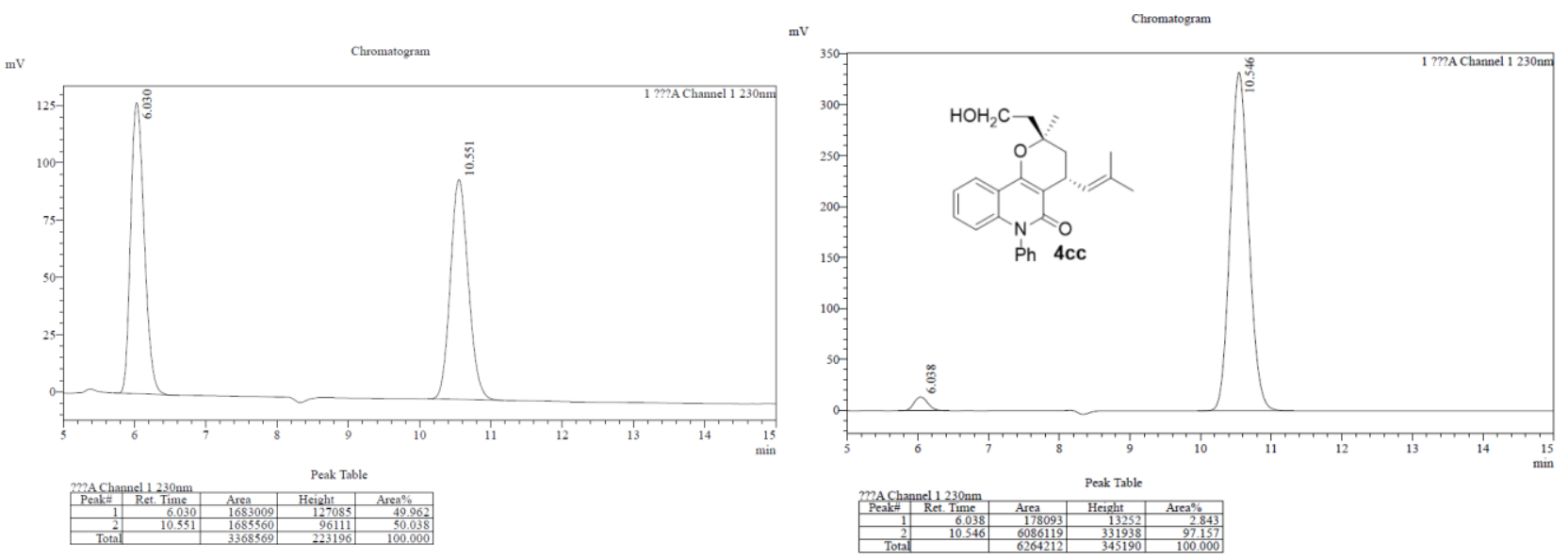
(2S,4R)-6-benzyl-9-fluoro-2-(2-hydroxyethyl)-2-methyl-4-(2-methylprop-1-en-1-yl)-2,3,4,6-tetrahydro-5H-pyrano [3,2-c]quinolin-5-one (4d):

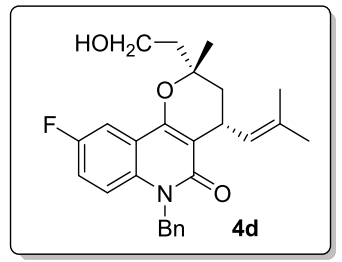

With catalyst 8e, $31 \%$ yield, $33 \mathrm{mg}$. White solid. $84 \%$ ee, determined by HPLC analysis [Chiralpak AD-H, $n$-hexane $/ \mathrm{i}-\mathrm{PrOH}=90 / 10,1.0 \mathrm{~mL} / \mathrm{min}, \lambda=230 \mathrm{~nm}, \mathrm{t}$ (major) $=11.55 \mathrm{~min}, \mathrm{t}$ (minor) $=13.64 \mathrm{~min}] .{ }^{1} \mathbf{H}$ NMR $\left(400 \mathrm{MHz} \mathrm{CDCl}_{3}\right) \delta=7.56(\mathrm{dd}, J=8.8,2.4 \mathrm{~Hz}, 1 \mathrm{H}), 7.29-$ $7.25(\mathrm{~m}, 2 \mathrm{H}), 7.22-7.18(\mathrm{~m}, 1 \mathrm{H}), 7.17-7.05(\mathrm{~m}, 4 \mathrm{H}), 5.54(\mathrm{~d}, J=16.4 \mathrm{~Hz}, 1 \mathrm{H}), 5.46(\mathrm{~d}, J=$ $16.4 \mathrm{~Hz}, 1 \mathrm{H}), 5.09(\mathrm{~d}, J=8.0 \mathrm{~Hz}, 1 \mathrm{H}), 3.95(\mathrm{t}, J=6.8 \mathrm{~Hz}, 2 \mathrm{H}), 3.79(\mathrm{dd}, J=16.0,8.4 \mathrm{~Hz}, 1 \mathrm{H})$, $2.11-2.06(\mathrm{~m}, 2 \mathrm{H}), 2.05-1.99(\mathrm{~m}, 1 \mathrm{H}), 1.89-1.83(\mathrm{~m}, 1 \mathrm{H}), 1.81(\mathrm{~s}, 3 \mathrm{H}), 1.75(\mathrm{~s}, 3 \mathrm{H}), 1.42$ (s, 3H). ${ }^{19} \mathbf{F}$ NMR $\left(376 \mathrm{MHz}, \mathrm{CDCl}_{3}\right) \delta=-121.2 .{ }^{13} \mathbf{C ~ N M R}\left(100 \mathrm{MHz}, \mathrm{CDCl}_{3}\right) \delta=162.3,157.7\left(\mathrm{~d}, J_{\mathrm{C}, \mathrm{F}}=239.9 \mathrm{~Hz}\right)$, $154.5\left(\mathrm{~d}, J_{\mathrm{C}, \mathrm{F}}=2.7 \mathrm{~Hz}\right), 136.7,134.8,131.7,128.7,127.1,126.9,126.4,117.9\left(\mathrm{~d}, J_{\mathrm{C}, \mathrm{F}}=23.9 \mathrm{~Hz}\right), 116.3\left(\mathrm{~d}, J_{\mathrm{C}, \mathrm{F}}=7.9 \mathrm{~Hz}\right)$ $110.3,108.5\left(\mathrm{~d}, J_{\mathrm{C}, \mathrm{F}}=24 \mathrm{~Hz}\right), 78.6,58.7,45.8,43.4,39.0,29.0,25.9,23.3,18.0 .[\mathbf{\alpha}]^{\mathbf{2 0}} \mathrm{D}=+12.29^{\circ}\left(c=1.0, \mathrm{CHCl}_{3}, 84 \%\right.$ ee). HRMS (ESI) m/z: $[\mathrm{M}+\mathrm{H}]^{+}$calcd. for $\mathrm{C}_{26} \mathrm{H}_{29} \mathrm{FNO}_{3} 422.2126$, found 422.2120 .
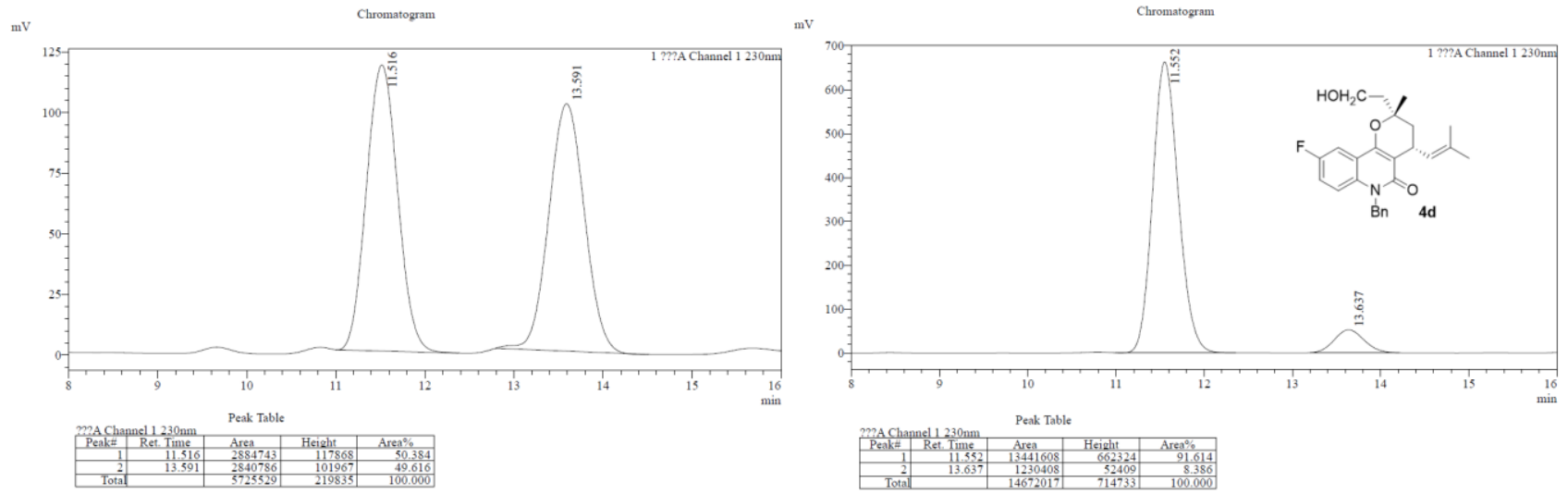

(2R,4R)-6-benzyl-9-fluoro-2-(2-hydroxyethyl)-2-methyl-4-(2-methylprop-1-en-1-yl)-2,3,4,6-tetrahydro-5H-pyrano [3,2-c]quinolin-5-one (4dd):

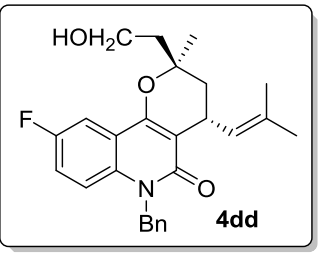

With catalyst 8e, $29 \%$ yield, $31 \mathrm{mg}$. White solid. $85 \%$ ee, determined by HPLC analysis [Chiralpak AD-H, $n$-hexane $/ i$-PrOH $=90 / 10,1.0 \mathrm{~mL} / \mathrm{min}, \lambda=230 \mathrm{~nm}, \mathrm{t}$ (major) $=10.79 \mathrm{~min}, \mathrm{t}$ $($ minor $)=18.05 \mathrm{~min}] .{ }^{1} \mathbf{H}$ NMR $\left(400 \mathrm{MHz}, \mathrm{CDCl}_{3}\right) \delta=7.58(\mathrm{dd}, J=8.8,2.8 \mathrm{~Hz}, 1 \mathrm{H}), 7.31-$ $7.24(\mathrm{~m}, 2 \mathrm{H}), 7.22-7.19(\mathrm{~m}, 1 \mathrm{H}), 7.18-7.05(\mathrm{~m}, 4 \mathrm{H}), 5.58(\mathrm{~d}, J=14.4 \mathrm{~Hz}, 1 \mathrm{H}), 5.42(\mathrm{~d}, J=$ $14.4 \mathrm{~Hz}, 1 \mathrm{H}), 5.12(\mathrm{~d}, J=8.4 \mathrm{~Hz}, 1 \mathrm{H}), 3.90-3.83(\mathrm{~m}, 2 \mathrm{H}), 3.83-3.77(\mathrm{~m}, 1 \mathrm{H}), 2.18-2.13(\mathrm{~m}$, 1H), $2.11-2.04(\mathrm{~m}, 1 \mathrm{H}), 1.99-1.92(\mathrm{~m}, 1 \mathrm{H}), 1.82-1.77(\mathrm{~m}, 4 \mathrm{H}), 1.74(\mathrm{~s}, 3 \mathrm{H}), 1.51$ (s, 3H). ${ }^{19} \mathbf{F ~ N M R}\left(376 \mathrm{MHz} \mathrm{CDCl}_{3}\right)$ $\delta=-121.2 .{ }^{13} \mathrm{C}$ NMR $\left(100 \mathrm{MHz}, \mathrm{CDCl}_{3}\right) \delta=162.4,157.8\left(\mathrm{~d}, J_{\mathrm{C}, \mathrm{F}}=239.9 \mathrm{~Hz}\right), 154.4\left(\mathrm{~d}, J_{\mathrm{C}, \mathrm{F}}=2.9 \mathrm{~Hz}\right), 136.8,134.8$, 131.7, 128.8, 127.2, 127.1, 126.5, $117.9\left(\mathrm{~d}, J_{\mathrm{C}, \mathrm{F}}=23.8 \mathrm{~Hz}\right), 116.3\left(\mathrm{~d}, J_{\mathrm{C}, \mathrm{F}}=7.9 \mathrm{~Hz}\right), 110.3,108.4\left(\mathrm{~d}, J_{\mathrm{C}, \mathrm{F}}=24 \mathrm{~Hz}\right), 78.4$ $58.7,45.9,40.8,39.4,29.0,26.1,25.9,18.0 .[\alpha]^{20} \mathbf{D}=+8.80^{\circ}\left(c=1.0, \mathrm{CHCl}_{3}, 85 \%\right.$ ee $) . \mathbf{H R M S}(\mathrm{ESI}) \mathrm{m} / \mathrm{z}:[\mathrm{M}+\mathrm{Na}]^{+} \mathrm{calcd}$ for $\mathrm{C}_{26} \mathrm{H}_{28} \mathrm{FNO}_{3} \mathrm{Na} 444.1945$, found 444.1954.
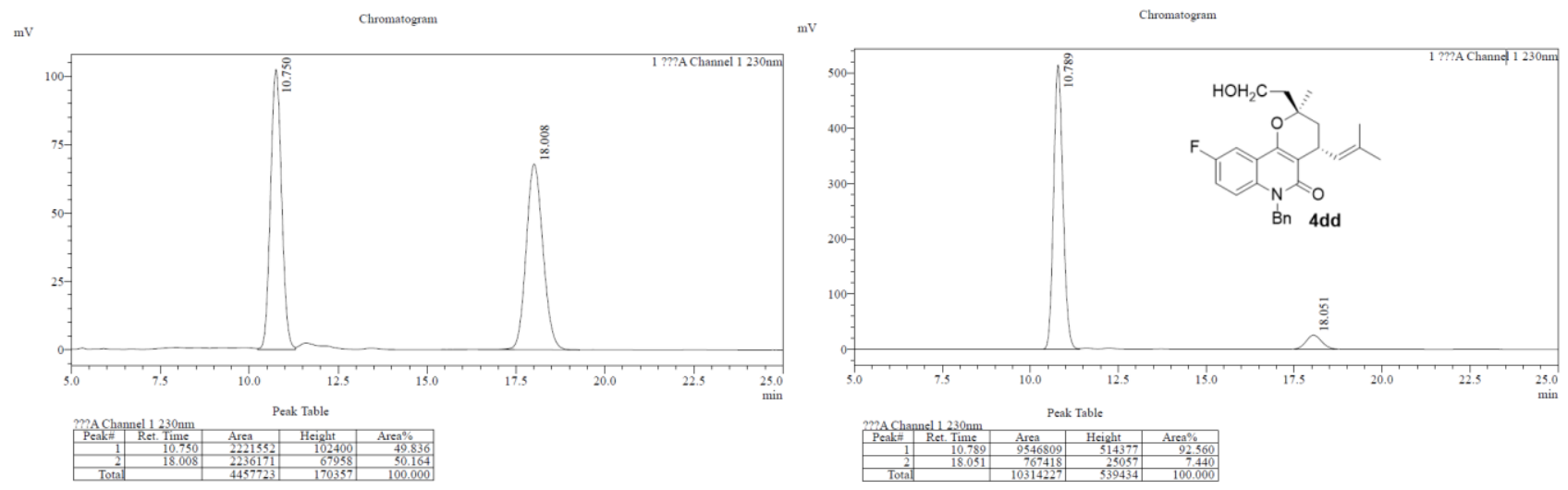
(2S,4R)-6-benzyl-9-chloro-2-(2-hydroxyethyl)-2-methyl-4-(2-methylprop-1-en-1-yl)-2,3,4,6-tetrahydro-5H-pyrano [3,2-c]quinolin-5-one (4e):

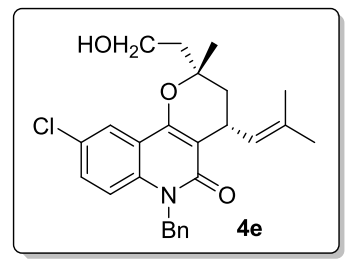

With catalyst 8e, $34 \%$ yield, $37 \mathrm{mg}$. White solid. $85 \%$ ee, determined by HPLC analysis [Chiralpak AD-H, $n$-hexane $/ i-\mathrm{PrOH}=90 / 10,1.0 \mathrm{~mL} / \mathrm{min}, \lambda=230 \mathrm{~nm}, \mathrm{t}$ (major) $=10.62 \mathrm{~min}, \mathrm{t}$ $($ minor $)=14.06 \mathrm{~min}] .{ }^{1} \mathbf{H}$ NMR $\left(400 \mathrm{MHz}, \mathrm{CDCl}_{3}\right) \delta=7.85(\mathrm{~d}, J=2.4 \mathrm{~Hz}, 1 \mathrm{H}), 7.34-7.23$ (m, 3H), $7.22-7.18(\mathrm{~m}, 1 \mathrm{H}), 7.14-7.09(\mathrm{~m}, 3 \mathrm{H}), 5.49$ (dd, $J=39.6,15.2 \mathrm{~Hz}, 2 \mathrm{H}), 5.07$ (d, $J$ $=8.4 \mathrm{~Hz}, 1 \mathrm{H}), 3.95(\mathrm{t}, J=6.8 \mathrm{~Hz}, 2 \mathrm{H}), 3.78(\mathrm{dd}, J=16.0,8.4 \mathrm{~Hz}, 1 \mathrm{H}), 2.13-2.05(\mathrm{~m}, 2 \mathrm{H})$, $2.05-1.98(\mathrm{~m}, 1 \mathrm{H}), 1.89-1.82(\mathrm{~m}, 1 \mathrm{H}), 1.80(\mathrm{~s}, 3 \mathrm{H}), 1.75(\mathrm{~s}, 3 \mathrm{H}), 1.42(\mathrm{~s}, 3 \mathrm{H}) .{ }^{13} \mathbf{C} \mathbf{N M R}$ $\left(100 \mathrm{MHz}, \mathrm{CDCl}_{3}\right) \delta=162.3,154.4,136.8,136.6,131.8,130.2,128.8,127.3,127.2,126.9,126.4,122.4,117.9,116.1$, $110.3,78.7,58.7,45.7,43.4,39.0,29.0,25.9,23.3,18.0 .[\alpha]^{20} \mathbf{D}=+6.32^{\circ}\left(c=1.0, \mathrm{CHCl}_{3}, 85 \%\right.$ ee $) . \mathbf{H R M S}(\mathrm{ESI}) \mathrm{m} / \mathrm{z}:$ $[\mathrm{M}+\mathrm{H}]^{+}$calcd. for $\mathrm{C}_{26} \mathrm{H}_{29} \mathrm{ClNO}_{3} 438.1830$, found 438.1836 .
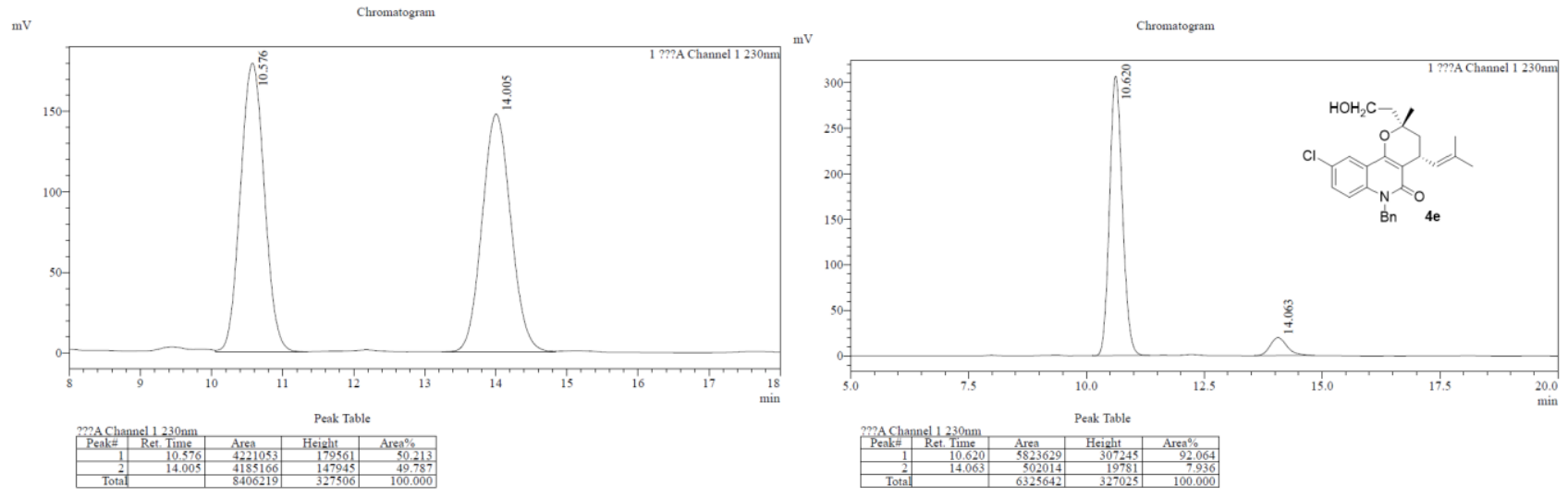

(2R,4R)-6-benzyl-9-chloro-2-(2-hydroxyethyl)-2-methyl-4-(2-methylprop-1-en-1-yl)-2,3,4,6-tetrahydro-5H-pyrano [3,2-c]quinolin-5-one (4ee):

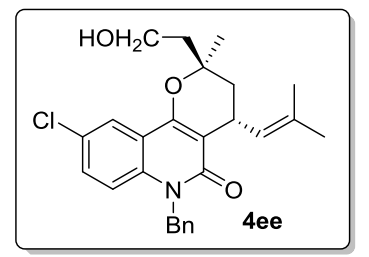

With catalyst 8e, 27\% yield, $30 \mathrm{mg}$. White solid. $85 \%$ ee, determined by HPLC analysis [Chiralpak AD-H, $n$-hexane $/ i$-PrOH $=90 / 10,1.0 \mathrm{~mL} / \mathrm{min}, \lambda=230 \mathrm{~nm}, \mathrm{t}$ (major) $=10.79 \mathrm{~min}$, $\mathrm{t}$ (minor) $=18.06 \mathrm{~min}] .{ }^{1} \mathbf{H}$ NMR $\left(400 \mathrm{MHz}, \mathrm{CDCl}_{3}\right) \delta=7.88(\mathrm{~d}, J=2.4 \mathrm{~Hz}, 1 \mathrm{H}), 7.34-7.23$ $(\mathrm{m}, 3 \mathrm{H}), 7.22-7.18(\mathrm{~m}, 1 \mathrm{H}), 7.15-7.09(\mathrm{~m}, 3 \mathrm{H}), 5.56(\mathrm{~d}, J=12.4 \mathrm{~Hz}, 1 \mathrm{H}), 5.39(\mathrm{~d}, J=12.4$ $\mathrm{Hz}, 1 \mathrm{H}), 5.10(\mathrm{~d}, J=8.4 \mathrm{~Hz}, 1 \mathrm{H}), 3.89-3.82(\mathrm{~m}, 2 \mathrm{H}), 3.82-3.76(\mathrm{~m}, 1 \mathrm{H}), 2.16(\mathrm{dd}, J=14.4$, $7.2 \mathrm{~Hz}, 1 \mathrm{H}), 2.10-2.03(\mathrm{~m}, 1 \mathrm{H}), 1.99-1.92(\mathrm{~m}, 1 \mathrm{H}), 1.82-1.78(\mathrm{~m}, 4 \mathrm{H}), 1.74(\mathrm{~s}, 3 \mathrm{H}), 1.51(\mathrm{~s}, 3 \mathrm{H}) .{ }^{13} \mathbf{C ~ N M R}(100$ $\left.\mathrm{MHz}, \mathrm{CDCl}_{3}\right) \delta=162.4,154.3,136.8,136.6,131.8,130.2,128.8,127.3,127.2,127.1,126.5,122.4,117.8,116.2,110.3$, $78.5,58.7,45.8,40.7,39.3,29.0,26.1,25.8,18.0 .[\alpha]^{20} \mathbf{D}=+6.21^{\circ}\left(c=1.0, \mathrm{CHCl}_{3}, 85 \%\right.$ ee $) . \mathbf{H R M S}(\mathrm{ESI}) \mathrm{m} / \mathrm{z}:[\mathrm{M}+\mathrm{H}]^{+}$ calcd. for $\mathrm{C}_{26} \mathrm{H}_{29} \mathrm{ClNO}_{3} 438.1830$, found 438.1832 .
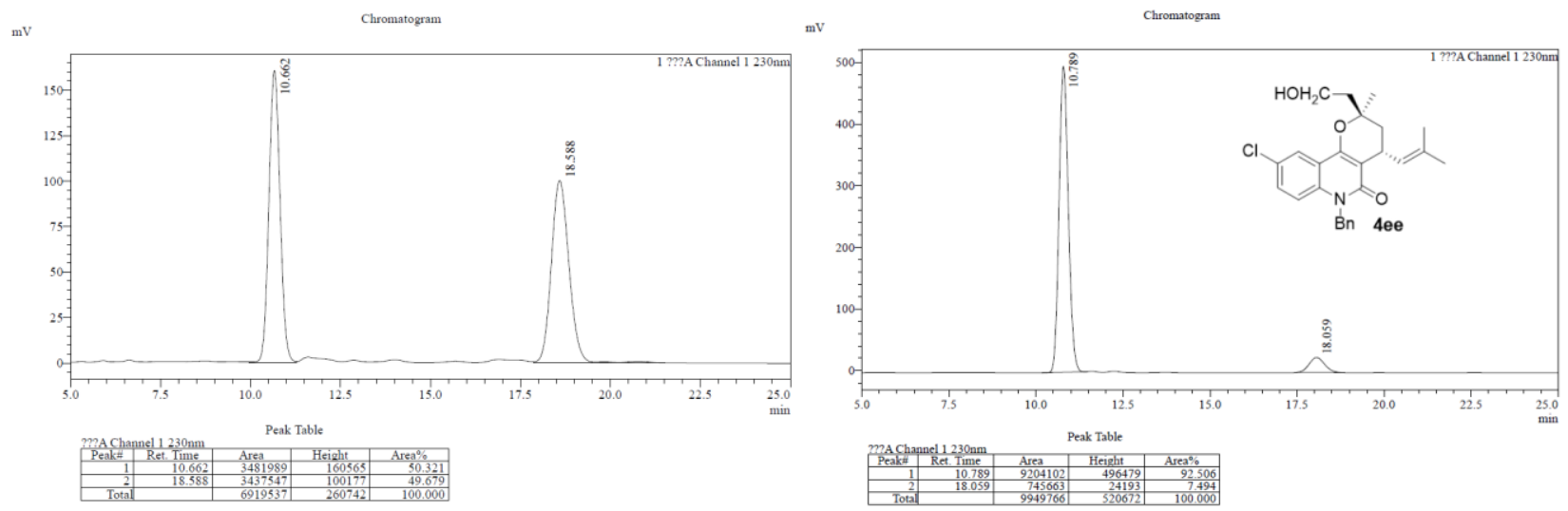
(2S,4R)-6-benzyl-9-bromo-2-(2-hydroxyethyl)-2-methyl-4-(2-methylprop-1-en-1-yl)-2,3,4,6-tetrahydro-5H-pyrano [3,2-c]quinolin-5-one (4f):

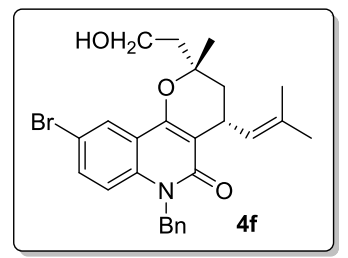

With catalyst 8e, 37\% yield, $45 \mathrm{mg}$. White solid. $87 \%$ ee, determined by HPLC analysis [Chiralpak AD-H, $n$-hexane $/ i$-PrOH $=90 / 10,1.0 \mathrm{~mL} / \mathrm{min}, \lambda=230 \mathrm{~nm}, \mathrm{t}$ (major) $=11.19 \mathrm{~min}, \mathrm{t}$ (minor) $=16.06 \mathrm{~min}] .{ }^{1} \mathbf{H}$ NMR $\left(400 \mathrm{MHz}, \mathrm{CDCl}_{3}\right) \delta=7.99(\mathrm{~d}, J=2.4 \mathrm{~Hz}, 1 \mathrm{H}), 7.43(\mathrm{dd}, J=$ 9.2, $2.4 \mathrm{~Hz}, 1 \mathrm{H}), 7.28-7.25(\mathrm{~m}, 2 \mathrm{H}), 7.22-7.18(\mathrm{~m}, 1 \mathrm{H}), 7.13(\mathrm{~d}, J=7.2 \mathrm{~Hz}, 2 \mathrm{H}), 7.04(\mathrm{~d}, J$ $=8.8 \mathrm{~Hz}, 1 \mathrm{H}), 5.53(\mathrm{~d}, J=15.2 \mathrm{~Hz}, 1 \mathrm{H}), 5.43(\mathrm{~d}, J=15.2 \mathrm{~Hz}, 1 \mathrm{H}), 5.07(\mathrm{~d}, J=8.4 \mathrm{~Hz}, 1 \mathrm{H})$, $3.95(\mathrm{t}, J=6.8 \mathrm{~Hz}, 2 \mathrm{H}), 3.78(\mathrm{dd}, J=15.6,8.0 \mathrm{~Hz}, 1 \mathrm{H}), 2.09(\mathrm{t}, J=6.4 \mathrm{~Hz}, 2 \mathrm{H}), 2.02(\mathrm{dd}, J=14.0,7.0 \mathrm{~Hz}, 1 \mathrm{H}), 1.89-$ $1.83(\mathrm{~m}, 1 \mathrm{H}), 1.80(\mathrm{~s}, 3 \mathrm{H}), 1.75(\mathrm{~s}, 3 \mathrm{H}), 1.42(\mathrm{~s}, 3 \mathrm{H}) .{ }^{13} \mathbf{C ~ N M R}\left(100 \mathrm{MHz}, \mathrm{CDCl}_{3}\right) \delta=162.3,154.3,137.2,136.5,133.0$, 131.8, 128.8, 127.2, 126.8, 126.4, 125.4, 118.3, 116.4, 114.6, 110.3, 78.7, 58.7, 45.7, 43.4, 38.9, 29.0, 25.9, 23.3, 18.0. $[\alpha]^{20} \mathbf{D}=+6.21^{\circ}\left(c=1.0, \mathrm{CHCl}_{3}, 87 \%\right.$ ee). HRMS (ESI) $\mathrm{m} / \mathrm{z}:[\mathrm{M}+\mathrm{Na}]^{+}$calcd. for $\mathrm{C}_{26} \mathrm{H}_{28} \mathrm{BrNO}_{3} \mathrm{Na} 504.1145$, found 504.1143.
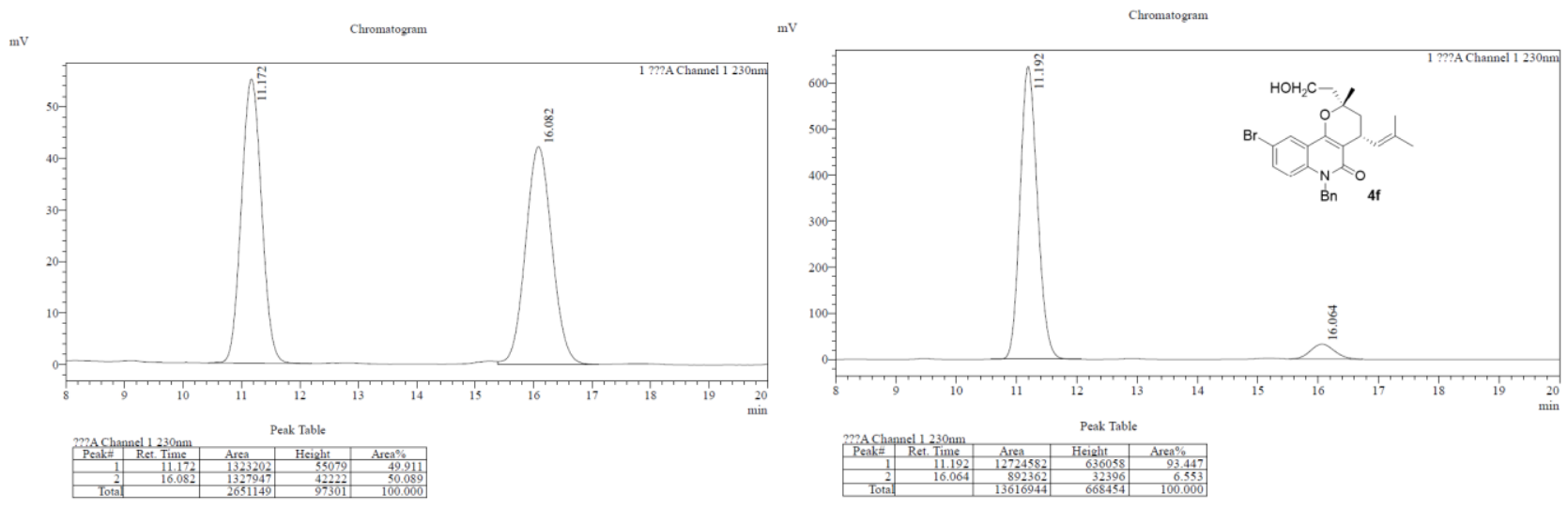

$(2 R, 4 R)-6$-benzyl-9-bromo-2-(2-hydroxyethyl)-2-methyl-4-(2-methylprop-1-en-1-yl)-2,3,4,6-tetrahydro-5H-pyrano [3,2-c]quinolin-5-one (4ff):

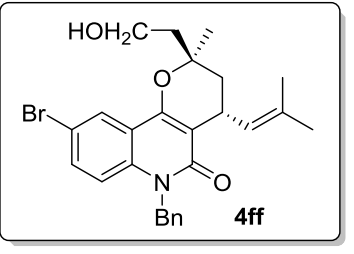

With catalyst $\mathbf{8 e}, 36 \%$ yield, $43 \mathrm{mg}$. White solid. $87 \%$ ee, determined by HPLC analysis [Chiralpak AD-H, $n$-hexane $/ i-\mathrm{PrOH}=90 / 10,1.0 \mathrm{~mL} / \mathrm{min}, \lambda=230 \mathrm{~nm}, \mathrm{t}$ (major) $=11.41 \mathrm{~min}$, $\mathrm{t}($ minor $)=19.92 \mathrm{~min}] .{ }^{1} \mathbf{H}$ NMR $\left(400 \mathrm{MHz} \mathrm{CDCl}_{3}\right) \delta=8.02(\mathrm{~d}, J=2.4 \mathrm{~Hz}, 1 \mathrm{H}), 7.43(\mathrm{dd}, J$ $=9.2,2.4 \mathrm{~Hz}, 1 \mathrm{H}), 7.29-7.25(\mathrm{~m}, 2 \mathrm{H}), 7.22-7.18(\mathrm{~m}, 1 \mathrm{H}), 7.14(\mathrm{~d}, J=7.2 \mathrm{~Hz}, 2 \mathrm{H}), 7.05(\mathrm{~d}$, $J=8.8 \mathrm{~Hz}, 1 \mathrm{H}), 5.55(\mathrm{~d}, J=15.2 \mathrm{~Hz}, 1 \mathrm{H}), 5.39(\mathrm{~d}, J=15.2 \mathrm{~Hz}, 1 \mathrm{H}), 5.10(\mathrm{~d}, J=8.0 \mathrm{~Hz}, 1 \mathrm{H})$, $3.92-3.83(\mathrm{~m}, 2 \mathrm{H}), 3.83-3.76(\mathrm{~m}, 1 \mathrm{H}), 2.16(\mathrm{dd}, J=14.0,7.0 \mathrm{~Hz}, 1 \mathrm{H}), 2.10-2.03(\mathrm{~m}, 1 \mathrm{H}), 1.99-1.92(\mathrm{~m}, 1 \mathrm{H}), 1.82-$ $1.76(\mathrm{~m}, 4 \mathrm{H}), 1.74(\mathrm{~s}, 3 \mathrm{H}), 1.51(\mathrm{~s}, 3 \mathrm{H}) .{ }^{13} \mathrm{C} \mathrm{NMR}\left(100 \mathrm{MHz}, \mathrm{CDCl}_{3}\right) \delta=162.4,154.2,137.2,136.6,133.0,131.8,128.8$, $127.2,127.1,126.5,125.4,118.3,116.4,114.7,110.3,78.5,58.7,45.7,40.7,39.4,29.0,26.2,25.8,18.0 .[\alpha]^{20} \mathrm{D}=+5.02^{\circ}$ $\left(c=1.0, \mathrm{CHCl}_{3}, 87 \%\right.$ ee). HRMS (ESI) m/z: $[\mathrm{M}+\mathrm{H}]^{+}$calcd. for $\mathrm{C}_{26} \mathrm{H}_{29} \mathrm{BrNO}_{3} 482.1325$, found 482.1346 .
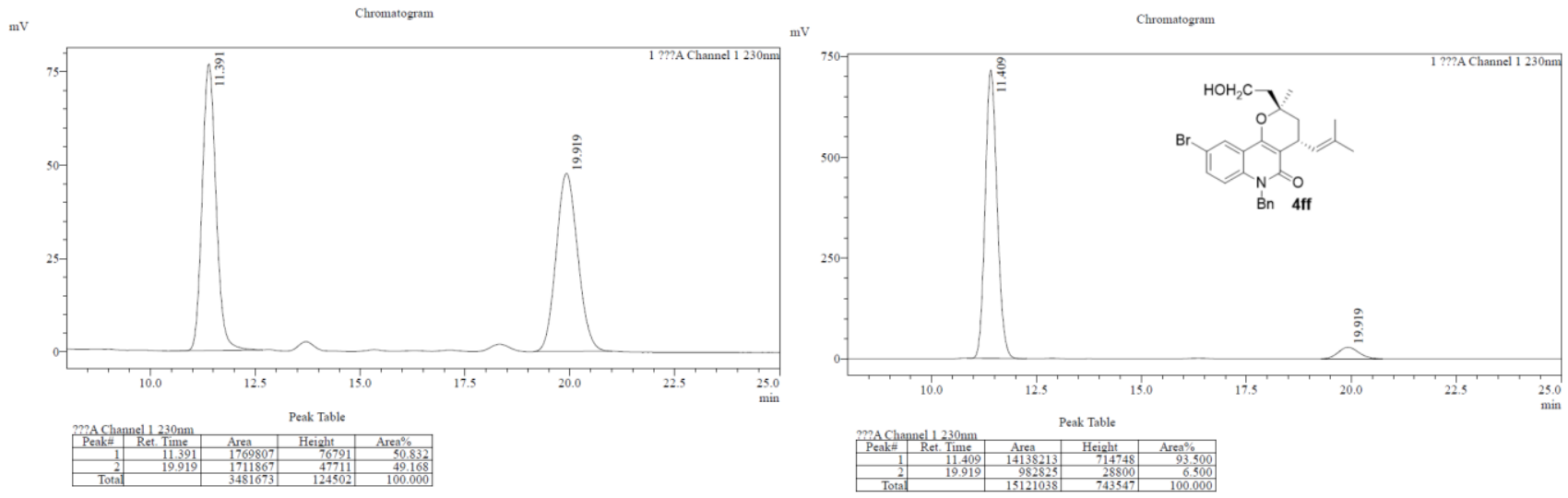
(2S,4R)-6-benzyl-2-(2-hydroxyethyl)-2-methyl-4-(2-methylprop-1-en-1-yl)-9-(trifluoromethyl)-2,3,4,6-tetrahydro-5 H-pyrano[3,2-c]quinolin-5-one (4g):

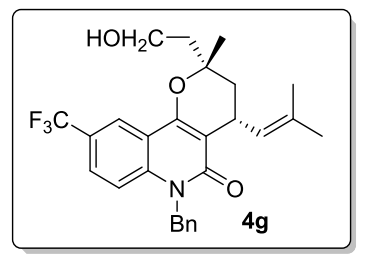

With catalyst 8e, 39\% yield, $46 \mathrm{mg}$. White solid. 89\% ee, determined by HPLC analysis [Chiralpak AD-H, $n$-hexane $/ i-\mathrm{PrOH}=90 / 10,1.0 \mathrm{~mL} / \mathrm{min}, \lambda=230 \mathrm{~nm}, \mathrm{t}$ (major) $=7.63 \mathrm{~min}, \mathrm{t}$ (minor) $=9.17 \mathrm{~min}] .{ }^{1} \mathbf{H}$ NMR $\left(400 \mathrm{MHz}, \mathrm{CDCl}_{3}\right) \delta=8.15(\mathrm{~d}, J=1.2 \mathrm{~Hz}, 1 \mathrm{H}), 7.58(\mathrm{dd}, J=$ 8.8, $2.0 \mathrm{~Hz}, 1 \mathrm{H}), 7.31-7.13(\mathrm{~m}, 6 \mathrm{H}), 5.53(\mathrm{dd}, J=40.4,14.8 \mathrm{~Hz}, 2 \mathrm{H}), 5.07$ (d, $J=8.4 \mathrm{~Hz}$, $1 \mathrm{H}), 3.96(\mathrm{t}, J=6.8 \mathrm{~Hz}, 2 \mathrm{H}), 3.79(\mathrm{dd}, J=16.0,8.4 \mathrm{~Hz}, 1 \mathrm{H}), 2.14-2.08(\mathrm{~m}, 2 \mathrm{H}), 2.05(\mathrm{dd}, J$ $=14.0,7.0 \mathrm{~Hz}, 1 \mathrm{H}), 1.89-1.84(\mathrm{~m}, 1 \mathrm{H}), 1.82(\mathrm{~s}, 3 \mathrm{H}), 1.76(\mathrm{~s}, 3 \mathrm{H}), 1.44(\mathrm{~s}, 3 \mathrm{H}) .{ }^{19} \mathbf{F ~ N M R}\left(376 \mathrm{MHz}, \mathrm{CDCl}_{3}\right) \delta=-61.7$. ${ }^{13} \mathbf{C ~ N M R}\left(100 \mathrm{MHz}, \mathrm{CDCl}_{3}\right) \delta=162.6,154.9,140.3,136.3,132.0,128.8,127.3,126.6\left(\mathrm{q}, J_{\mathrm{C}, \mathrm{F}}=6.7 \mathrm{~Hz}\right), 126.4,125.5$, $123.7\left(\mathrm{q}, J_{\mathrm{C}, \mathrm{F}}=66.2 \mathrm{~Hz}\right), 120.6\left(\mathrm{q}, J_{\mathrm{C}, \mathrm{F}}=8.0 \mathrm{~Hz}\right), 116.6,115.1,110.4,78.9,58.6,45.8,43.4,38.9,29.0,25.8,23.3,18.0$ $[\boldsymbol{\alpha}]^{\mathbf{2 0}} \mathbf{D}=+13.81^{\circ}\left(c=1.0, \mathrm{CHCl}_{3}, 89 \%\right.$ ee). HRMS (ESI) $\mathrm{m} / \mathrm{z}:[\mathrm{M}+\mathrm{Na}]^{+}$calcd. for $\mathrm{C}_{27} \mathrm{H}_{28} \mathrm{~F}_{3} \mathrm{NO}_{3} \mathrm{Na} 494.1914$, found 494.1890.

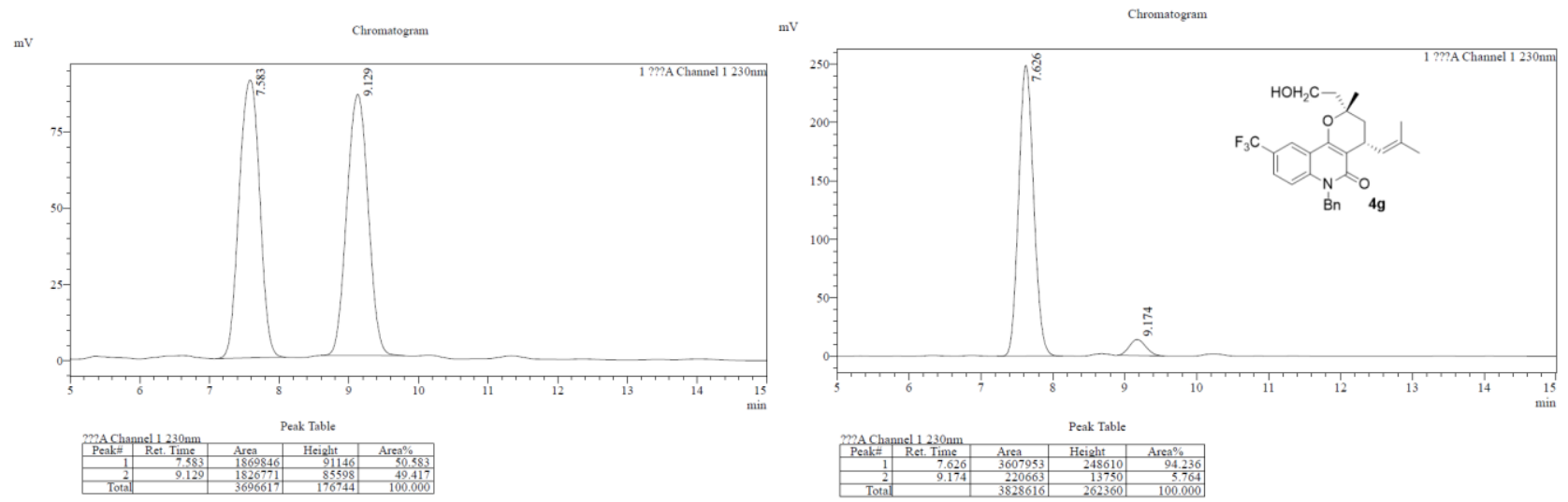

(2R,4R)-6-benzyl-2-(2-hydroxyethyl)-2-methyl-4-(2-methylprop-1-en-1-yl)-9-(trifluoromethyl)-2,3,4,6-tetrahydro-5 H-pyrano[3,2-c]quinolin-5-one (4gg):

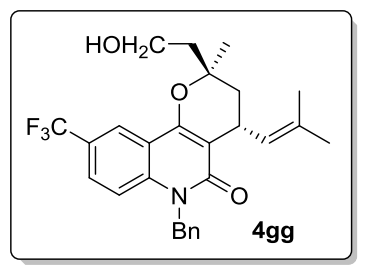

With catalyst 8e, 34\% yield, $40 \mathrm{mg}$. White solid. $87 \%$ ee, determined by HPLC analysis [Chiralpak AD-H, $n$-hexane $/ i-\mathrm{PrOH}=90 / 10,1.0 \mathrm{~mL} / \mathrm{min}, \lambda=230 \mathrm{~nm}, \mathrm{t}$ (major) $=8.18 \mathrm{~min}, \mathrm{t}$ $($ minor $)=12.90 \mathrm{~min}] .{ }^{1} \mathbf{H}$ NMR $\left(400 \mathrm{MHz}, \mathrm{CDCl}_{3}\right) \delta=8.17(\mathrm{~s}, 1 \mathrm{H}), 7.65-7.51(\mathrm{~m}, 1 \mathrm{H}), 7.32$ $-7.13(\mathrm{~m}, 6 \mathrm{H}), 5.60(\mathrm{~d}, J=14.4 \mathrm{~Hz}, 1 \mathrm{H}), 5.43(\mathrm{~d}, J=15.6 \mathrm{~Hz}, 1 \mathrm{H}), 5.10(\mathrm{~d}, J=8.4 \mathrm{~Hz}, 1 \mathrm{H})$, $3.93-3.75(\mathrm{~m}, 3 \mathrm{H}), 2.18(\mathrm{dd}, J=14.2,7.1 \mathrm{~Hz}, 1 \mathrm{H}), 2.08(\mathrm{dd}, J=13.9,7.0 \mathrm{~Hz}, 1 \mathrm{H}), 1.98(\mathrm{dt}$, $J=14.0,6.8 \mathrm{~Hz}, 1 \mathrm{H}), 1.84-1.77(\mathrm{~m}, 4 \mathrm{H}), 1.75(\mathrm{~s}, 3 \mathrm{H}), 1.54(\mathrm{~s}, 3 \mathrm{H}) .{ }^{19} \mathbf{F}$ NMR $(376 \mathrm{MHz}$, $\left.\mathrm{CDCl}_{3}\right) \delta=-61.7 .{ }^{13} \mathrm{C} \mathrm{NMR}\left(100 \mathrm{MHz}, \mathrm{CDCl}_{3}\right) \delta=162.6,154.8,140.3,136.4,132.0,128.8,127.3,126.9,126.6(\mathrm{q}, J \mathrm{C}, \mathrm{F}$ $=6.8 \mathrm{~Hz}), 126.5,123.4\left(\mathrm{q}, J_{\mathrm{C}, \mathrm{F}}=33 \mathrm{~Hz}\right), 120.6\left(\mathrm{q}, J_{\mathrm{C}, \mathrm{F}}=8.0 \mathrm{~Hz}\right), 116.5,115.1,110.5,78.8,58.7,45.9,40.7,39.4,29.0$, 26.2, 25.8, 18.0. $[\boldsymbol{\alpha}]^{20} \mathbf{D}=+15.36^{\circ}\left(c=1.0, \mathrm{CHCl}_{3}, 87 \%\right.$ ee). HRMS (ESI) m/z: $[\mathrm{M}+\mathrm{Na}]^{+}$calcd. for $\mathrm{C}_{27} \mathrm{H}_{28} \mathrm{~F}_{3} \mathrm{NO}_{3} \mathrm{Na}$ 494.1914, found 494.1900.

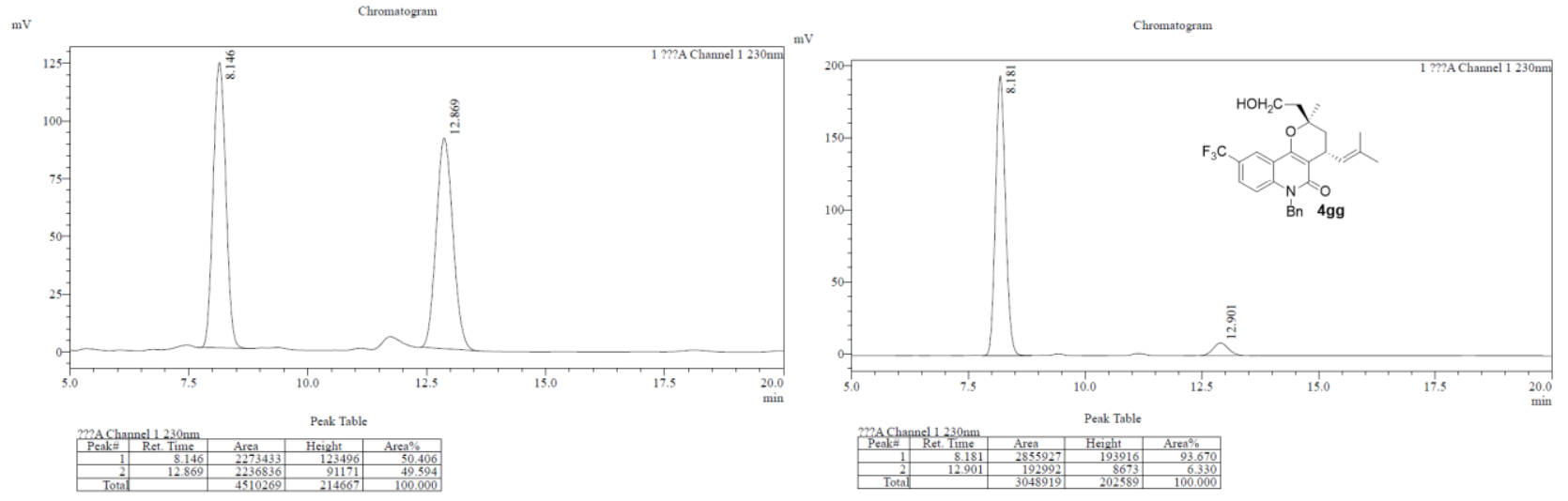


(2S,4R)-6-benzyl-2-(2-hydroxyethyl)-2-methyl-4-(2-methylprop-1-en-1-yl)-9-nitro-2,3,4,6-tetrahydro-5H-pyrano[3, 2-c]quinolin-5-one (4h):

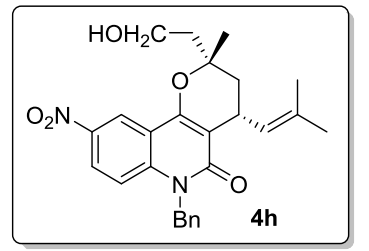

With catalyst 8e, $25 \%$ yield, $28 \mathrm{mg}$. White solid. $73 \%$ ee, determined by HPLC analysis [Chiralpak OD-H, $n$-hexane $/ i$-PrOH $=90 / 10,1.0 \mathrm{~mL} / \mathrm{min}, \lambda=230 \mathrm{~nm}, \mathrm{t}$ (major) $=26.87 \mathrm{~min}$, $\mathrm{t}$ (minor) $=45.24 \mathrm{~min}] .{ }^{1} \mathbf{H}$ NMR $\left(400 \mathrm{MHz}, \mathrm{CDCl}_{3}\right) \delta=8.77(\mathrm{~d}, J=2.8 \mathrm{~Hz}, 1 \mathrm{H}), 8.19(\mathrm{dd}, J$ $=9.6,2.8 \mathrm{~Hz}, 1 \mathrm{H}), 7.33-7.20(\mathrm{~m}, 4 \mathrm{H}), 7.16(\mathrm{~d}, J=7.2 \mathrm{~Hz}, 2 \mathrm{H}), 5.59(\mathrm{~d}, J=15.2 \mathrm{~Hz}, 1 \mathrm{H})$, $5.48(\mathrm{~d}, J=15.2 \mathrm{~Hz}, 1 \mathrm{H}), 5.06(\mathrm{~d}, J=8.4 \mathrm{~Hz}, 1 \mathrm{H}), 3.97(\mathrm{t}, J=6.8 \mathrm{~Hz}, 2 \mathrm{H}), 3.79(\mathrm{dd}, J=16.0$, $8.4 \mathrm{~Hz}, 1 \mathrm{H}), 2.16-2.04(\mathrm{~m}, 3 \mathrm{H}), 1.90-1.80(\mathrm{~m}, 1 \mathrm{H}), 1.82(\mathrm{~s}, 3 \mathrm{H}), 1.76(\mathrm{~s}, 3 \mathrm{H}), 1.46(\mathrm{~s}, 3 \mathrm{H}) .{ }^{13} \mathbf{C ~ N M R}\left(100 \mathrm{MHz}, \mathrm{CDCl}_{3}\right)$ $\delta=162.4,154.9,142.2,141.8,135.9,132.4,128.9,127.5,126.4,126.2,124.9,119.6,116.6,115.2,110.9,79.3,58.6,46.1$, $43.3,38.8,29.0,25.8,23.4,18.0 .[\alpha]^{20} \mathbf{D}=+11.53^{\circ}\left(c=1.0, \mathrm{CHCl}_{3}, 73 \%\right.$ ee $) . \mathbf{H R M S}(\mathrm{ESI}) \mathrm{m} / \mathrm{z}:[\mathrm{M}+\mathrm{Na}]^{+}$calcd. for $\mathrm{C}_{26} \mathrm{H}_{28} \mathrm{~N}_{2} \mathrm{O}_{5} \mathrm{Na} 471.1890$, found 471.1874 .
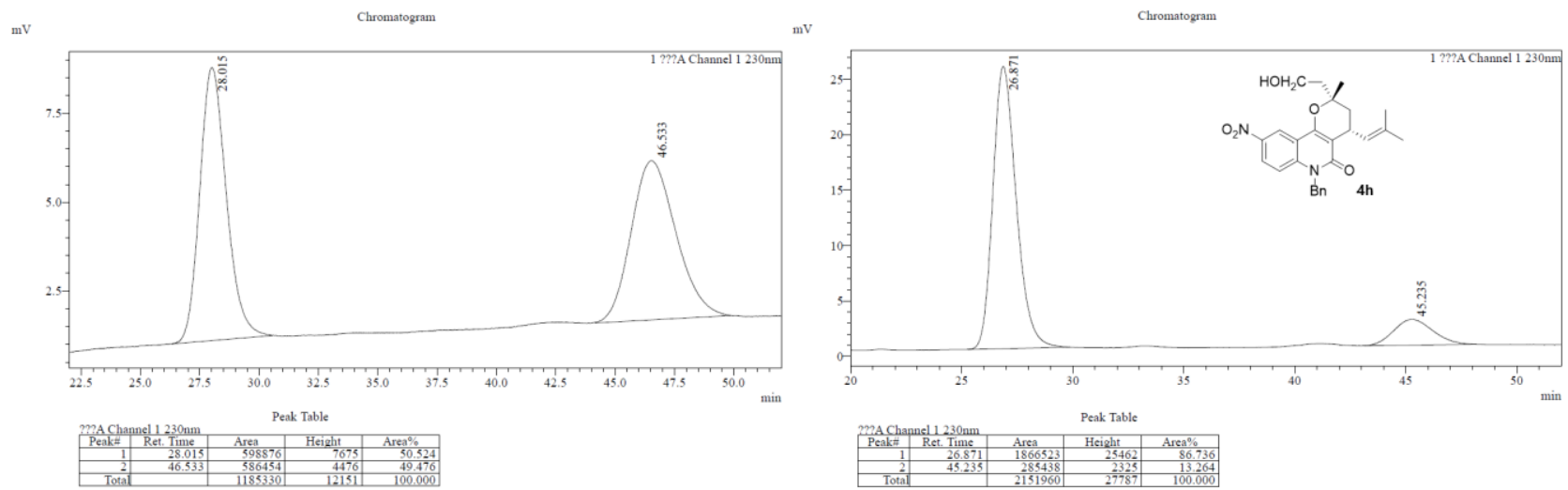

(2R,4R)-6-benzyl-2-(2-hydroxyethyl)-2-methyl-4-(2-methylprop-1-en-1-yl)-9-nitro-2,3,4,6-tetrahydro-5H-pyrano[3, 2-c]quinolin-5-one (4hh):

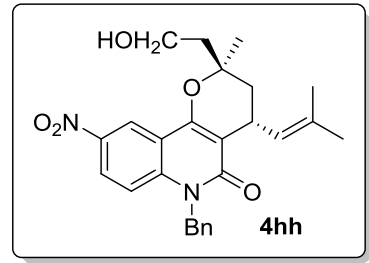

With catalyst 8e, $20 \%$ yield, $23 \mathrm{mg}$. White solid. $68 \%$ ee, determined by HPLC analysis [Chiralpak AD-H, $n$-hexane $/ i$-PrOH $=90 / 10,1.0 \mathrm{~mL} / \mathrm{min}, \lambda=230 \mathrm{~nm}, \mathrm{t}$ (major) $=19.89 \mathrm{~min}$, $\mathrm{t}($ minor $)=32.60 \mathrm{~min}] .{ }^{1} \mathbf{H}$ NMR $\left(400 \mathrm{MHz} \mathrm{CDCl}_{3}\right) \delta=8.79(\mathrm{~d}, J=2.4 \mathrm{~Hz}, 1 \mathrm{H}), 8.19(\mathrm{dd}, J$ $=9.2,2.4 \mathrm{~Hz}, 1 \mathrm{H}), 7.33-7.20(\mathrm{~m}, 4 \mathrm{H}), 7.16(\mathrm{~d}, J=7.2 \mathrm{~Hz}, 2 \mathrm{H}), 5.62(\mathrm{~d}, J=15.6 \mathrm{~Hz}, 1 \mathrm{H})$, $5.44(\mathrm{~d}, J=15.6 \mathrm{~Hz}, 1 \mathrm{H}), 5.08(\mathrm{~d}, J=8.0 \mathrm{~Hz}, 1 \mathrm{H}), 3.95-3.85(\mathrm{~m}, 2 \mathrm{H}), 3.81(\mathrm{dd}, J=15.6$, $7.6 \mathrm{~Hz}, 1 \mathrm{H}), 2.20(\mathrm{dd}, J=14.4,7.2 \mathrm{~Hz}, 1 \mathrm{H}), 2.12-2.05(\mathrm{~m}, 1 \mathrm{H}), 2.05-1.95(\mathrm{~m}, 1 \mathrm{H}), 1.86-$ $1.78(\mathrm{~m}, 4 \mathrm{H}), 1.75(\mathrm{~s}, 3 \mathrm{H}), 1.56(\mathrm{~s}, 3 \mathrm{H}) .{ }^{13} \mathbf{C ~ N M R}\left(100 \mathrm{MHz}, \mathrm{CDCl}_{3}\right) \delta=162.5,154.8,142.2,141.9,135.9,132.4,128.9$, $127.5,126.4,126.4,124.9,119.6,116.6,115.2,111.0,79.2,58.6,46.1,40.5,39.3,29.0,26.2,25.8,18.0 .[\alpha]^{20} \mathbf{D}=+11.01^{\circ}$ $\left(c=1.0, \mathrm{CHCl}_{3}, 68 \%\right.$ ee). HRMS (ESI) m/z: $[\mathrm{M}+\mathrm{H}]^{+}$calcd. for $\mathrm{C}_{26} \mathrm{H}_{29} \mathrm{~N}_{2} \mathrm{O}_{5} 449.2071$, found 449.2088.
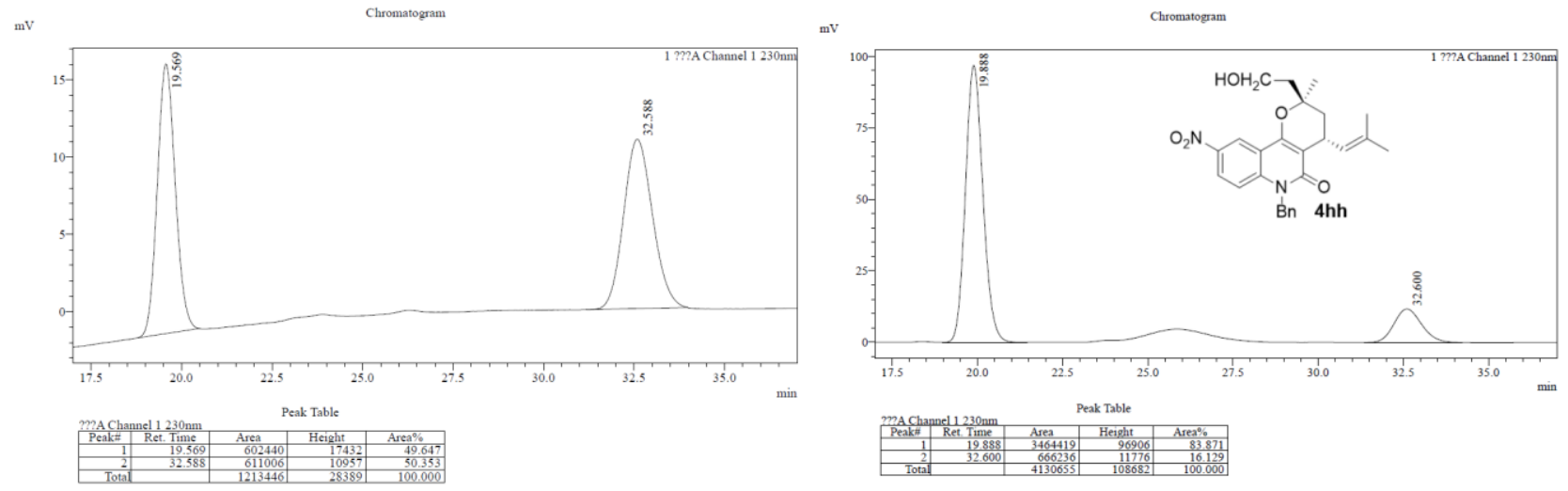
(2S,4R)-6-benzyl-2-(2-hydroxyethyl)-2,9-dimethyl-4-(2-methylprop-1-en-1-yl)-2,3,4,6-tetrahydro-5H-pyrano[3,2-c] quinolin-5-one (4i):

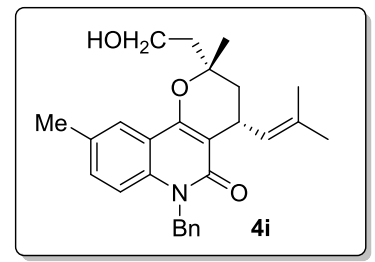

With catalyst 8e, $32 \%$ yield, $33 \mathrm{mg}$. White solid. 90\% ee, determined by HPLC analysis [Chiralpak AD-H, $n$-hexane $/ i$-PrOH $=90 / 10,1.0 \mathrm{~mL} / \mathrm{min}, \lambda=230 \mathrm{~nm}, \mathrm{t}$ (major) $=11.06 \mathrm{~min}$, $\mathrm{t}($ minor $)=18.70 \mathrm{~min}] .{ }^{1} \mathbf{H}$ NMR $\left(400 \mathrm{MHz}^{\mathrm{C}} \mathrm{CDCl}_{3}\right) \delta=7.66(\mathrm{~s}, 1 \mathrm{H}), 7.28-7.23(\mathrm{~m}, 4 \mathrm{H})$, $7.21-7.14(\mathrm{~m}, 4 \mathrm{H}), 7.08(\mathrm{~d}, J=8.4 \mathrm{~Hz}, 1 \mathrm{H}), 5.51(\mathrm{dd}, J=30.8,16.8 \mathrm{~Hz}, 2 \mathrm{H}), 5.10(\mathrm{~d}, J=$ $8.4 \mathrm{~Hz}, 1 \mathrm{H}), 3.98(\mathrm{t}, J=6.8 \mathrm{~Hz}, 2 \mathrm{H}), 3.79(\mathrm{dd}, J=16.0,8.4 \mathrm{~Hz}, 1 \mathrm{H}), 2.37(\mathrm{~s}, 3 \mathrm{H}), 2.10(\mathrm{t}, J=$ $6.4 \mathrm{~Hz}, 2 \mathrm{H}), 2.01(\mathrm{dd}, J=14.0,6.8 \mathrm{~Hz}, 1 \mathrm{H}), 1.89-1.82(\mathrm{~m}, 1 \mathrm{H}), 1.80(\mathrm{~s}, 3 \mathrm{H}), 1.75(\mathrm{~s}, 3 \mathrm{H})$, $1.43(\mathrm{~s}, 3 \mathrm{H}) .{ }^{13} \mathrm{C}$ NMR $\left(100 \mathrm{MHz}, \mathrm{CDCl}_{3}\right) \delta=162.6,155.1,137.1,136.3,131.5,131.4,131.1,128.6,127.4,126.9,126.5$, $122.5,116.6,114.7,109.3,78.4,58.9,45.6,43.4,39.1,29.0,25.9,23.3,20.8,18.0 .[\alpha]^{20} \mathrm{D}=+9.61^{\circ}\left(c=1.0, \mathrm{CHCl}_{3}, 90 \%\right.$ ee). HRMS (ESI) m/z: [M+H] ${ }^{+}$calcd. for $\mathrm{C}_{27} \mathrm{H}_{32} \mathrm{NO}_{3} 418.2377$, found 418.2382.
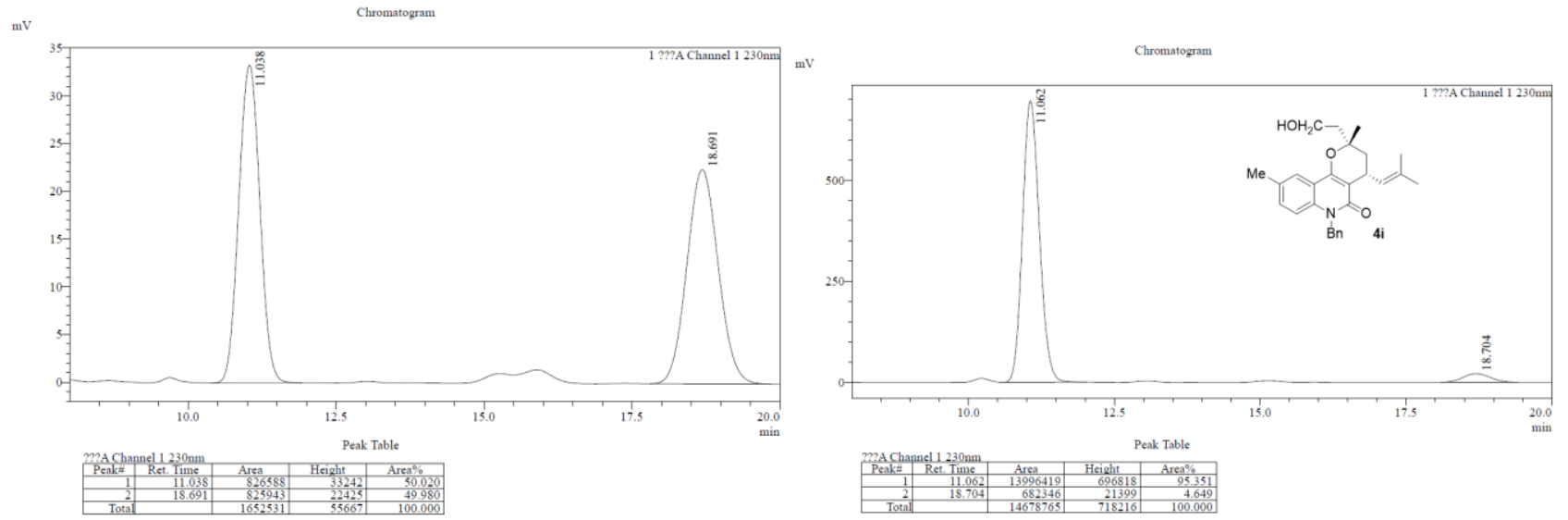

(2R,4R)-6-benzyl-2-(2-hydroxyethyl)-2,9-dimethyl-4-(2-methylprop-1-en-1-yl)-2,3,4,6-tetrahydro-5H-pyrano[3,2-c] quinolin-5-one (4ii):

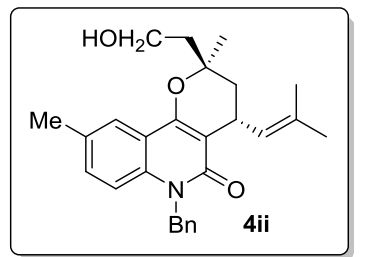

With catalyst 8e, $32 \%$ yield, $33 \mathrm{mg}$. White solid. $90 \%$ ee, determined by HPLC analysis [Chiralpak AD-H, $n$-hexane $/ i-\mathrm{PrOH}=90 / 10,1.0 \mathrm{~mL} / \mathrm{min}, \lambda=230 \mathrm{~nm}, \mathrm{t}$ (major) $=10.09 \mathrm{~min}$, $\mathrm{t}$ (minor) $=17.06 \mathrm{~min}] .{ }^{1} \mathbf{H}$ NMR $\left(400 \mathrm{MHz}, \mathrm{CDCl}_{3}\right) \delta=7.70(\mathrm{~s}, 1 \mathrm{H}), 7.30-7.23(\mathrm{~m}, 2 \mathrm{H})$, $7.20-7.15(\mathrm{~m}, 4 \mathrm{H}), 7.09(\mathrm{~d}, J=8.4 \mathrm{~Hz}, 1 \mathrm{H}), 5.58(\mathrm{~d}, J=14.0 \mathrm{~Hz}, 1 \mathrm{H}), 5.42$ (d, $J=14.0 \mathrm{~Hz}$, $1 \mathrm{H}), 5.13(\mathrm{~d}, J=8.0 \mathrm{~Hz}, 1 \mathrm{H}), 3.89(\mathrm{t}, J=6.4 \mathrm{~Hz}, 2 \mathrm{H}), 3.81(\mathrm{dd}, J=14.8,7.6 \mathrm{~Hz}, 1 \mathrm{H}), 2.37(\mathrm{~s}$, $3 \mathrm{H}), 2.18-2.06(\mathrm{~m}, 2 \mathrm{H}), 2.00-1.92(\mathrm{~m}, 1 \mathrm{H}), 1.82-1.76(\mathrm{~m}, 4 \mathrm{H}), 1.74(\mathrm{~s}, 3 \mathrm{H}), 1.52(\mathrm{~s}, 3 \mathrm{H})$. ${ }^{13} \mathrm{C} \mathrm{NMR}\left(100 \mathrm{MHz}, \mathrm{CDCl}_{3}\right) \delta=162.7,155.0,137.2,136.3,131.5,131.4,131.1,128.6,127.8,126.9,126.5,122.5,116.5$, 114.7, 109.3, 78.2, 58.9, 45.6, 40.8, 39.5, 29.0, 26.1, 25.9, 20.8, 18.0. $[\boldsymbol{\alpha}]^{\mathbf{2 0}} \mathbf{D}=+9.70^{\circ}\left(c=1.0, \mathrm{CHCl}_{3}, 90 \%\right.$ ee $) . \mathbf{H R M S}$ (ESI) $\mathrm{m} / \mathrm{z}:[\mathrm{M}+\mathrm{Na}]^{+}$calcd. for $\mathrm{C}_{27} \mathrm{H}_{31} \mathrm{NO}_{3} \mathrm{Na} 440.2196$, found 440.2200 .
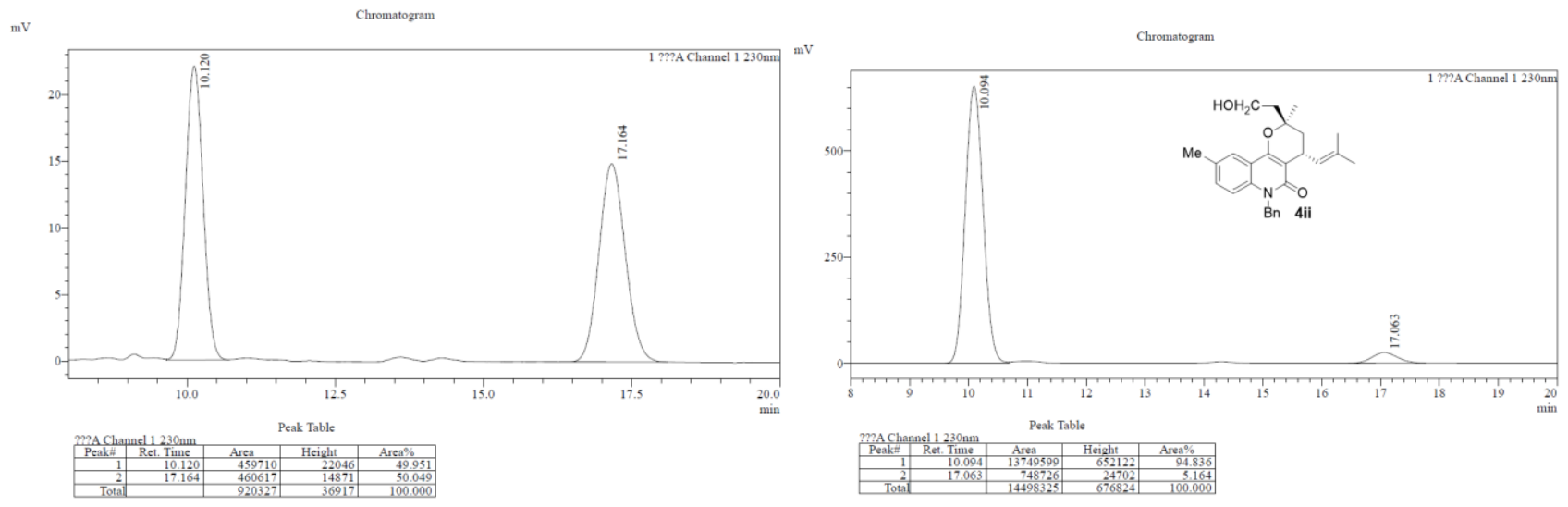
(2S,4R)-6-benzyl-2-(2-hydroxyethyl)-9-isopropyl-2-methyl-4-(2-methylprop-1-en-1-yl)-2,3,4,6-tetrahydro-5H-pyra no[3,2-c]quinolin-5-one $(4 \mathbf{j})$ :

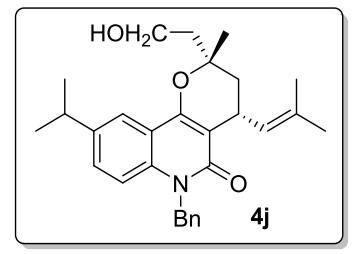

With catalyst 8e, 29\% yield, $32 \mathrm{mg}$. White solid. 93\% ee, determined by HPLC analysis [Chiralpak AD-H, $n$-hexane $/ i-\mathrm{PrOH}=90 / 10,1.0 \mathrm{~mL} / \mathrm{min}, \lambda=230 \mathrm{~nm}, \mathrm{t}$ (major) $=8.69 \mathrm{~min}, \mathrm{t}$ $($ minor $)=16.75 \mathrm{~min}] .{ }^{1} \mathbf{H}$ NMR $\left(400 \mathrm{MHz}, \mathrm{CDCl}_{3}\right) \delta=7.70(\mathrm{~d}, J=2.0 \mathrm{~Hz}, 1 \mathrm{H}), 7.30-7.23$ $(\mathrm{m}, 3 \mathrm{H}), 7.23-7.10(\mathrm{~m}, 4 \mathrm{H}), 5.51(\mathrm{~s}, 2 \mathrm{H}), 5.08(\mathrm{~d}, J=8.0 \mathrm{~Hz}, 1 \mathrm{H}), 3.98(\mathrm{t}, J=6.0 \mathrm{~Hz}, 2 \mathrm{H})$, $3.79(\mathrm{dd}, J=16.4,8.8 \mathrm{~Hz}, 1 \mathrm{H}), 2.99-2.90(\mathrm{~m}, 1 \mathrm{H}), 2.13-2.07(\mathrm{~m}, 2 \mathrm{H}), 2.01(\mathrm{dd}, J=14.0$, $7.2 \mathrm{~Hz}, 1 \mathrm{H}), 1.88-1.83(\mathrm{~m}, 1 \mathrm{H}), 1.81(\mathrm{~d}, J=1.2 \mathrm{~Hz}, 3 \mathrm{H}), 1.75(\mathrm{~s}, 3 \mathrm{H}), 1.43(\mathrm{~s}, 3 \mathrm{H}), 1.24(\mathrm{~d}, J=7.2 \mathrm{~Hz}, 7 \mathrm{H}) .{ }^{13} \mathbf{C ~ N M R}$ $\left(100 \mathrm{MHz}, \mathrm{CDCl}_{3}\right) \delta=162.6,155.3,142.1,137.2,136.6,131.3,128.9,128.6,127.4,126.9,126.5,120.0,116.5,114.8$, $109.2,78.5,58.9,45.6,43.4,39.2,33.6,29.0,25.9,24.1,24.0,23.1,18.0 .[\alpha]^{20} \mathbf{D}=+15.23^{\circ}\left(c=1.0, \mathrm{CHCl}_{3}, 93 \%\right.$ ee $)$. HRMS (ESI) m/z: [M+H] ${ }^{+}$calcd. for $\mathrm{C}_{29} \mathrm{H}_{36} \mathrm{NO}_{3} 446.2690$, found 446.2692.
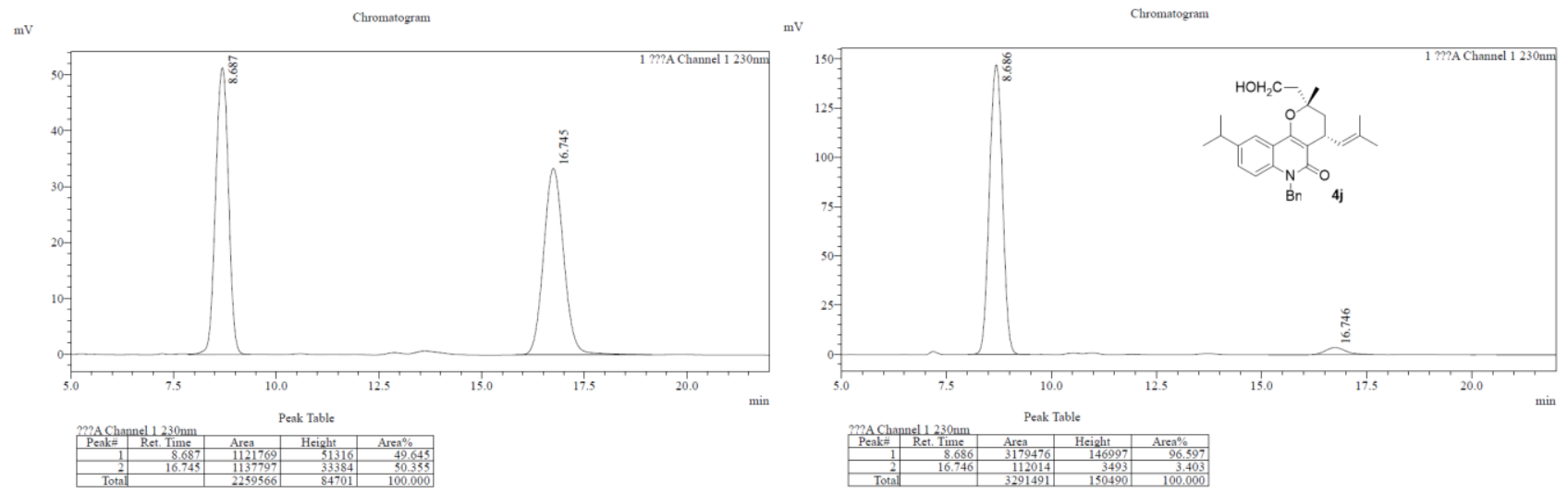

$(2 R, 4 R)$-6-benzyl-2-(2-hydroxyethyl)-9-isopropyl-2-methyl-4-(2-methylprop-1-en-1-yl)-2,3,4,6-tetrahydro-5H-pyra no[3,2-c]quinolin-5-one $(4 \mathbf{j j})$ :

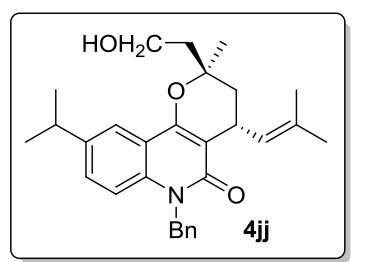

With catalyst $\mathbf{8 e}, 27 \%$ yield, $30 \mathrm{mg}$. White solid. $87 \%$ ee, determined by HPLC analysis [Chiralpak AD-H, $n$-hexane $/ i-\mathrm{PrOH}=90 / 10,1.0 \mathrm{~mL} / \mathrm{min}, \lambda=230 \mathrm{~nm}, \mathrm{t}$ (major) $=7.28 \mathrm{~min}, \mathrm{t}$ $($ minor $)=11.95 \mathrm{~min}] .{ }^{1} \mathbf{H} \mathbf{N M R}\left(400 \mathrm{MHz} \mathrm{CDCl}_{3}\right) \delta=7.73(\mathrm{~d}, J=2.0 \mathrm{~Hz}, 1 \mathrm{H}), 7.30-7.24$ $(\mathrm{m}, 3 \mathrm{H}), 7.23-7.11(\mathrm{~m}, 4 \mathrm{H}), 5.56(\mathrm{~d}, J=15.6 \mathrm{~Hz}, 1 \mathrm{H}), 5.43(\mathrm{~d}, J=14.0 \mathrm{~Hz}, 1 \mathrm{H}), 5.19-5.08$ $(\mathrm{m}, 1 \mathrm{H}), 3.89(\mathrm{t}, J=6.8 \mathrm{~Hz}, 2 \mathrm{H}), 3.82(\mathrm{q}, J=7.4 \mathrm{~Hz}, 1 \mathrm{H}), 3.00-2.90(\mathrm{~m}, 1 \mathrm{H}), 2.19-2.06(\mathrm{~m}$, $2 \mathrm{H}), 2.00-1.92(\mathrm{~m}, 1 \mathrm{H}), 1.85-1.77(\mathrm{~m}, 4 \mathrm{H}), 1.74(\mathrm{~s}, 3 \mathrm{H}), 1.53(\mathrm{~s}, 3 \mathrm{H}), 1.26(\mathrm{~s}, 3 \mathrm{H}), 1.24(\mathrm{~s}$, 3H). ${ }^{13} \mathrm{C} \mathrm{NMR}\left(100 \mathrm{MHz}, \mathrm{CDCl}_{3}\right) \delta=162.7,155.2,142.2,137.2,136.6,131.4,128.8,128.6,127.8,127.0,126.6,120.0$, $116.5,114.8,109.2,78.2,58.9,45.7,40.8,39.6,33.6,29.0,26.1,25.9,24.1,18.1 . \quad[\alpha]^{20} \mathbf{D}=+6.23^{\circ}\left(c=1.0, \mathrm{CHCl}_{3}, 87 \%\right.$ ee). HRMS (ESI) m/z: [M+H] ${ }^{+}$calcd. for $\mathrm{C}_{29} \mathrm{H}_{36} \mathrm{NO}_{3} 446.2690$, found 446.2694 .
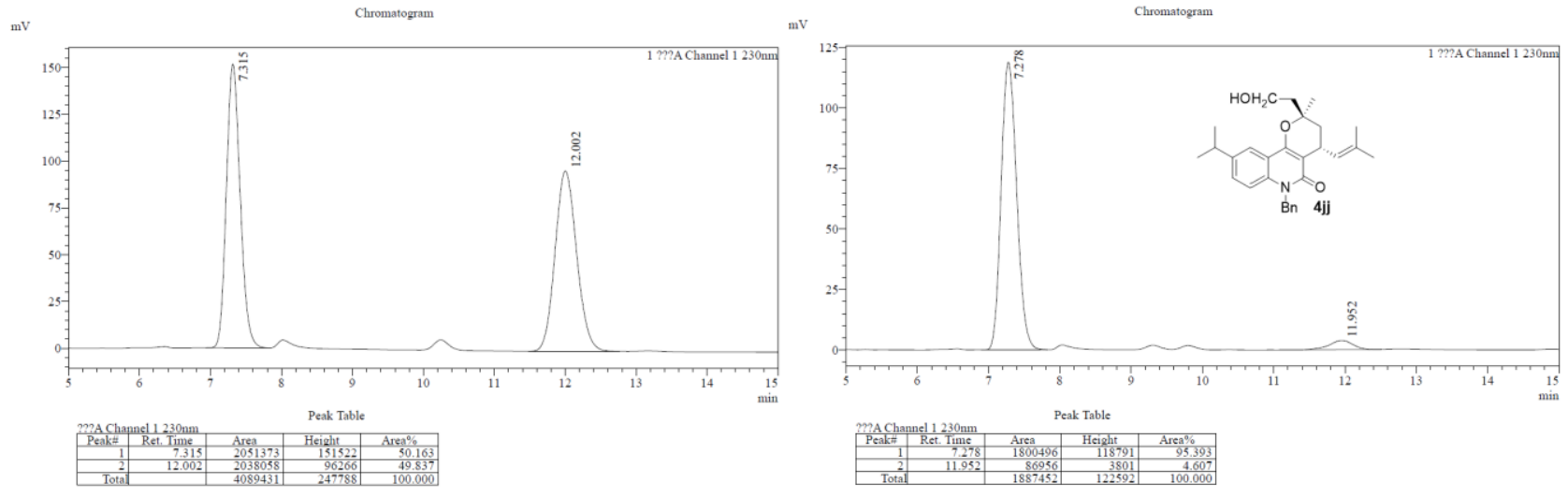
(2S,4R)-6-benzyl-9-(tert-butyl)-2-(2-hydroxyethyl)-2-methyl-4-(2-methylprop-1-en-1-yl)-2,3,4,6-tetrahydro-5H-pyr ano[3,2-c]quinolin-5-one (4k):

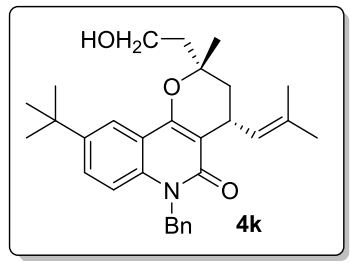

With catalyst 8e, $28 \%$ yield, $32 \mathrm{mg}$. White solid. 91\% ee, determined by HPLC analysis [Chiralpak AD-H, $n$-hexane $/ i$-PrOH $=90 / 10,1.0 \mathrm{~mL} / \mathrm{min}, \lambda=230 \mathrm{~nm}, \mathrm{t}$ (major) $=8.05 \mathrm{~min}, \mathrm{t}$ (minor) $=10.12 \mathrm{~min}] .{ }^{1} \mathbf{H} \mathbf{~ N M R}\left(400 \mathrm{MHz}, \mathrm{CDCl}_{3}\right) \delta=7.85(\mathrm{~d}, J=2.4 \mathrm{~Hz}, 1 \mathrm{H}), 7.43(\mathrm{dd}, J=$ 9.2, $2.4 \mathrm{~Hz}, 1 \mathrm{H}), 7.29-7.24(\mathrm{~m}, 2 \mathrm{H}), 7.21-7.12(\mathrm{~m}, 4 \mathrm{H}), 5.51(\mathrm{~s}, 2 \mathrm{H}), 5.08$ (d, J = 8.4 Hz, $1 \mathrm{H}), 4.00-3.95(\mathrm{~m}, 2 \mathrm{H}), 3.80(\mathrm{dd}, J=16.0,8.4 \mathrm{~Hz}, 1 \mathrm{H}), 2.15-1.97(\mathrm{~m}, 3 \mathrm{H}), 1.87-1.83(\mathrm{~m}$, 1H), $1.81(\mathrm{~s}, 3 \mathrm{H}), 1.75(\mathrm{~s}, 3 \mathrm{H}), 1.44(\mathrm{~s}, 3 \mathrm{H}), 1.32(\mathrm{~s}, 9 \mathrm{H}),{ }^{13} \mathbf{C ~ N M R}\left(100 \mathrm{MHz}, \mathrm{CDCl}_{3}\right) \delta=$ 162.6, 155.4, 144.4, 137.2, 136.3, 131.3, 128.6, 128.1, 127.4, 127.0, 126.6, 118.6, 116.1, 114.5, 109.2, 78.5, 58.9, 45.6, 43.5, 39.4, 34.4, 31.3, 29.0, 25.9, 23.0, 18.0. $[\mathbf{\alpha}]^{\mathbf{2 0}} \mathbf{D}=+4.66^{\circ}\left(c=1.0, \mathrm{CHCl}_{3}, 91 \%\right.$ ee $) . \mathbf{H R M S}(\mathrm{ESI}) \mathrm{m} / \mathrm{z}:[\mathrm{M}+\mathrm{H}]^{+} \mathrm{calcd}$. for $\mathrm{C}_{30} \mathrm{H}_{38} \mathrm{NO}_{3} 460.2846$, found 460.2838 .
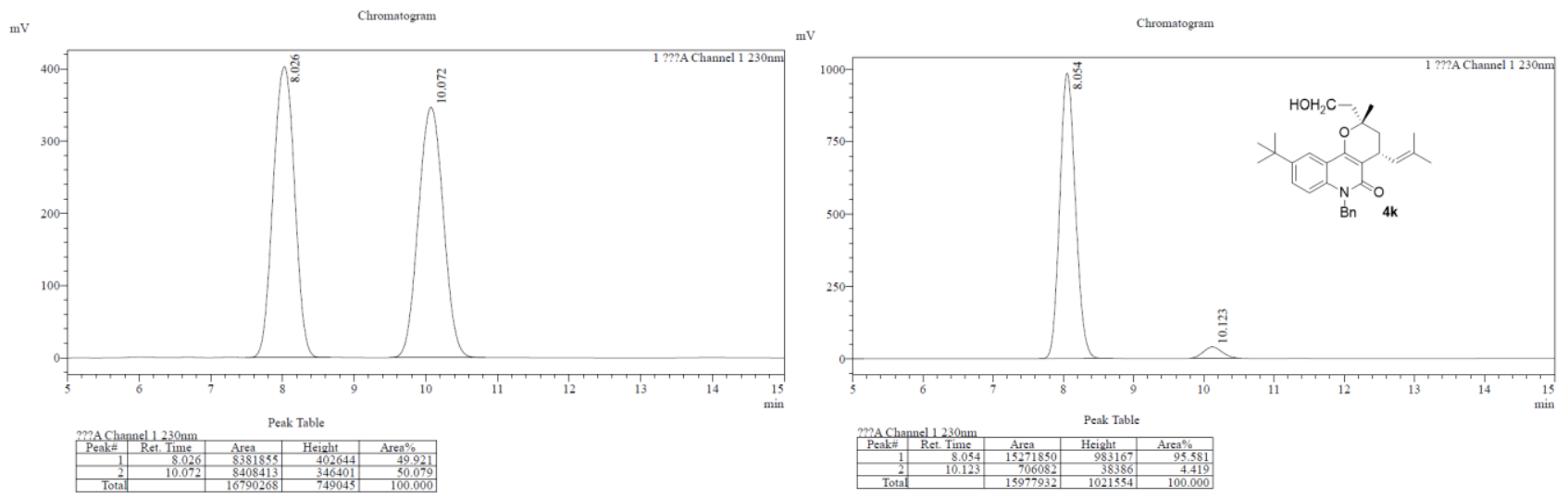

(2R,4R)-6-benzyl-9-(tert-butyl)-2-(2-hydroxyethyl)-2-methyl-4-(2-methylprop-1-en-1-yl)-2,3,4,6-tetrahydro-5H-py rano[3,2-c]quinolin-5-one (4kk):

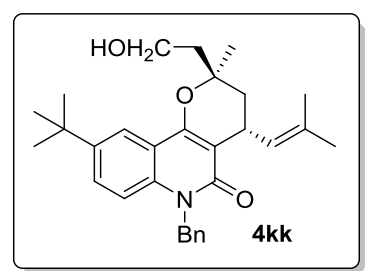

With catalyst 8e, $23 \%$ yield, $26 \mathrm{mg}$. White solid. 85\% ee, determined by HPLC analysis [Chiralpak AD-H, $n$-hexane $/ i-\mathrm{PrOH}=90 / 10,1.0 \mathrm{~mL} / \mathrm{min}, \lambda=230 \mathrm{~nm}, \mathrm{t}$ (major) $=7.73 \mathrm{~min}, \mathrm{t}$ (minor) $=10.08 \mathrm{~min}] .{ }^{1} \mathbf{H}$ NMR $\left(400 \mathrm{MHz}, \mathrm{CDCl}_{3}\right) \delta=7.88(\mathrm{~d}, J=2.4 \mathrm{~Hz}, 1 \mathrm{H}), 7.43(\mathrm{dd}, J=$ 9.2, $2.4 \mathrm{~Hz}, 1 \mathrm{H}), 7.29-7.25(\mathrm{~m}, 2 \mathrm{H}), 7.22-7.11(\mathrm{~m}, 4 \mathrm{H}), 5.56(\mathrm{~d}, J=15.6 \mathrm{~Hz}, 1 \mathrm{H}), 5.44(\mathrm{~d}$, $J=14.4 \mathrm{~Hz}, 1 \mathrm{H}), 5.14(\mathrm{~d}, J=8.0 \mathrm{~Hz}, 1 \mathrm{H}), 3.89(\mathrm{t}, J=6.8 \mathrm{~Hz}, 2 \mathrm{H}), 3.82(\mathrm{dd}, J=14.8,7.2 \mathrm{~Hz}$, $1 \mathrm{H}), 2.20-2.06(\mathrm{~m}, 2 \mathrm{H}), 2.01-1.91(\mathrm{~m}, 1 \mathrm{H}), 1.85-1.77(\mathrm{~m}, 4 \mathrm{H}), 1.74(\mathrm{~s}, 3 \mathrm{H}), 1.53(\mathrm{~s}, 3 \mathrm{H})$, $1.33(\mathrm{~s}, 9 \mathrm{H}) .{ }^{13} \mathrm{C} \mathbf{N M R}\left(100 \mathrm{MHz}, \mathrm{CDCl}_{3}\right) \delta=162.8,155.3,144.5,137.2,136.3,131.4,128.7,128.1,127.9,127.0,126.6$, $118.6,116.1,114.5,109.2,78.2,59.0,45.7,41.0,39.7,34.4,31.4,29.0,26.0,25.9,18.1 .[\alpha]^{20} \mathbf{D}=+4.14^{\circ}\left(c=1.0, \mathrm{CHCl}_{3}\right.$, $85 \%$ ee). HRMS (ESI) m/z: $[\mathrm{M}+\mathrm{H}]^{+}$calcd. for $\mathrm{C}_{30} \mathrm{H}_{38} \mathrm{NO}_{3} 460.2846$, found 460.2864.
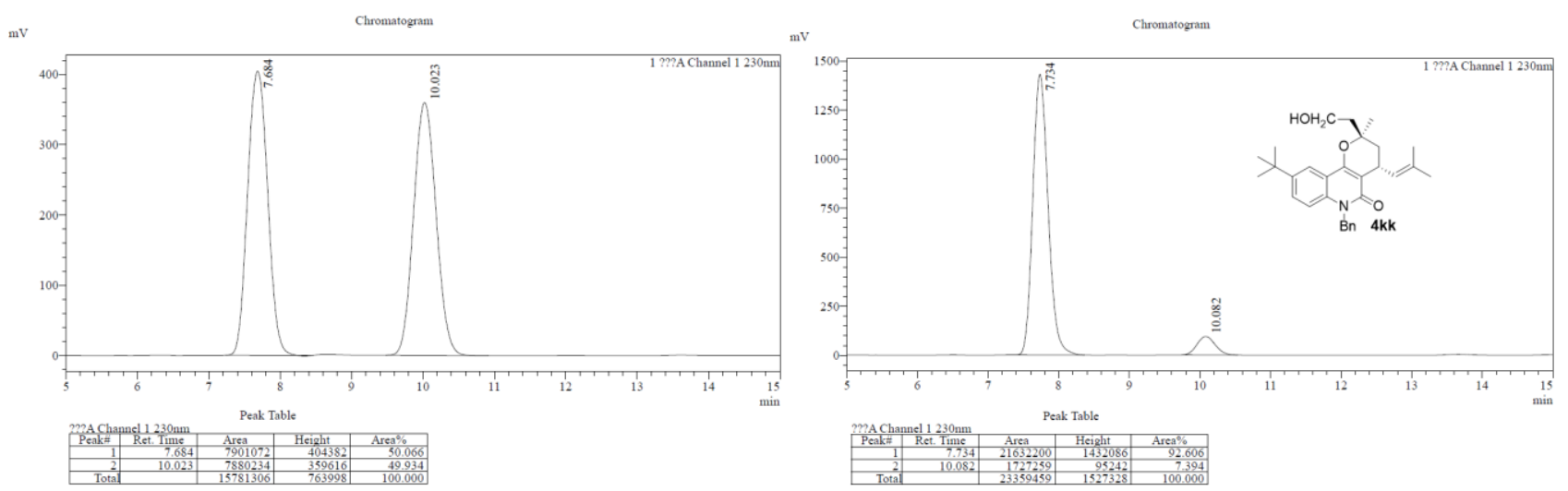
(2S,4R)-6-benzyl-2-(2-hydroxyethyl)-2-methyl-4-(2-methylprop-1-en-1-yl)-9-phenyl-2,3,4,6-tetrahydro-5H-pyrano [3,2-c]quinolin-5-one (4l):

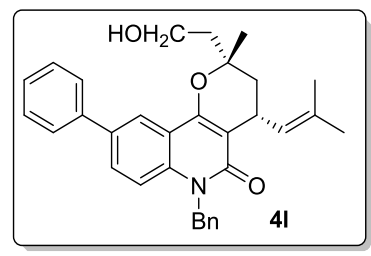

With catalyst $\mathbf{8 e}, 32 \%$ yield, $39 \mathrm{mg}$. White solid. $92 \%$ ee, determined by HPLC analysis [Chiralpak AD-H, $n$-hexane $/ i$-PrOH $=90 / 10,1.0 \mathrm{~mL} / \mathrm{min}, \lambda=230 \mathrm{~nm}, \mathrm{t}$ (major) $=13.74 \mathrm{~min}$, $\mathrm{t}($ minor $)=15.69 \mathrm{~min}] .{ }^{1} \mathbf{H}$ NMR $\left(400 \mathrm{MHz}, \mathrm{CDCl}_{3}\right) \delta=8.09(\mathrm{~d}, J=2.4 \mathrm{~Hz}, 1 \mathrm{H}), 7.65-7.55$ $(\mathrm{m}, 3 \mathrm{H}), 7.46-7.41(\mathrm{~m}, 2 \mathrm{H}), 7.36-7.25(\mathrm{~m}, 5 \mathrm{H}), 7.23-7.18(\mathrm{~m}, 3 \mathrm{H}), 5.56(\mathrm{dd}, J=34,17.6$ $\mathrm{Hz}, 2 \mathrm{H}), 5.11(\mathrm{~d}, J=8.4 \mathrm{~Hz}, 1 \mathrm{H}), 3.97(\mathrm{t}, J=6.4 \mathrm{~Hz}, 2 \mathrm{H}), 3.82(\mathrm{dd}, J=16.0,8.4 \mathrm{~Hz}, 1 \mathrm{H})$, $2.14-2.09(\mathrm{~m}, 2 \mathrm{H}), 2.04(\mathrm{dd}, J=14.0,7.0 \mathrm{~Hz}, 1 \mathrm{H}), 1.91-1.84(\mathrm{~m}, 1 \mathrm{H}), 1.82(\mathrm{~s}, 3 \mathrm{H}), 1.76(\mathrm{~s}, 3 \mathrm{H}), 1.44(\mathrm{~s}, 3 \mathrm{H}) .{ }^{\mathbf{1 3}} \mathrm{C}$ NMR $\left(100 \mathrm{MHz}, \mathrm{CDCl}_{3}\right) \delta=162.60,155.3,140.2,137.6,137.0,134.6,131.5,129.3,128.9,128.7,127.3,127.2,127.1$, 127.0, 126.5, 121.0, 117.0, 115.2, 109.6, 78.6, 58.8, 45.7, 43.4, 39.1, 29.0, 25.9, 23.3, 18.0. $[\alpha]^{\mathbf{2 0}} \mathbf{D}=+8.51^{\circ}\left(c=1.0, \mathrm{CHCl}_{3}\right.$, $92 \%$ ee). HRMS (ESI) m/z: $[\mathrm{M}+\mathrm{H}]^{+}$calcd. for $\mathrm{C}_{32} \mathrm{H}_{34} \mathrm{NO}_{3} 480.2533$, found 480.2527 .
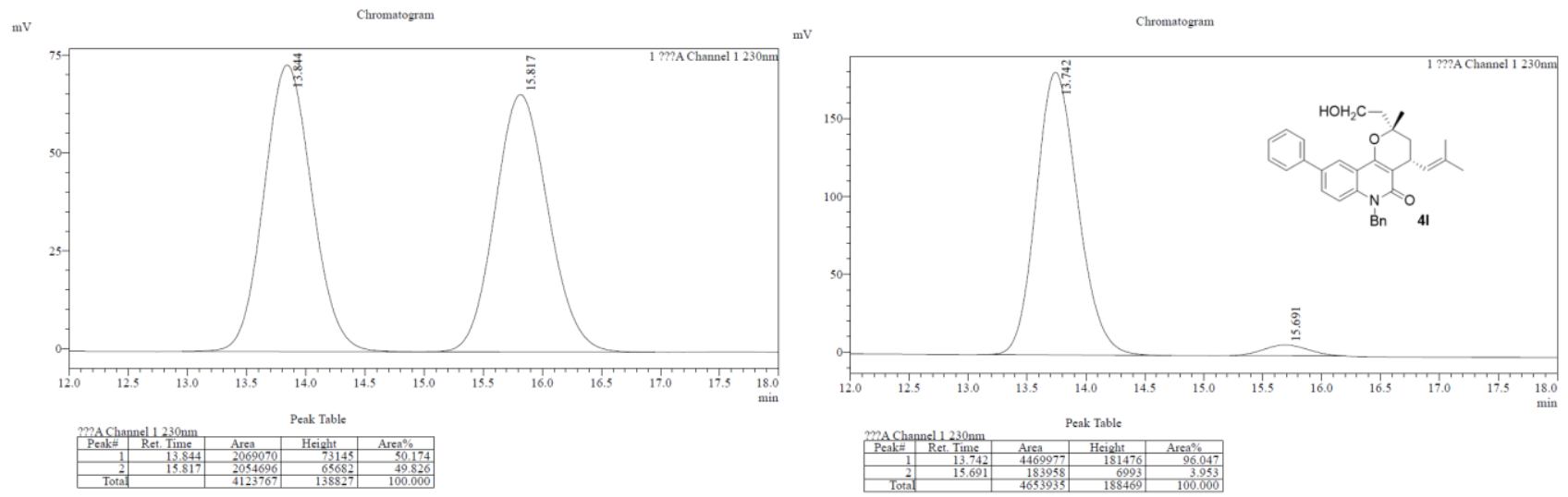

(2R,4R)-6-benzyl-2-(2-hydroxyethyl)-2-methyl-4-(2-methylprop-1-en-1-yl)-9-phenyl-2,3,4,6-tetrahydro-5H-pyrano [3,2-c]quinolin-5-one (4l1):

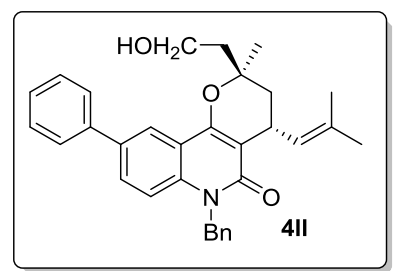

With catalyst $\mathbf{8 e}, 26 \%$ yield, $31 \mathrm{mg}$. White solid. $85 \%$ ee, determined by HPLC analysis [Chiralpak AD-H, $n$-hexane $/ i-\mathrm{PrOH}=90 / 10,1.0 \mathrm{~mL} / \mathrm{min}, \lambda=230 \mathrm{~nm}, \mathrm{t}$ (major) $=11.71$ $\min , \mathrm{t}($ minor $)=18.42 \mathrm{~min}] .{ }^{1} \mathbf{H}$ NMR $\left(400 \mathrm{MHz}, \mathrm{CDCl}_{3}\right) \delta=8.12(\mathrm{~d}, J=2.0 \mathrm{~Hz}, 1 \mathrm{H}), 7.64$ $-7.56(\mathrm{~m}, 3 \mathrm{H}), 7.44(\mathrm{t}, J=7.2 \mathrm{~Hz}, 2 \mathrm{H}), 7.37-7.19(\mathrm{~m}, 7 \mathrm{H}), 5.62(\mathrm{~d}, J=14.4 \mathrm{~Hz}, 1 \mathrm{H}), 5.47$ $(\mathrm{d}, J=14.4 \mathrm{~Hz}, 1 \mathrm{H}), 5.15(\mathrm{~d}, J=8.4 \mathrm{~Hz}, 1 \mathrm{H}), 3.90-3.80(\mathrm{~m}, 3 \mathrm{H}), 2.22-2.07(\mathrm{~m}, 2 \mathrm{H}), 2.01$ $-1.94(\mathrm{~m}, 1 \mathrm{H}), 1.85-1.79(\mathrm{~m}, 4 \mathrm{H}), 1.75(\mathrm{~s}, 3 \mathrm{H}), 1.54(\mathrm{~s}, 3 \mathrm{H}),{ }^{13} \mathbf{C} \mathbf{N M R}\left(100 \mathrm{MHz}, \mathrm{CDCl}_{3}\right)$ $\delta=162.7,155.3,140.3,137.6,137.0,134.7,131.6,129.3,128.9,128.7,127.6,127.3,127.1,127.0,126.6,121.0,117.0$, $115.2,109.7,78.3,58.9,45.8,40.8,39.5,29.0,26.2,25.9,18.1 . \quad[\alpha]^{20} \mathbf{D}=+6.08^{\circ}\left(c=1.0, \mathrm{CHCl}_{3}, 85 \%\right.$ ee $)$. HRMS $(\mathrm{ESI})$ $\mathrm{m} / \mathrm{z}:[\mathrm{M}+\mathrm{H}]^{+}$calcd. for $\mathrm{C}_{32} \mathrm{H}_{34} \mathrm{NO}_{3} 480.2533$, found 480.2513 .
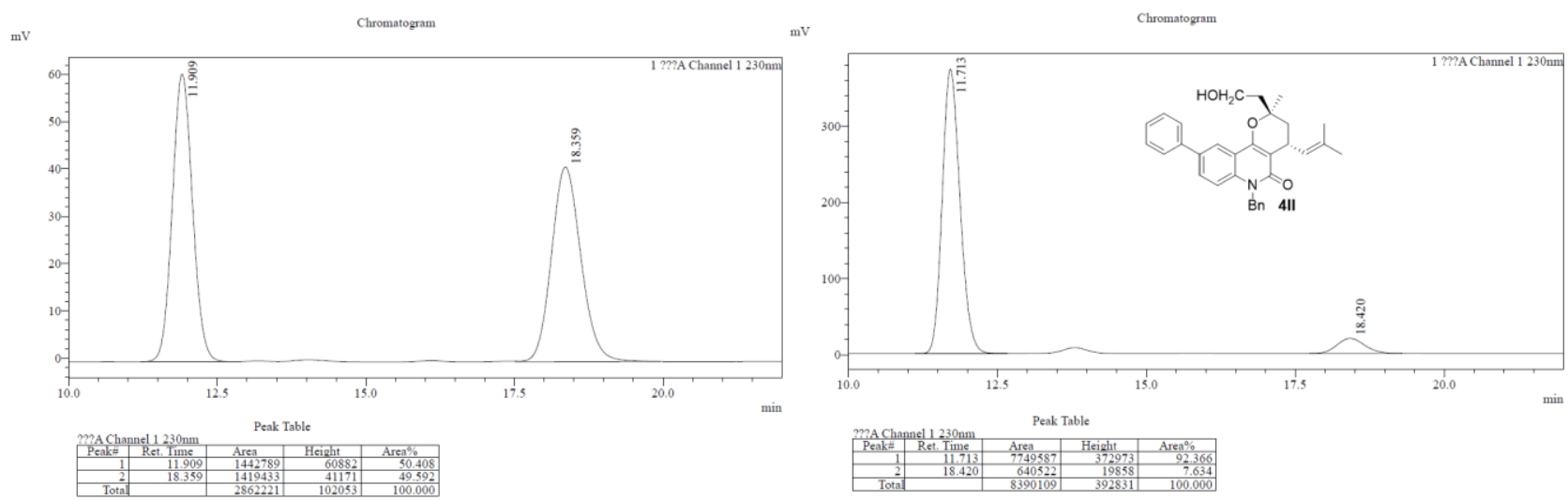
(2S,4R)-6-benzyl-2-(2-hydroxyethyl)-9-methoxy-2-methyl-4-(2-methylprop-1-en-1-yl)-2,3,4,6-tetrahydro-5H-pyran o[3,2-c]quinolin-5-one $(4 \mathrm{~m})$ :

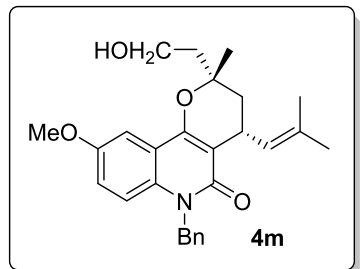

With catalyst 8e, $36 \%$ yield, $39 \mathrm{mg}$. White solid. $90 \%$ ee, determined by HPLC analysis [Chiralpak AD-H, $n$-hexane $/ i-\operatorname{PrOH}=90 / 10,1.0 \mathrm{~mL} / \mathrm{min}, \lambda=230 \mathrm{~nm}, \mathrm{t}$ (major) $=16.00 \mathrm{~min}$, $\mathrm{t}($ minor $)=22.47 \mathrm{~min}] .{ }^{1} \mathbf{H}$ NMR $\left(400 \mathrm{MHz}, \mathrm{CDCl}_{3}\right) \delta=7.34(\mathrm{~d}, J=3.2 \mathrm{~Hz}, 1 \mathrm{H}), 7.30-7.22$ $(\mathrm{m}, 2 \mathrm{H}), 7.22-7.08(\mathrm{~m}, 4 \mathrm{H}), 7.00-6.97(\mathrm{~m}, 1 \mathrm{H}), 5.55(\mathrm{~d}, J=16.4 \mathrm{~Hz}, 1 \mathrm{H}), 5.46(\mathrm{~d}, J=16.4$ $\mathrm{Hz}, 1 \mathrm{H}), 5.10(\mathrm{~d}, J=8.4 \mathrm{~Hz}, 1 \mathrm{H}), 3.96(\mathrm{t}, J=6.8 \mathrm{~Hz}, 2 \mathrm{H}), 3.82(\mathrm{~s}, 3 \mathrm{H}), 3.81-3.76(\mathrm{~m}, 1 \mathrm{H})$, $2.11-2.07(\mathrm{~m}, 2 \mathrm{H}), 2.04-1.99(\mathrm{~m}, 1 \mathrm{H}), 1.88-1.83(\mathrm{~m}, 1 \mathrm{H}), 1.80(\mathrm{~s}, 3 \mathrm{H}), 1.75(\mathrm{~s}, 3 \mathrm{H}), 1.42$ (s, 3H). ${ }^{13} \mathbf{C ~ N M R}\left(100 \mathrm{MHz}, \mathrm{CDCl}_{3}\right) \delta=162.2,154.7,154.5,137.1,132.9,131.4,128.7,127.3,127.0,126.5,118.6,117.4$, $116.1,109.8,105.1,78.5,58.8,55.7,45.7,43.4,39.2,29.0,25.9,23.2,18.0 .[\alpha]^{20} \mathbf{D}=+8.79^{\circ}\left(c=1.0, \mathrm{CHCl}_{3}, 90 \%\right.$ ee $)$. HRMS (ESI) m/z: [M+H] ${ }^{+}$calcd. for $\mathrm{C}_{27} \mathrm{H}_{32} \mathrm{NO}_{4} 434.2326$, found 434.2336 .
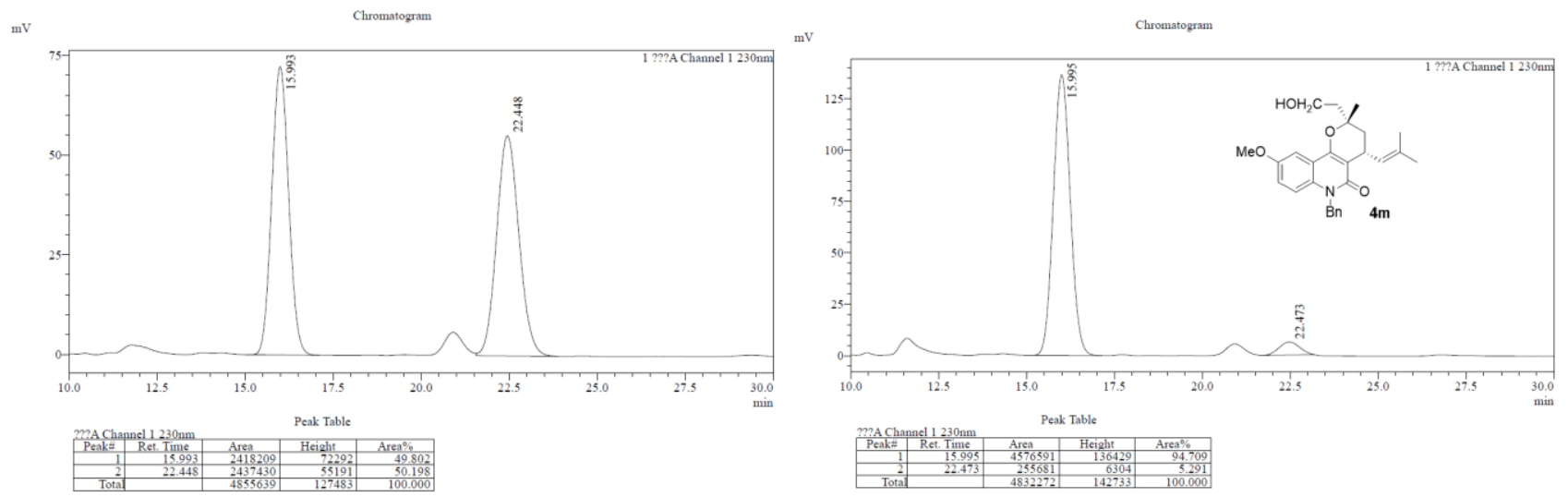

(2R,4R)-6-benzyl-2-(2-hydroxyethyl)-9-methoxy-2-methyl-4-(2-methylprop-1-en-1-yl)-2,3,4,6-tetrahydro-5H-pyran o[3,2-c]quinolin-5-one $(4 \mathrm{~mm})$ :

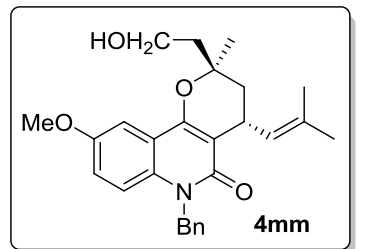

With catalyst 8e, $32 \%$ yield, $35 \mathrm{mg}$. White solid. $83 \%$ ee, determined by HPLC analysis [Chiralpak AD-H, $n$-hexane $/ i$-PrOH $=90 / 10,1.0 \mathrm{~mL} / \mathrm{min}, \lambda=230 \mathrm{~nm}, \mathrm{t}$ (major) $=14.41 \mathrm{~min}$, $\mathrm{t}($ minor $)=23.11 \mathrm{~min}] .{ }^{1} \mathbf{H}$ NMR $\left(400 \mathrm{MHz}, \mathrm{CDCl}_{3}\right) \delta=7.36(\mathrm{~d}, J=2.8 \mathrm{~Hz}, 1 \mathrm{H}), 7.31-7.23$ (m, 2H), $7.22-7.09(\mathrm{~m}, 4 \mathrm{H}), 7.00-6.97(\mathrm{~m}, 1 \mathrm{H}), 5.57(\mathrm{~d}, J=14.8 \mathrm{~Hz}, 1 \mathrm{H}), 5.42(\mathrm{~d}, J=14.8$ $\mathrm{Hz}, 1 \mathrm{H}), 5.13(\mathrm{~d}, J=8.4 \mathrm{~Hz}, 1 \mathrm{H}), 3.87(\mathrm{t}, J=6.8 \mathrm{~Hz}, 2 \mathrm{H}), 3.84-3.78(\mathrm{~m}, 4 \mathrm{H}), 2.18-2.13(\mathrm{~m}$, $1 \mathrm{H}), 2.10-2.05(\mathrm{~m}, 1 \mathrm{H}), 2.00-1.91(\mathrm{~m}, 1 \mathrm{H}), 1.80(\mathrm{~s}, 3 \mathrm{H}), 1.74(\mathrm{~s}, 4 \mathrm{H}), 1.52(\mathrm{~s}, 3 \mathrm{H}) .{ }^{13} \mathrm{C}$ NMR $\left(100 \mathrm{MHz}, \mathrm{CDCl}_{3}\right) \delta=162.3,154.7,154.5,137.1,132.9,131.4,128.7,127.7,127.0,126.5,118.6,117.4,116.1$, $109.8,105.1,78.2,58.9,55.7,45.8,40.8,39.5,29.0,26.1,25.9,18.1 .[\alpha]^{20} \mathbf{D}=+6.21^{\circ}\left(c=1.0, \mathrm{CHCl}_{3}, 83 \%\right.$ ee $) . \mathbf{H R M S}$ (ESI) $\mathrm{m} / \mathrm{z}$ : $[\mathrm{M}+\mathrm{Na}]^{+}$calcd. for $\mathrm{C}_{27} \mathrm{H}_{31} \mathrm{NO}_{4} \mathrm{Na} 456.2145$, found 456.2140 .
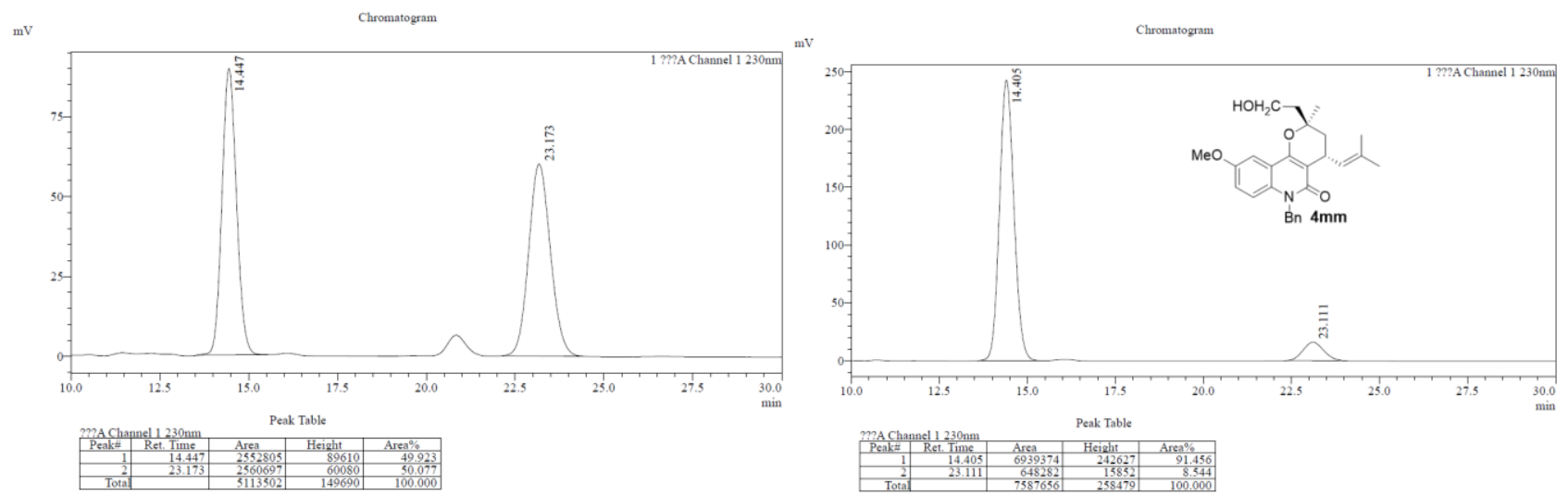
(2S,4R)-2-(2-hydroxyethyl)-7-methoxy-2,6-dimethyl-4-(2-methylprop-1-en-1-yl)-2,3,4,6-tetrahydro-5H-pyrano[3,2c]quinolin-5-one (4n):

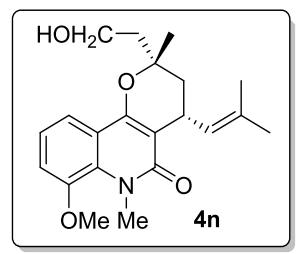

With catalyst 8e, 33\% yield, $30 \mathrm{mg}$. White solid. $91 \%$ ee, determined by HPLC analysis [Chiralpak $\mathrm{AD}-\mathrm{H}, n$-hexane $/ i$-PrOH $=95 / 5,1.0 \mathrm{~mL} / \mathrm{min}, \lambda=230 \mathrm{~nm}, \mathrm{t}$ (major) $=39.39 \mathrm{~min}, \mathrm{t}($ minor $)=42.33$ $\min ] .{ }^{1} \mathbf{H}$ NMR $\left(400 \mathrm{MHz}, \mathrm{CDCl}_{3}\right) \delta=7.50(\mathrm{dd}, J=8.0,1.2 \mathrm{~Hz}, 1 \mathrm{H}), 7.12(\mathrm{t}, J=8.0 \mathrm{~Hz}, 1 \mathrm{H}), 7.04$ $(\mathrm{dd}, J=8.0,1.2 \mathrm{~Hz}, 1 \mathrm{H}), 5.01-4.94(\mathrm{~m}, 1 \mathrm{H}), 3.95(\mathrm{t}, J=6.8 \mathrm{~Hz}, 2 \mathrm{H}), 3.88(\mathrm{~d}, J=1.6 \mathrm{~Hz}, 6 \mathrm{H})$, $3.71(\mathrm{dd}, J=17.2,8.0 \mathrm{~Hz}, 1 \mathrm{H}), 2.06(\mathrm{t}, J=6.8 \mathrm{~Hz}, 2 \mathrm{H}), 1.95(\mathrm{dd}, J=14.0,8.0 \mathrm{~Hz}, 2 \mathrm{H}), 1.81(\mathrm{~d}, J$ $=1.2 \mathrm{~Hz}, 3 \mathrm{H}), 1.74(\mathrm{~s}, 3 \mathrm{H}), 1.36(\mathrm{~s}, 3 \mathrm{H}) .{ }^{13} \mathbf{C ~ N M R}\left(100 \mathrm{MHz}, \mathrm{CDCl}_{3}\right) \delta=163.7,154.7,148.5,131.4,130.6,127.0,122.1$, $118.9,115.1,113.6,109.7,78.4,58.7,56.7,43.6,39.0,34.9,28.9,25.9,22.7,18.0 .[\alpha]^{20} \mathbf{D}=+27.52^{\circ}\left(c=1.0, \mathrm{CHCl}_{3}, 91 \%\right.$ ee). HRMS (ESI) m/z: $[\mathrm{M}+\mathrm{H}]^{+}$calcd. for $\mathrm{C}_{21} \mathrm{H}_{28} \mathrm{NO}_{4} 358.2013$, found 358.2001.
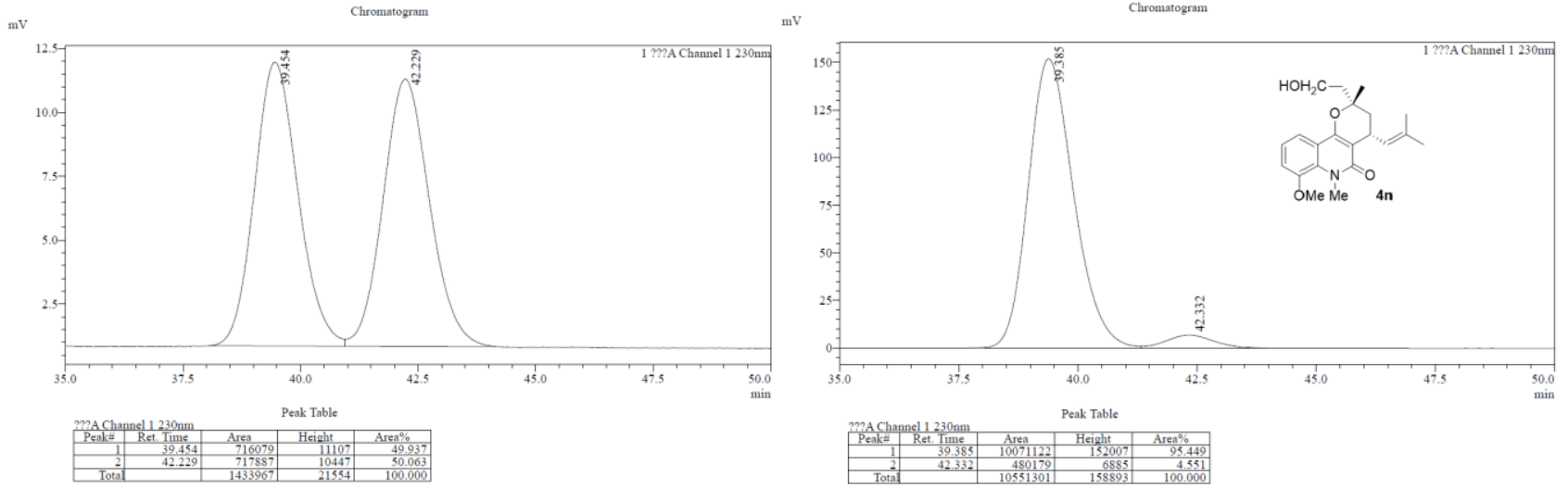

(2R,4R)-2-(2-hydroxyethyl)-7-methoxy-2,6-dimethyl-4-(2-methylprop-1-en-1-yl)-2,3,4,6-tetrahydro-5H-pyrano[3,2 -c]quinolin-5-one (4nn):

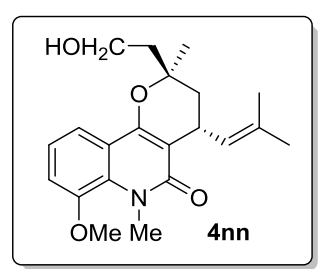

With catalyst 8e, 30\% yield, $26 \mathrm{mg}$. White solid. $91 \%$ ee, determined by HPLC analysis [Chiralpak AD-H, $n$-hexane $/ i$-PrOH $=90 / 10,1.0 \mathrm{~mL} / \mathrm{min}, \lambda=230 \mathrm{~nm}, \mathrm{t}$ (major) $=15.89 \mathrm{~min}, \mathrm{t}$ (minor) $=13.87 \mathrm{~min}] .{ }^{1} \mathbf{H}$ NMR $\left(400 \mathrm{MHz}, \mathrm{CDCl}_{3}\right) \delta=7.55(\mathrm{dd}, J=8.0,1.2 \mathrm{~Hz}, 1 \mathrm{H}), 7.12(\mathrm{t}, J$ $=8.0 \mathrm{~Hz}, 1 \mathrm{H}), 7.04(\mathrm{dd}, J=8.0,1.2 \mathrm{~Hz}, 1 \mathrm{H}), 5.02(\mathrm{~d}, J=8.4 \mathrm{~Hz}, 1 \mathrm{H}), 3.87(\mathrm{~d}, J=2.0 \mathrm{~Hz}, 6 \mathrm{H})$, $3.82(\mathrm{t}, J=6.4 \mathrm{~Hz}, 2 \mathrm{H}), 3.72(\mathrm{dd}, J=16.0,8.0 \mathrm{~Hz}, 1 \mathrm{H}), 2.14-2.02(\mathrm{~m}, 2 \mathrm{H}), 1.88(\mathrm{dd}, J=13.6$, $6.8 \mathrm{~Hz}, 1 \mathrm{H}), 1.81(\mathrm{~s}, 3 \mathrm{H}), 1.74-1.70(\mathrm{~m}, 4 \mathrm{H}), 1.49(\mathrm{~s}, 3 \mathrm{H}),{ }^{13} \mathbf{C} \mathbf{~ N M R}\left(100 \mathrm{MHz}, \mathrm{CDCl}_{3}\right) \delta=$ 163.8, 154.7, 148.5, 131.4, 130.7, 127.4, 122.1, 118.9, 115.2, 113.8, 109.7, 78.1, 58.8, 56.7, 40.0, 39.7, 34.9, 29.0, 26.1, $25.8,18.0 .[\alpha]^{20} \mathrm{D}=+17.91^{\circ}\left(c=1.0, \mathrm{CHCl}_{3}, 91 \%\right.$ ee $)$. HRMS $(\mathrm{ESI}) \mathrm{m} / \mathrm{z}:[\mathrm{M}+\mathrm{H}]^{+}$calcd. for $\mathrm{C}_{21} \mathrm{H}_{28} \mathrm{NO}_{4} 358.2013$, found 358.2006 .
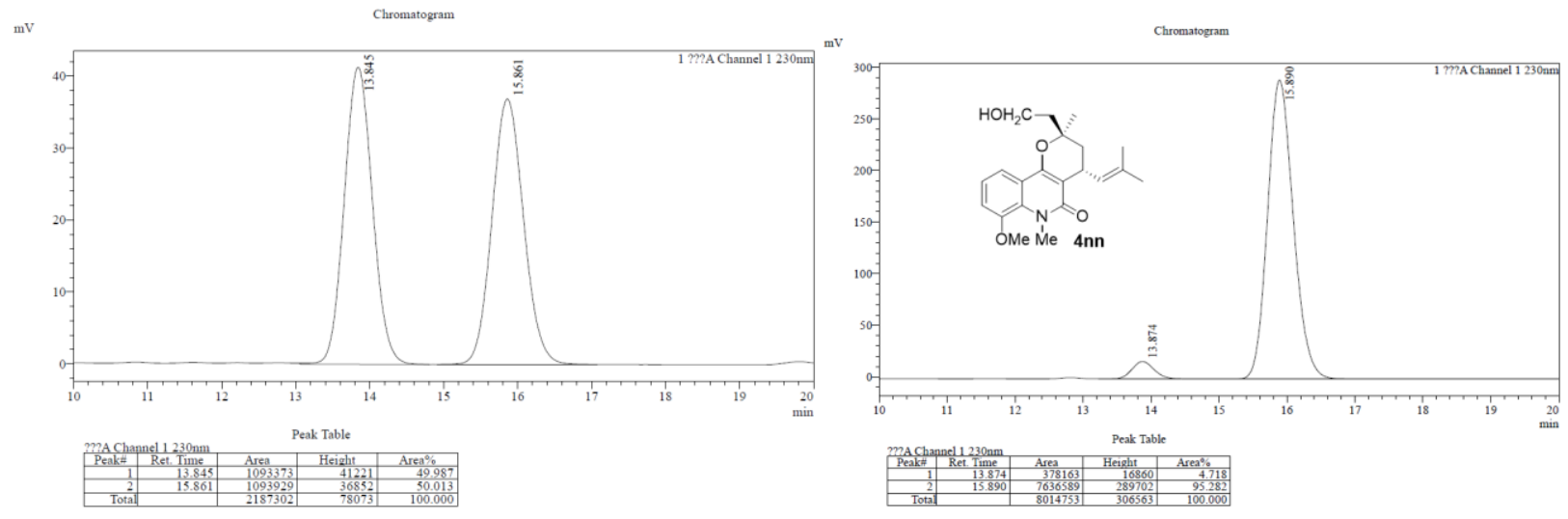
(2S,4R)-6-benzyl-2-(2-hydroxyethyl)-8,9-dimethoxy-2-methyl-4-(2-methylprop-1-en-1-yl)-2,3,4,6-tetrahydro-5H-py rano[3,2-c]quinolin-5-one (4o):

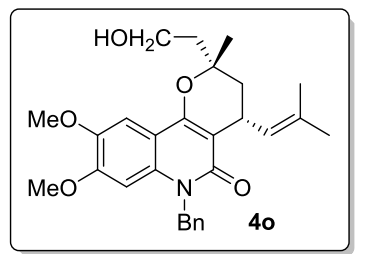

With catalyst 8e, 30\% yield, $34 \mathrm{mg}$. White solid. $81 \%$ ee, determined by HPLC analysis [Chiralpak AD-H, $n$-hexane $/ i-\mathrm{PrOH}=90 / 10,1.0 \mathrm{~mL} / \mathrm{min}, \lambda=230 \mathrm{~nm}, \mathrm{t}$ (major) $=16.75 \mathrm{~min}$, $\mathrm{t}($ minor $)=22.85 \mathrm{~min}] .{ }^{1} \mathbf{H}$ NMR $\left(400 \mathrm{MHz}^{\mathrm{CDCl}}{ }_{3}\right) \delta=7.31-7.16(\mathrm{~m}, 7 \mathrm{H}), 6.65(\mathrm{~s}, 1 \mathrm{H})$, $5.58(\mathrm{~d}, J=16.0 \mathrm{~Hz}, 1 \mathrm{H}), 5.46(\mathrm{~d}, J=16.0 \mathrm{~Hz}, 1 \mathrm{H}), 5.11(\mathrm{~d}, J=8.4 \mathrm{~Hz}, 1 \mathrm{H}), 3.96(\mathrm{t}, J=6.0$ $\mathrm{Hz}, 2 \mathrm{H}), 3.90(\mathrm{~s}, 3 \mathrm{H}), 3.79(\mathrm{dd}, J=16.4,8.4 \mathrm{~Hz}, 1 \mathrm{H}), 3.72(\mathrm{~s}, 3 \mathrm{H}), 2.09$ (dd, $J=12.8,6.8 \mathrm{~Hz}$, 2H), $2.01(\mathrm{dd}, J=14.0,7.2 \mathrm{~Hz}, 1 \mathrm{H}), 1.87-1.83(\mathrm{~m}, 1 \mathrm{H}), 1.81(\mathrm{~s}, 3 \mathrm{H}), 1.76(\mathrm{~s}, 3 \mathrm{H}), 1.43(\mathrm{~s}, 3 \mathrm{H}) .{ }^{13} \mathbf{C} \mathbf{N M R}(100 \mathrm{MHz}$ $\left.\mathrm{CDCl}_{3}\right) \delta=162.7,155.1,151.5,144.7,137.3,133.8,131.2,128.7,127.6,127.1,126.6,109.4,107.4,103.8,98.3,78.5$, 58.9, 56.2, 55.9, 46.0, 43.4, 39.2, 28.9, 25.9, 23.2, 18.0. $[\alpha]^{\mathbf{2 0}} \mathrm{D}=+8.05^{\circ}\left(c=1.0, \mathrm{CHCl}_{3}, 81 \%\right.$ ee $) . \mathbf{H R M S}(\mathrm{ESI}) \mathrm{m} / \mathrm{z}$ : $[\mathrm{M}+\mathrm{H}]^{+}$calcd. for $\mathrm{C}_{28} \mathrm{H}_{34} \mathrm{NO}_{5} 464.2431$, found 464.2410 .
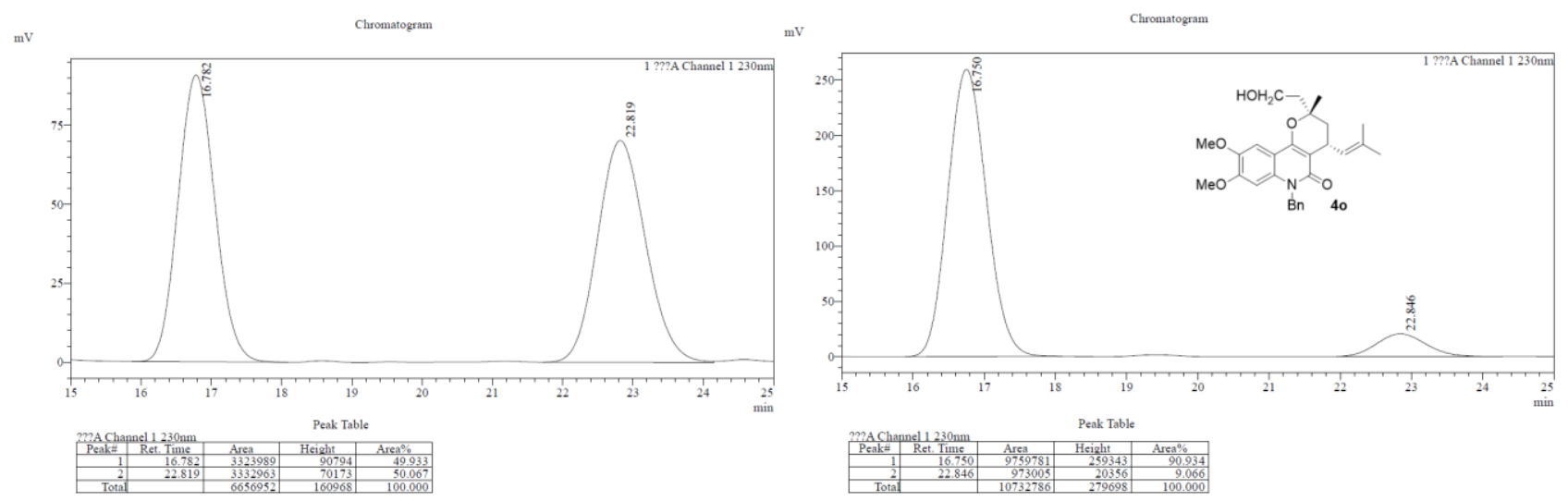

(2R,4R)-6-benzyl-2-(2-hydroxyethyl)-8,9-dimethoxy-2-methyl-4-(2-methylprop-1-en-1-yl)-2,3,4,6-tetrahydro-5H-p yrano[3,2-c]quinolin-5-one (40o):

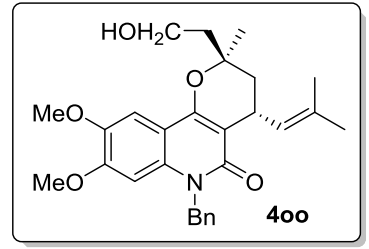

With catalyst 8e, $24 \%$ yield, $28 \mathrm{mg}$. White solid. $79 \%$ ee, determined by HPLC analysis [Chiralpak AD-H, $n$-hexane $/ i-\mathrm{PrOH}=90 / 10,1.0 \mathrm{~mL} / \mathrm{min}, \lambda=230 \mathrm{~nm}, \mathrm{t}$ (major) $=19.25 \mathrm{~min}$, $\mathrm{t}($ minor $)=14.58 \mathrm{~min}] .{ }^{1} \mathbf{H}$ NMR $\left(400 \mathrm{MHz} \mathrm{CDCl}_{3}\right) \delta=7.32-7.24(\mathrm{~m}, 3 \mathrm{H}), 7.22-7.18(\mathrm{~m}$, $3 \mathrm{H}), 6.66(\mathrm{~s}, 1 \mathrm{H}), 5.63(\mathrm{~d}, J=15.6 \mathrm{~Hz}, 1 \mathrm{H}), 5.41(\mathrm{~d}, J=15.6 \mathrm{~Hz}, 1 \mathrm{H}), 5.15(\mathrm{~d}, J=8.4 \mathrm{~Hz}$, $1 \mathrm{H}), 3.91(\mathrm{~s}, 3 \mathrm{H}), 3.88(\mathrm{t}, J=6.4 \mathrm{~Hz}, 2 \mathrm{H}), 3.81(\mathrm{dd}, J=15.2,7.6 \mathrm{~Hz}, 1 \mathrm{H}), 3.72(\mathrm{~s}, 3 \mathrm{H}), 2.19$ - $2.00(\mathrm{~m}, 2 \mathrm{H}), 2.00-1.92(\mathrm{~m}, 1 \mathrm{H}), 1.84-1.77(\mathrm{~m}, 4 \mathrm{H}), 1.75(\mathrm{~s}, 3 \mathrm{H}), 1.52(\mathrm{~s}, 3 \mathrm{H}) .{ }^{13} \mathbf{C ~ N M R}\left(100 \mathbf{M H z} \mathrm{CDCl}_{3}\right) \delta=$ $162.8,155.0,151.5,144.8,137.3,133.8,131.3,128.7,128.0,127.1,126.7,109.4,107.4,103.8,98.3,78.2,58.9,56.2,55.9$, $46.1,40.8,39.6,28.9,26.1,25.9,18.0 .[\alpha]^{20} \mathbf{D}=+7.22^{\circ}\left(c=1.0, \mathrm{CHCl}_{3}, 79 \%\right.$ ee $) . \mathbf{H R M S}(\mathrm{ESI}) \mathrm{m} / \mathrm{z}:[\mathrm{M}+\mathrm{H}]^{+}$calcd. for $\mathrm{C}_{28} \mathrm{H}_{34} \mathrm{NO}_{5} 464.2431$, found 464.2447 .
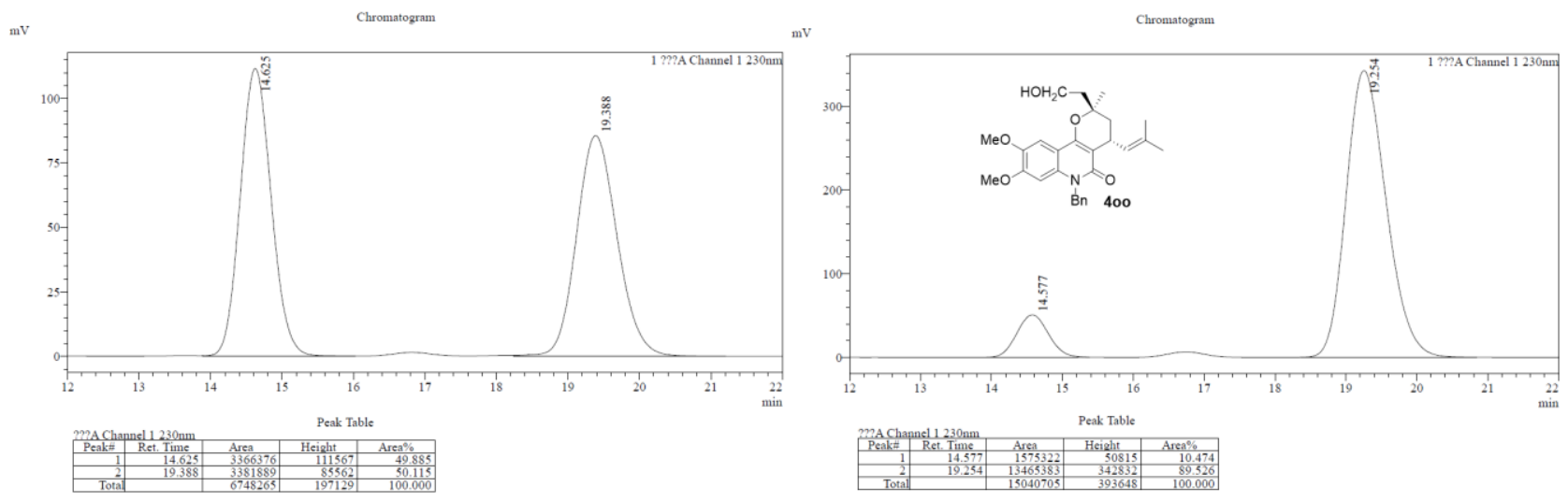
(1R,3S)-3-(2-hydroxyethyl)-3,11-dimethyl-1-(2-methylprop-1-en-1-yl)-1,2,3,11-tetrahydro-12H-benzo[h]pyrano[3,2 -c]quinolin-12-one (4p):

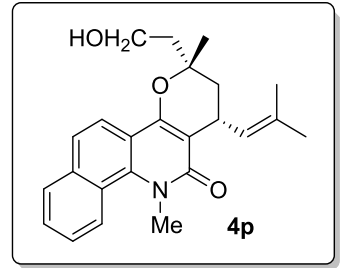

With catalyst 8e, $28 \%$ yield, $26 \mathrm{mg}$. White solid. 95\% ee, determined by HPLC analysis [Chiralpak AD-H, $n$-hexane $/ i$-PrOH $=90 / 10,1.0 \mathrm{~mL} / \mathrm{min}, \lambda=230 \mathrm{~nm}, \mathrm{t}$ (major) $=17.76 \mathrm{~min}, \mathrm{t}$ (minor) $=19.41 \mathrm{~min}] .{ }^{1} \mathbf{H}$ NMR $\left(400 \mathrm{MHz}, \mathrm{CDCl}_{3}\right) \delta=8.38(\mathrm{~d}, J=8.4 \mathrm{~Hz}, 1 \mathrm{H}), 7.88(\mathrm{~d}, J=8.8$ $\mathrm{Hz}, 2 \mathrm{H}), 7.63-7.47(\mathrm{~m}, 3 \mathrm{H}), 5.01(\mathrm{~d}, J=8.4 \mathrm{~Hz}, 1 \mathrm{H}), 4.02-3.97(\mathrm{~m}, 5 \mathrm{H}), 3.78(\mathrm{dd}, J=17.2$, $8.8 \mathrm{~Hz}, 1 \mathrm{H}), 2.11(\mathrm{t}, J=6.4 \mathrm{~Hz}, 2 \mathrm{H}), 2.00(\mathrm{dd}, J=14.0,7.2 \mathrm{~Hz}, 1 \mathrm{H}), 1.86(\mathrm{~s}, 3 \mathrm{H}), 1.87-1.77$ $(\mathrm{m}, 4 \mathrm{H}), 1.42(\mathrm{~s}, 3 \mathrm{H}) .{ }^{13} \mathbf{C}$ NMR $\left(100 \mathrm{MHz} \mathrm{CDCl}_{3}\right) \delta=164.9,155.7,138.4,135.4,132.0$, 128.6, 126.9, 126.6, 125.7, 124.9, 123.8, 123.2, 119.1, 114.5, 109.3, 78.7, 58.7, 43.9, 39.8, 39.0, 28.8, 25.9, 22.7, 18.1. $[\alpha]^{20}{ }_{D}=+81.26^{\circ}\left(c=1.0, \mathrm{CHCl}_{3}, 95 \%\right.$ ee $)$. HRMS (ESI) $\mathrm{m} / \mathrm{z}:[\mathrm{M}+\mathrm{H}]^{+}$calcd. for $\mathrm{C}_{24} \mathrm{H}_{28} \mathrm{NO}_{3} 378.2064$, found 378.2051 .
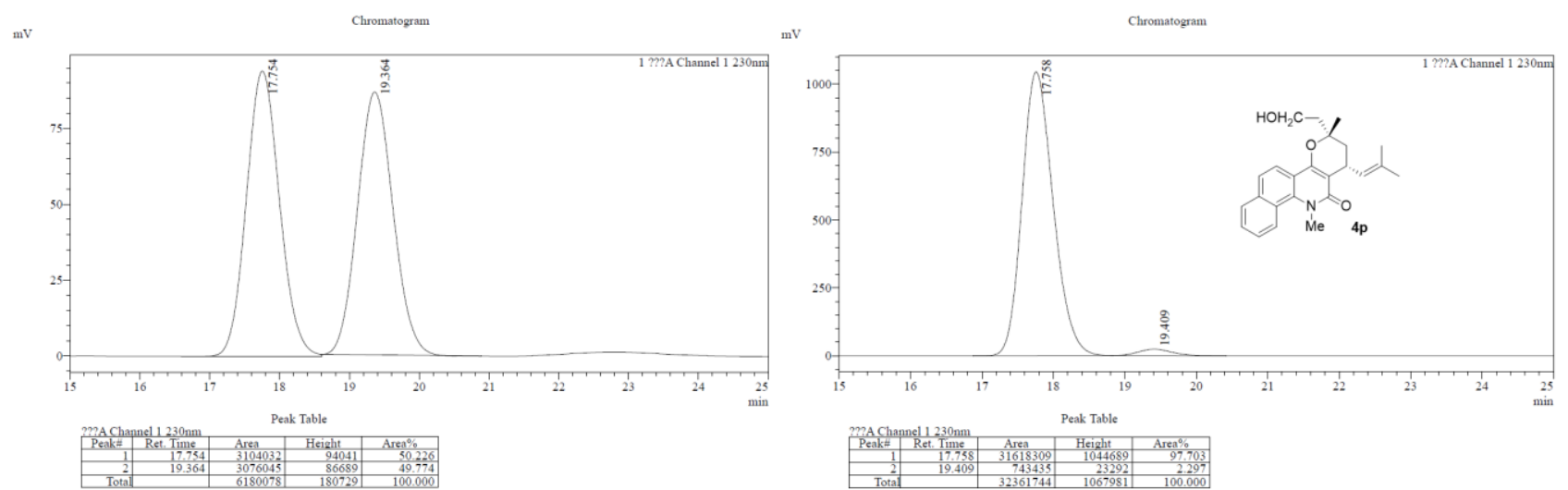

(1R,3R)-3-(2-hydroxyethyl)-3,11-dimethyl-1-(2-methylprop-1-en-1-yl)-1,2,3,11-tetrahydro-12H-benzo[h]pyrano[3,2 -c]quinolin-12-one (4pp):

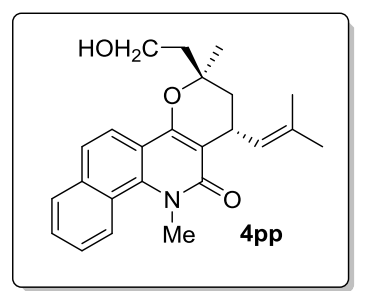

With catalyst 8e, $26 \%$ yield, $24 \mathrm{mg}$. White solid. $96 \%$ ee, determined by HPLC analysis [Chiralpak AS-H, $n$-hexane $/ i$-PrOH $=90 / 5,1.0 \mathrm{~mL} / \mathrm{min}, \lambda=230 \mathrm{~nm}, \mathrm{t}$ (major) $=15.81 \mathrm{~min}, \mathrm{t}$ $($ minor $)=17.53 \mathrm{~min}] .{ }^{1} \mathbf{H}$ NMR $\left(400 \mathrm{MHz}, \mathrm{CDCl}_{3}\right) \delta=8.38(\mathrm{~d}, J=8.4 \mathrm{~Hz}, 1 \mathrm{H}), 7.92(\mathrm{~d}, J=$ $8.4 \mathrm{~Hz}, 1 \mathrm{H}), 7.88(\mathrm{~d}, J=8.0 \mathrm{~Hz}, 1 \mathrm{H}), 7.60(\mathrm{~d}, J=8.8 \mathrm{~Hz}, 1 \mathrm{H}), 7.57-7.47(\mathrm{~m}, 2 \mathrm{H}), 5.04(\mathrm{~d}, J$ $=8.4 \mathrm{~Hz}, 1 \mathrm{H}), 3.98(\mathrm{~s}, 3 \mathrm{H}), 3.87(\mathrm{t}, J=6.8 \mathrm{~Hz}, 2 \mathrm{H}), 3.79(\mathrm{dd}, J=16.4,8.4 \mathrm{~Hz}, 1 \mathrm{H}), 2.18-$ $2.08(\mathrm{~m}, 2 \mathrm{H}), 1.93(\mathrm{dd}, J=13.6,6.8 \mathrm{~Hz}, 1 \mathrm{H}), 1.85(\mathrm{~s}, 3 \mathrm{H}), 1.76-1.70(\mathrm{~m}, 4 \mathrm{H}), 1.54(\mathrm{~s}, 3 \mathrm{H})$. ${ }^{13} \mathbf{C}$ NMR $\left(100 \mathrm{MHz}, \mathrm{CDCl}_{3}\right) \delta=165.0,155.7,138.3,135.5,131.9,128.7,126.9,126.9,125.7,124.8,123.8,123.2,119.1$, $114.4,109.3,78.3,58.9,39.8,39.7,39.6,28.8,26.3,25.9,18.1 .[\alpha]^{\mathbf{2 0}} \mathrm{D}=+55.82^{\circ}\left(c=1.0, \mathrm{CHCl}_{3}, 96 \%\right.$ ee $)$. HRMS (ESI) $\mathrm{m} / \mathrm{z}:[\mathrm{M}+\mathrm{H}]^{+}$calcd. for $\mathrm{C}_{24} \mathrm{H}_{28} \mathrm{NO}_{3} 378.2064$, found 378.2071.
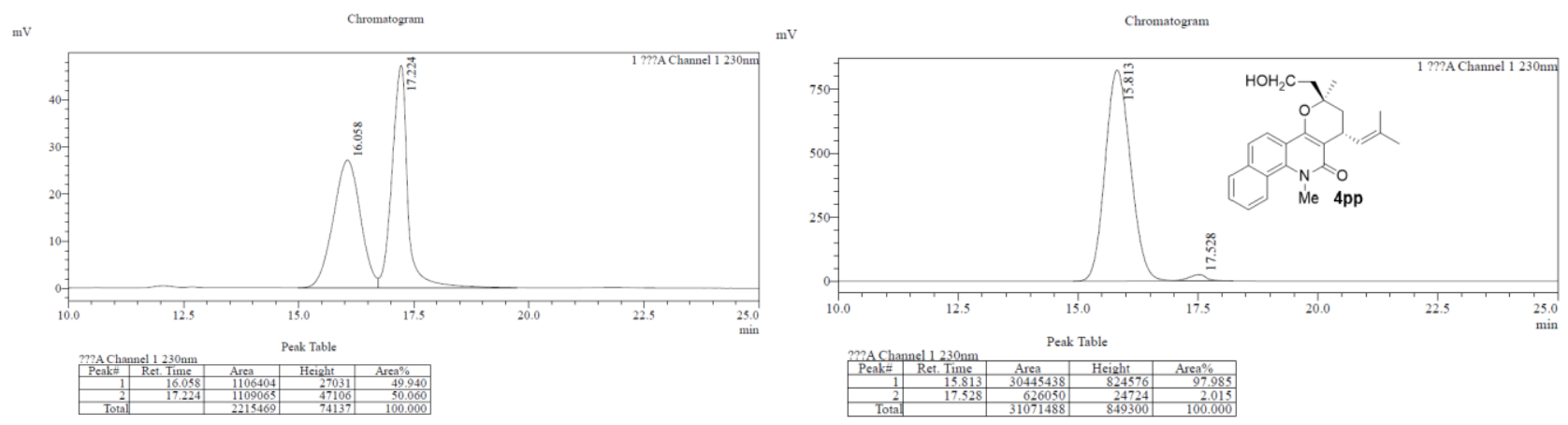
(2S,4R)-6-benzyl-2-(2-hydroxyethyl)-2-methyl-4-(2-methylprop-1-en-1-yl)-2,3,4,6-tetrahydro-5H-benzo[f]pyrano[3, 2-c]quinolin-5-one (4q):

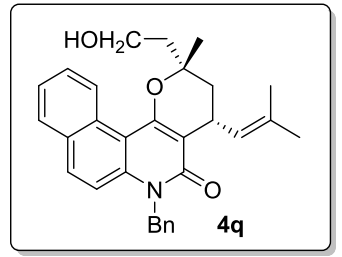

With catalyst 8e, 30\% yield, $34 \mathrm{mg}$. White solid. 89\% ee, determined by HPLC analysis [Chiralpak AD-H, $n$-hexane $/ i$-PrOH $=90 / 10,1.0 \mathrm{~mL} / \mathrm{min}, \lambda=230 \mathrm{~nm}, \mathrm{t}$ (major) $=17.15 \mathrm{~min}, \mathrm{t}$ (minor) $=29.43 \mathrm{~min}] .{ }^{1} \mathbf{H}$ NMR $\left(400 \mathrm{MHz}, \mathrm{CDCl}_{3}\right) \delta=9.41(\mathrm{~d}, J=8.8 \mathrm{~Hz}, 1 \mathrm{H}), 7.83-7.73(\mathrm{~m}$, $2 \mathrm{H}), 7.62-7.55(\mathrm{~m}, 1 \mathrm{H}), 7.46-7.40(\mathrm{~m}, 2 \mathrm{H}), 7.29-7.14(\mathrm{~m}, 5 \mathrm{H}), 5.73(\mathrm{~s}, 2 \mathrm{H}), 5.12(\mathrm{~d}, J=$ $8.4 \mathrm{~Hz}, 1 \mathrm{H}), 4.01(\mathrm{t}, J=6.8 \mathrm{~Hz}, 2 \mathrm{H}), 3.91(\mathrm{dd}, J=16.4,8.4 \mathrm{~Hz}, 1 \mathrm{H}), 2.32-2.20(\mathrm{~m}, 2 \mathrm{H}), 2.12$ $(\mathrm{dd}, J=14.0,7.6 \mathrm{~Hz}, 1 \mathrm{H}), 1.95(\mathrm{dd}, J=14.0,8.8 \mathrm{~Hz}, 1 \mathrm{H}), 1.84(\mathrm{~s}, 3 \mathrm{H}), 1.76(\mathrm{~s}, 4 \mathrm{H}), 1.51$ (s, 3H). ${ }^{13} \mathrm{C}$ NMR $\left(100 \mathrm{MHz}, \mathrm{CDCl}_{3}\right) \delta=162.1,159.3,138.6,137.0,132.0,131.6,129.9,129.7,128.8,128.6,127.6,127.6$, $127.1,126.8,126.3,124.8,115.5,110.3,110.2,79.0,58.9,46.5,43.8,38.7,29.5,25.9,23.2,18.1 .[\alpha]^{20} \mathbf{D}=+8.95^{\circ}(c=$ 1.0, $\mathrm{CHCl}_{3}, 89 \%$ ee). HRMS (ESI) m/z: $[\mathrm{M}+\mathrm{H}]^{+}$calcd. for $\mathrm{C}_{30} \mathrm{H}_{32} \mathrm{NO}_{3} 454.2377$, found 454.2361.
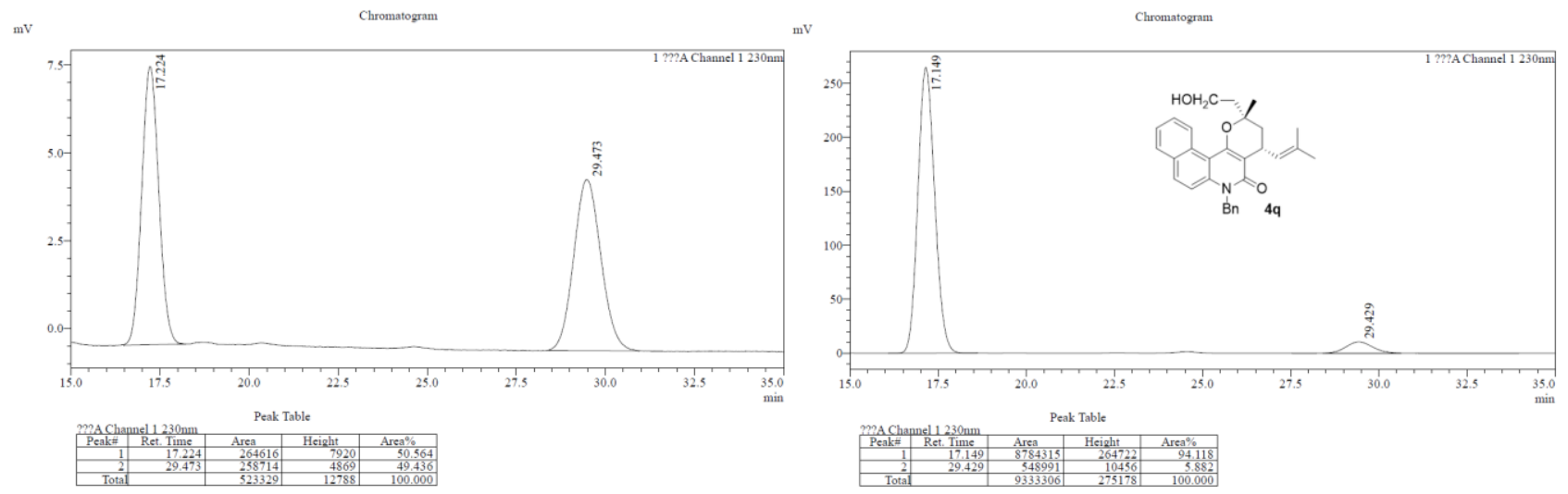

(2R,4R)-6-benzyl-2-(2-hydroxyethyl)-2-methyl-4-(2-methylprop-1-en-1-yl)-2,3,4,6-tetrahydro-5H-benzo[f]pyrano [3,2-c]quinolin-5-one (4qq):

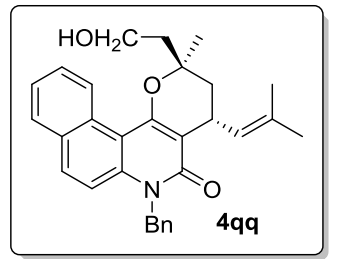

With catalyst 8e, $20 \%$ yield, $23 \mathrm{mg}$. White solid. $77 \%$ ee, determined by HPLC analysis [Chiralpak AD-H, $n$-hexane $/ i-\mathrm{PrOH}=90 / 10,1.0 \mathrm{~mL} / \mathrm{min}, \lambda=230 \mathrm{~nm}, \mathrm{t}$ (major) $=23.71 \mathrm{~min}, \mathrm{t}$ $($ minor $)=28.91 \mathrm{~min}] .{ }^{1} \mathbf{H}$ NMR $\left(400 \mathrm{MHz}, \mathrm{CDCl}_{3}\right) \delta=9.44(\mathrm{~d}, J=8.8 \mathrm{~Hz}, 1 \mathrm{H}), 7.78(\mathrm{~d}, J=9.2$ $\mathrm{Hz}, 2 \mathrm{H}), 7.59(\mathrm{t}, J=8.0 \mathrm{~Hz}, 1 \mathrm{H}), 7.45(\mathrm{dd}, J=16.0,8.6 \mathrm{~Hz}, 2 \mathrm{H}), 7.31-7.14(\mathrm{~m}, 6 \mathrm{H}), 5.70(\mathrm{~s}$, $2 \mathrm{H}), 5.13(\mathrm{~d}, J=8.4 \mathrm{~Hz}, 1 \mathrm{H}), 3.97-3.76(\mathrm{~m}, 3 \mathrm{H}), 2.28-2.14(\mathrm{~m}, 2 \mathrm{H}), 2.06-2.00(\mathrm{~m}, 1 \mathrm{H})$, $1.92-1.86(\mathrm{~m}, 1 \mathrm{H}), 1.83(\mathrm{~s}, 3 \mathrm{H}), 1.76(\mathrm{~s}, 3 \mathrm{H}), 1.70(\mathrm{~s}, 3 \mathrm{H}) .{ }^{13} \mathbf{C ~ N M R}\left(100 \mathrm{MHz}, \mathrm{CDCl}_{3}\right) \delta=$ 162.1 159.3, 138.7, 137.1, 132.1, 131.4, 129.9, 129.7, 128.8, 128.7, 127.7, 127.1, 126.8, 126.3, 124.8, 115.5, 110.4, 110.1, 78.8, 58.9, 46.6, 39.7, 39.3, 29.5, 26.7, 25.9, 18.1. $[\alpha]^{20} \mathbf{D}=+8.86^{\circ}\left(c=1.0, \mathrm{CHCl}_{3}, 77 \%\right.$ ee $) . \mathbf{H R M S}(\mathrm{ESI}) \mathrm{m} / \mathrm{z}:[\mathrm{M}+\mathrm{H}]^{+}$ calcd. for $\mathrm{C}_{30} \mathrm{H}_{32} \mathrm{NO}_{3} 454.2377$, found 454.2359 .
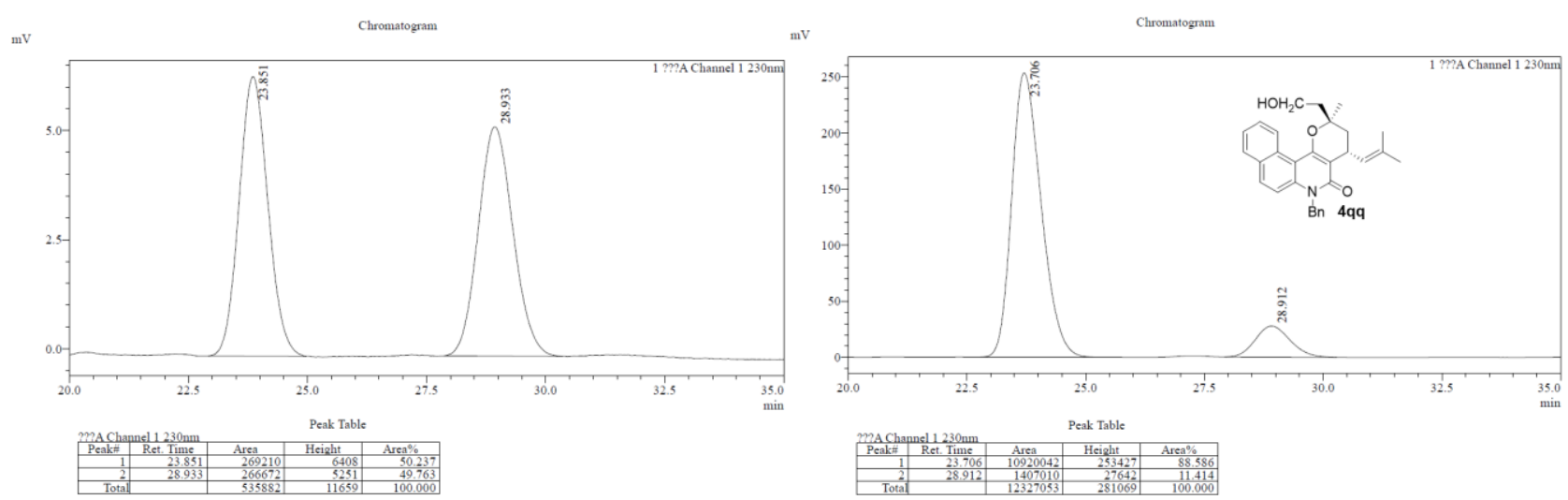
(2S,4R)-6-benzyl-2-(2-hydroxyethyl)-2-methyl-4-(2-methylprop-1-en-1-yl)-2,3,4,6-tetrahydro-5H-pyrano[3,2-c][1, 8]naphthyridin-5-one (4r):

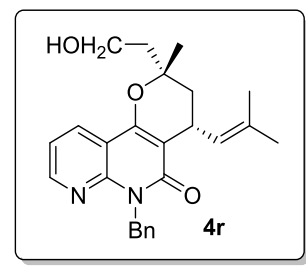

With catalyst 8e, $29 \%$ yield, $29 \mathrm{mg}$. White solid. $70 \%$ ee, determined by HPLC analysis [Chiralpak AD-H, $n$-hexane $/ i$-PrOH $=90 / 10,1.0 \mathrm{~mL} / \mathrm{min}, \lambda=230 \mathrm{~nm}, \mathrm{t}$ (major) $=10.93 \mathrm{~min}, \mathrm{t}$ (minor) $=$ $12.63 \mathrm{~min}] .{ }^{1} \mathbf{H}$ NMR $\left(400 \mathrm{MHz}, \mathrm{CDCl}_{3}\right) \delta=8.59-8.46(\mathrm{~m}, 1 \mathrm{H}), 8.19-8.04(\mathrm{~m}, 1 \mathrm{H}), 7.43(\mathrm{~d}, J$ $=7.6 \mathrm{~Hz}, 2 \mathrm{H}), 7.25-7.07(\mathrm{~m}, 4 \mathrm{H}), 5.78(\mathrm{~d}, J=14.0 \mathrm{~Hz}, 1 \mathrm{H}), 5.64(\mathrm{~d}, J=14.0 \mathrm{~Hz}, 1 \mathrm{H}), 5.07(\mathrm{~d}$, $J=8.0 \mathrm{~Hz}, 1 \mathrm{H}), 3.92(\mathrm{t}, J=6.8 \mathrm{~Hz}, 2 \mathrm{H}), 3.75(\mathrm{dd}, J=16.0,8.0 \mathrm{~Hz}, 1 \mathrm{H}), 2.08-1.97(\mathrm{~m}, 3 \mathrm{H}), 1.86$ - $1.80(\mathrm{~m}, 1 \mathrm{H}), 1.79(\mathrm{~s}, 3 \mathrm{H}), 1.75(\mathrm{~s}, 3 \mathrm{H}), 1.39$ (s, 3H). ${ }^{13} \mathbf{C} \mathbf{~ N M R}\left(100 \mathrm{MHz}, \mathrm{CDCl}_{3}\right) \delta=162.9$, 154.0, 149.7, 148.8, 138.3, 131.8, 131.1, 128.4, 128.2, 126.9, 126.8, 117.4, 111.9, 110.6, 78.8, 58.8, 43.9, 43.3, 39.0, 28.8, 25.8, 23.3, 18.1. $[\boldsymbol{\alpha}]^{20}{ }_{D}=+28.70^{\circ}\left(c=1.0, \mathrm{CHCl}_{3}, 70 \%\right.$ ee). HRMS (ESI) m/z: $[\mathrm{M}+\mathrm{Na}]^{+}$calcd. for $\mathrm{C}_{25} \mathrm{H}_{28} \mathrm{~N}_{2} \mathrm{O}_{3} \mathrm{Na}$ 427.1992, found 427.2004.
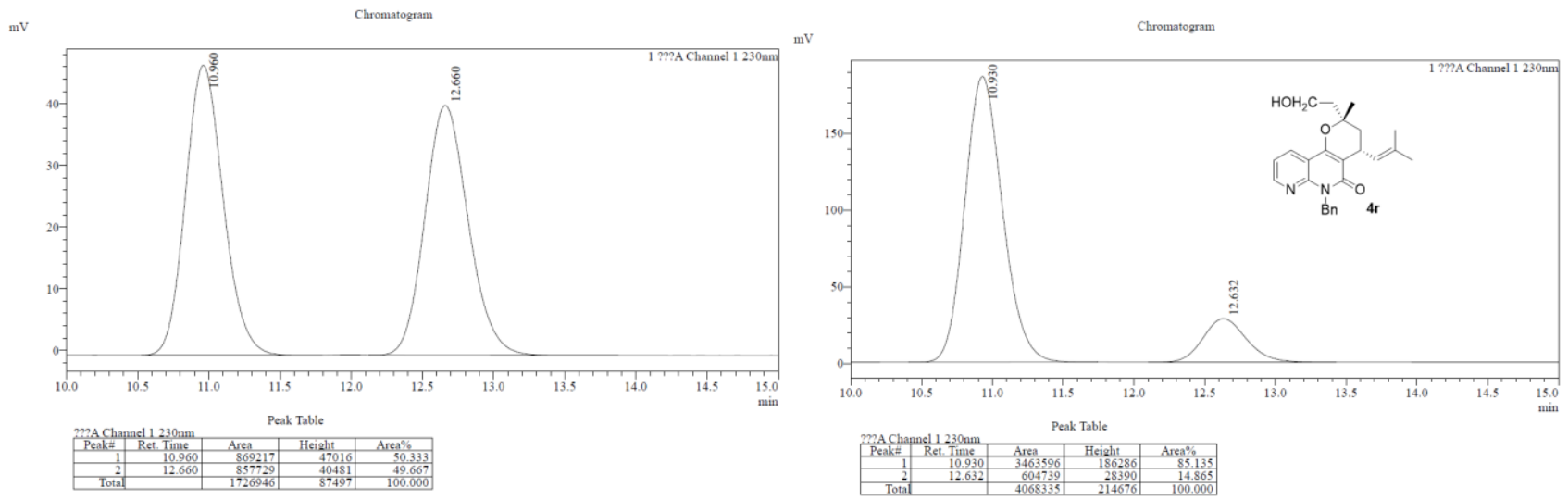

(2R,4R)-6-benzyl-2-(2-hydroxyethyl)-2-methyl-4-(2-methylprop-1-en-1-yl)-2,3,4,6-tetrahydro-5H-pyrano[3,2-c][1, 8]naphthyridin-5-one (4rr)

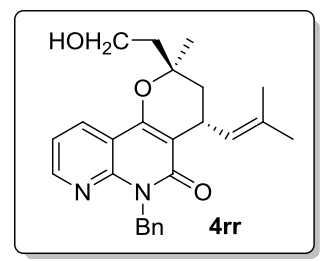

With catalyst 8e, 27\% yield, $27 \mathrm{mg}$. White solid. $66 \%$ ee, determined by HPLC analysis [Chiralpak AD-H, $n$-hexane $/ i$-PrOH $=90 / 10,1.0 \mathrm{~mL} / \mathrm{min}, \lambda=230 \mathrm{~nm}, \mathrm{t}$ (major) $=10.90 \mathrm{~min}, \mathrm{t}$ $($ minor $)=11.97 \mathrm{~min}] .{ }^{1} \mathbf{H}$ NMR $\left(400 \mathrm{MHz}, \mathrm{CDCl}_{3}\right) \delta=8.54-8.53(\mathrm{~m}, 1 \mathrm{H}), 8.15-8.13(\mathrm{~m}, 1 \mathrm{H})$, $7.44(\mathrm{~d}, J=7.2 \mathrm{~Hz}, 2 \mathrm{H}), 7.26-7.09(\mathrm{~m}, 4 \mathrm{H}), 5.78(\mathrm{~d}, J=14.0 \mathrm{~Hz}, 1 \mathrm{H}), 5.63(\mathrm{~d}, J=14.0 \mathrm{~Hz}, 1 \mathrm{H})$, $5.10(\mathrm{~d}, J=8.4 \mathrm{~Hz}, 1 \mathrm{H}), 3.84(\mathrm{t}, J=6.8 \mathrm{~Hz}, 2 \mathrm{H}), 3.77(\mathrm{dd}, J=14.8,7.2 \mathrm{~Hz}, 1 \mathrm{H}), 2.13(\mathrm{dd}, J=$ 14.0, $6.8 \mathrm{~Hz}, 1 \mathrm{H}), 2.07-2.00(\mathrm{~m}, 1 \mathrm{H}), 1.96-1.91(\mathrm{~m}, 1 \mathrm{H}), 1.83-1.76(\mathrm{~m}, 4 \mathrm{H}), 1.75(\mathrm{~s}, 3 \mathrm{H})$, $1.48(\mathrm{~s}, 3 \mathrm{H}) .{ }^{13} \mathrm{C} \mathbf{N M R}\left(100 \mathrm{MHz}, \mathrm{CDCl}_{3}\right) \delta=163.0,153.9,149.6,148.8,138.3,131.8,131.1,128.5,128.2,127.3,126.9$, $117.5,111.9,110.6,78.6,58.8,43.9,40.9,39.4,28.8,26.0,25.8,18.1 .[\alpha]^{20} \mathbf{D}=+18.80^{\circ}\left(c=1.0, \mathrm{CHCl}_{3}, 66 \%\right.$ ee $) . \mathbf{H R M S}$ (ESI) $\mathrm{m} / \mathrm{z}$ : $[\mathrm{M}+\mathrm{Na}]^{+}$calcd. for $\mathrm{C}_{25} \mathrm{H}_{28} \mathrm{~N}_{2} \mathrm{O}_{3} \mathrm{Na} 427.1992$, found 427.1998.
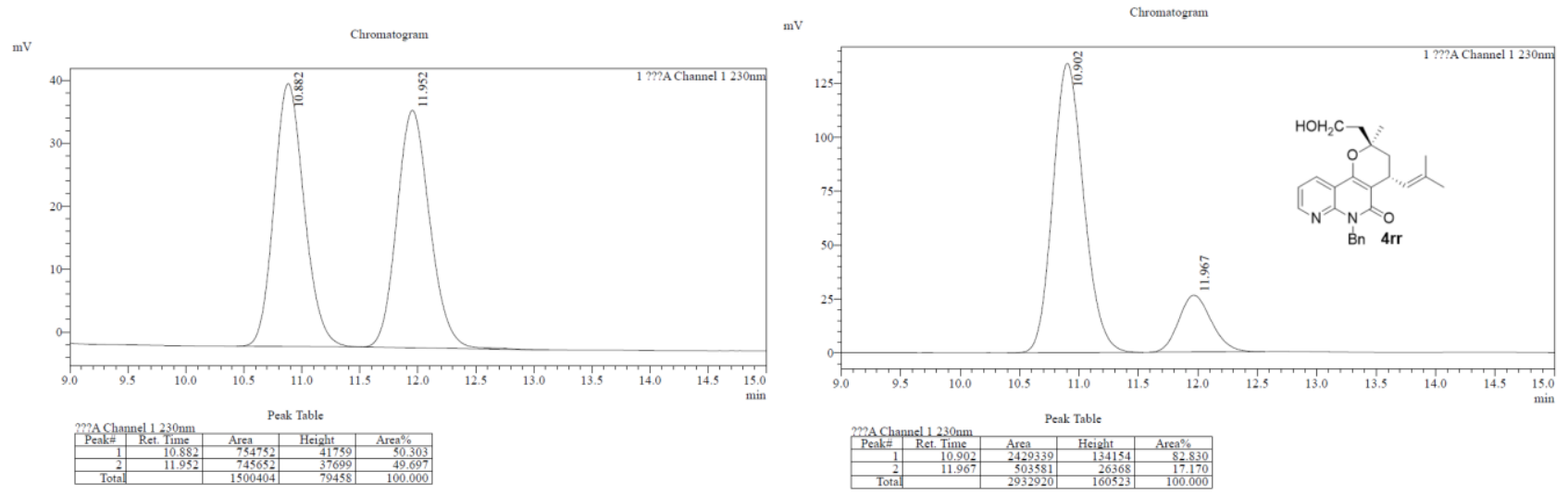
$(2 S, 4 R)-6$-benzyl-2-(2-hydroxyethyl)-2-methyl-4-(2-methylprop-1-en-1-yl)-2,3,4,6-tetrahydro-5H-pyrano[2,3-a][4, 7]phenanthrolin-5-one (4s):

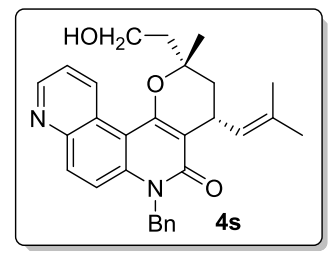

With catalyst 8e, $30 \%$ yield, $34 \mathrm{mg}$. White solid. $72 \%$ ee, determined by HPLC analysis [Chiralpak AD-H, $n$-hexane $/ i$-PrOH $=85 / 15,1.0 \mathrm{~mL} / \mathrm{min}, \lambda=230 \mathrm{~nm}, \mathrm{t}$ (major) $=20.01 \mathrm{~min}, \mathrm{t}$ (minor) $=21.38 \mathrm{~min}] .{ }^{1} \mathbf{H}$ NMR $\left(400 \mathrm{MHz}, \mathrm{CDCl}_{3}\right) \delta=9.75(\mathrm{~d}, J=9.2 \mathrm{~Hz}, 1 \mathrm{H}), 8.84(\mathrm{~d}, J=4.0$ $\mathrm{Hz}, 1 \mathrm{H}), 8.05(\mathrm{~d}, J=9.6 \mathrm{~Hz}, 1 \mathrm{H}), 7.68(\mathrm{~d}, J=9.6 \mathrm{~Hz}, 1 \mathrm{H}), 7.47$ (dd, $J=9.2,4.4 \mathrm{~Hz}, 1 \mathrm{H}), 7.31-$ $7.15(\mathrm{~m}, 5 \mathrm{H}), 5.69(\mathrm{~s}, 2 \mathrm{H}), 5.11(\mathrm{~d}, J=7.6 \mathrm{~Hz}, 1 \mathrm{H}), 4.02-3.97(\mathrm{~m}, 2 \mathrm{H}), 3.94-3.87(\mathrm{~m}, 1 \mathrm{H})$, $2.31-2.21(\mathrm{~m}, 2 \mathrm{H}), 2.14(\mathrm{dd}, J=14.0,7.6 \mathrm{~Hz}, 1 \mathrm{H}), 1.97(\mathrm{dd}, J=14.0,9.2 \mathrm{~Hz}, 1 \mathrm{H}), 1.84(\mathrm{~s}, 3 \mathrm{H}), 1.77(\mathrm{~s}, 3 \mathrm{H}), 1.52(\mathrm{~s}$, 3H). ${ }^{13} \mathbf{C ~ N M R ~}\left(100 \mathrm{MHz}, \mathrm{CDCl}_{3}\right) \delta=162.0,158.9,148.5,144.8,138.3,136.7,135.0,133.0,132.0,128.9,127.2,126.3$, $125.5,121.9,119.0,111.1,109.7,79.3,58.8,46.6,43.7,38.6,29.5,25.9,23.4,18.1 .[\alpha]^{20} \mathbf{D}=+8.16^{\circ}\left(c=1.0, \mathrm{CHCl}_{3}, 72 \%\right.$ ee). HRMS (ESI) m/z: $[\mathrm{M}+\mathrm{H}]^{+}$calcd. for $\mathrm{C}_{29} \mathrm{H}_{31} \mathrm{~N}_{2} \mathrm{O}_{3} 455.2329$, found 455.2315.
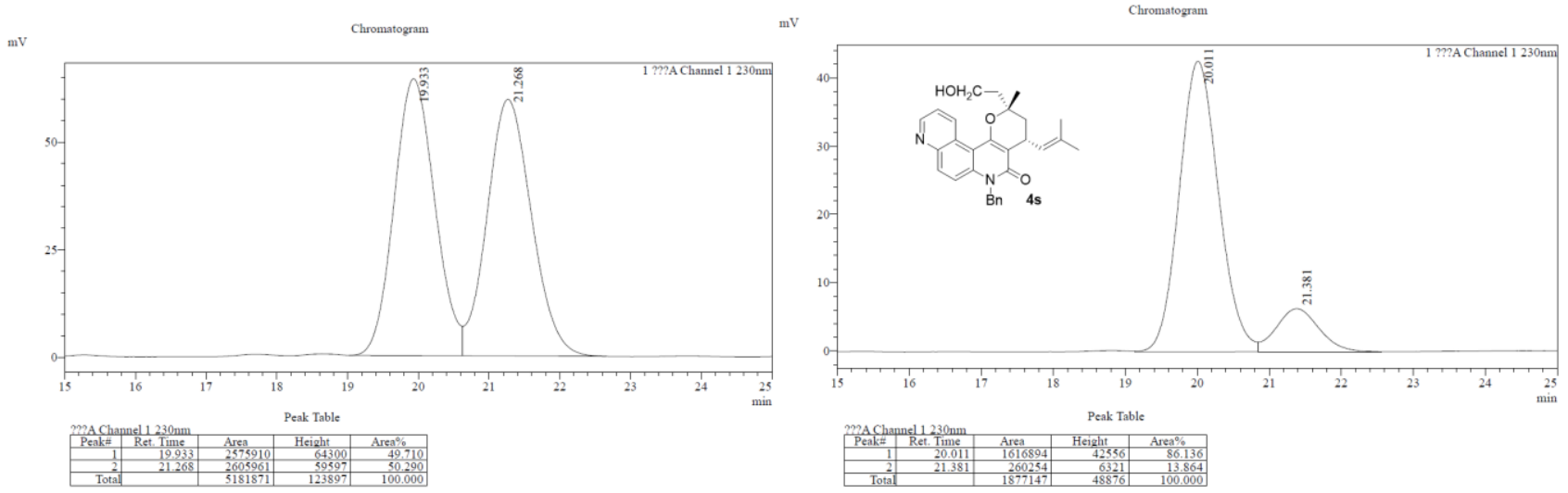

(2R,4R)-6-benzyl-2-(2-hydroxyethyl)-2-methyl-4-(2-methylprop-1-en-1-yl)-2,3,4,6-tetrahydro-5H-pyrano[2,3-a][4, 7]phenanthrolin-5-one (4ss):

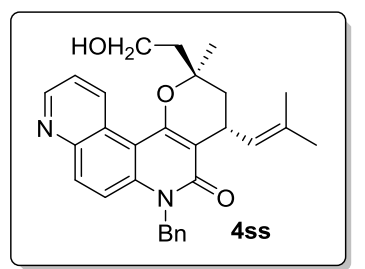

With catalyst 8e, 22\% yield, $25 \mathrm{mg}$. White solid. $62 \%$ ee, determined by HPLC analysis [Chiralpak AD-H, $n$-hexane $/ i$-PrOH $=85 / 15,1.0 \mathrm{~mL} / \mathrm{min}, \lambda=230 \mathrm{~nm}, \mathrm{t}$ (major) $=19.75 \mathrm{~min}$, $\mathrm{t}$ (minor) $=17.76 \mathrm{~min}] .{ }^{1} \mathbf{H}$ NMR $\left(400 \mathrm{MHz}, \mathrm{CDCl}_{3}\right) \delta=9.75(\mathrm{~d}, J=8.8 \mathrm{~Hz}, 1 \mathrm{H}), 8.83(\mathrm{~d}, J=$ $3.6 \mathrm{~Hz}, 1 \mathrm{H}), 8.05(\mathrm{~d}, J=9.6 \mathrm{~Hz}, 1 \mathrm{H}), 7.68(\mathrm{~d}, J=9.6 \mathrm{~Hz}, 1 \mathrm{H}), 7.47(\mathrm{dd}, J=8.8,4.0 \mathrm{~Hz}, 1 \mathrm{H})$, $7.31-7.16(\mathrm{~m}, 5 \mathrm{H}), 5.70(\mathrm{~s}, 2 \mathrm{H}), 5.11(\mathrm{~d}, J=8.4 \mathrm{~Hz}, 1 \mathrm{H}), 3.96-3.74(\mathrm{~m}, 3 \mathrm{H}), 2.29-2.11(\mathrm{~m}$, $2 \mathrm{H}), 2.08-2.00(\mathrm{~m}, 1 \mathrm{H}), 1.91-1.82(\mathrm{~m}, 1 \mathrm{H}), 1.83(\mathrm{~s}, 3 \mathrm{H}), 1.76(\mathrm{~s}, 3 \mathrm{H}), 1.69(\mathrm{~s}, 3 \mathrm{H}) .{ }^{13} \mathrm{C}$ NMR $\left(100 \mathrm{MHz}, \mathrm{CDCl}_{3}\right) \delta=162.0,158.9,148.5,144.8,138.4,136.7,135.0,133.0,131.8,128.9,127.2,126.4,125.5$, $121.9,119.1,111.1,109.5,79.2,58.8,46.6,39.7,39.2,29.5,26.7,25.9,18.1 .[\alpha]^{20} \mathbf{D}=+5.06^{\circ}\left(c=1.0, \mathrm{CHCl}_{3}, 62 \%\right.$ ee $)$. HRMS (ESI) m/z: [M+H] $]^{+}$calcd. for $\mathrm{C}_{29} \mathrm{H}_{31} \mathrm{~N}_{2} \mathrm{O}_{3} 455.2329$, found 455.2336.
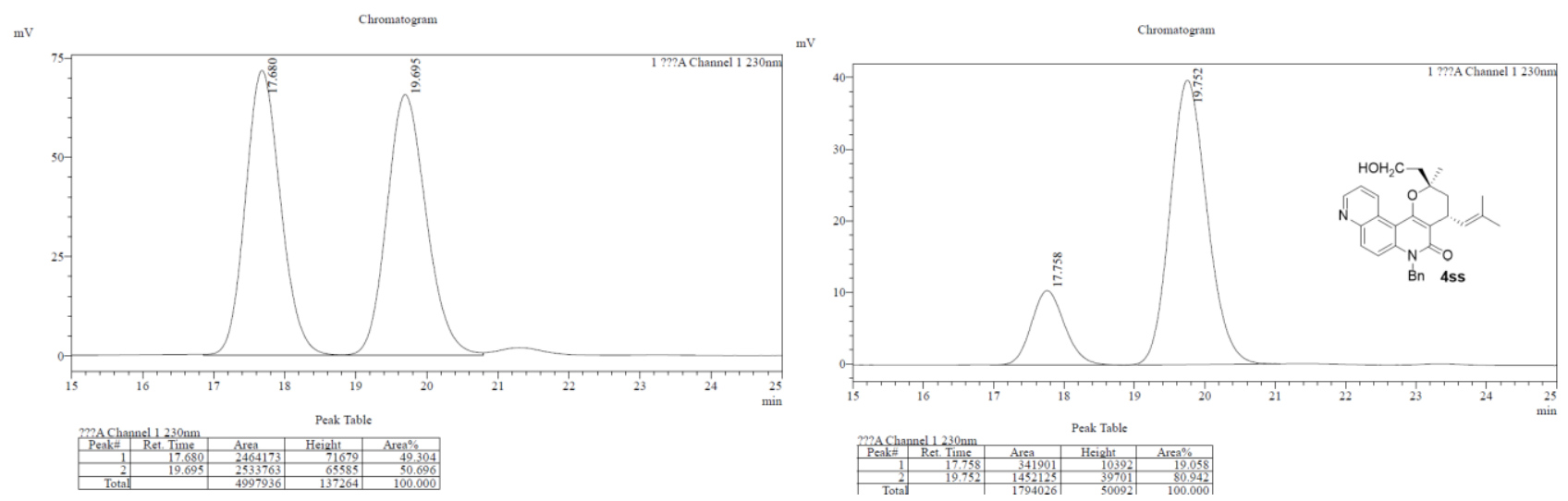
(2S,4R)-6-benzyl-4-(2-ethylbut-1-en-1-yl)-2-(2-hydroxyethyl)-2-methyl-2,3,4,6-tetrahydro-5H-pyrano[3,2-c]quinoli n-5-one (4t):

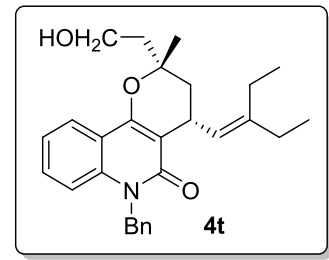

With catalyst 8e, 20\% yield, $22 \mathrm{mg}$. White solid. 93\% ee, determined by HPLC analysis [Chiralpak AD-H, $n$-hexane $/ i$-PrOH $=90 / 10,1.0 \mathrm{~mL} / \mathrm{min}, \lambda=230 \mathrm{~nm}, \mathrm{t}$ (major) $=9.54 \mathrm{~min}, \mathrm{t}$ $($ minor $)=12.55 \mathrm{~min}] .{ }^{1} \mathbf{H}$ NMR $\left(400 \mathrm{MHz}, \mathrm{CDCl}_{3}\right) \delta=7.91-7.86(\mathrm{~m}, 1 \mathrm{H}), 7.39-7.33(\mathrm{~m}, 1 \mathrm{H})$, $7.28-7.23(\mathrm{~m}, 2 \mathrm{H}), 7.23-7.11(\mathrm{~m}, 5 \mathrm{H}), 5.53(\mathrm{~s}, 2 \mathrm{H}), 5.01(\mathrm{~d}, J=8.4 \mathrm{~Hz}, 1 \mathrm{H}), 3.97$ (t, $J=6.8$ $\mathrm{Hz}, 2 \mathrm{H}), 3.84(\mathrm{dd}, J=16.0,8.4 \mathrm{~Hz}, 1 \mathrm{H}), 2.38-2.27(\mathrm{~m}, 1 \mathrm{H}), 2.26-2.17(\mathrm{~m}, 1 \mathrm{H}), 2.14-1.97$ $(\mathrm{m}, 5 \mathrm{H}), 1.87(\mathrm{dd}, J=14.0,8.8 \mathrm{~Hz}, 1 \mathrm{H}), 1.43(\mathrm{~s}, 3 \mathrm{H}), 1.11(\mathrm{t}, J=7.6 \mathrm{~Hz}, 3 \mathrm{H}), 0.99(\mathrm{t}, J=7.6$ $\mathrm{Hz}, 3 \mathrm{H}) .{ }^{13} \mathrm{C}$ NMR $\left(100 \mathrm{MHz}, \mathrm{CDCl}_{3}\right) \delta=162.6,155.1,142.5,138.3,137.0,130.2,128.6,127.0,126.5,125.3,122.8$, $121.5,116.7,114.7,109.6,78.5,58.9,45.6,43.5,39.9,28.7,28.5,23.6,23.2,13.3,12.7 .[\alpha]^{20} \mathbf{D}=-4.66^{\circ}\left(c=1.0, \mathrm{CHCl}_{3}\right.$, $93 \%$ ee). HRMS (ESI) m/z: $[\mathrm{M}+\mathrm{H}]^{+}$calcd. for $\mathrm{C}_{28} \mathrm{H}_{34} \mathrm{NO}_{3} 432.2533$, found 432.2526 .
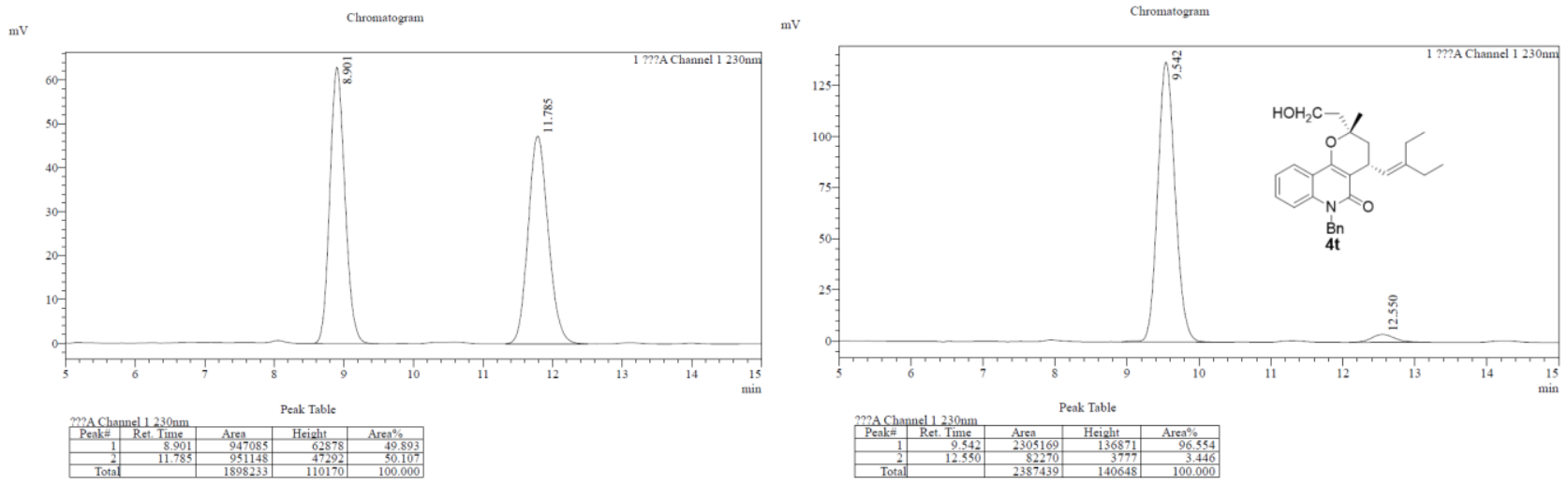

(2R,4R)-6-benzyl-4-(2-ethylbut-1-en-1-yl)-2-(2-hydroxyethyl)-2-methyl-2,3,4,6-tetrahydro-5H-pyrano[3,2-c]quinoli n-5-one (4tt):

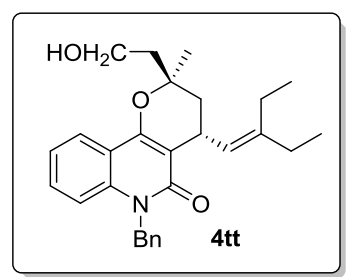

With catalyst 8e, $19 \%$ yield, $20 \mathrm{mg}$. White solid. 93\% ee, determined by HPLC analysis [Chiralpak AD-H, $n$-hexane $/ i-\mathrm{PrOH}=90 / 10,1.0 \mathrm{~mL} / \mathrm{min}, \lambda=230 \mathrm{~nm}, \mathrm{t}$ (major) $=8.80 \mathrm{~min}, \mathrm{t}$ (minor) $=13.14 \mathrm{~min}] .{ }^{1} \mathbf{H}$ NMR $\left(400 \mathrm{MHz}, \mathrm{CDCl}_{3}\right) \delta=7.92(\mathrm{~d}, J=8.0 \mathrm{~Hz}, 1 \mathrm{H}), 7.37(\mathrm{t}, J=7.6$ $\mathrm{Hz}, 1 \mathrm{H}), 7.29-7.23(\mathrm{~m}, 3 \mathrm{H}), 7.28-7.11(\mathrm{~m}, 5 \mathrm{H}), 5.51(\mathrm{~s}, 2 \mathrm{H}), 5.05(\mathrm{~d}, J=8.4 \mathrm{~Hz}, 1 \mathrm{H}), 3.91$ $-3.83(\mathrm{~m}, 3 \mathrm{H}), 2.35-2.26(\mathrm{~m}, 1 \mathrm{H}), 2.26-2.02(\mathrm{~m}, 5 \mathrm{H}), 2.01-1.92(\mathrm{~m}, 1 \mathrm{H}), 1.82(\mathrm{dd}, J=$ 14.0, 7.2 Hz, 1H), $1.53(\mathrm{~s}, 3 \mathrm{H}), 1.11(\mathrm{t}, J=7.2 \mathrm{~Hz}, 3 \mathrm{H}), 0.99(\mathrm{t}, J=7.6 \mathrm{~Hz}, 3 \mathrm{H}) .{ }^{13} \mathbf{C ~ N M R}$ $\left(100 \mathrm{MHz}, \mathrm{CDCl}_{3}\right) \delta=162.7,155.1,142.5,138.4,137.1,130.2,128.6,127.0,126.5,125.7,122.8,121.5,116.7,114.7$,

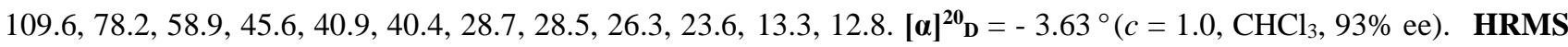
(ESI) $\mathrm{m} / \mathrm{z}:[\mathrm{M}+\mathrm{H}]^{+}$calcd. for $\mathrm{C}_{28} \mathrm{H}_{34} \mathrm{NO}_{3} 432.2533$, found 432.2542 .
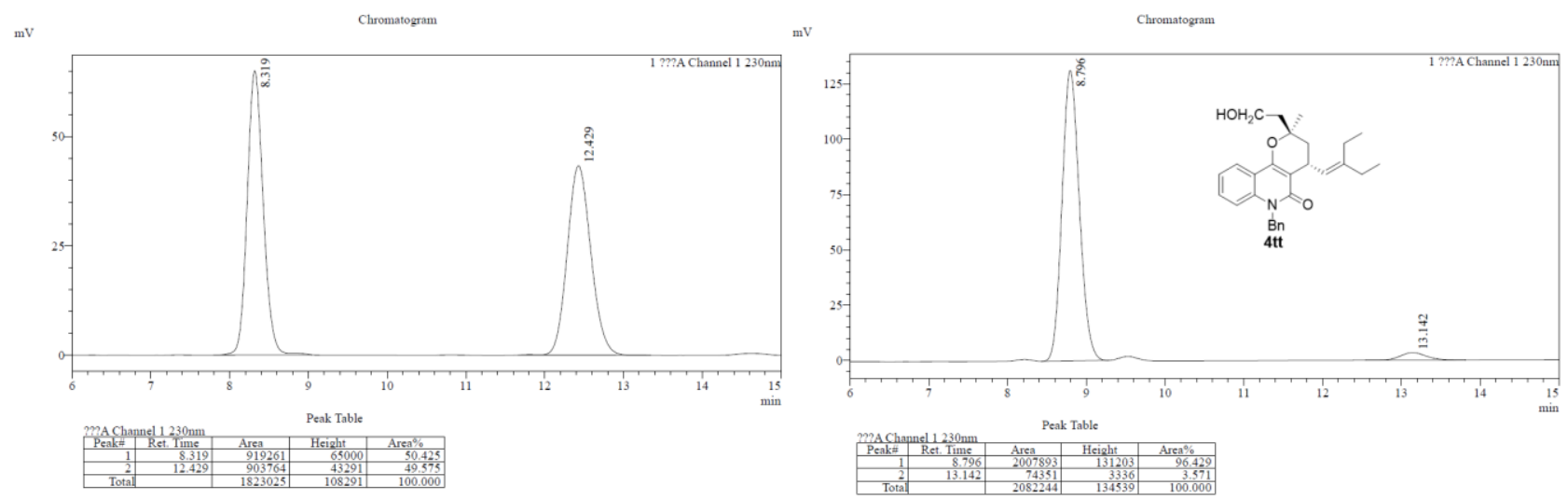


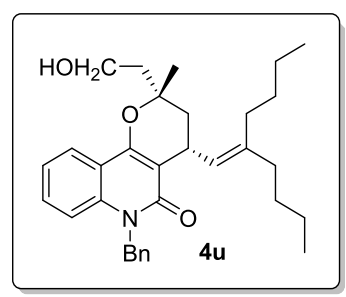

With catalyst 8e, $18 \%$ yield, $22 \mathrm{mg}$. White solid. 94\% ee, determined by HPLC analysis [Chiralpak AD-H, $n$-hexane $/ i$-PrOH $=90 / 10,1.0 \mathrm{~mL} / \mathrm{min}, \lambda=230 \mathrm{~nm}, \mathrm{t}$ (major) $=7.00 \mathrm{~min}, \mathrm{t}$ (minor) $=10.36 \mathrm{~min}] .{ }^{1} \mathbf{H}$ NMR $\left(400 \mathrm{MHz}, \mathrm{CDCl}_{3}\right) \delta=7.88(\mathrm{dd}, J=8.0,1.2 \mathrm{~Hz}, 1 \mathrm{H}), 7.39-$ $7.32(\mathrm{~m}, 1 \mathrm{H}), 7.28-7.23(\mathrm{~m}, 2 \mathrm{H}), 7.22-7.10(\mathrm{~m}, 5 \mathrm{H}), 5.62(\mathrm{~d}, J=12.8 \mathrm{~Hz}, 1 \mathrm{H}), 5.41(\mathrm{~d}, J=$ $13.2 \mathrm{~Hz}, 1 \mathrm{H}), 5.01(\mathrm{~d}, J=8.8 \mathrm{~Hz}, 1 \mathrm{H}), 3.97(\mathrm{t}, J=6.8 \mathrm{~Hz}, 2 \mathrm{H}), 3.82(\mathrm{dd}, J=16.0,8.4 \mathrm{~Hz}, 1 \mathrm{H})$, $2.39-2.28(\mathrm{~m}, 1 \mathrm{H}), 2.18-2.06(\mathrm{~m}, 3 \mathrm{H}), 2.06-1.95(\mathrm{~m}, 3 \mathrm{H}), 1.86(\mathrm{dd}, J=14.0,9.2 \mathrm{~Hz}, 2 \mathrm{H})$, $1.60-1.52(\mathrm{~m}, 1 \mathrm{H}), 1.42(\mathrm{~s}, 3 \mathrm{H}), 1.41-1.22(\mathrm{~m}, 8 \mathrm{H}), 0.94(\mathrm{t}, J=7.2 \mathrm{~Hz}, 3 \mathrm{H}), 0.86(\mathrm{t}, J=7.2 \mathrm{~Hz}, 3 \mathrm{H}) .{ }^{13} \mathbf{C ~ N M R}(100$ $\left.\mathrm{MHz}, \mathrm{CDCl}_{3}\right) \delta=162.5,155.0,139.9,138.3,137.1,130.2,128.6,127.0,126.7,126.5,122.8,121.4,116.7,114.6,109.6$, $78.5,58.8,45.6,43.5,39.9,36.2,30.8,30.3,30.3,28.6,23.2,23.1,22.4,14.1,14.1 .[\alpha]^{\mathbf{2 0}} \mathbf{D}=-26.14^{\circ}\left(c=1.0, \mathrm{CHCl}_{3}, 94 \%\right.$ ee). HRMS (ESI) m/z: $[\mathrm{M}+\mathrm{Na}]^{+}$calcd. for $\mathrm{C}_{32} \mathrm{H}_{41} \mathrm{NO}_{3} \mathrm{Na} 510.2979$, found 510.2961.
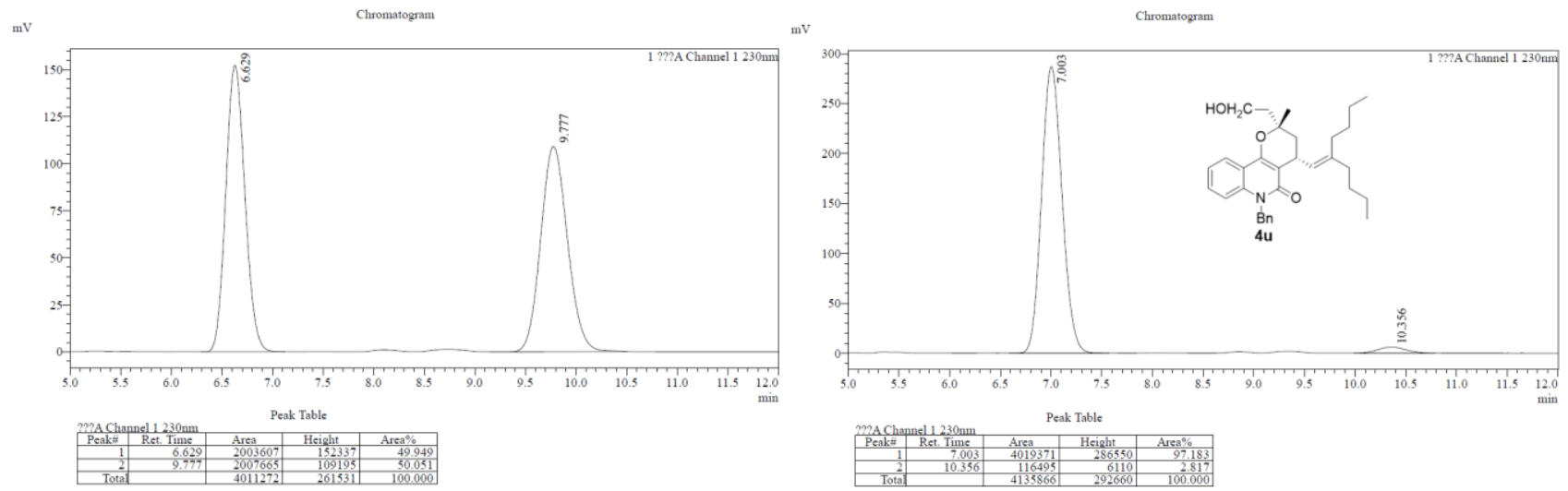

(2R,4R)-6-benzyl-4-(2-butylhex-1-en-1-yl)-2-(2-hydroxyethyl)-2-methyl-2,3,4,6-tetrahydro-5H-pyrano[3,2-c]quinol in-5-one (4uu):

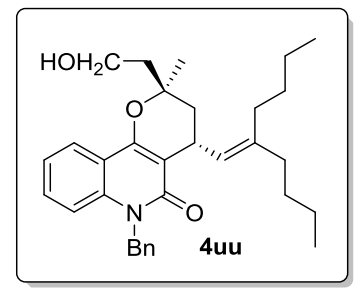

With catalyst $\mathbf{8 e}, 18 \%$ yield, $21 \mathrm{mg}$. White solid. $93 \%$ ee, determined by HPLC analysis [Chiralpak AD-H, $n$-hexane $/ i-\mathrm{PrOH}=90 / 10,1.0 \mathrm{~mL} / \mathrm{min}, \lambda=230 \mathrm{~nm}, \mathrm{t}$ (major) $=6.38 \mathrm{~min}, \mathrm{t}$ $($ minor $)=8.46 \mathrm{~min}] .{ }^{1} \mathbf{H} \mathbf{N M R}\left(400 \mathrm{MHz}, \mathrm{CDCl}_{3}\right) \delta=7.92(\mathrm{dd}, J=8.0,1.2 \mathrm{~Hz}, 1 \mathrm{H}), 7.39-$ $7.33(\mathrm{~m}, 1 \mathrm{H}), 7.28-7.23(\mathrm{~m}, 3 \mathrm{H}), 7.22-7.11(\mathrm{~m}, 5 \mathrm{H}), 5.50(\mathrm{~d}, J=25.2 \mathrm{~Hz}, 2 \mathrm{H}), 5.06(\mathrm{~d}, J=$ $8.4 \mathrm{~Hz}, 1 \mathrm{H}), 3.92-3.79(\mathrm{~m}, 3 \mathrm{H}), 2.36-2.26(\mathrm{~m}, 1 \mathrm{H}), 2.19-2.05(\mathrm{~m}, 3 \mathrm{H}), 2.02(\mathrm{t}, J=7.2 \mathrm{~Hz}$, 2H), $1.99-1.90(\mathrm{~m}, 1 \mathrm{H}), 1.81(\mathrm{dd}, J=14.0,7.2 \mathrm{~Hz}, 1 \mathrm{H}), 1.52(\mathrm{~s}, 3 \mathrm{H}), 1.44-1.23(\mathrm{~m}, 8 \mathrm{H})$, $0.94(\mathrm{t}, J=6.8 \mathrm{~Hz}, 3 \mathrm{H}), 0.86(\mathrm{t}, J=7.2 \mathrm{~Hz}, 3 \mathrm{H}) .{ }^{13} \mathrm{C}$ NMR $\left(100 \mathrm{MHz}, \mathrm{CDCl}_{3}\right) \delta=162.6,155.0,139.9,138.4,137.1$, 130.2 , 128.6, 127.2, 126.9, 126.5, 122.8, 121.5, 116.7, 114.6, 109.6, 78.1, 58.9, 45.6, 40.7, 40.3, 36.2, 30.8, 30.4, 30.3, 28.5, 26.3, 23.3, 22.4, 14.2, 14.1. $[\boldsymbol{\alpha}]^{20} \mathrm{D}=-19.48^{\circ}\left(c=1.0, \mathrm{CHCl}_{3}, 93 \%\right.$ ee $) . \mathbf{H R M S}(\mathrm{ESI}) \mathrm{m} / \mathrm{z}:[\mathrm{M}+\mathrm{H}]^{+}$calcd. for $\mathrm{C}_{32} \mathrm{H}_{42} \mathrm{NO}_{3} 488.3159$, found 488.3160 .
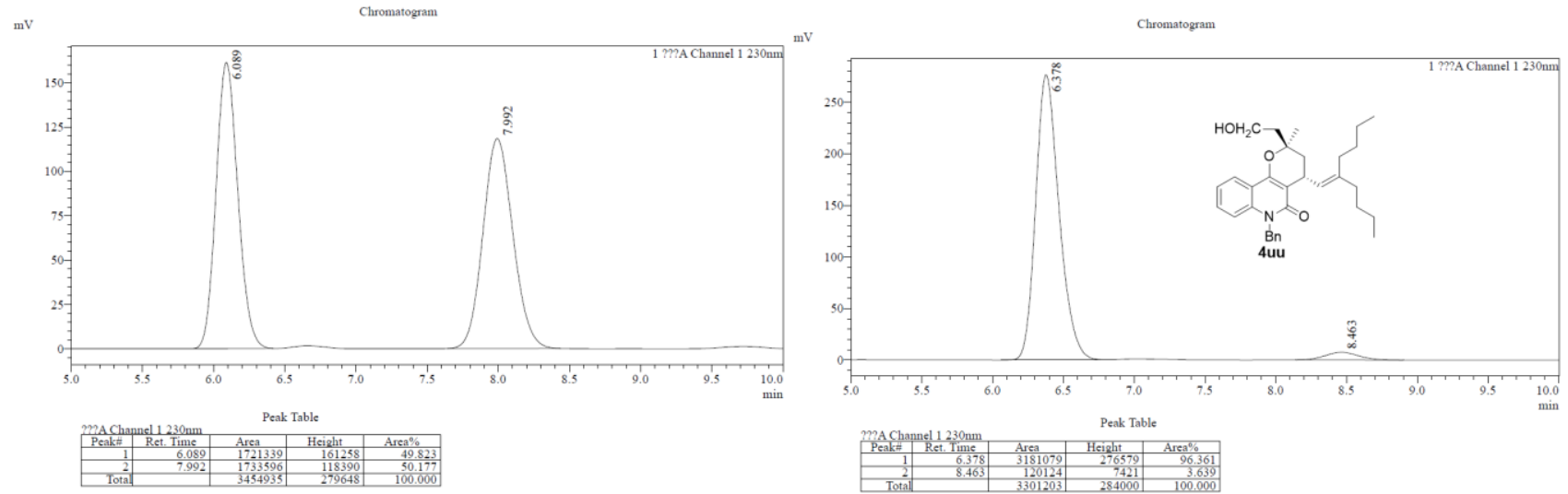
(2S,4R)-6-benzyl-4-(cyclopentylidenemethyl)-2-(2-hydroxyethyl)-2-methyl-2,3,4,6-tetrahydro-5H-pyrano[3,2-c]qui nolin-5-one (4v):

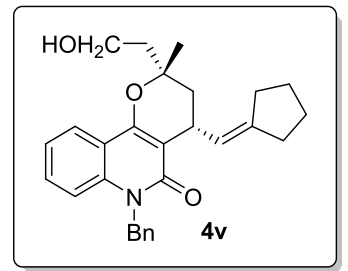

With catalyst 8e, $29 \%$ yield, $31 \mathrm{mg}$. White solid. $92 \%$ ee, determined by HPLC analysis [Chiralpak IA, $n$-hexane $/ i$-PrOH $=90 / 10,1.0 \mathrm{~mL} / \mathrm{min}, \lambda=230 \mathrm{~nm}, \mathrm{t}$ (major) $=16.15 \mathrm{~min}, \mathrm{t}$ $($ minor $)=14.60 \mathrm{~min}] .{ }^{1} \mathbf{H}$ NMR $\left(400 \mathrm{MHz}, \mathrm{CDCl}_{3}\right) \delta=7.90(\mathrm{~d}, J=7.6 \mathrm{~Hz}, 1 \mathrm{H}), 7.37(\mathrm{t}, J=8.0$ $\mathrm{Hz}, 1 \mathrm{H}), 7.29-7.23(\mathrm{~m}, 3 \mathrm{H}), 7.21-7.11(\mathrm{~m}, 5 \mathrm{H}), 5.53(\mathrm{dd}, J=24.4,13.6 \mathrm{~Hz}, 2 \mathrm{H}), 5.25(\mathrm{~d}, J$ $=8.0 \mathrm{~Hz}, 1 \mathrm{H}), 3.96(\mathrm{t}, J=6.8 \mathrm{~Hz}, 2 \mathrm{H}), 3.69(\mathrm{dd}, J=16.0,6.4 \mathrm{~Hz}, 1 \mathrm{H}), 2.62-2.49(\mathrm{~m}, 1 \mathrm{H})$, $2.46-2.33(\mathrm{~m}, 1 \mathrm{H}), 2.27-2.19(\mathrm{~m}, 2 \mathrm{H}), 2.14-2.00(\mathrm{~m}, 3 \mathrm{H}), 1.90(\mathrm{dd}, J=14.0,8.8 \mathrm{~Hz}, 1 \mathrm{H})$, $1.76-1.59(\mathrm{~m}, 4 \mathrm{H}), 1.42(\mathrm{~s}, 3 \mathrm{H}) .{ }^{13} \mathbf{C} \mathbf{N M R}\left(100 \mathrm{MHz}, \mathrm{CDCl}_{3}\right) \delta=162.7,155.2,143.2,138.3,137.0,130.3,128.7,127.0$, $126.5,122.9,122.7,121.5,116.7,114.7,109.4,78.5,58.9,45.6,43.3,38.6,33.7,30.4,28.6,26.5,26.3,23.4 .[\alpha]^{\mathbf{2 0}} \mathbf{D}=+$ $13.33^{\circ}\left(c=1.0, \mathrm{CHCl}_{3}, 92 \%\right.$ ee). HRMS (ESI) m/z: $[\mathrm{M}+\mathrm{H}]^{+}$calcd. for $\mathrm{C}_{28} \mathrm{H}_{32} \mathrm{NO}_{3} 430.2377$, found 430.2369 .
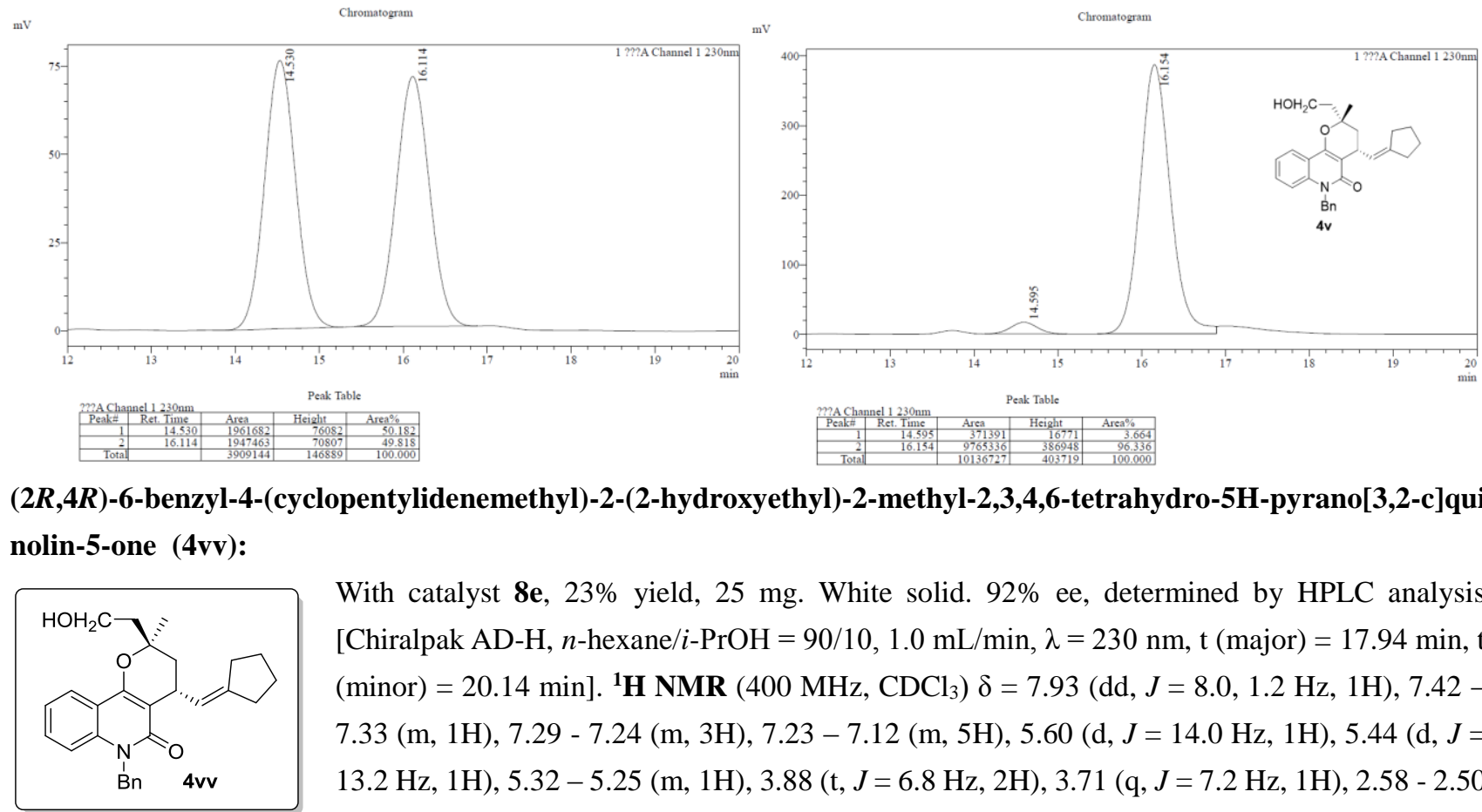

$(2 R, 4 R)$-6-benzyl-4-(cyclopentylidenemethyl)-2-(2-hydroxyethyl)-2-methyl-2,3,4,6-tetrahydro-5H-pyrano[3,2-c]qui nolin-5-one $(4 \mathrm{vv})$ :

With catalyst 8e, 23\% yield, $25 \mathrm{mg}$. White solid. 92\% ee, determined by HPLC analysis [Chiralpak AD-H, $n$-hexane $/ i$-PrOH $=90 / 10,1.0 \mathrm{~mL} / \mathrm{min}, \lambda=230 \mathrm{~nm}, \mathrm{t}$ (major) $=17.94 \mathrm{~min}, \mathrm{t}$ $($ minor $)=20.14 \mathrm{~min}] .{ }^{1} \mathbf{H}$ NMR $\left(400 \mathrm{MHz}, \mathrm{CDCl}_{3}\right) \delta=7.93(\mathrm{dd}, J=8.0,1.2 \mathrm{~Hz}, 1 \mathrm{H}), 7.42-$ $7.33(\mathrm{~m}, 1 \mathrm{H}), 7.29-7.24(\mathrm{~m}, 3 \mathrm{H}), 7.23-7.12(\mathrm{~m}, 5 \mathrm{H}), 5.60(\mathrm{~d}, J=14.0 \mathrm{~Hz}, 1 \mathrm{H}), 5.44(\mathrm{~d}, J=$ $13.2 \mathrm{~Hz}, 1 \mathrm{H}), 5.32-5.25(\mathrm{~m}, 1 \mathrm{H}), 3.88(\mathrm{t}, J=6.8 \mathrm{~Hz}, 2 \mathrm{H}), 3.71(\mathrm{q}, J=7.2 \mathrm{~Hz}, 1 \mathrm{H}), 2.58-2.50$ $(\mathrm{m}, 1 \mathrm{H}), 2.42-2.33(\mathrm{~m}, 1 \mathrm{H}), 2.29-2.14(\mathrm{~m}, 3 \mathrm{H}), 2.13-2.04(\mathrm{~m}, 1 \mathrm{H}), 2.01-1.91(\mathrm{~m}, 1 \mathrm{H}), 1.88$ - $1.81(\mathrm{~m}, 1 \mathrm{H}), 1.78-1.57(\mathrm{~m}, 4 \mathrm{H}), 1.52(\mathrm{~s}, 3 \mathrm{H}) .{ }^{13} \mathbf{C} \mathbf{N M R}\left(100 \mathrm{MHz}, \mathrm{CDCl}_{3}\right) \delta=162.8,155.1,143.1,138.3,137.0$, 130.2 , 128.7, 127.0, 126.5, 123.0, 122.9, 121.5, 116.7, 114.7, 109.3, 78.3, 58.8, 45.7, 41.1, 38.9, 33.7, 30.4, 28.6, 26.5, 26.3, 26.0. $[\alpha]^{20} \mathbf{D}=+11.80^{\circ}\left(c=1.0, \mathrm{CHCl}_{3}, 92 \%\right.$ ee $)$. HRMS (ESI) m/z: $[\mathrm{M}+\mathrm{H}]^{+}$calcd. for $\mathrm{C}_{28} \mathrm{H}_{32} \mathrm{NO}_{3} 430.2377$, found 430.2368 .
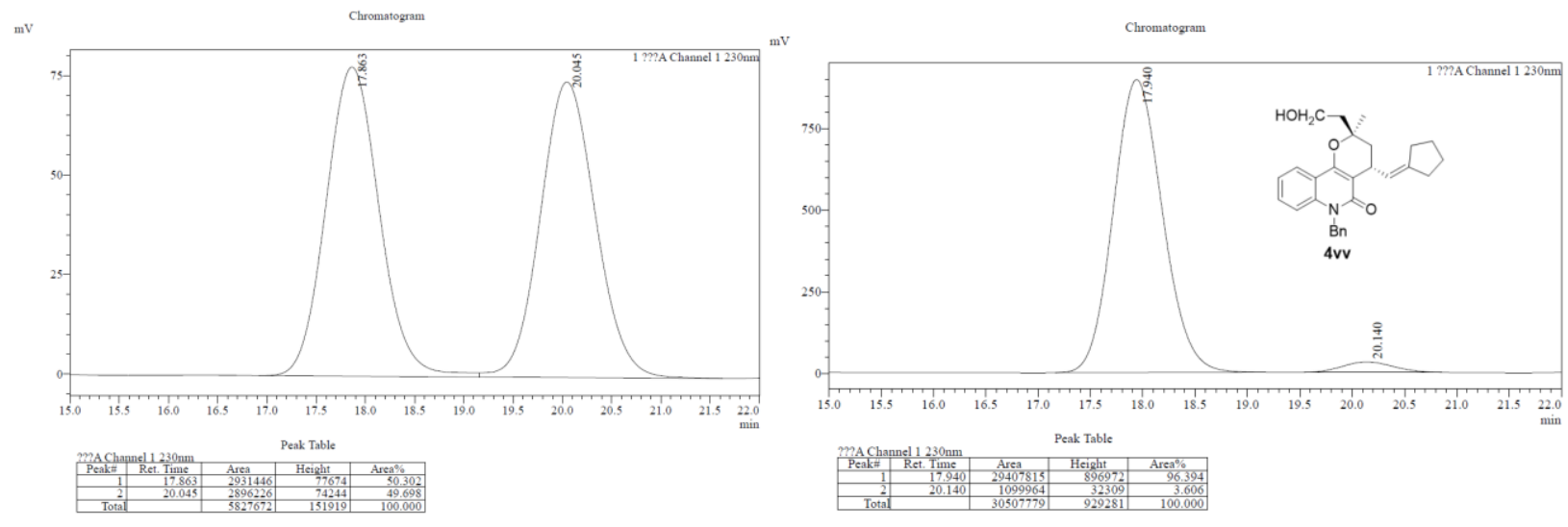
$(8 S, 10 R)$-10-(2-hydroxyethyl)-10-methyl-8-(2-methylprop-1-en-1-yl)-4,5,9,10-tetrahydro-7H,8H-pyrano[3,2-c]pyrr olo[3,2,1-ij]quinolin-7-one $(4 \mathrm{w})$ :

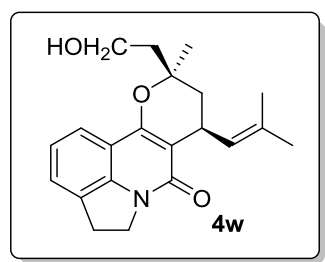

With catalyst 8f, $31 \%$ yield, $26 \mathrm{mg}$. White solid. $-95 \%$ ee, determined by HPLC analysis [Chiralpak OD-H, $n$-hexane $/ i$-PrOH $=90 / 10,1.0 \mathrm{~mL} / \mathrm{min}, \lambda=230 \mathrm{~nm}, \mathrm{t}$ (major) $=23.61 \mathrm{~min}, \mathrm{t}$ $($ minor $)=15.62 \mathrm{~min}] .{ }^{1} \mathbf{H}$ NMR $\left(400 \mathrm{MHz}, \mathrm{CDCl}_{3}\right) \delta=7.49(\mathrm{~d}, J=8.0 \mathrm{~Hz}, 1 \mathrm{H}), 7.28(\mathrm{~d}, J=8.0$ $\mathrm{Hz}, 1 \mathrm{H}), 7.09$ (t, $J=7.6 \mathrm{~Hz}, 1 \mathrm{H}), 5.02(\mathrm{~d}, J=8.4 \mathrm{~Hz}, 1 \mathrm{H}), 4.46-4.27(\mathrm{~m}, 2 \mathrm{H}), 4.01-3.89(\mathrm{~m}$, 2H), $3.73(\mathrm{dd}, J=16.4,8.8 \mathrm{~Hz}, 1 \mathrm{H}), 3.41-3.32(\mathrm{~m}, 2 \mathrm{H}), 2.13-1.93(\mathrm{~m}, 4 \mathrm{H}), 1.81(\mathrm{~s}, 3 \mathrm{H}), 1.75$ $(\mathrm{s}, 3 \mathrm{H}), 1.36(\mathrm{~s}, 3 \mathrm{H}) .{ }^{13} \mathrm{C} \mathbf{N M R}\left(100 \mathrm{MHz}, \mathrm{CDCl}_{3}\right) \delta=161.3,155.5,141.2,131.5,130.2,126.9$, $124.7,122.4,119.0,113.4,111.0,78.5,58.8,46.6,43.4,39.2,28.9,27.3,25.9,22.7,18.0 .[\alpha]^{20} \mathbf{D}=+6.60^{\circ}\left(c=1.0, \mathrm{CHCl}_{3}\right.$, $95 \%$ ee). HRMS (ESI) m/z: $[\mathrm{M}+\mathrm{H}]^{+}$calcd. for $\mathrm{C}_{21} \mathrm{H}_{26} \mathrm{NO}_{3} 340.1907$, found 340.1895 .
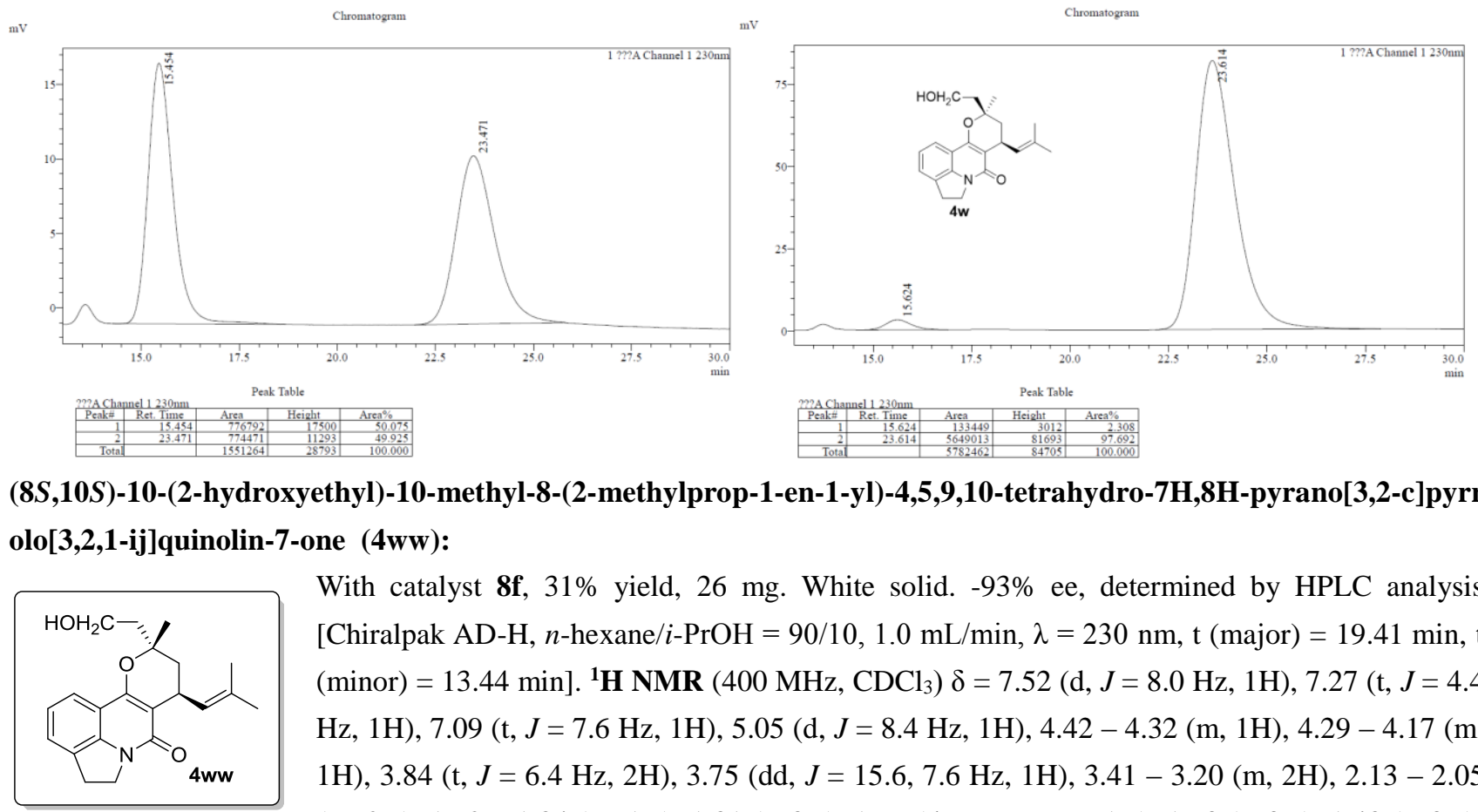

(8S,10S)-10-(2-hydroxyethyl)-10-methyl-8-(2-methylprop-1-en-1-yl)-4,5,9,10-tetrahydro-7H,8H-pyrano[3,2-c]pyrr olo[3,2,1-ij]quinolin-7-one (4ww):

With catalyst 8f, $31 \%$ yield, $26 \mathrm{mg}$. White solid. $-93 \%$ ee, determined by HPLC analysis [Chiralpak AD-H, $n$-hexane $/ i-\mathrm{PrOH}=90 / 10,1.0 \mathrm{~mL} / \mathrm{min}, \lambda=230 \mathrm{~nm}, \mathrm{t}$ (major) $=19.41 \mathrm{~min}, \mathrm{t}$ $($ minor $)=13.44 \mathrm{~min}] .{ }^{1} \mathbf{H}$ NMR $\left(400 \mathrm{MHz}, \mathrm{CDCl}_{3}\right) \delta=7.52(\mathrm{~d}, J=8.0 \mathrm{~Hz}, 1 \mathrm{H}), 7.27(\mathrm{t}, J=4.4$ $\mathrm{Hz}, 1 \mathrm{H}), 7.09$ (t, $J=7.6 \mathrm{~Hz}, 1 \mathrm{H}), 5.05(\mathrm{~d}, J=8.4 \mathrm{~Hz}, 1 \mathrm{H}), 4.42-4.32(\mathrm{~m}, 1 \mathrm{H}), 4.29-4.17$ (m, $1 \mathrm{H}), 3.84(\mathrm{t}, J=6.4 \mathrm{~Hz}, 2 \mathrm{H}), 3.75(\mathrm{dd}, J=15.6,7.6 \mathrm{~Hz}, 1 \mathrm{H}), 3.41-3.20(\mathrm{~m}, 2 \mathrm{H}), 2.13-2.05$ (m, 2H), $1.92-1.84(\mathrm{~m}, 1 \mathrm{H}), 1.81(\mathrm{~s}, 3 \mathrm{H}), 1.77(\mathrm{~d}, J=7.6 \mathrm{~Hz}, 1 \mathrm{H}), 1.73(\mathrm{~s}, 3 \mathrm{H}), 1.48(\mathrm{~s}, 3 \mathrm{H})$.

${ }^{13} \mathbf{C}$ NMR $\left(100 \mathrm{MHz}, \mathrm{CDCl}_{3}\right) \delta=161.3,155.5,141.2,131.6,130.2,127.3,124.6,122.4,119.0,113.4,110.9,78.1,58.8$, 46.6, 40.5, 39.6, 28.9, 27.3, 26.0, 25.9, 18.0. $[\boldsymbol{\alpha}]^{20} \mathrm{D}=+16.20^{\circ}\left(c=1.0, \mathrm{CHCl}_{3}, 93 \%\right.$ ee $) . \mathbf{H R M S}(\mathrm{ESI}) \mathrm{m} / \mathrm{z}:[\mathrm{M}+\mathrm{H}]^{+} \mathrm{calcd}$. for $\mathrm{C}_{21} \mathrm{H}_{26} \mathrm{NO}_{3} 340.1907$, found 340.1896.
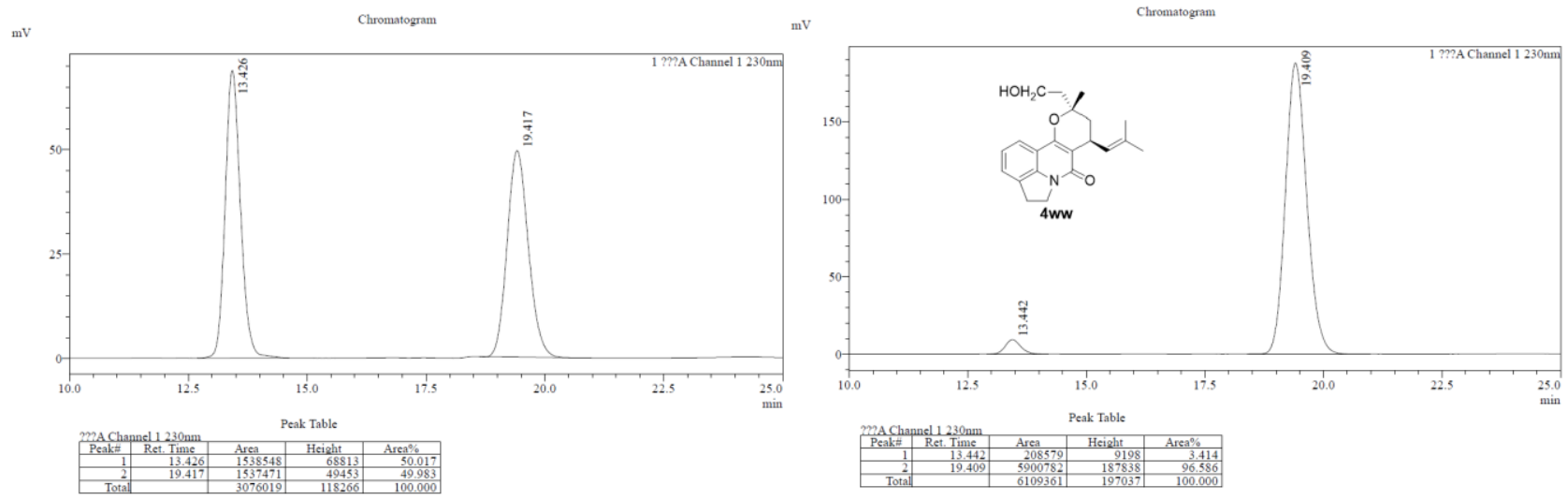
(9S,11R)-11-(2-hydroxyethyl)-11-methyl-9-(2-methylprop-1-en-1-yl)-5,6,10,11-tetrahydro-4H,8H,9H-pyrano[3,2-c] pyrido[3,2,1-ij]quinolin-8-one $(4 x)$ :

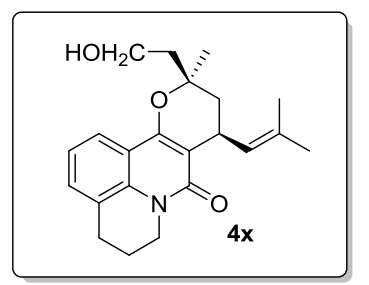

With catalyst 8f, 29\% yield, $25 \mathrm{mg}$. White solid. $-96 \%$ ee, determined by HPLC analysis [Chiralpak AD-H, $n$-hexane $/ i$-PrOH $=90 / 10,1.0 \mathrm{~mL} / \mathrm{min}, \lambda=230 \mathrm{~nm}, \mathrm{t}$ (major) $=11.55 \mathrm{~min}$, $\mathrm{t}($ minor $)=10.22 \mathrm{~min}] .{ }^{1} \mathbf{H} \mathbf{N M R}\left(400 \mathrm{MHz}, \mathrm{CDCl}_{3}\right) \delta=7.71(\mathrm{~d}, J=8.0 \mathrm{~Hz}, 1 \mathrm{H}), 7.27-7.24$ $(\mathrm{m}, 2 \mathrm{H}), 7.08(\mathrm{t}, J=8.0 \mathrm{~Hz}, 1 \mathrm{H}), 5.03(\mathrm{~d}, J=8.4 \mathrm{~Hz}, 1 \mathrm{H}), 4.30-4.22(\mathrm{~m}, 1 \mathrm{H}), 4.08-4.00(\mathrm{~m}$, $1 \mathrm{H}), 3.95(\mathrm{t}, J=6.4 \mathrm{~Hz}, 2 \mathrm{H}), 3.73(\mathrm{dd}, J=16.4,8.4 \mathrm{~Hz}, 1 \mathrm{H}), 2.95(\mathrm{t}, J=6.0 \mathrm{~Hz}, 2 \mathrm{H}), 2.09-$ $2.03(\mathrm{~m}, 4 \mathrm{H}), 1.96(\mathrm{dd}, J=14.0,7.2 \mathrm{~Hz}, 1 \mathrm{H}), 1.84-1.77(\mathrm{~m}, 4 \mathrm{H}), 1.75(\mathrm{~s}, 3 \mathrm{H}), 1.37$ (s, 3H). ${ }^{13} \mathrm{C}$ NMR $\left(100 \mathrm{MHz}, \mathrm{CDCl}_{3}\right) \delta=162.2,155.0,135.6,131.3,129.5,127.2,124.4,121.0,120.6,116.2,109.2,78.3,58.8$, $43.5,41.9,39.2,28.8,27.9,25.9,23.0,20.9,18.0 .[\alpha]^{20} \mathbf{D}=-13.10^{\circ}\left(c=1.0, \mathrm{CHCl}_{3}, 96 \%\right.$ ee $) . \mathbf{H R M S}(\mathrm{ESI}) \mathrm{m} / \mathrm{z}:[\mathrm{M}+\mathrm{H}]^{+}$ calcd. for $\mathrm{C}_{22} \mathrm{H}_{28} \mathrm{NO}_{3} 354.2064$, found 354.2063.
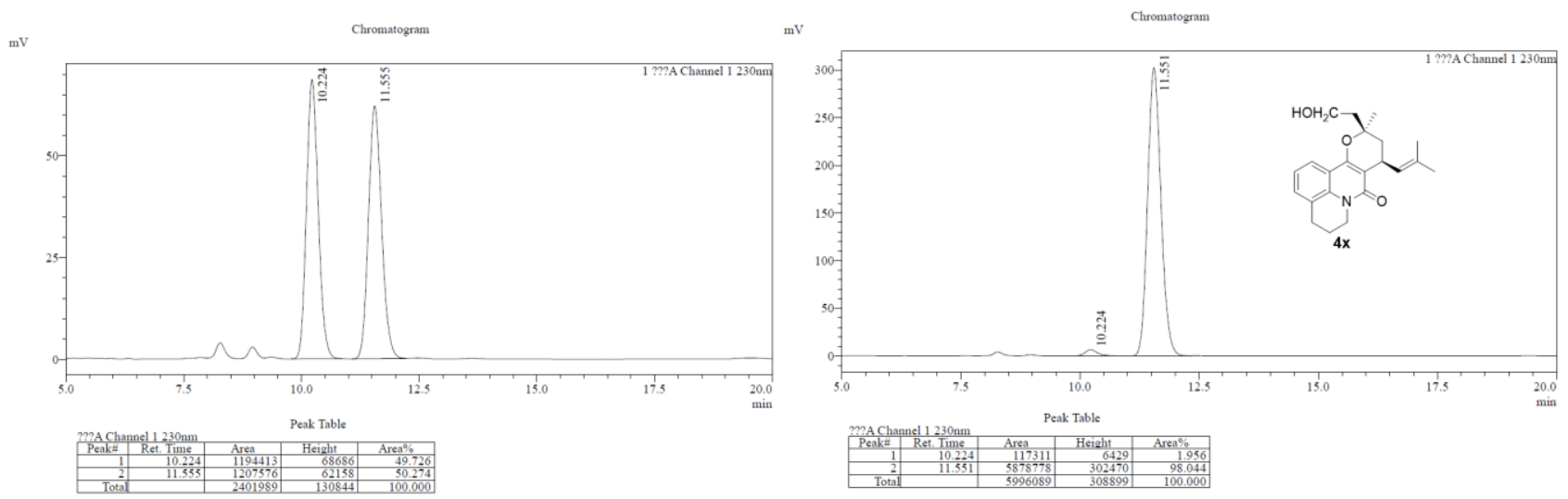

(9S,11S)-11-(2-hydroxyethyl)-11-methyl-9-(2-methylprop-1-en-1-yl)-5,6,10,11-tetrahydro-4H,8H,9H-pyrano[3,2-c] pyrido[3,2,1-ij]quinolin-8-one (4xx):

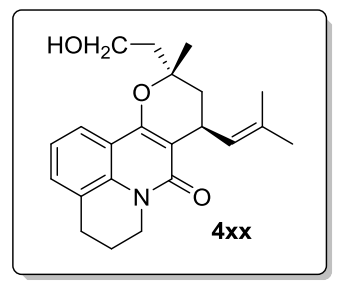

With catalyst 8f, $23 \%$ yield, $21 \mathrm{mg}$. White solid. $-95 \%$ ee, determined by HPLC analysis [Chiralpak AD-H, $n$-hexane $/ i-\mathrm{PrOH}=90 / 10,1.0 \mathrm{~mL} / \mathrm{min}, \lambda=230 \mathrm{~nm}, \mathrm{t}$ (major) $=14.42 \mathrm{~min}, \mathrm{t}$ (minor) $=9.64 \mathrm{~min}] .{ }^{1} \mathbf{H}$ NMR $\left(400 \mathrm{MHz}, \mathrm{CDCl}_{3}\right) \delta=7.74(\mathrm{~d}, J=8.0 \mathrm{~Hz}, 1 \mathrm{H}), 7.27-7.23(\mathrm{~m}$, $2 \mathrm{H}), 7.08(\mathrm{t}, J=8.0 \mathrm{~Hz}, 1 \mathrm{H}), 5.07(\mathrm{~d}, J=8.4 \mathrm{~Hz}, 1 \mathrm{H}), 4.28-4.19(\mathrm{~m}, 1 \mathrm{H}), 4.04-3.95(\mathrm{~m}, 1 \mathrm{H})$, $3.84(\mathrm{t}, J=6.8 \mathrm{~Hz}, 2 \mathrm{H}), 3.74(\mathrm{dd}, J=15.6,8.0 \mathrm{~Hz}, 1 \mathrm{H}), 2.93(\mathrm{t}, J=6.0 \mathrm{~Hz}, 2 \mathrm{H}), 2.14-2.03(\mathrm{~m}$, 4H), $1.93-1.84(\mathrm{~m}, 2 \mathrm{H}), 1.81(\mathrm{~s}, 3 \mathrm{H}), 1.74(\mathrm{~s}, 3 \mathrm{H}), 1.48(\mathrm{~s}, 3 \mathrm{H}) .{ }^{13} \mathbf{C ~ N M R}\left(100 \mathrm{MHz}, \mathrm{CDCl}_{3}\right)$ $\delta=162.2,154.9,135.6,131.3,129.5,127.6,124.4,121.0,120.6,116.2,109.2,77.9,58.8,41.9,40.5,39.7,28.8,27.9,26.0$, 25.9, 20.9, 18.0. $[\boldsymbol{\alpha}]^{\mathbf{2 0}} \mathbf{D}=-9.60^{\circ}\left(c=1.0, \mathrm{CHCl}_{3}, 95 \%\right.$ ee $)$. HRMS (ESI) m/z: $[\mathrm{M}+\mathrm{H}]^{+}$calcd. for $\mathrm{C}_{22} \mathrm{H}_{28} \mathrm{NO}_{3} 354.2064$, found 354.2060 .
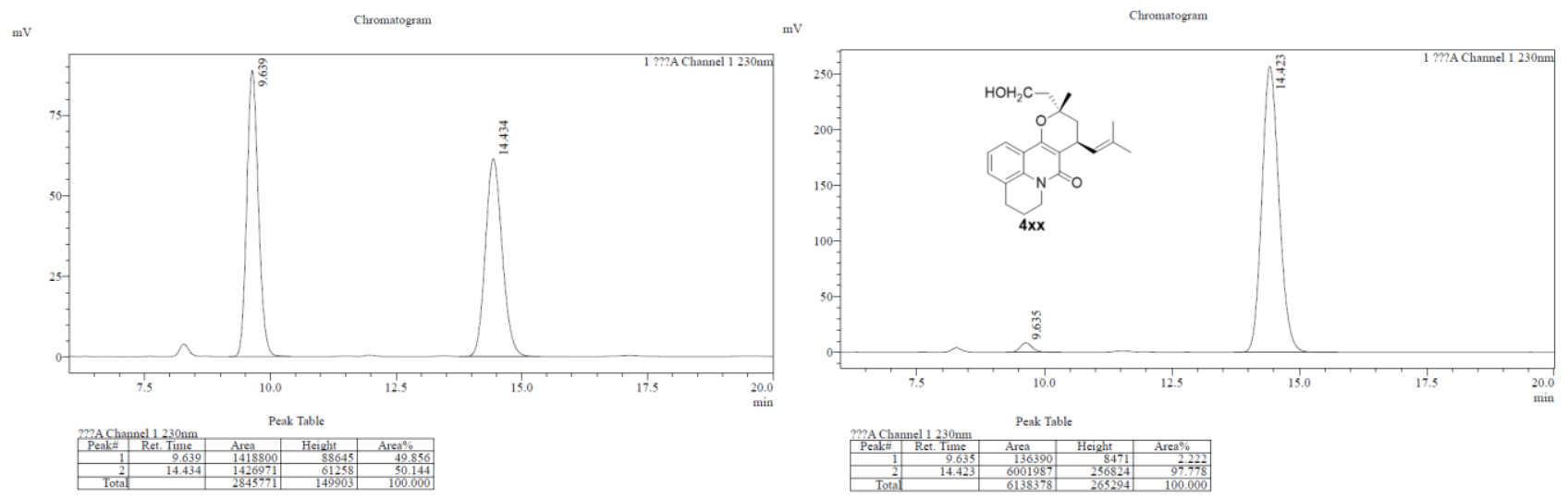


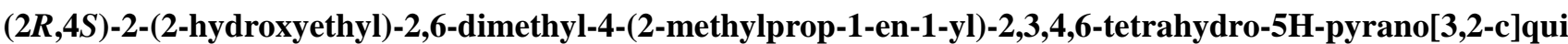
nolin-5-one (4a'):

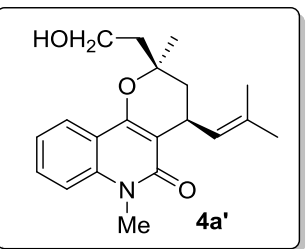

$\mathrm{mV}$

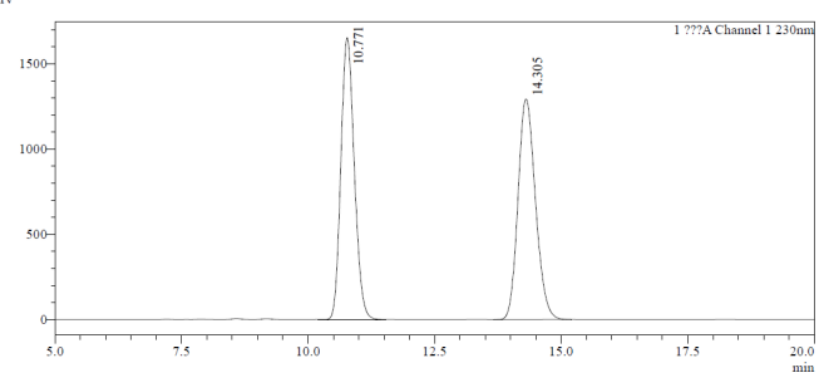

Peak Table $($ minor $)=10.73 \mathrm{~min}]$.

Spectral data of $\mathbf{4} \mathbf{a}^{\prime}$ is in agreement with $\mathbf{4 a}$.

With catalyst 8f, $31 \%$ yield, $25 \mathrm{mg}$. White solid. 91\% ee, determined by HPLC analysis [Chiralpak AD-H, $n$-hexane $/ i$-PrOH $=90 / 10,1.0 \mathrm{~mL} / \mathrm{min}, \lambda=230 \mathrm{~nm}, \mathrm{t}$ (major) $=13.89 \mathrm{~min}, \mathrm{t}$

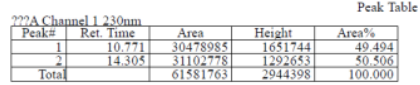

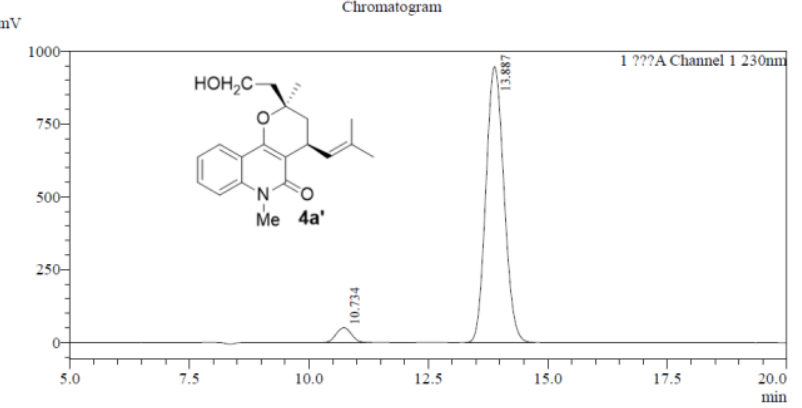

Peak Table

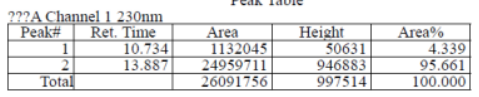

(2S,4S)-2-(2-hydroxyethyl)-2,6-dimethyl-4-(2-methylprop-1-en-1-yl)-2,3,4,6-tetrahydro-5H-pyrano[3,2-c]qui nolin-5-one (4aa'):
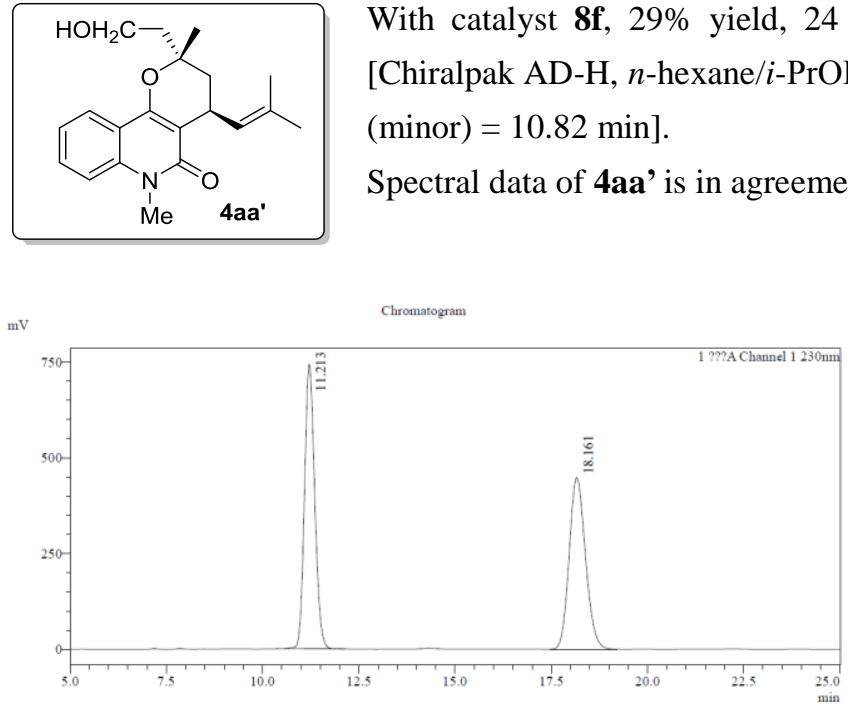

Peak Table

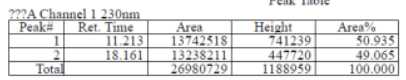
$($ minor $)=10.82 \mathrm{~min}]$.
With catalyst 8f, $29 \%$ yield, $24 \mathrm{mg}$. White solid. $91 \%$ ee, determined by HPLC analysis [Chiralpak AD-H, $n$-hexane $/ i-\mathrm{PrOH}=90 / 10,1.0 \mathrm{~mL} / \mathrm{min}, \lambda=230 \mathrm{~nm}, \mathrm{t}$ (major) $=17.13 \mathrm{~min}, \mathrm{t}$

Spectral data of $\mathbf{4 a a}$ ' is in agreement with $4 \mathbf{a a}$.

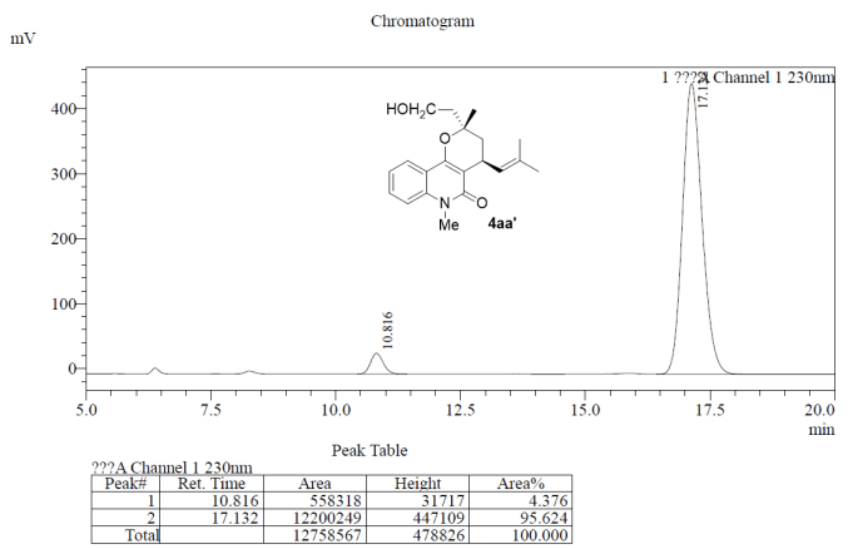




\section{Synthesis of acid 9 from $4 a a^{\prime}$}

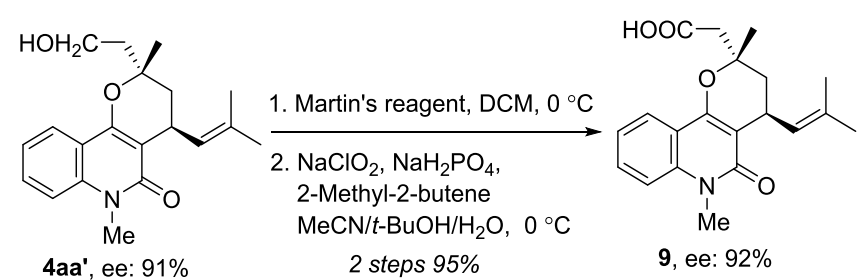

To a solution of 4aa' (109 mg, $0.33 \mathrm{mmol})$ in DCM (5 mL) were added Martin's reagent (170 mg, $0.40 \mathrm{mmol}, 1.2 \mathrm{equiv})$ under $0{ }^{\circ} \mathrm{C}$. After stirring at $0{ }^{\circ} \mathrm{C}$ for $2 \mathrm{~h}$, the reaction was quenched with saturated aqueous $\mathrm{Na}_{2} \mathrm{~S}_{2} \mathrm{O}_{3}$ solution (10 mL). The mixture was extracted with DCM and the combined organic phase was washed with brine, dried over anhydrous $\mathrm{Na}_{2} \mathrm{SO}_{4}$. The solvent was evaporated in vacuo, and the residue was purified by flash chromatography (petroleum ether/ethyl acetate $=3: 1$ ) to give $106 \mathrm{mg}$ of the aldehyde in $99 \%$ yield as a colorless oil. The above aldehyde (106 $\mathrm{mg}, 0.33 \mathrm{mmol}) \mathrm{was}$ then added to a solution $\left(9 \mathrm{~mL}, \mathrm{CH}_{3} \mathrm{CN} / t\right.$ - $\mathrm{BuOH} / 2$-methylbut-2-ene $\left.=4: 4: 1\right)$ and cooled to $0{ }^{\circ} \mathrm{C}$. Then a solution of $\mathrm{NaClO}_{2}$ (250mg, $2.77 \mathrm{mmol})$ and $\mathrm{NaH}_{2} \mathrm{PO}_{4}(241 \mathrm{mg}, 2.01 \mathrm{mmol})$ in water $(2 \mathrm{~mL})$ was added slowly. After being stirred at $0{ }^{\circ} \mathrm{C}$ for about 30 mins, the mixture was concentrated under reduced pressure. The residue was extracted with EtOAc, dried over anhydrous $\mathrm{Na}_{2} \mathrm{SO}_{4}$. The solvent was removed, and the residue was purified by flash column chromatography $(\mathrm{DCM} / \mathrm{MeOH}$ $=10: 1)$ to give $107 \mathrm{mg}$ of acid $\mathbf{9}$ in $95 \%$ yield as a white solid. 92\% ee, determined by HPLC analysis [Chiralpak AD-H, $n$-hexane $/ i$-PrOH $=90 / 10,1.0 \mathrm{~mL} / \mathrm{min}, \lambda=230 \mathrm{~nm}, \mathrm{t}$ (major) $=27.80 \mathrm{~min}, \mathrm{t}$ (minor) $=22.07 \mathrm{~min}] .{ }^{1} \mathbf{H} \mathbf{~ N M R}(400 \mathrm{MHz}$, $\left.\mathrm{CDCl}_{3}\right) \delta=7.98-7.92(\mathrm{~m}, 1 \mathrm{H}), 7.57-7.50(\mathrm{~m}, 1 \mathrm{H}), 7.30(\mathrm{~d}, J=8.4 \mathrm{~Hz}, 1 \mathrm{H}), 7.20(\mathrm{t}, J=7.6 \mathrm{~Hz}, 1 \mathrm{H}), 5.02(\mathrm{~d}, J=8.4 \mathrm{~Hz}$ $1 \mathrm{H}), 3.76(\mathrm{dd}, J=16.0,8.0 \mathrm{~Hz}, 1 \mathrm{H}), 3.64(\mathrm{~s}, 3 \mathrm{H}), 2.73(\mathrm{q}, J=14.0 \mathrm{~Hz}, 2 \mathrm{H}), 2.31$ (dd, $J=14.0,7.0 \mathrm{~Hz}, 1 \mathrm{H}), 1.84-1.75$ $(\mathrm{m}, 4 \mathrm{H}), 1.73$ (s, 3H), 1.63 (s, 3H). ${ }^{13} \mathbf{C} \mathbf{N M R}\left(100 \mathrm{MHz}, \mathrm{CDCl}_{3}\right) \delta=174.1,162.7,155.0,138.8,131.8,130.4,126.8,123.1$, 121.6, 116.4, 113.8, 109.4, 76.6, 42.3, 38.6, 29.3, 28.9, 26.3, 25.9, 17.9. $[\boldsymbol{\alpha}]^{\mathbf{2 0}} \mathbf{\mathbf { D }}=-19.20^{\circ}\left(c=1.0, \mathrm{CHCl}_{3}, 92 \%\right.$ ee $) . \mathbf{H R M S}$ (ESI) m/z: $[\mathrm{M}+\mathrm{H}]^{+}$calcd. for $\mathrm{C}_{20} \mathrm{H}_{24} \mathrm{NO}_{2} 342.1700$, found 342.1709.

$\mathrm{m}$
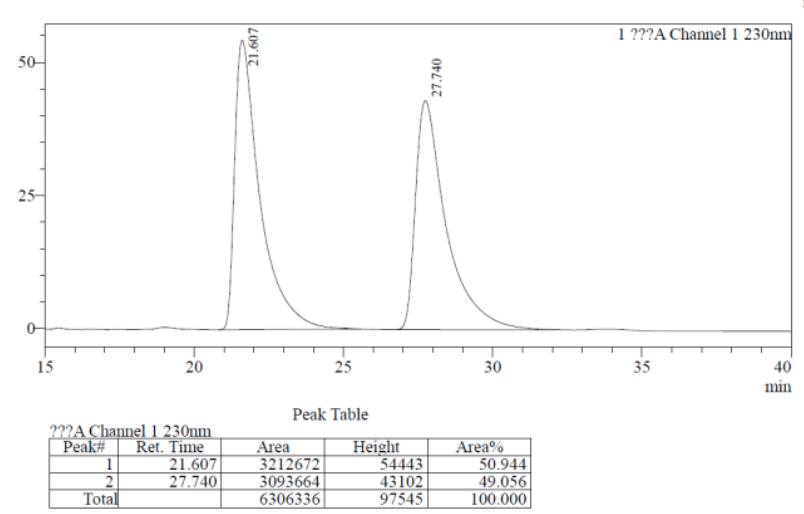

$\mathrm{mV}$

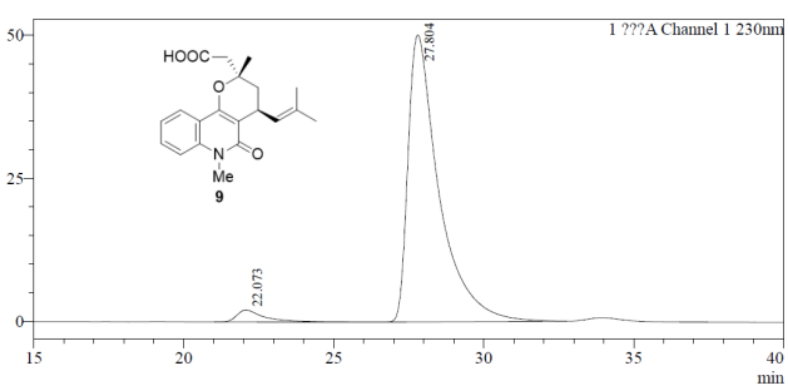

Peak Table

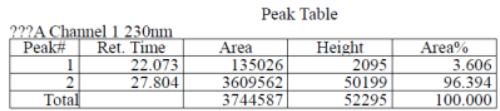




\section{Total synthesis of toddacoumalone}

\section{Synthesis of toddacoumalone (1a) from 4a:}

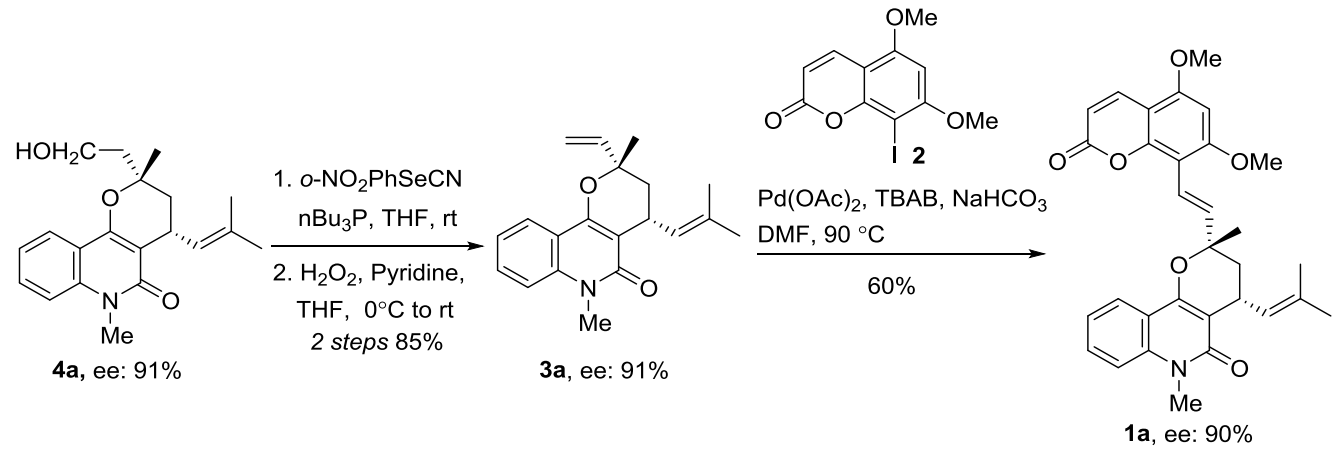

To a stirred solution of the alcohol $4 \mathbf{a}(65 \mathrm{mg}, 0.2 \mathrm{mmol})$ and $o$-nitrophenylselenocyanate ( $90 \mathrm{mg}, 0.4 \mathrm{mmol})$ in dry THF ( $2 \mathrm{ml}$ ) under $\mathrm{Ar}$, was added $n-\mathrm{Bu}_{3} \mathrm{P}(80 \mathrm{mg}, 0.4 \mathrm{mmol})$ at room temperature. The reaction was completed after stirring for $3 \mathrm{~h}$ at room temperature. Silica gel $(2 \mathrm{~g})$ was added to the reaction mixture and the solvent was evaporated under reduced pressure (CAUTION: toxic HCN gas present in the fumes). The residue was transferred onto a column of silica gel and eluted with petroleum ether/ethyl acetate (10:1 to 4:1). This afforded the selenide as a brightly yellow colour oil. To a stirred solution of this selenide in THF $(6 \mathrm{~mL})$, was added pyridine (16 mg, $0.2 \mathrm{mmol})$ and $30 \%$ aqueous $\mathrm{H}_{2} \mathrm{O}_{2}$ solution $(0.06 \mathrm{ml}, 0.6 \mathrm{mmol})$ dropwise at $0{ }^{\circ} \mathrm{C}$. The reaction showed a significant and varying induction period. The elimination was completed when the yellow colour disappeared. Then, reaction was quenched with ice cold water $(10 \mathrm{~mL})$ and extracted with ethyl acetate $(3 \times 10 \mathrm{~mL})$, and organic layer was washed with brine $(10 \mathrm{~mL})$, dried over anhydrous $\mathrm{Na}_{2} \mathrm{SO}_{4}$, concentrated under reduce pressure. The residue was purified by flash chromatography (neutral $\mathrm{Al}_{2} \mathrm{O}_{3}$, petroleum ether/ ethyl acetate $=95: 5)$. This afforded the alkene product 3a in $85 \%$ yield as a white solid. 91\% ee, determined by HPLC analysis [Chiralpak AD-H, $n$-hexane $/ i-\mathrm{PrOH}=95 / 5,1.0 \mathrm{~mL} / \mathrm{min}, \lambda=230 \mathrm{~nm}, \mathrm{t}$ (major) $=7.31 \mathrm{~min}, \mathrm{t}(\operatorname{minor})=8.44 \mathrm{~min}$ ] ${ }^{1} \mathbf{H}$ NMR $\left(500 \mathrm{MHz}, \mathrm{CDCl}_{3}\right) \delta=8.08(\mathrm{~d}, J=7.5 \mathrm{~Hz}, 1 \mathrm{H}), 7.60-7.52(\mathrm{~m}, 1 \mathrm{H}), 7.33-7.22(\mathrm{~m}, 2 \mathrm{H}), 6.01-5.90(\mathrm{~m}, 1 \mathrm{H})$, $5.21(\mathrm{~d}, J=17.0 \mathrm{~Hz}, 1 \mathrm{H}), 5.12(\mathrm{~d}, J=8.0 \mathrm{~Hz}, 1 \mathrm{H}), 5.04(\mathrm{~d}, J=10.5 \mathrm{~Hz}, 1 \mathrm{H}), 3.74(\mathrm{~s}, 1 \mathrm{H}), 3.67(\mathrm{~s}, 3 \mathrm{H}), 2.10-2.04(\mathrm{~m}$, 1H), $1.98-1.94(\mathrm{~m}, 1 \mathrm{H}), 1.82(\mathrm{~s}, 3 \mathrm{H}), 1.71(\mathrm{~s}, 3 \mathrm{H}), 1.48(\mathrm{~s}, 3 \mathrm{H}) .{ }^{13} \mathbf{C} \mathbf{N M R}\left(125 \mathrm{MHz}, \mathrm{CDCl}_{3}\right) \delta=162.8,155.0,142.3$, $138.8,131.3,130.2,127.6,122.9,121.4,116.4,113.7,112.4,109.6,78.0,39.7,29.2,29.2,26.7,25.9,17.9 .[\boldsymbol{\alpha}]^{\mathbf{2 0}} \mathbf{D}=-$ $110.90^{\circ}\left(c=1.0, \mathrm{CHCl}_{3}, 91 \%\right.$ ee). HRMS (ESI) m/z: $[\mathrm{M}+\mathrm{H}]^{+}$calcd. for $\mathrm{C}_{20} \mathrm{H}_{24} \mathrm{NO}_{2} 310.1802$, found 310.1801 .
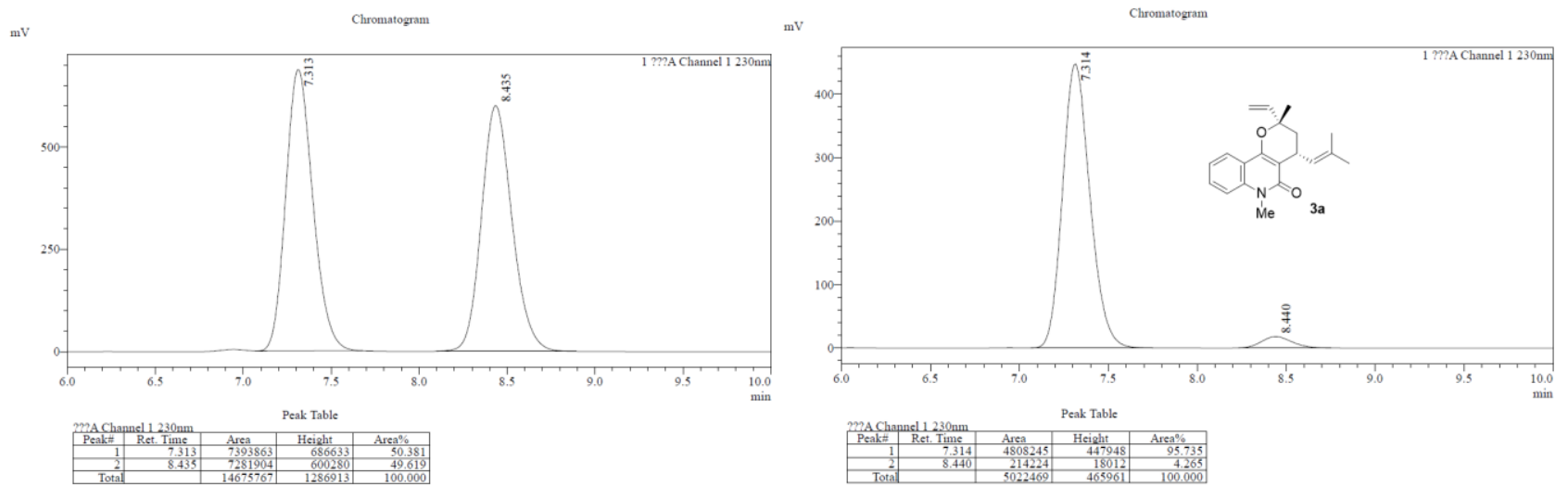
To a solution of $\mathbf{3 a}(40 \mathrm{mg}, 0.13 \mathrm{mmol})$ in dry DMF $(1.5 \mathrm{ml})$, coumarin-derivative 2 (43 $\mathrm{mg}, 0.13 \mathrm{mmol})$, tetrabutylammonium bromide (41 mg, $0.13 \mathrm{mmol}), \mathrm{NaHCO}_{3}(44 \mathrm{mg}, 0.52 \mathrm{mmol})$ and $\mathrm{Pd}(\mathrm{OAc})_{2}(11 \mathrm{mg}, 0.05 \mathrm{mmol})$ were added under Ar. The resulting mixture was degassed and heated to $90^{\circ} \mathrm{C}$ for $24 \mathrm{~h}$. The reaction mixture was cooled to room temperature and added with $\mathrm{H}_{2} \mathrm{O}(5 \mathrm{~mL})$ and ethyl acetate $(5 \mathrm{~mL})$. The organic layer was washed with brine and $\mathrm{H}_{2} \mathrm{O}(3 \times$ $5 \mathrm{~mL}$ ), and dried over anhydrous $\mathrm{Na}_{2} \mathrm{SO}_{4}$. The product $1 \mathbf{a}$ was purified by column chromatography (silica gel, petroleum ether/ethyl acetate $=4: 1$ to $1: 1$ ). This afforded toddacoumalone (1a) in $60 \%$ yield as a faint yellow solid. $90 \%$ ee, determined by HPLC analysis [Chiralpak AS-H, $n$-hexane $/ i$-PrOH $=90 / 10,1.0 \mathrm{~mL} / \mathrm{min}, \lambda=230 \mathrm{~nm}, \mathrm{t}$ (major) $=18.05$ $\min , \mathrm{t}($ minor $)=15.59 \mathrm{~min}] .{ }^{1} \mathbf{H}$ NMR $\left(400 \mathrm{MHz}, \mathrm{CDCl}_{3}\right) \delta=8.23(\mathrm{~d}, J=8.0 \mathrm{~Hz}, 1 \mathrm{H}), 7.96(\mathrm{~d}, J=9.6 \mathrm{~Hz}, 1 \mathrm{H}), 7.55(\mathrm{t}, J$ $=7.2 \mathrm{~Hz}, 1 \mathrm{H}), 7.34-7.27(\mathrm{~m}, 2 \mathrm{H}), 6.94(\mathrm{~s}, 2 \mathrm{H}), 6.27(\mathrm{~s}, 1 \mathrm{H}), 6.13(\mathrm{~d}, J=9.6 \mathrm{~Hz}, 1 \mathrm{H}), 5.12(\mathrm{~d}, J=8.8 \mathrm{~Hz}, 1 \mathrm{H}), 3.92(\mathrm{~s}$, $3 \mathrm{H}), 3.82(\mathrm{~d}, J=5.1 \mathrm{~Hz}, 3 \mathrm{H}), 3.81-3.75(\mathrm{~m}, 1 \mathrm{H}), 3.67(\mathrm{~s}, 3 \mathrm{H}), 2.21-2.06(\mathrm{~m}, 2 \mathrm{H}), 1.84(\mathrm{~s}, 3 \mathrm{H}), 1.64(\mathrm{~s}, 3 \mathrm{H}), 1.61(\mathrm{~s}$, 3H). ${ }^{13} \mathrm{C}$ NMR $\left(100 \mathrm{MHz}, \mathrm{CDCl}_{3}\right) \delta=162.9,161.2,160.8,155.8,155.3,153.7,138.8,138.6,137.4,131.1,130.1,127.7$,

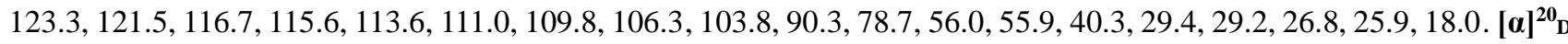
$=-140.20^{\circ}\left(c=1.0, \mathrm{CHCl}_{3}, 90 \%\right.$ ee $)$. HRMS (ESI) $\mathrm{m} / \mathrm{z}:[\mathrm{M}+\mathrm{Na}]^{+}$calcd. for $\mathrm{C}_{31} \mathrm{H}_{31} \mathrm{NO}_{6} \mathrm{Na} 536.2044$, found 536.2045
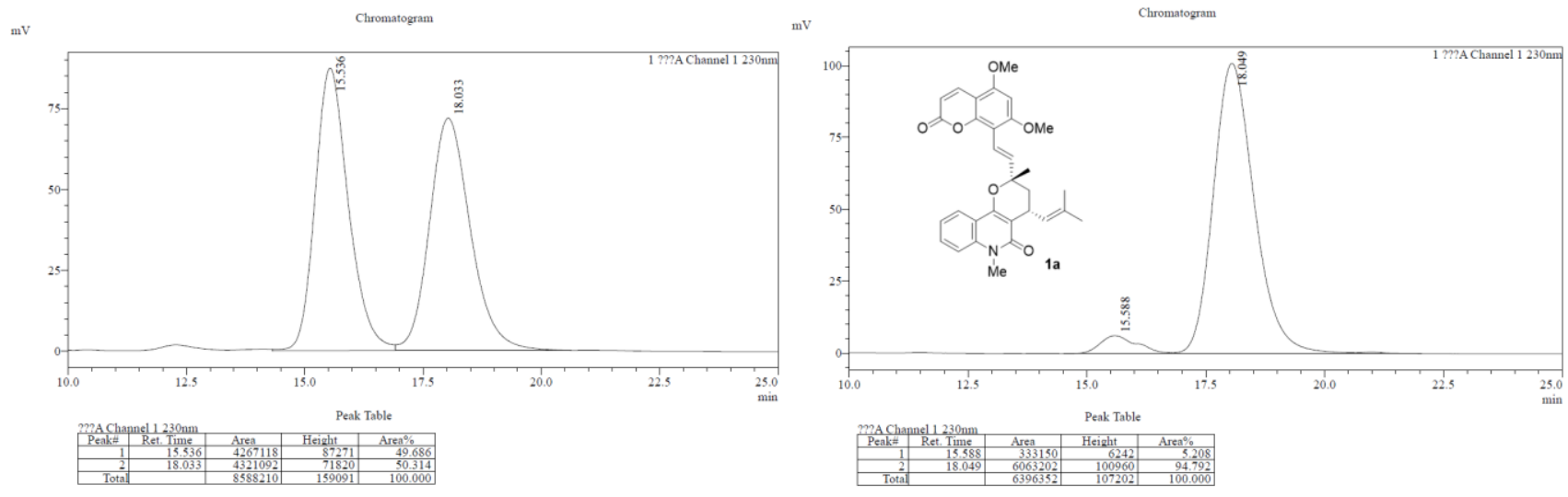

\section{Synthesis of toddacoumalone (1aa) from 4aa:}

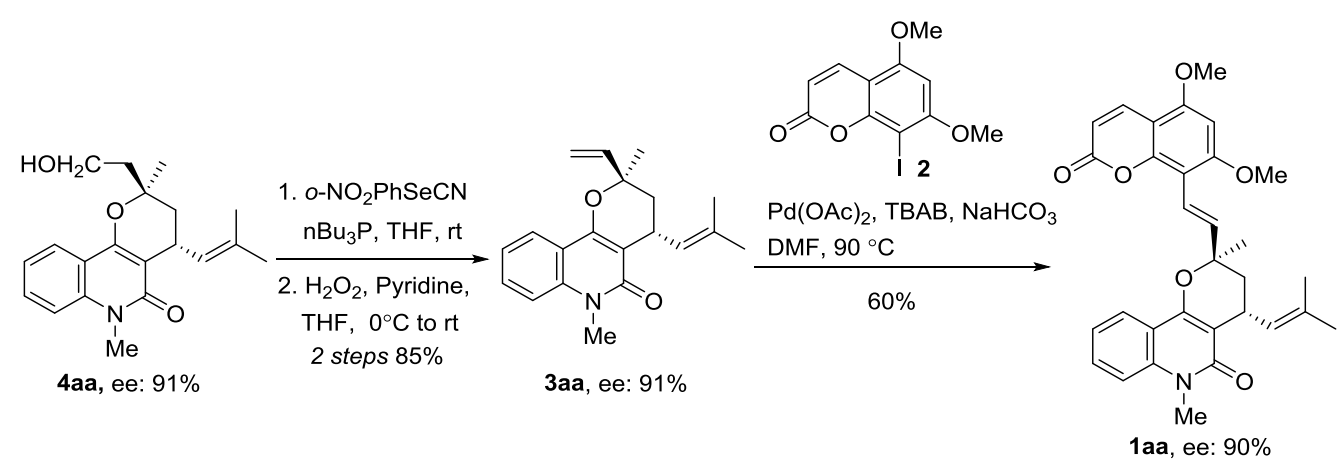

The product 3aa was prepared through the same process from the alcohol 4aa according to the synthesis of $\mathbf{3 a}$ in $85 \%$ yield as a white solid. 91\% ee, determined by HPLC analysis [Chiralpak AD-H, $n$-hexane $/ i$-PrOH $=95 / 5,1.0 \mathrm{~mL} / \mathrm{min}, \lambda=$ $230 \mathrm{~nm}, \mathrm{t}$ (major) $=6.94 \mathrm{~min}, \mathrm{t}($ minor $)=8.24 \mathrm{~min}] .{ }^{1} \mathbf{H} \mathbf{N M R}\left(400 \mathrm{MHz}, \mathrm{CDCl}_{3}\right) \delta=8.05(\mathrm{~d}, J=8.0 \mathrm{~Hz}, 1 \mathrm{H}), 7.53(\mathrm{t}, J$ $=8.4 \mathrm{~Hz}, 1 \mathrm{H}), 7.30(\mathrm{~d}, J=8.4 \mathrm{~Hz}, 1 \mathrm{H}), 7.22(\mathrm{t}, J=8.0 \mathrm{~Hz}, 1 \mathrm{H}), 5.86(\mathrm{dd}, J=17.2,10.8 \mathrm{~Hz}, 1 \mathrm{H}), 5.12-5.00(\mathrm{~m}, 3 \mathrm{H}), 3.66$ (s, 3H), $3.62-3.56(\mathrm{~m}, 1 \mathrm{H}), 2.12(\mathrm{dd}, J=14.0,7.2 \mathrm{~Hz}, 1 \mathrm{H}), 1.80(\mathrm{~s}, 3 \mathrm{H}), 1.78-1.71(\mathrm{~m}, 4 \mathrm{H}), 1.54(\mathrm{~s}, 3 \mathrm{H}) .{ }^{13} \mathbf{C ~ N M R}$ $\left(100 \mathrm{MHz} \mathrm{CDCl}_{3}\right) \delta=162.6,155.5,140.0,138.9,130.9,130.2,127.3,123.0,121.3,116.4,113.8,113.7,109.9,78.7,39.5$, 29.5, 29.1, 27.8, 25.9, 17.9. $[\boldsymbol{\alpha}]^{\mathbf{2 0}} \mathbf{D}=57.77^{\circ}\left(c=1.0, \mathrm{CHCl}_{3}, 91 \%\right.$ ee $) . \mathbf{H R M S}(\mathrm{ESI}) \mathrm{m} / \mathrm{z}:[\mathrm{M}+\mathrm{H}]^{+}$calcd. for $\mathrm{C}_{20} \mathrm{H}_{24} \mathrm{NO}_{2}$ 310.1802 , found 310.1799 . 

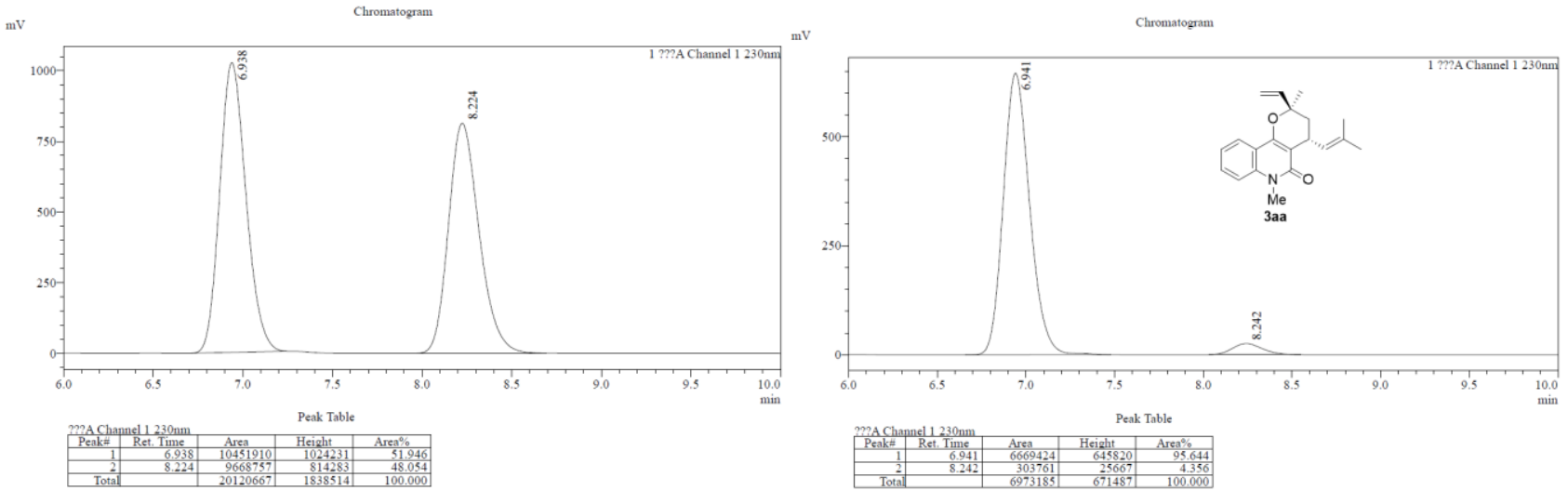

The toddacoumalone (1aa) was prepared through the same process from 3aa according to the synthesis of $\mathbf{1 a}$ in $60 \%$ yield as a faint yellow solid. 90\% ee, determined by HPLC analysis [Chiralpak AS-H, $n$-hexane/i-PrOH $=90 / 10,1.0$ $\mathrm{mL} / \mathrm{min}, \lambda=230 \mathrm{~nm}, \mathrm{t}$ (major) $=15.21 \mathrm{~min}, \mathrm{t}($ minor $)=12.46 \mathrm{~min}] .{ }^{1} \mathbf{H} \mathbf{N M R}\left(400 \mathrm{MHz}, \mathrm{CDCl}_{3}\right) \delta=8.19-8.14(\mathrm{~m}, 1 \mathrm{H})$, $7.93(\mathrm{~d}, J=9.6 \mathrm{~Hz}, 1 \mathrm{H}), 7.57-7.50(\mathrm{~m}, 1 \mathrm{H}), 7.33-7.24(\mathrm{~m}, 2 \mathrm{H}), 6.74(\mathrm{~s}, 2 \mathrm{H}), 6.21(\mathrm{~s}, 1 \mathrm{H}), 6.11(\mathrm{~d}, J=10.0 \mathrm{~Hz}, 1 \mathrm{H})$, $5.10(\mathrm{~d}, J=8.8 \mathrm{~Hz}, 1 \mathrm{H}), 3.89(\mathrm{~s}, 3 \mathrm{H}), 3.76-3.68(\mathrm{~m}, 4 \mathrm{H}), 3.64(\mathrm{~s}, 3 \mathrm{H}), 2.31(\mathrm{dd}, J=13.6,6.8 \mathrm{~Hz}, 1 \mathrm{H}), 1.89-1.80(\mathrm{~m}$, 4H), 1.77 (s, 3H), 1.67 (s, 3H). $\left.{ }^{13} \mathbf{C ~ N M R ~ ( 1 0 0 ~ M H z , ~} \mathrm{CDCl}_{3}\right) \delta=162.7,161.0,160.9,155.9,155.8,153.5,138.8,138.6$, 135.7, 130.9, 130.1, 127.5, 123.3, 121.4, 116.9, 116.7, 113.5, 111.0, 110.2, 106.2, 103.7, 90.3, 79.4, 55.9, 55.9, 40.0, 29.8, 29.2, 28.2, 25.9, 18.0. $[\boldsymbol{\alpha}]^{20} \mathbf{D}=86.80^{\circ}\left(c=1.0, \mathrm{CHCl}_{3}, 90 \%\right.$ ee). HRMS (ESI) m/z: $[\mathrm{M}+\mathrm{Na}]^{+}$calcd. for $\mathrm{C}_{31} \mathrm{H}_{31} \mathrm{NO}_{6} \mathrm{Na}$ 536.2044 , found 536.2034 .
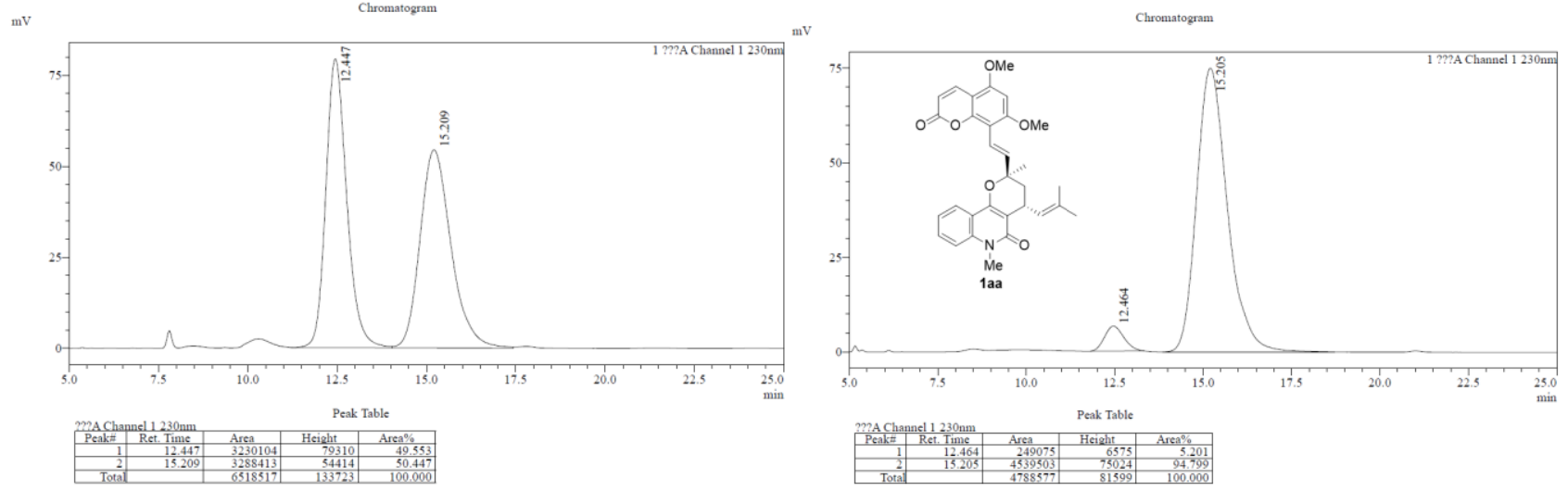

\section{Synthesis of toddacoumalone (1a') from 4a':}

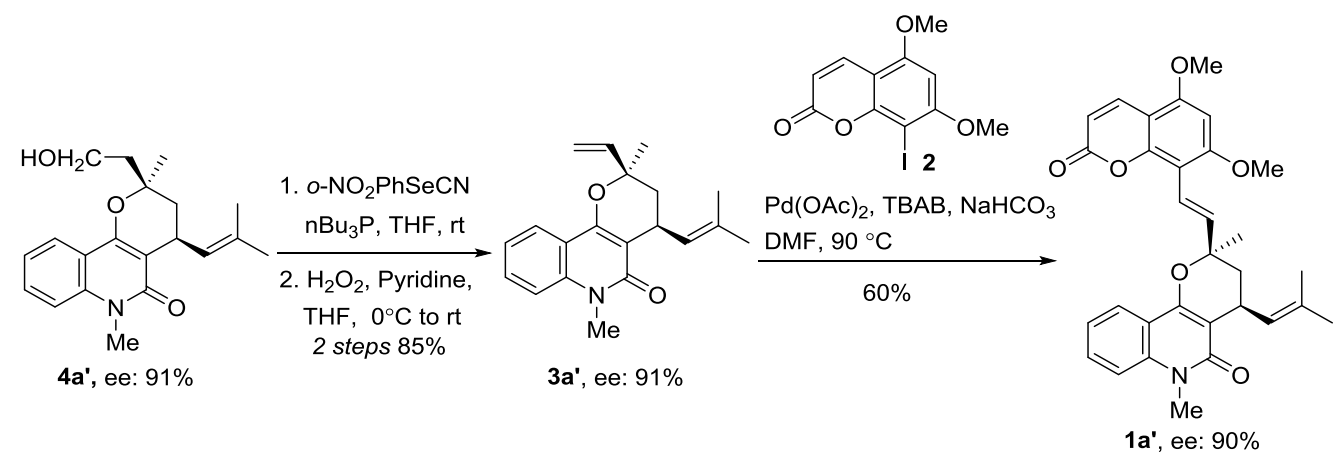

The product 3a' was prepared through the same process from the alcohol 4a' according to the synthesis of $\mathbf{3 a}$ in $85 \%$ yield as a white solid. 91\% ee, determined by HPLC analysis [Chiralpak AD-H, $n$-hexane $/ i$-PrOH $=95 / 5,1.0 \mathrm{~mL} / \mathrm{min}, \lambda=$ $230 \mathrm{~nm}, \mathrm{t}$ (major) $=8.44 \mathrm{~min}, \mathrm{t}($ minor $)=7.31 \mathrm{~min}]$. Spectral data of $\mathbf{3 a} \mathbf{a}^{\prime}$ is in agreement with $\mathbf{3 a} .[\mathbf{\alpha}]^{\mathbf{2 0}} \mathbf{D}=120.37^{\circ}(c=1.0$, $\mathrm{CHCl}_{3}, 91 \%$ ee). 

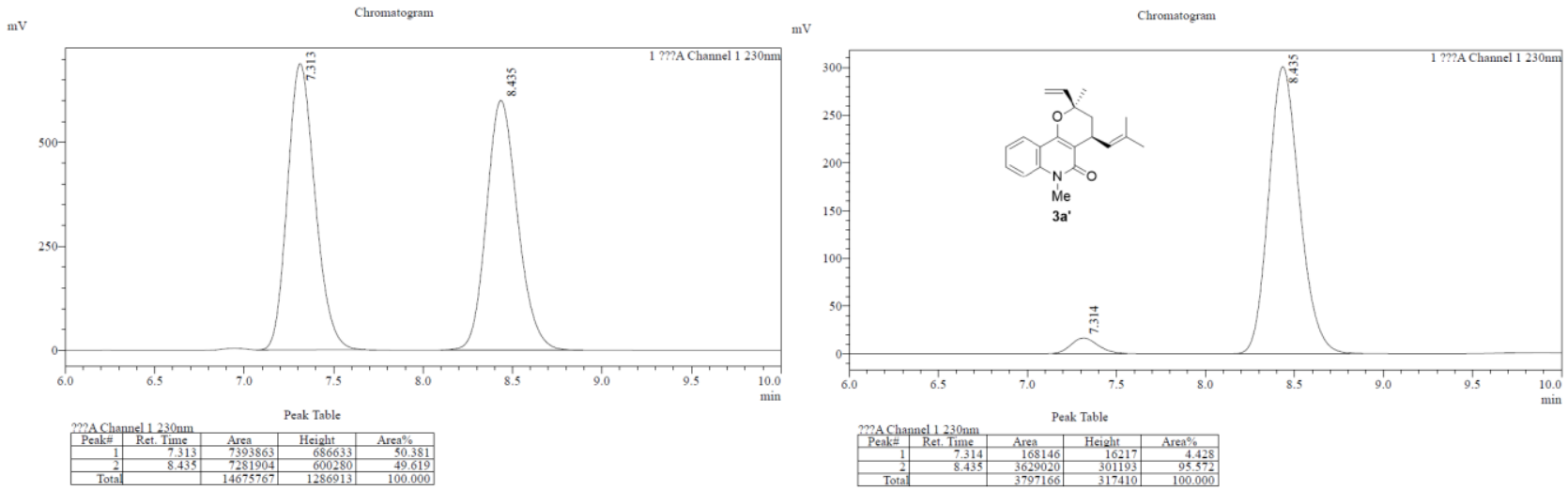

The toddacoumalone (19') was prepared through the same process from 3a' according to the synthesis of $1 \mathbf{a}$ in $60 \%$ yield as a faint yellow solid. 90\% ee, determined by HPLC analysis [Chiralpak AS-H, $n$-hexane/ $i$-PrOH $=90 / 10,1.0$ $\mathrm{mL} / \mathrm{min}, \lambda=230 \mathrm{~nm}, \mathrm{t}$ (major) $=15.57 \mathrm{~min}, \mathrm{t}$ (minor) $=18.09 \mathrm{~min}]$. Spectral data of $\mathbf{1 a}{ }^{\prime}$ is in agreement with $\mathbf{1 a}$. $[\boldsymbol{\alpha}]^{\mathbf{2 0}} \mathbf{D}=$ $250.80^{\circ}\left(c=1.0, \mathrm{CHCl}_{3}, 90 \%\right.$ ee $)$.
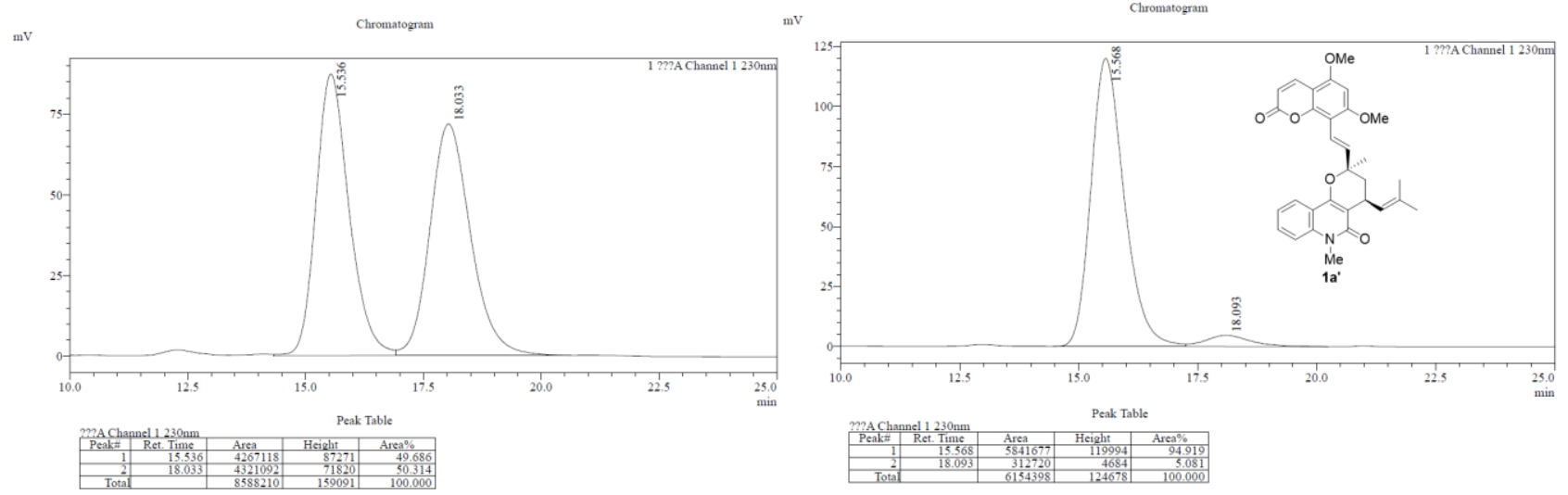

Synthesis of toddacoumalone (1aa') from 4aa':

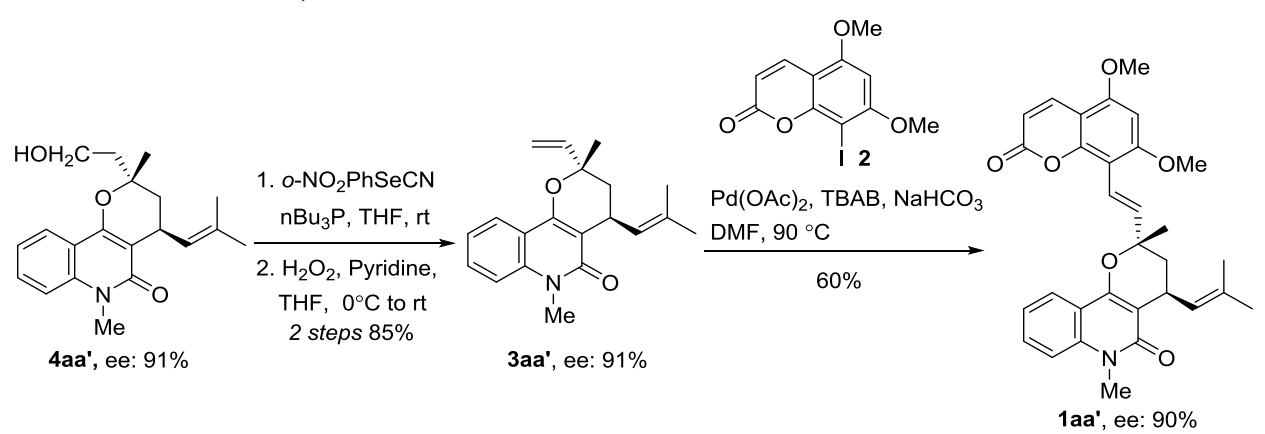

The product 3aa' was prepared through the same process from the alcohol 4aa' according to the synthesis of $\mathbf{3 a}$ in $85 \%$ yield as a white solid. 91\% ee, determined by HPLC analysis [Chiralpak AD-H, $n$-hexane $/ i$-PrOH $=95 / 5,1.0 \mathrm{~mL} / \mathrm{min}, \lambda=$ $230 \mathrm{~nm}, \mathrm{t}($ major $)=8.22 \mathrm{~min}, \mathrm{t}($ minor $)=6.94 \mathrm{~min}]$. Spectral data of 3aa' is in agreement with 3aa. $[\boldsymbol{\alpha}]^{\mathbf{2 0}} \mathbf{D}=-60.30^{\circ}(c=$ $1.0, \mathrm{CHCl}_{3}, 91 \%$ ee). 

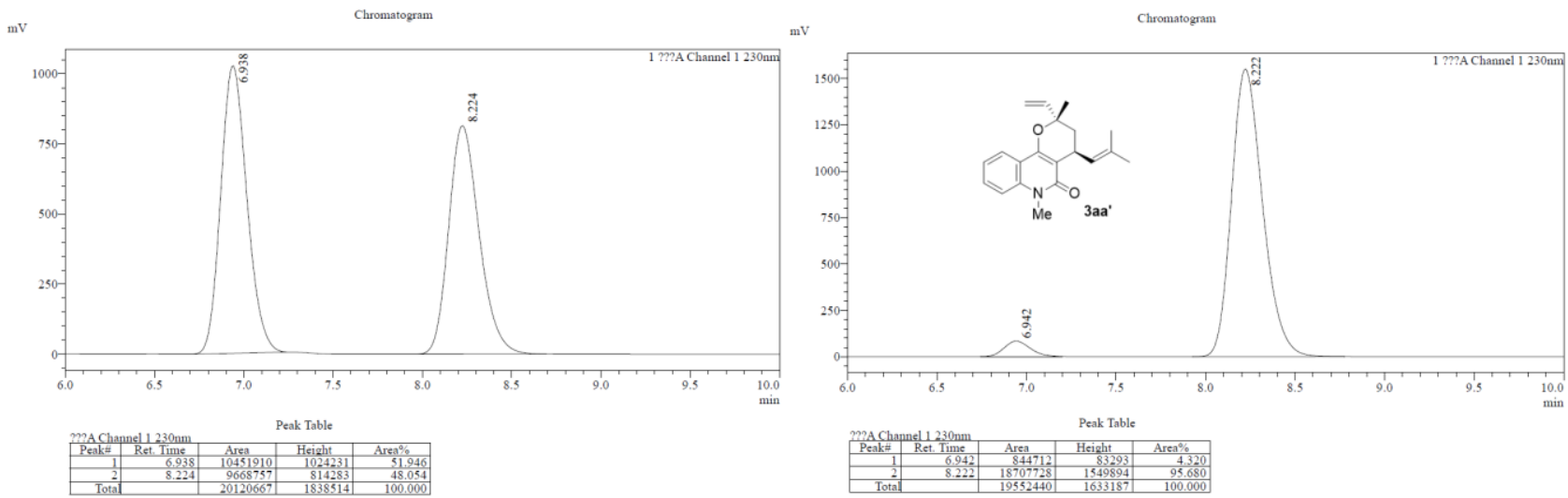

The toddacoumalone (1aa') was prepared through a similar process according to the product 1a from 3aa' in $60 \%$ yield as a faint yellow solid. 90\% ee, determined by HPLC analysis [Chiralpak AS-H, $n$-hexane $/ i-\mathrm{PrOH}=90 / 10,1.0 \mathrm{~mL} / \mathrm{min}, \lambda$ $=230 \mathrm{~nm}, \mathrm{t}($ major $)=12.41 \mathrm{~min}, \mathrm{t}($ minor $)=15.20 \mathrm{~min}]$. Spectral data of 1aa' is in agreement with $\mathbf{1} \mathbf{a a} \cdot[\boldsymbol{\alpha}]^{\mathbf{2 0}} \mathbf{D}=-93.10^{\circ}$ $\left(c=1.0, \mathrm{CHCl}_{3}, 90 \%\right.$ ee $)$.

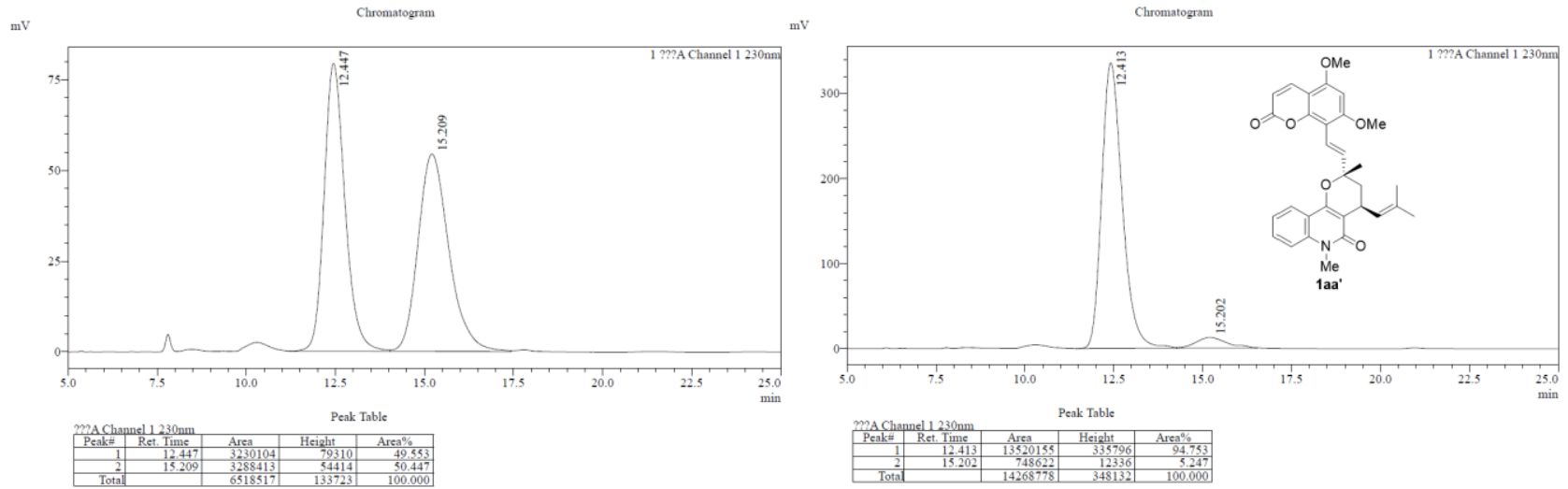

\section{PDE4 inhibition potency in enzymatic assays}

\section{Protein Expression and Purification}

The subcloning and protein expressing of human PDE4D2 (catalytic domain, residues 86-413) were reported previously and are briefly described here. ${ }^{[8]}$ The recombined vector pET15b-PDE4D2 (86-413) was transformed into E. coli competent cell BL21 (Codonplus) for over expression. The E. coli cells carrying these plasmids were grown in LB medium at $37^{\circ} \mathrm{C}$ to absorption A600 = 0.6-0.8, and then $0.1 \mathrm{mM}$ isopropyl $\beta$-D-thiogalactopyranoside was added for further growth at $15^{\circ} \mathrm{C}$ for $24 \mathrm{~h}$. These recombinant proteins were purified by Ni-NTA column (Qiagen). A typical batch of purification yielded over $50 \mathrm{mg}$ of PDE4D2 and the purity of these recombinant proteins was greater than 95\% as shown by SDS-PAGE.

\section{Enzymatic Assays}

The enzymatic activities of the catalytic domains of PDE4D2 were measured by using ${ }^{3} \mathrm{H}$-cAMP as substrates and rolipram as the reference compound. The assay buffer contains $20 \mathrm{mM}$ Tris- $\mathrm{HCl}(\mathrm{pH} 7.5), 10 \mathrm{mM} \mathrm{MgCl}, 1 \mathrm{mM}$ DTT, 10-30 nM ${ }^{3} \mathrm{H}$-cAMP (20,000-30,000 cpm/assay, GE Healthcare). The reaction was carried out at room temperature for $15 \mathrm{~min}$ and then terminated by addition of $0.2 \mathrm{M} \mathrm{ZnSO}_{4}$. The reaction product ${ }^{3} \mathrm{H}-\mathrm{AMP}$ was precipitated by $0.2 \mathrm{~N} \mathrm{Ba}(\mathrm{OH})_{2}$, while unreacted ${ }^{3} \mathrm{H}$-cAMP was remained in the supernatant. Radioactivity of the supernatant was measured in $2.5 \mathrm{ml}$ Ultima Gold liquid scintillation cocktails (Fisher Scientific) by a PerkinElmer 2910 liquid scintillation counter. For the measurement of $\mathrm{IC}_{50}$, at least eight concentrations of inhibitors were used in the presence of suitable concentrations of ${ }^{3} \mathrm{H}-$ cAMP and the enzymes that hydrolyze about $50 \%$ of the substrates. Each measurement was repeated at least three times and the $\mathrm{IC}_{50}$ values were calculated by nonlinear regression. 


\section{Transition state model for the reaction}

\section{Figure S4. Transition state model for the formal [4+2] cycloaddition reaction}

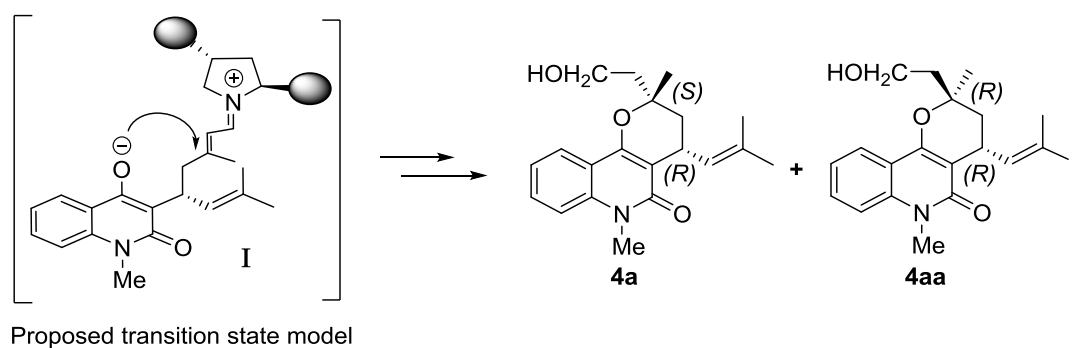

As most of the inverse-electron-demand Diels-Alder (IEDDA) reactions generally gave excellent diastereoselectivities, we speculated that this reaction was not occurred as the IEDDA reaction, but might be a step-wised formal [4+2] cycloaddition reaction. A proposed transition state model was provided in Figure S4. So far we do not know the direct evidence why the reactions exclusively gave the $R$ configuration at the 4-position of the dihydropyran. One possible explanation about the poor diasteroselectivity is that the oxa-Michael addition (transition state I) could not effectively control the stereoselectivity at the remote methyl site. Further mechanistic studies to fully understand the reaction mechanism are currently underway.

\section{References:}

[1] L. Caruana, F. Kniep, T. K. Johansen, P. H. Poulsen, K. A. Jørgensen, J. Am. Chem. Soc. 2014, 136, 15929-15932.

[2] I. Arenas, A. Ferrali, C. Rodríguez-Escrich, F. Bravo, M. A. Pericàs, Adv. Synth. Catal. 2017, 359, $2414-2424$.

[3] E. Gomez-Bengoa, M. Maestro, A. Mielgo, I. Otazo, C. Palomo, I. Velilla, Chem. Eur. J. 2010, 16, 5333-5342.

[4] a) J. Li, Q.-L. Hu, X.-P. Chen, K.-Q. Hou, A. S. C. Chan, X.-F. Xiong, Chinese Chemical Letters 2019, doi: 10.1016/j.cclet.2019.08.040. b) C. D. Haffner, J. D. Becherer, E. E. Boros, R. Cadilla, T. Carpenter, D. Cowan, D. N. Deaton, Y. Guo, W. Harrington, B. R. Henke, M. R Jeune, I. Kaldor, N. Milliken, K. G. Petrov, F. Preugschat, C. Schulte, B. G. Shearer, T. Shearer, T. L. Smalley, Jr., E. L. Stewart, J. D. Stuart, J. C. Ulrich, J. Med. Chem. 2015, 58, 3548-3571.

[5] J. D. Pettigrew, J. A. Cadieux, S. S. So, P. D. Wilson, Org. Lett. 2005, 7, 467-470.

[6] J. E. Neve, H. P. Wijesekera, S. Duffy, I. D. Jenkins, J. A. Ripper, S. J. Teague, M. Campitelli, A. Garavelas, G. Nikolakopoulos, P. V. Le, P. de A. Leone, N. B. Pham, P. Shelton, N. Fraser, A. R. Carroll, V. M. Avery, C. McCrae, N. Williams, R. J. Quinn, J. Med. Chem. 2014, 57, $1252-1275$

[7] W. E. Campbell, B. Davidowitz, G. E. Jackson, Phytochemistry 1990, 29, 1303-1306.

[8] a) Z. Wang, Y. Wang, B. Wang, W. Li, L. Huang, X. Li, J. Med. Chem. 2015, 58, 8616-8637; b) Y. H. Cai, Y. Guo, Z. Li, D. Wu, X. Li, H. Zhang, J. Yang, H. Lu, Z. Sun, H. B. Luo, S. Yin, Y. Wu, Eur. J. Med. Chem. 2016, 114, 134-140. 


\section{Crystal data for 6a, acid 9 and toddacoumalone (1a)}

The structure of $\mathbf{6 a}$ was assigned by the single-crystal X-ray analysis. (CCDC 1952168 contains the supplementary crystallographic data for this structure. These data can be obtained free of charge from The Cambridge Crystallographic Data Centre)

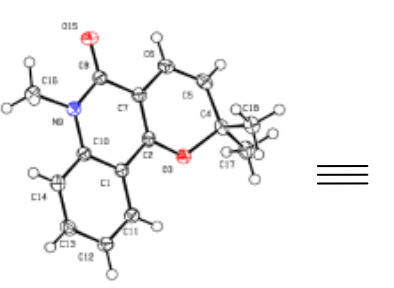

CCDC 1952168<smiles>Cn1c(=O)c2c(c3ccccc31)OC(C)(C)C=C2</smiles>

$6 a$

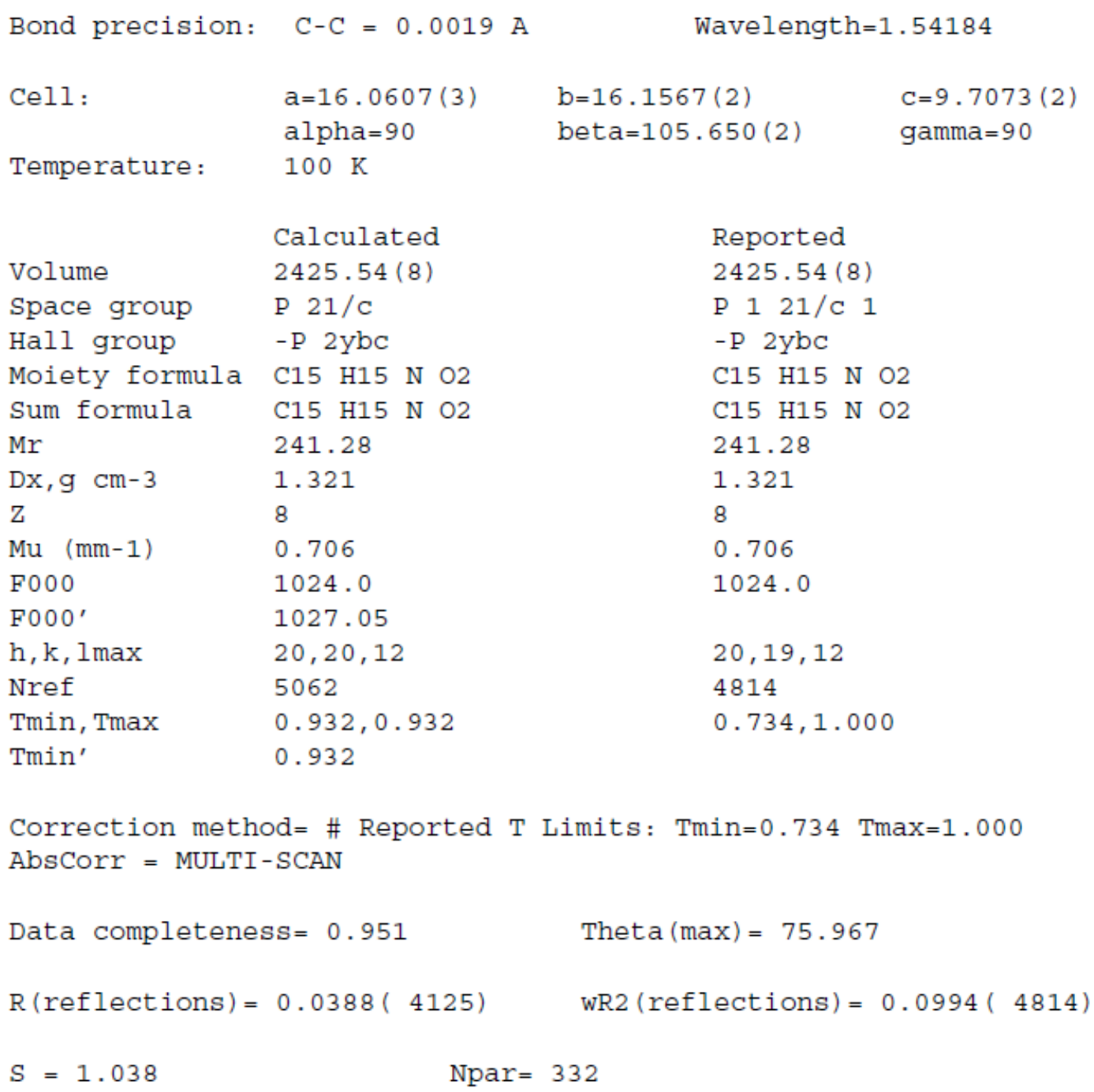


The absolute configuration of acid $\mathbf{9}$ was determined by single crystal X-ray analysis. (CCDC 1903773 contains the supplementary crystallographic data for this structure. These data can be obtained free of charge from The Cambridge Crystallographic Data Centre)

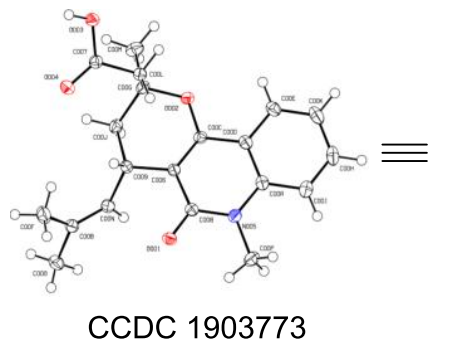<smiles>CC(C)=CC1c2c(c3ccccc3n(C)c2=O)O[C@](C)(CC(=O)O)[C@@H]1O</smiles>

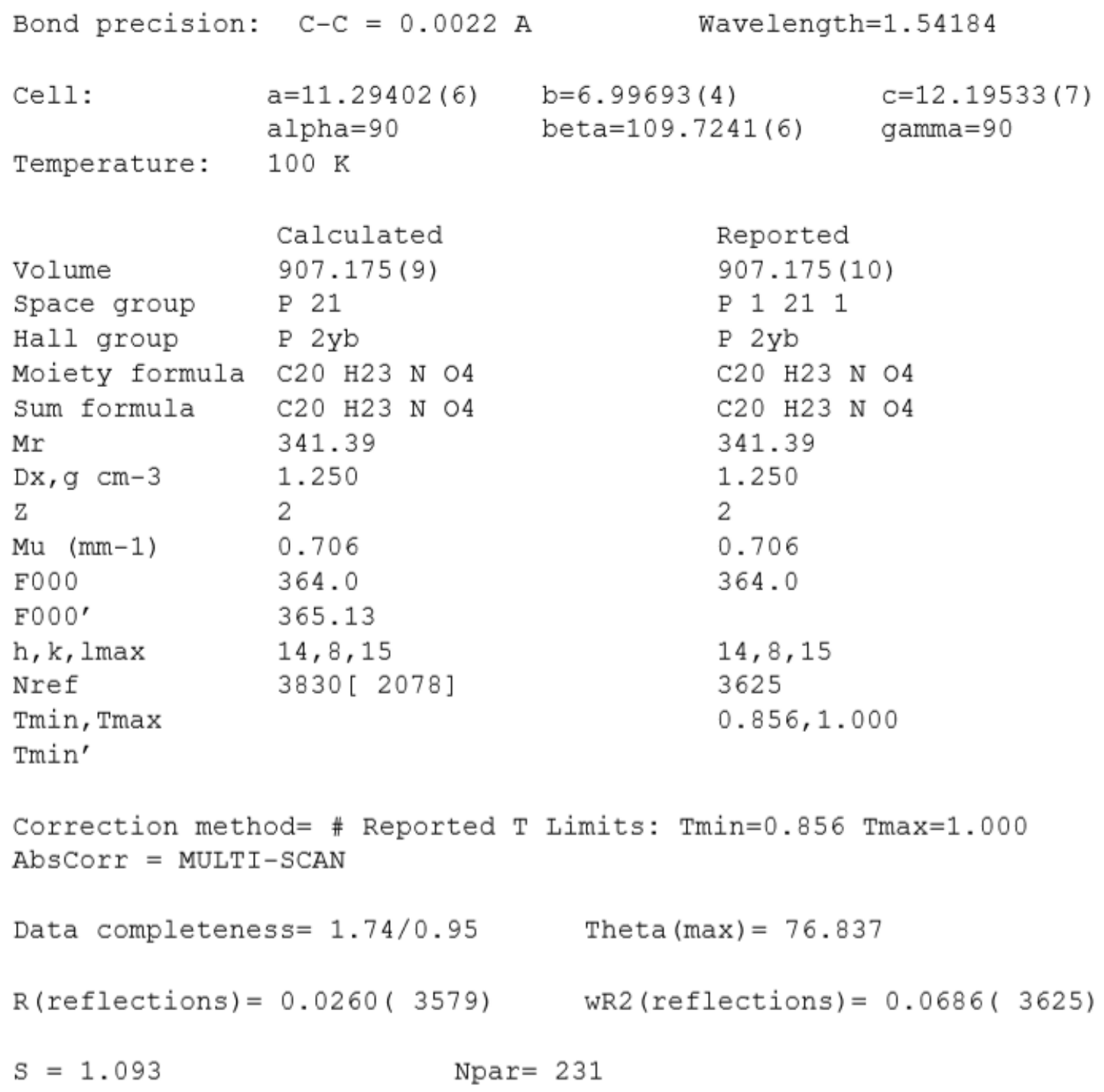


The absolute configuration of toddacoumalone 1a was determined by single crystal X-ray analysis. (CCDC 1903774 contains the supplementary crystallographic data for this structure. These data can be obtained free of charge from The Cambridge Crystallographic Data Centre)

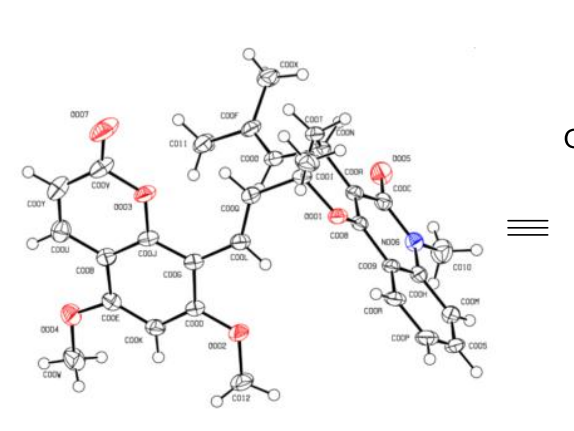

CCDC 1903774

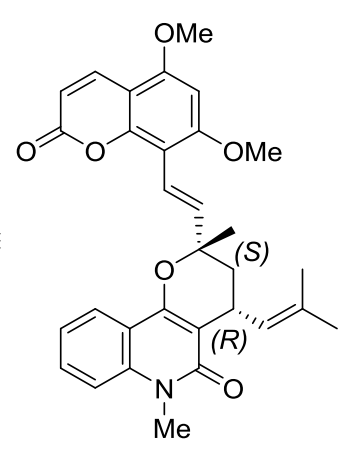

$1 \mathrm{a}$

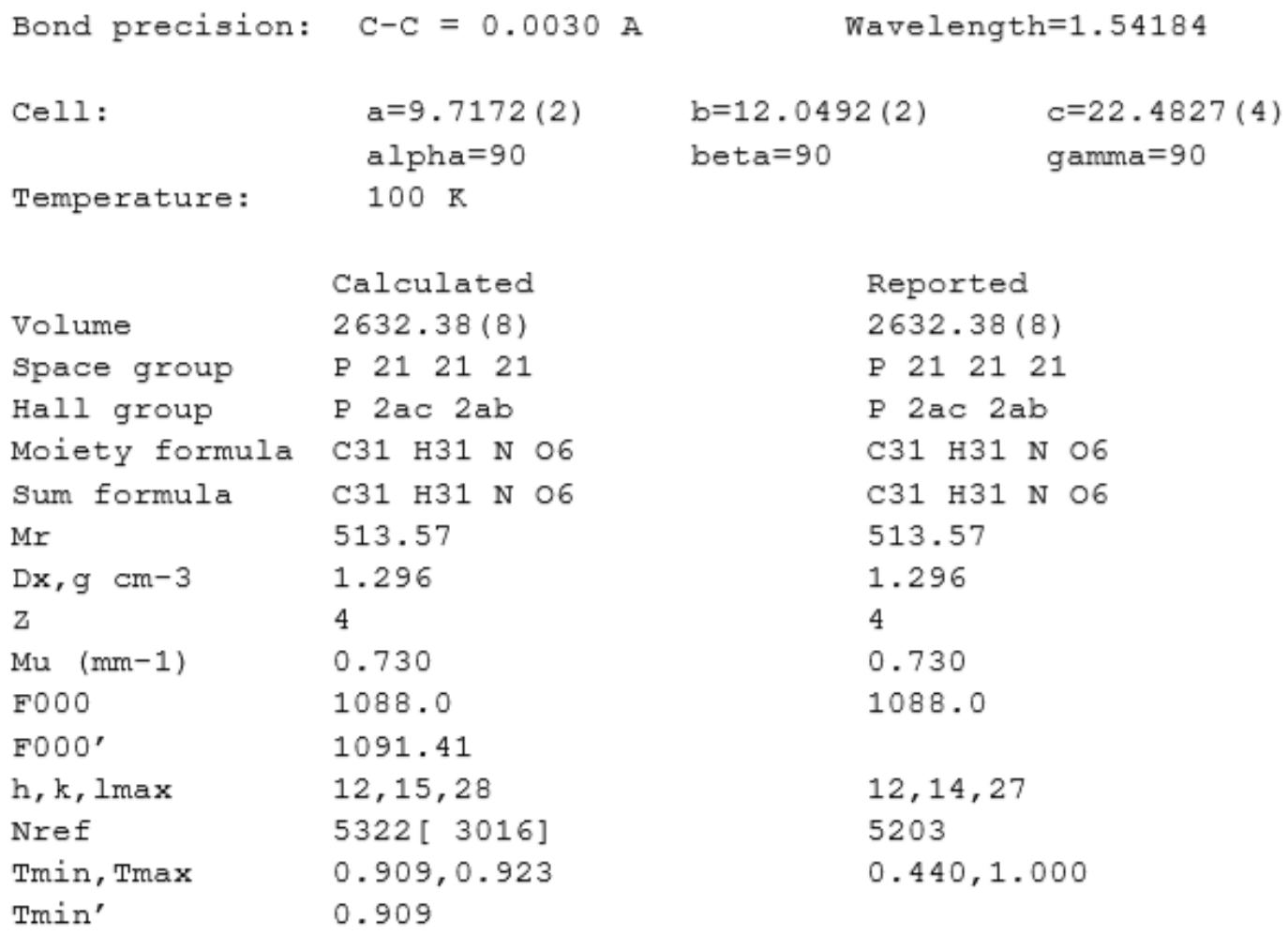

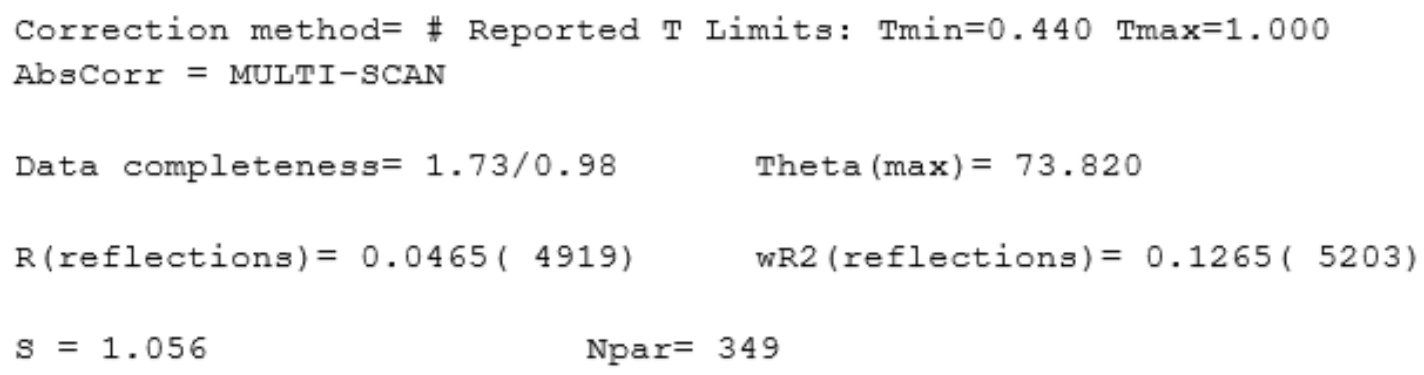




\section{NMR spectra.}

(2S,4R)-2-(diphenyl((triethylsilyl)oxy)methyl)-4-((triethylsilyl)oxy)pyrrolidine (8c)

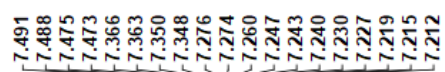

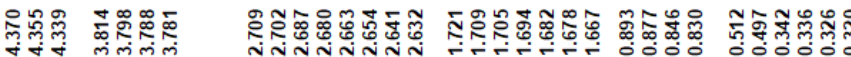

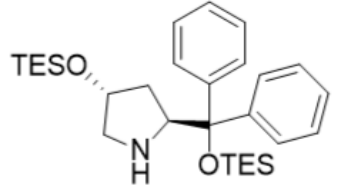

$8 c$

$500 \mathrm{MHz}, \mathrm{CDCl}_{3}$

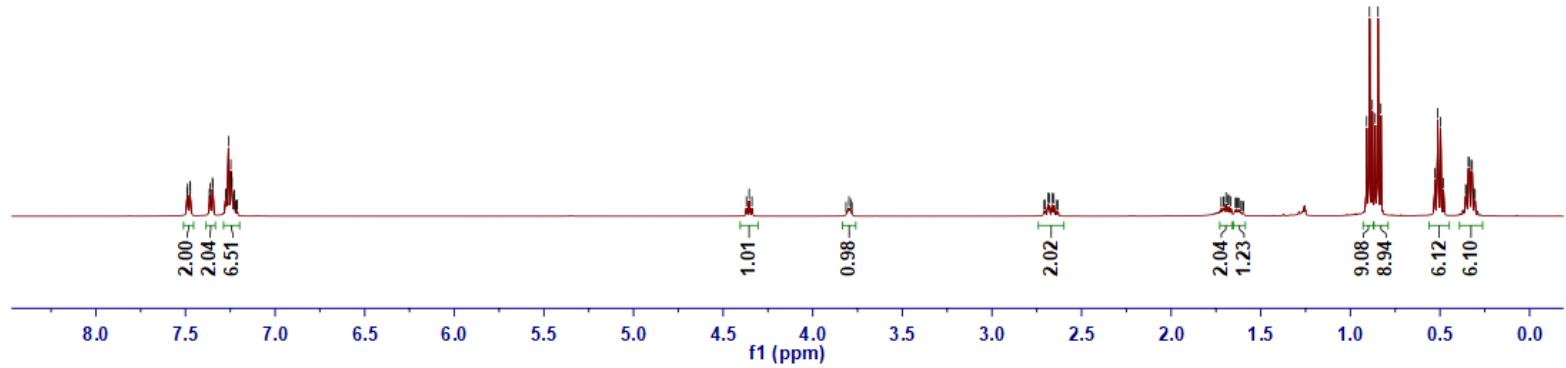

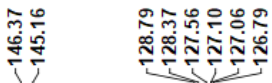

₹

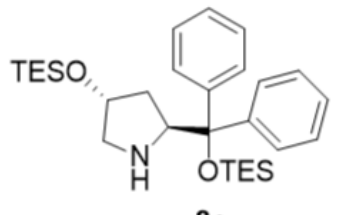

$8 \mathrm{c}$

$100 \mathrm{MHz}, \mathrm{CDCl}_{3}$

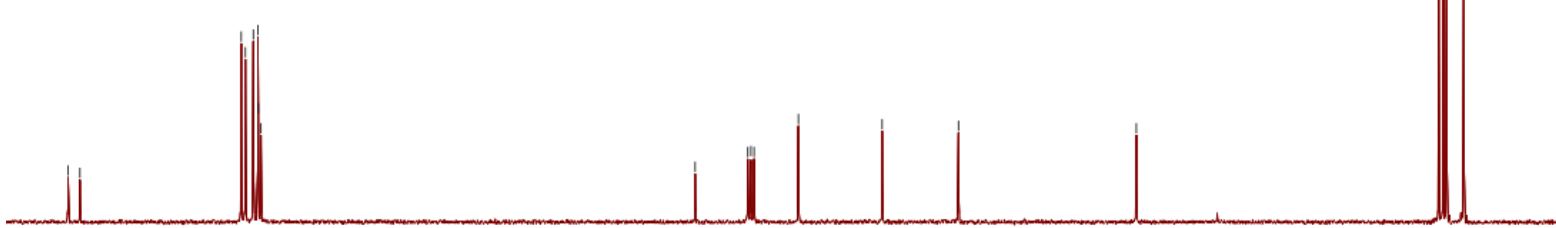

150

$80 \begin{array}{r}70 \\ \mathrm{f} 1(\mathrm{ppm})\end{array}$

$51 / 146$ 
((2S,4R)-4-((tert-butyldimethylsilyl)oxy)pyrrolidin-2-yl)diphenylmethanol (10)
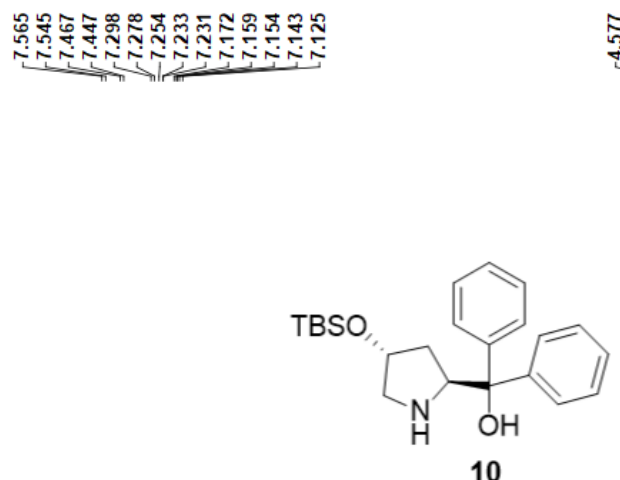

$400 \mathrm{MHz}, \mathrm{CDCl}_{3}$

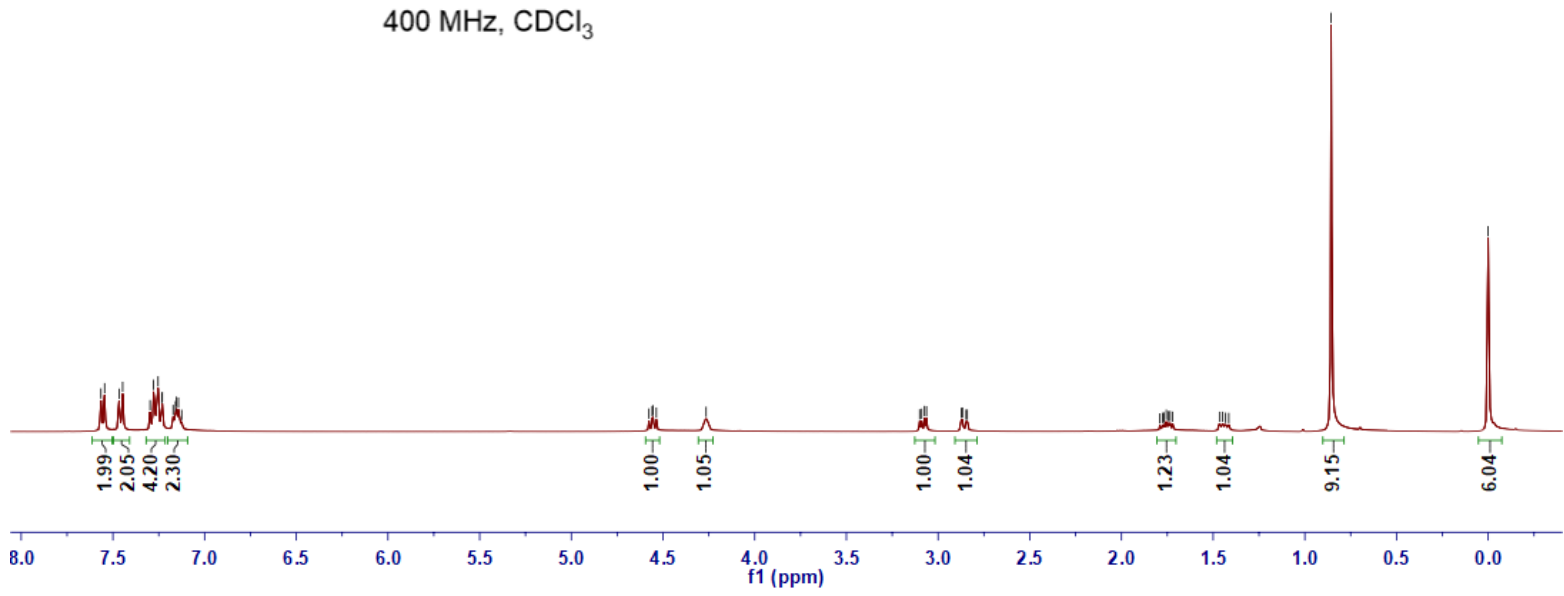

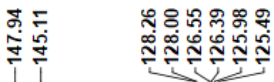

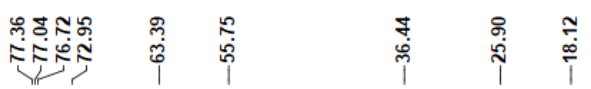

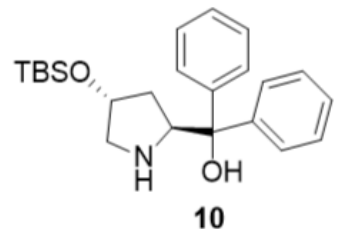

$100 \mathrm{MHz}, \mathrm{CDCl}_{3}$

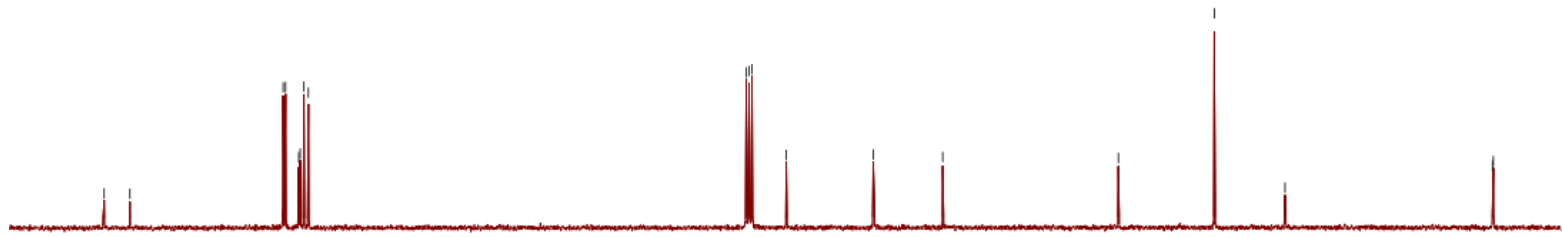

$\begin{array}{llllllll}150 & 140 & 130 & 120 & 110 & 100 & 90 & 80 \begin{array}{r}70 \\ \mathrm{f} 1(\mathrm{ppm})\end{array}\end{array}$ 
(2S,4R)-4-((tert-butyldimethylsilyl)oxy)-2-(((tert-butyldimethylsilyl)oxy)diphenylmethyl)pyrrolidine (8d)

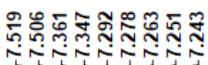

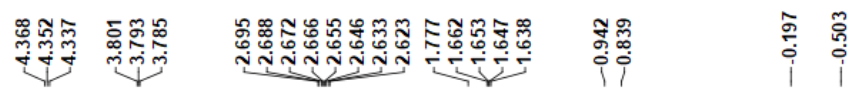

TBSO

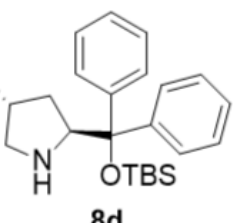

$400 \mathrm{MHz}, \mathrm{CDCl}_{3}$

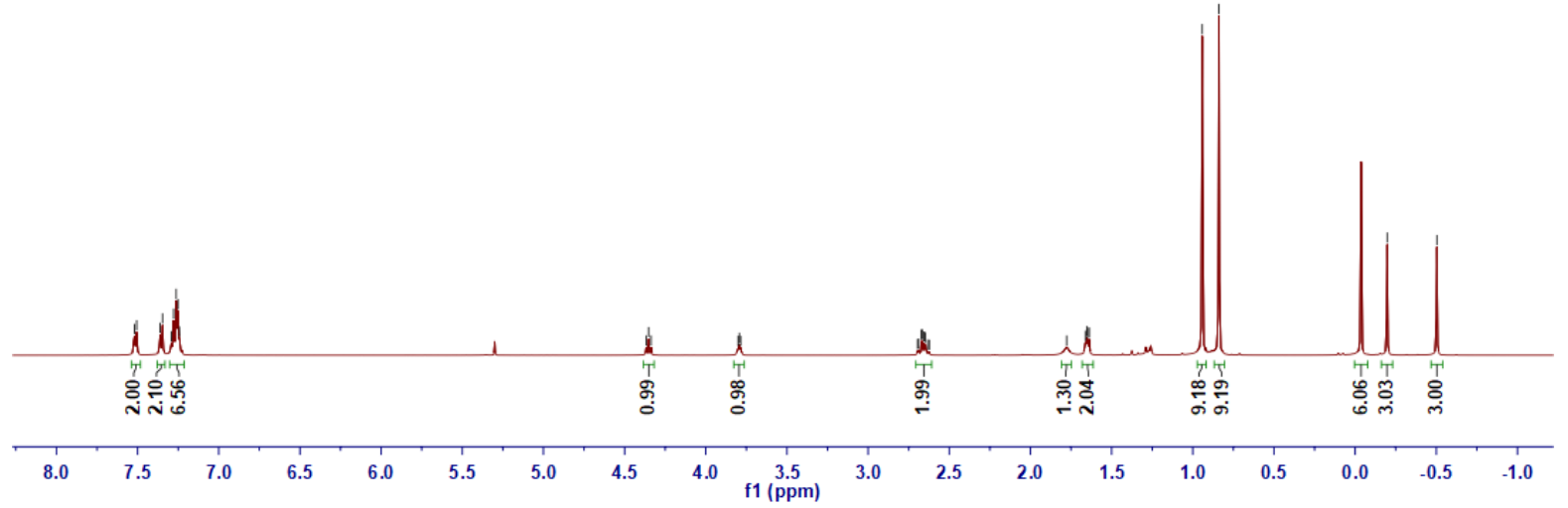

方竞

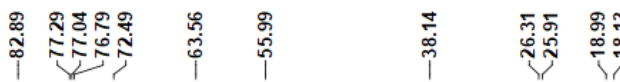

我

TBSO

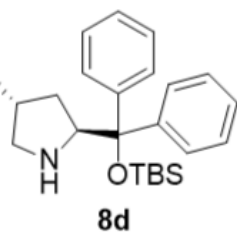

$125 \mathrm{MHz}, \mathrm{CDCl}_{3}$

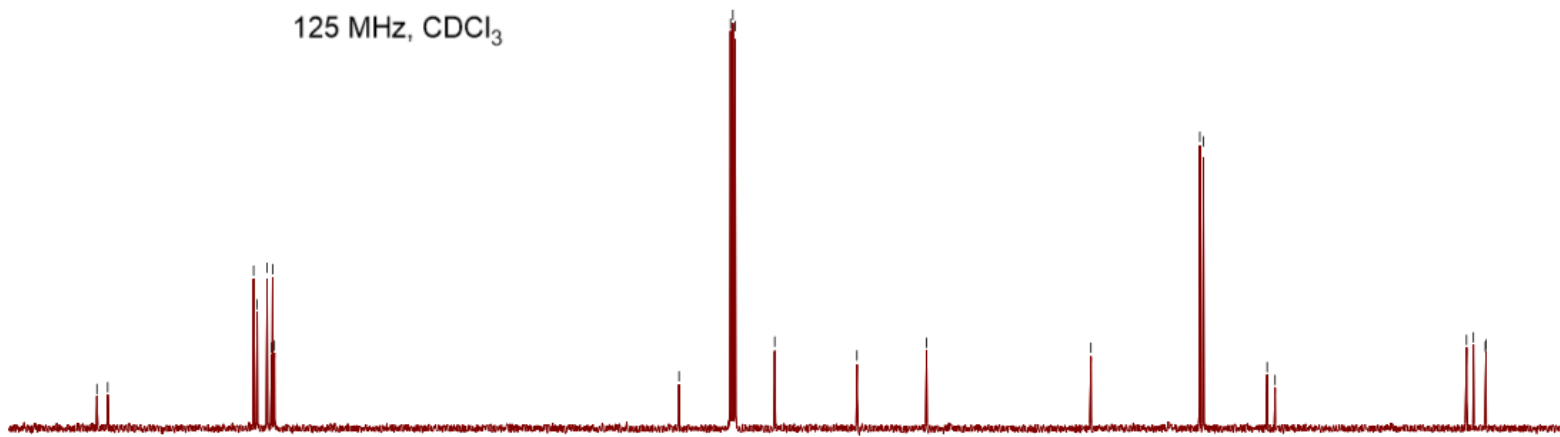

100

90

$80 \quad \begin{gathered}70 \\ \mathrm{f} 1(\mathrm{ppm})\end{gathered}$ 
Methyl (2S,4R)-1-benzyl-4-((triethylsilyl)oxy)pyrrolidine-2-carboxylate (12)

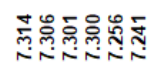

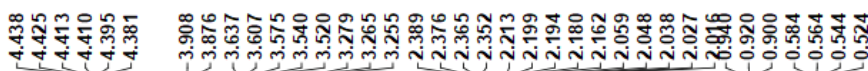

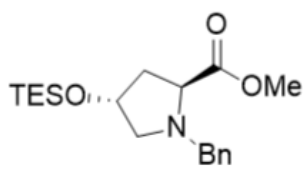

12

$400 \mathrm{MHz}, \mathrm{CDCl}_{3}$

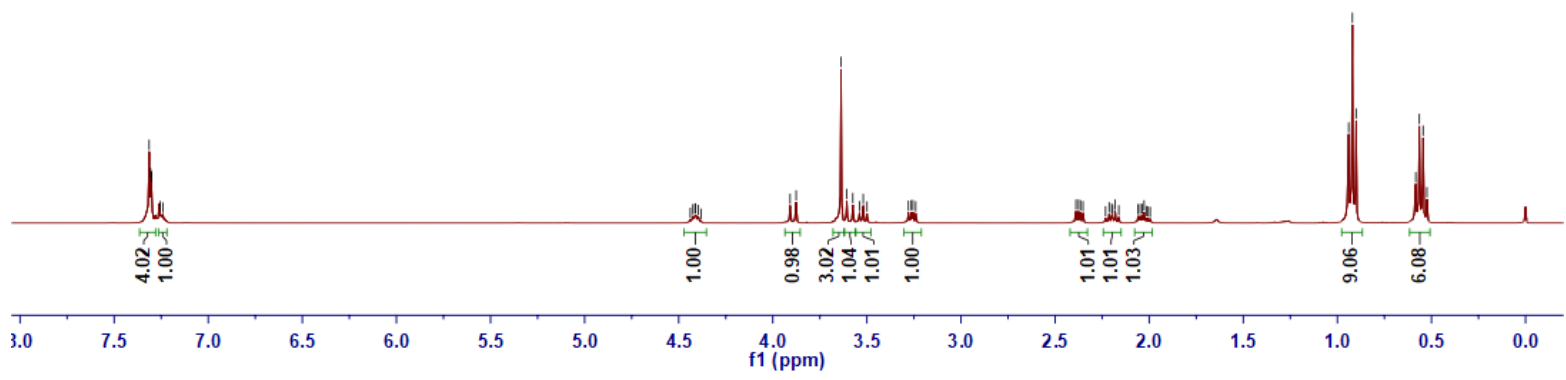

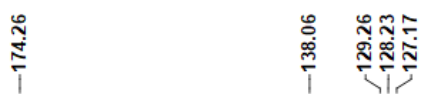

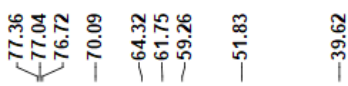

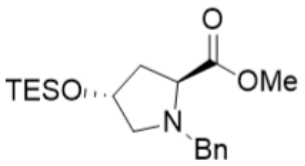

12

$100 \mathrm{MHz}, \mathrm{CDCl}_{3}$

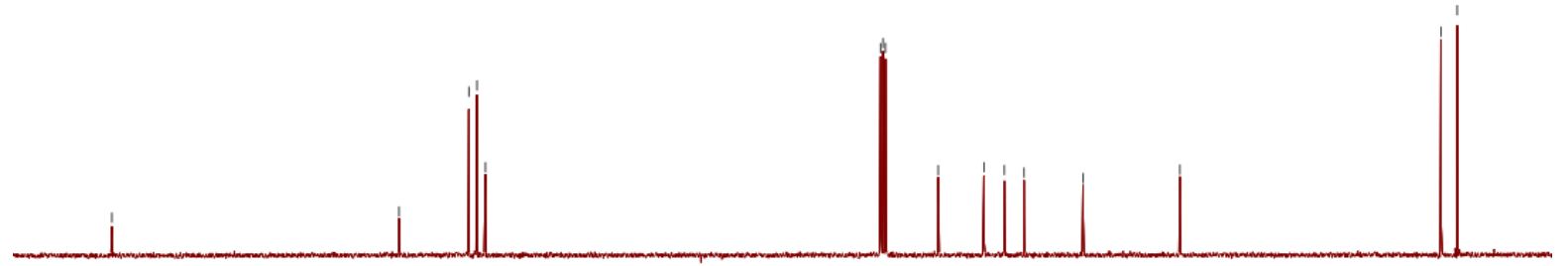

180

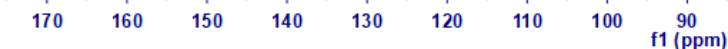

80

60

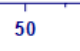

$40 \quad 3$

$2010 \quad 0$ 
$((2 S, 4 R)-1-b e n z y l-4-(($ triethylsilyl)oxy)pyrrolidin-2-yl)bis(3,5-bis(trifluoromethyl)phenyl)methanol (14) 궁

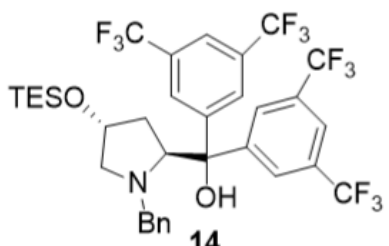

$400 \mathrm{MHz}, \mathrm{CDCl}_{3}$

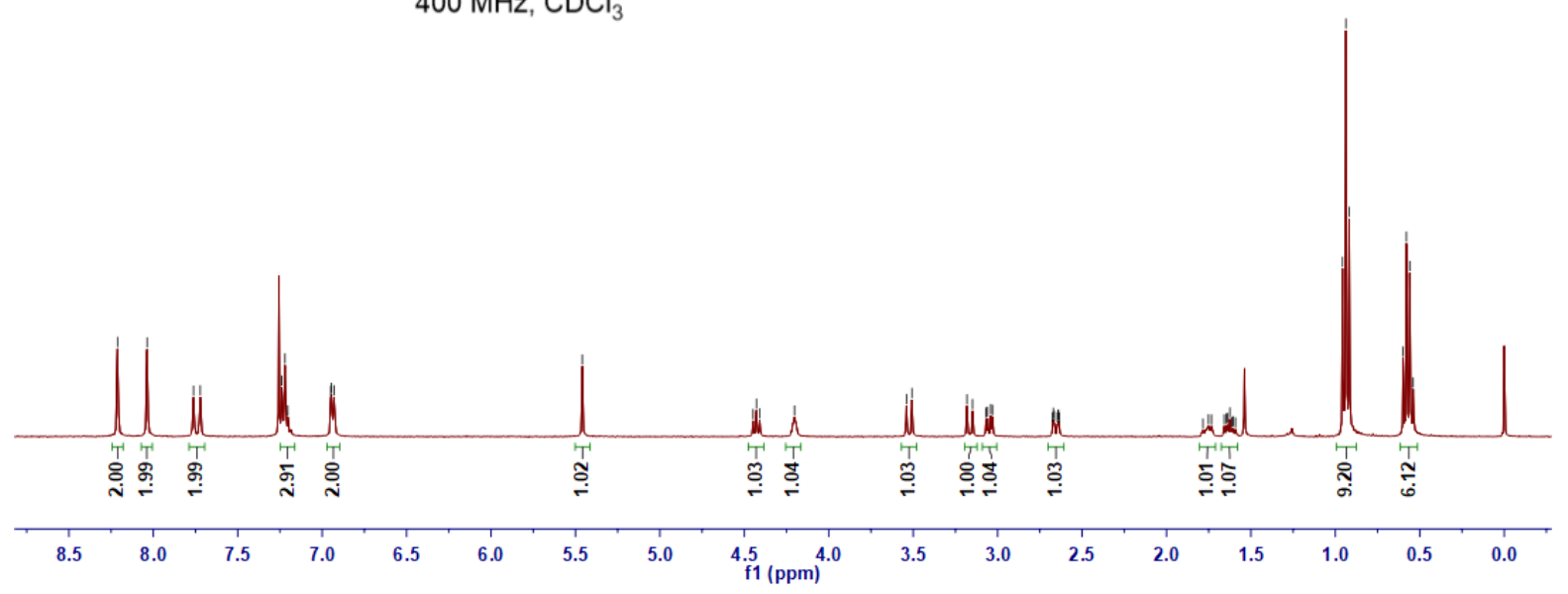

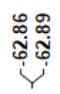

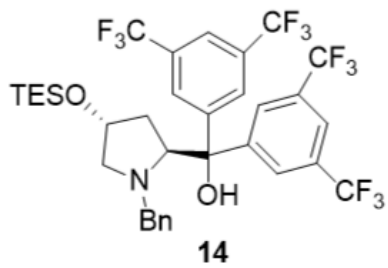

$376 \mathrm{MHz} C D C l_{3}$

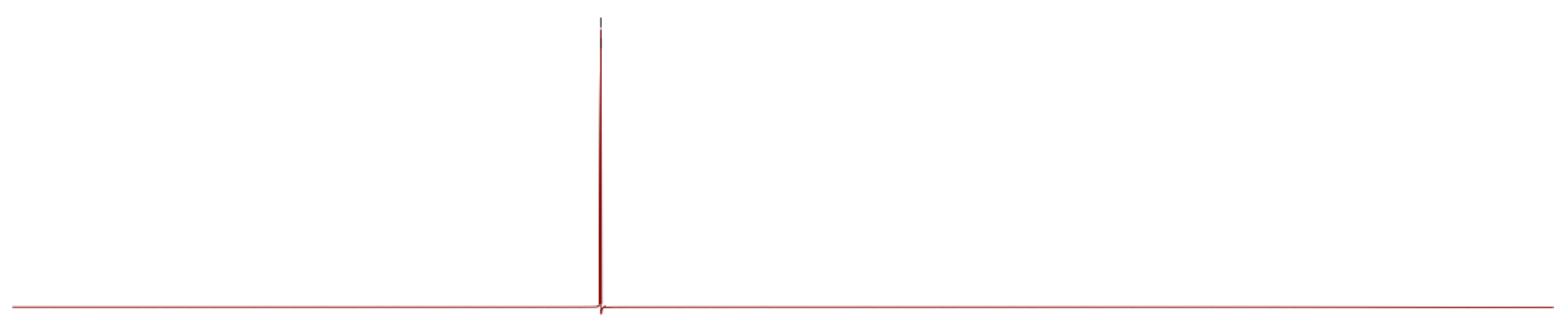

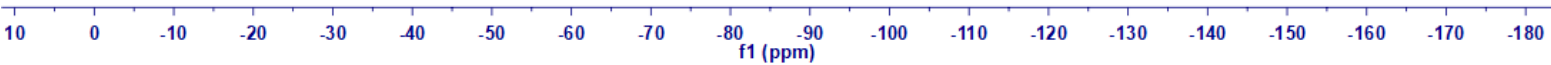




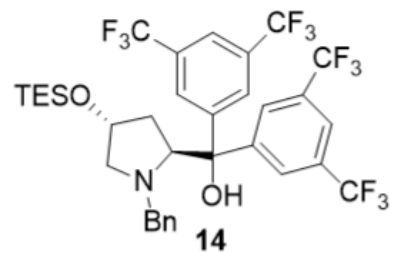

$100 \mathrm{MHz}, \mathrm{CDCl}_{3}$

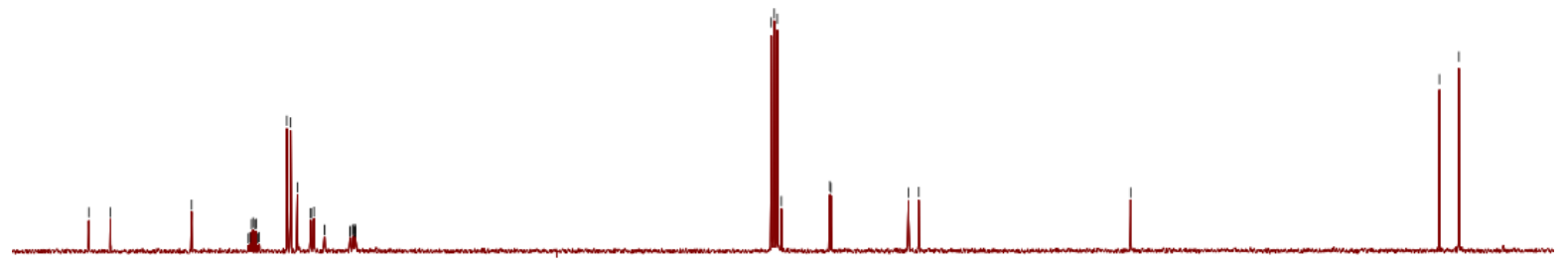

bis(3,5-bis(trifluoromethyl)phenyl)((2S,4R)-4-((triethylsilyl)oxy)pyrrolidin-2-yl)methanol (15)

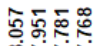

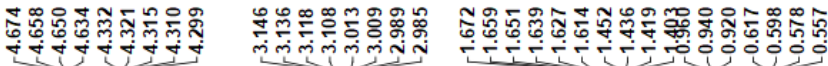
isi

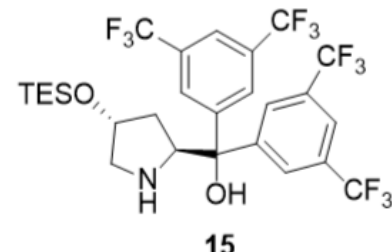

$400 \mathrm{MHz}, \mathrm{CDCl}_{3}$

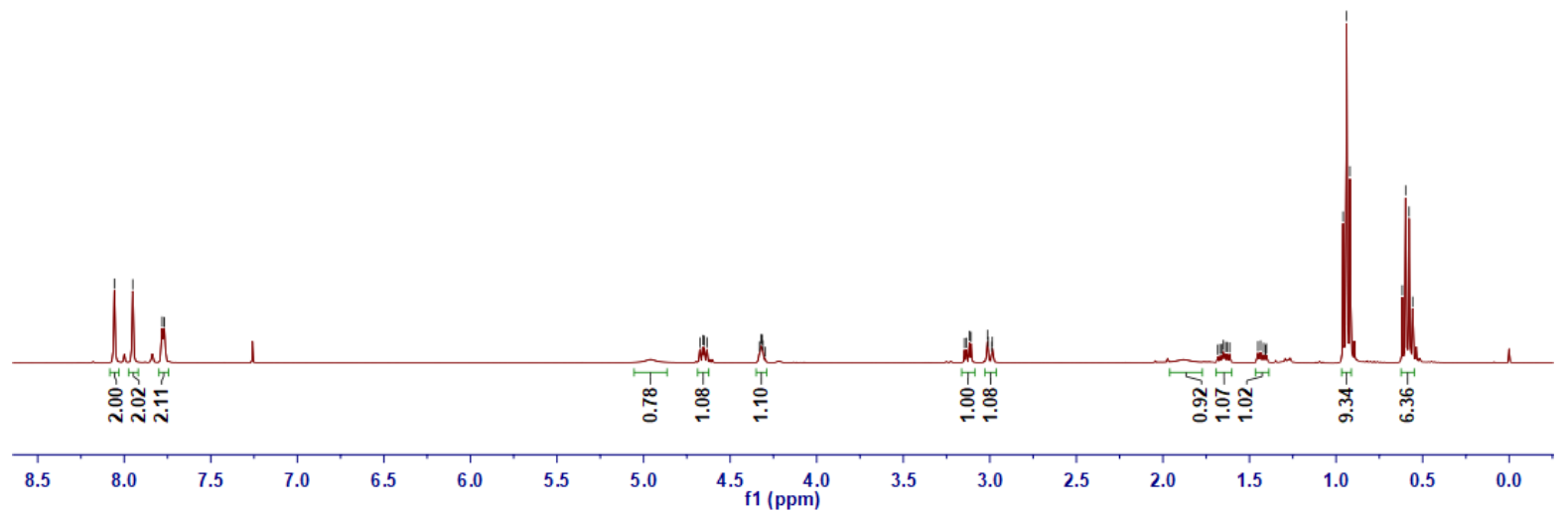




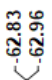

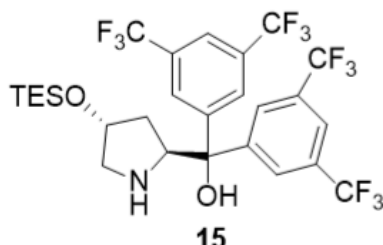

$376 \mathrm{MHz} \mathrm{CDCl}_{3}$

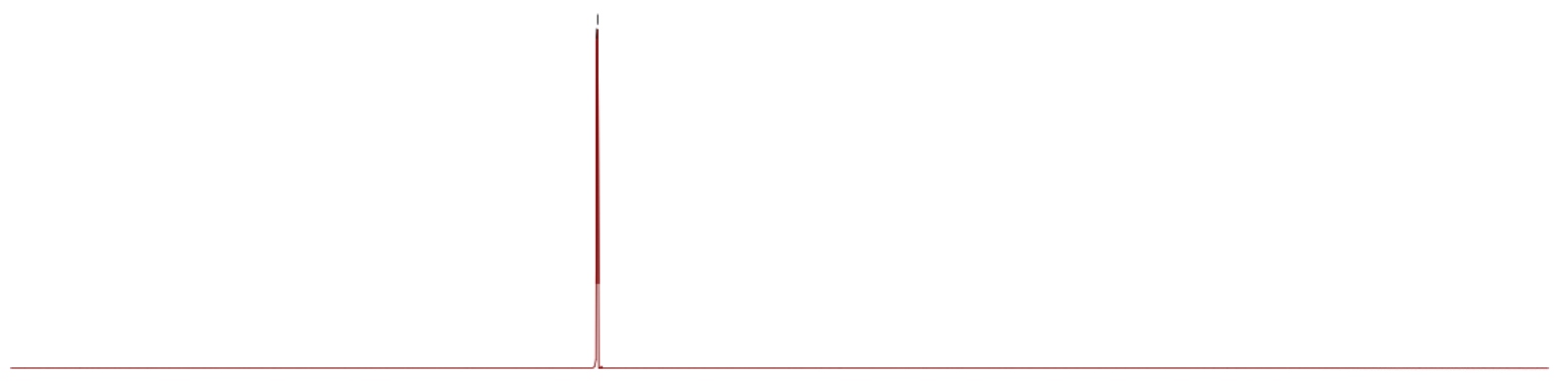

$\begin{array}{llllllllllllllllllllll}1 & 0 & 0 & -10 & -20 & -30 & -40 & -50 & -60 & -70 & -80 & -90 & -100 & -110 & -120 & -130 & -140 & -150 & -160 & -170 & -180 & -15\end{array}$

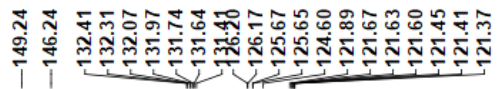

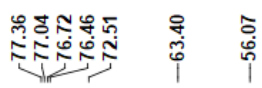

$\stackrel{\infty}{\oplus}$

$\stackrel{i}{\substack{0 \\ \hdashline}}$
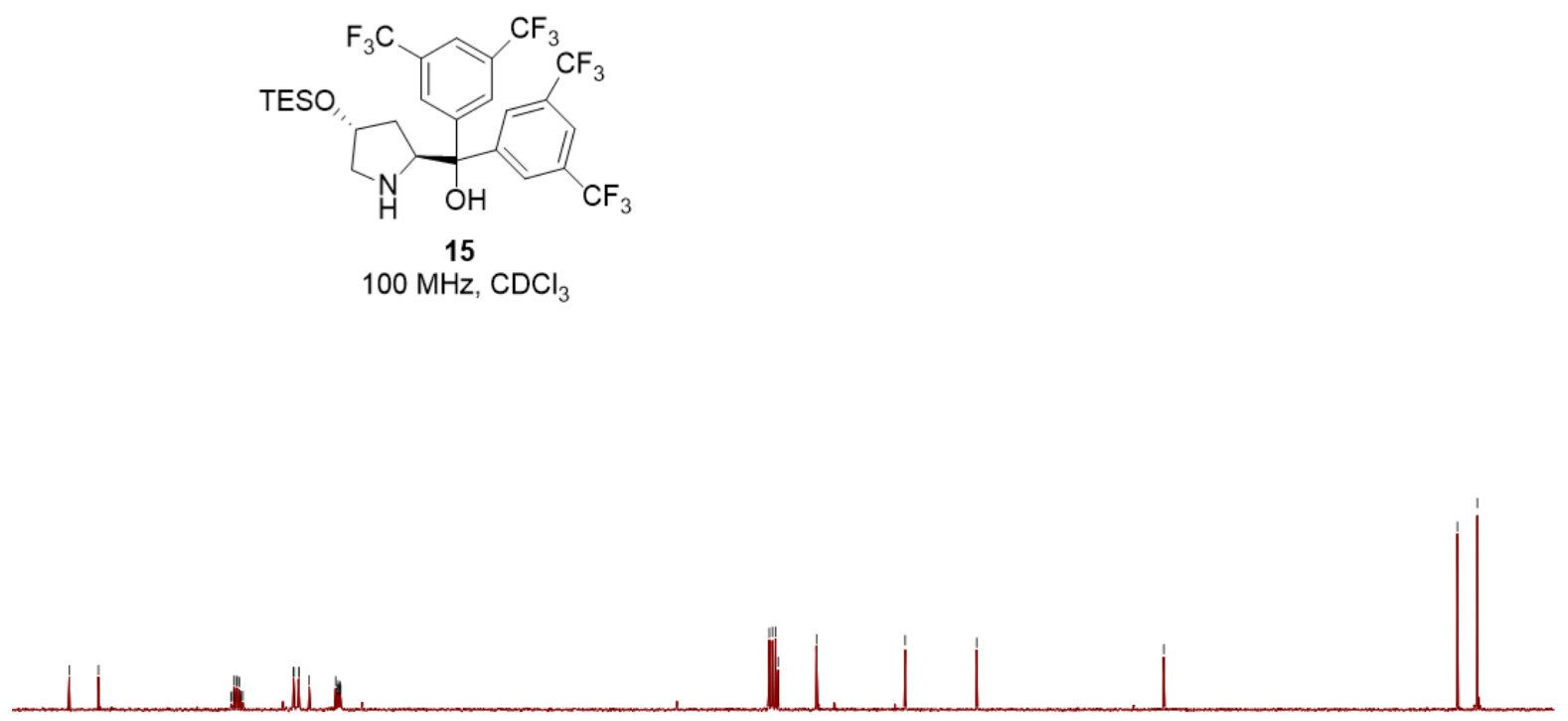

\begin{tabular}{|c|c|c|c|c|c|c|c|c|c|c|c|c|c|c|}
\hline 150 & 140 & 130 & 120 & 110 & 100 & 90 & ${ }_{\mathrm{f} 1}^{80}(\mathrm{ppm})$ & 70 & 60 & 50 & 40 & 30 & 20 & 10 \\
\hline
\end{tabular}


(2S,4R)-2-(bis(3,5-bis(trifluoromethyl)phenyl)((triethylsilyl)oxy)methyl)-4-((triethylsilyl)oxy)pyrrolidine (8e)

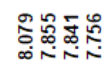

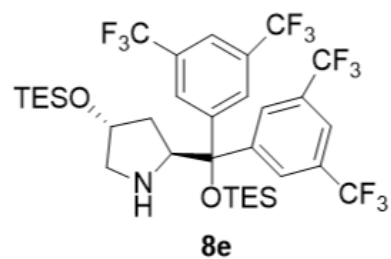

$400 \mathrm{MHz}, \mathrm{CDCl}_{3}$

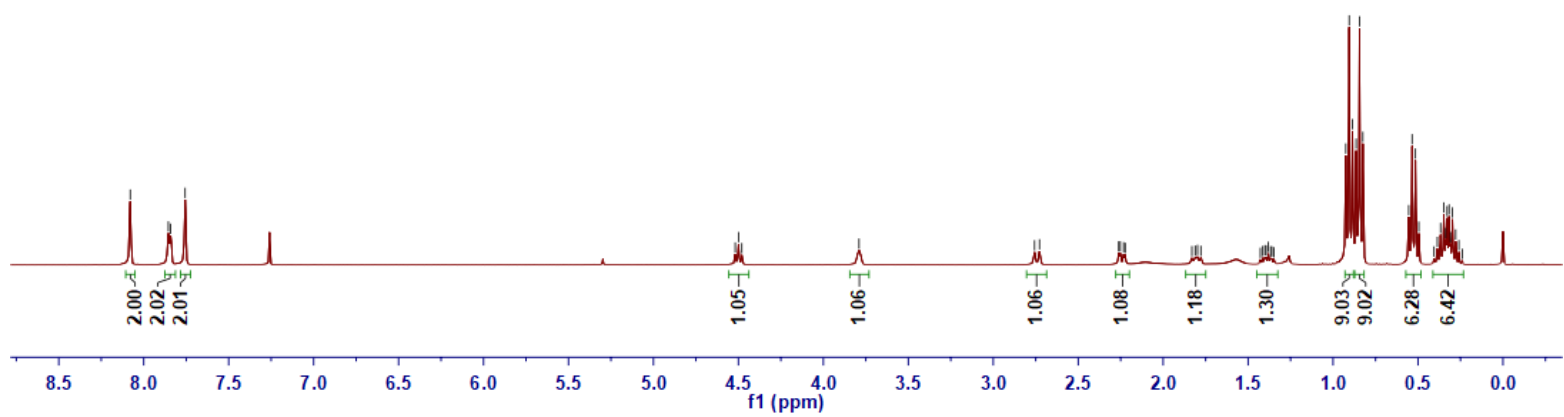

ஸ̛̣

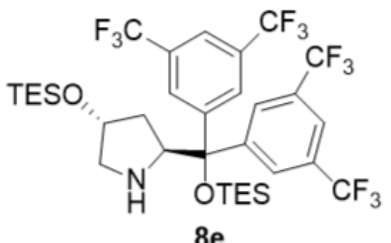

$376 \mathrm{MHz} \mathrm{CDCl}_{3}$

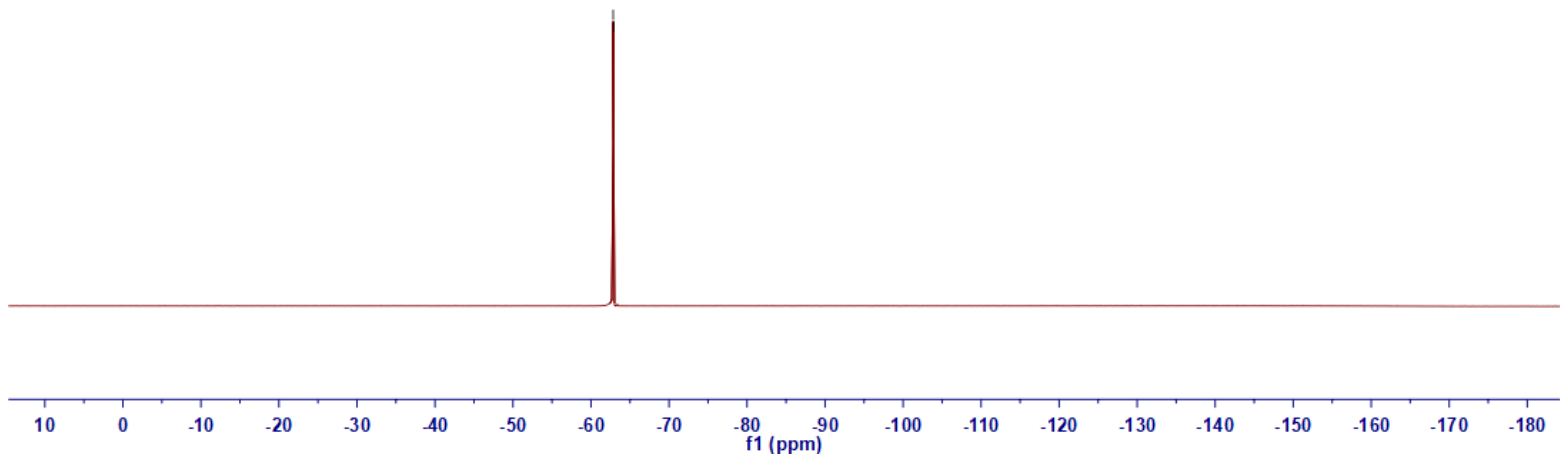




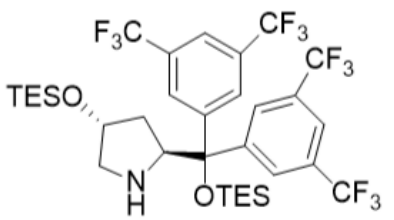

$8 \mathrm{e}$

$100 \mathrm{MHz}, \mathrm{CDCl}_{3}$

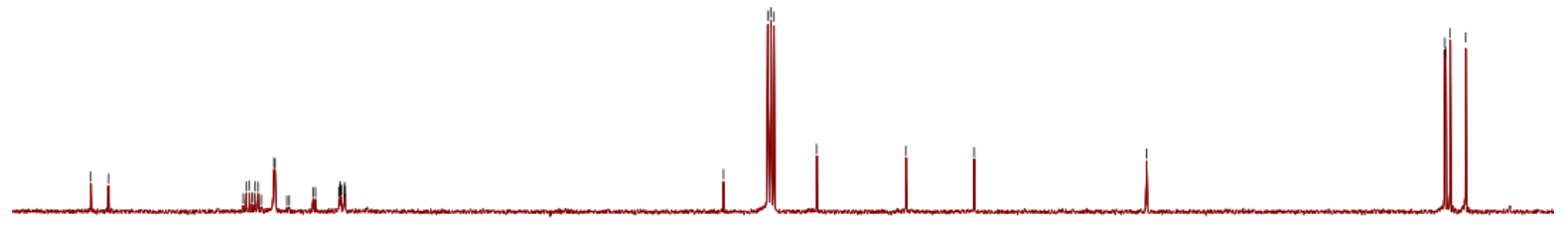

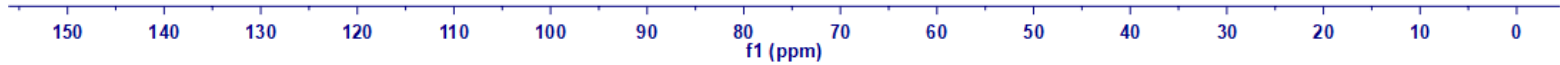

((2S,4R)-1-benzyl-4-((triisopropylsilyl)oxy)pyrrolidin-2-yl)bis(3,5-bis(trifluoromethyl)phenyl)methanol (17)

융

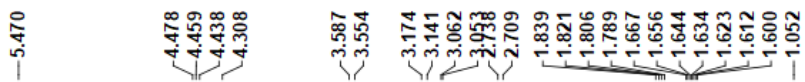

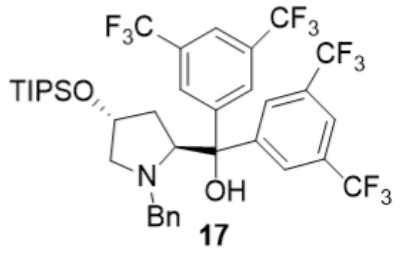

$400 \mathrm{MHz}, \mathrm{CDCl}_{3}$

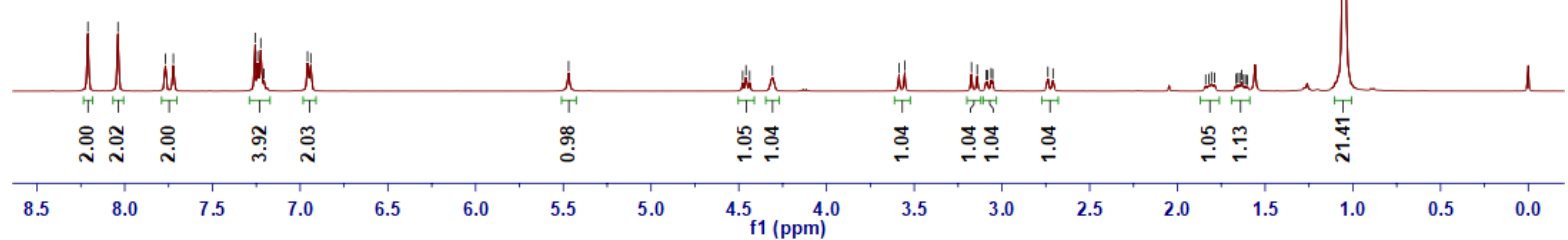




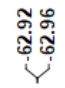

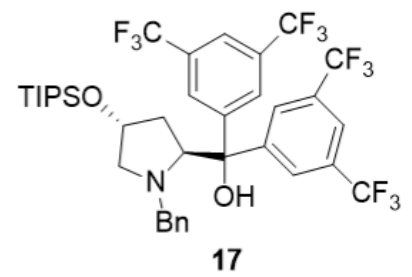

$376 \mathrm{MHz} C D C l_{3}$

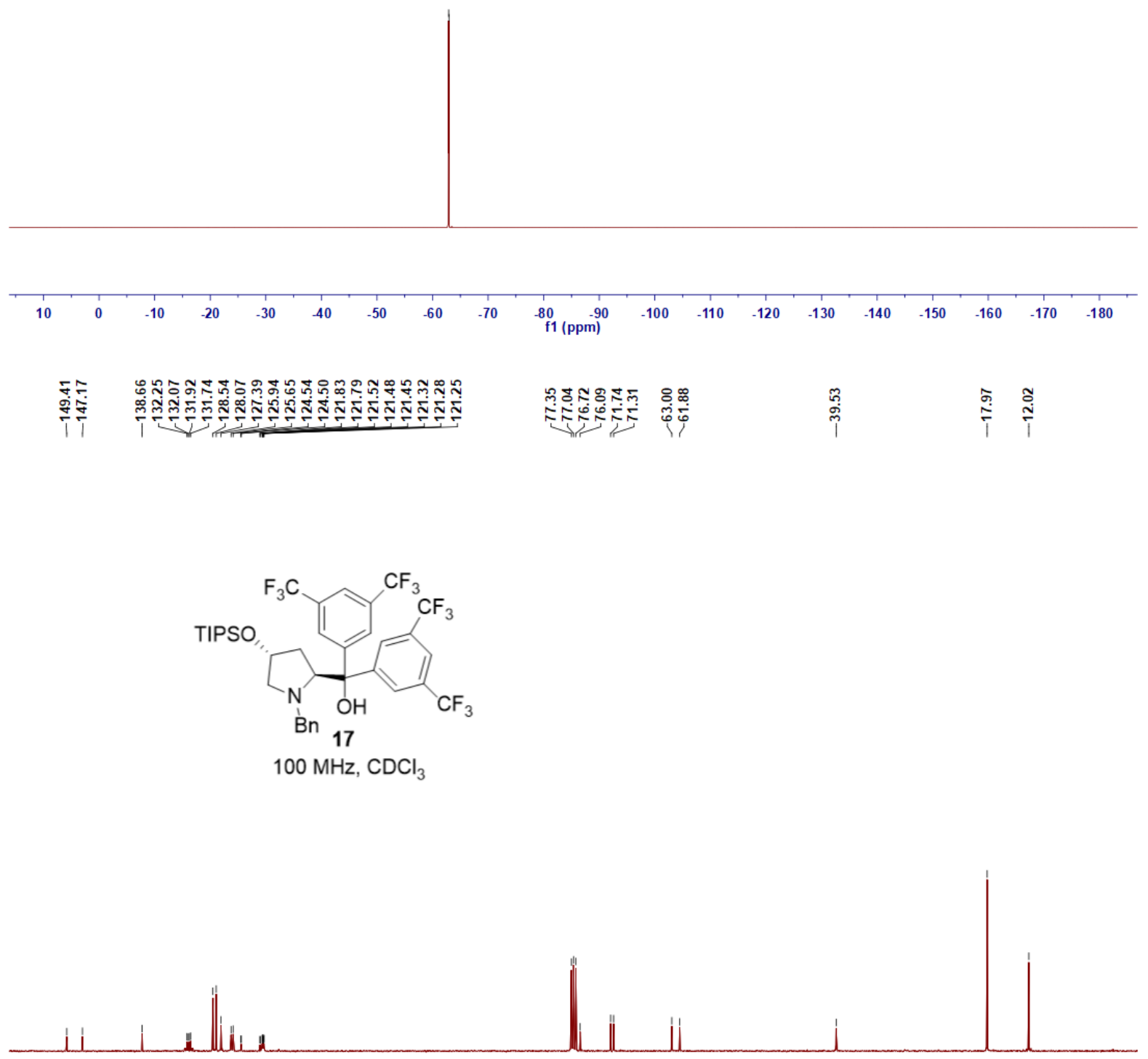

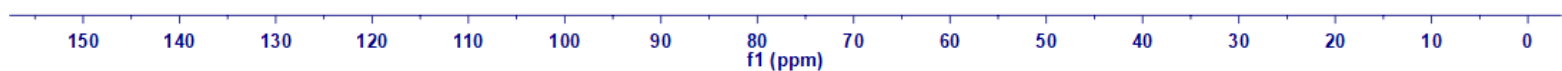


bis(3,5-bis(trifluoromethyl)phenyl)((2S,4R)-4-((triisopropylsilyl)oxy)pyrrolidin-2-yl)methanol (18)
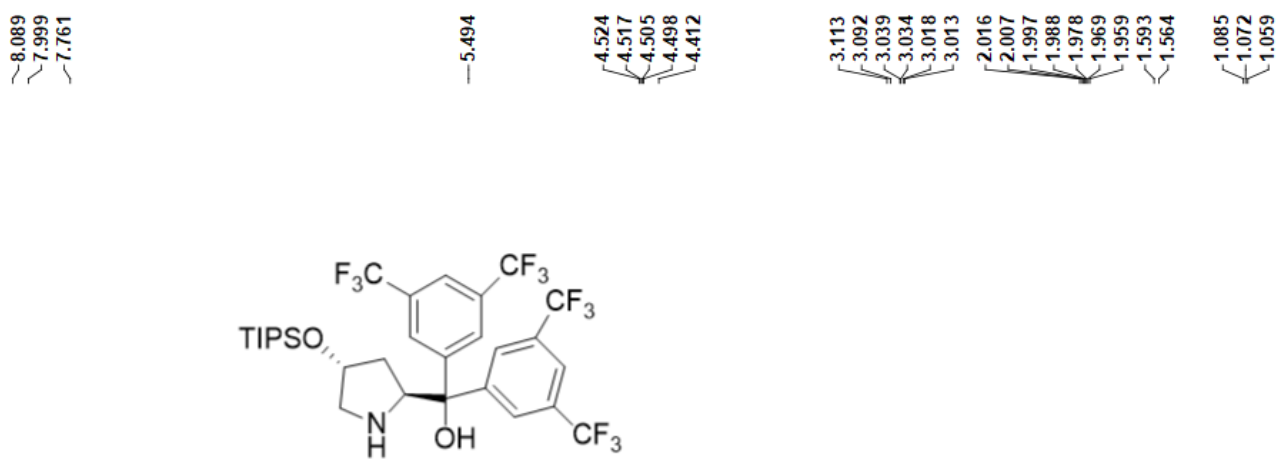

18

$500 \mathrm{MHz}, \mathrm{CDCl}_{3}$

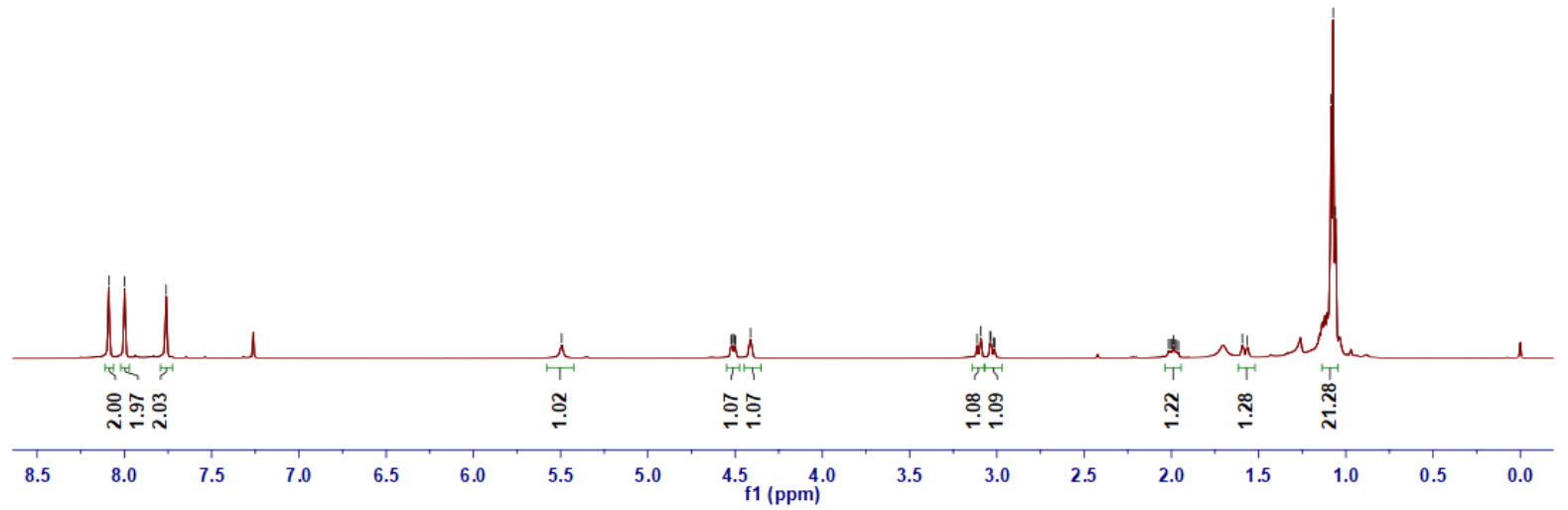

ํํำ

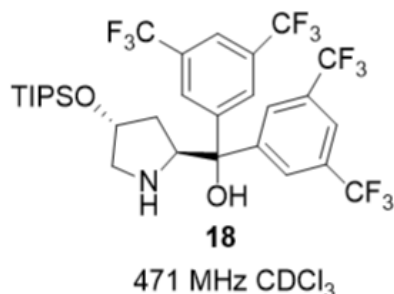

$471 \mathrm{MHz} \mathrm{CDCl}_{3}$

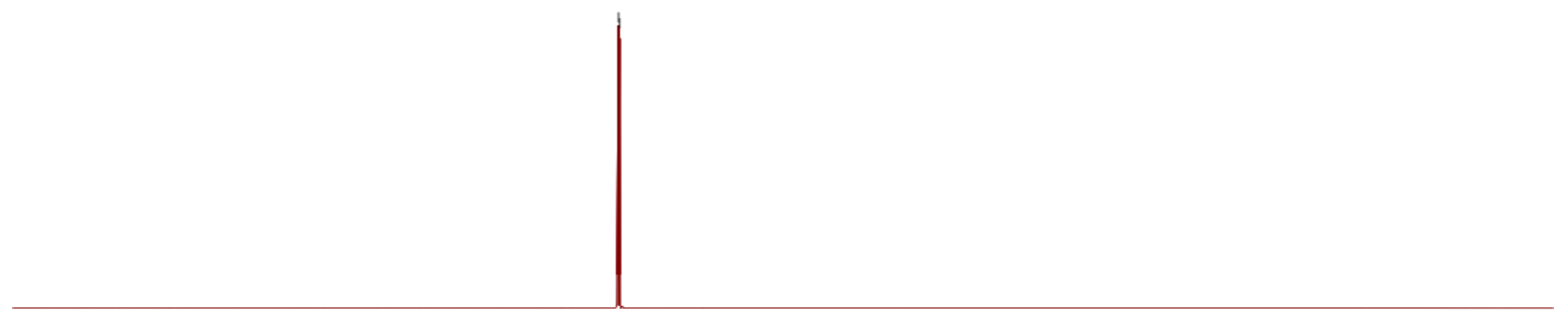

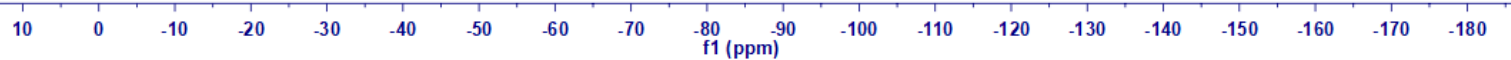




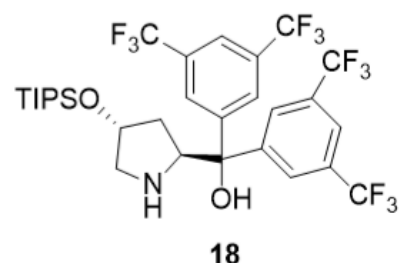

$125 \mathrm{MHz}, \mathrm{CDCl}_{3}$

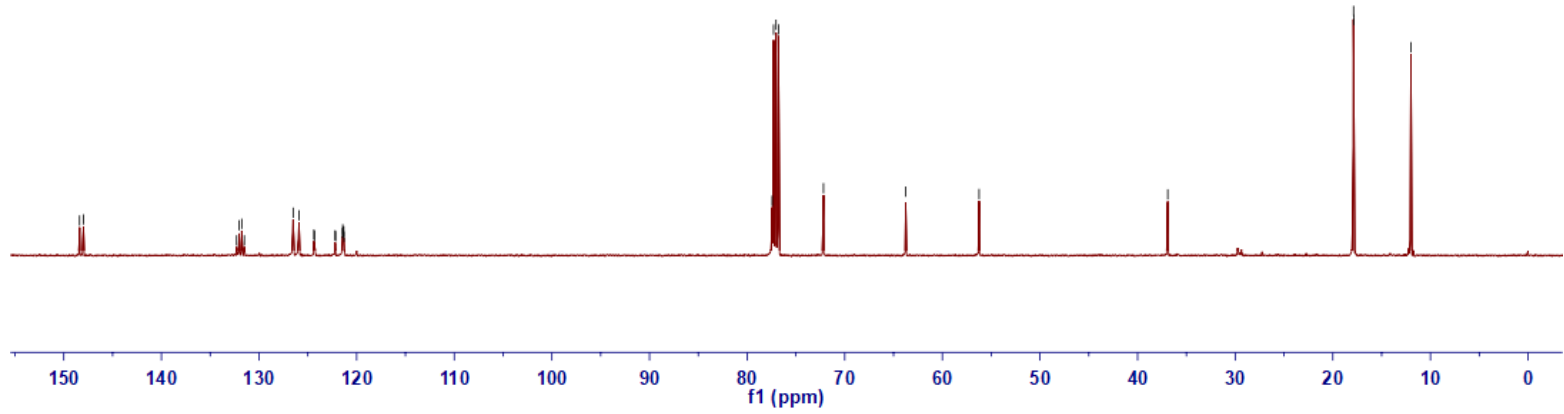

$(2 S, 4 R)-2-(b i s(3,5-b i s($ trifluoromethyl)phenyl)((triethylsilyl)oxy)methyl)-4-((triisopropylsilyl)oxy)pyrrolidine (8g)

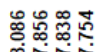

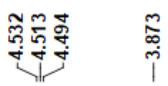

兽
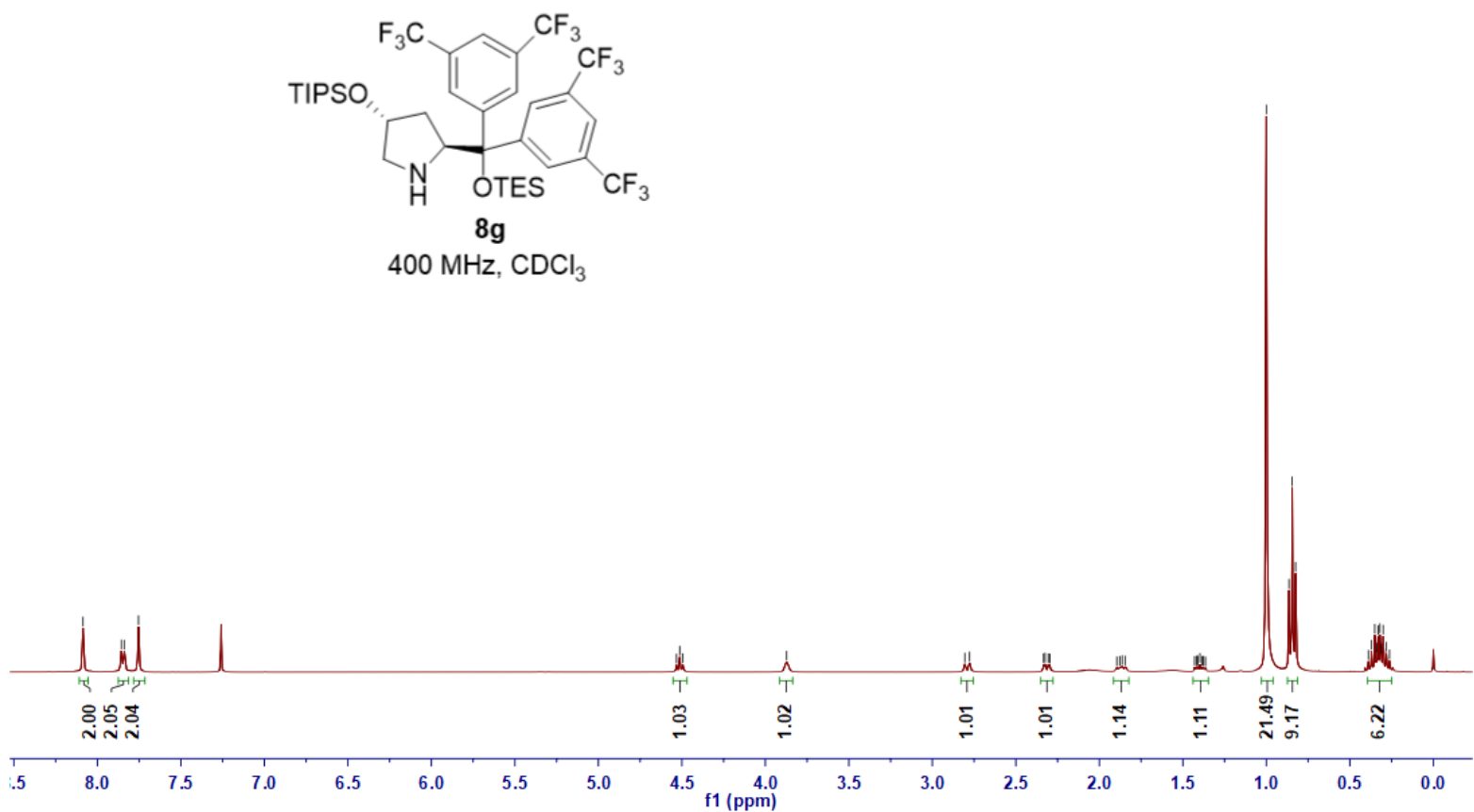


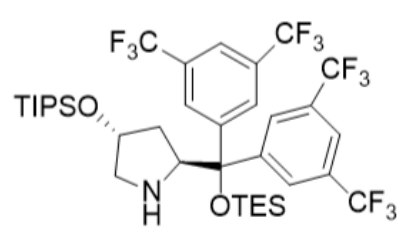

$8 \mathrm{~g}$

$376 \mathrm{MHz} \mathrm{CDCl}_{3}$

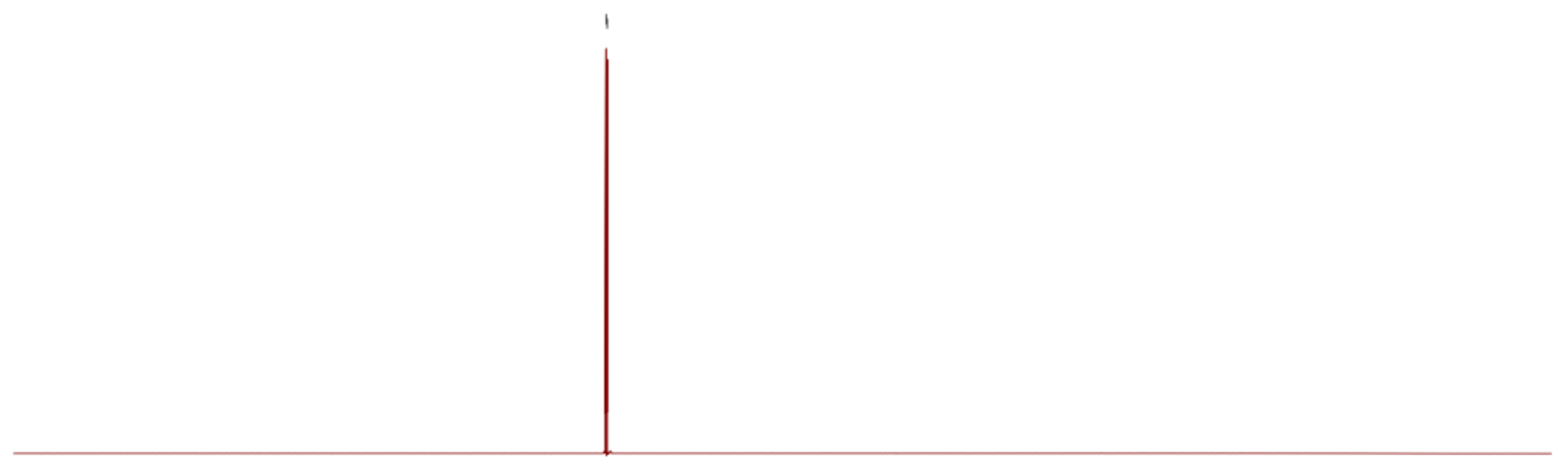

\begin{tabular}{llllllllllllllllllll}
\hline 10 & 0 & -10 & -20 & -30 & -40 & -50 & -60 & -70 & -80 & -90 & -100 & -110 & -120 & -130 & -140 & -150 & -160 & -170 & -180
\end{tabular}

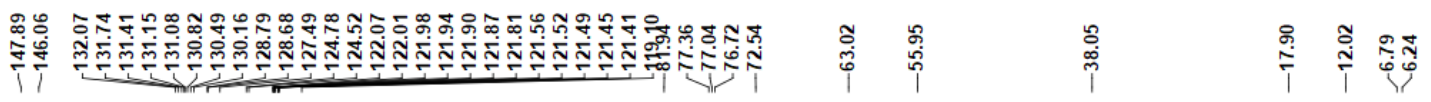

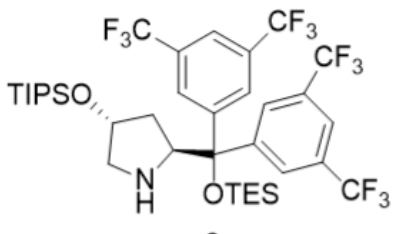

$8 \mathrm{~g}$

$100 \mathrm{MHz}, \mathrm{CDCl}_{3}$

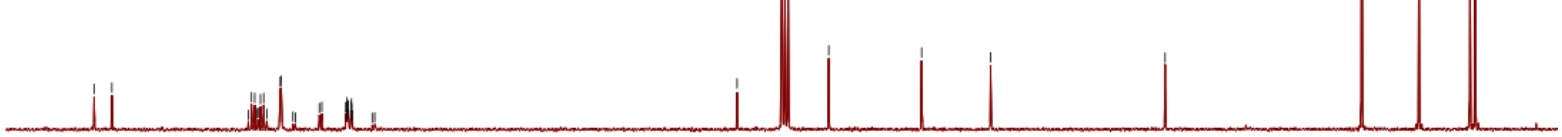


1-benzyl-6-bromo-4-hydroxyquinolin-2(1H)-one (a6)

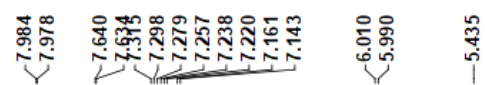

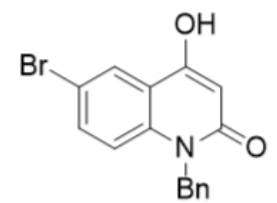

a6

$400 \mathrm{MHz}, \mathrm{DMSO}_{-} \mathrm{d}_{6}$

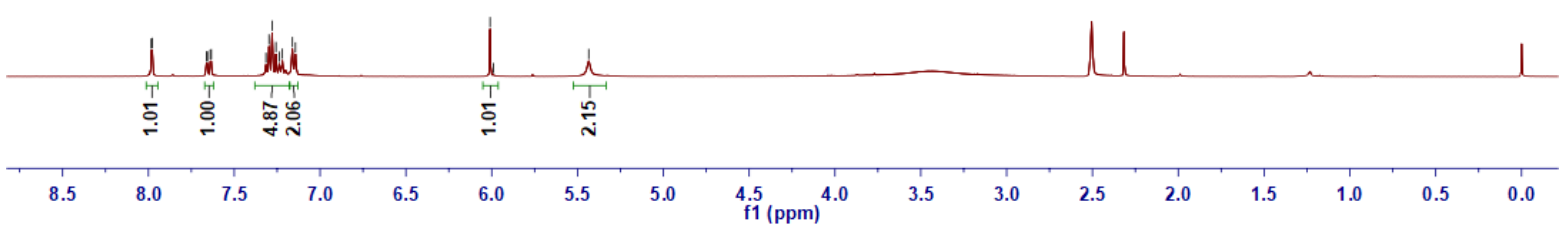

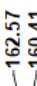

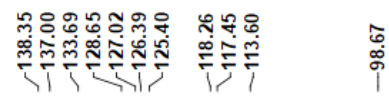

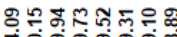

क़ी

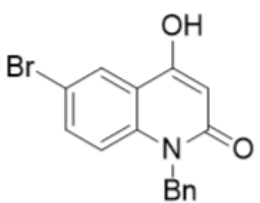

a6

$100 \mathrm{MHz}, \mathrm{DMSO}-\mathrm{d}_{6}$

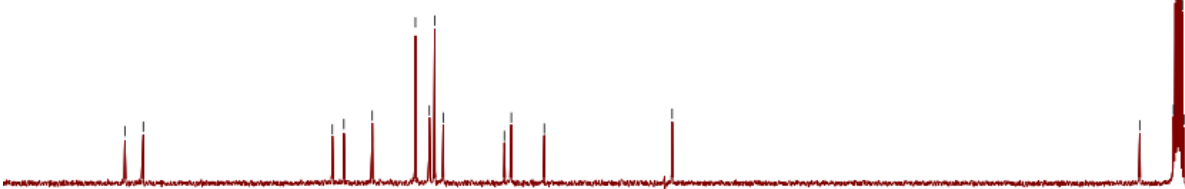

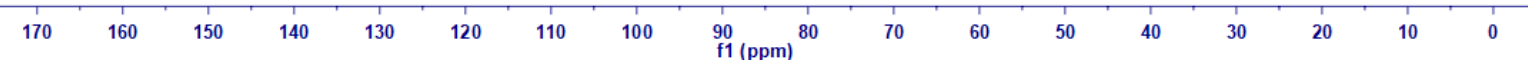


1-benzyl-4-hydroxy-6-nitroquinolin-2(1H)-one (a8)

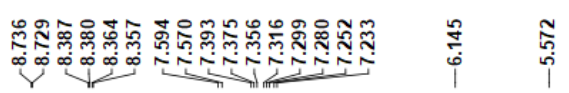

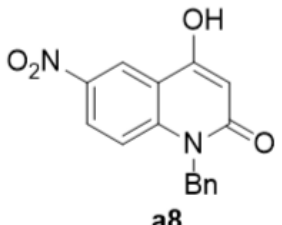

$400 \mathrm{MHz}, \mathrm{DMSO}^{-\mathrm{d}_{6}}$

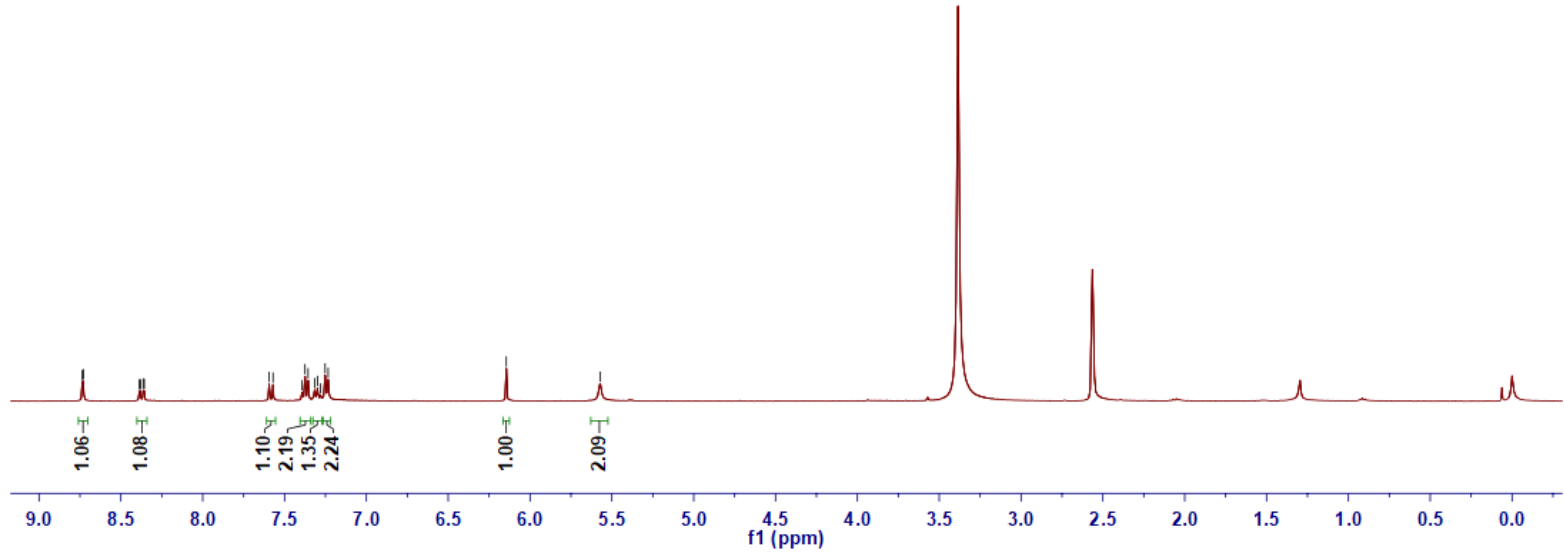

\begin{tabular}{|c|c|}
\hline \multirow[t]{2}{*}{ 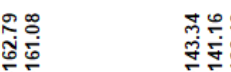 } & \\
\hline & 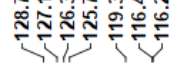 \\
\hline
\end{tabular}

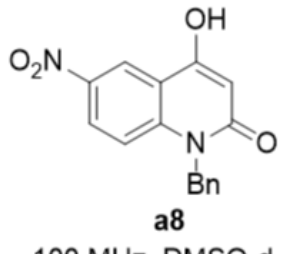

$100 \mathrm{MHz}, \mathrm{DMSO}-\mathrm{d}_{6}$

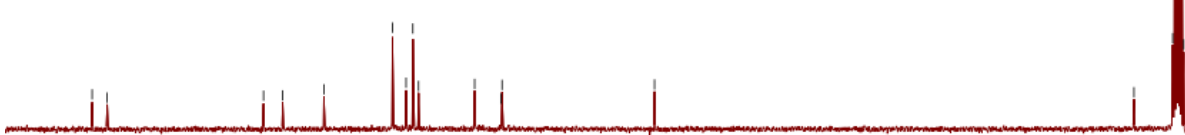

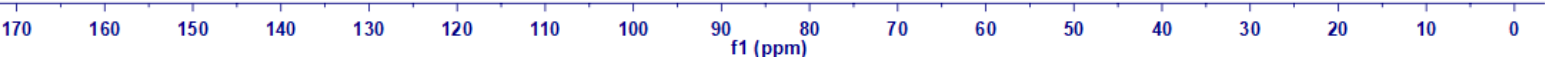


1-benzyl-4-hydroxy-6-methoxyquinolin-2(1H)-one (a13)

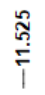

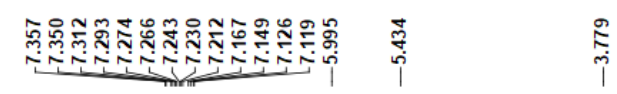

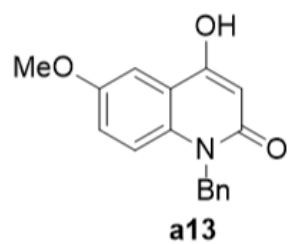

$400 \mathrm{MHz}$, DMSO-d

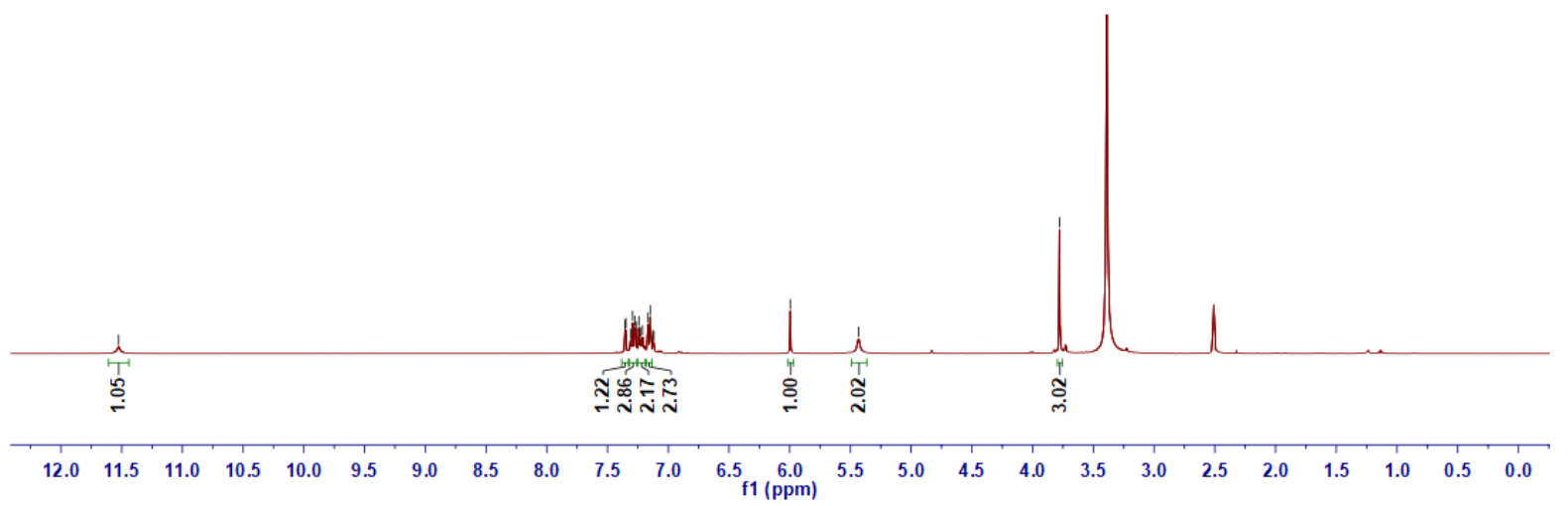

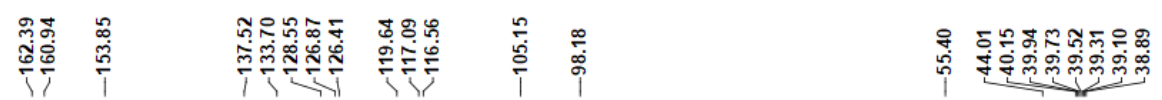

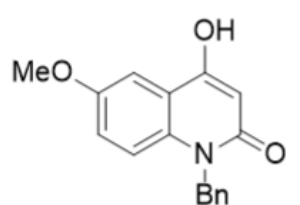

a13

$100 \mathrm{MHz}, \mathrm{DMSO}-\mathrm{d}_{6}$

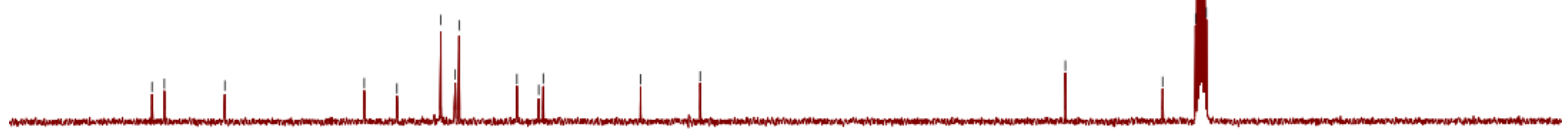

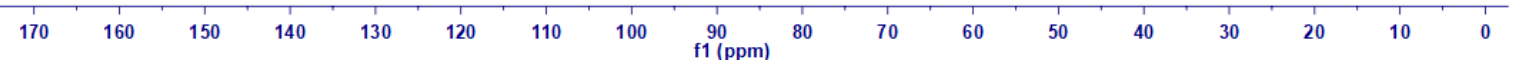


4-benzyl-1-hydroxybenzo[f]quinolin-3(4H)-one (a17)

$\stackrel{\stackrel{\circ}{\circ}}{\stackrel{\circ}{i}}$

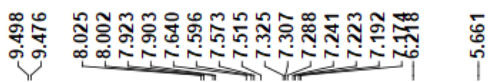

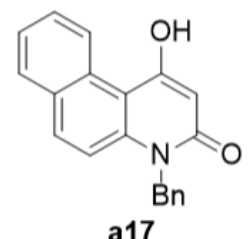

$400 \mathrm{MHz}, \mathrm{DMSO}-\mathrm{d}_{6}$

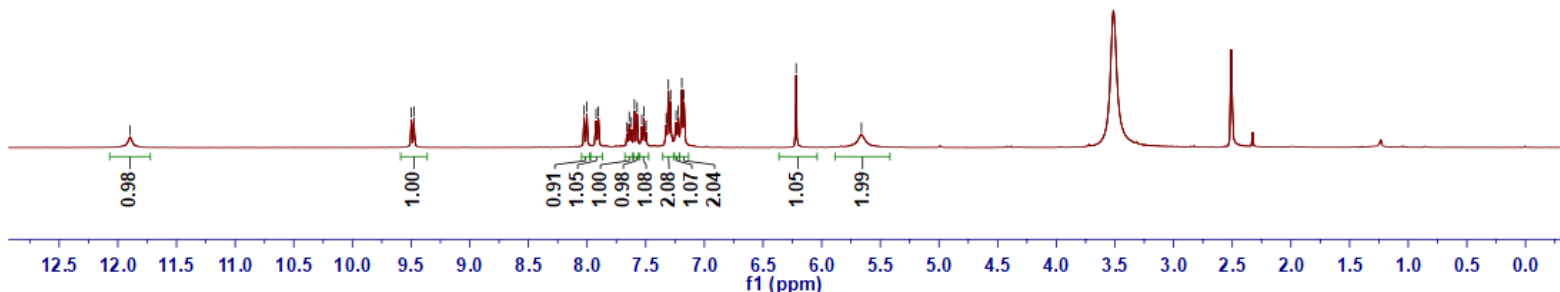

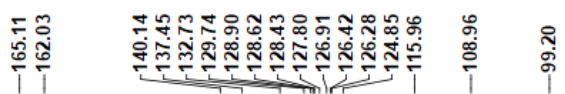

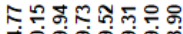

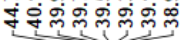

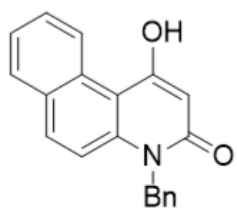

a17

$100 \mathrm{MHz}$, DMSO-d

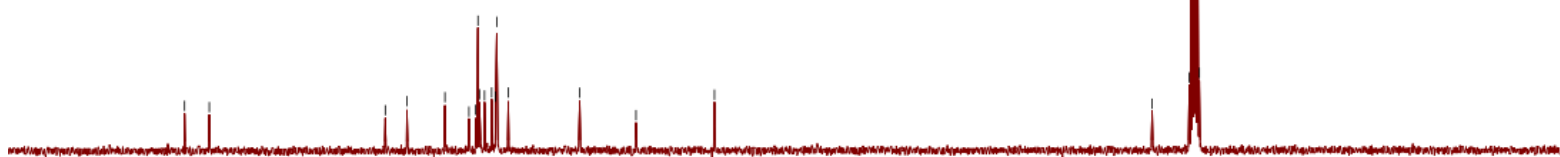

$\begin{array}{llllllllll}180 & 170 & 160 & 150 & 140 & 130 & 120 & 110 & 100 & \begin{array}{c}90 \\ \mathrm{f} 1(\mathrm{ppm})\end{array}\end{array}$ 
4-benzyl-1-hydroxy-4,7-phenanthrolin-3(4H)-one (a19)

$\stackrel{\sum}{\stackrel{i}{i}}$

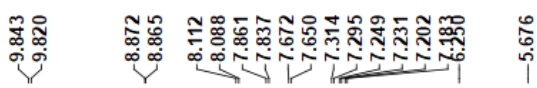

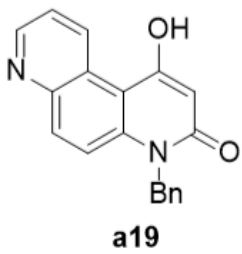

$400 \mathrm{MHz}$, DMSO-d 6

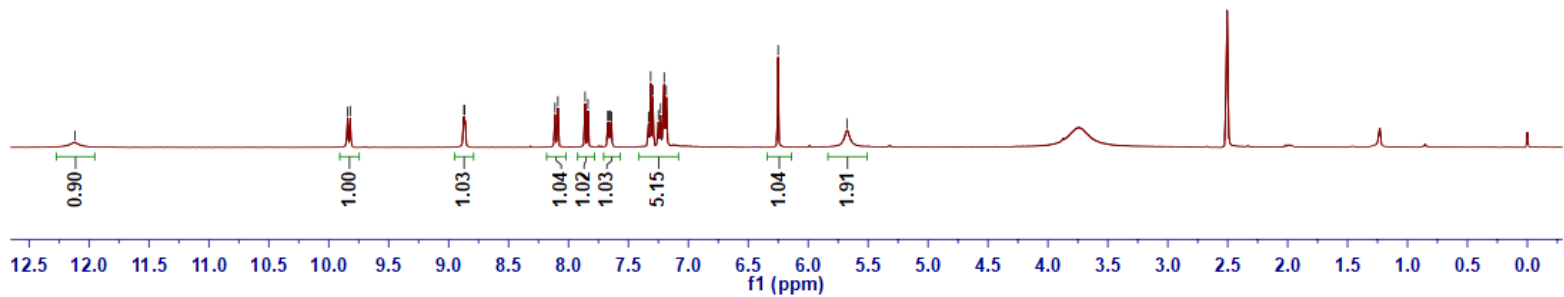

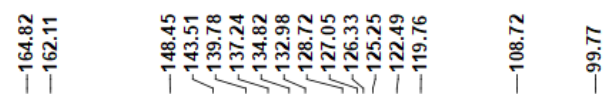

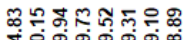

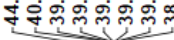

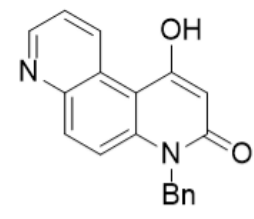

a19

$100 \mathrm{MHz}, \mathrm{DMSO}^{-\mathrm{d}_{6}}$

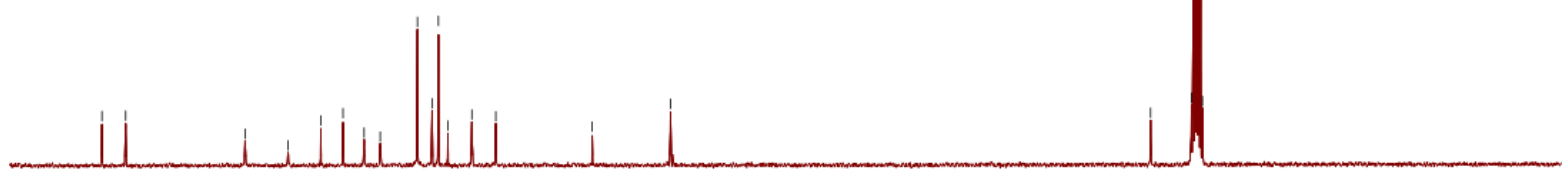

$\begin{array}{llllllllllllllllll}170 & 160 & 150 & 140 & 130 & 120 & 110 & 100 & 90 & 90 & 70 & 60 & 50 & 40 & 30 & 20 & 10 & 0\end{array}$ 
6-benzyl-2,2-dimethyl-2,6-dihydro-5H-pyrano[3,2-c]quinolin-5-one (6b)
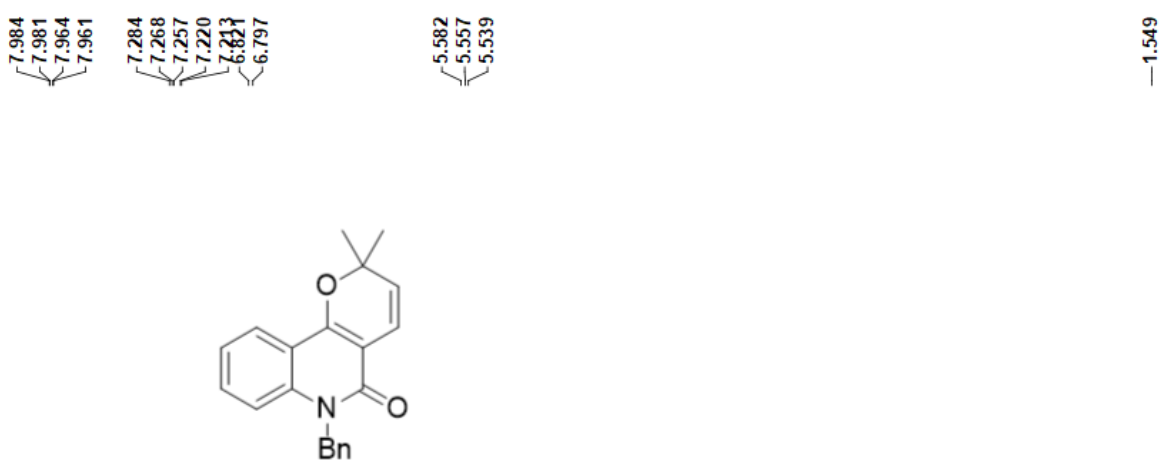

$6 \mathrm{~b}$

$400 \mathrm{MHz} C D C l_{3}$

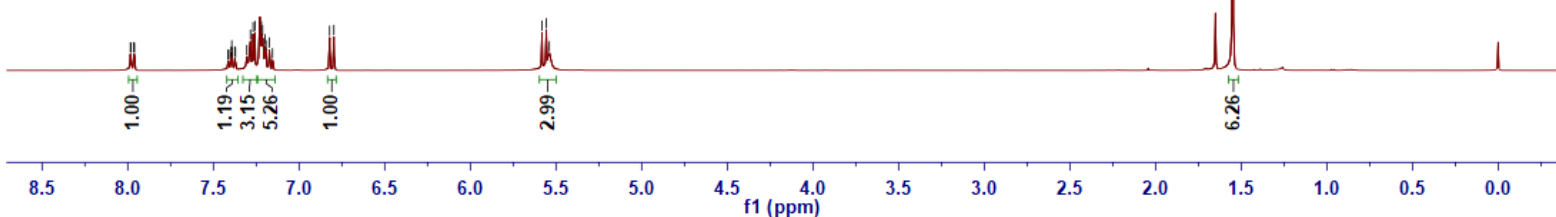

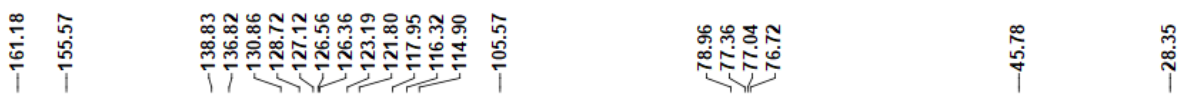

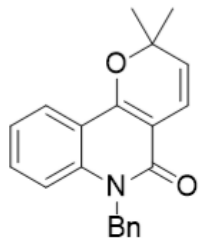

$6 b$

$100 \mathrm{MHz} C D C l_{3}$

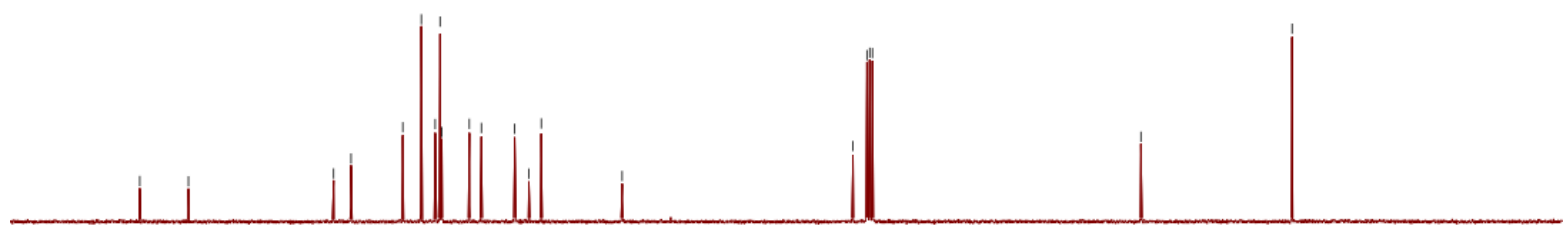

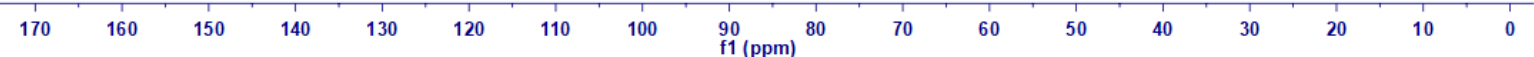


2,2-dimethyl-6-phenyl-2,6-dihydro-5H-pyrano[3,2-c]quinolin-5-one (6c)

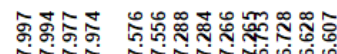 \\ 近}

เกํำ

$\stackrel{5}{i}$

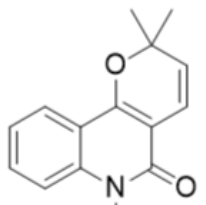

$\mathrm{Ph}$

6c

$400 \mathrm{MHz} \mathrm{CDCl}_{3}$

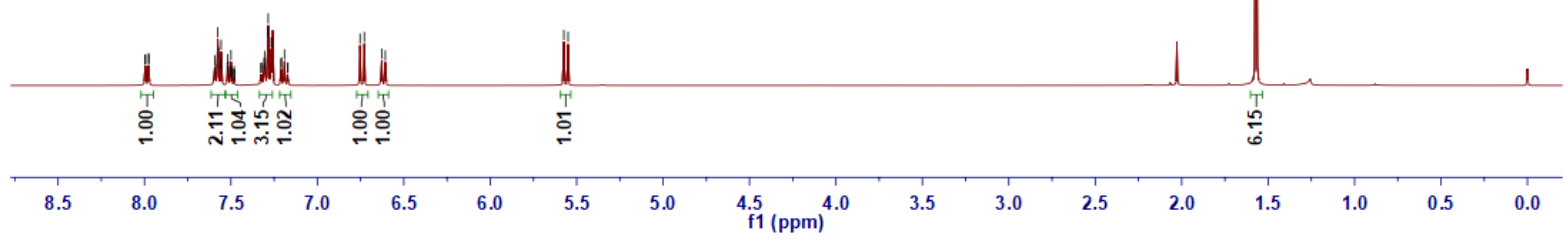

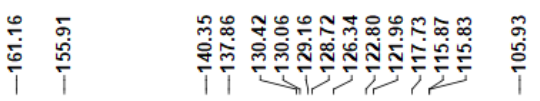

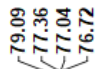

$\stackrel{\substack{\infty \\ \text { ஸे }}}{1}$

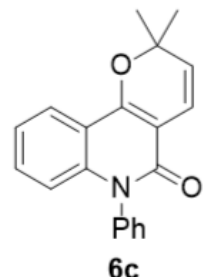

$100 \mathrm{MHz} \mathrm{CDCl}_{3}$

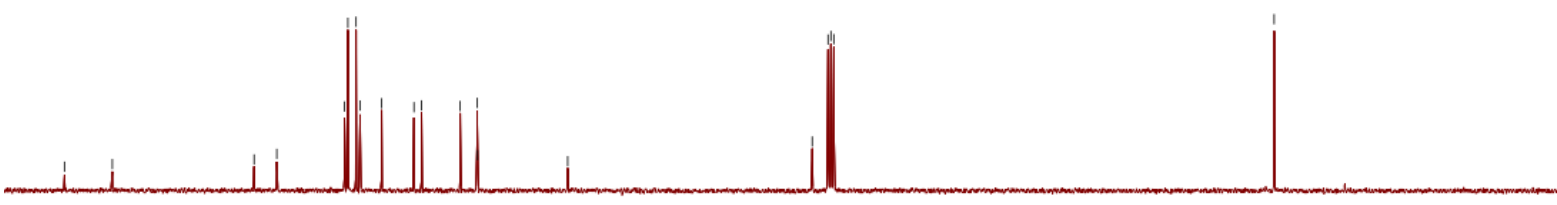

$\begin{array}{rrrrrrrr}160 & 150 & 140 & 130 & 120 & 110 & 100 & 90 \begin{array}{r}80 \\ \mathrm{f} 1(\mathrm{ppm})\end{array}\end{array}$ 
6-benzyl-9-fluoro-2,2-dimethyl-2,6-dihydro-5H-pyrano[3,2-c]quinolin-5-one (6d)

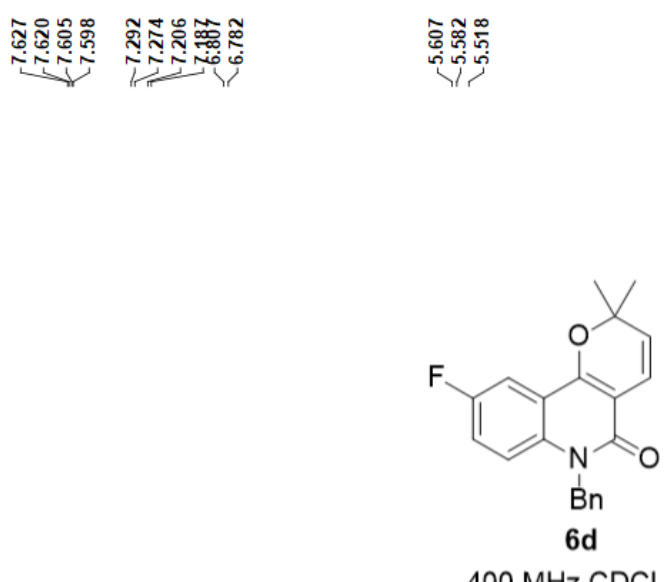

$400 \mathrm{MHz} \mathrm{CDCl}_{3}$
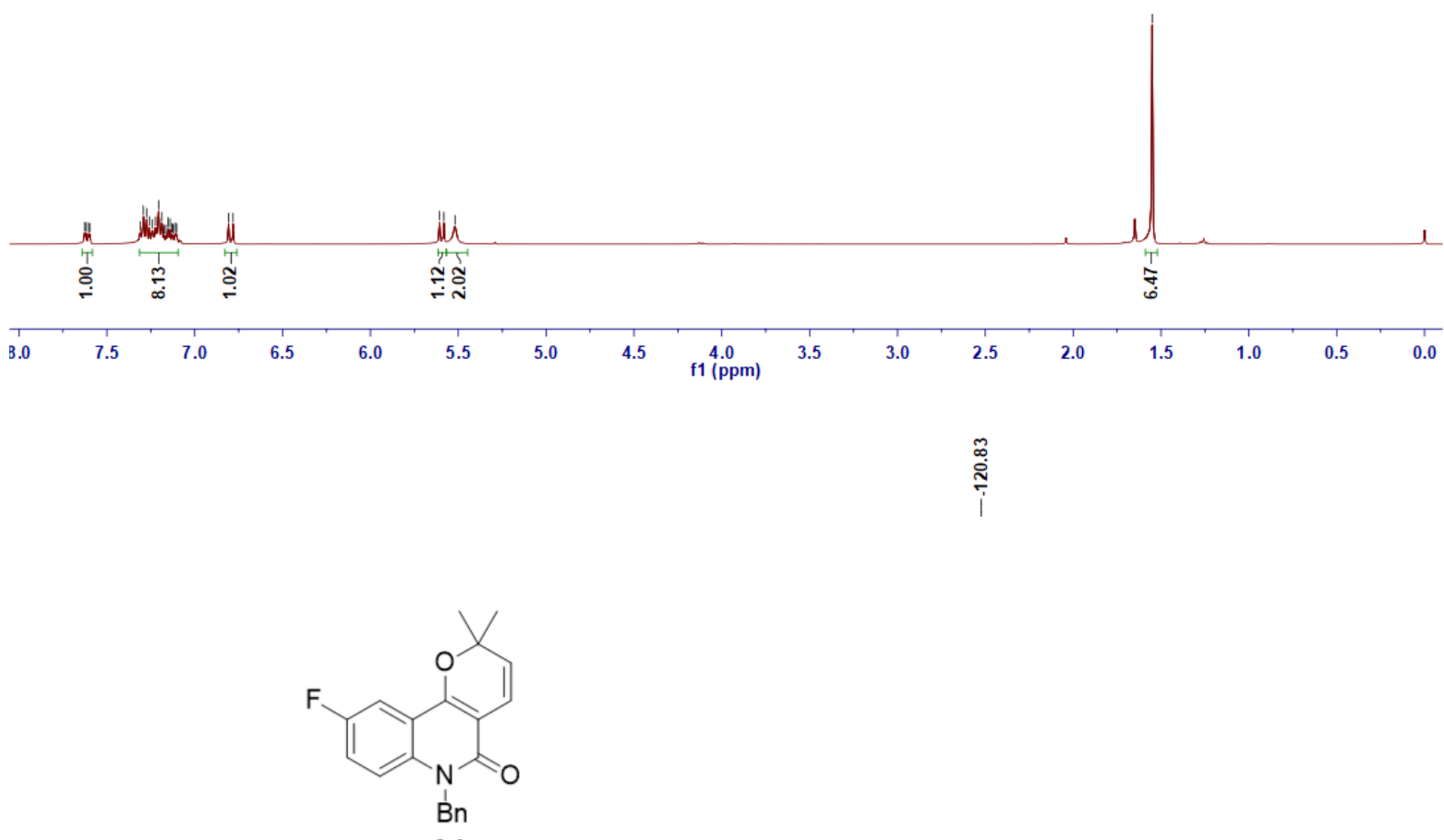

6d

$376 \mathrm{MHz} \mathrm{CDCl}_{3}$

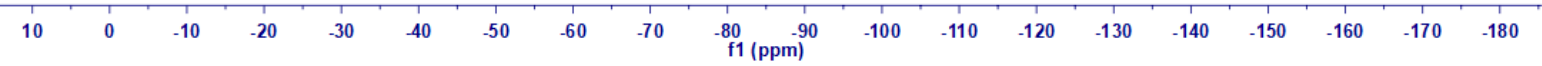




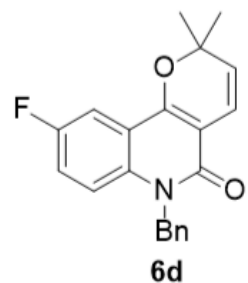

$100 \mathrm{MHz} \mathrm{CDCl}_{3}$

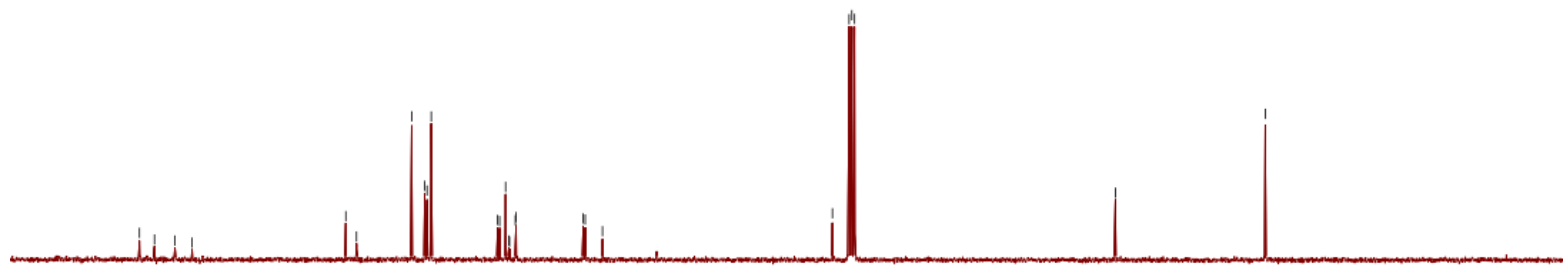

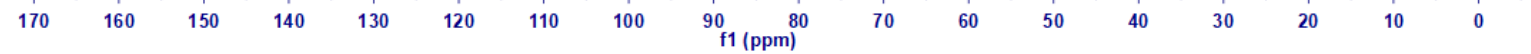

6-benzyl-9-chloro-2,2-dimethyl-2,6-dihydro-5H-pyrano[3,2-c]quinolin-5-one (6e)

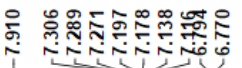

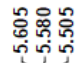

莡

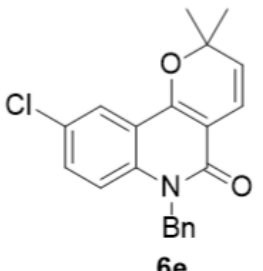

$400 \mathrm{MHz} \mathrm{CDCl}_{3}$

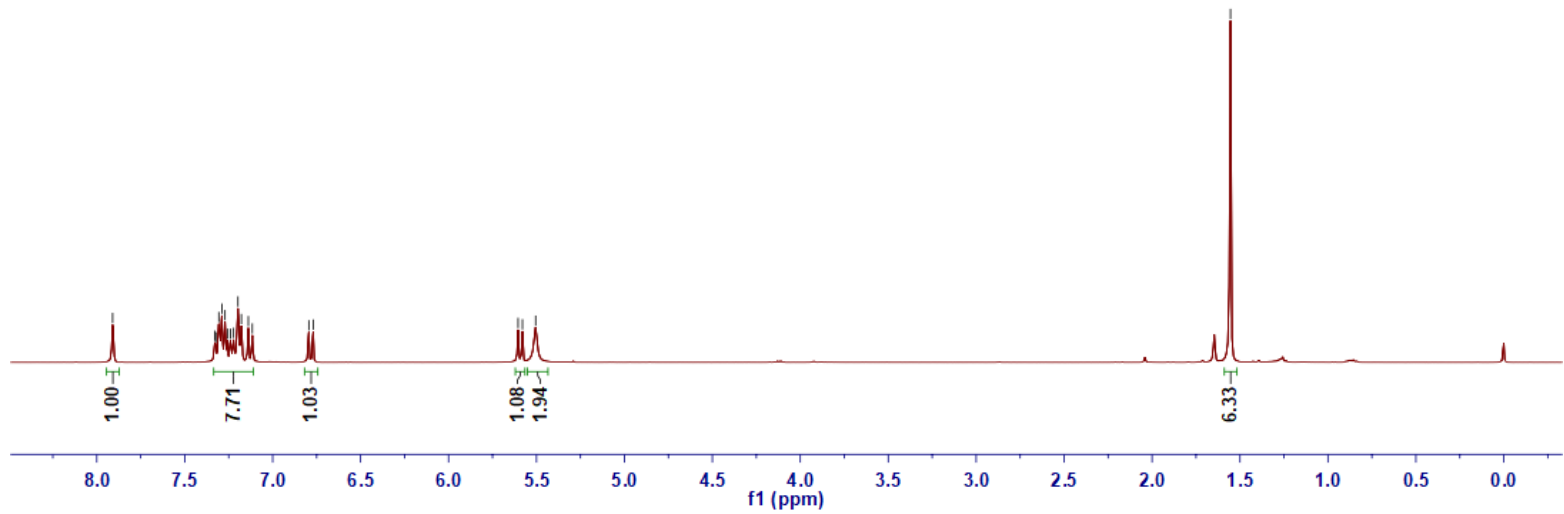

72 / 146 

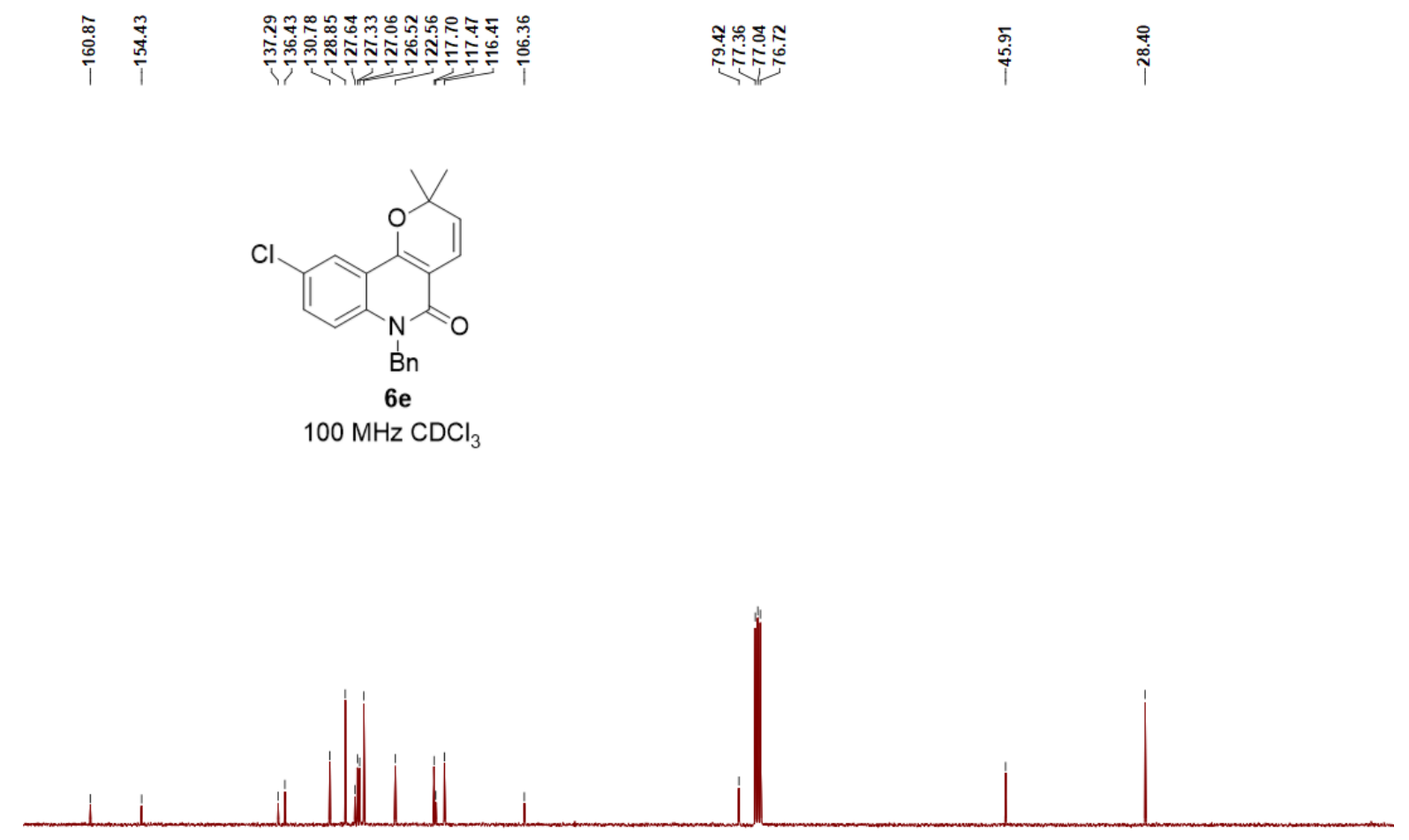

\begin{tabular}{|c|c|c|c|c|c|c|c|c|c|c|c|c|c|c|c|c|}
\hline 160 & 150 & 140 & 130 & 120 & 110 & 100 & & $\begin{array}{r}80 \\
\mathrm{f} 1(\mathrm{ppm})\end{array}$ & 70 & 60 & 50 & 40 & 30 & 20 & 10 & 0 \\
\hline
\end{tabular}

6-benzyl-9-bromo-2,2-dimethyl-2,6-dihydro-5H-pyrano[3,2-c]quinolin-5-one (6f)

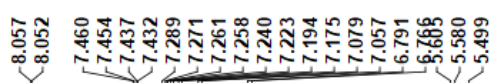<smiles>CC1(C)C=Cc2c(c3cc(Br)ccc3n(Cc3ccccc3Br)c2=O)O1</smiles>

$400 \mathrm{MHz} \mathrm{CDCl}_{3}$

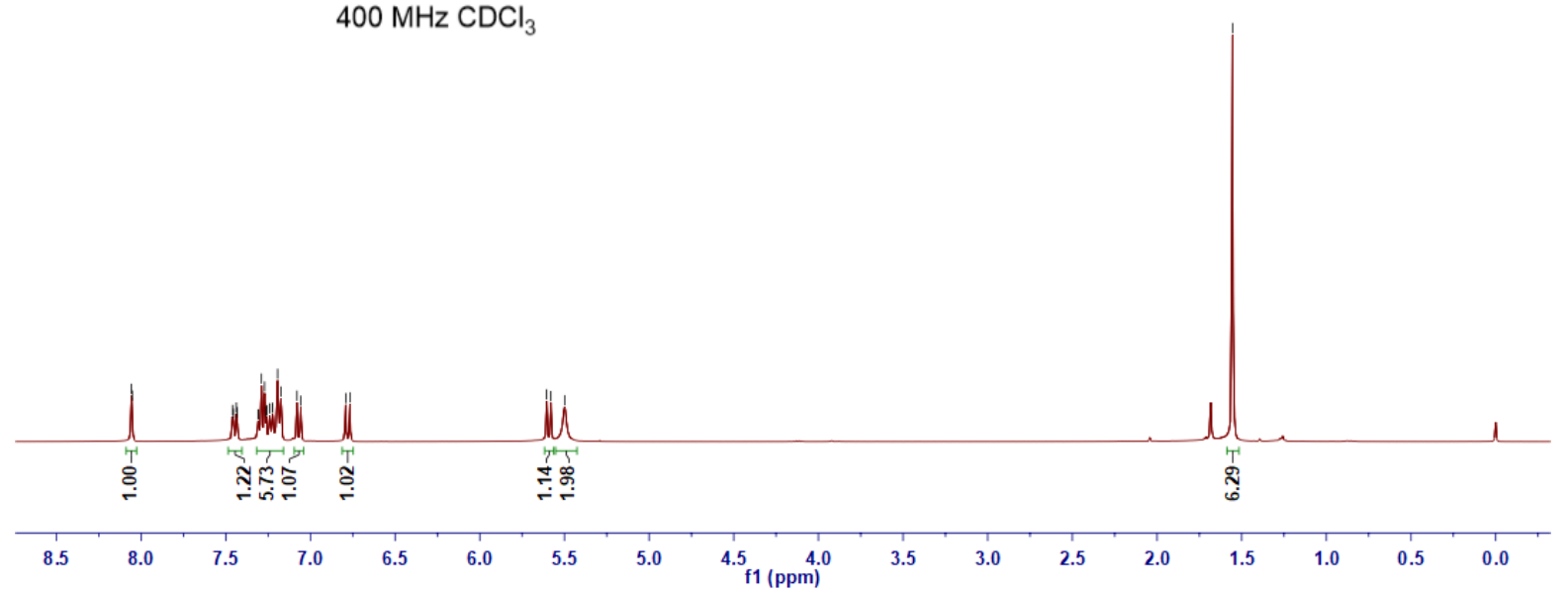




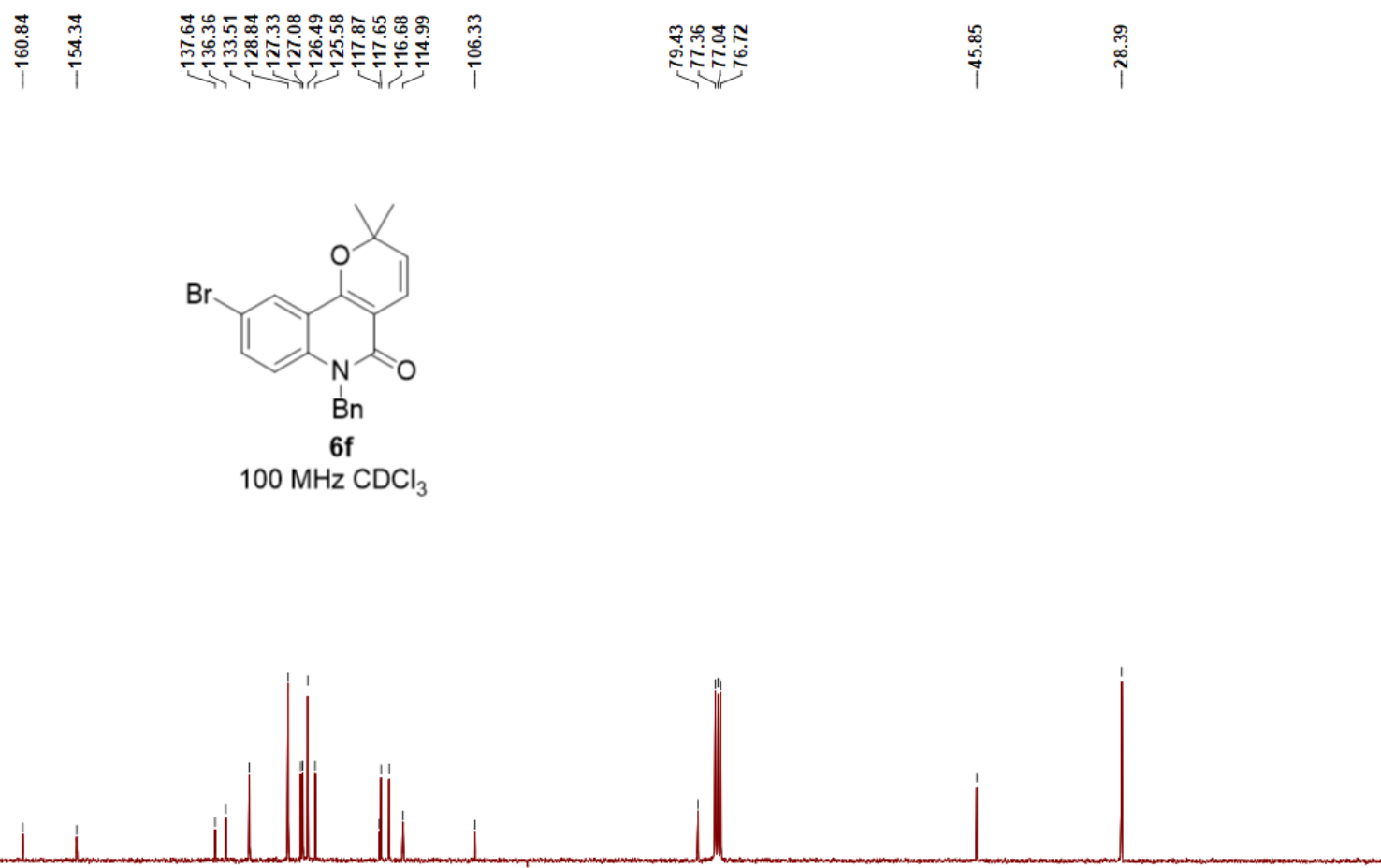

\begin{tabular}{|c|c|c|c|c|c|c|c|c|c|c|c|c|c|c|c|}
\hline 170 & 160 & 150 & 140 & 130 & 120 & 110 & 100 & $\begin{array}{c}90 \\
\mathrm{f} 1(\mathrm{ppm})\end{array}$ & 70 & 60 & 50 & 40 & 30 & 20 & 10 \\
\hline
\end{tabular}

6-benzyl-2,2-dimethyl-9-(trifluoromethyl)-2,6-dihydro-5H-pyrano[3,2-c]quinolin-5-one (6g)

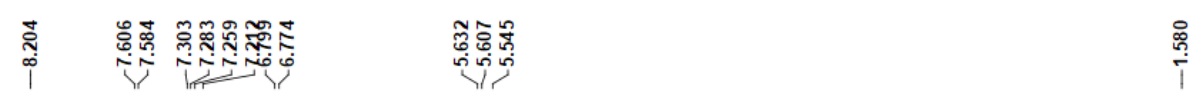<smiles>CC1(C)C=Cc2c(c3cc(C(F)(F)F)ccc3n(Cc3ccccc3)c2=O)O1</smiles>

$6 \mathrm{~g}$

$400 \mathrm{MHz} \mathrm{CDCl}_{3}$

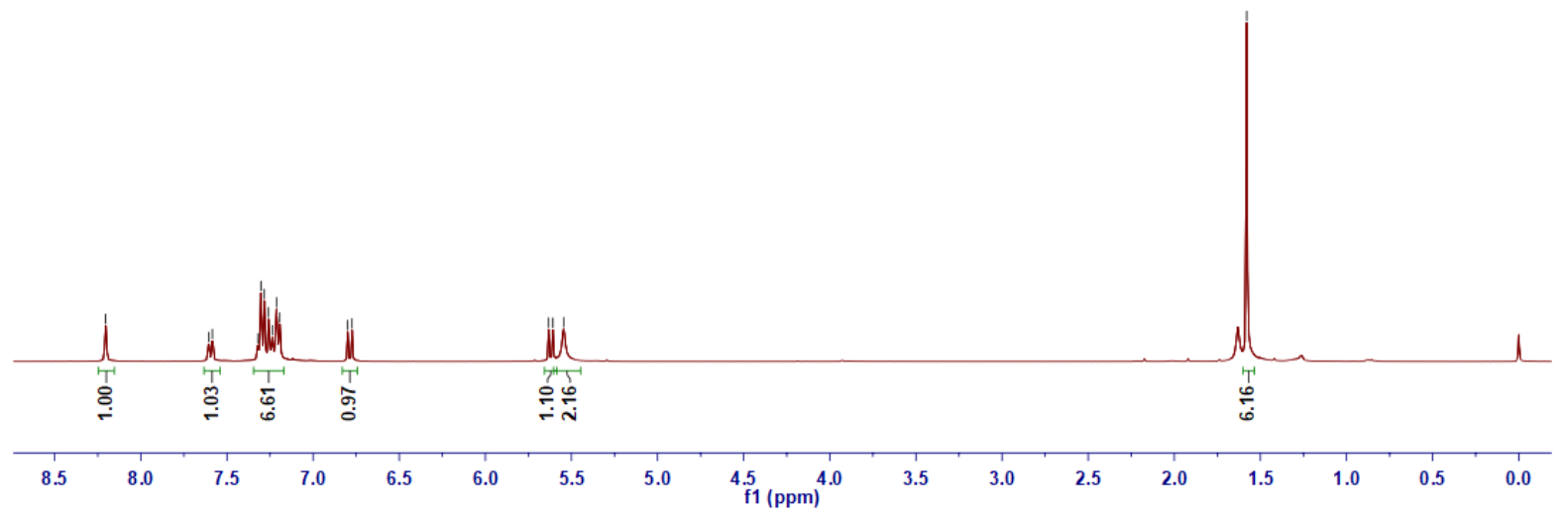



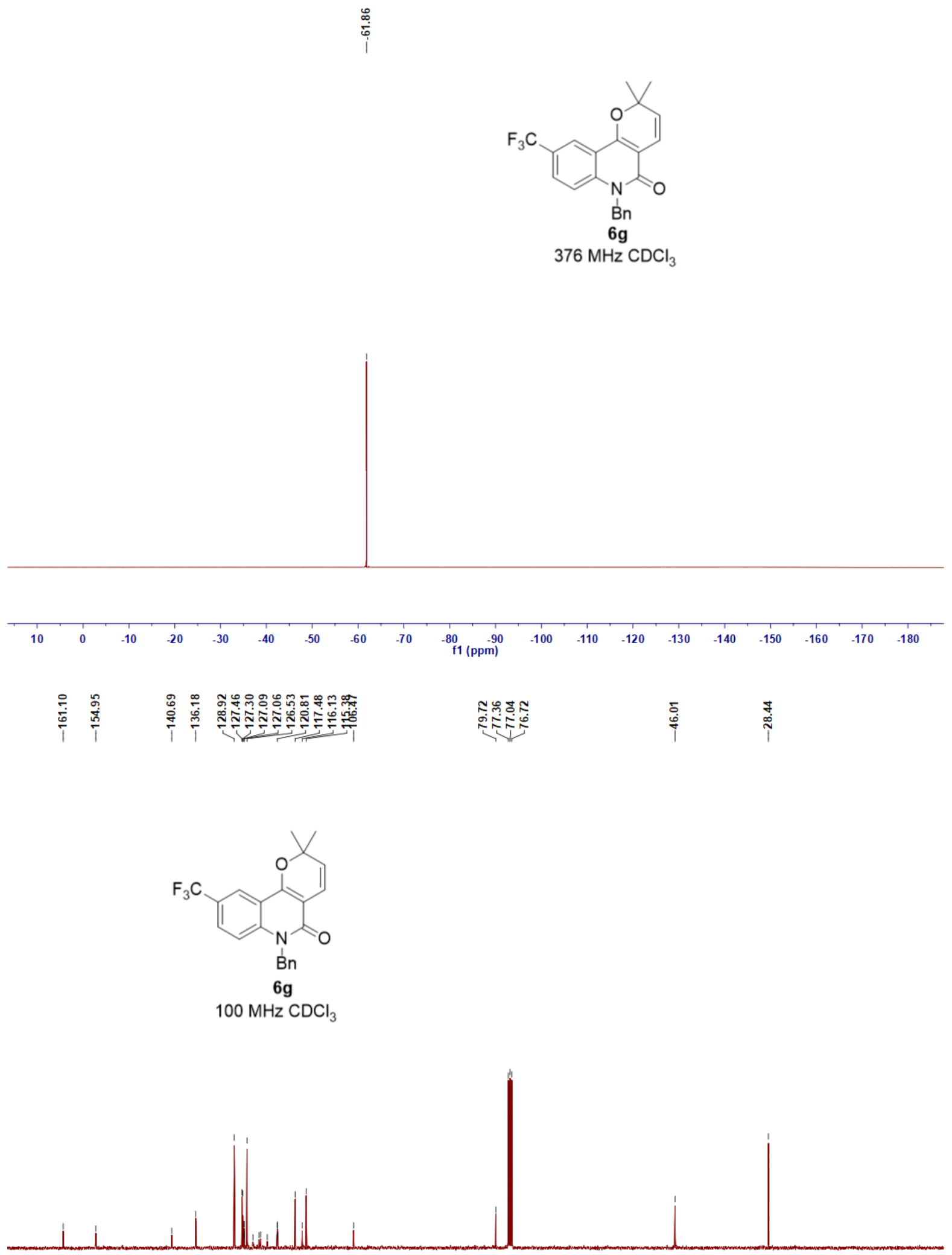

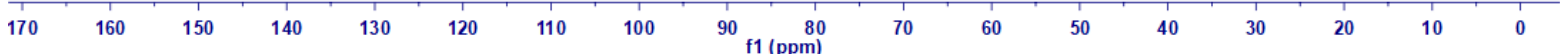


6-benzyl-2,2-dimethyl-9-nitro-2,6-dihydro-5H-pyrano[3,2-c]quinolin-5-one (6h)

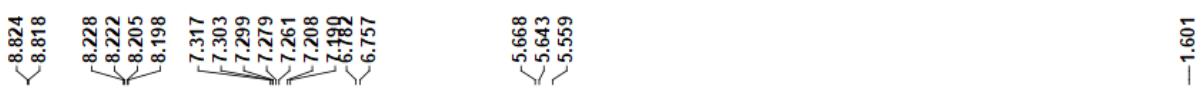

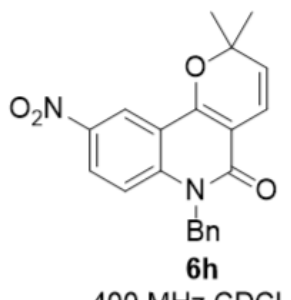

$400 \mathrm{MHz} \mathrm{CDCl}_{3}$

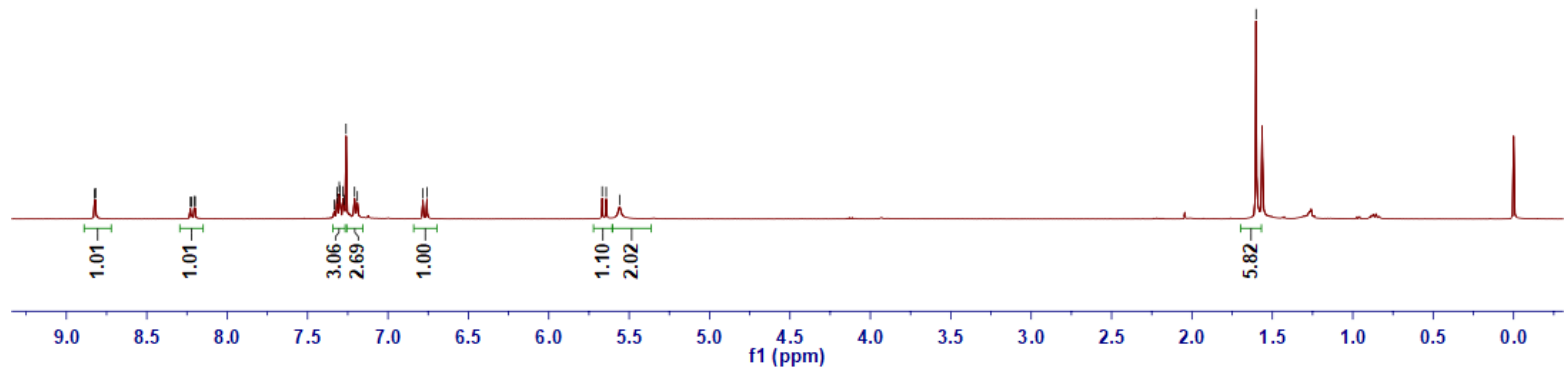

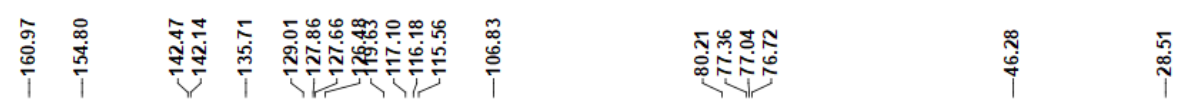

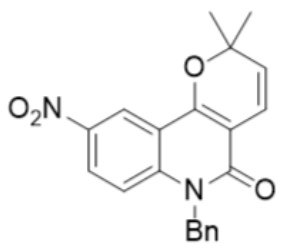

$6 \mathrm{~h}$

$100 \mathrm{MHz} \mathrm{CDCl}_{3}$

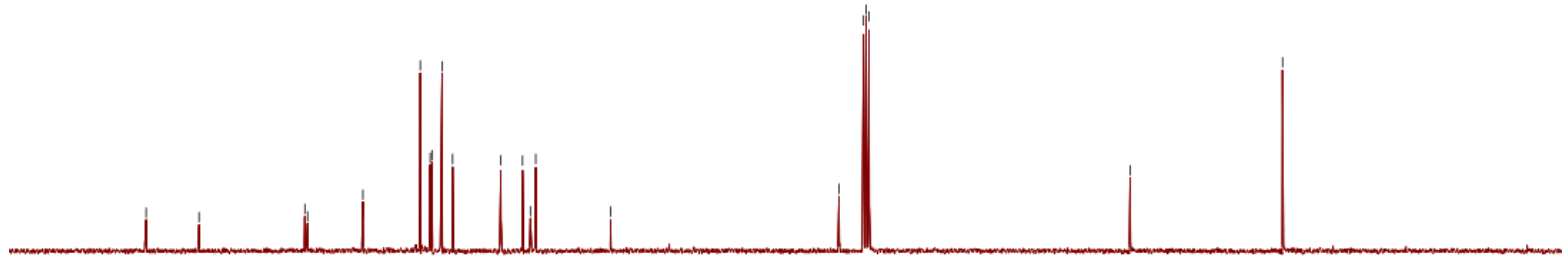

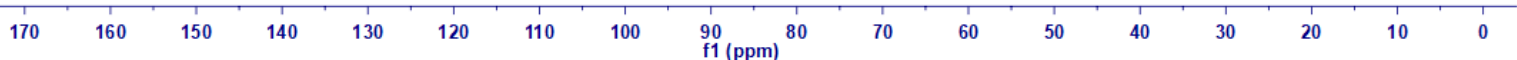


6-benzyl-2,2,9-trimethyl-2,6-dihydro-5H-pyrano[3,2-c]quinolin-5-one (6i)

芦袋

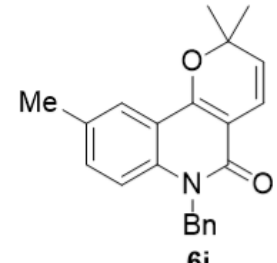

$400 \mathrm{MHz} \mathrm{CDCl}_{3}$

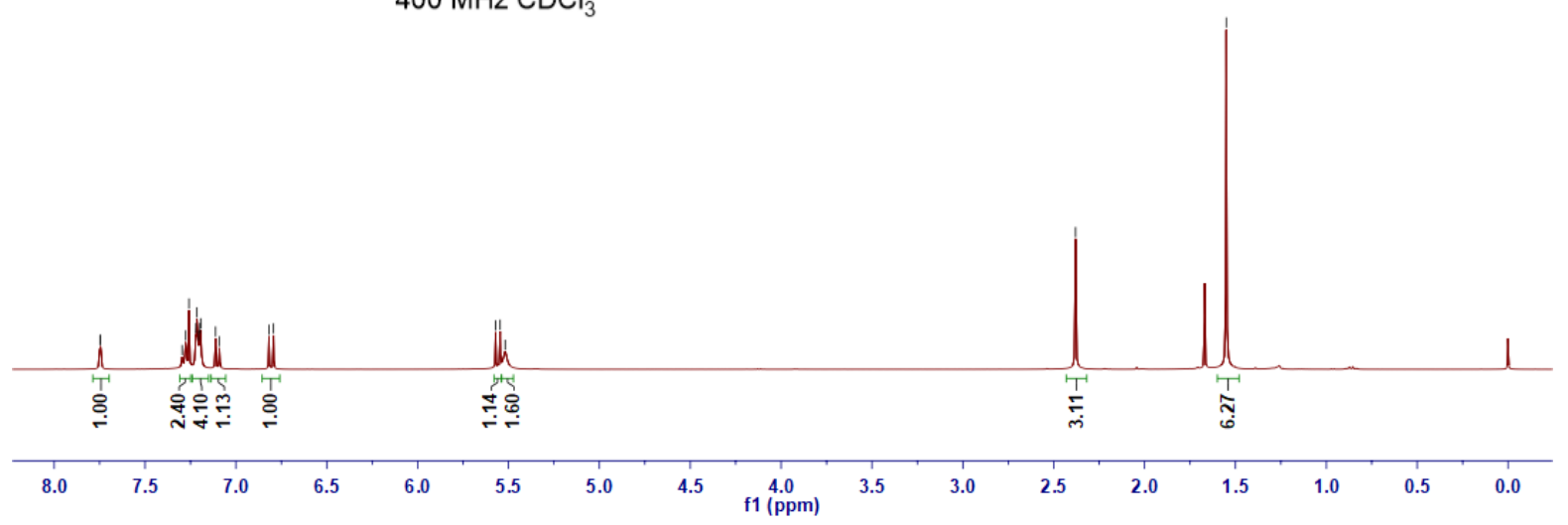

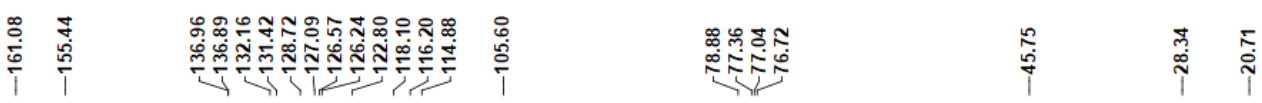
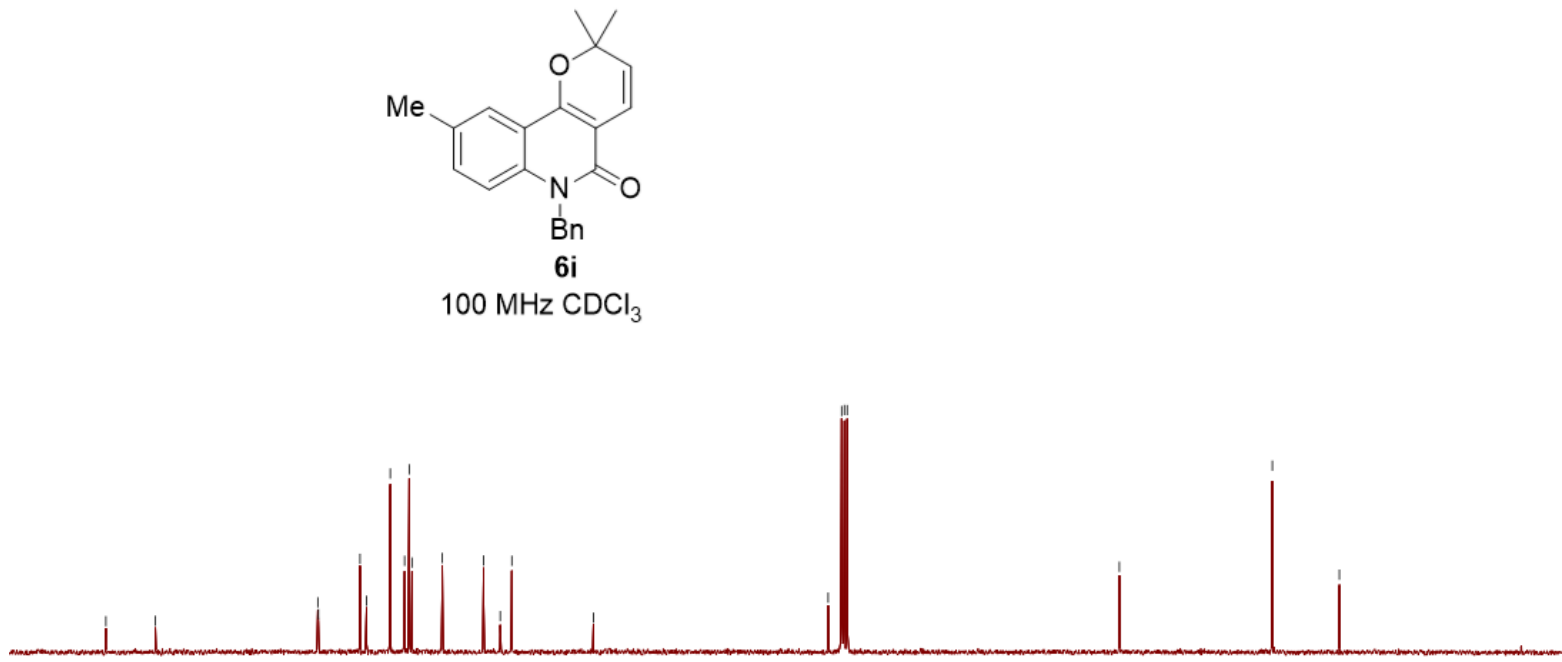

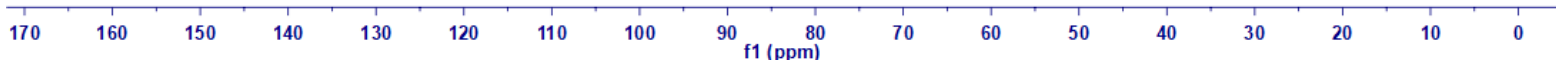


6-benzyl-9-isopropyl-2,2-dimethyl-2,6-dihydro-5H-pyrano[3,2-c]quinolin-5-one (6j)
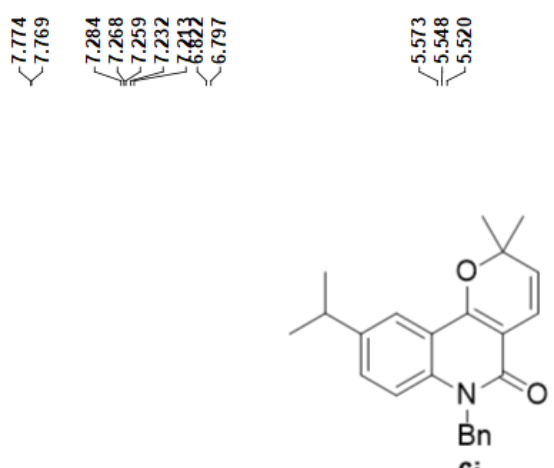

$400 \mathrm{MHz} \mathrm{CDCl}_{3}$

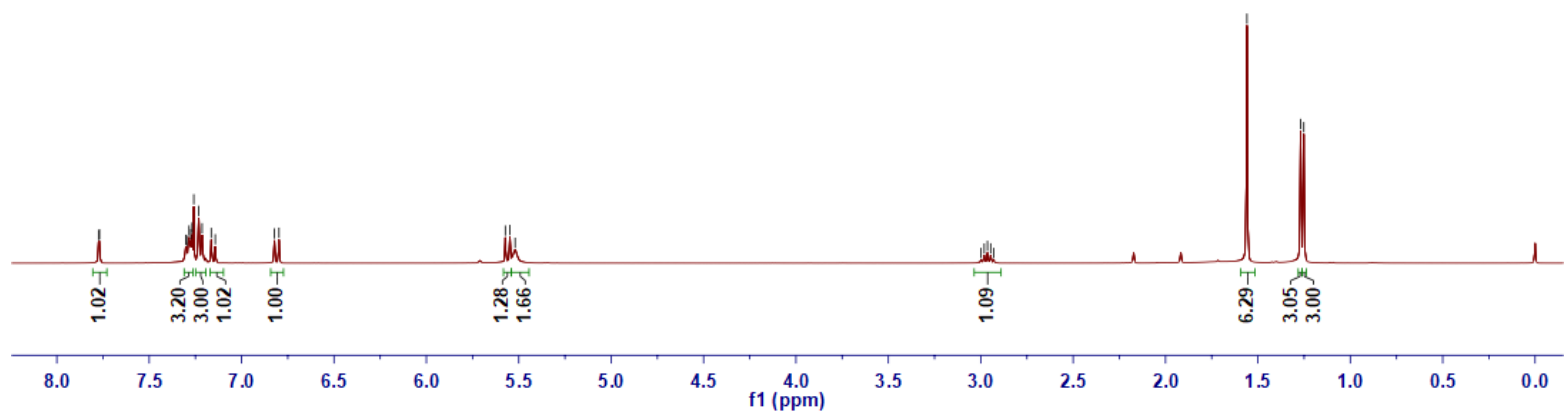

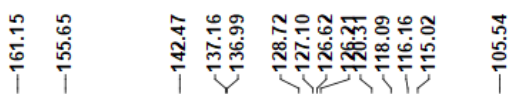

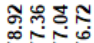

永篎

ஊั
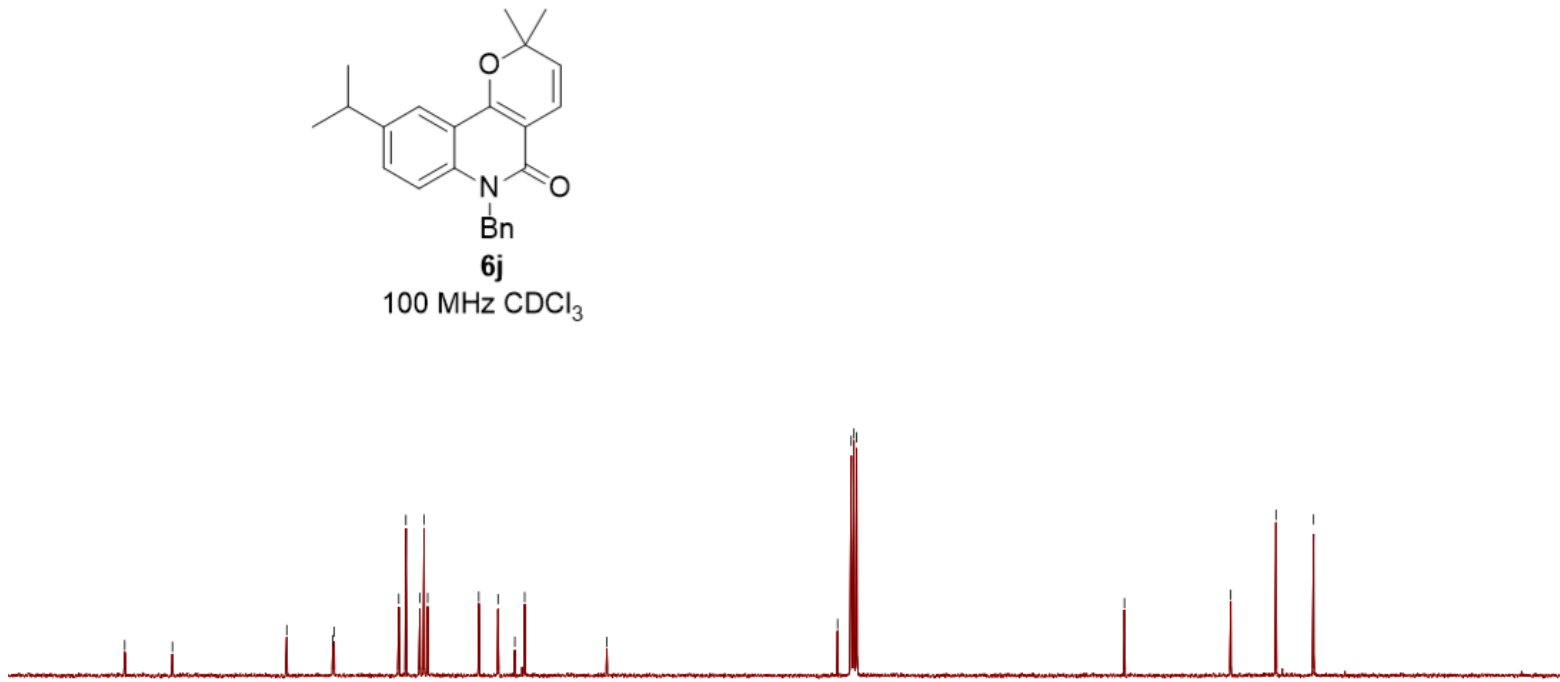

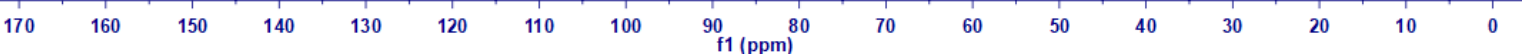


6-benzyl-9-(tert-butyl)-2,2-dimethyl-2,6-dihydro-5H-pyrano[3,2-c]quinolin-5-one (6k)
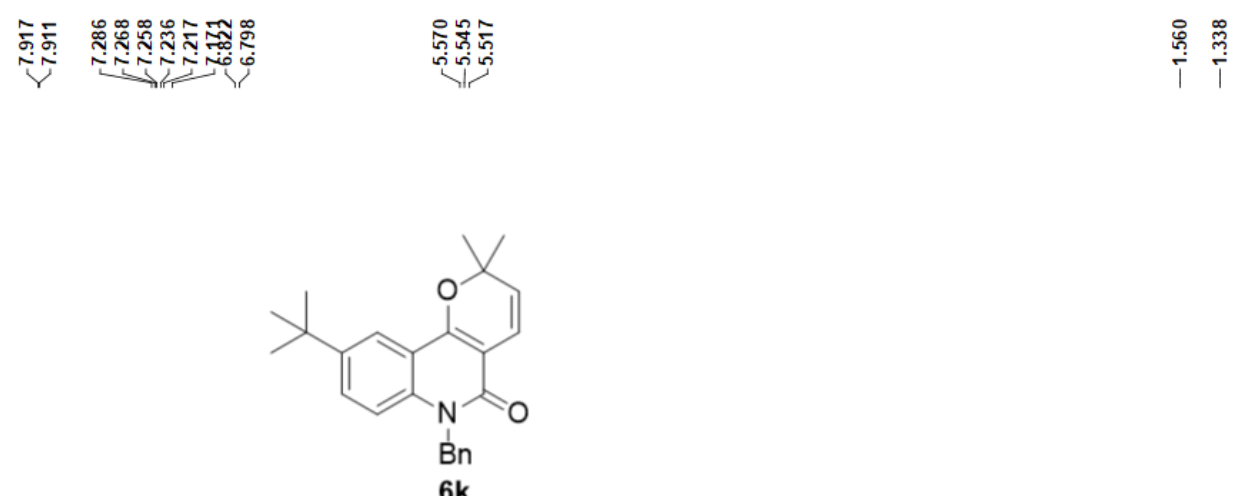

$400 \mathrm{MHz} \mathrm{CDCl}_{3}$

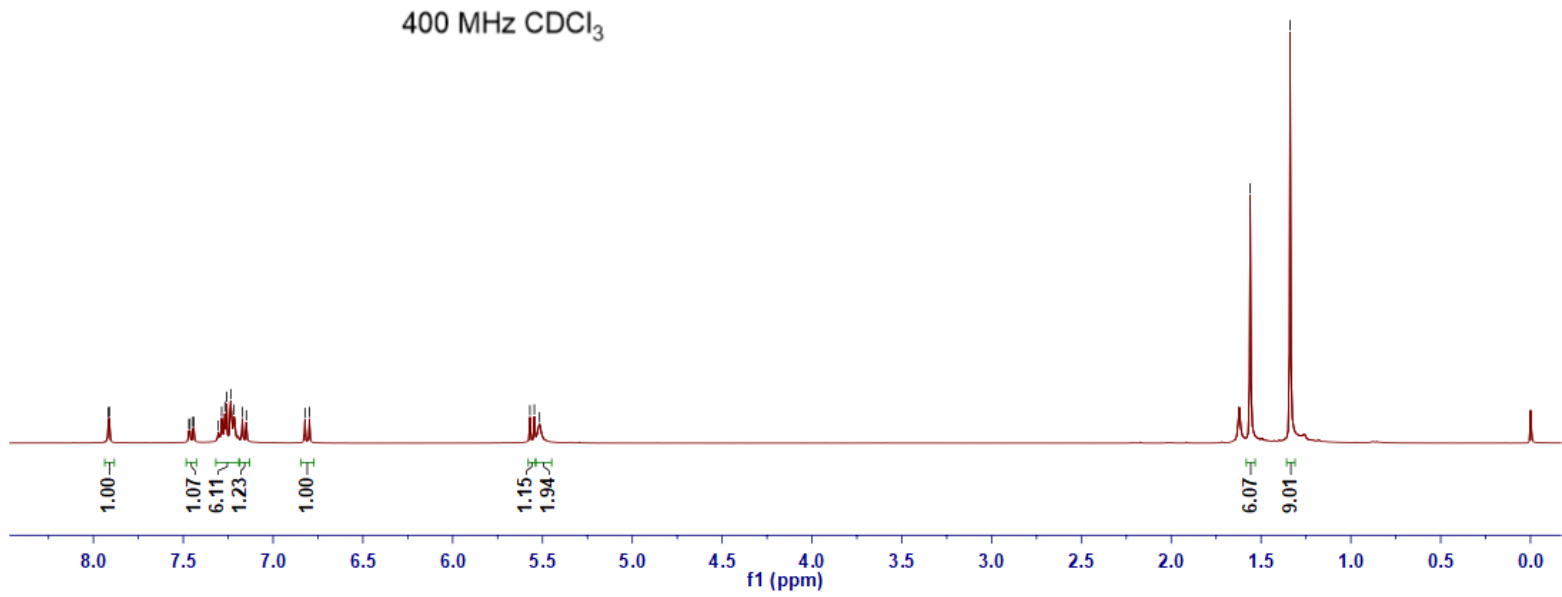

至

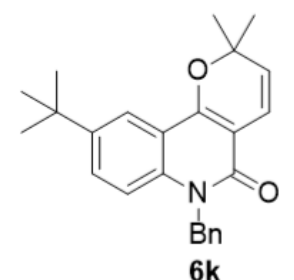

$100 \mathrm{MHz} \mathrm{CDCl}_{3}$

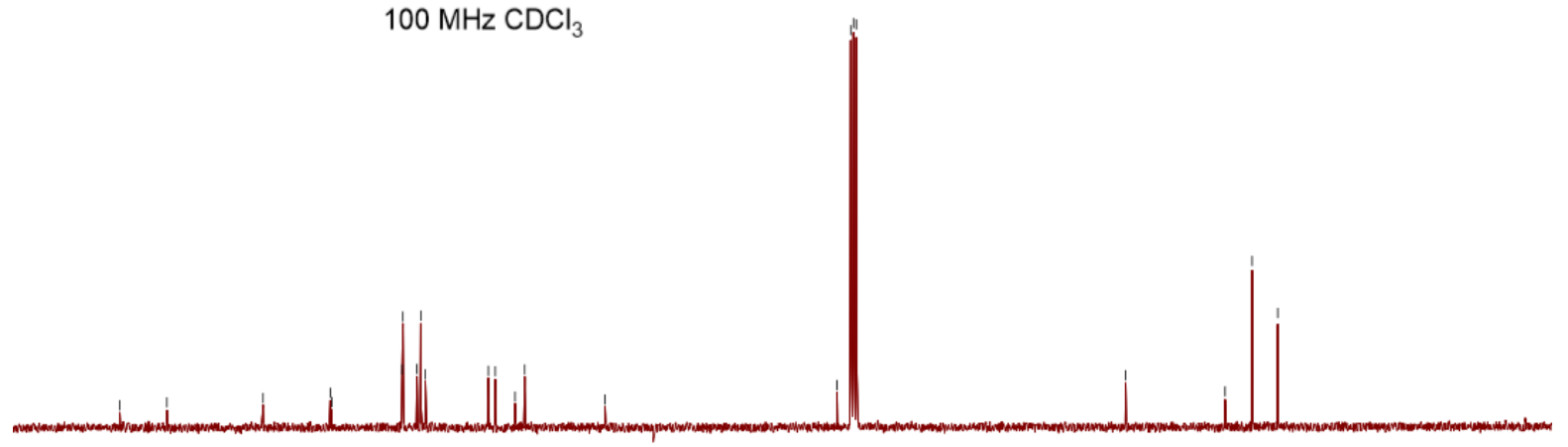

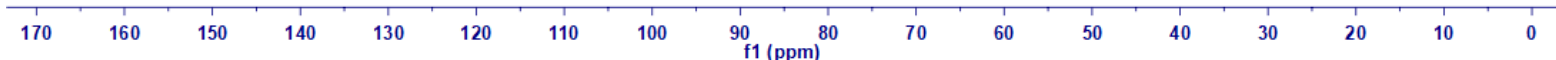


6-benzyl-2,2-dimethyl-9-phenyl-2,6-dihydro-5H-pyrano[3,2-c]quinolin-5-one (6l)

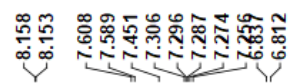

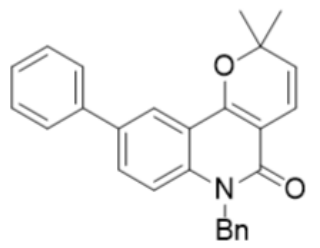

6I

$400 \mathrm{MHz} \mathrm{CDCl}_{3}$

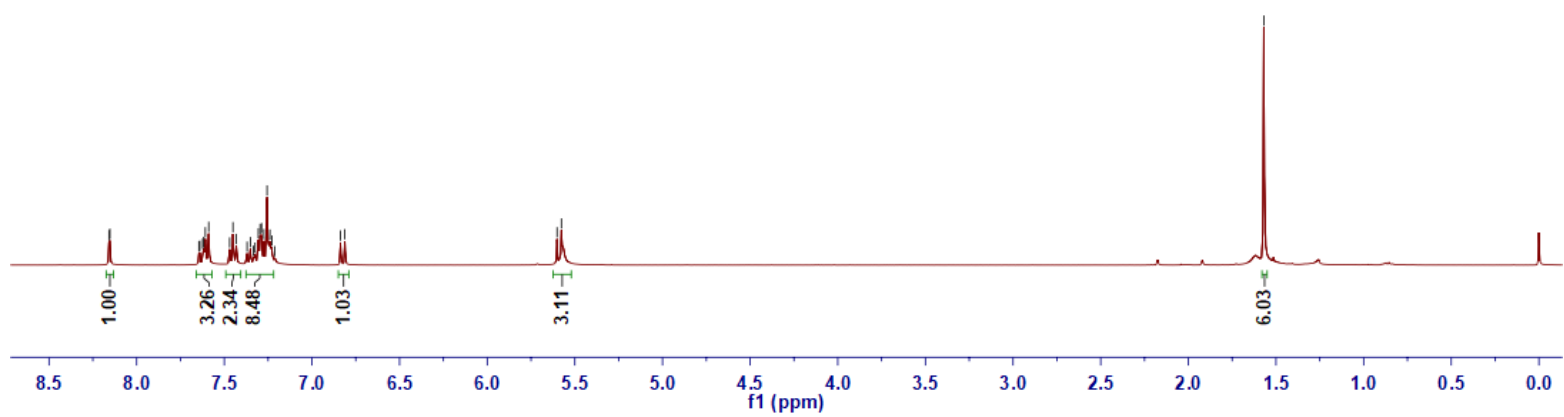

†

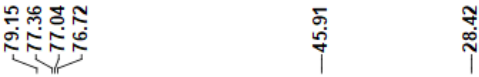

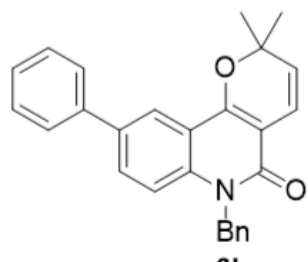

$100 \mathrm{MHz} C D C l_{3}$

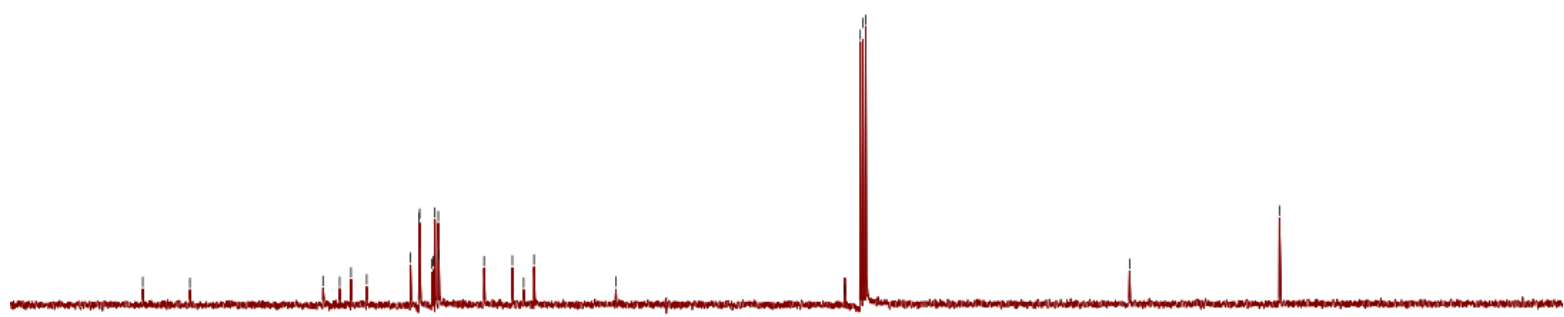

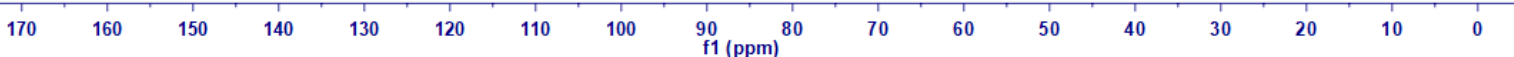


6-benzyl-9-methoxy-2,2-dimethyl-2,6-dihydro-5H-pyrano[3,2-c]quinolin-5-one (6m)

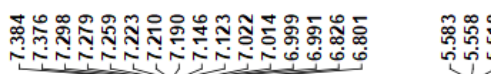
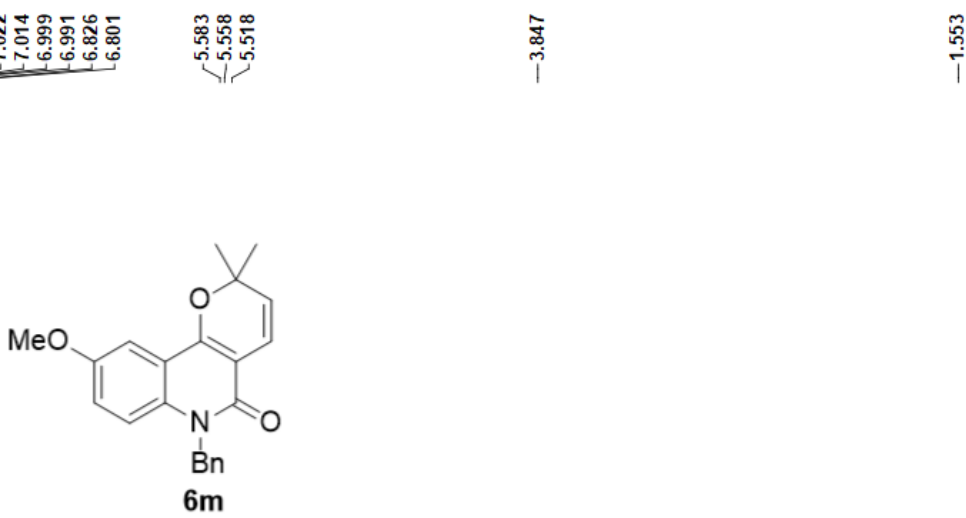

$400 \mathrm{MHz} \mathrm{CDCl}_{3}$

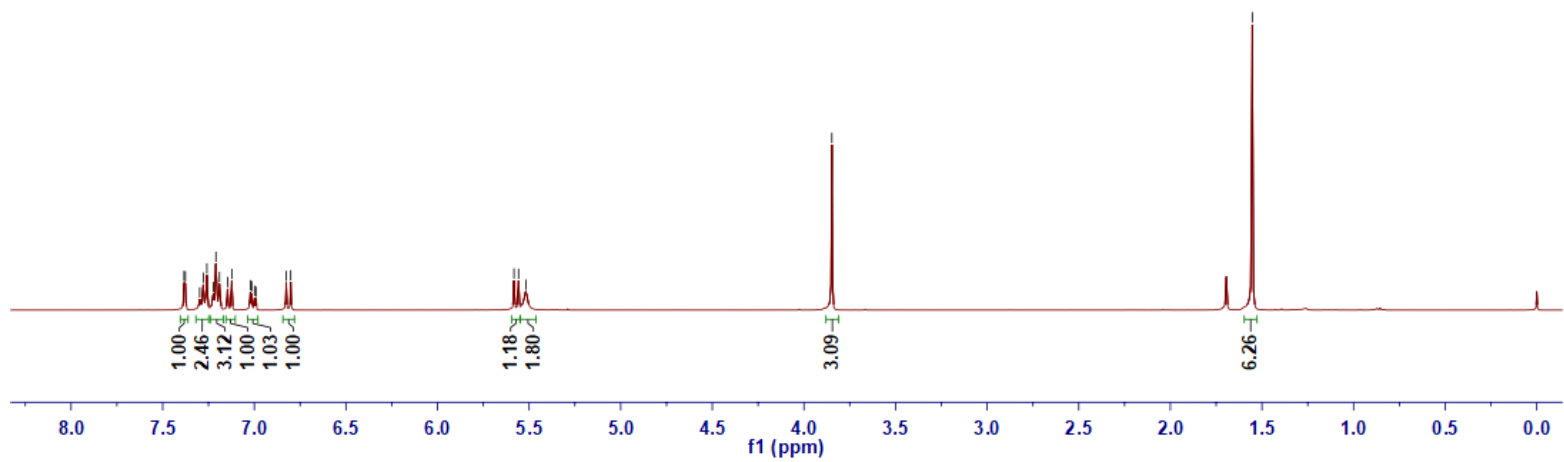

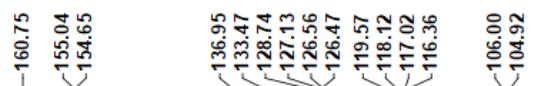

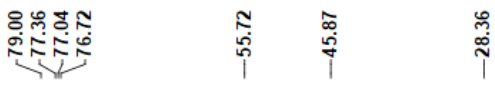

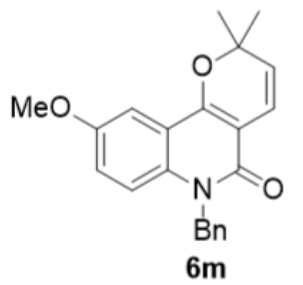

$100 \mathrm{MHz} \mathrm{CDCl}_{3}$

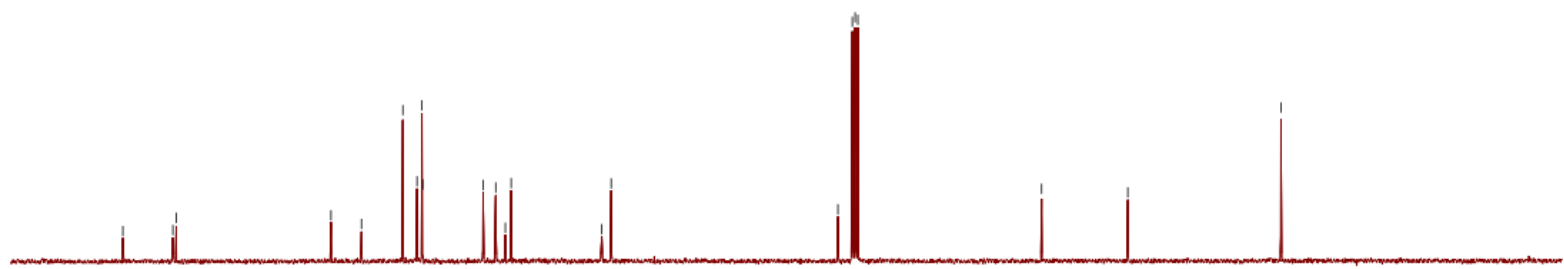

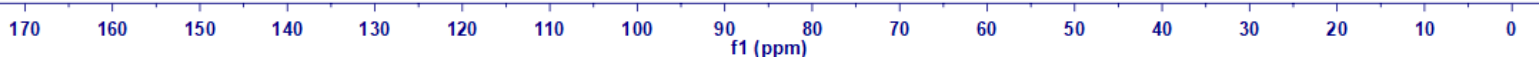


6-benzyl-8,9-dimethoxy-2,2-dimethyl-2,6-dihydro-5H-pyrano[3,2-c]quinolin-5-one (6o)

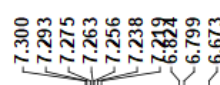

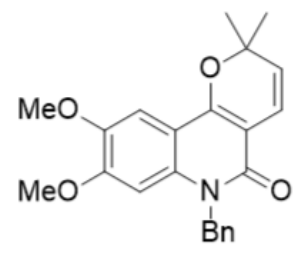

$400 \mathrm{MHz} \mathrm{CDCl}_{3}$

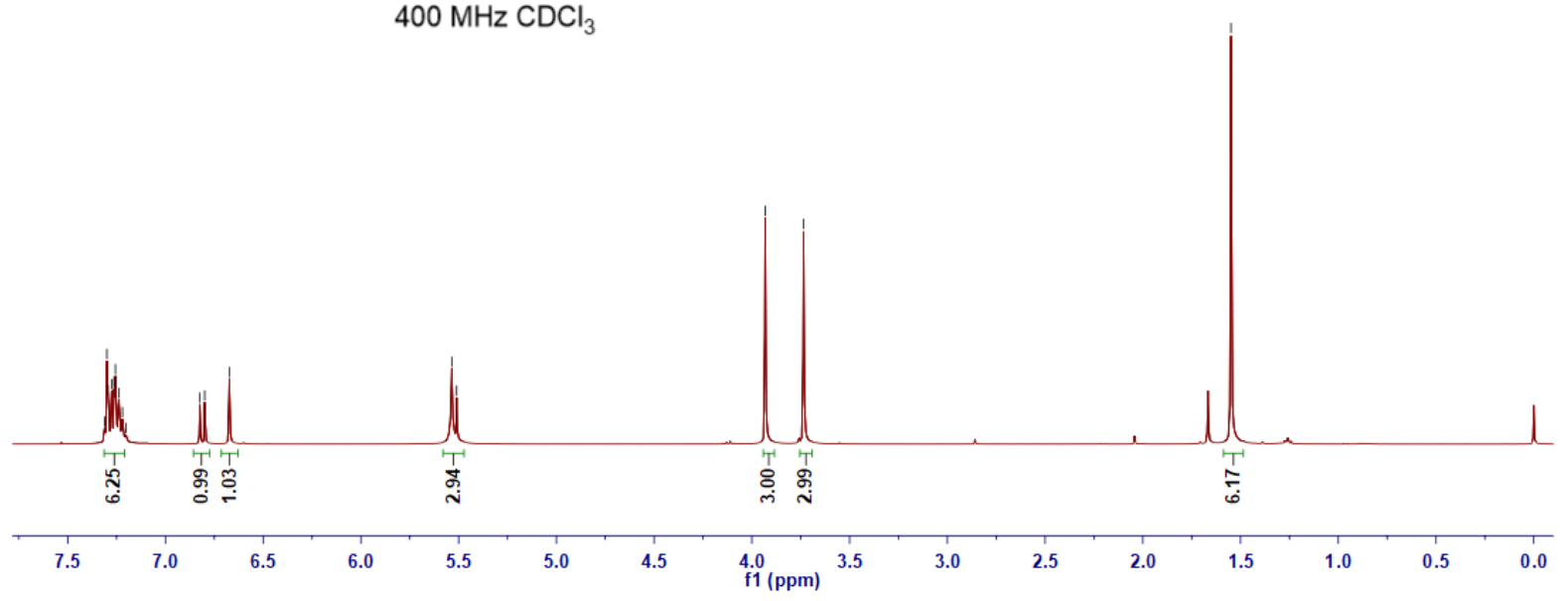

†

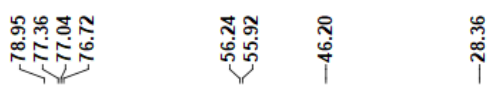
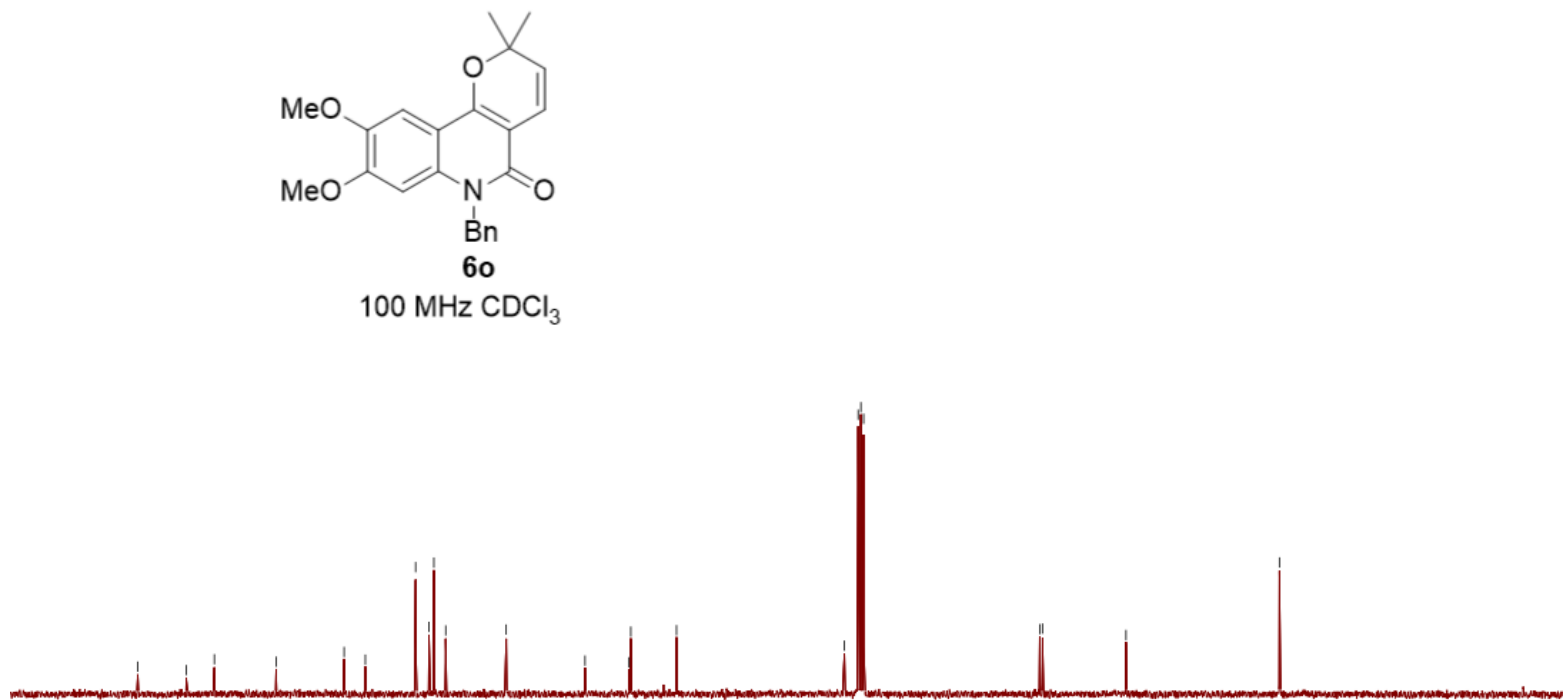

$\begin{array}{lllllllll}170 & 160 & 150 & 140 & 130 & 120 & 110 & 100 & 90 \\ \mathrm{f} 1(\mathrm{ppm})\end{array}$ 
3,3,11-trimethyl-3,11-dihydro-12H-benzo[h]pyrano[3,2-c]quinolin-12-one (6p)

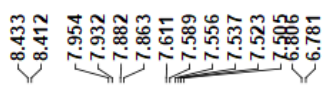

象

$\stackrel{\substack{0 \\+}}{+}$

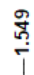

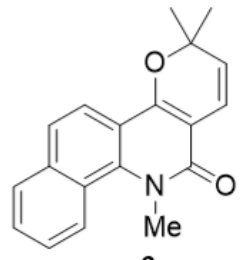

6p

$400 \mathrm{MHz} \mathrm{CDCl}_{3}$

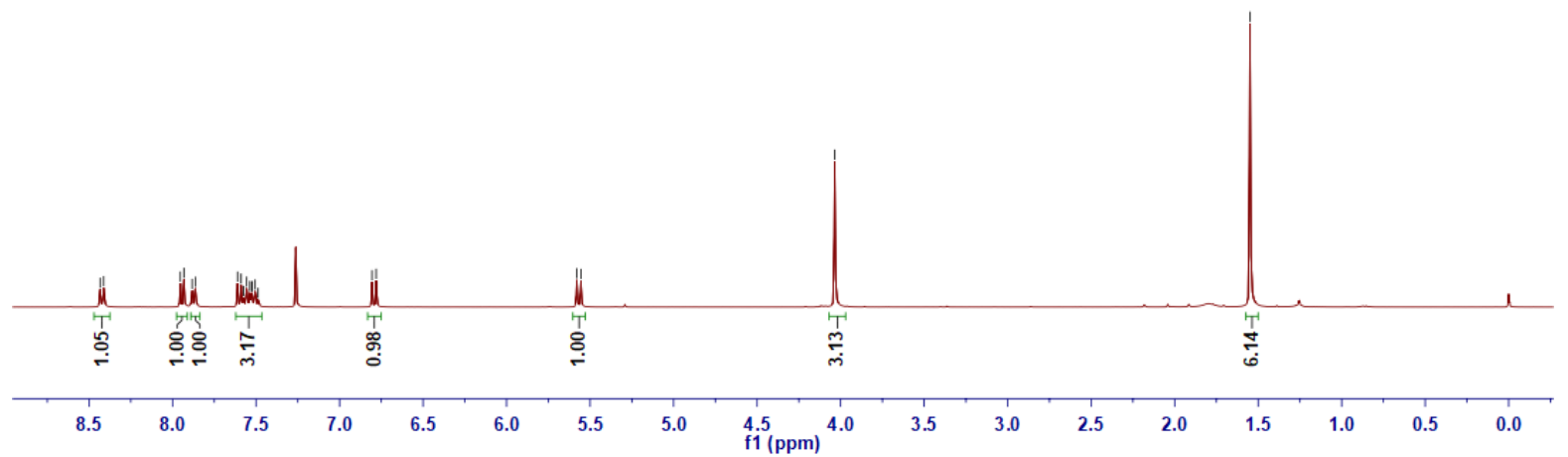

M.

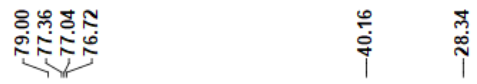

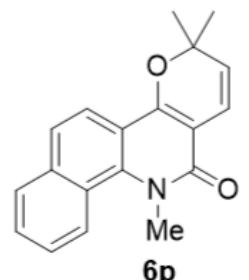

$100 \mathrm{MHz} \mathrm{CDCl}_{3}$

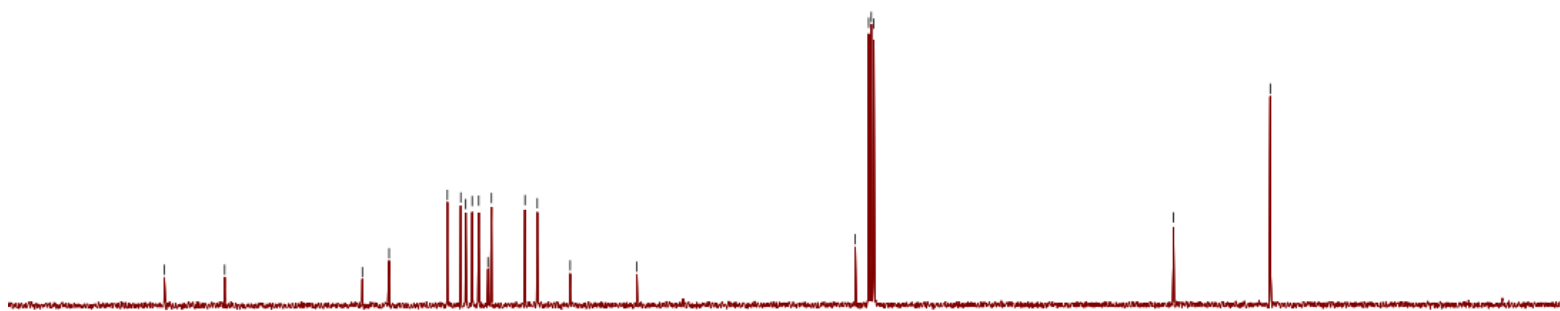

180

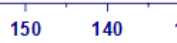

$\stackrel{90}{\mathrm{f1}(\mathrm{ppm})}$ 
6-benzyl-2,2-dimethyl-2,6-dihydro-5H-benzo[f]pyrano[3,2-c]quinolin-5-one (6q)

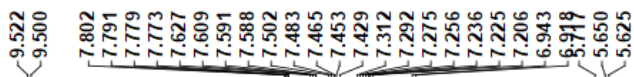

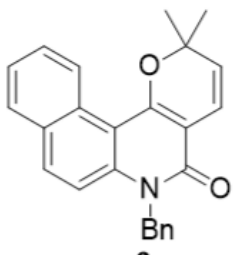

$400 \mathrm{MHz} \mathrm{CDCl}_{3}$

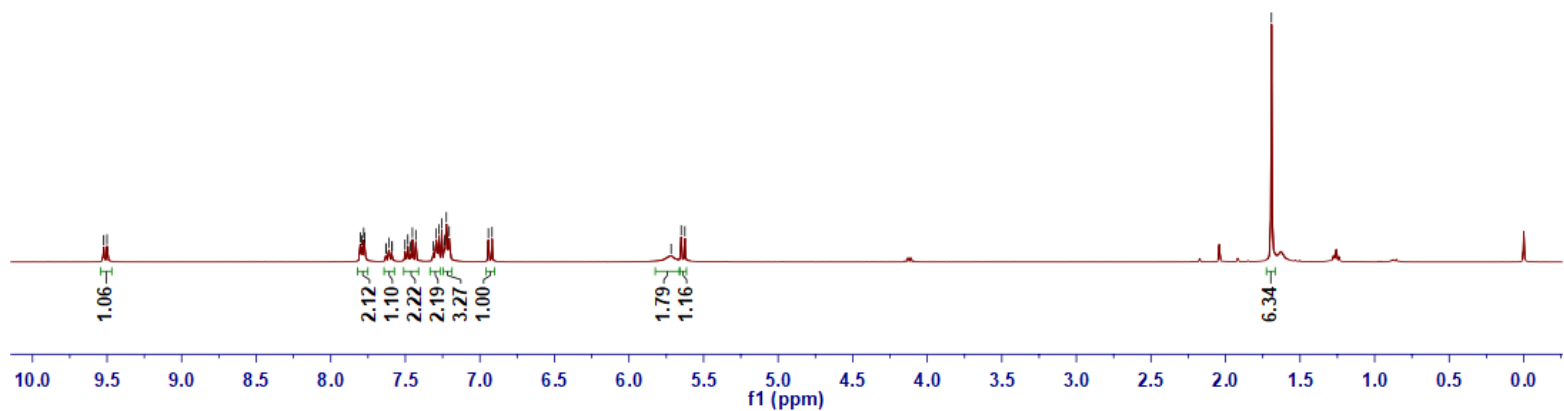

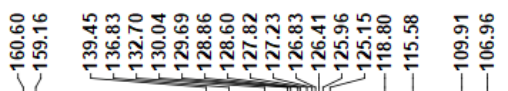

هిల్లి

TR̃N

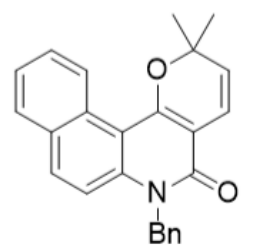

$6 q$

$100 \mathrm{MHz} \mathrm{CDCl}_{3}$

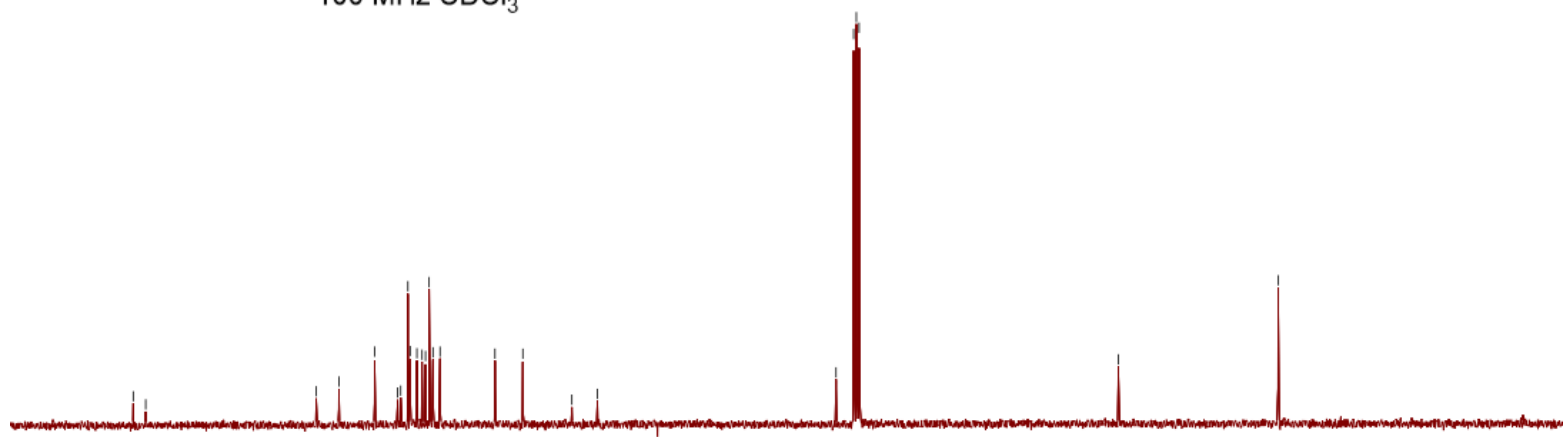

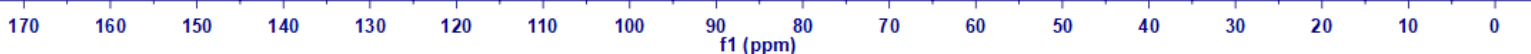


6-benzyl-2,2-dimethyl-2,6-dihydro-5H-pyrano[3,2-c][1,8]naphthyridin-5-one (6r)

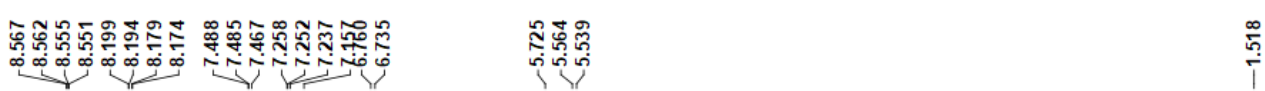

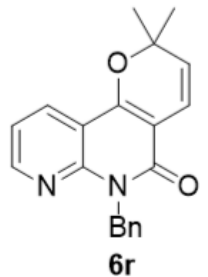

$400 \mathrm{MHz} C D C l_{3}$

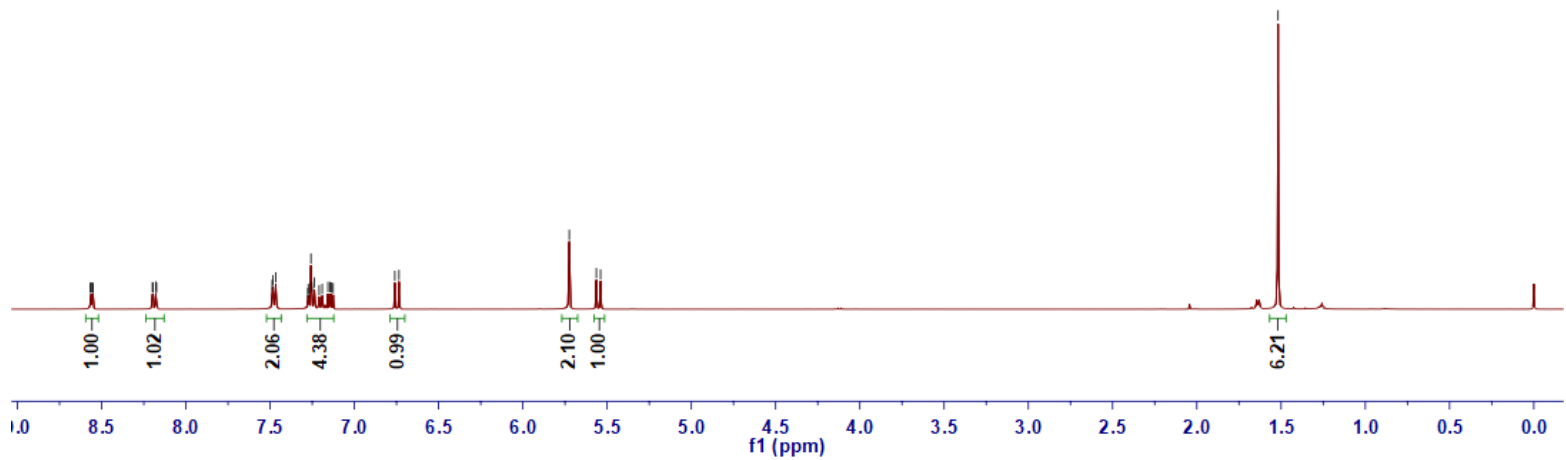
₹

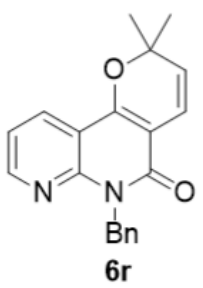

$100 \mathrm{MHz} \mathrm{CDCl}_{3}$

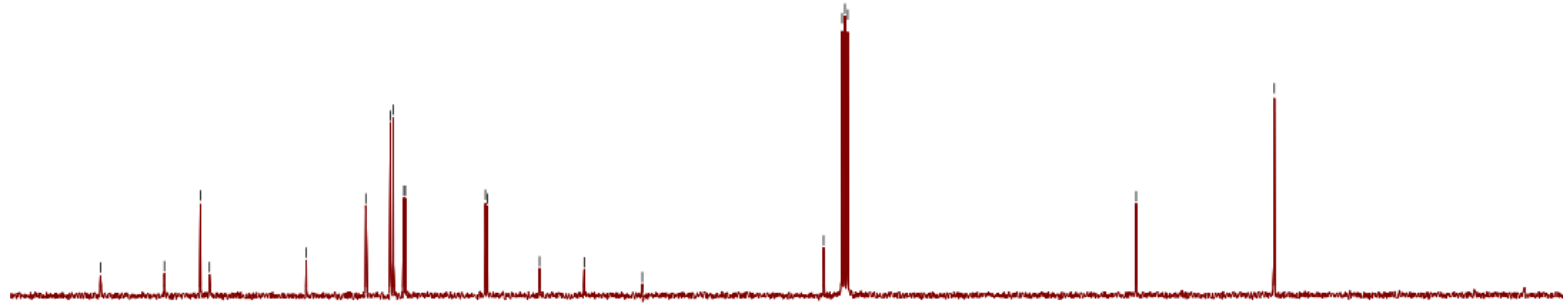

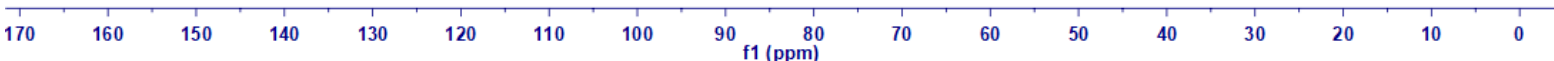


6-benzyl-2,2-dimethyl-2,6-dihydro-5H-pyrano[2,3-a][4,7]phenanthrolin-5-one (6s)

幽

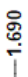

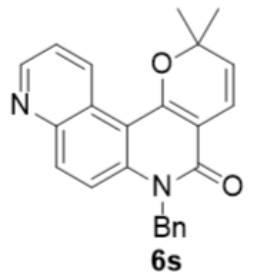

$400 \mathrm{MHz} \mathrm{CDCl}_{3}$

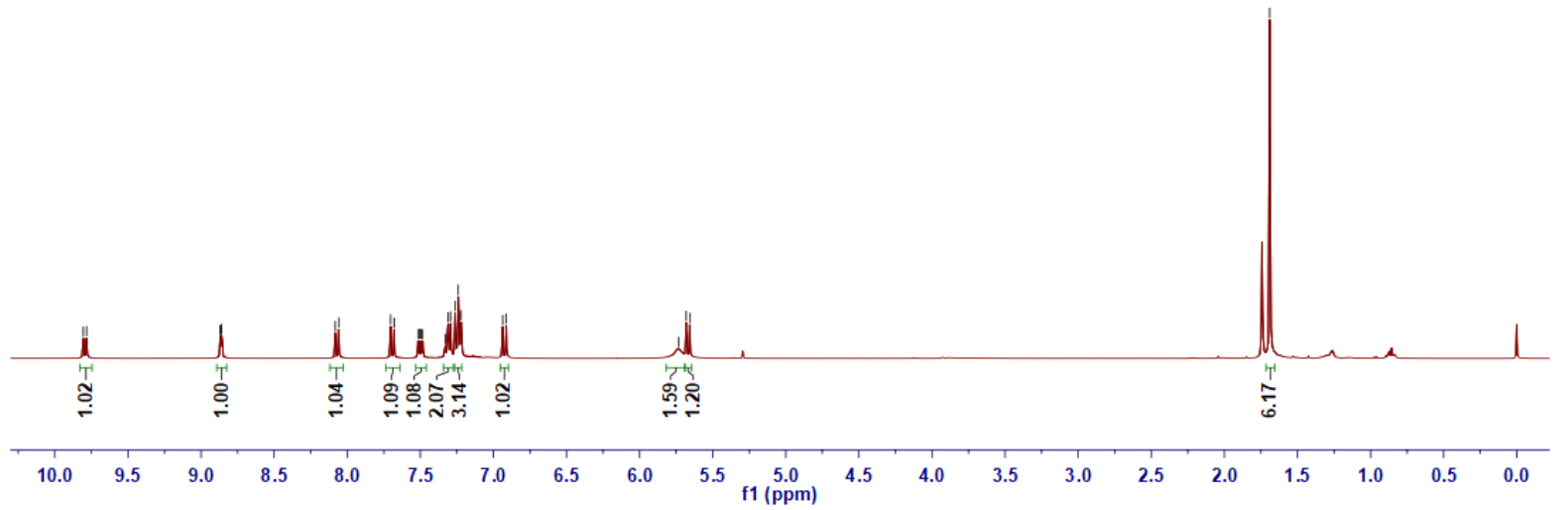

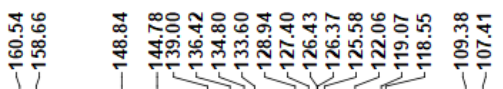

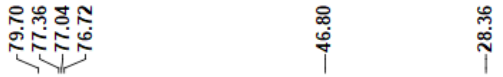

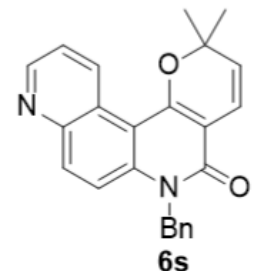

$100 \mathrm{MHz} \mathrm{CDCl}_{3}$
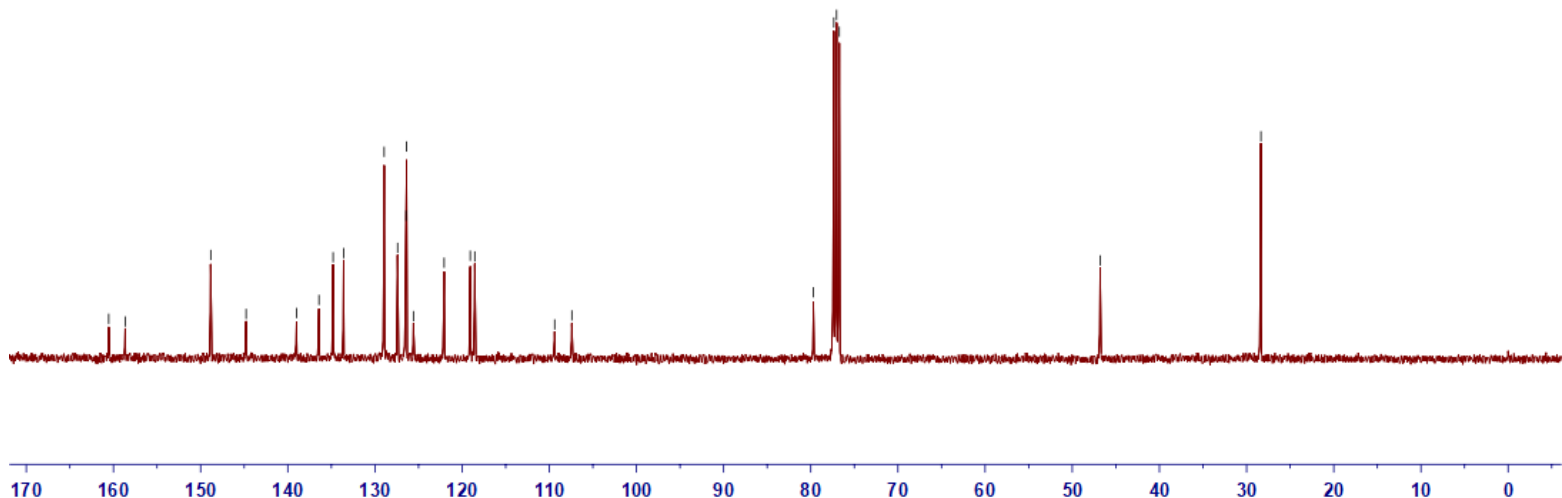

140130

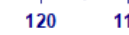

100

$90 \begin{array}{r}80 \\ \mathrm{f} 1(\mathrm{ppm})\end{array}$ 
6-benzyl-2,2-diethyl-2,6-dihydro-5H-pyrano[3,2-c]quinolin-5-one (6t)

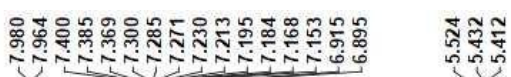

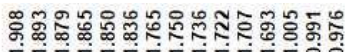

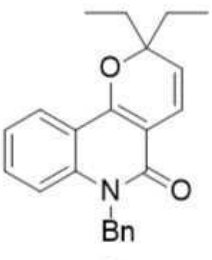

$6 \mathbf{t}$

$500 \mathrm{MHz} \mathrm{CDCl}_{3}$

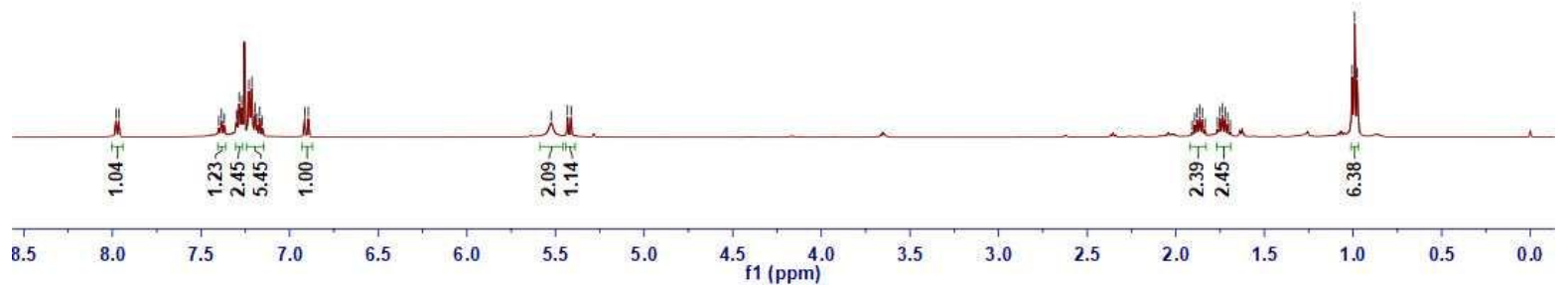

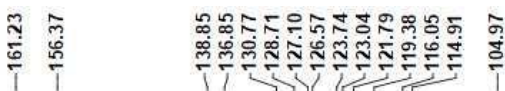

包

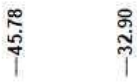

$\stackrel{2}{i}$
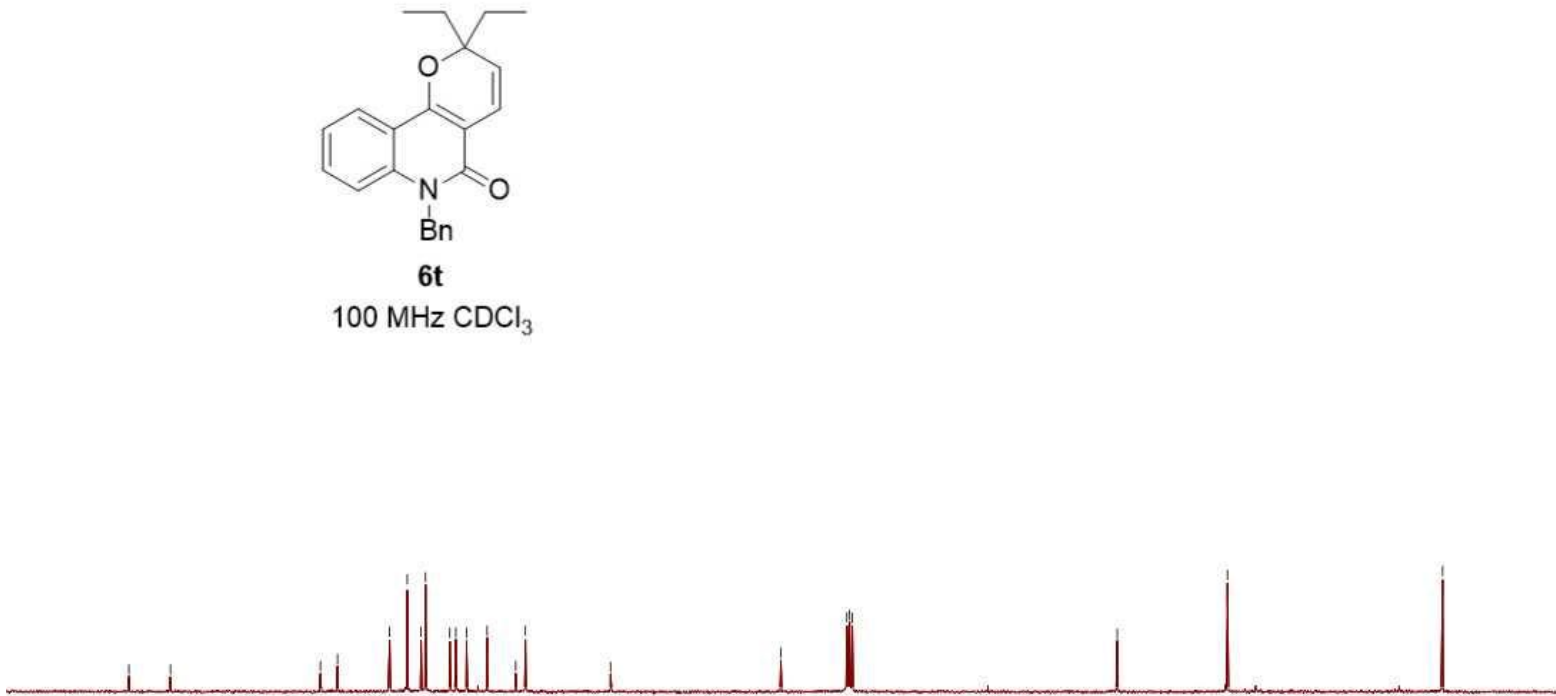

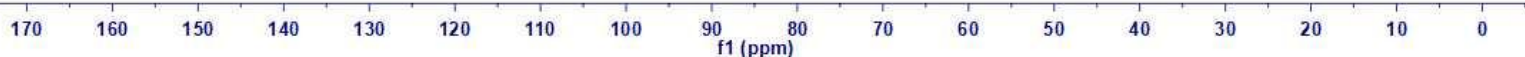


6-benzyl-2,2-dibutyl-2,6-dihydro-5H-pyrano[3,2-c]quinolin-5-one (6u)
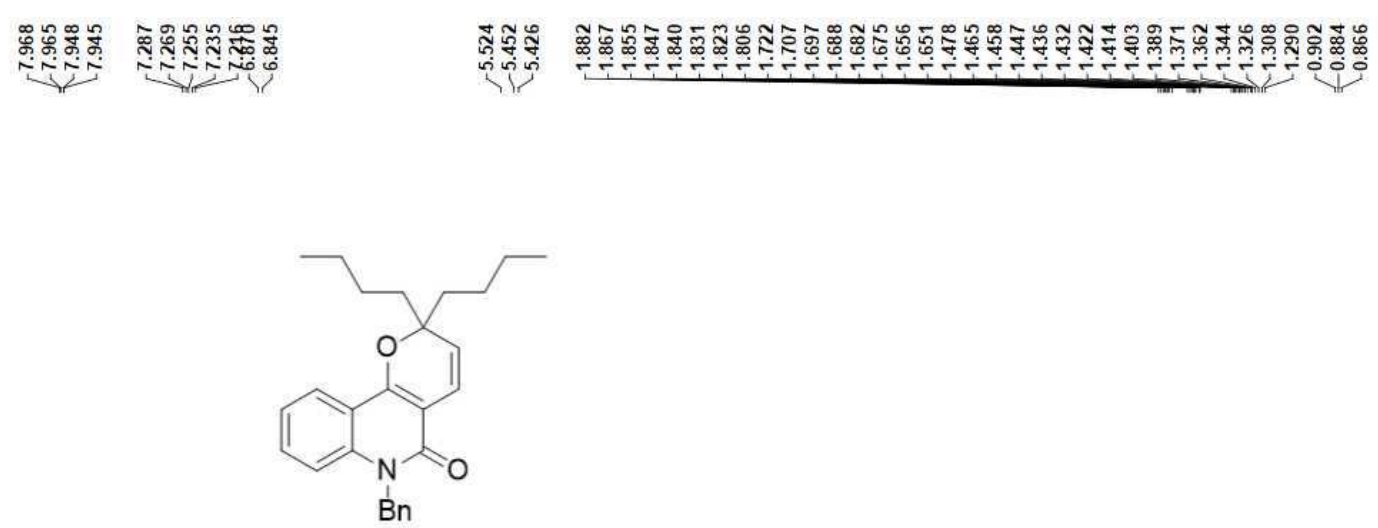

$6 \mathrm{u}$

$400 \mathrm{MHz} \mathrm{CDCl}_{3}$

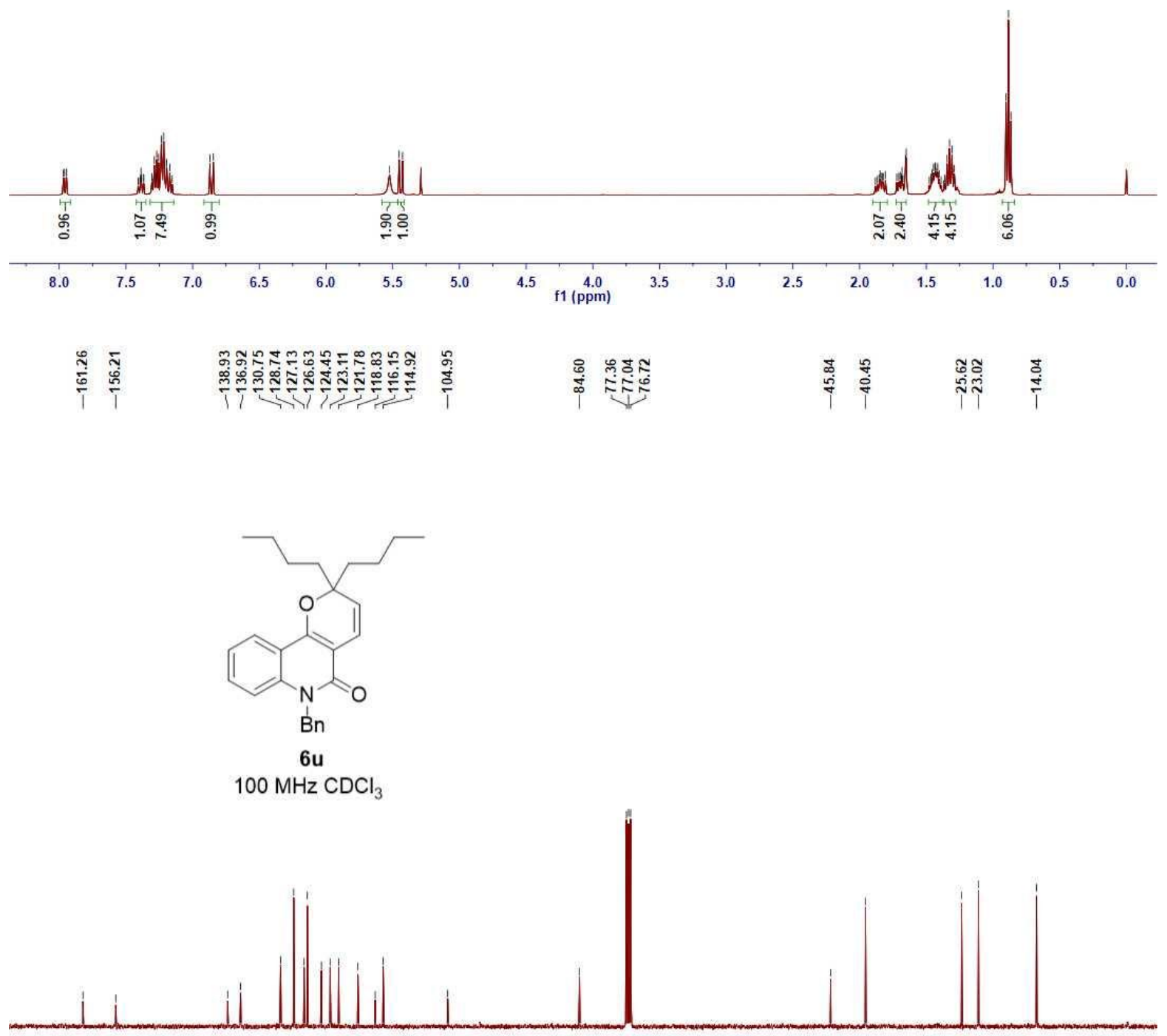

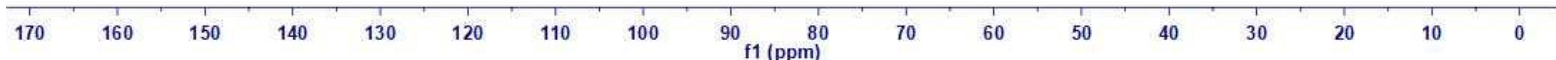


6'-benzylspiro[cyclopentane-1,2'-pyrano[3,2-c]quinolin]-5'(6'H)-one (6v)

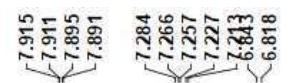

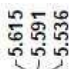

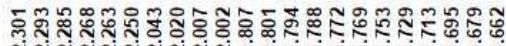

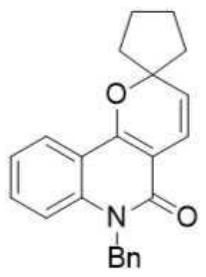

6v

$400 \mathrm{MHz} \mathrm{CDCl}_{3}$

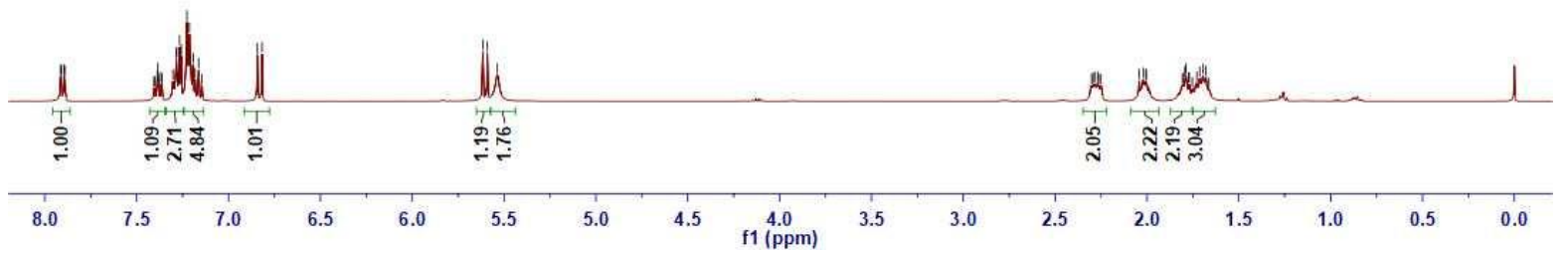

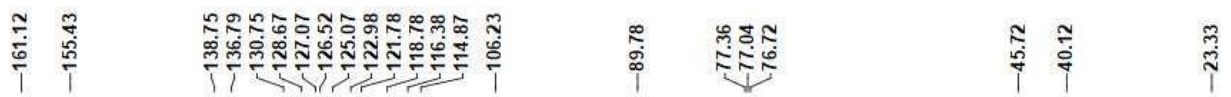

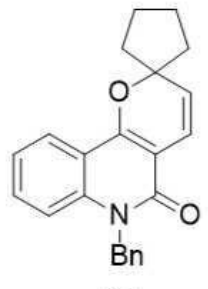

6v

$100 \mathrm{MHz} \mathrm{CDCl}_{3}$

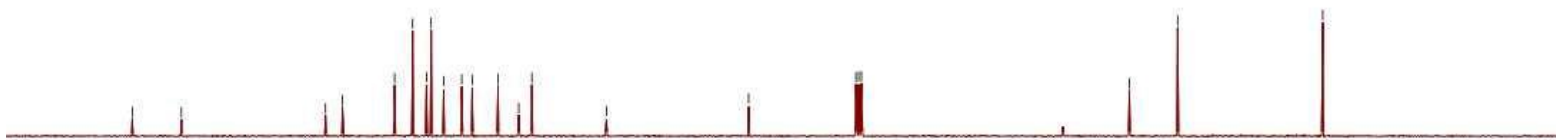


10,10-dimethyl-4,5-dihydro-7H,10H-pyrano[3,2-c]pyrrolo[3,2,1-ij]quinolin-7-one (6w)
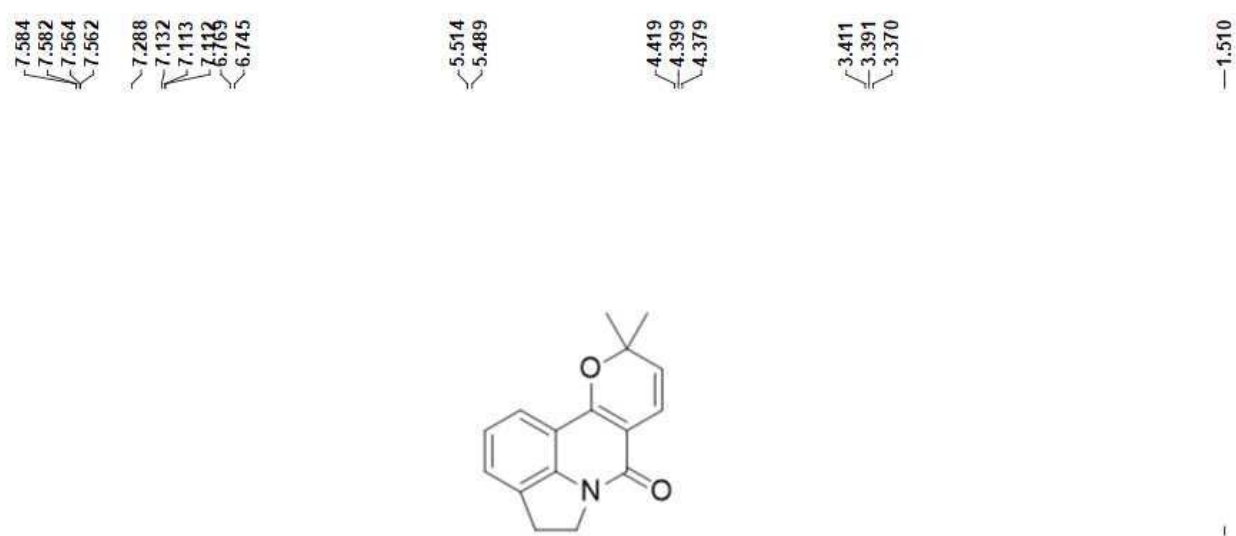

$6 \mathrm{w}$

$400 \mathrm{MHz}^{\mathrm{CDCl}} \mathrm{C}_{3}$

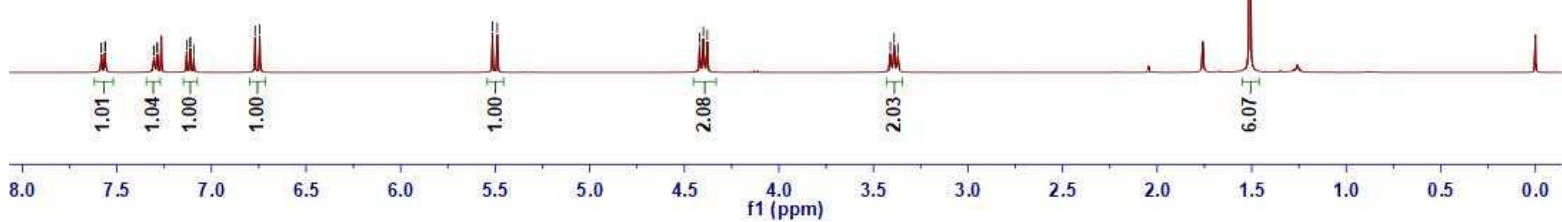

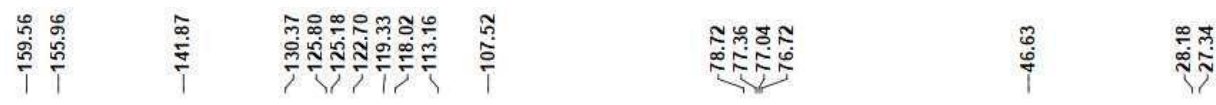

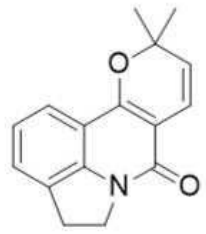

$6 w$

$100 \mathrm{MHz} \mathrm{CDCl}_{3}$

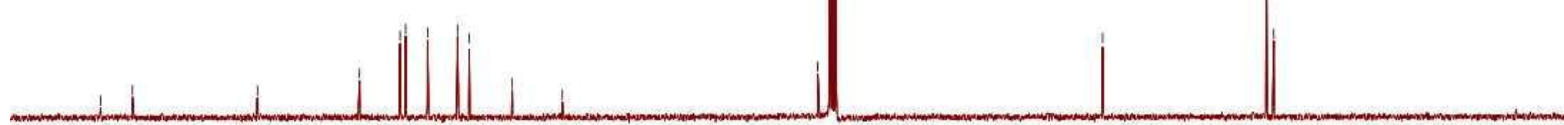

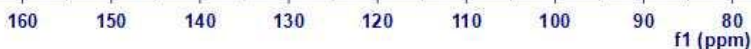


11,11-dimethyl-5,6-dihydro-4H,8H,11H-pyrano[3,2-c]pyrido[3,2,1-ij]quinolin-8-one (6x)
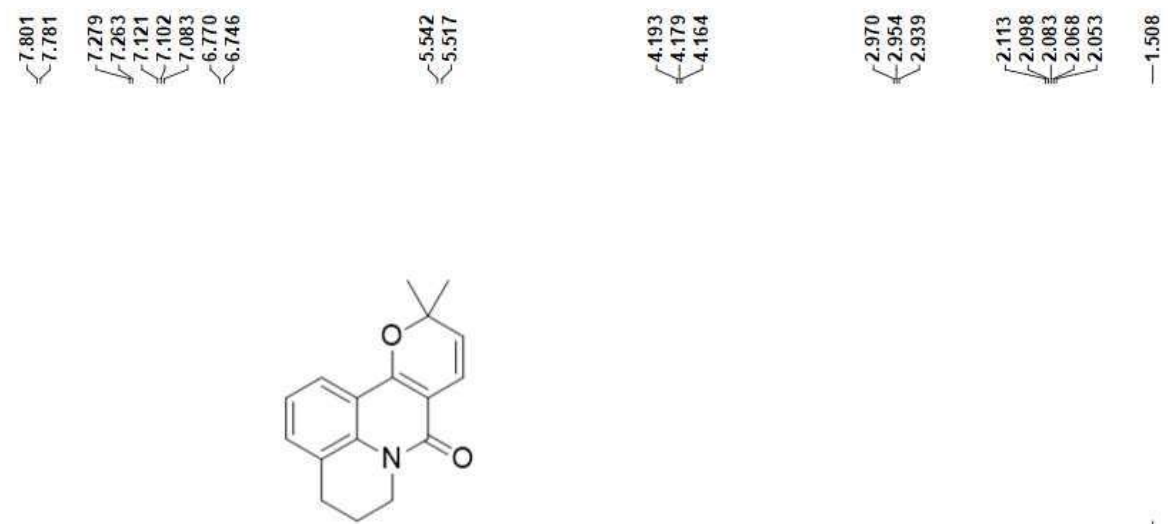

$6 x$

$400 \mathrm{MHz} \mathrm{CDCl}_{3}$

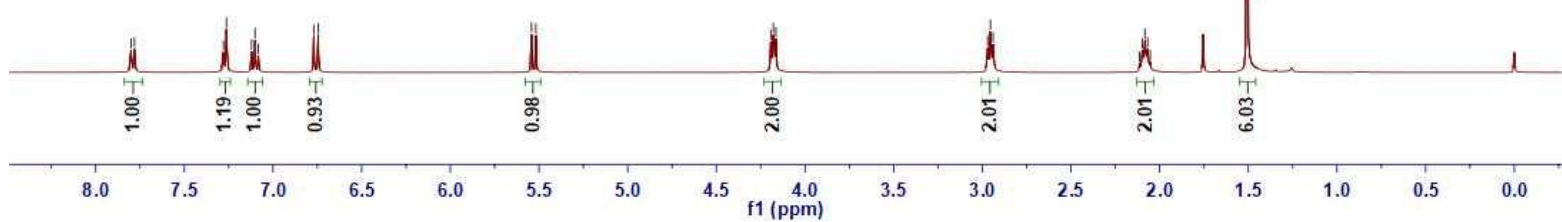

菅 芯

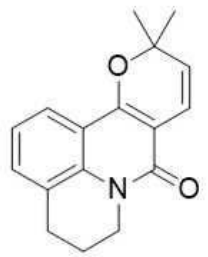

$6 \mathrm{x}$

$100 \mathrm{MHz} \mathrm{CDCl}_{3}$

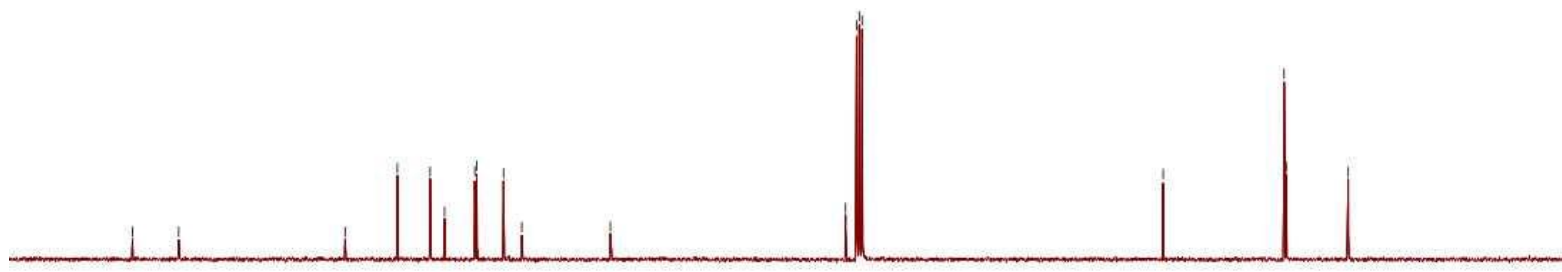

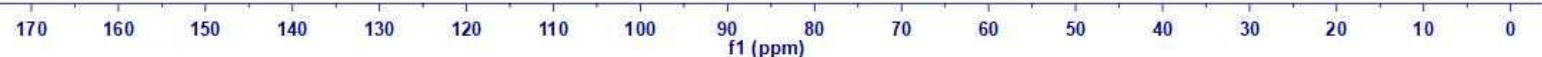


(2S,4R)-2-(2-hydroxyethyl)-2,6-dimethyl-4-(2-methylprop-1-en-1-yl)-2,3,4,6-tetrahydro-5H-pyrano[3,2-c]qui nolin-5-one (4a)

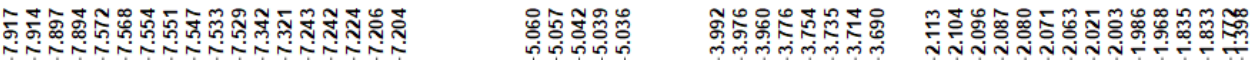
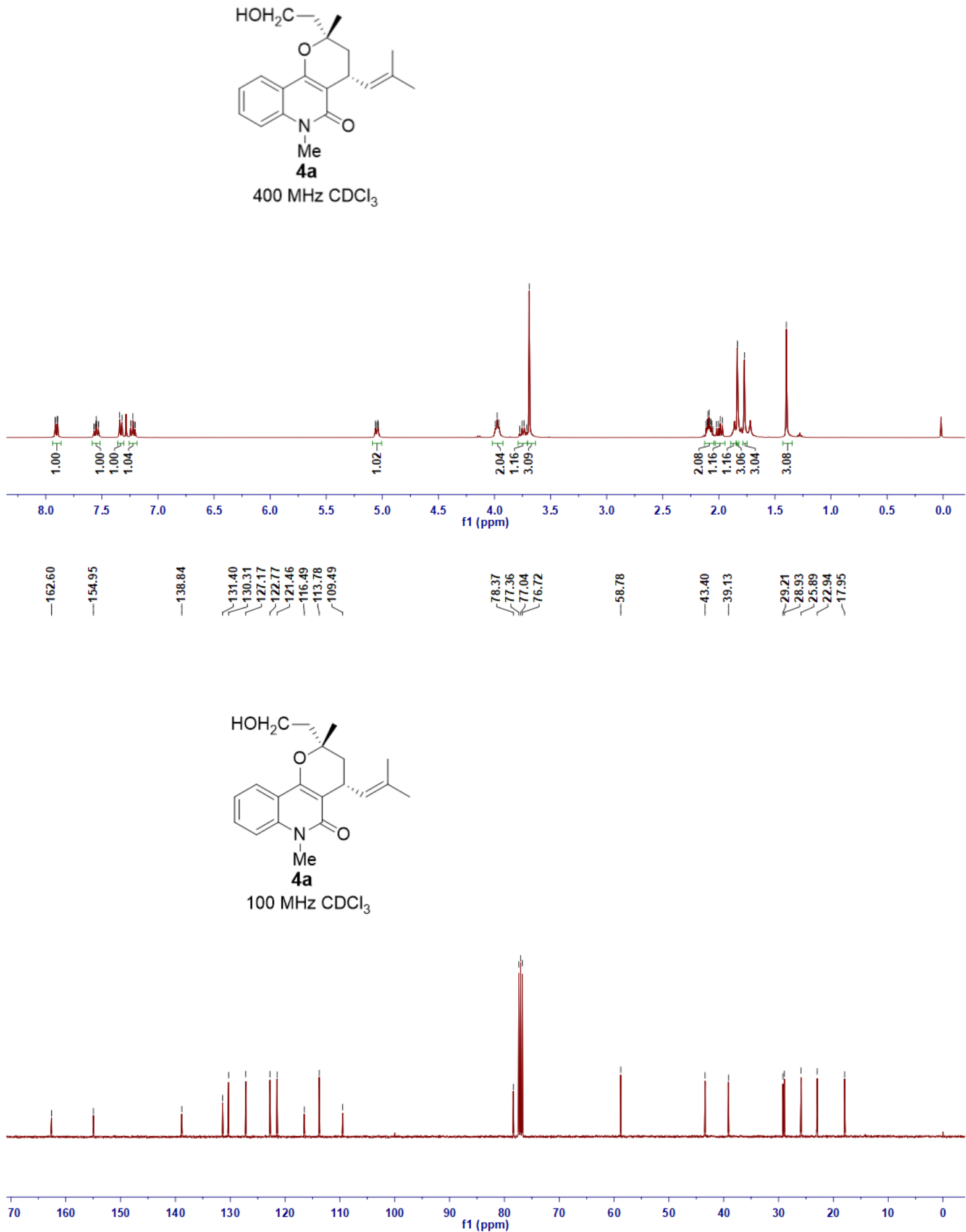
(2R,4R)-2-(2-hydroxyethyl)-2,6-dimethyl-4-(2-methylprop-1-en-1-yl)-2,3,4,6-tetrahydro-5H-pyrano[3,2-c]qui nolin-5-one (4aa)
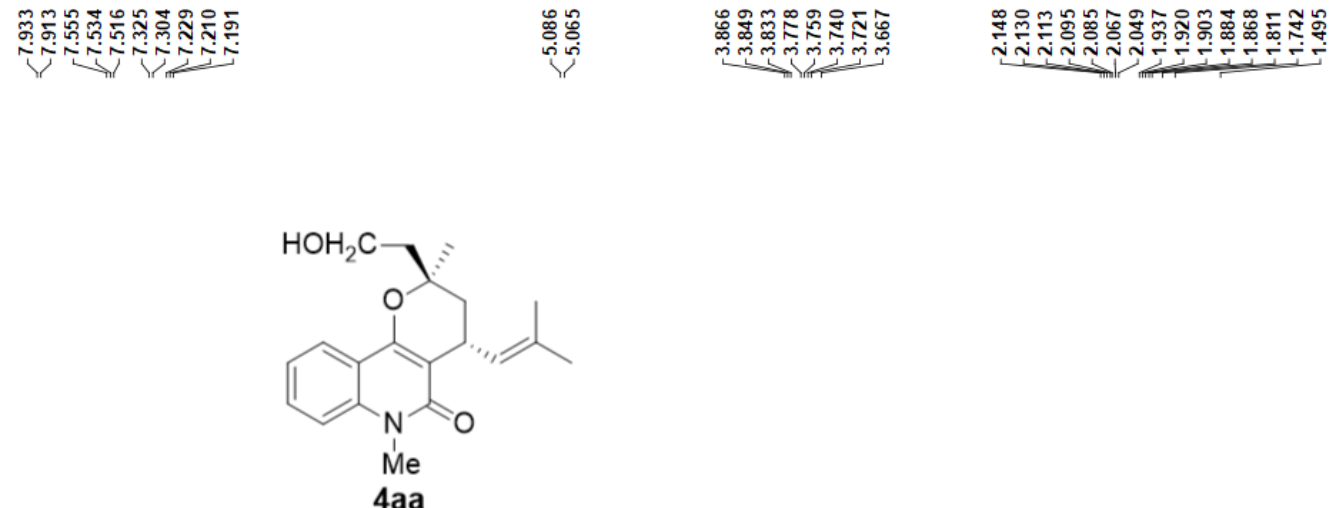

$400 \mathrm{MHz} \mathrm{CDCl}_{3}$
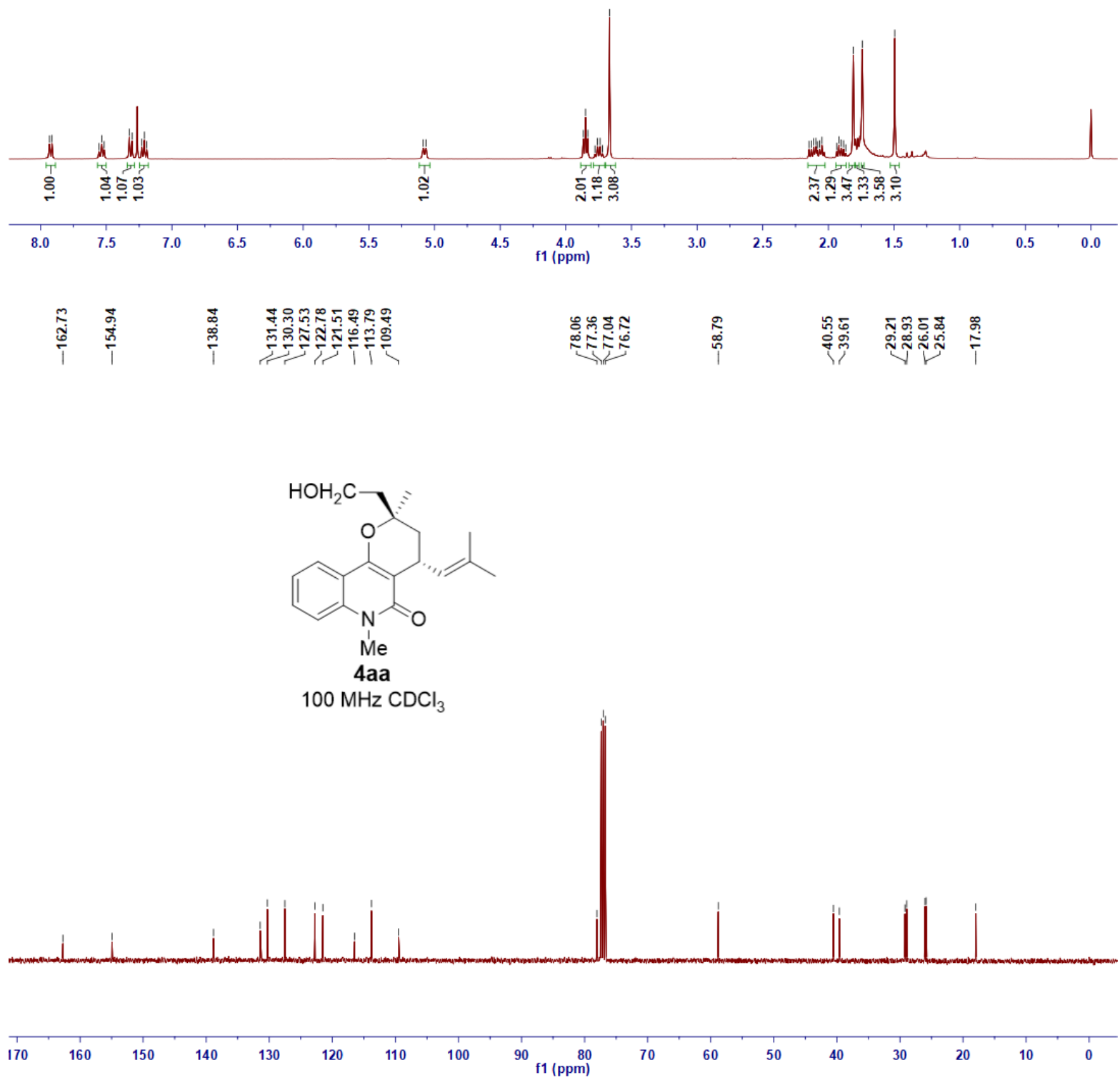

$93 / 146$ 
(2S,4R)-6-benzyl-2-(2-hydroxyethyl)-2-methyl-4-(2-methylprop-1-en-1-yl)-2,3,4,6-tetrahydro-5H-pyrano[3,2-c]qui nolin-5-one (4b)
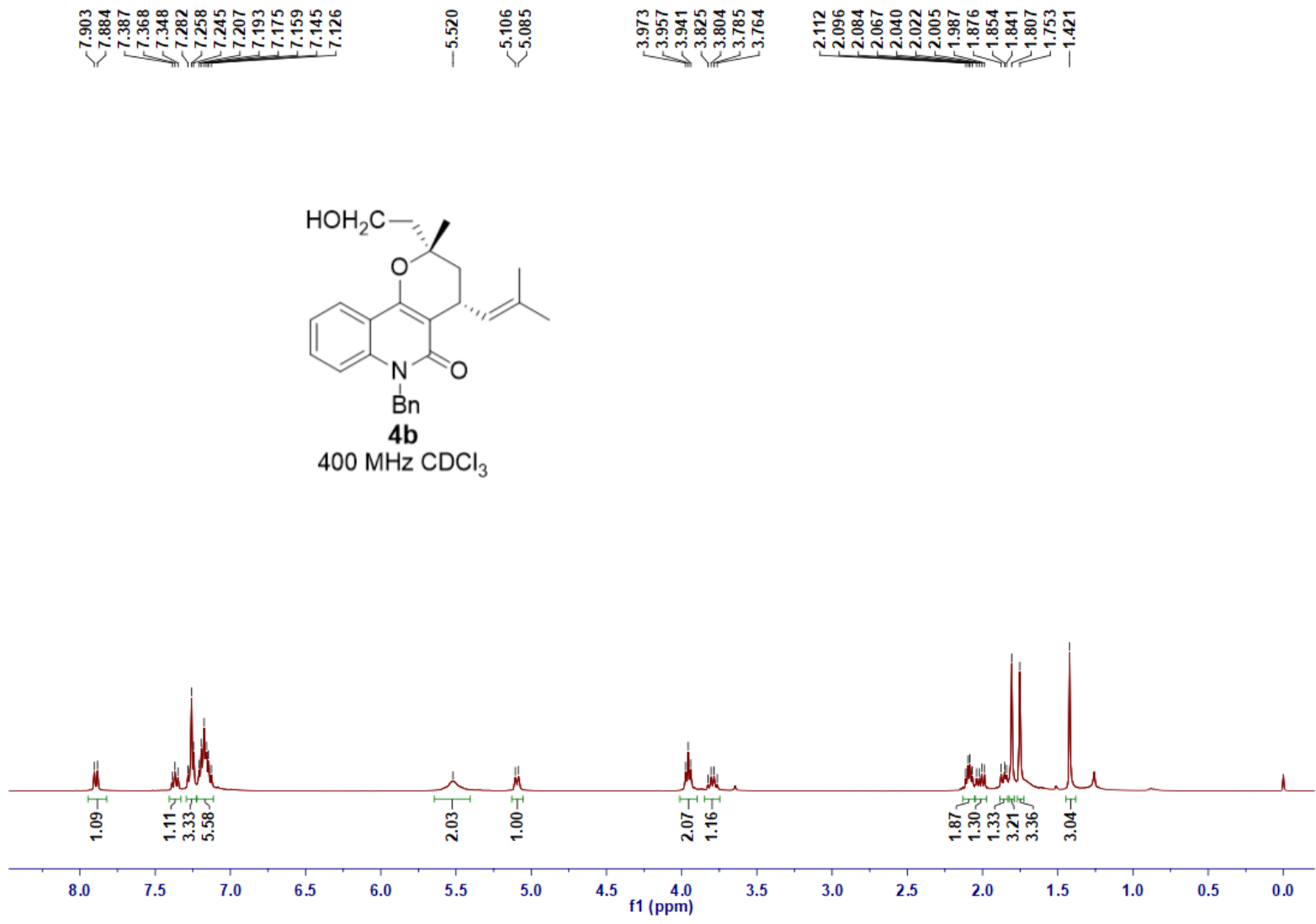

\begin{tabular}{|c|c|c|}
\hline$\stackrel{\stackrel{\infty}{乛}}{\stackrel{\Gamma}{\Gamma}}$ & బु. & 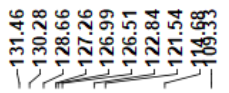 \\
\hline
\end{tabular}

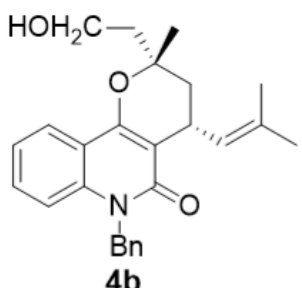

$100 \mathrm{MHz} \mathrm{CDCl}_{3}$

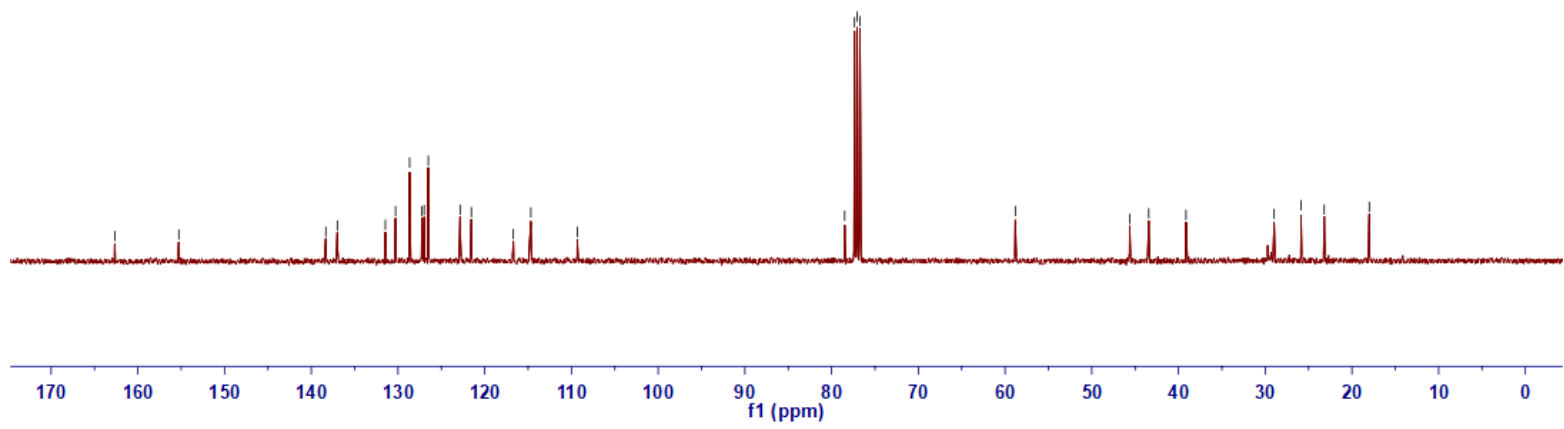


(2R,4R)-6-benzyl-2-(2-hydroxyethyl)-2-methyl-4-(2-methylprop-1-en-1-yl)-2,3,4,6-tetrahydro-5H-pyrano[3,2-c]qui nolin-5-one (4bb)

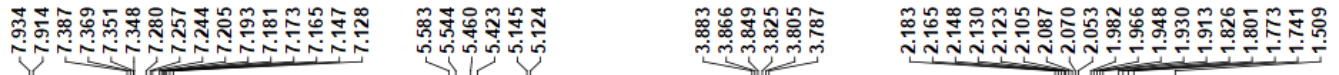

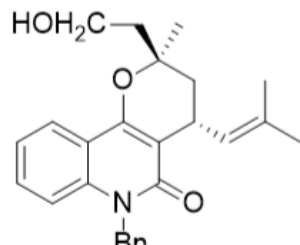

$400 \mathrm{MHz} \mathrm{CDCl}_{3}$
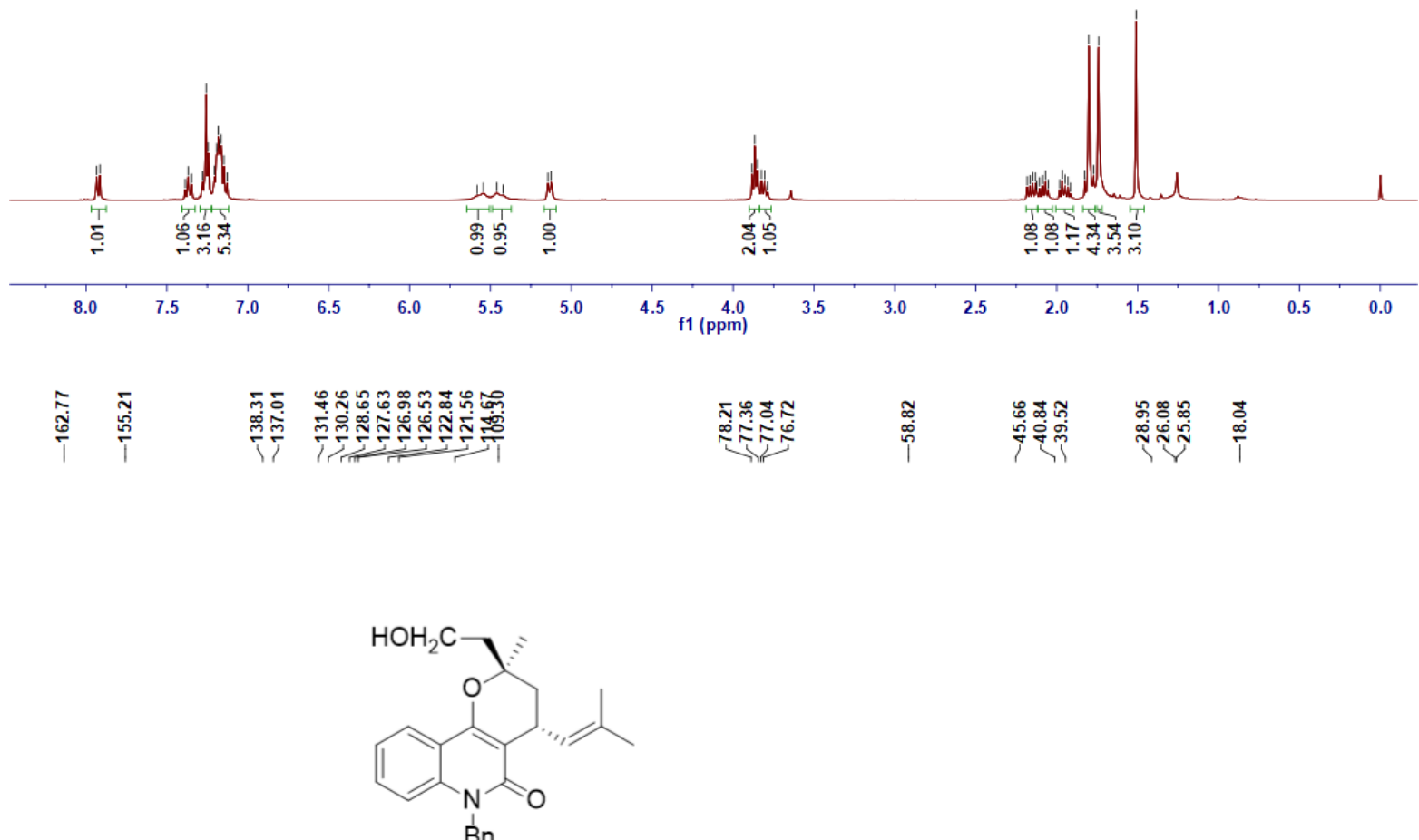

$4 \mathrm{bb}$

$100 \mathrm{MHz} \mathrm{CDCl}_{3}$

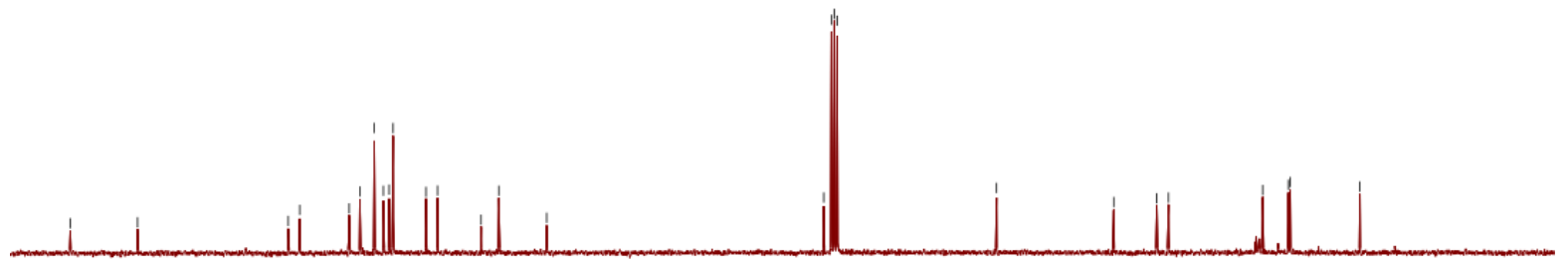

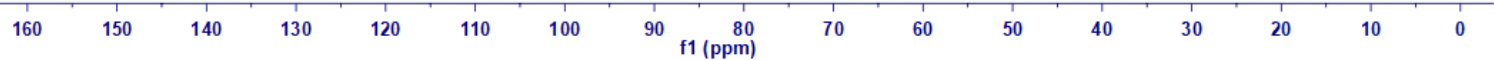


(2S,4R)-2-(2-hydroxyethyl)-2-methyl-4-(2-methylprop-1-en-1-yl)-6-phenyl-2,3,4,6-tetrahydro-5H-pyrano[3,2-c]qui nolin-5-one (4c)

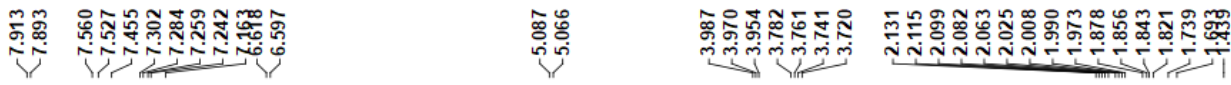
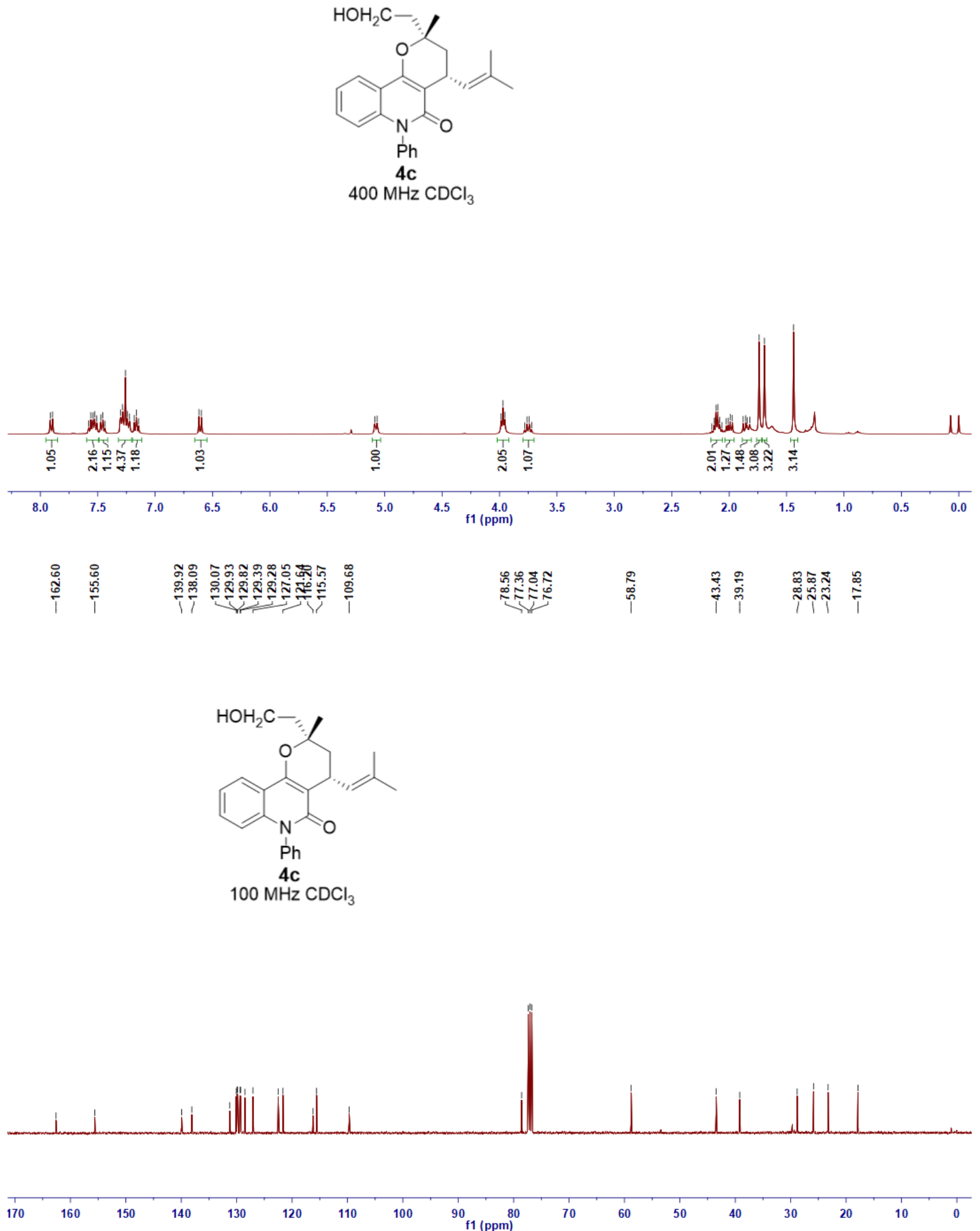


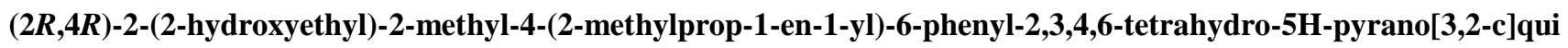
nolin-5-one (4cc)

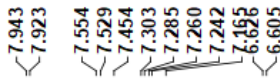

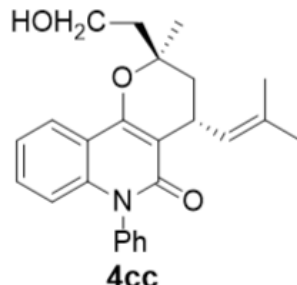

$400 \mathrm{MHz} \mathrm{CDCl}_{3}$
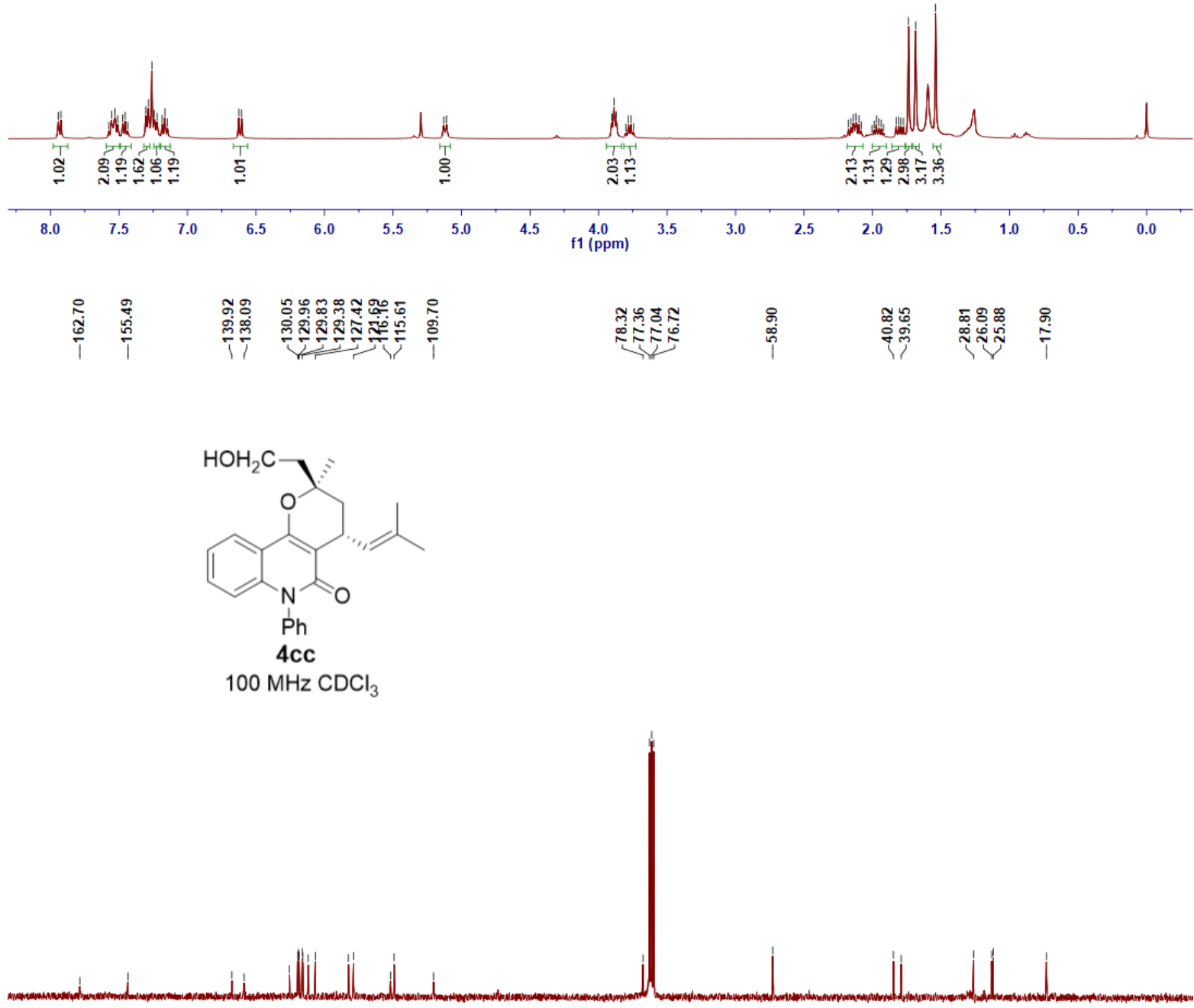

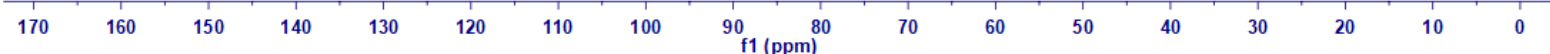


(2S,4R)-6-benzyl-9-fluoro-2-(2-hydroxyethyl)-2-methyl-4-(2-methylprop-1-en-1-yl)-2,3,4,6-tetrahydro-5H-pyrano [3,2-c]quinolin-5-one (4d)

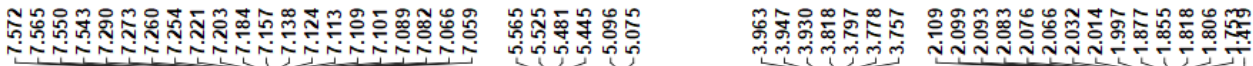
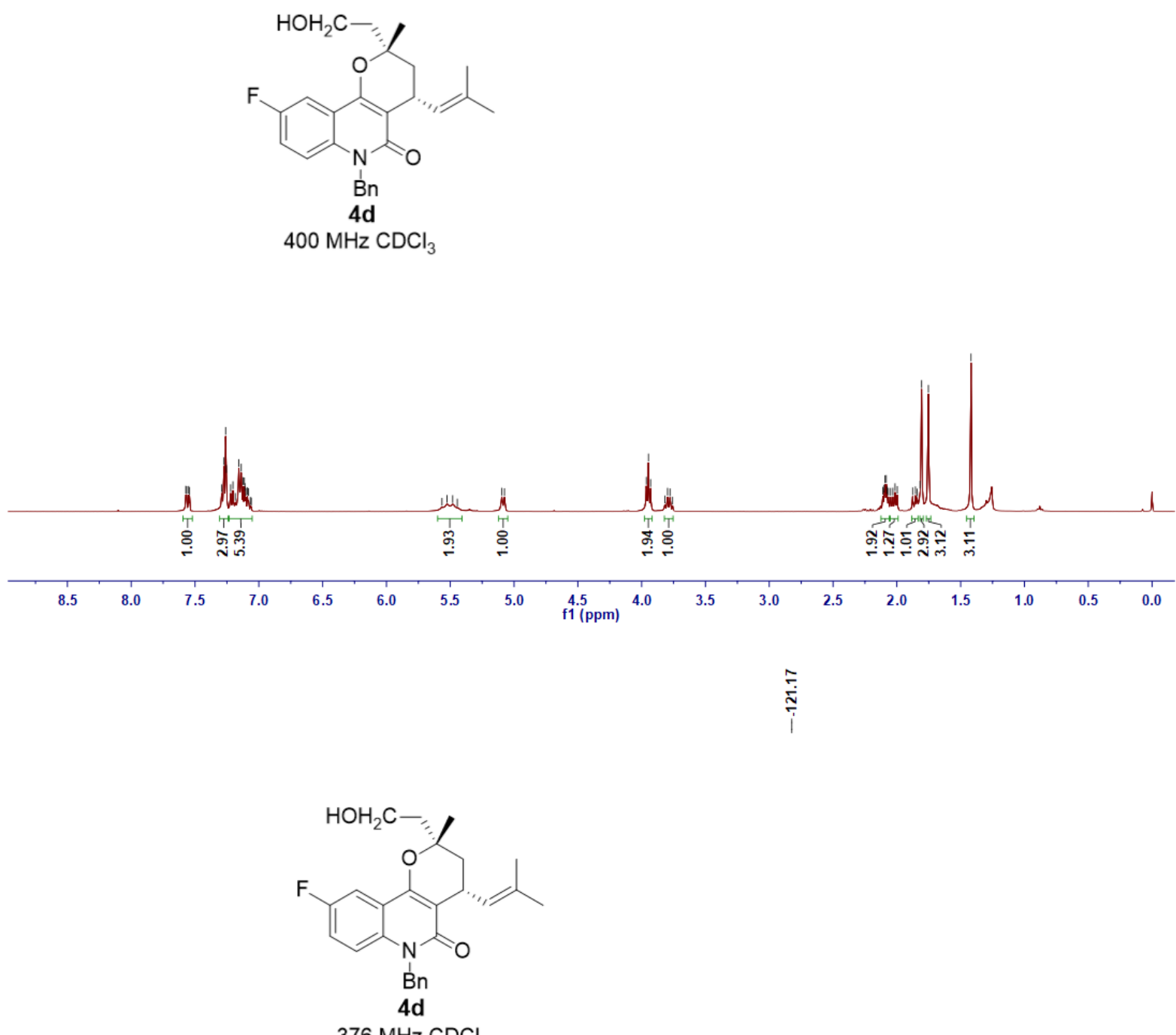

$376 \mathrm{MHz} \mathrm{CDCl}_{3}$

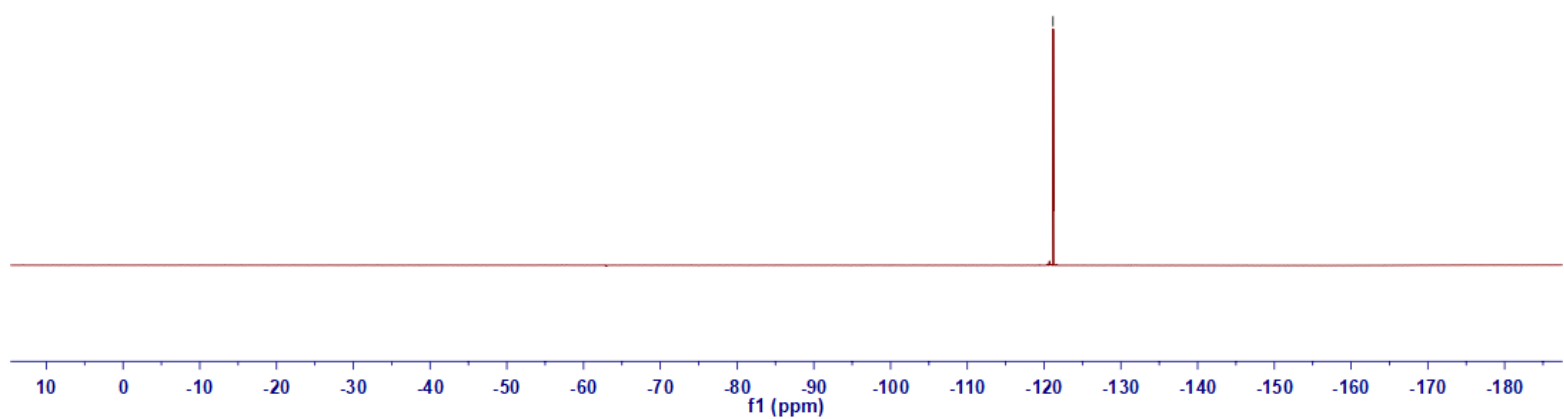




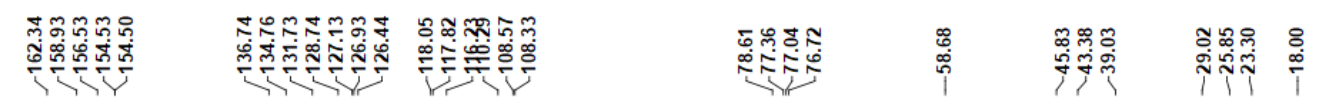<smiles>CC(C)=CC1CC(C)(C)Oc2c1c(=O)n([13C]([O-])([O-])[O-])c1ccc(F)cc21</smiles>

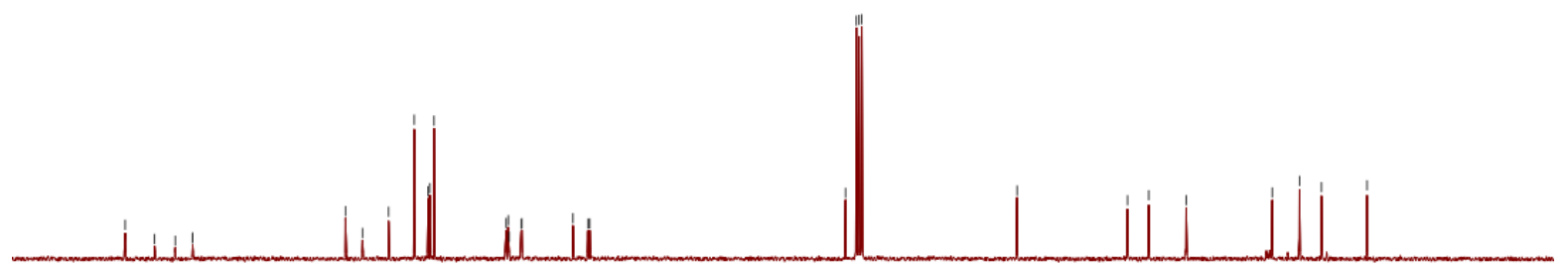

\begin{tabular}{|lllllllllllllllll}
\hline 170 & 160 & 150 & 140 & 130 & 120 & 110 & 100 & $\underset{\mathrm{f} 1(\mathrm{ppm})}{1} 80$ & 70 & 60 & 50 & 40 & 30 & 20 & 10 & 0
\end{tabular}

(2R,4R)-6-benzyl-9-fluoro-2-(2-hydroxyethyl)-2-methyl-4-(2-methylprop-1-en-1-yl)-2,3,4,6-tetrahydro-5H-pyrano [3,2-c]quinolin-5-one (4dd)

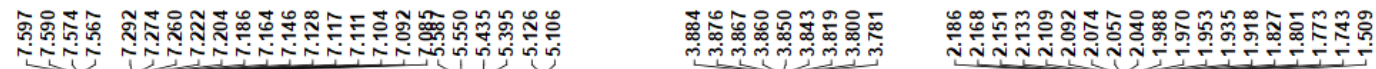
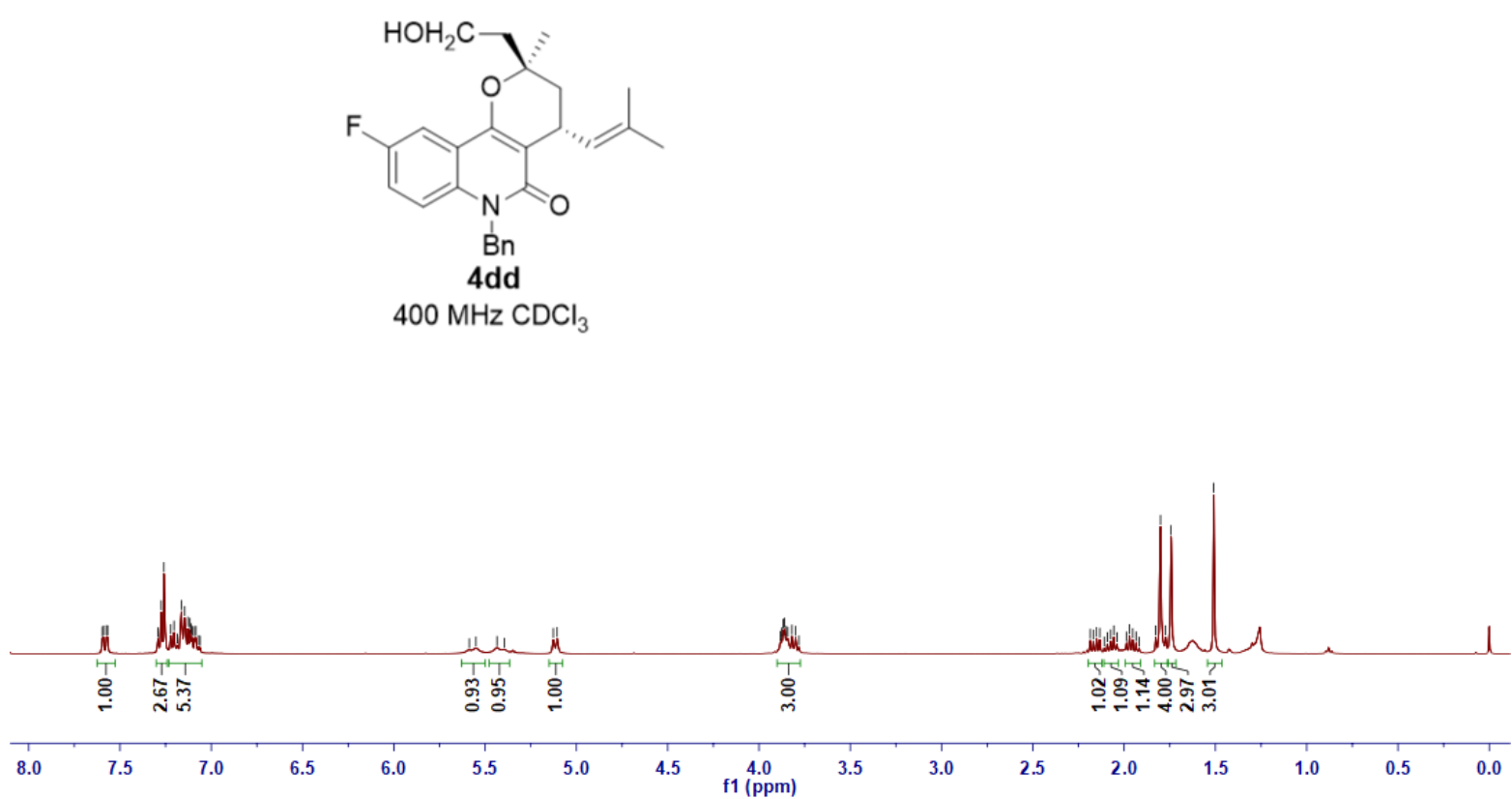


\section{$\underset{i}{\stackrel{i}{i}}$}<smiles>CC[C@]1(C)C[C@H](C=C(C)C)c2c(c3cc(F)ccc3n(Cc3ccccc3)c2=O)O1</smiles>

\begin{tabular}{llllllllllllllllllllll}
\hline 10 & 0 & -10 & -20 & -30 & -40 & -50 & -60 & -70 & -80 & -90 & -100 & -110 & -120 & -130 & -140 & -150 & -160 & -170 & -180 \\
\hline
\end{tabular}

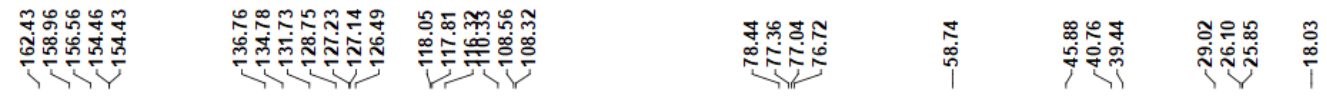
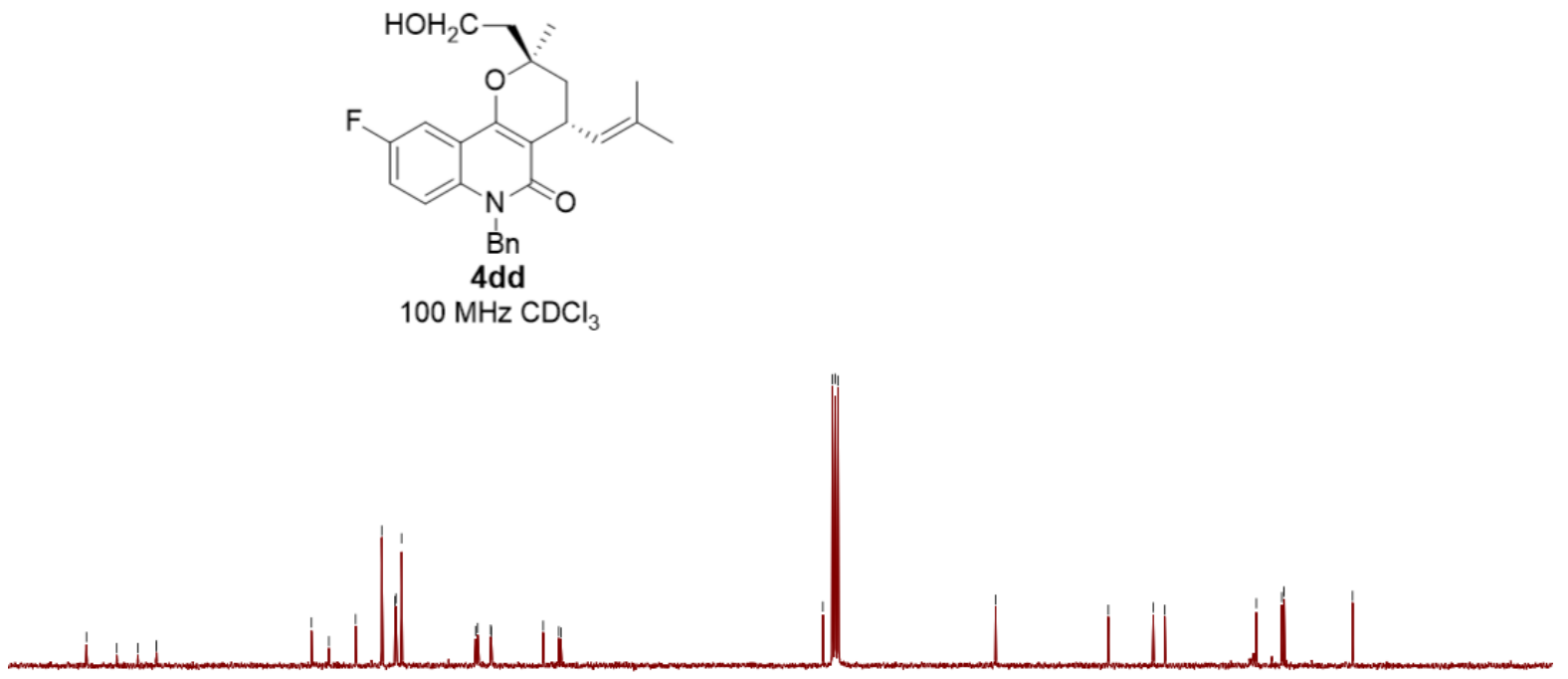

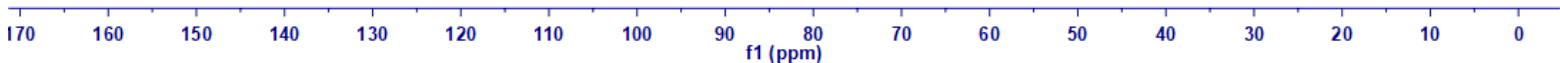


(2S,4R)-6-benzyl-9-chloro-2-(2-hydroxyethyl)-2-methyl-4-(2-methylprop-1-en-1-yl)-2,3,4,6-tetrahydro-5H-pyrano $[3,2-c] q u i n o l i n-5-o n e(4 e)$

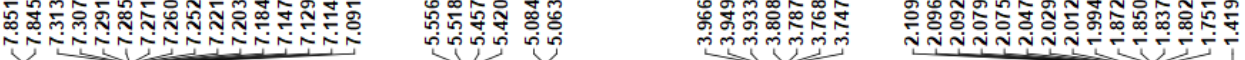

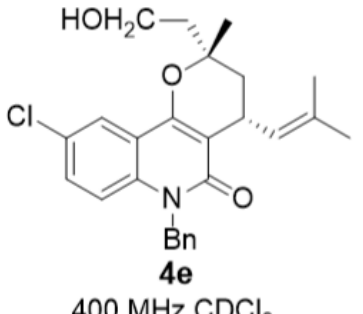

$400 \mathrm{MHz} C D C l_{3}$
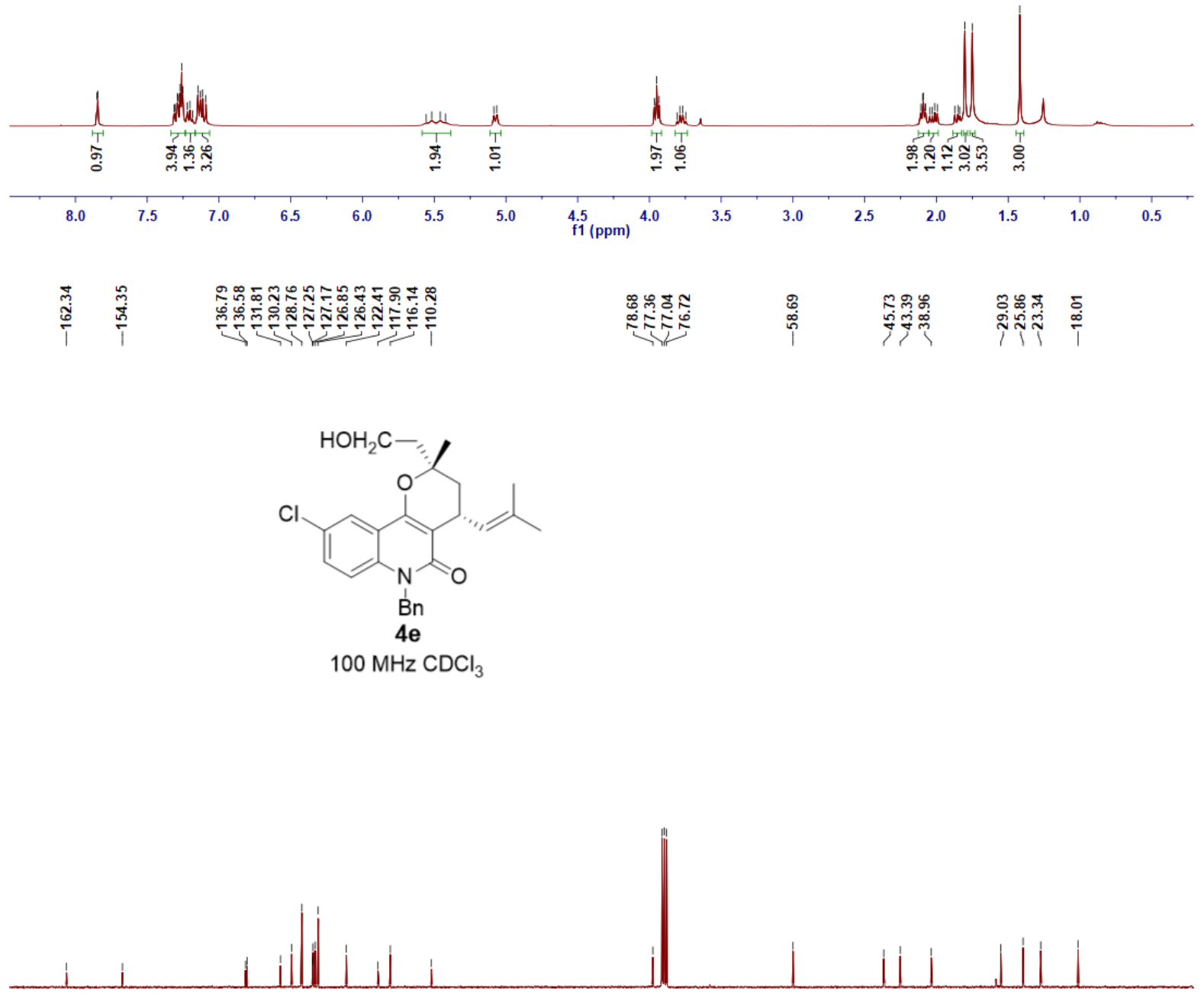

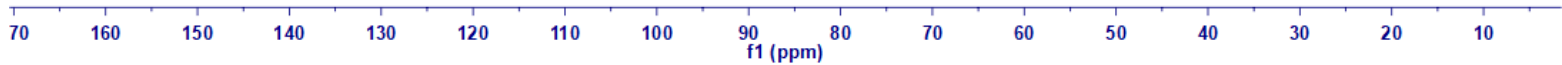


(2R,4R)-6-benzyl-9-chloro-2-(2-hydroxyethyl)-2-methyl-4-(2-methylprop-1-en-1-yl)-2,3,4,6-tetrahydro-5H-pyrano [3,2-c]quinolin-5-one (4ee)

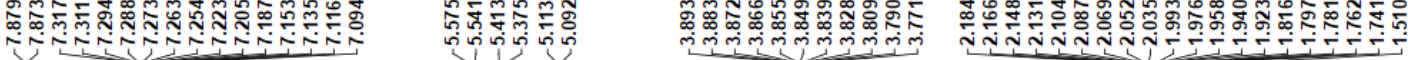
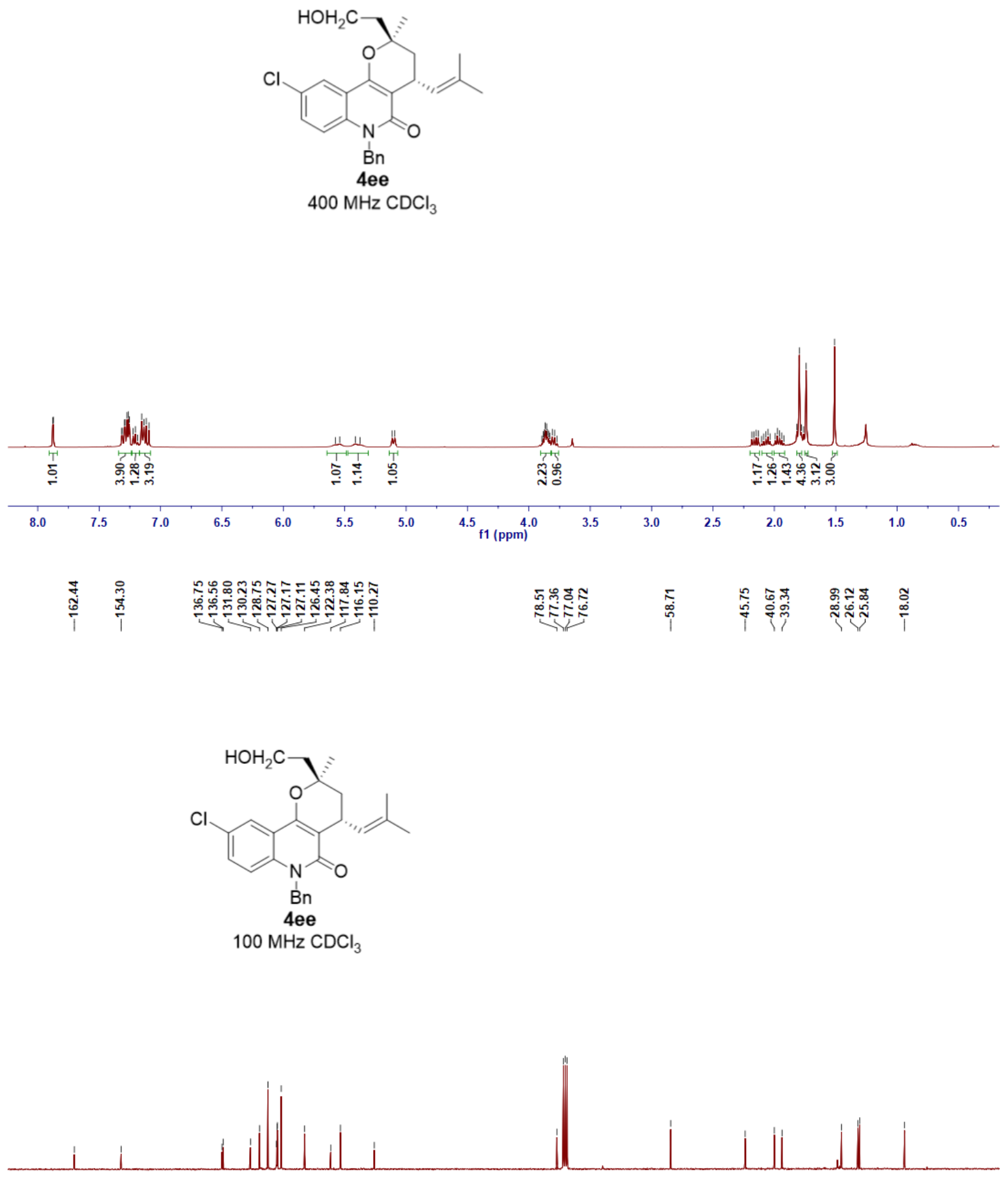

\begin{tabular}{|c|c|c|c|c|c|c|c|c|c|c|c|c|c|c|c|}
\hline 170 & 160 & 150 & 140 & 130 & 120 & 110 & 100 & $\begin{array}{l}90 \\
\mathrm{f} 1(\mathrm{ppm})\end{array}$ & 80 & 70 & 60 & 50 & 40 & 30 & 20 \\
\hline
\end{tabular}


(2S,4R)-6-benzyl-9-bromo-2-(2-hydroxyethyl)-2-methyl-4-(2-methylprop-1-en-1-yl)-2,3,4,6-tetrahydro-5H-pyrano [3,2-c]quinolin-5-one (4f)
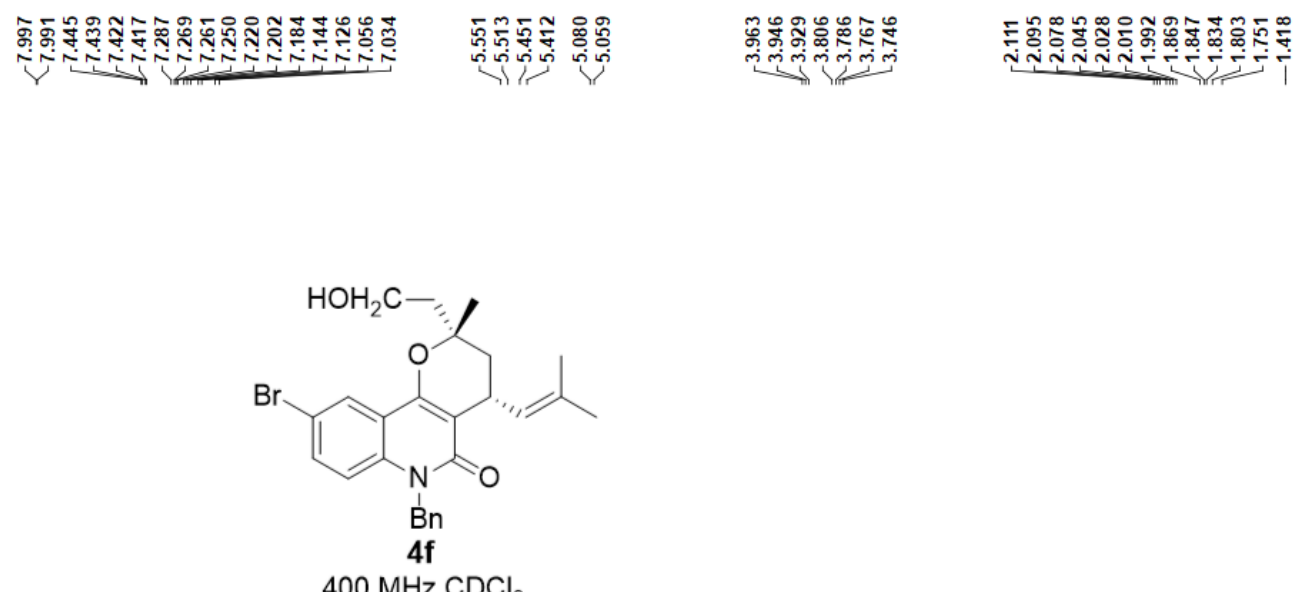

$400 \mathrm{MHz} \mathrm{CDCl}_{3}$
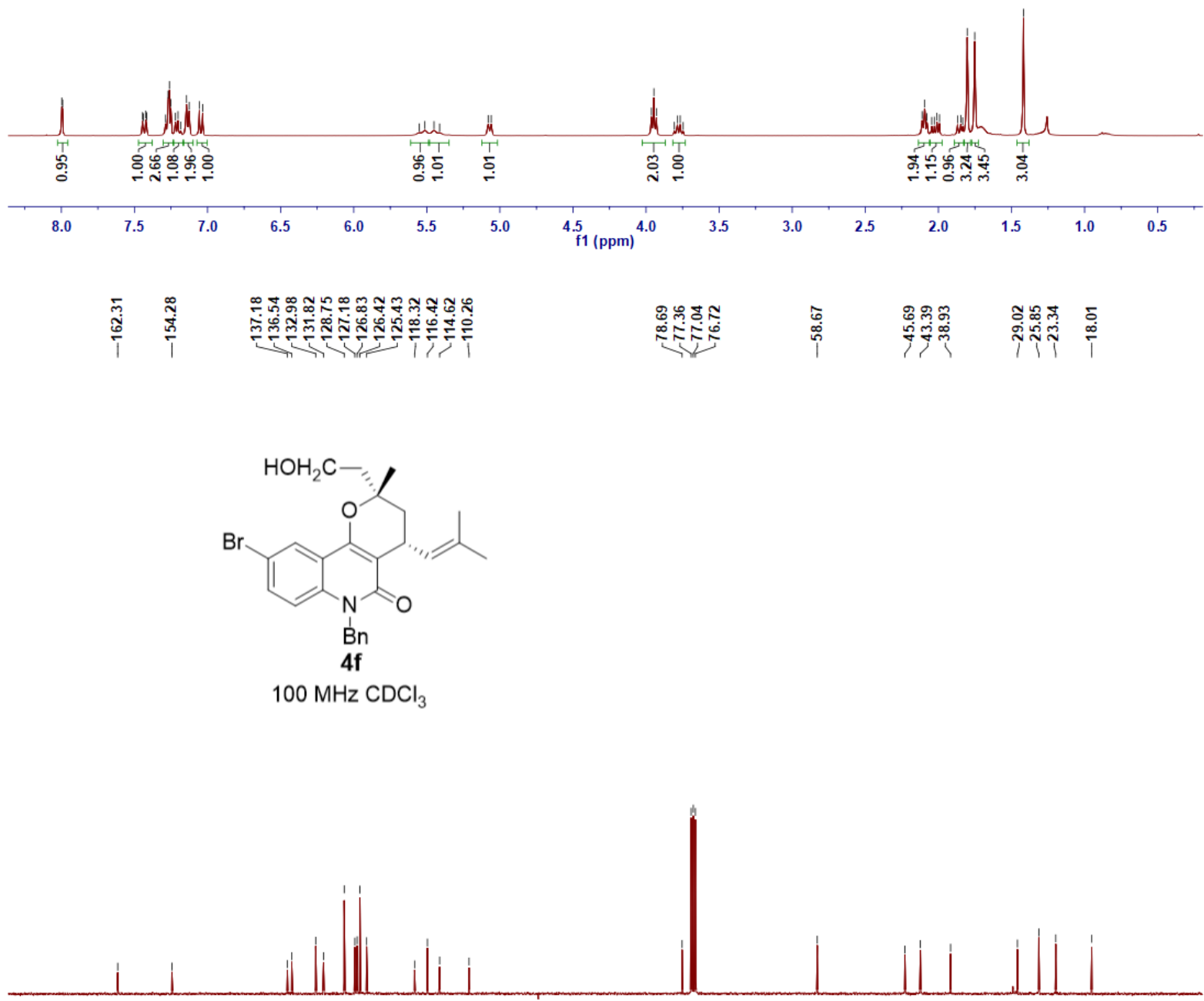

\begin{tabular}{|c|c|c|c|c|c|c|c|c|c|c|c|c|c|c|c|c|}
\hline 170 & 160 & 150 & 140 & 130 & 120 & 110 & 100 & $\begin{array}{c}90 \\
\mathrm{f} 1(\mathrm{ppm})\end{array}$ & 80 & 70 & 60 & 50 & 40 & 30 & 20 & 10 \\
\hline
\end{tabular}


(2R,4R)-6-benzyl-9-bromo-2-(2-hydroxyethyl)-2-methyl-4-(2-methylprop-1-en-1-yl)-2,3,4,6-tetrahydro-5H-pyrano [3,2-c]quinolin-5-one (4ff)

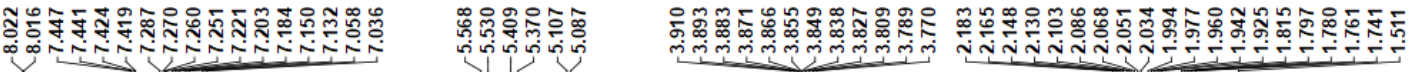
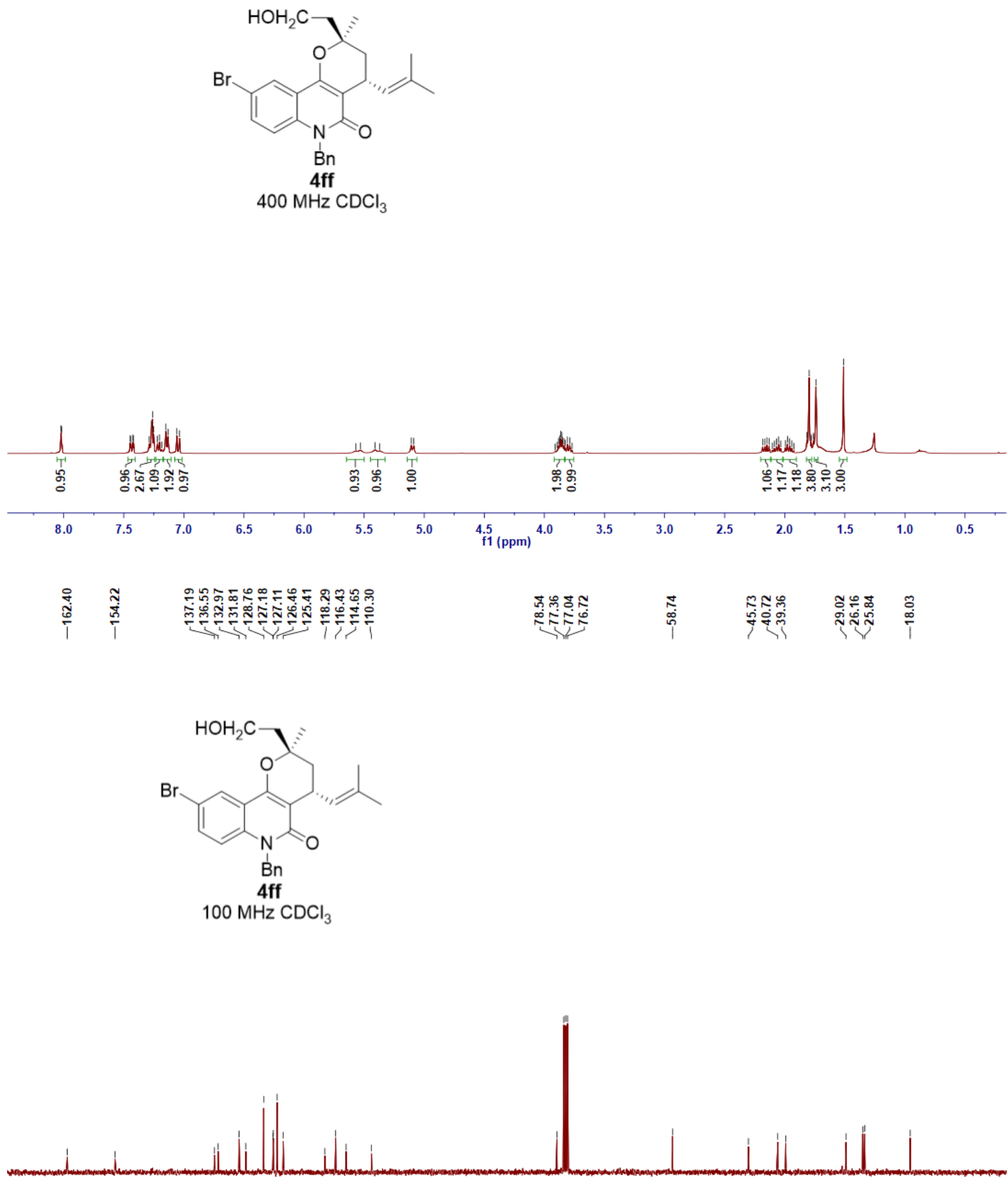

\begin{tabular}{|c|c|c|c|c|c|c|c|c|c|c|c|c|c|c|c|}
\hline 170 & 160 & 150 & 140 & 130 & 120 & 110 & 100 & $\begin{array}{l}90 \\
\mathrm{f} 1(\mathrm{ppm})\end{array}$ & 80 & 70 & 60 & 50 & 40 & 30 & 20 \\
\hline
\end{tabular}


(2S,4R)-6-benzyl-2-(2-hydroxyethyl)-2-methyl-4-(2-methylprop-1-en-1-yl)-9-(trifluoromethyl)-2,3,4,6-tetrahydro-5 H-pyrano[3,2-c]quinolin-5-one (4g)
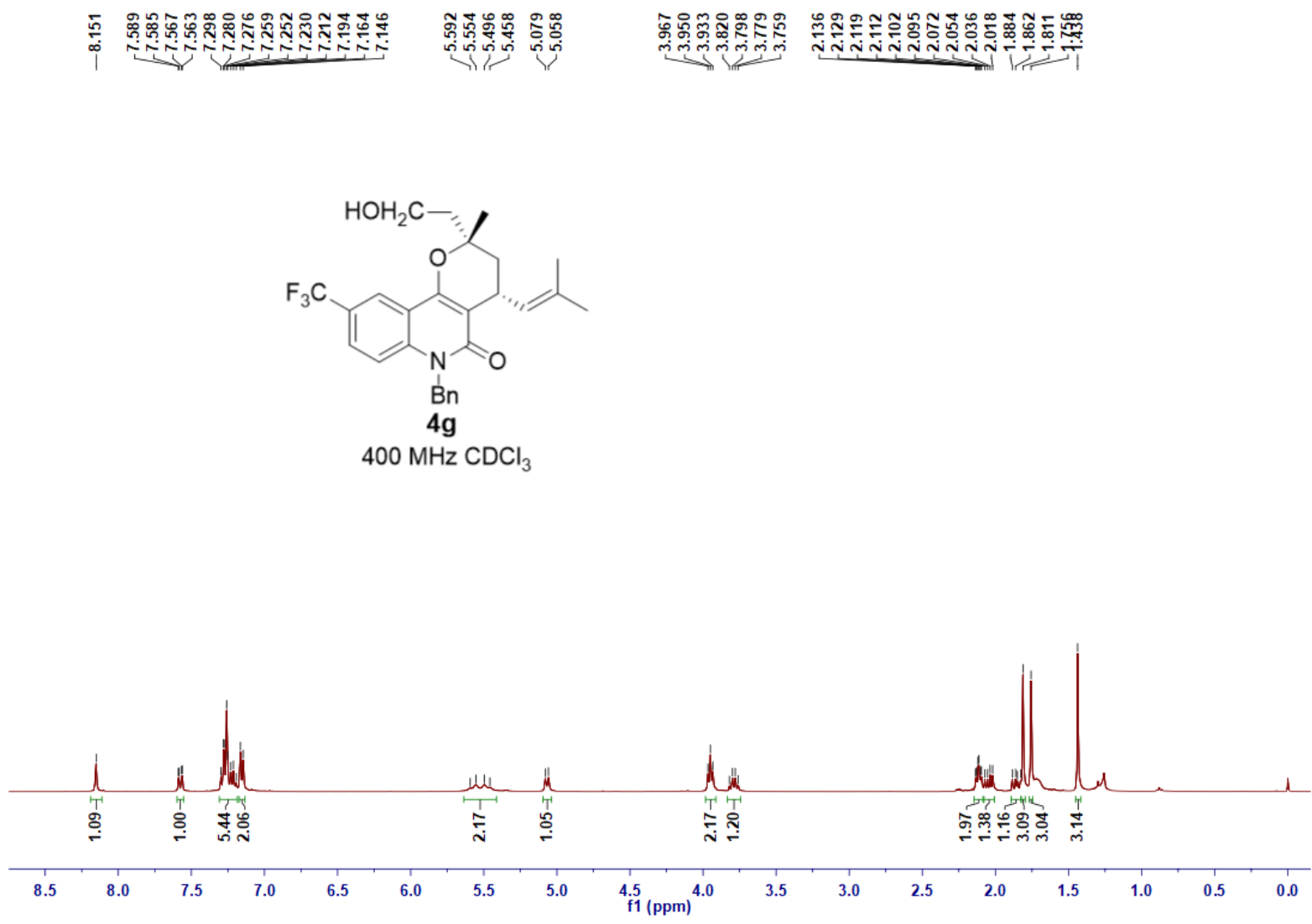

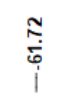

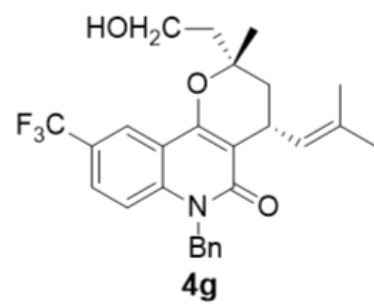

$376 \mathrm{MHz} \mathrm{CDCl}_{3}$

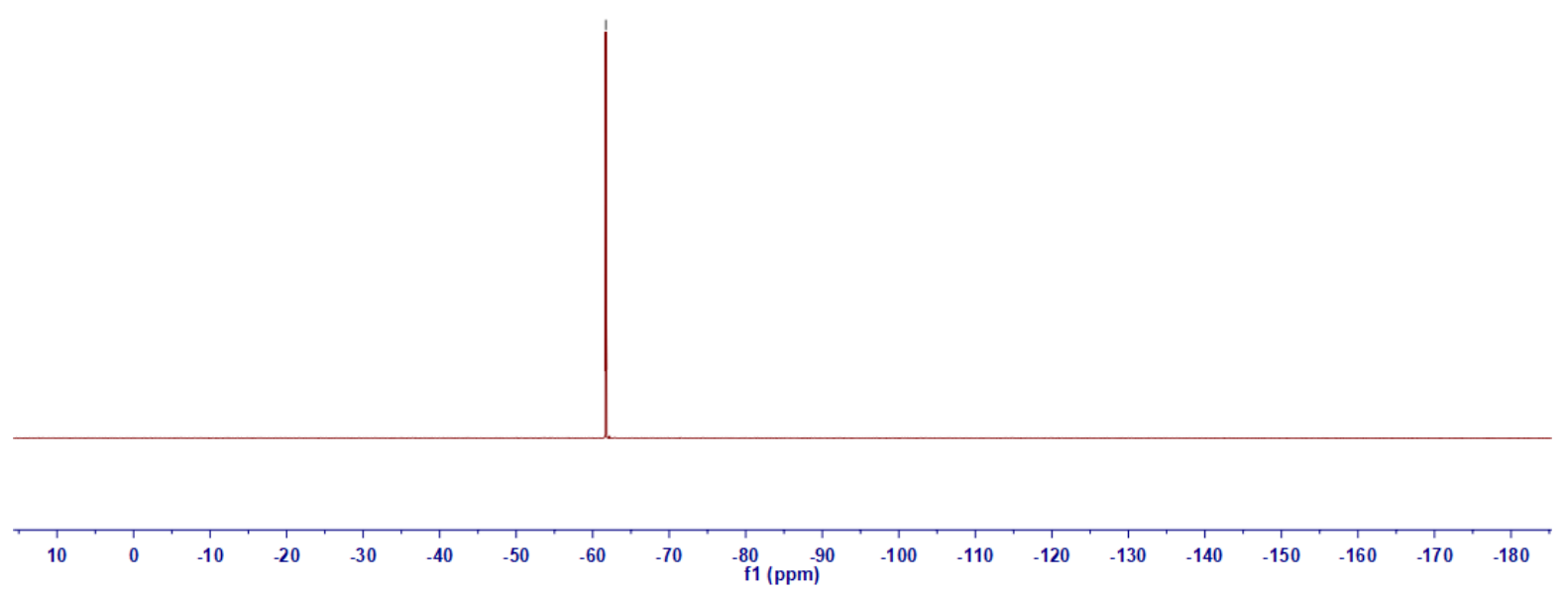




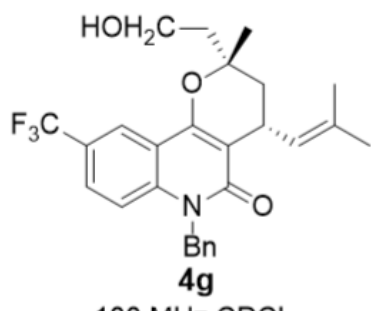

$100 \mathrm{MHz} \mathrm{CDCl}_{3}$

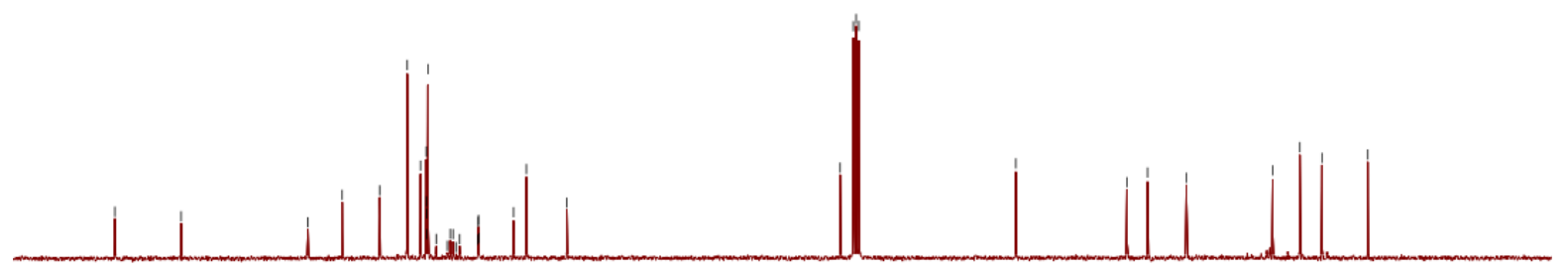

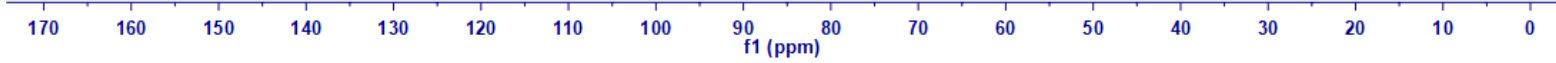

(2R,4R)-6-benzyl-2-(2-hydroxyethyl)-2-methyl-4-(2-methylprop-1-en-1-yl)-9-(trifluoromethyl)-2,3,4,6-tetrahydro5 H-pyrano[3,2-c]quinolin-5-one (4gg)
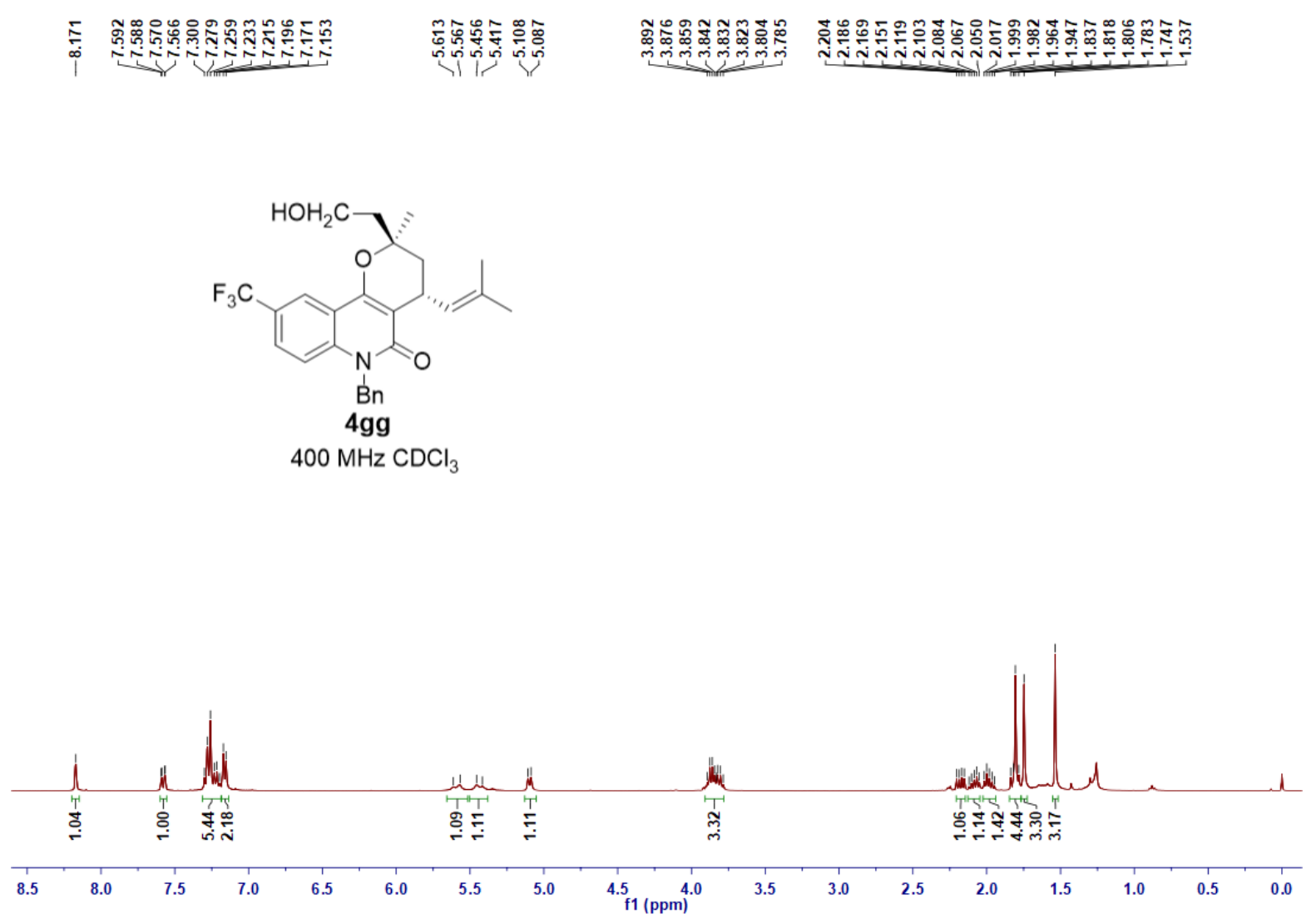
$\stackrel{\substack{0 \\:}}{i}$
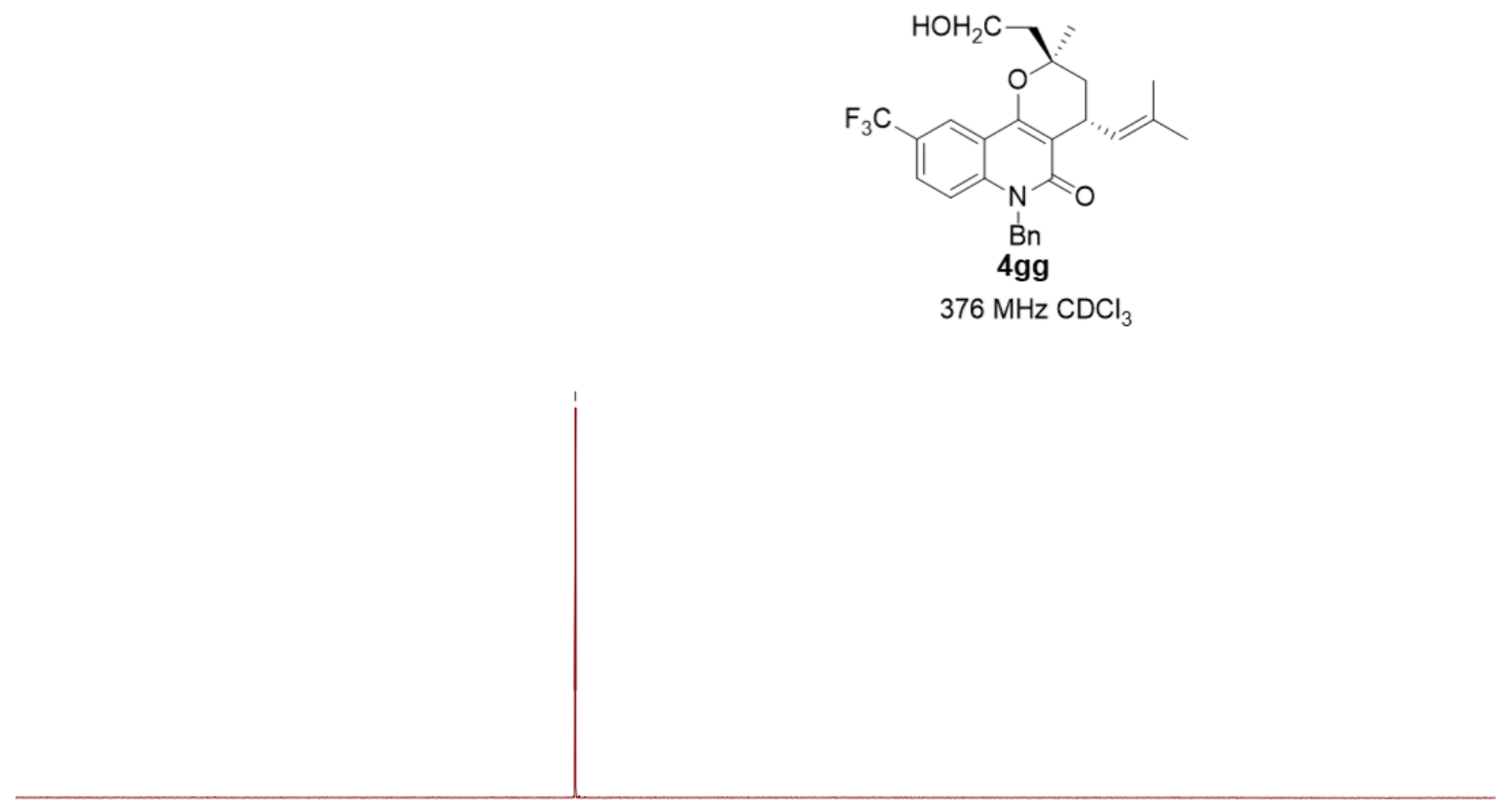

\begin{tabular}{llllllllllllllllllllll}
\hline 10 & 0 & -10 & -20 & -30 & -40 & -50 & -60 & -70 & -80 & -90 & -100 & -110 & -120 & -130 & -140 & -150 & -160 & -170 & -180
\end{tabular}

I

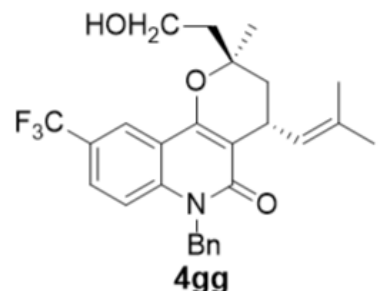

$100 \mathrm{MHz} \mathrm{CDCl}_{3}$

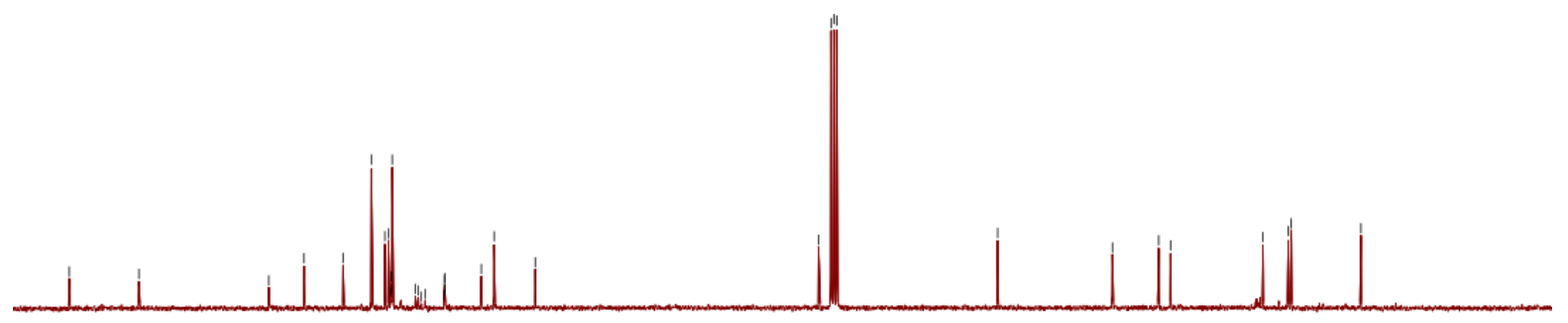

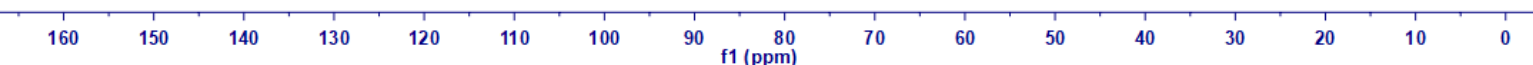


(2S,4R)-6-benzyl-2-(2-hydroxyethyl)-2-methyl-4-(2-methylprop-1-en-1-yl)-9-nitro-2,3,4,6-tetrahydro-5H-pyrano[3, 2-c]quinolin-5-one (4h)
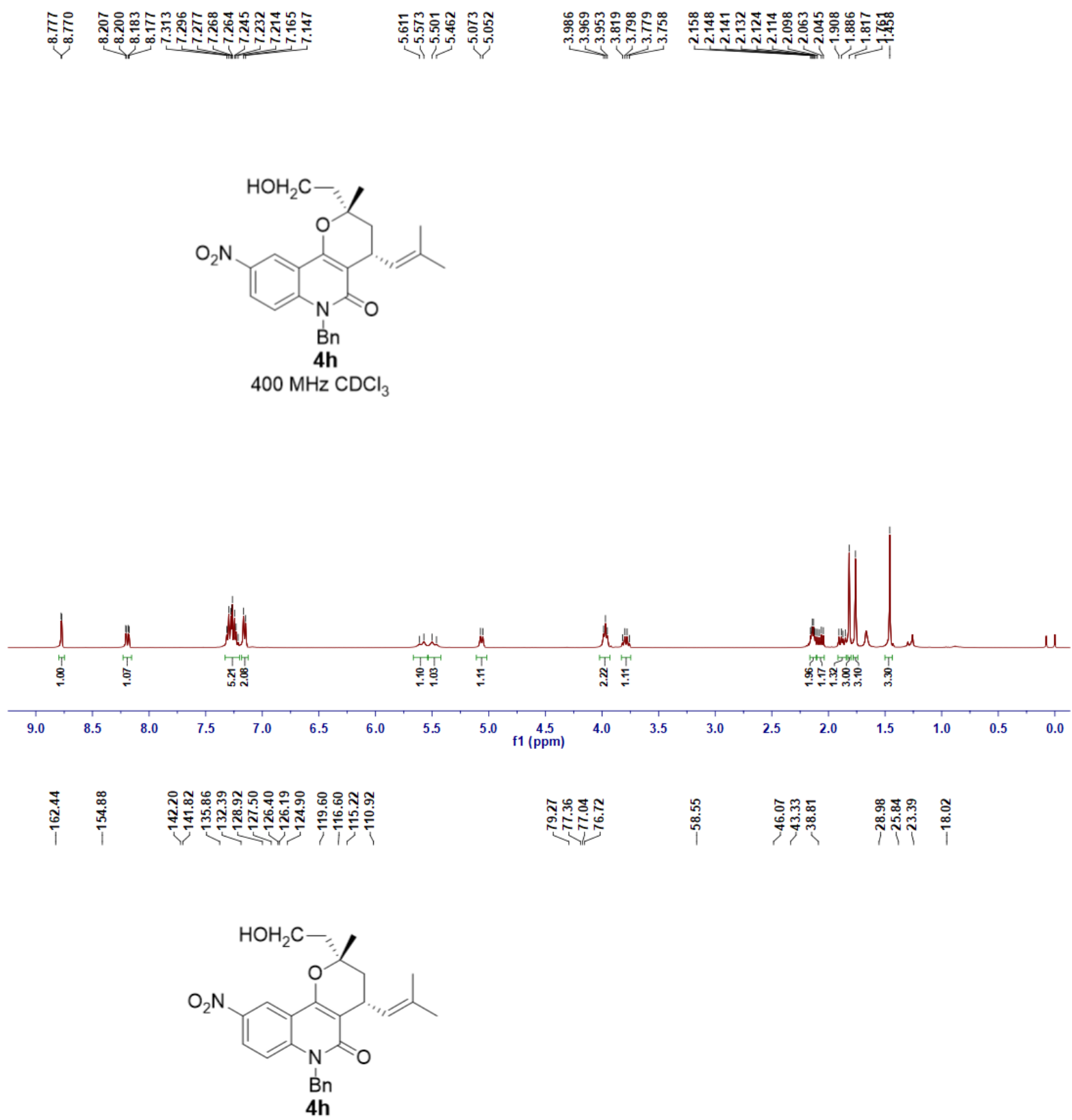

$100 \mathrm{MHz} \mathrm{CDCl}_{3}$

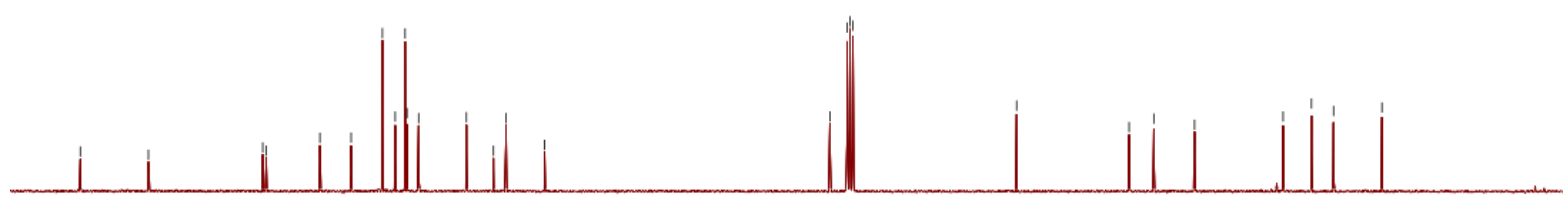

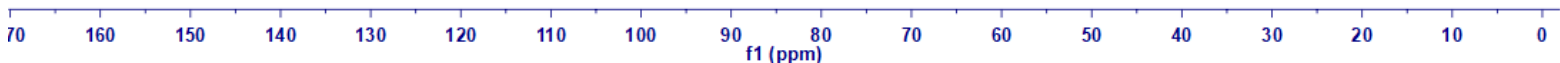


(2R,4R)-6-benzyl-2-(2-hydroxyethyl)-2-methyl-4-(2-methylprop-1-en-1-yl)-9-nitro-2,3,4,6-tetrahydro-5H-pyrano[3, 2-c]quinolin-5-one (4hh)

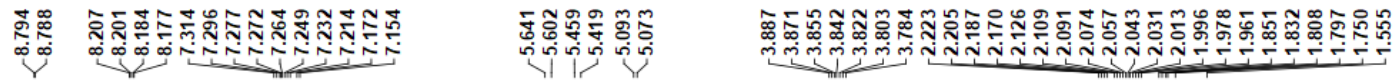

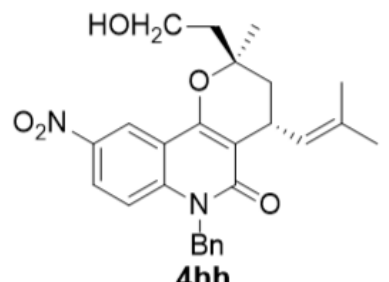

$400 \mathrm{MHz} \mathrm{CDCl}_{3}$
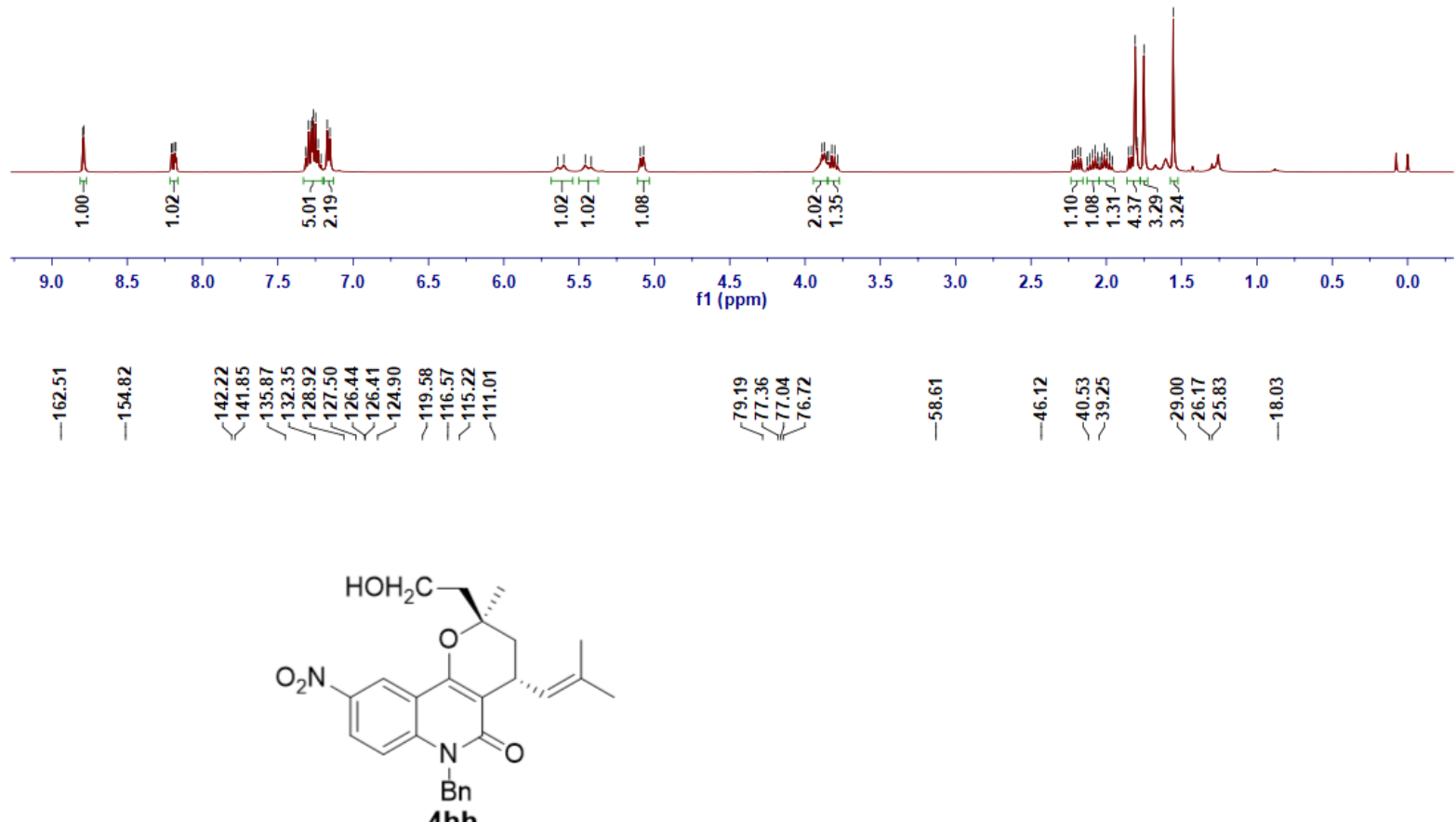

4hh

$100 \mathrm{MHz} \mathrm{CDCl}_{3}$

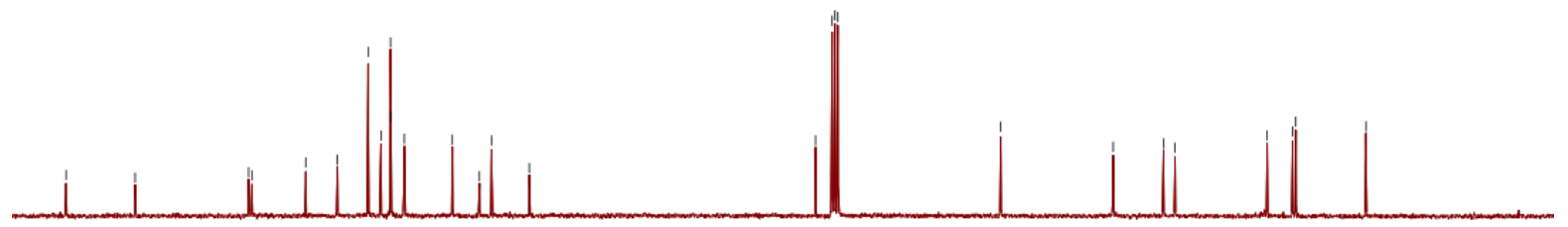

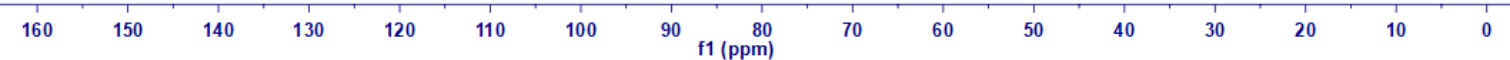


(2S,4R)-6-benzyl-2-(2-hydroxyethyl)-2,9-dimethyl-4-(2-methylprop-1-en-1-yl)-2,3,4,6-tetrahydro-5H-pyrano[3,2c]quinolin-5-one (4i)
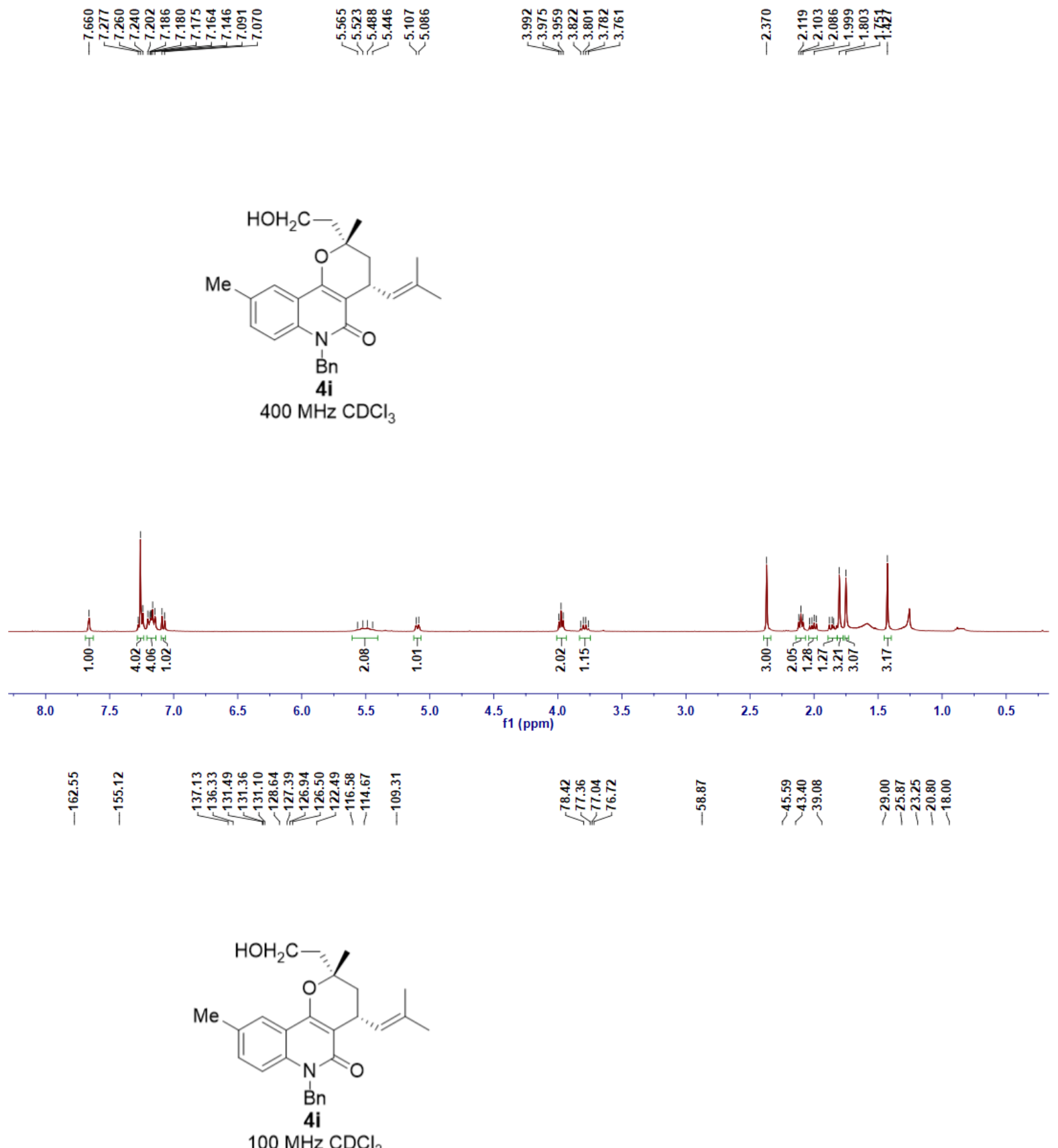

$100 \mathrm{MHz} \mathrm{CDCl}_{3}$

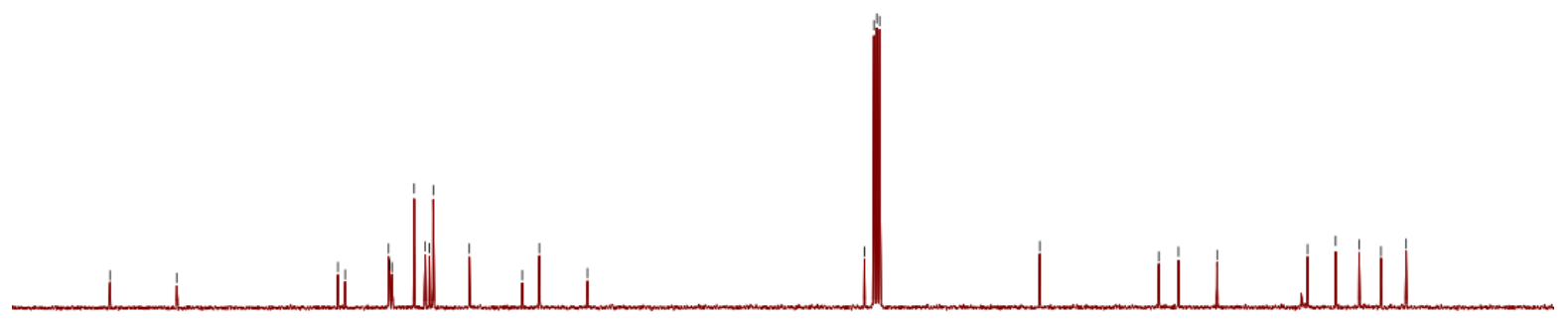

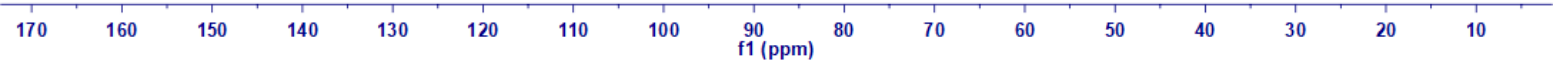


(2R,4R)-6-benzyl-2-(2-hydroxyethyl)-2,9-dimethyl-4-(2-methylprop-1-en-1-yl)-2,3,4,6-tetrahydro-5H-pyrano[3,2c]quinolin-5-one (4ii)
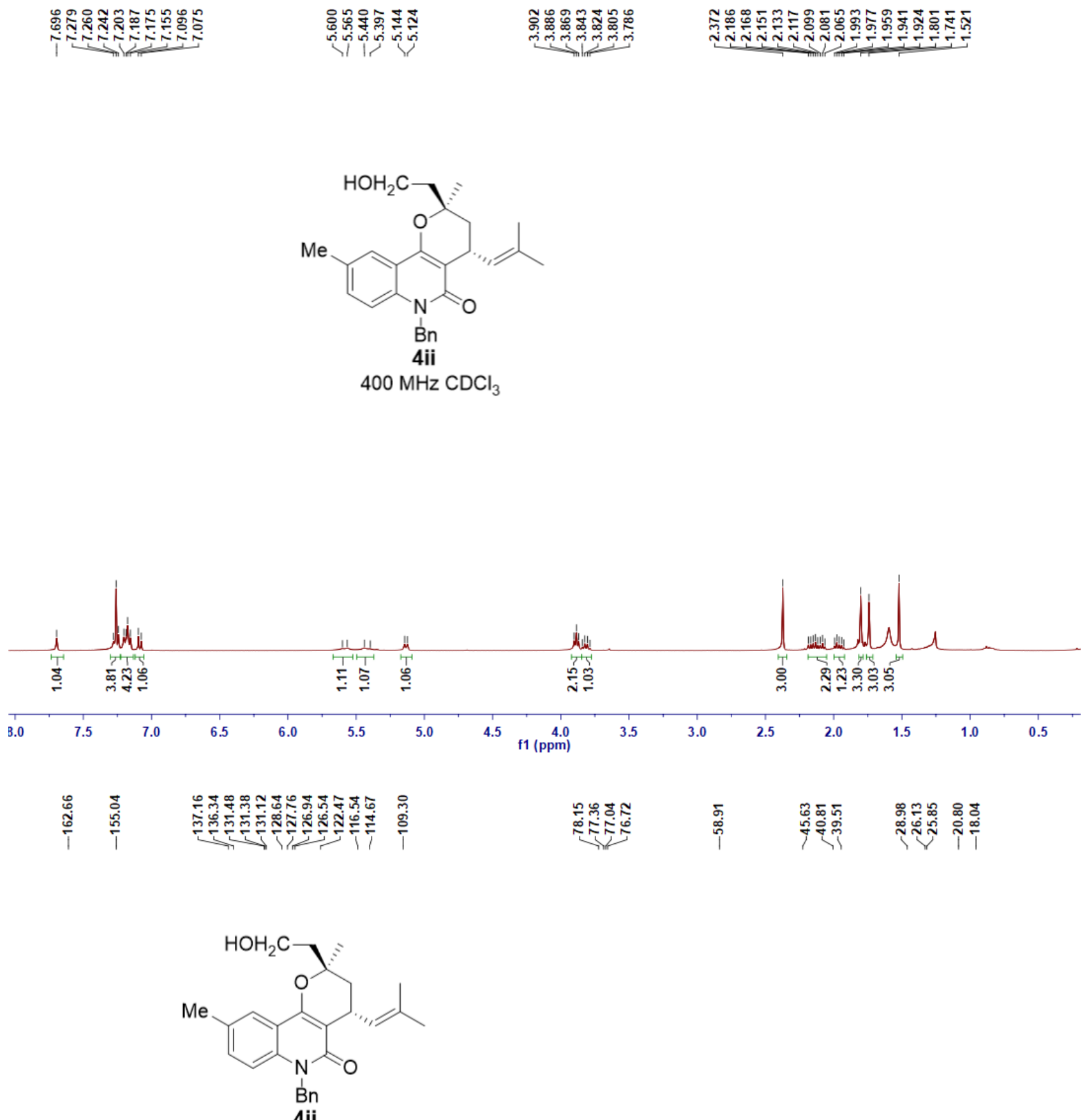

$100 \mathrm{MHz} \mathrm{CDCl}_{3}$

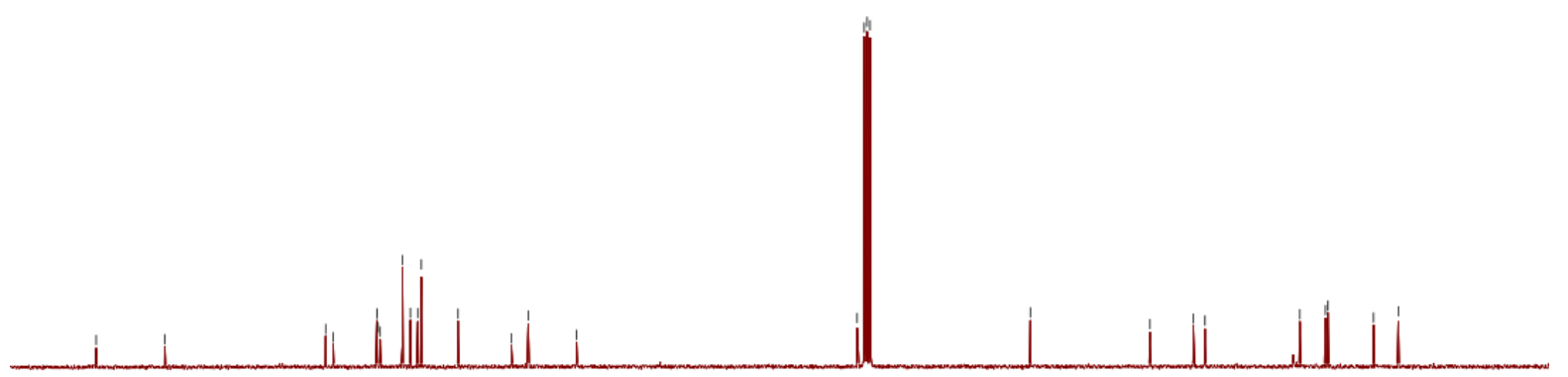

\begin{tabular}{|c|c|c|c|c|c|c|c|c|c|c|c|c|c|c|c|}
\hline 170 & 160 & 150 & 140 & 130 & 120 & 110 & 100 & $\begin{array}{l}90 \\
\text { f1 (ppm) }\end{array}$ & 80 & 70 & 60 & 50 & 40 & 30 & 20 \\
\hline
\end{tabular}


(2S,4R)-6-benzyl-2-(2-hydroxyethyl)-9-isopropyl-2-methyl-4-(2-methylprop-1-en-1-yl)-2,3,4,6-tetrahydro-5Hpyrano[3,2-c]quinolin-5-one $(4 \mathbf{j})$

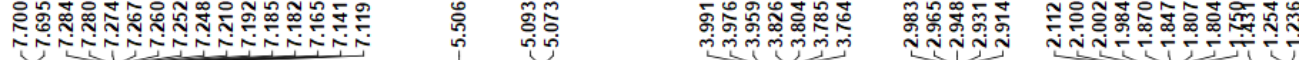<smiles>CC[C@]1(C)C[C@H](C=C(C)C)c2c(c3cc(C(C)C)ccc3n(Cc3ccccc3)c2=O)O1</smiles>

4j

$400 \mathrm{MHz} \mathrm{CDCl}_{3}$

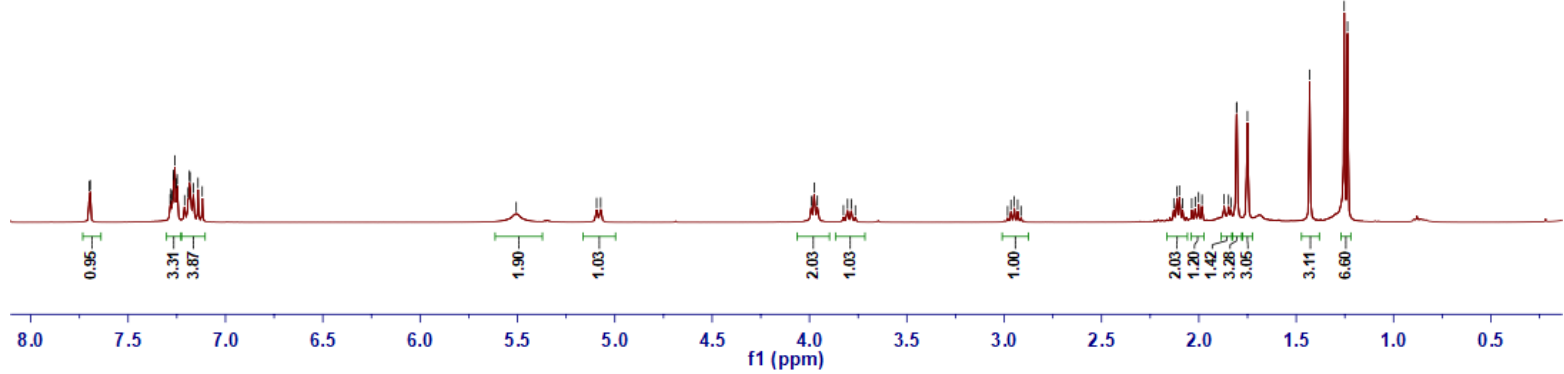

常
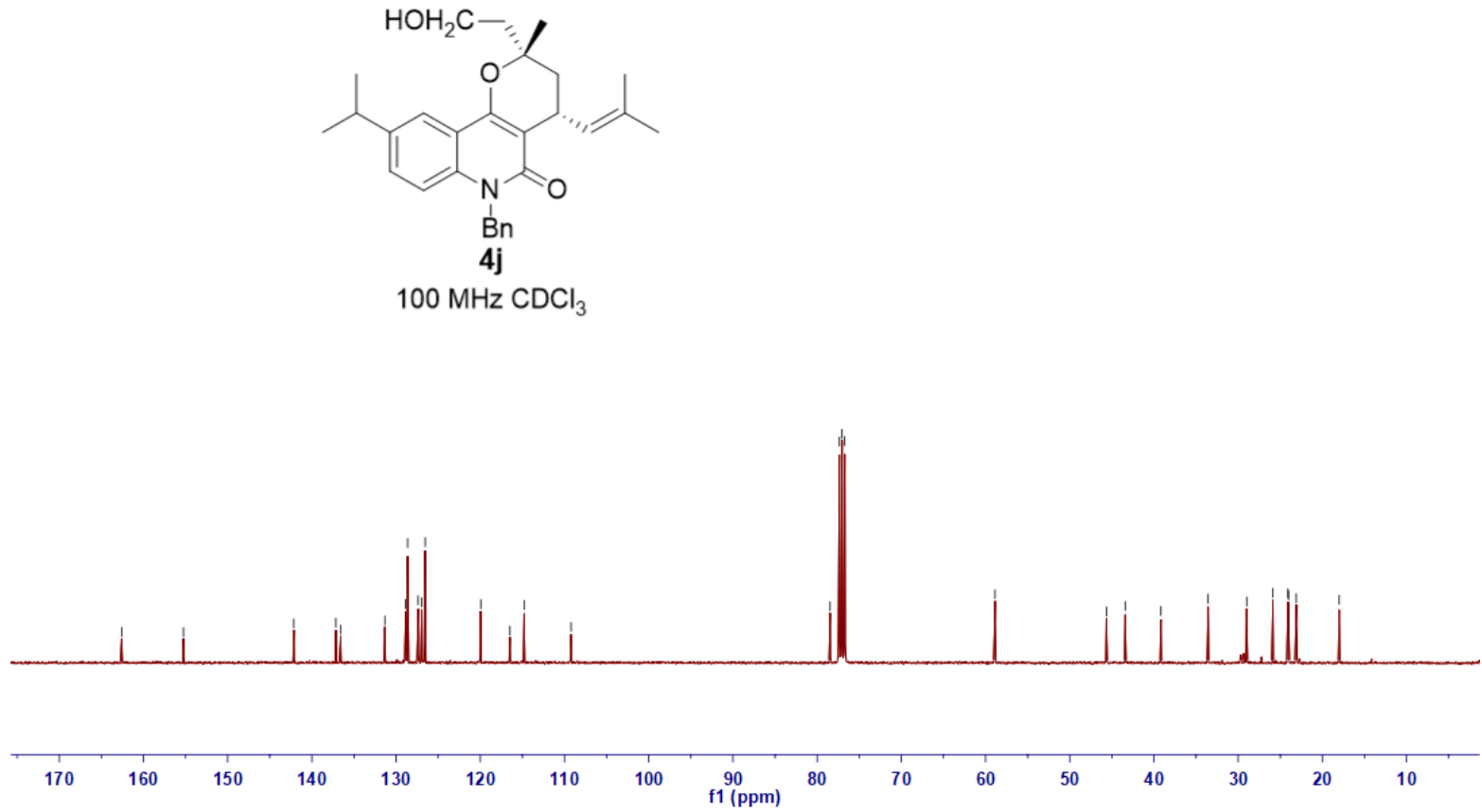
$(2 R, 4 R)-6$-benzyl-2-(2-hydroxyethyl)-9-isopropyl-2-methyl-4-(2-methylprop-1-en-1-yl)-2,3,4,6-tetrahydro-5Hpyrano[3,2-c]quinolin-5-one $(4 \mathbf{j j})$

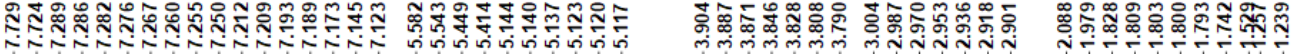<smiles>CC[C@]1(C)C[C@H](C=C(C)C)c2c(c3cc(C(C)C)ccc3n(Cc3ccccc3)c2=O)O1</smiles>

4jj

$400 \mathrm{MHz} \mathrm{CDCl}_{3}$
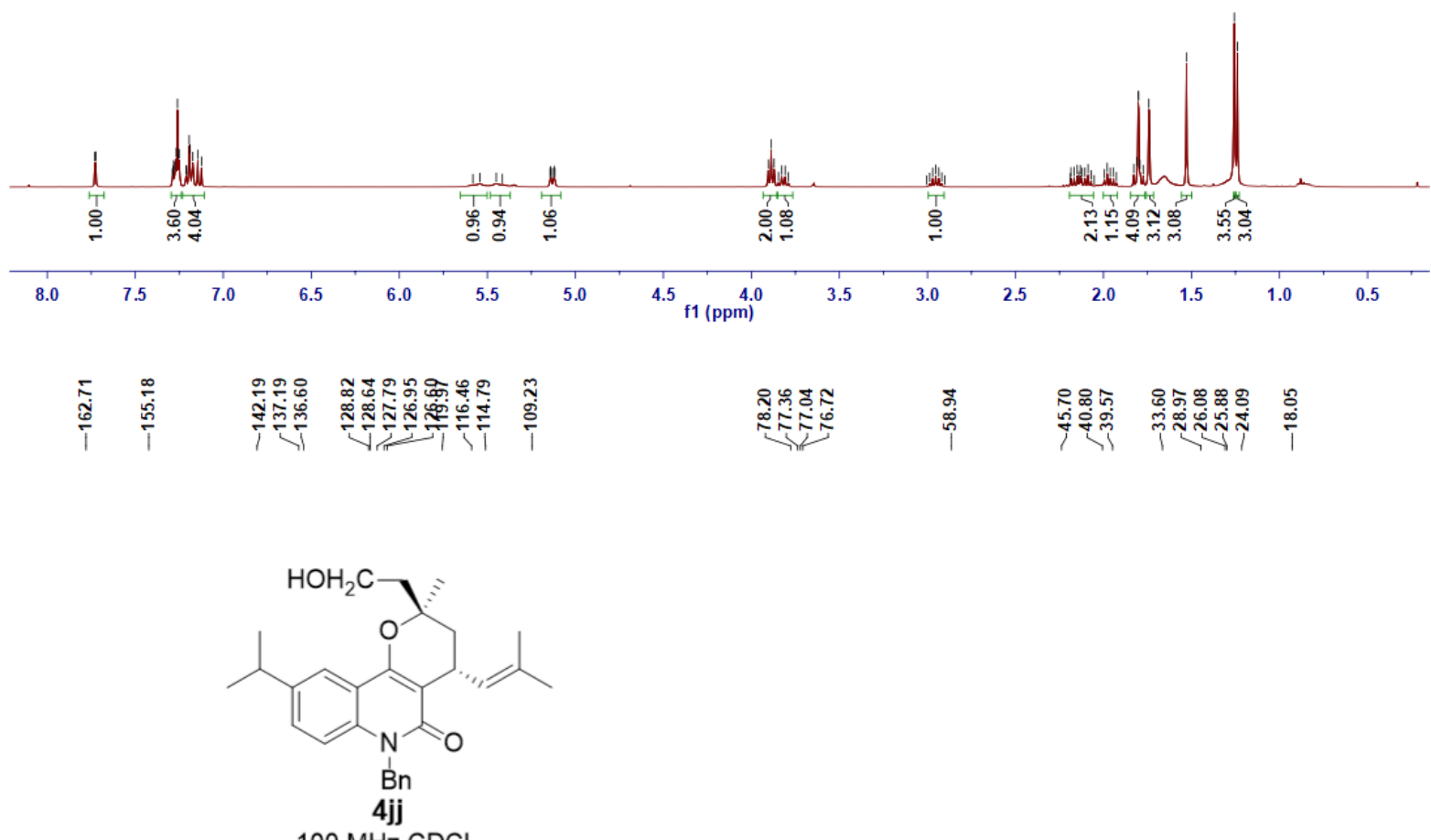

$100 \mathrm{MHz} C D C l_{3}$

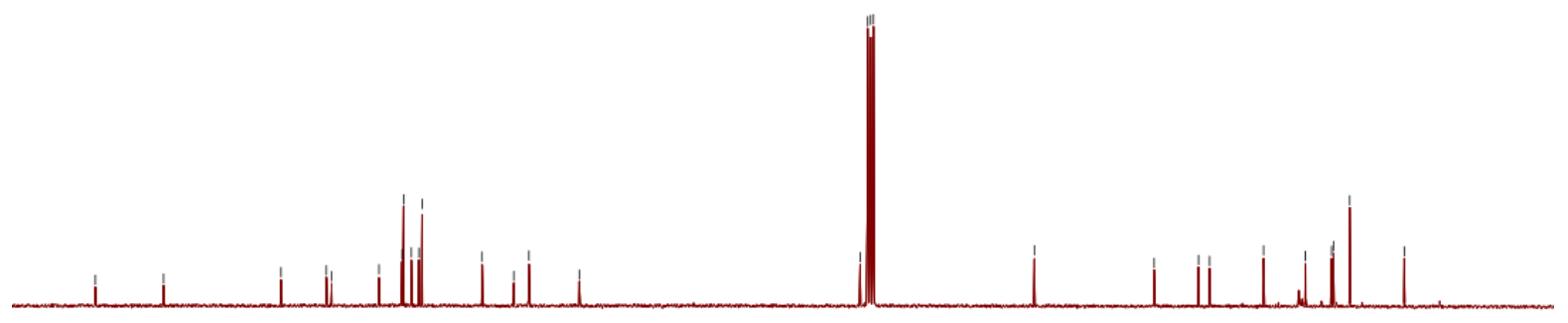

$160 \quad 150 \quad 140$

$130 \quad 120$

$110 \quad 100$

${ }_{\mathrm{f} 1(\mathrm{ppm})}^{80}$ 
(2S,4R)-6-benzyl-9-(tert-butyl)-2-(2-hydroxyethyl)-2-methyl-4-(2-methylprop-1-en-1-yl)-2,3,4,6-tetrahydro-5Hpyrano[3,2-c]quinolin-5-one (4k)
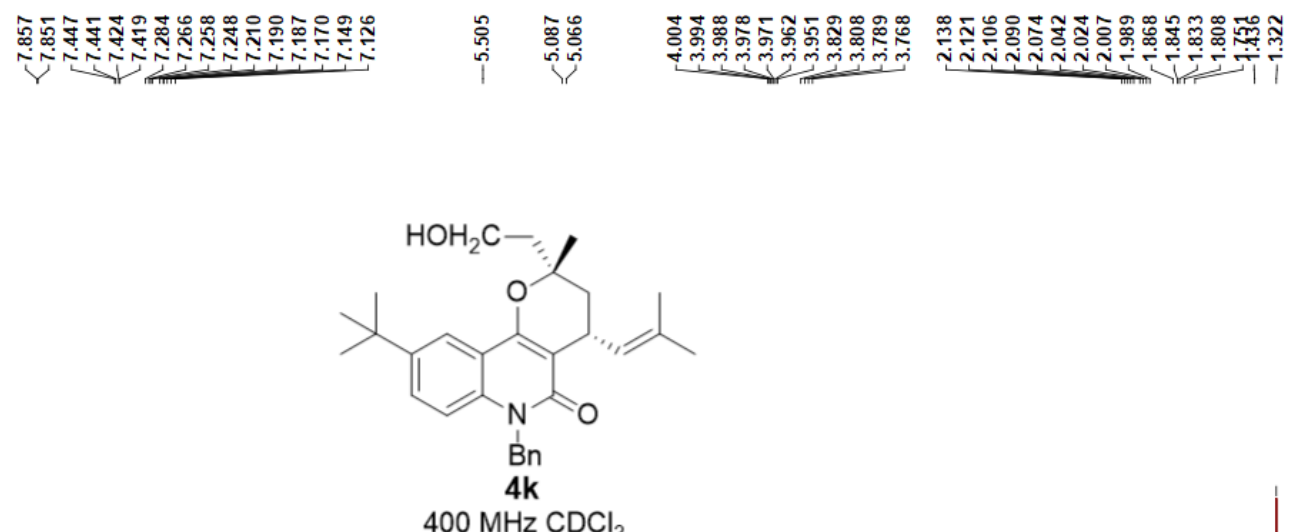

$400 \mathrm{MHz} \mathrm{CDCl}_{3}$
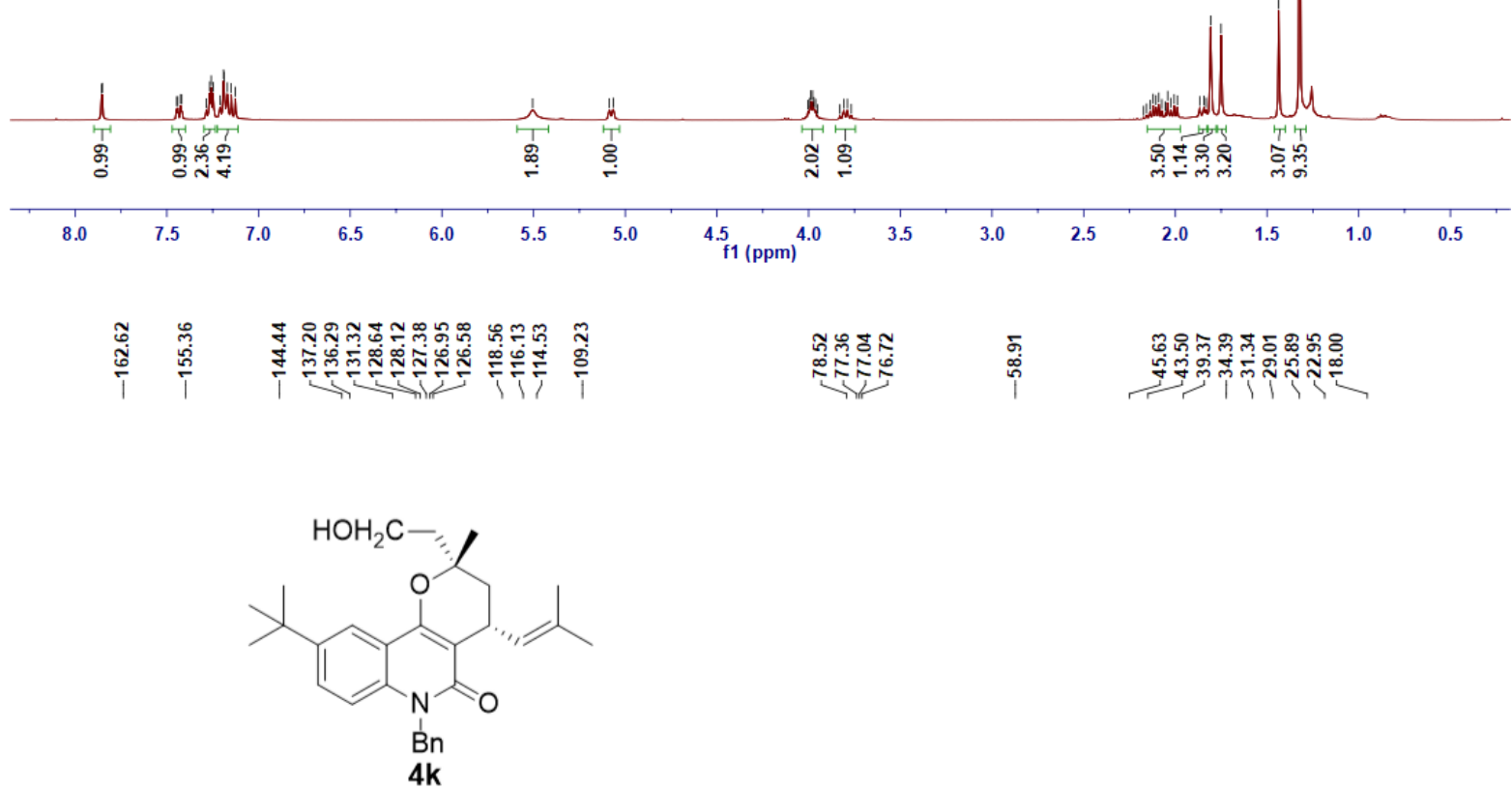

$100 \mathrm{MHz} \mathrm{CDCl}_{3}$

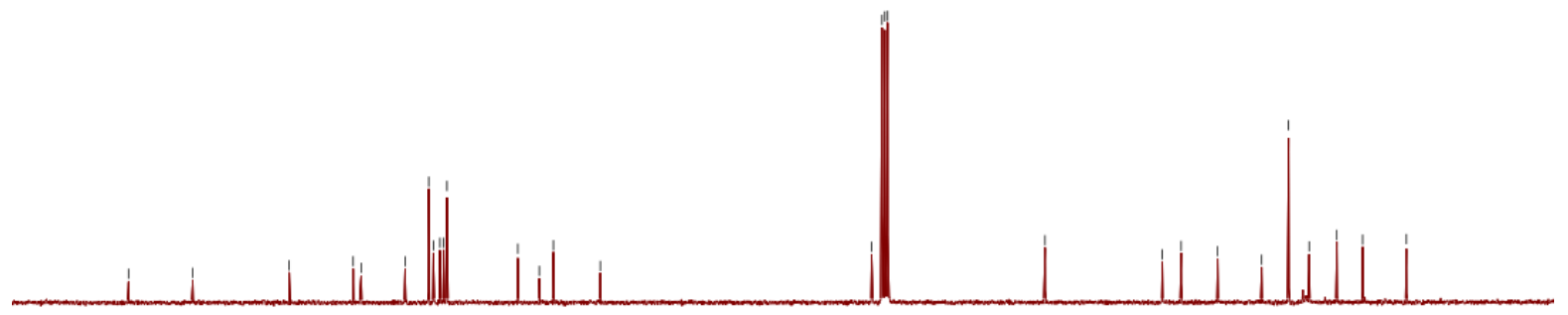

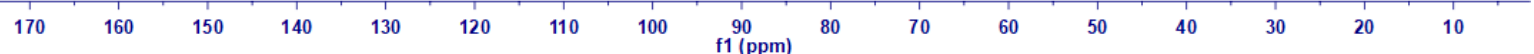




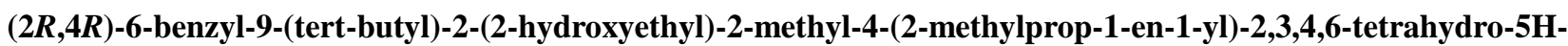
pyrano[3,2-c]quinolin-5-one (4kk)

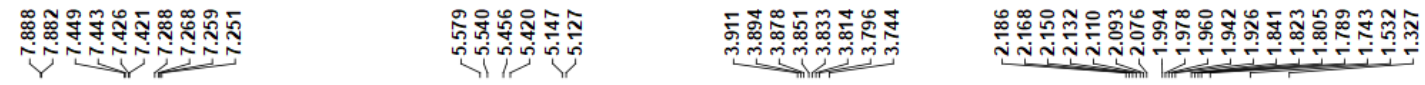

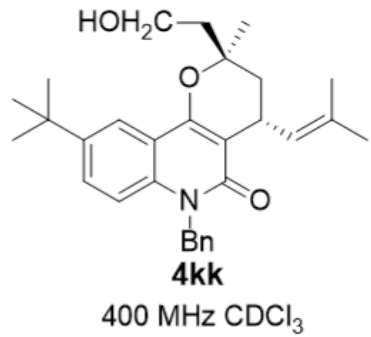

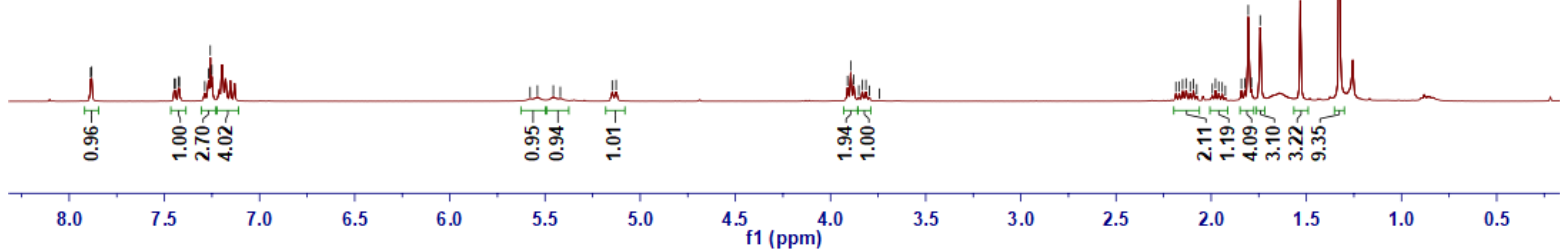

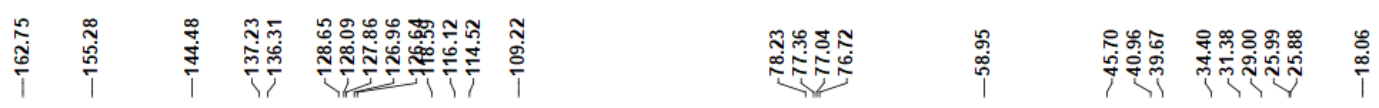<smiles>CC[C@]1(C)C[C@H](C=C(C)C)c2c(c3cc(C(C)(C)C)ccc3n(Cc3ccccc3)c2=O)O1</smiles>

4kk

$100 \mathrm{MHz} \mathrm{CDCl}_{3}$

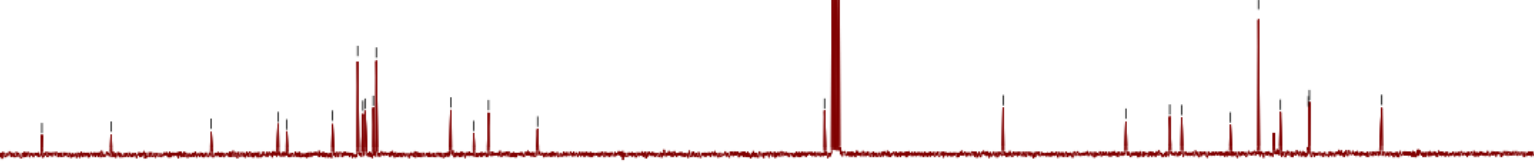

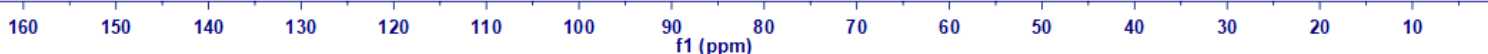


(2S,4R)-6-benzyl-2-(2-hydroxyethyl)-2-methyl-4-(2-methylprop-1-en-1-yl)-9-phenyl-2,3,4,6-tetrahydro-5H-pyrano [3,2-c]quinolin-5-one (41)

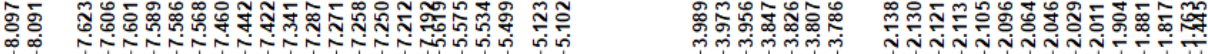

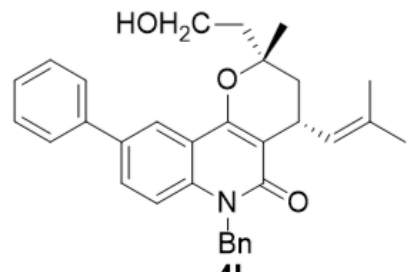

$400 \mathrm{MHz} \mathrm{CDCl}_{3}$

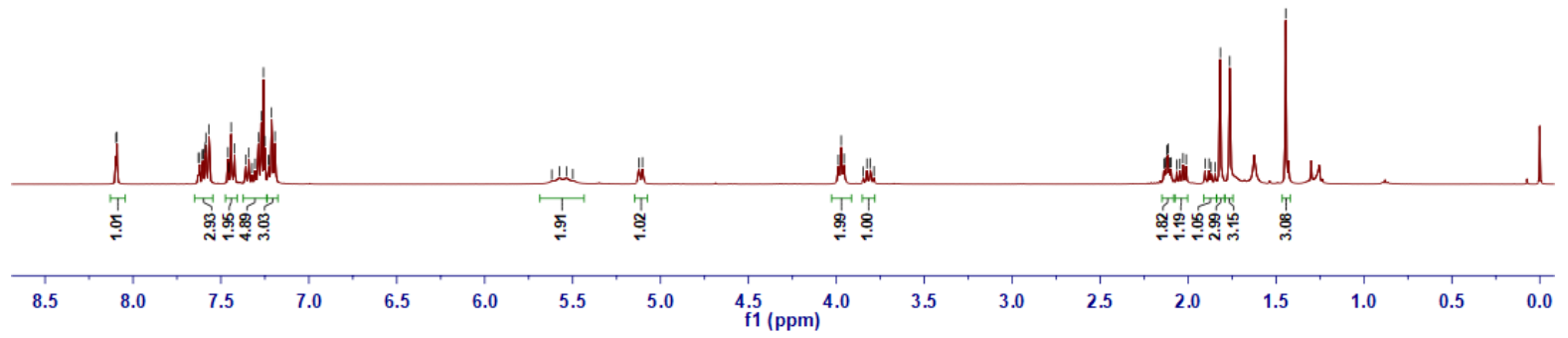

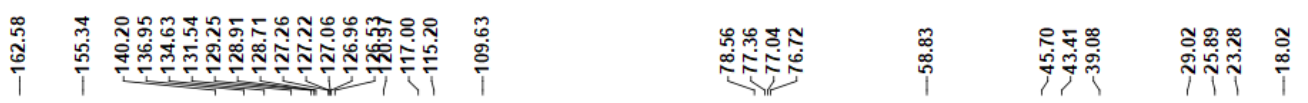
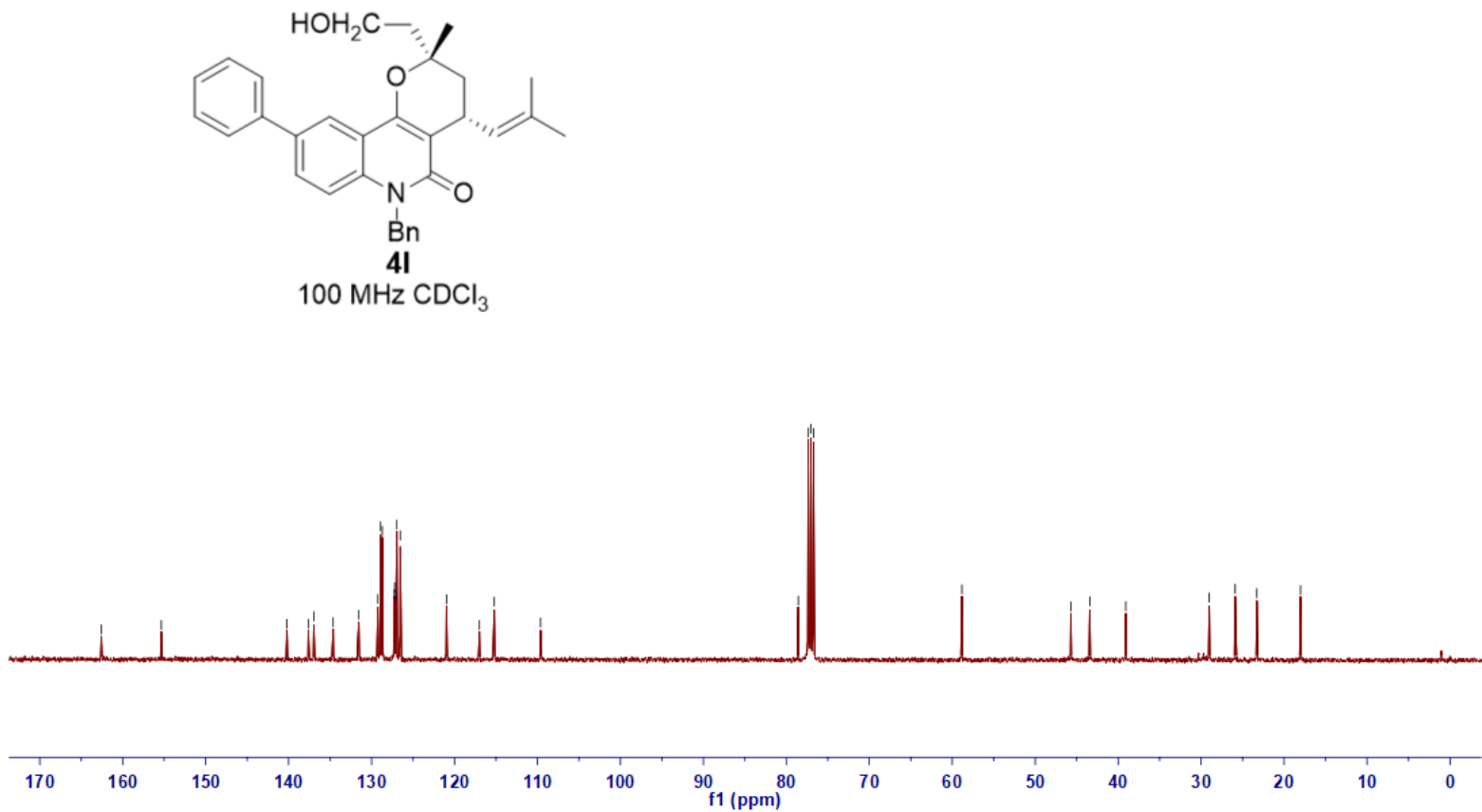
(2R,4R)-6-benzyl-2-(2-hydroxyethyl)-2-methyl-4-(2-methylprop-1-en-1-yl)-9-phenyl-2,3,4,6-tetrahydro-5H-pyrano $[3,2-c] q u i n o l i n-5-o n e(411)$
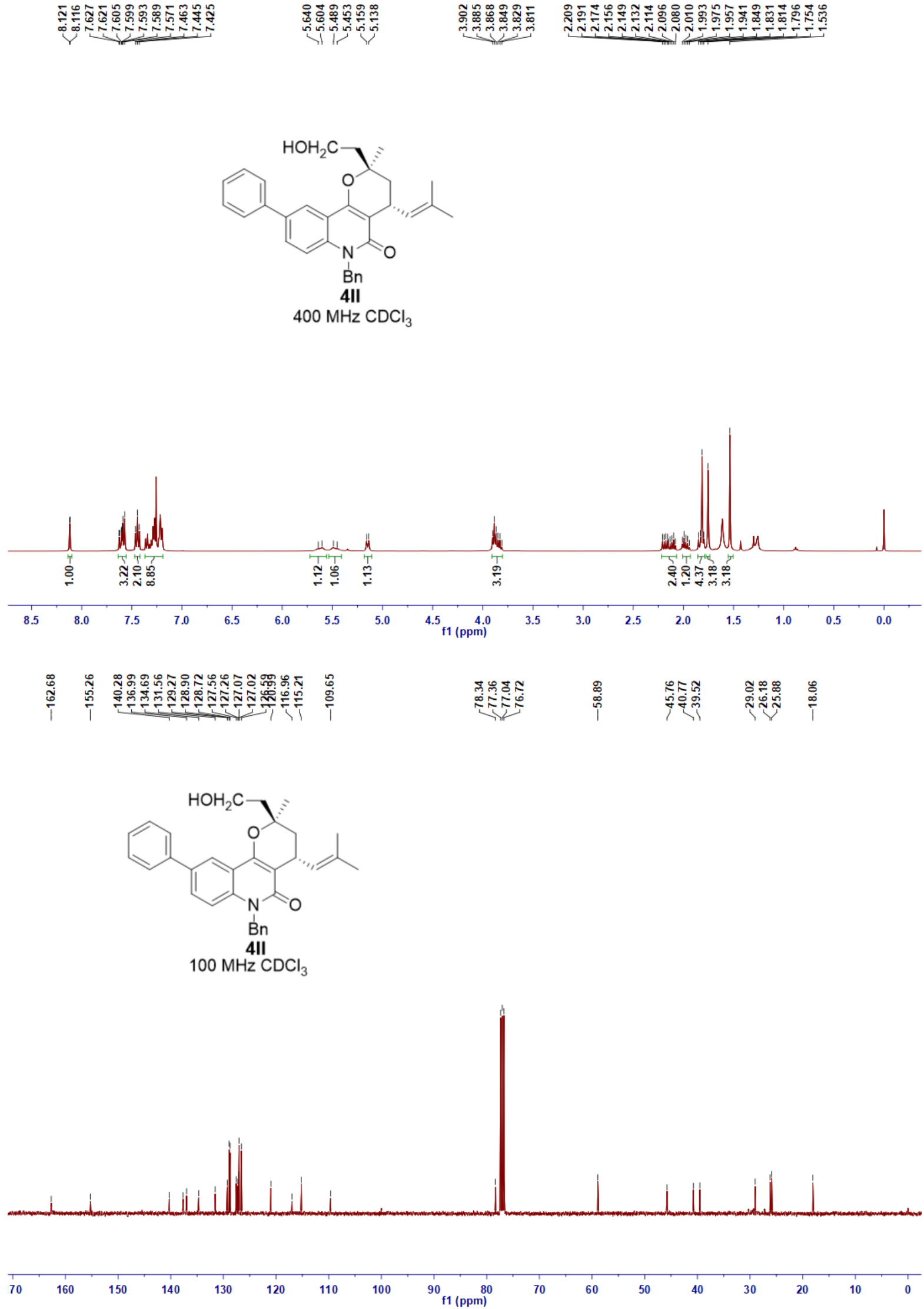
(2S,4R)-6-benzyl-2-(2-hydroxyethyl)-9-methoxy-2-methyl-4-(2-methylprop-1-en-1-yl)-2,3,4,6-tetrahydro-5H-pyran o[3,2-c]quinolin-5-one $(4 \mathrm{~m})$

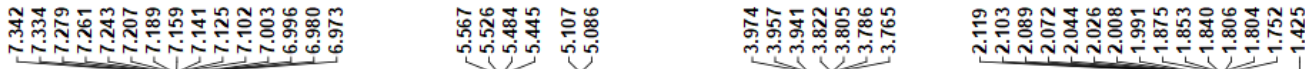

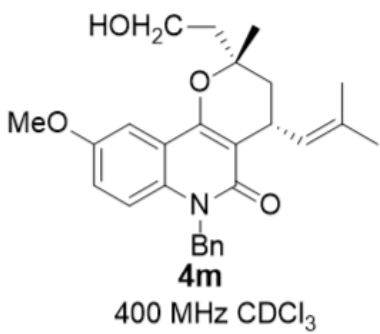

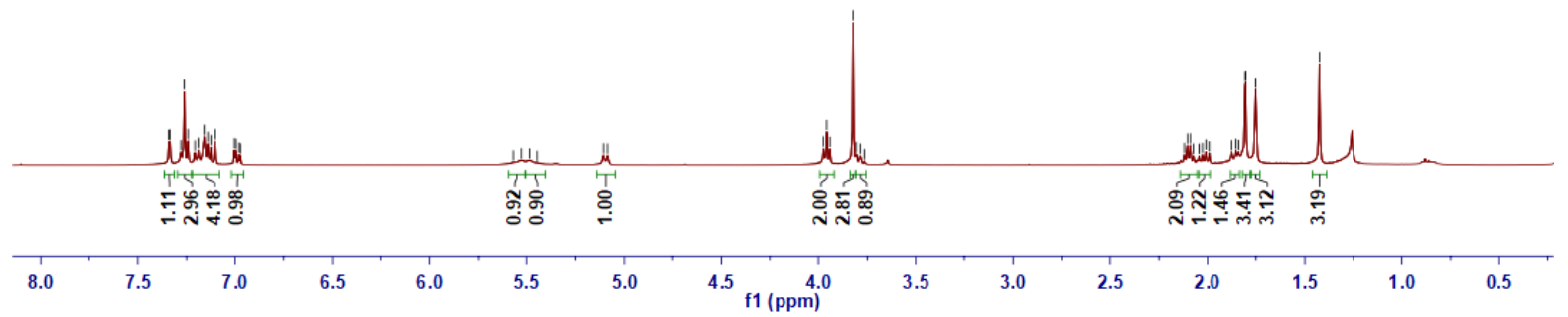

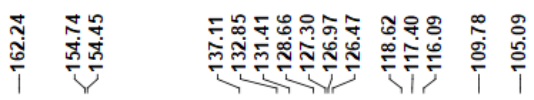

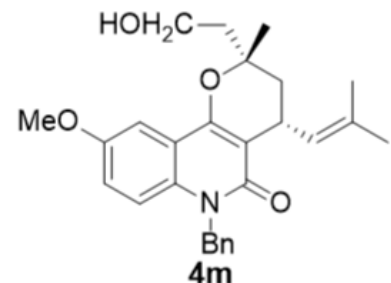

$100 \mathrm{MHz} \mathrm{CDCl}_{3}$

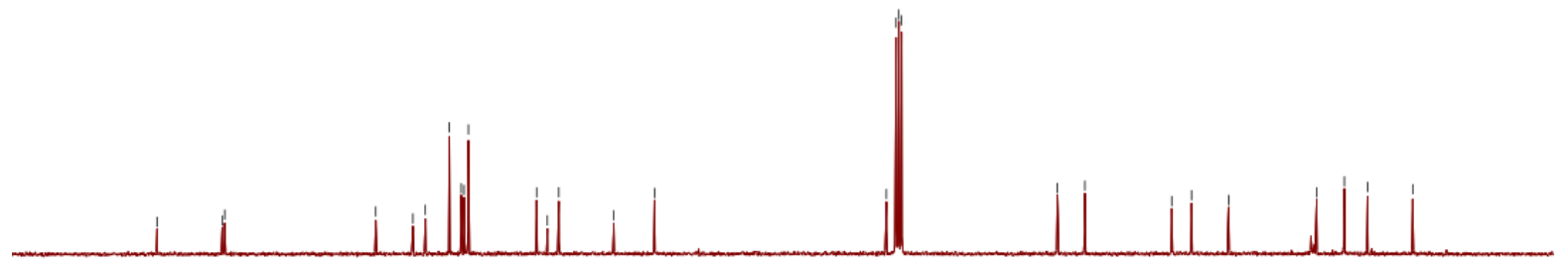

$\begin{array}{lllllllll}170 & 160 & 150 & 140 & 130 & 120 & 110 & 100 & \begin{array}{c}90 \\ \mathrm{f} 1(\mathrm{ppm})\end{array}\end{array}$ 
(2R,4R)-6-benzyl-2-(2-hydroxyethyl)-9-methoxy-2-methyl-4-(2-methylprop-1-en-1-yl)-2,3,4,6-tetrahydro-5H-pyran o[3,2-c]quinolin-5-one $(4 \mathrm{~mm})$

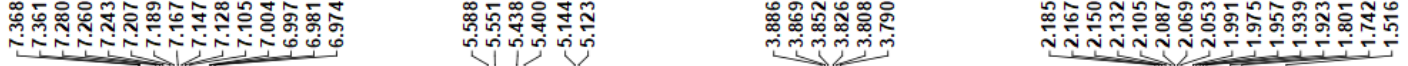

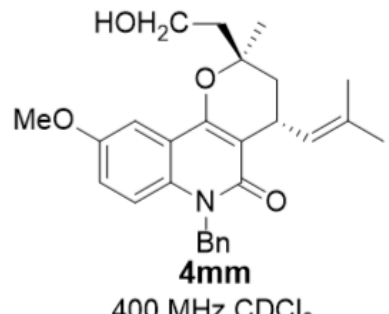

$400 \mathrm{MHz} \mathrm{CDCl}_{3}$

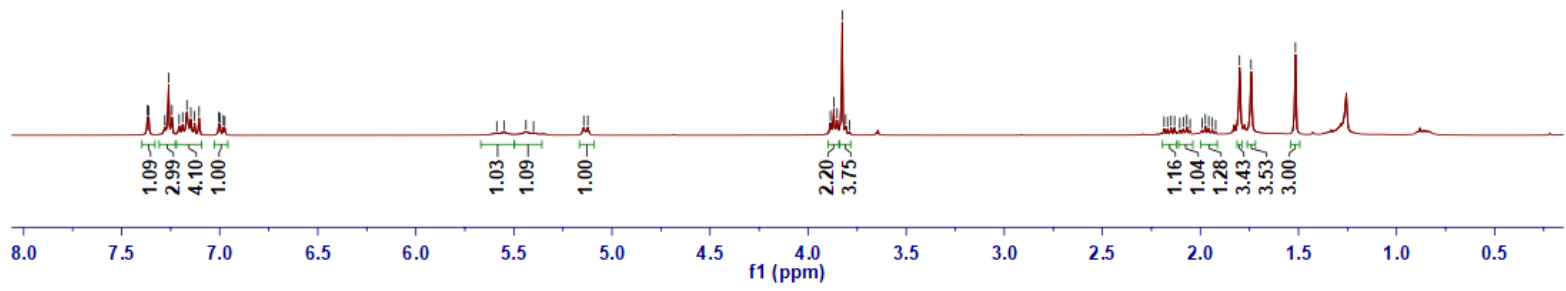

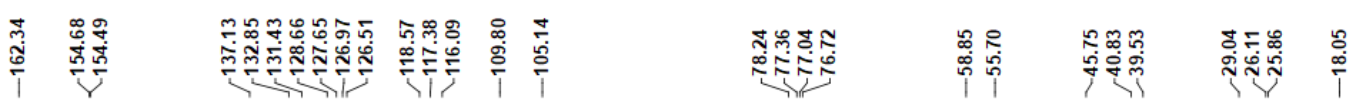
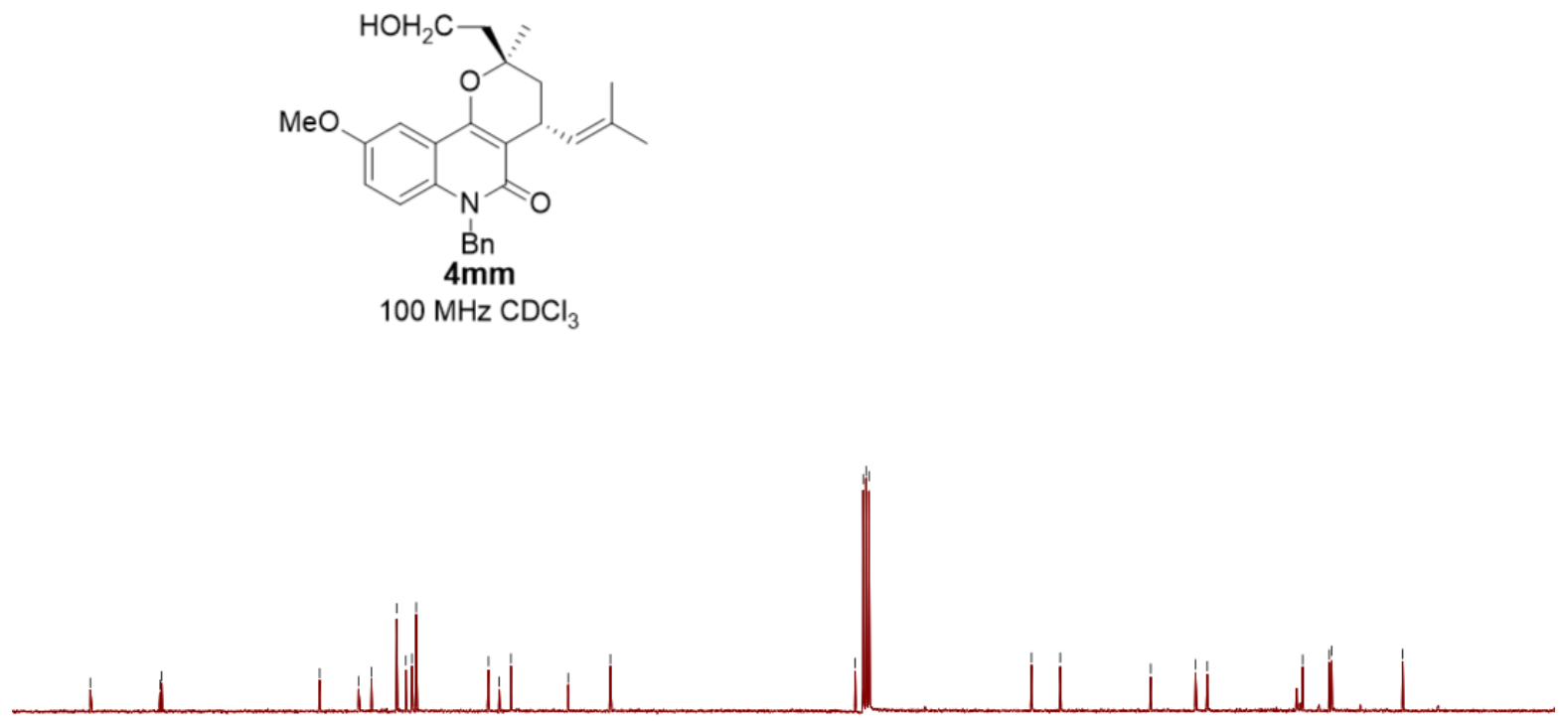

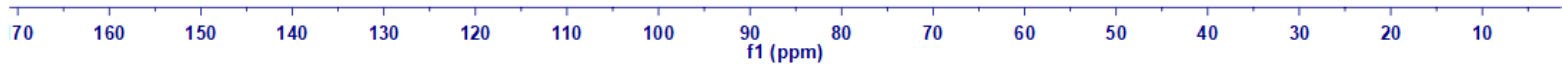


(2S,4R)-2-(2-hydroxyethyl)-7-methoxy-2,6-dimethyl-4-(2-methylprop-1-en-1-yl)-2,3,4,6-tetrahydro-5H-pyrano[3,2c]quinolin-5-one (4n)
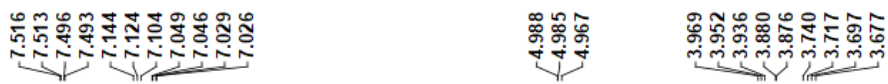

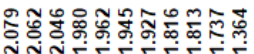

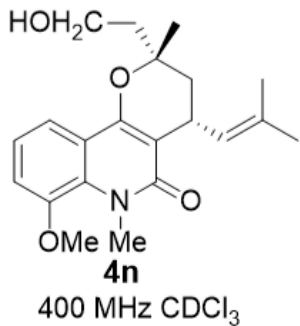

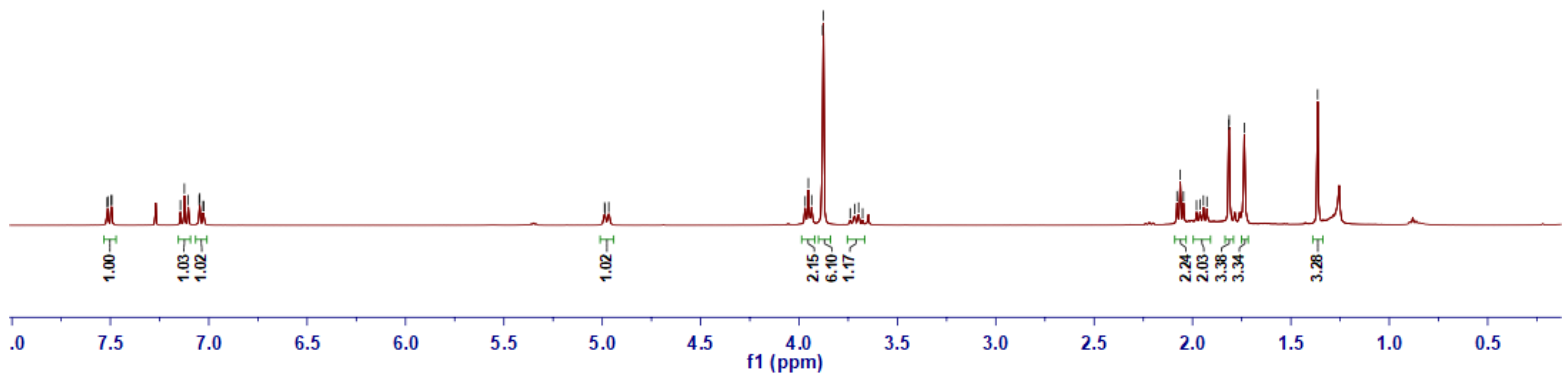

它<smiles>COC(=O)[C@@H](N)n1c(=O)c2c(c3cccc(OC)c31)O[C@](C)(CCO)C[C@@H]2C=C(C)C</smiles>
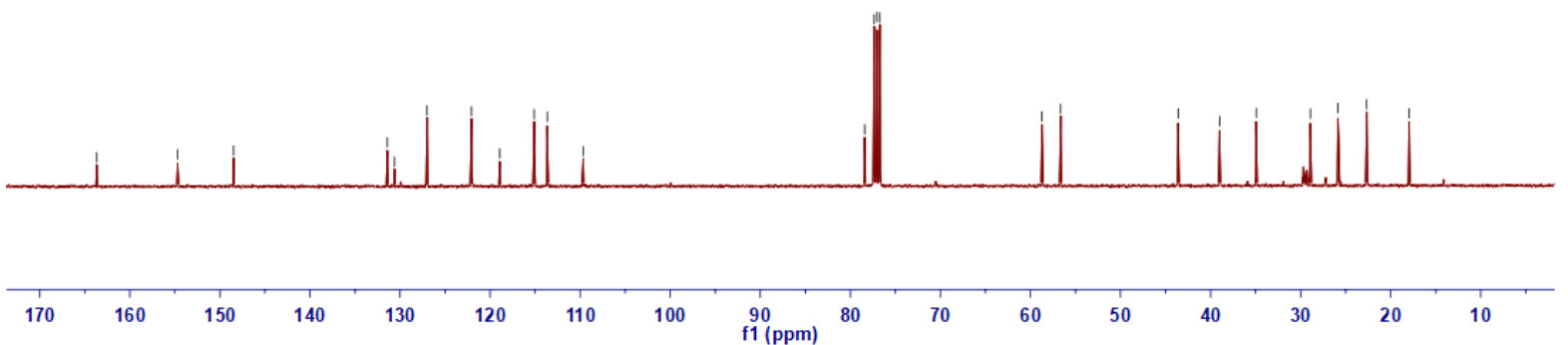
(2R,4R)-2-(2-hydroxyethyl)-7-methoxy-2,6-dimethyl-4-(2-methylprop-1-en-1-yl)-2,3,4,6-tetrahydro-5H-pyrano[3,2 -c]quinolin-5-one (4nn)

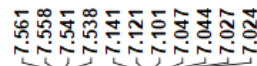

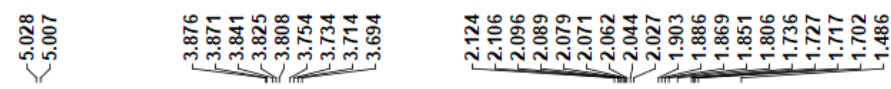
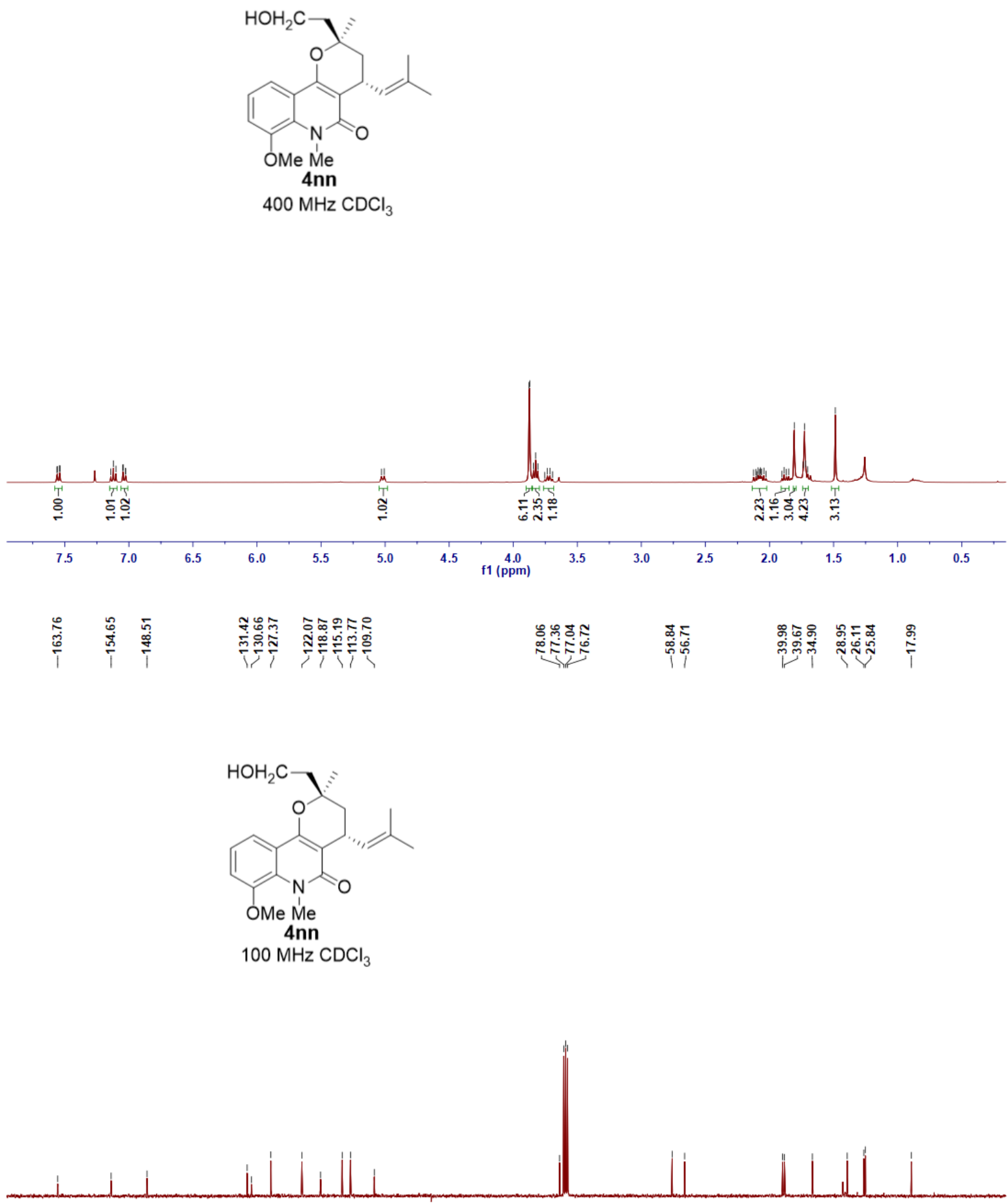

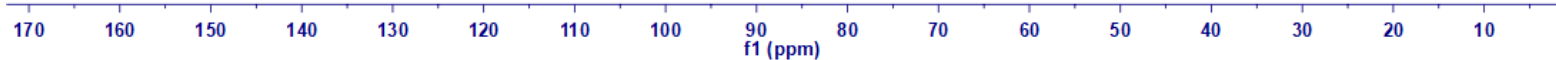


(2S,4R)-6-benzyl-2-(2-hydroxyethyl)-8,9-dimethoxy-2-methyl-4-(2-methylprop-1-en-1-yl)-2,3,4,6-tetrahydro-5H-py rano[3,2-c]quinolin-5-one $(40)$
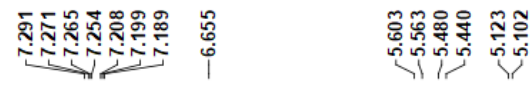

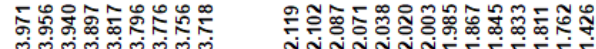
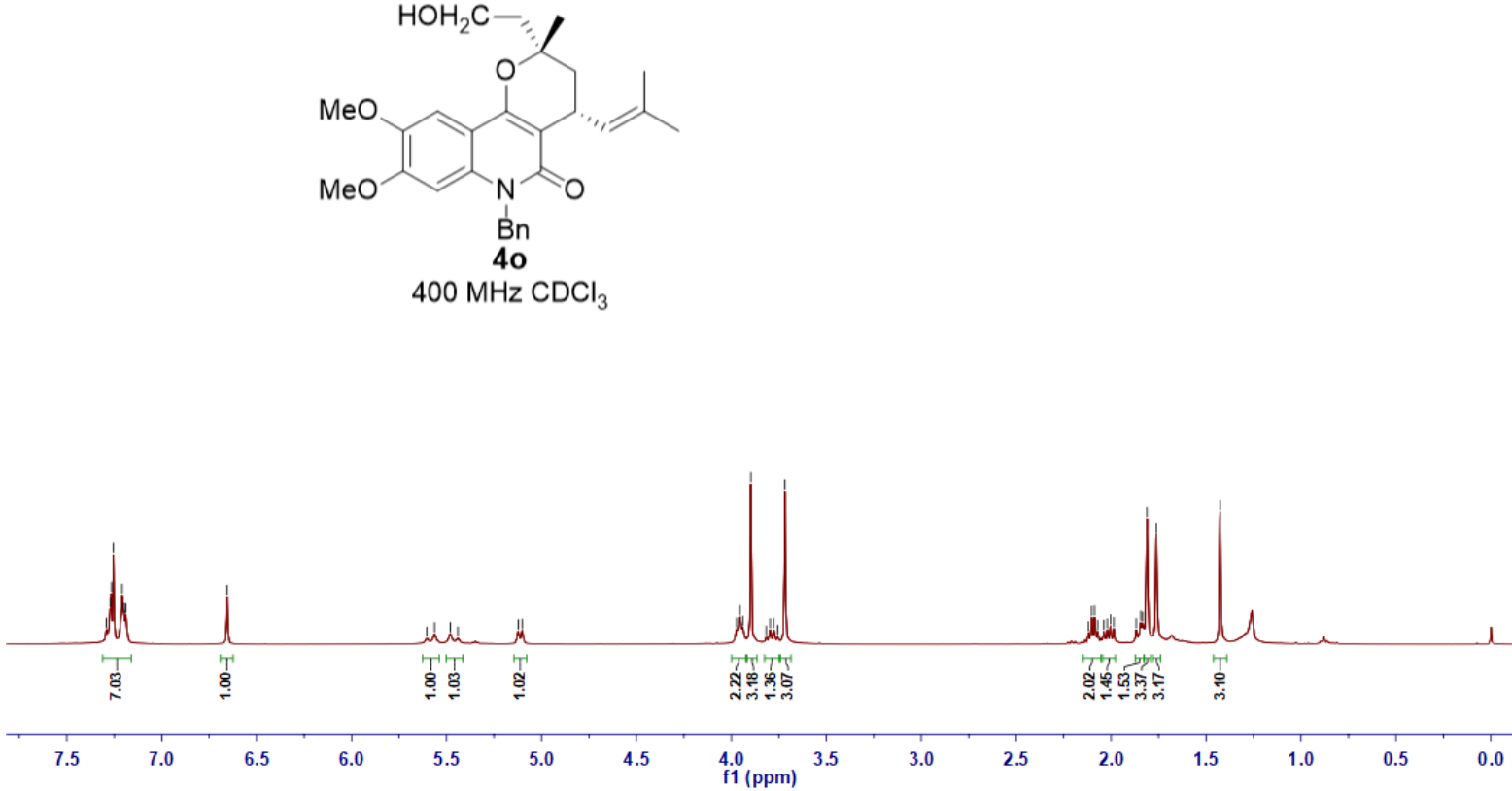

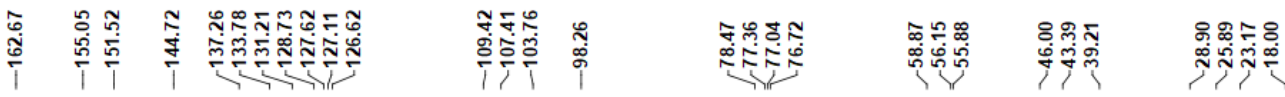
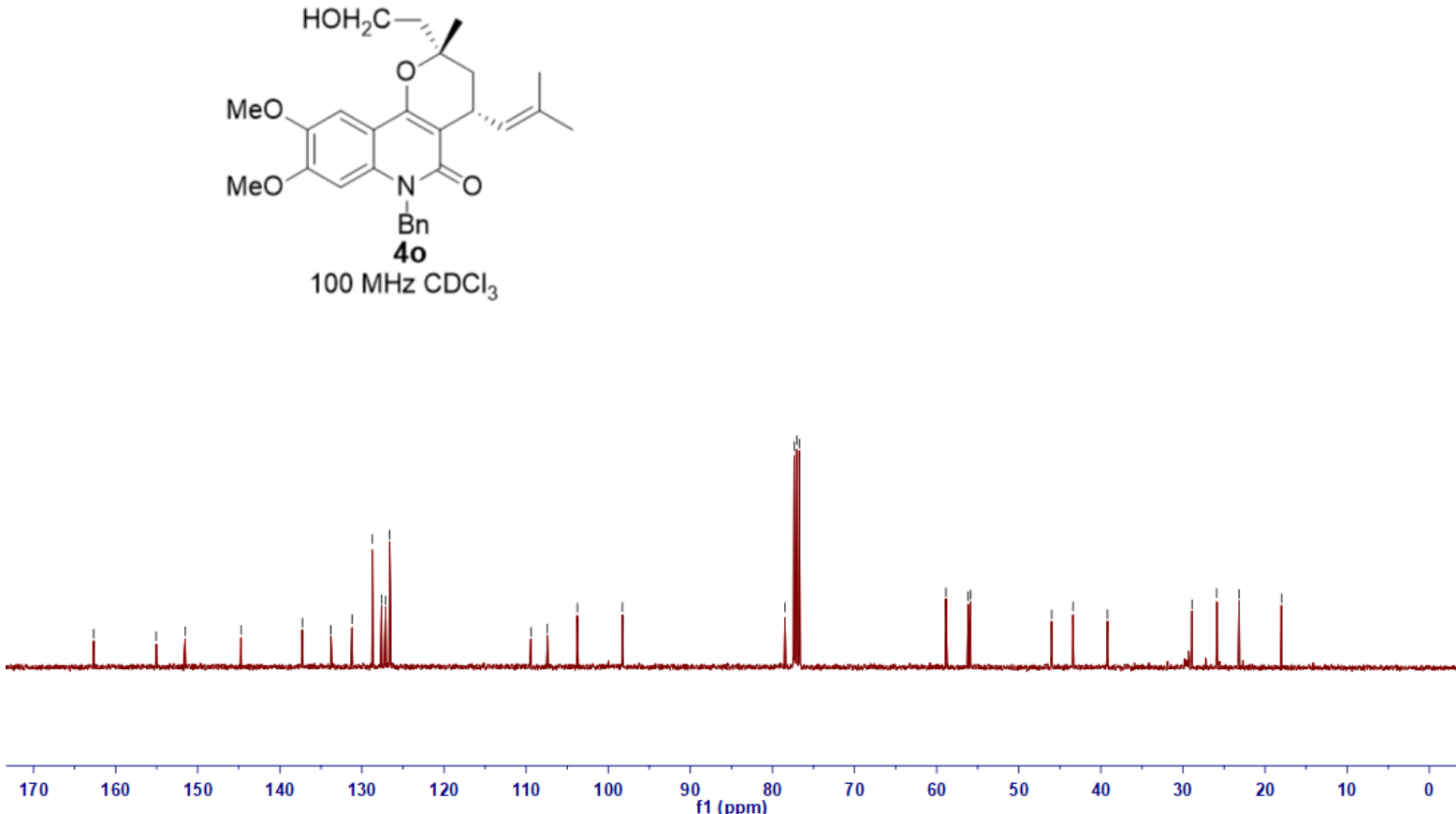
(2R,4R)-6-benzyl-2-(2-hydroxyethyl)-8,9-dimethoxy-2-methyl-4-(2-methylprop-1-en-1-yl)-2,3,4,6-tetrahydro-5H-p yrano[3,2-c]quinolin-5-one (40o)
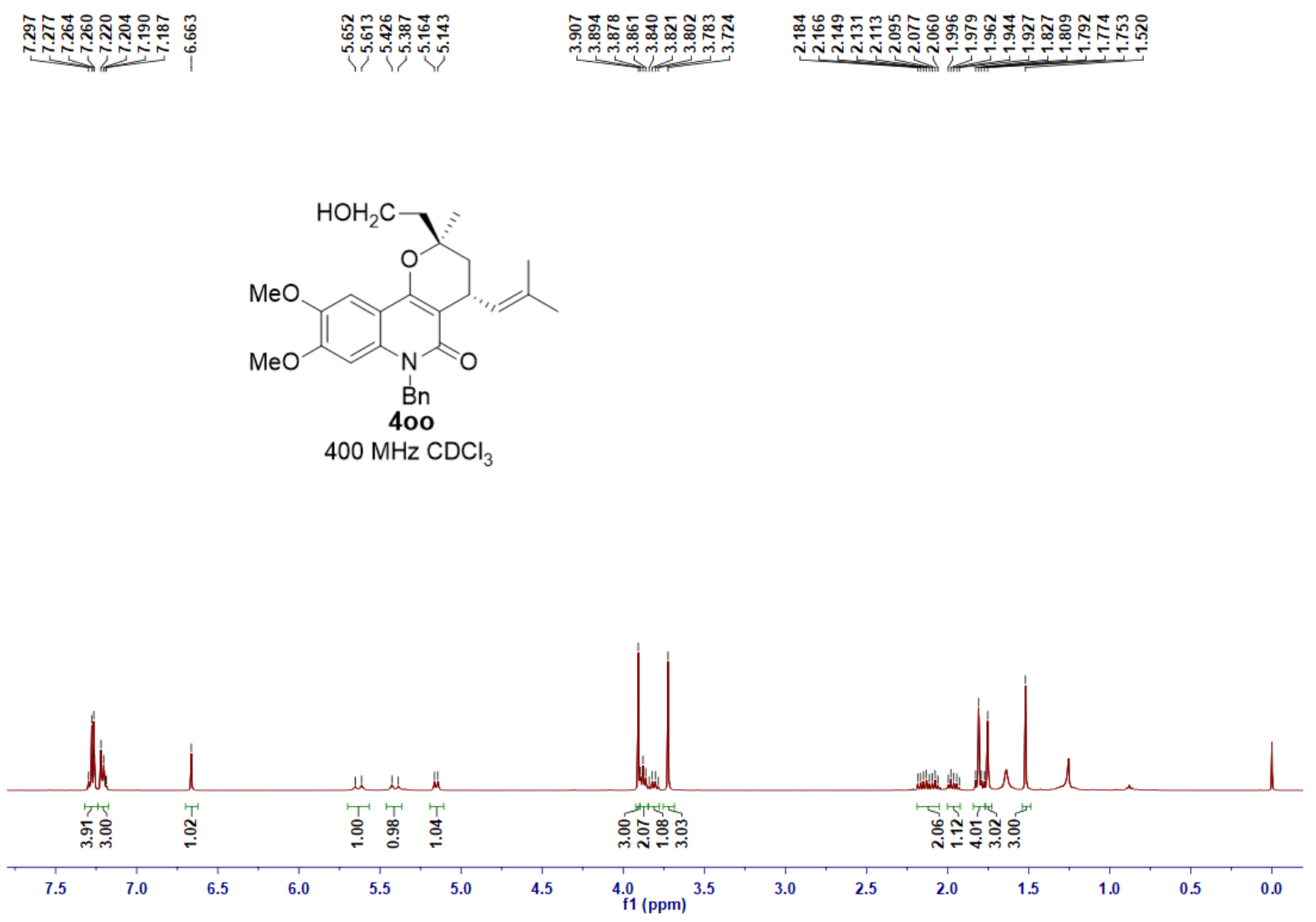

它

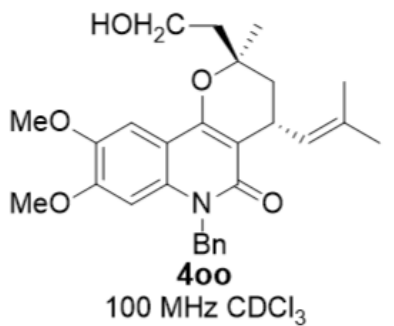

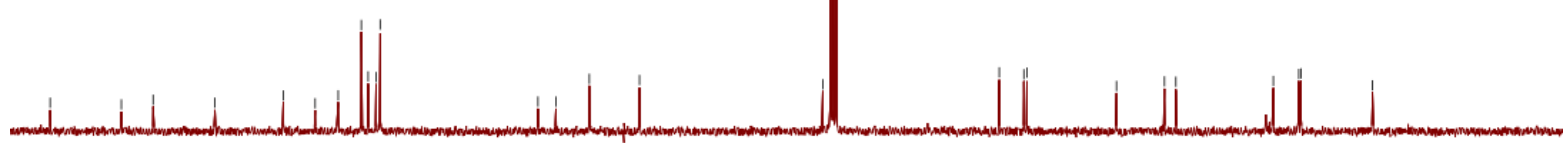

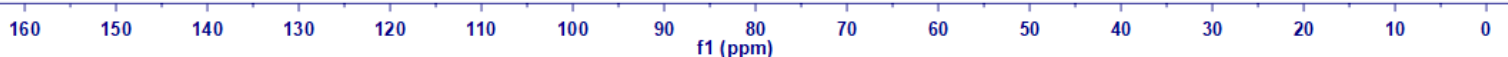


(1R,3S)-3-(2-hydroxyethyl)-3,11-dimethyl-1-(2-methylprop-1-en-1-yl)-1,2,3,11-tetrahydro-12H-benzo[h]pyrano[3,2 -c]quinolin-12-one (4p)
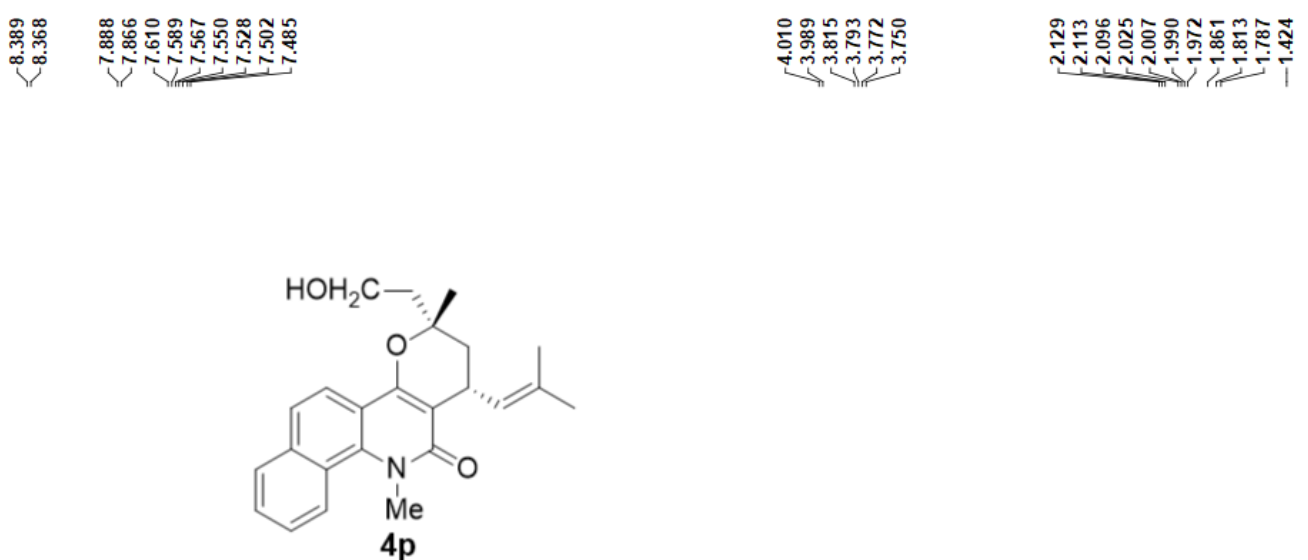

$400 \mathrm{MHz} \mathrm{CDCl}_{3}$

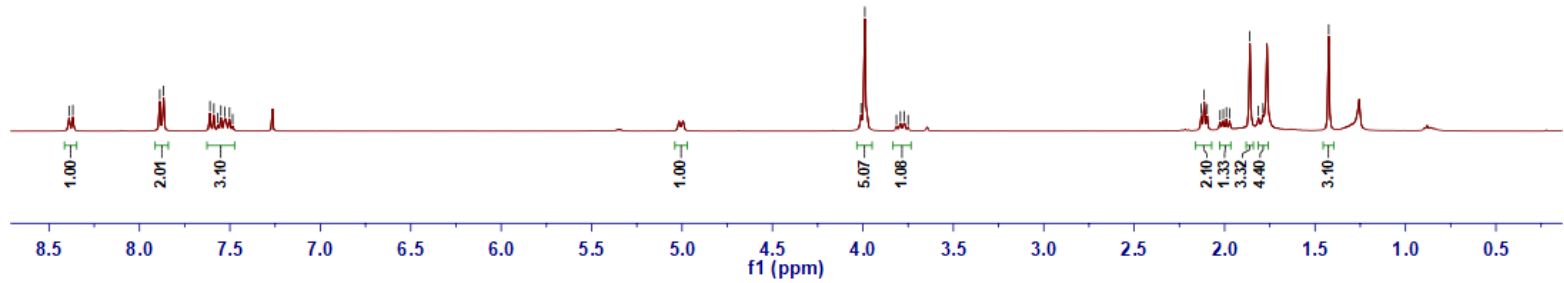

管

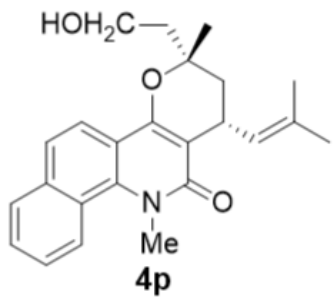

$100 \mathrm{MHz} \mathrm{CDCl}_{3}$

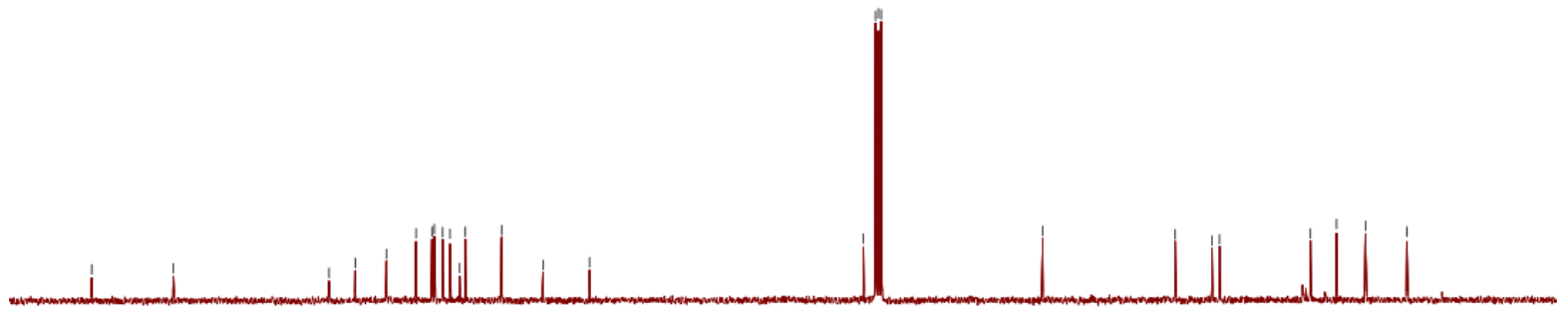

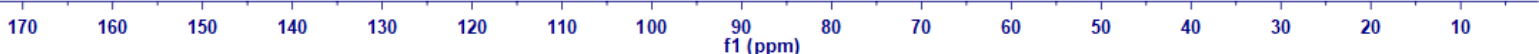


(1R,3R)-3-(2-hydroxyethyl)-3,11-dimethyl-1-(2-methylprop-1-en-1-yl)-1,2,3,11-tetrahydro-12H-benzo[h]pyrano[3,2 -c]quinolin-12-one (4pp)
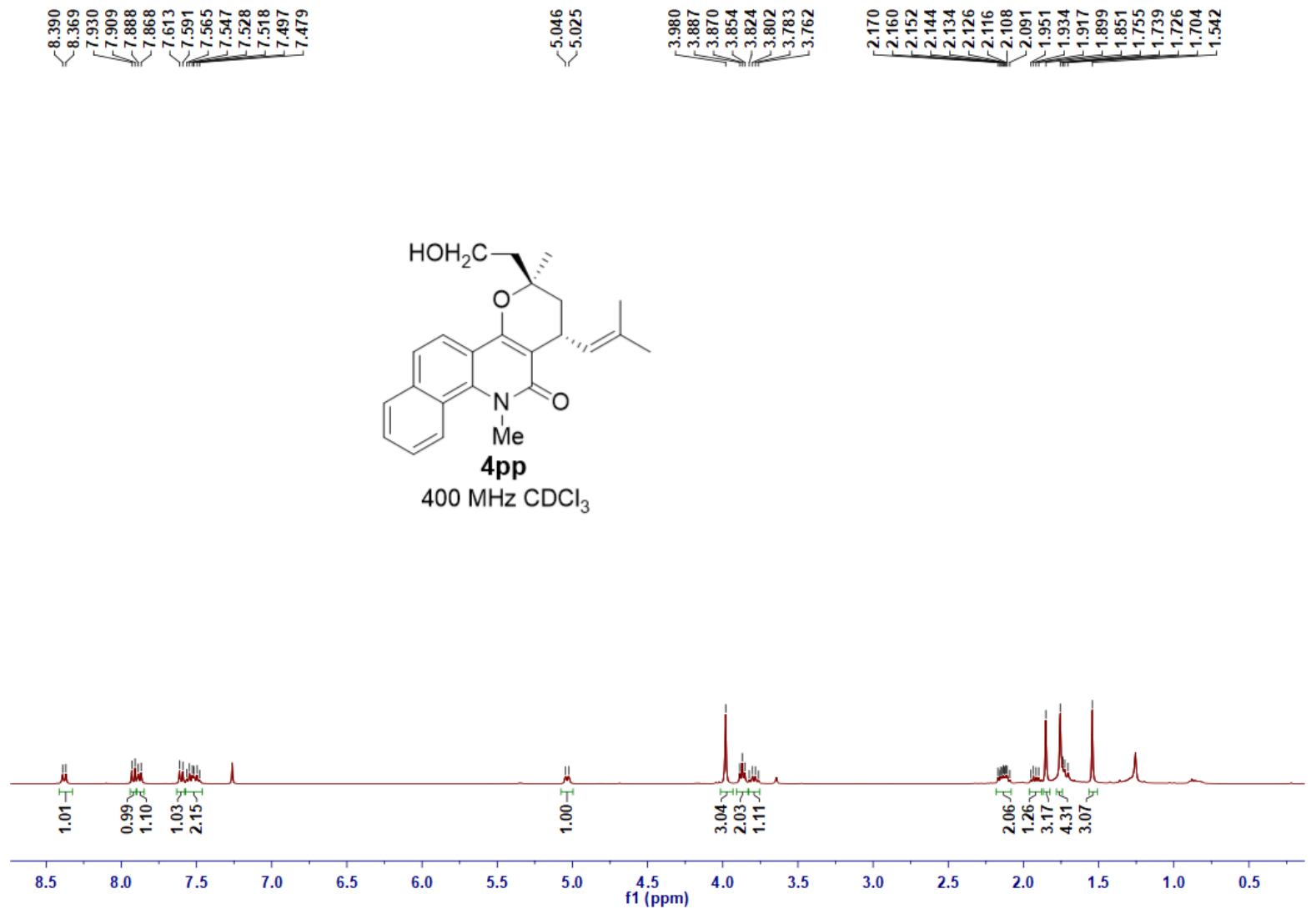

\begin{tabular}{|c|c|c|}
\hline 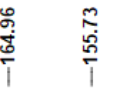 & 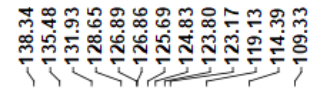 & 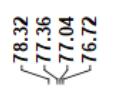 \\
\hline
\end{tabular}

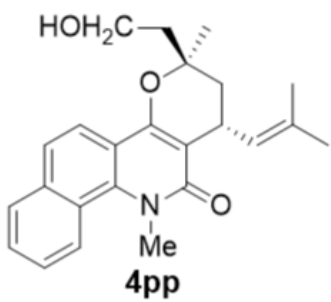

$100 \mathrm{MHz} \mathrm{CDCl}_{3}$

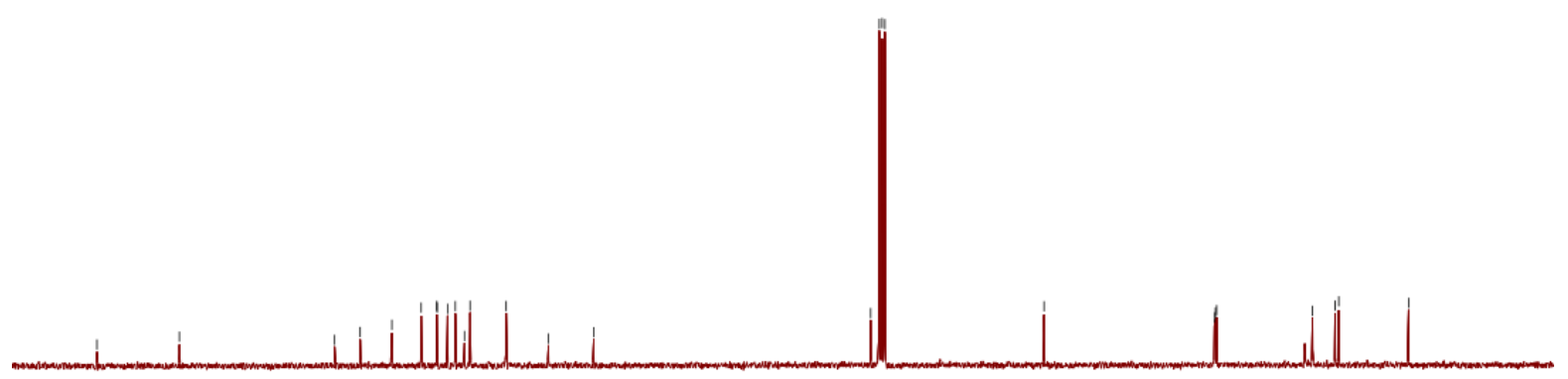

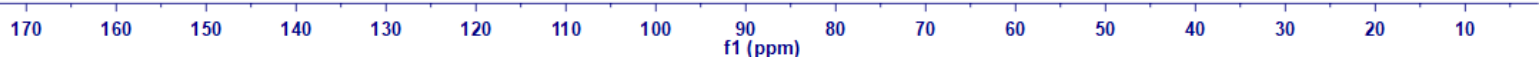


(2S,4R)-6-benzyl-2-(2-hydroxyethyl)-2-methyl-4-(2-methylprop-1-en-1-yl)-2,3,4,6-tetrahydro-5H-benzo[f]pyrano[3, 2-c]quinolin-5-one (4q)

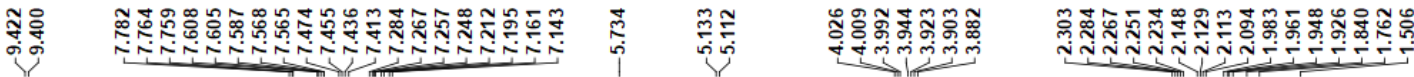

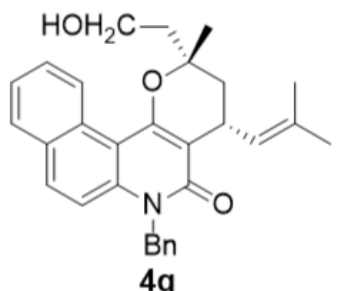

$400 \mathrm{MHz} \mathrm{CDCl}_{3}$

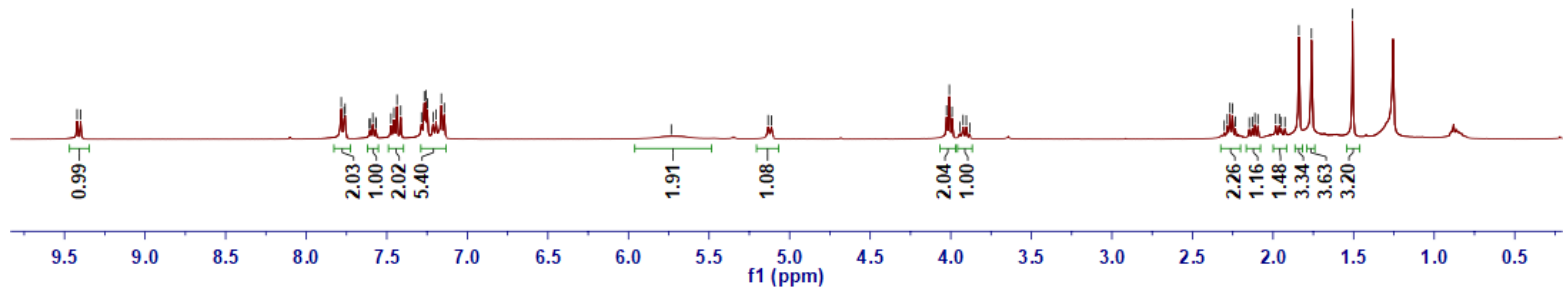

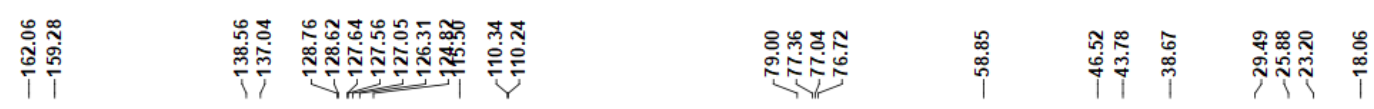
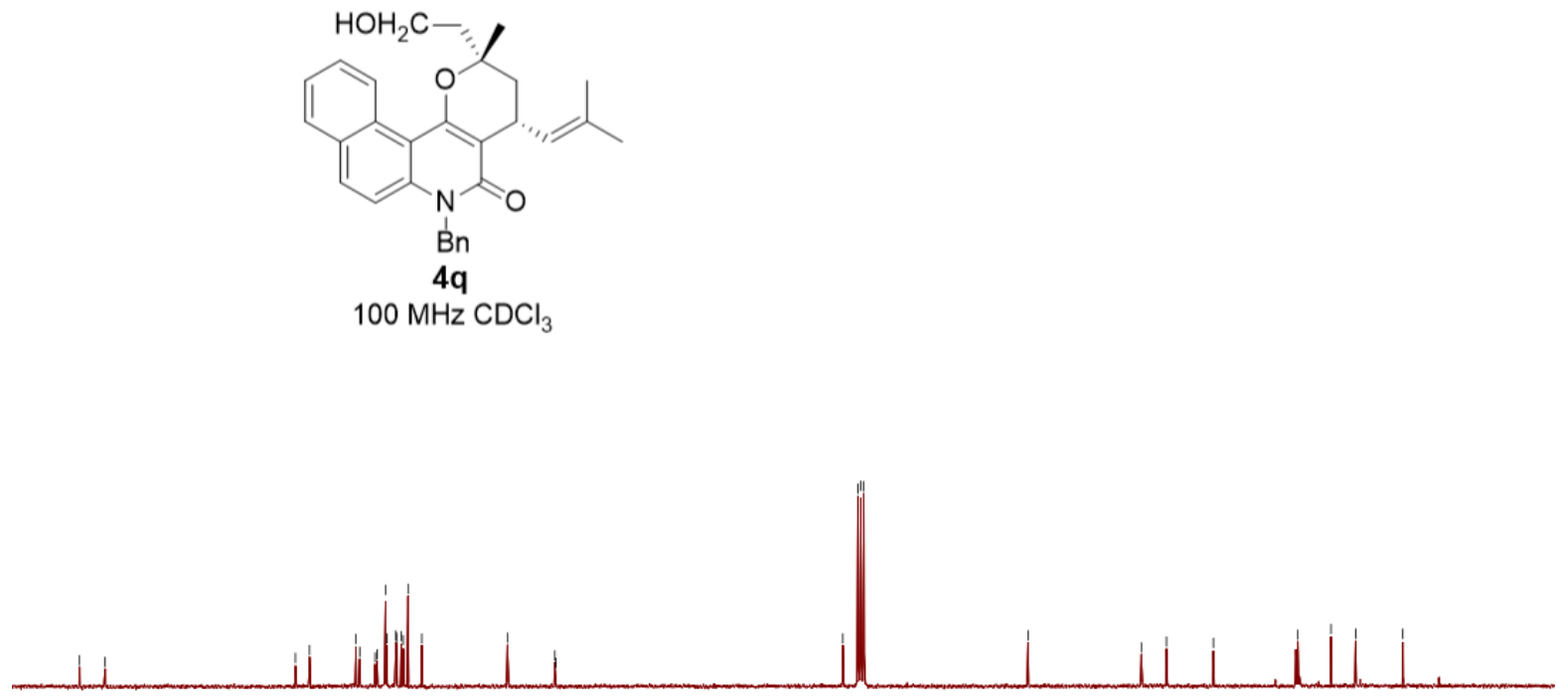

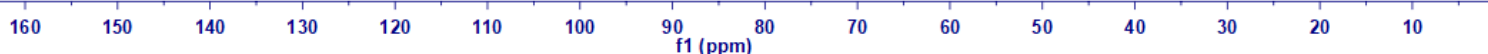


(2R,4R)-6-benzyl-2-(2-hydroxyethyl)-2-methyl-4-(2-methylprop-1-en-1-yl)-2,3,4,6-tetrahydro-5H-benzo[f]pyrano [3,2-c]quinolin-5-one (4qq)

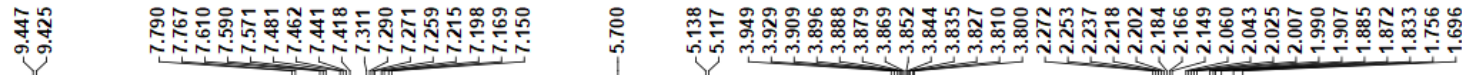
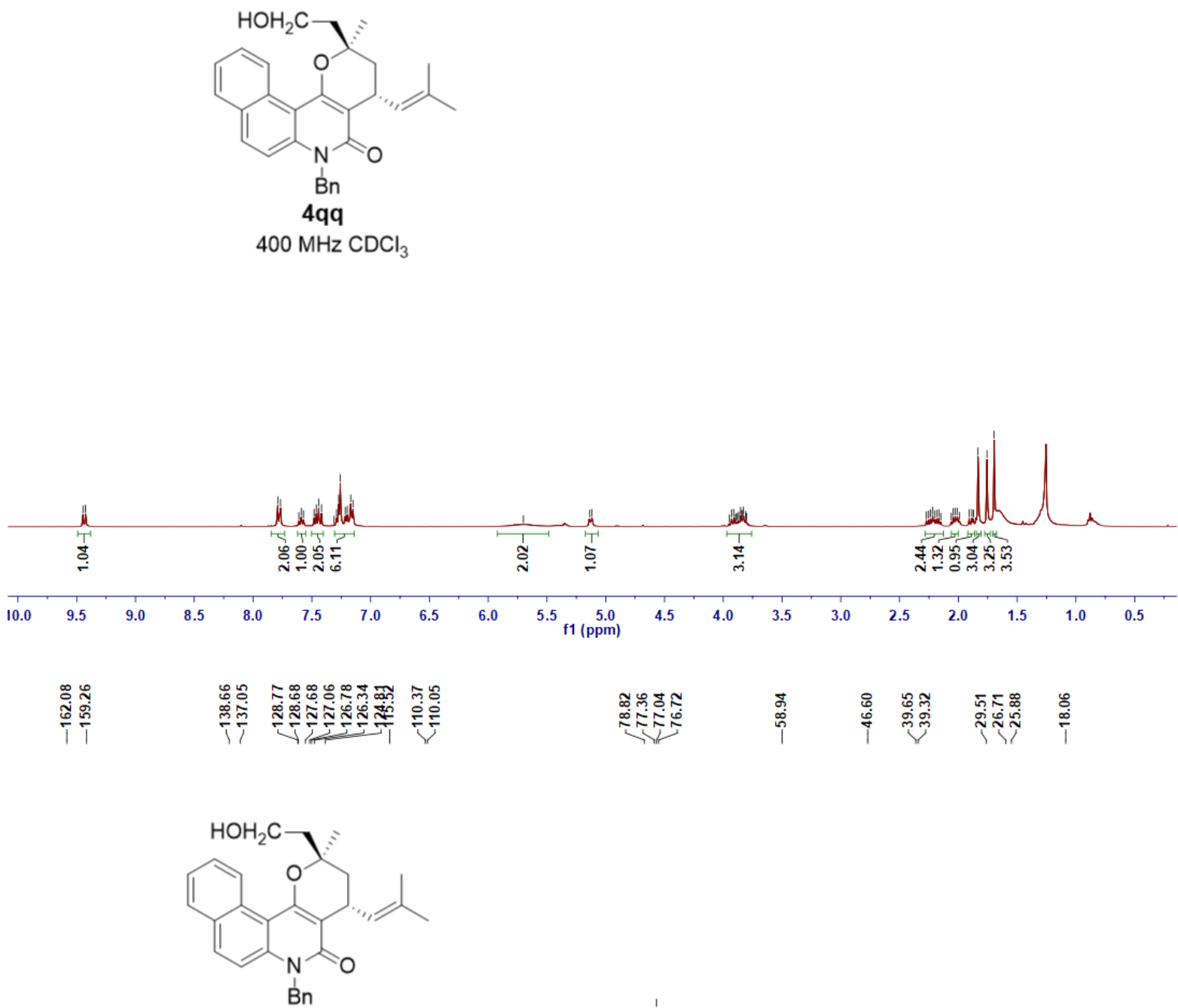

$4 \mathrm{qq}$ $100 \mathrm{MHz} \mathrm{CDCl}_{3}$

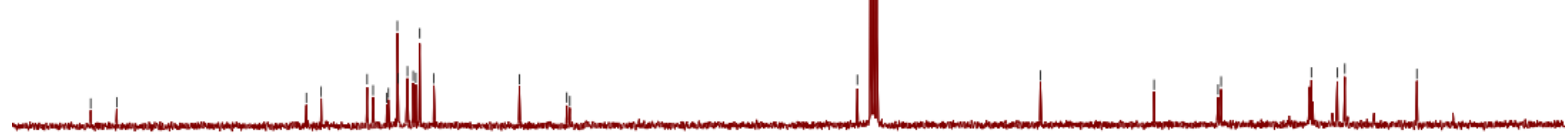

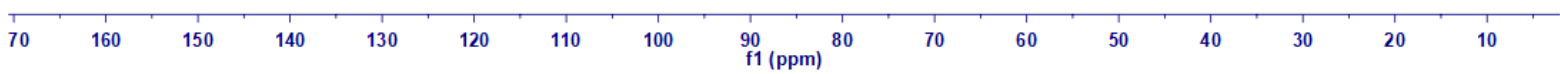


(2S,4R)-6-benzyl-2-(2-hydroxyethyl)-2-methyl-4-(2-methylprop-1-en-1-yl)-2,3,4,6-tetrahydro-5H-pyrano[3,2-c][1, 8]naphthyridin-5-one $(4 r)$

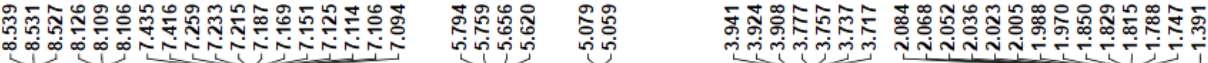
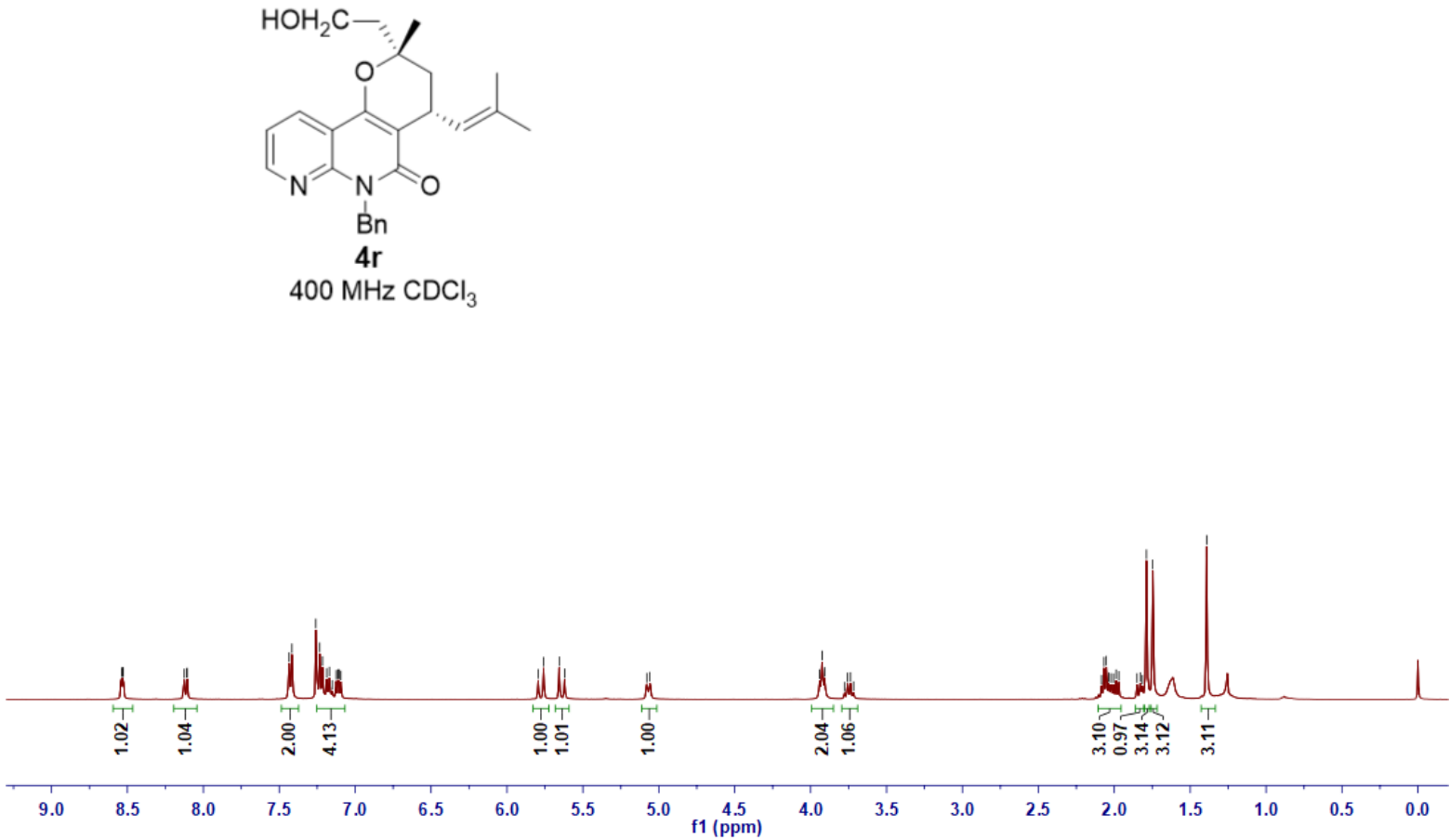

†

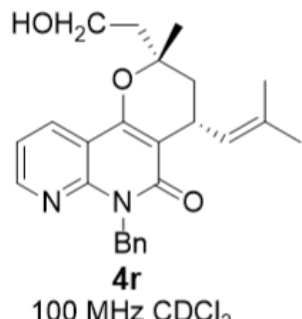

$100 \mathrm{MHz} \mathrm{CDCl}_{3}$

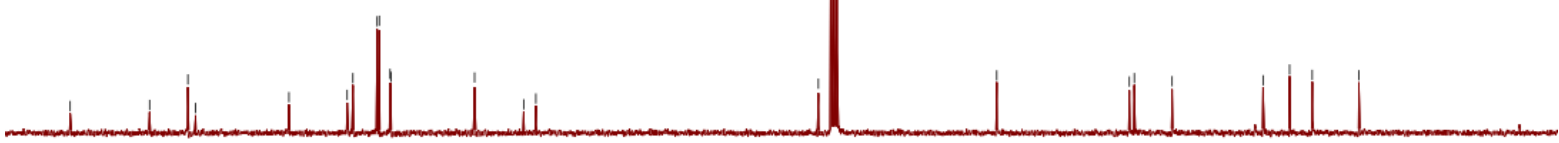

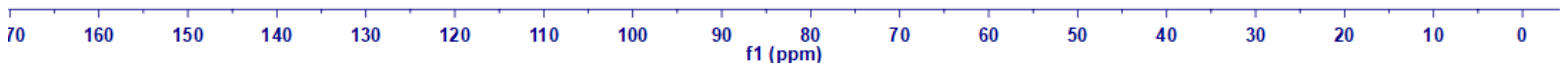


(2R,4R)-6-benzyl-2-(2-hydroxyethyl)-2-methyl-4-(2-methylprop-1-en-1-yl)-2,3,4,6-tetrahydro-5H-pyrano[3,2-c][1, 8]naphthyridin-5-one (4rr)
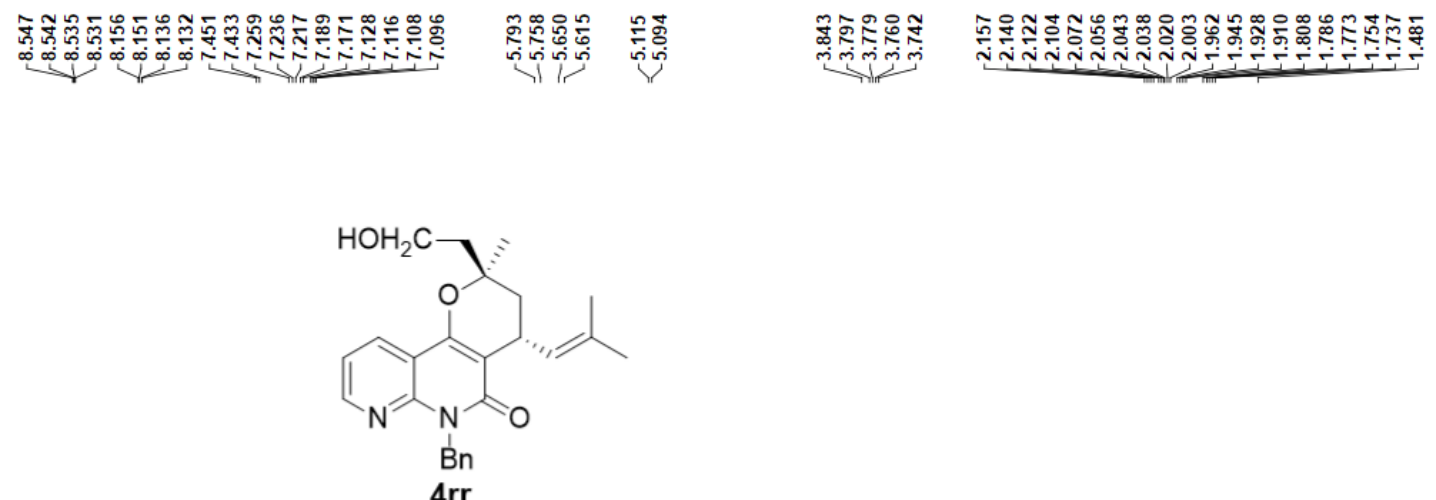

$400 \mathrm{MHz} \mathrm{CDCl}_{3}$

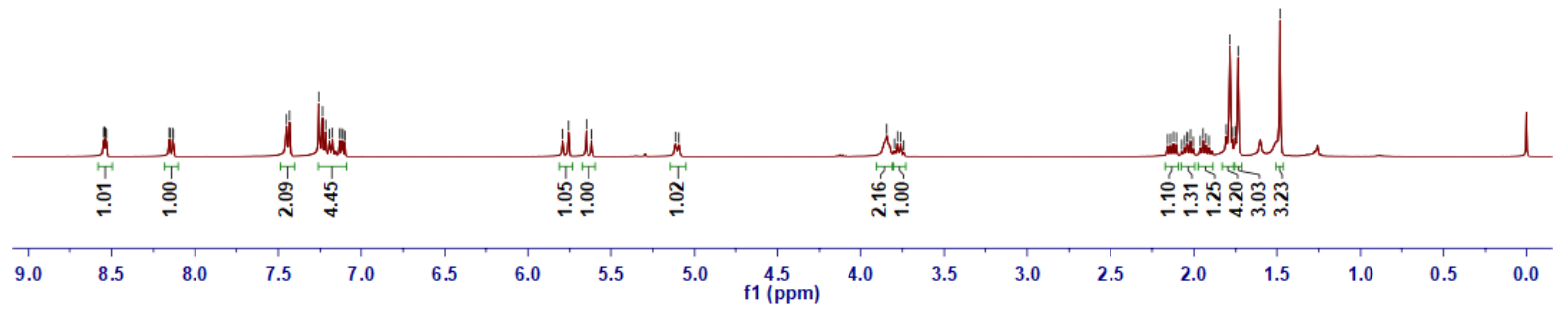

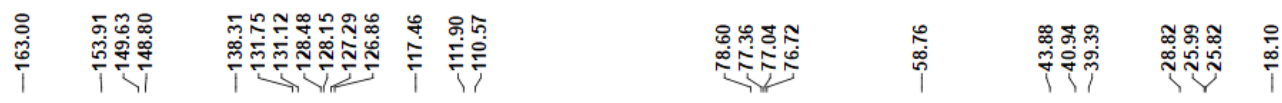

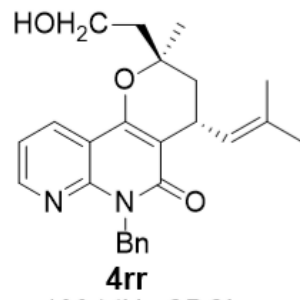

$100 \mathrm{MHz} \mathrm{CDCl}_{3}$

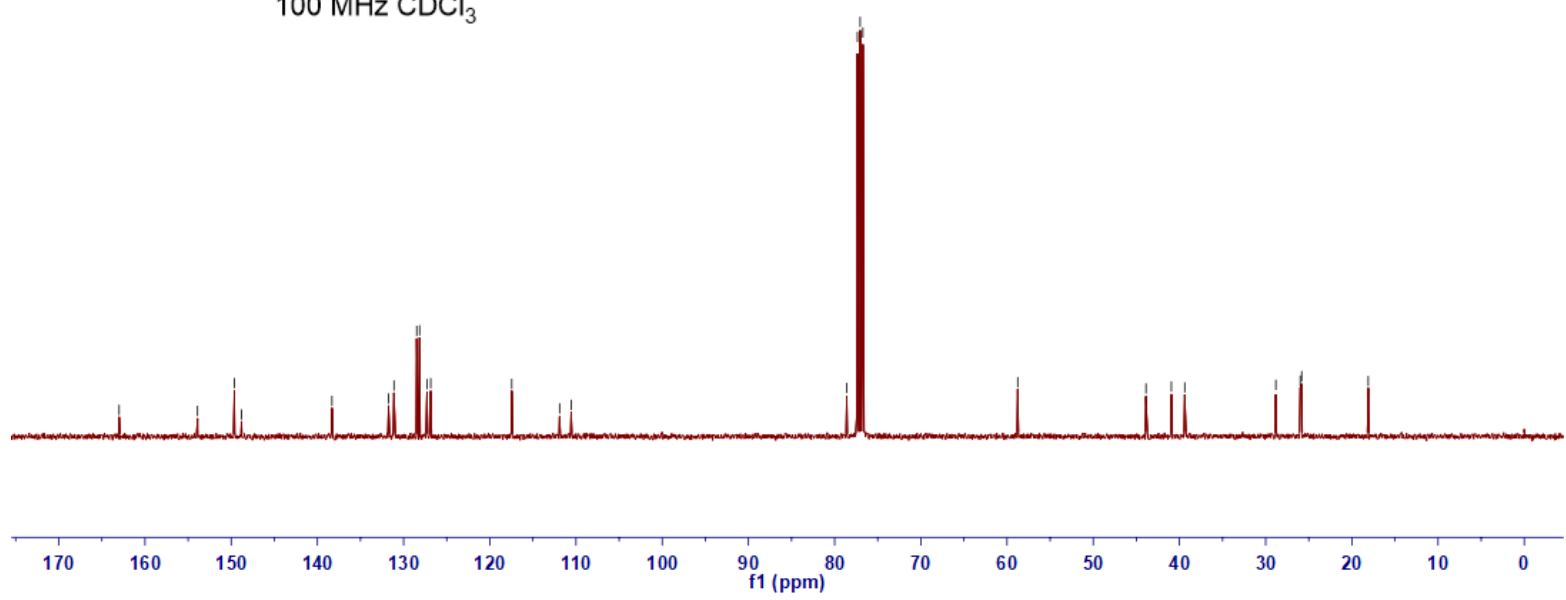


(2S,4R)-6-benzyl-2-(2-hydroxyethyl)-2-methyl-4-(2-methylprop-1-en-1-yl)-2,3,4,6-tetrahydro-5H-pyrano[2,3-a][4, 7]phenanthrolin-5-one (4s)
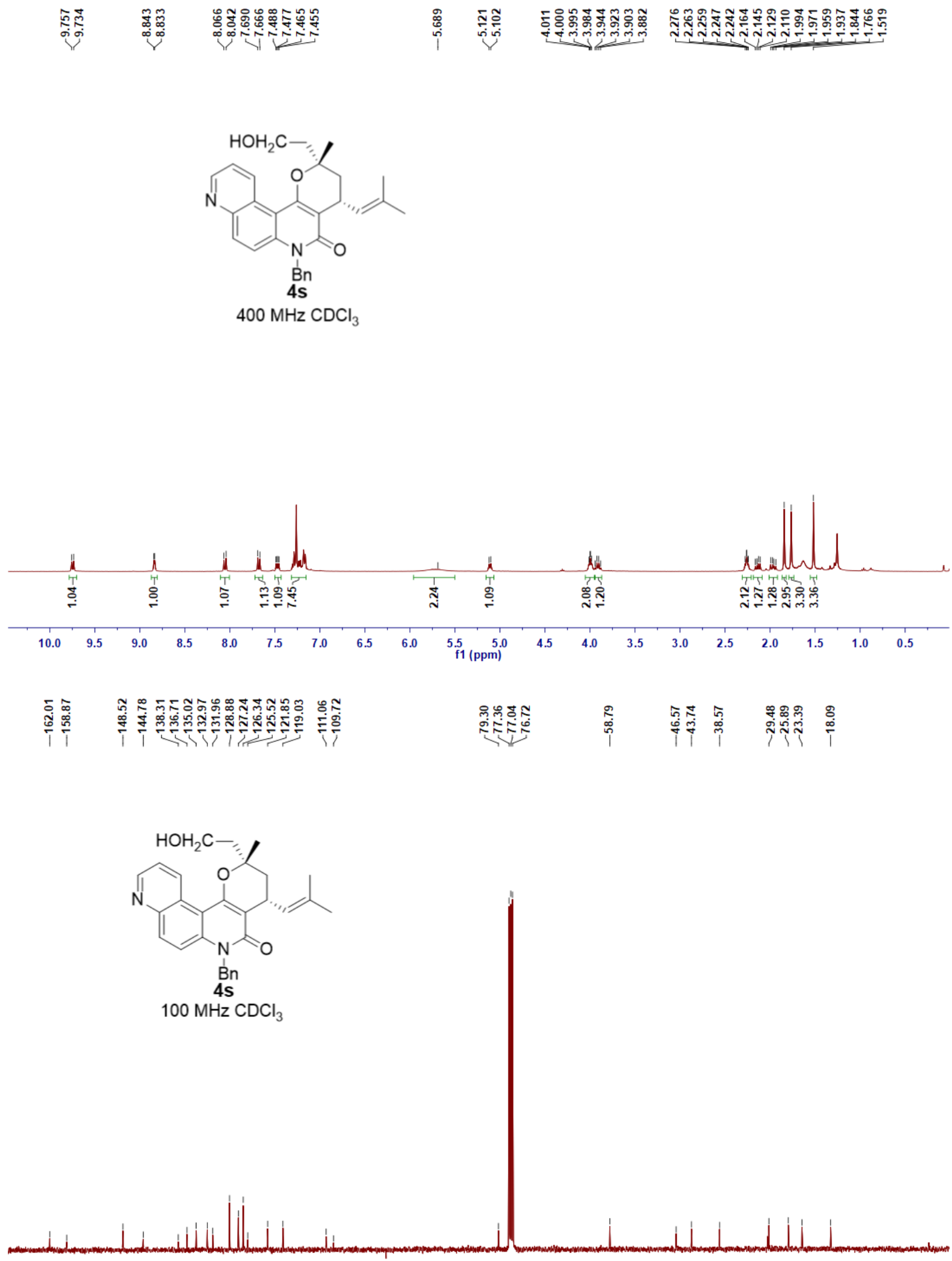

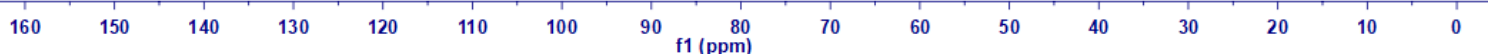


(2R,4R)-6-benzyl-2-(2-hydroxyethyl)-2-methyl-4-(2-methylprop-1-en-1-yl)-2,3,4,6-tetrahydro-5H-pyrano[2,3-a][4, 7]phenanthrolin-5-one (4ss)

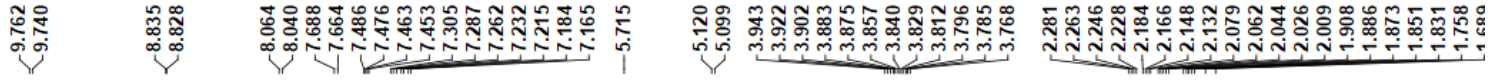<smiles>CC(C)=CC1C[C@H](C)Oc2c1c(=O)n([13C]([14CH3])[14CH3])c1ccc3ncccc3c21</smiles>

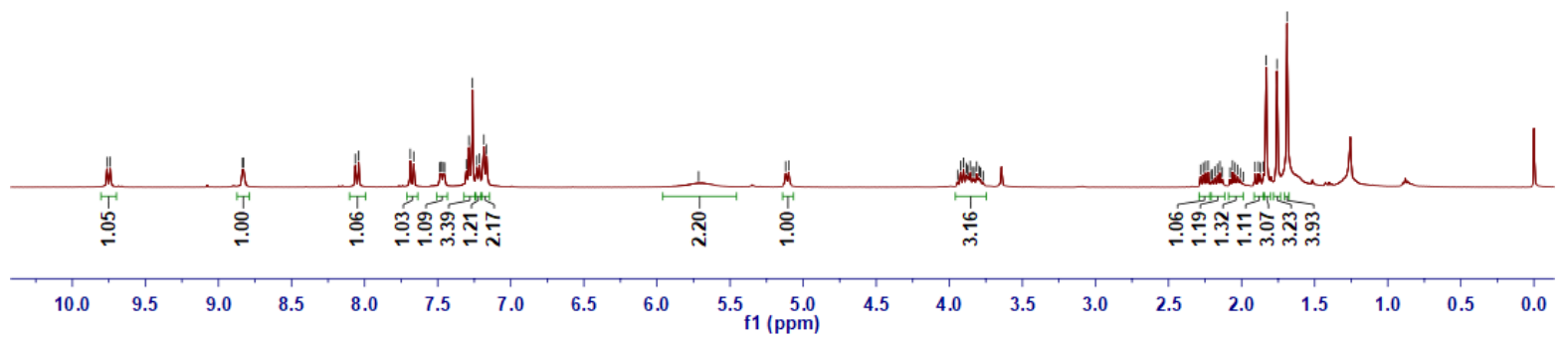

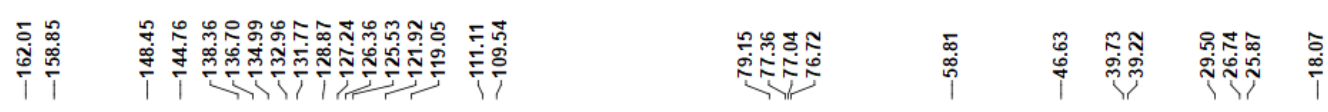

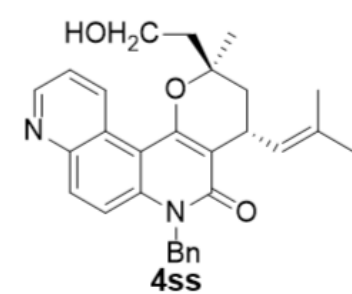

$100 \mathrm{MHz} \mathrm{CDCl}_{3}$

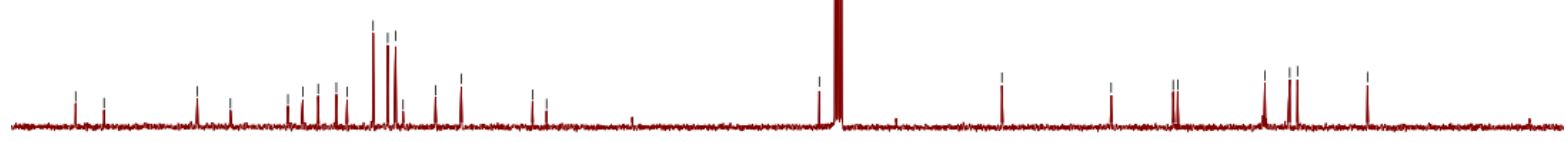

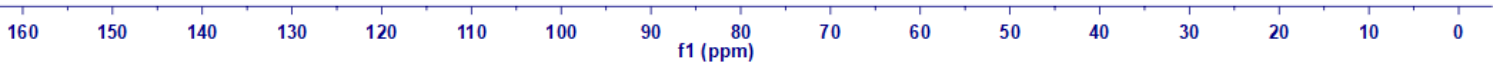


(2S,4R)-6-benzyl-4-(2-ethylbut-1-en-1-yl)-2-(2-hydroxyethyl)-2-methyl-2,3,4,6-tetrahydro-5H-pyrano[3,2-c]quinoli n-5-one (4t)

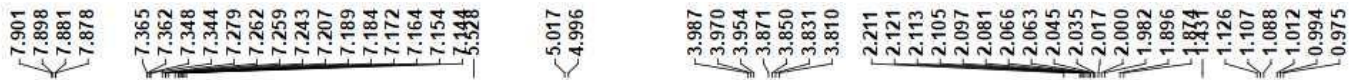
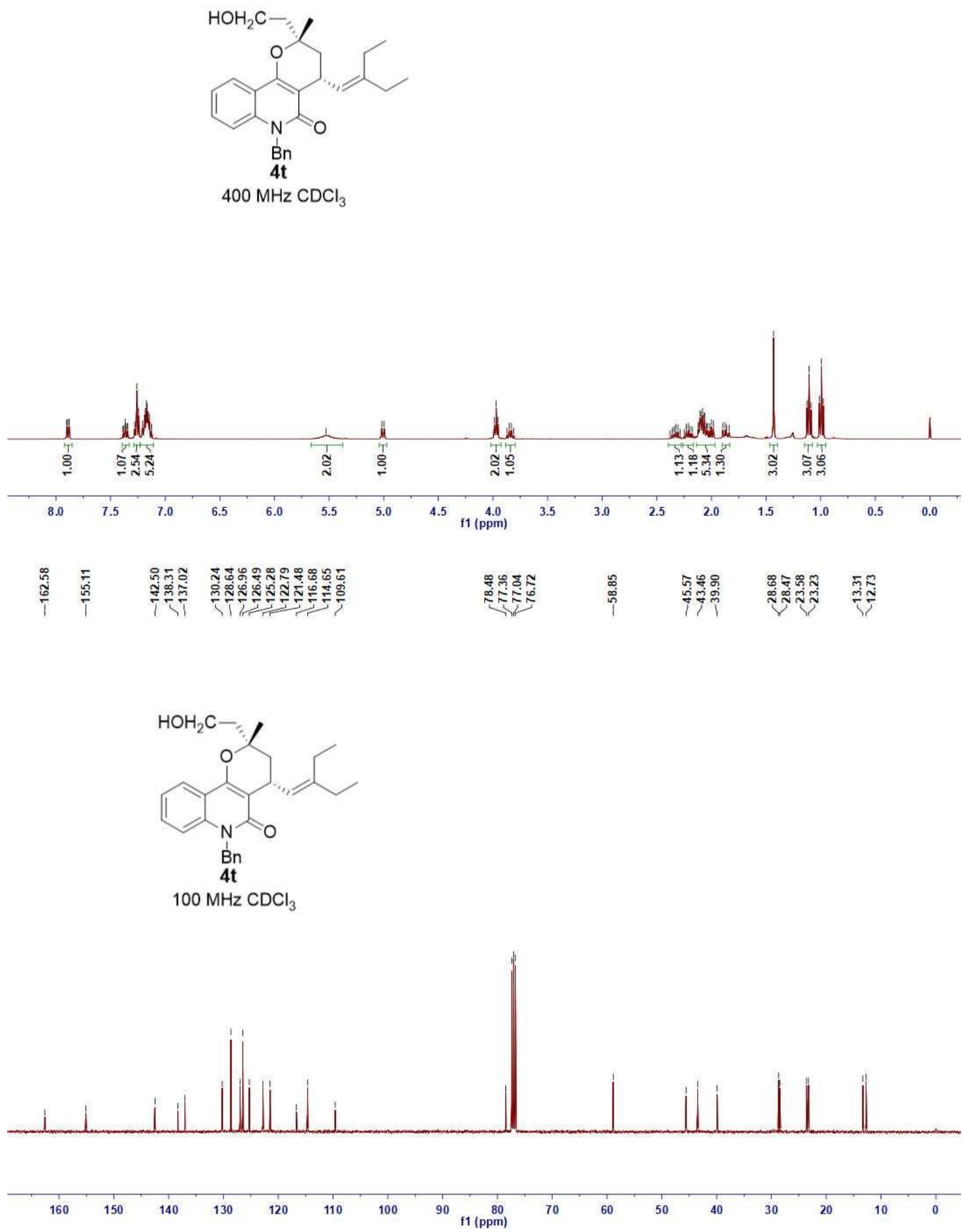
(2R,4R)-6-benzyl-4-(2-ethylbut-1-en-1-yl)-2-(2-hydroxyethyl)-2-methyl-2,3,4,6-tetrahydro-5H-pyrano[3,2-c]quinoli n-5-one (4tt)
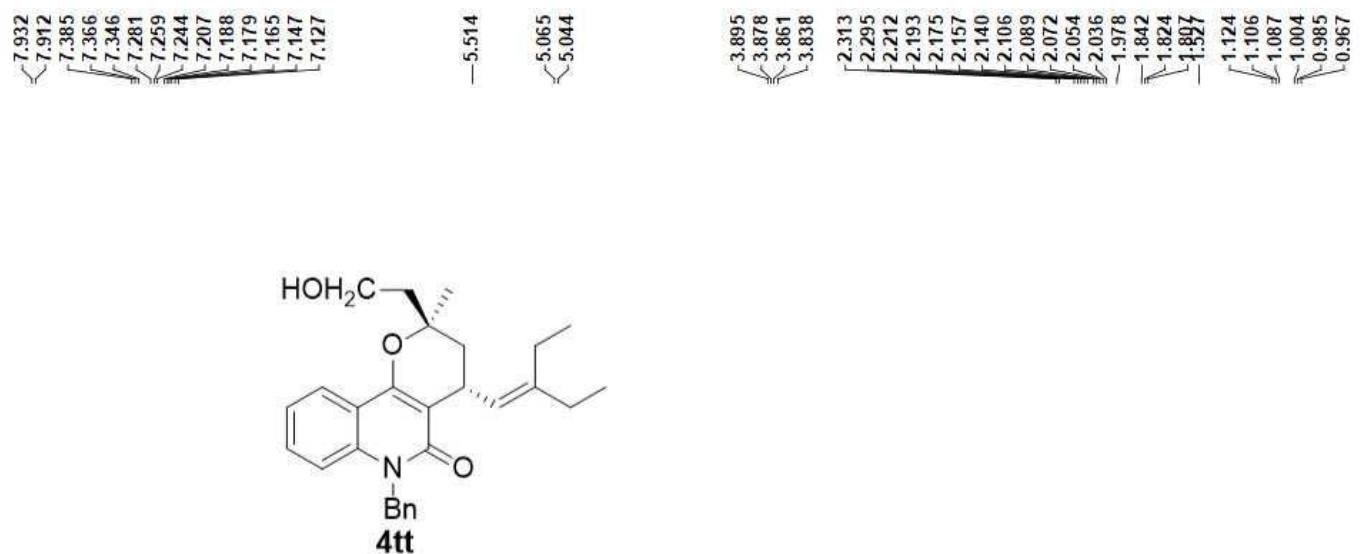

$400 \mathrm{MHz} \mathrm{CDCl}_{3}$

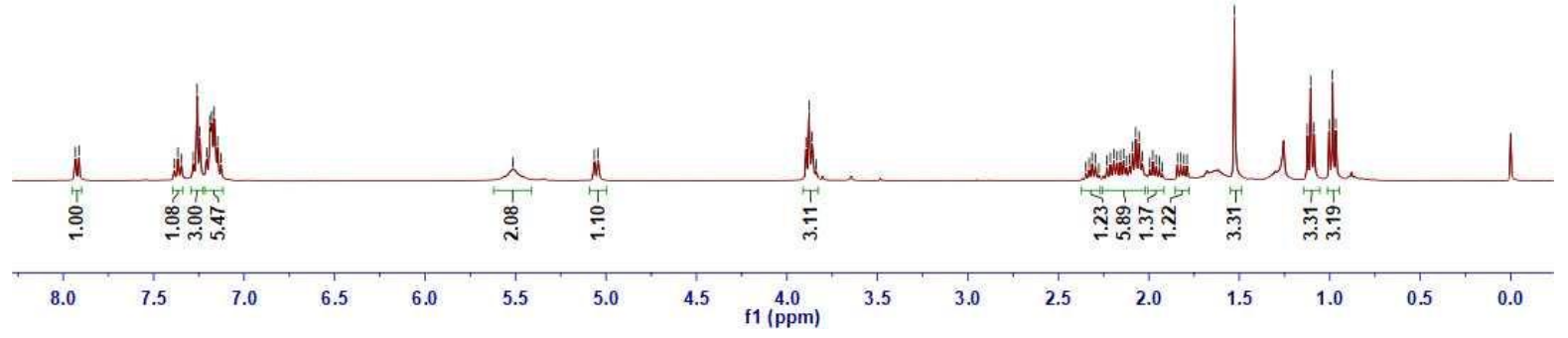

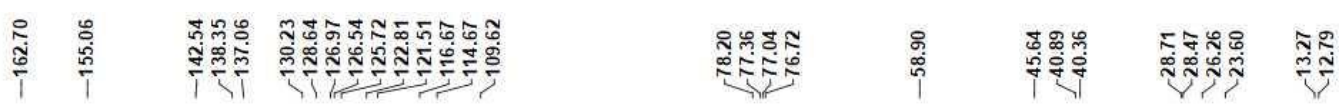

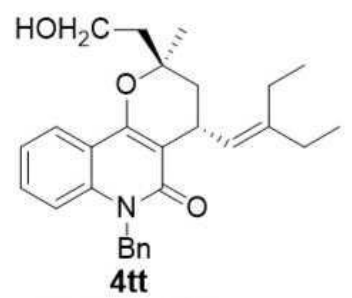

$100 \mathrm{MHz} \mathrm{CDCl}_{3}$

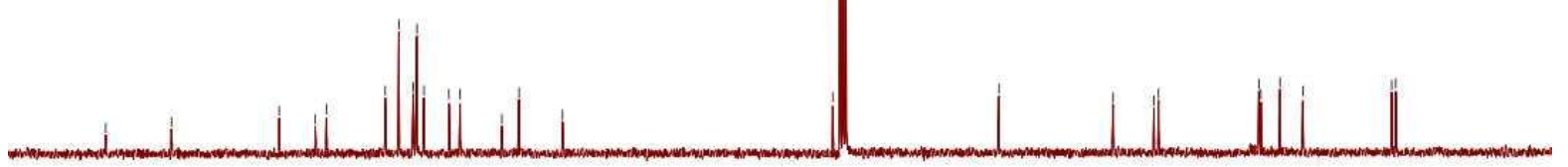

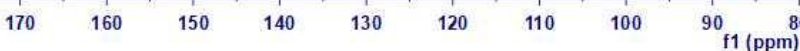


(2S,4R)-6-benzyl-4-(2-butylhex-1-en-1-yl)-2-(2-hydroxyethyl)-2-methyl-2,3,4,6-tetrahydro-5H-pyrano[3,2-c]quinoli n-5-one (4u)

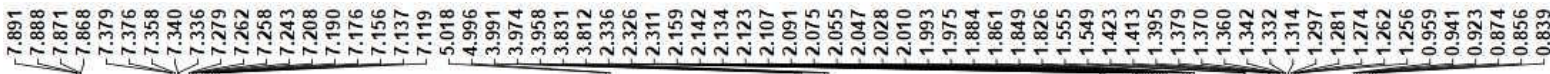

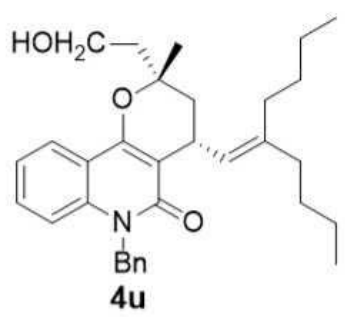

$400 \mathrm{MHz} \mathrm{CDCl}_{3}$
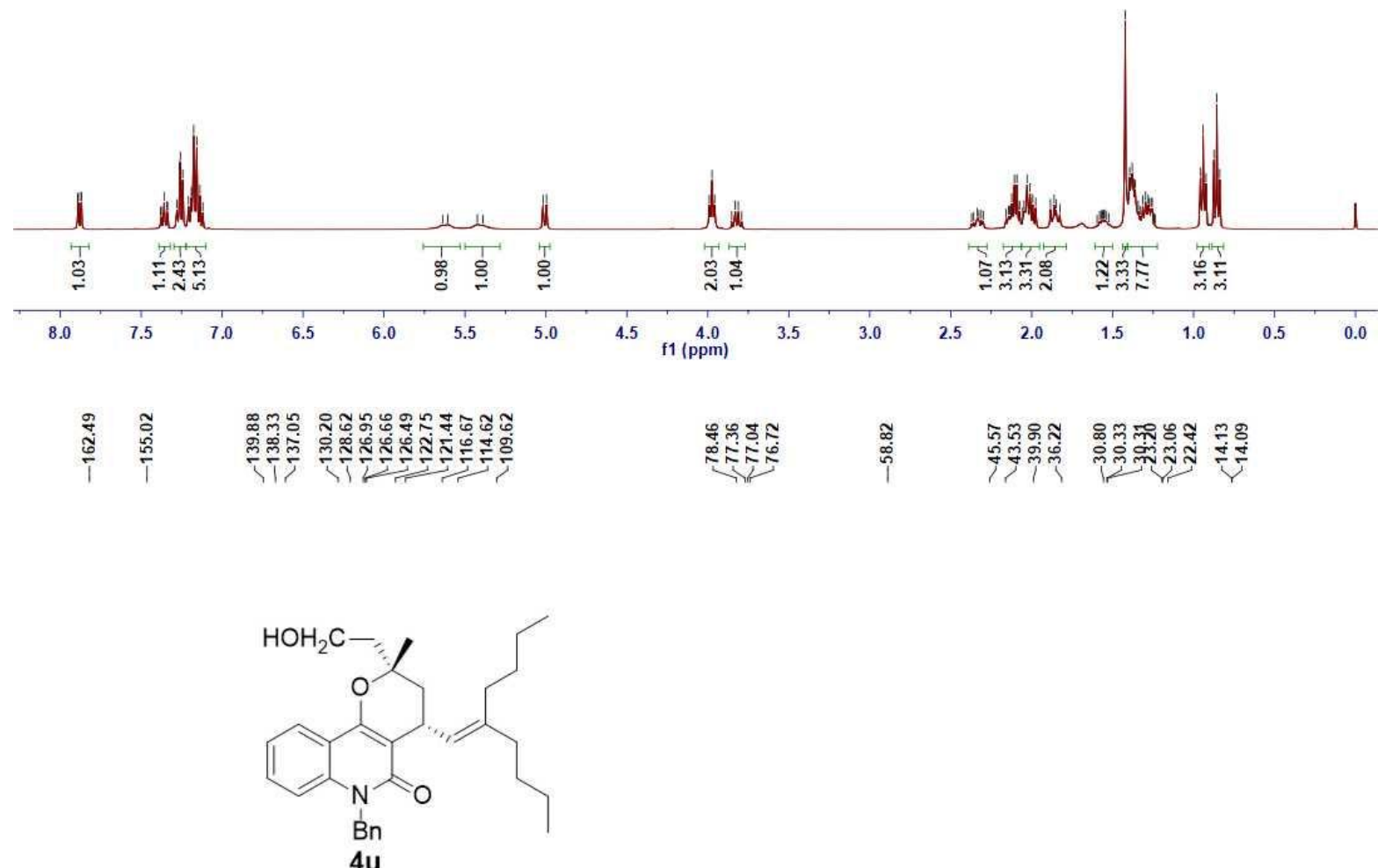

$100 \mathrm{MHz} \mathrm{CDCl}_{3}$

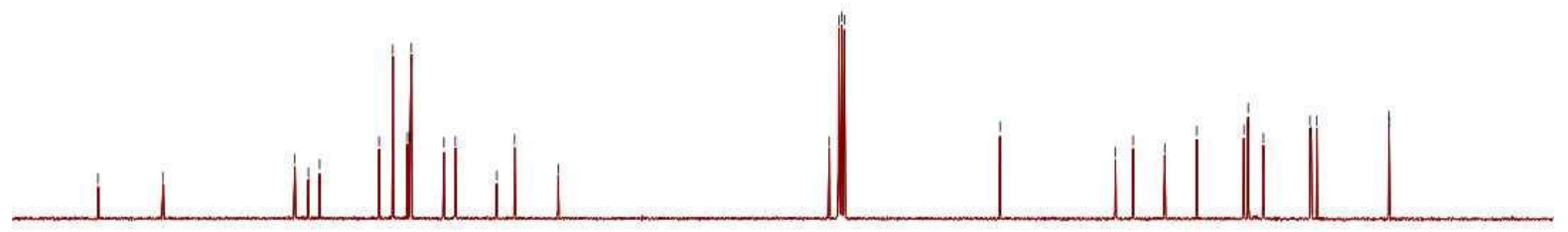

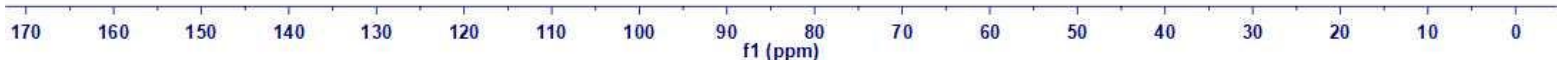


(2R,4R)-6-benzyl-4-(2-butylhex-1-en-1-yl)-2-(2-hydroxyethyl)-2-methyl-2,3,4,6-tetrahydro-5H-pyrano[3,2-c]quinol in-5-one (4uu)

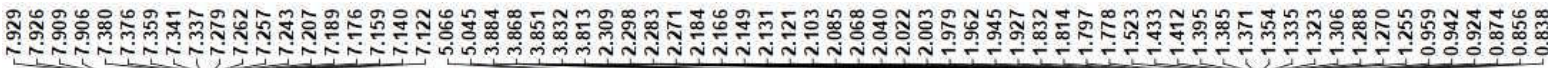
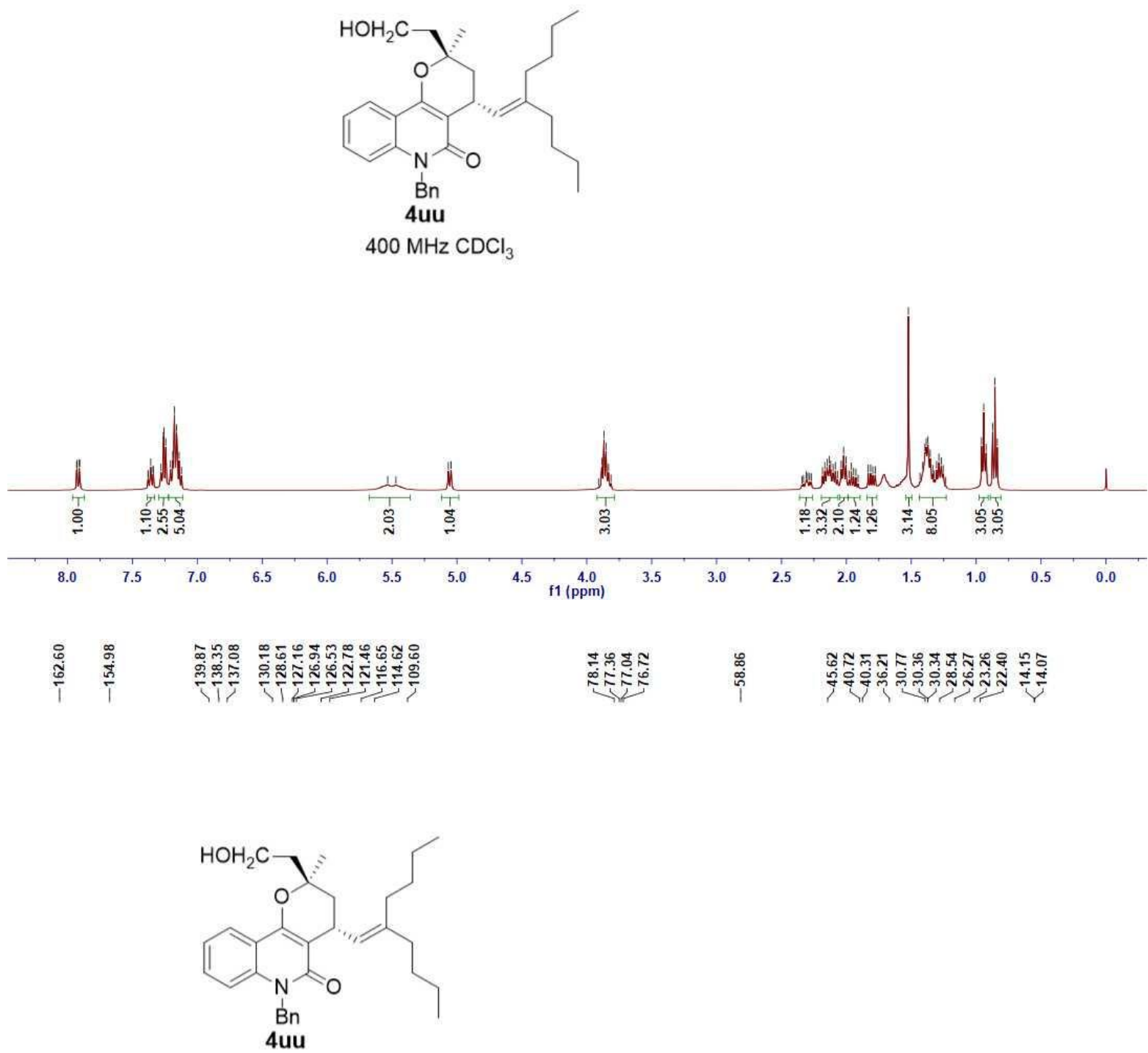

$100 \mathrm{MHz} \mathrm{CDCl}_{3}$
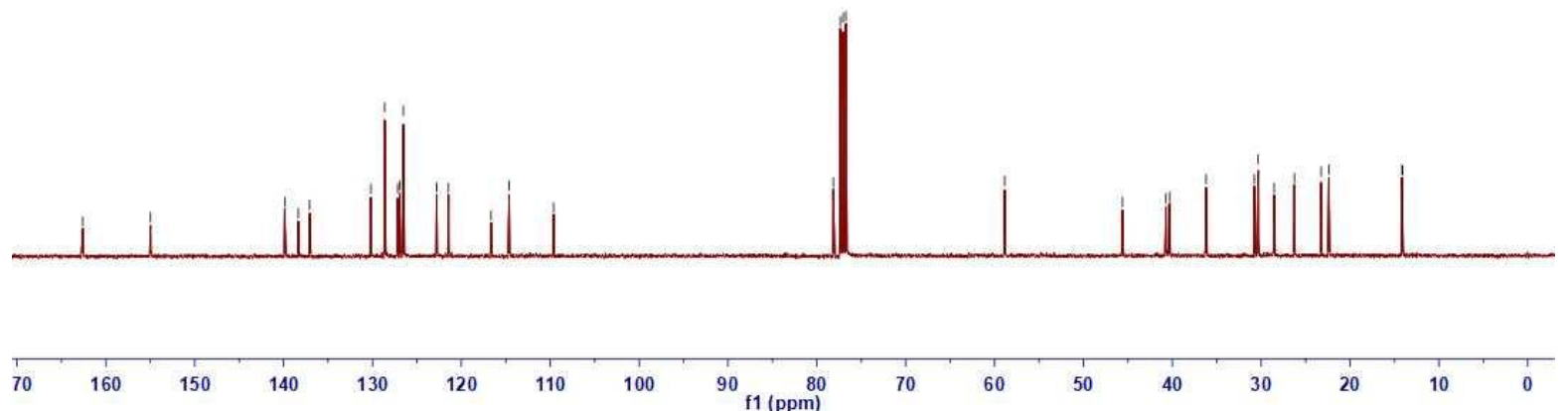
(2S,4R)-6-benzyl-4-(cyclopentylidenemethyl)-2-(2-hydroxyethyl)-2-methyl-2,3,4,6-tetrahydro-5H-pyrano[3,2-c]qui nolin-5-one (4v)

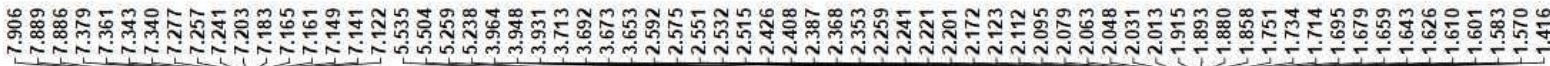

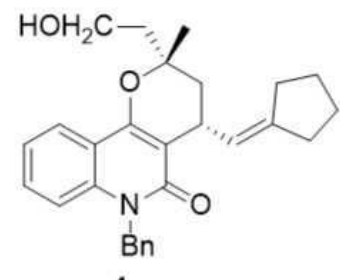

$4 v$

$400 \mathrm{MHz} \mathrm{CDCl}_{3}$

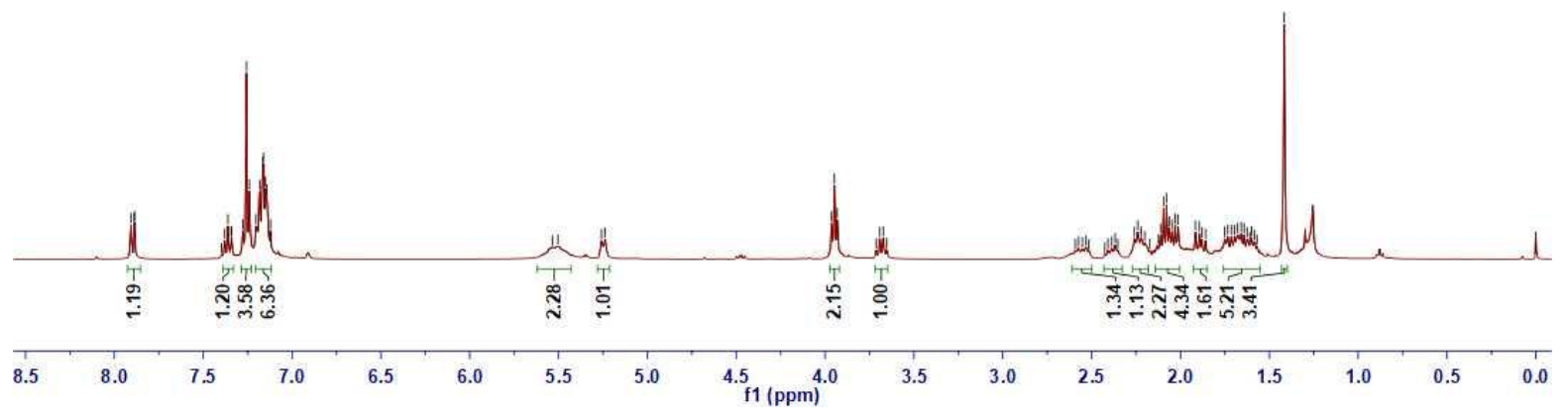

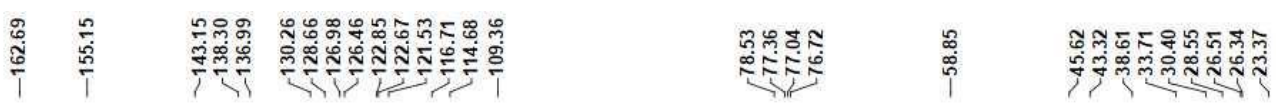

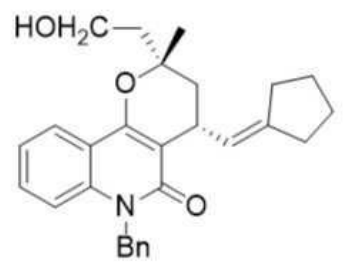

$4 \mathrm{v}$

$100 \mathrm{MHz} \mathrm{CDCl}_{3}$

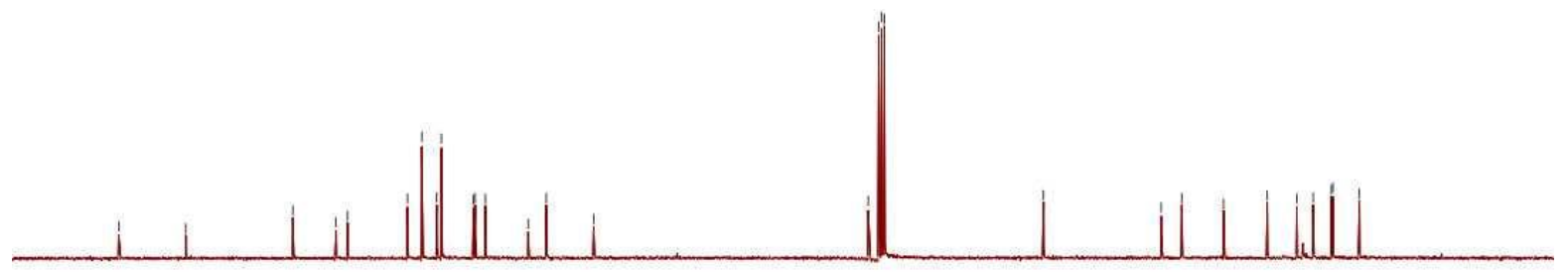

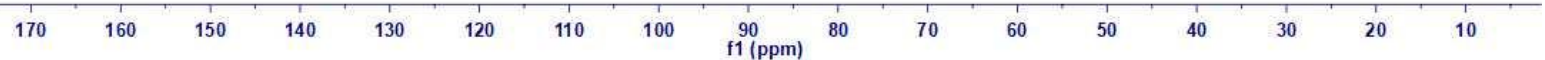


(2R,4R)-6-benzyl-4-(cyclopentylidenemethyl)-2-(2-hydroxyethyl)-2-methyl-2,3,4,6-tetrahydro-5H-pyrano[3,2-c]qui nolin-5-one $(4 \mathrm{vv})$

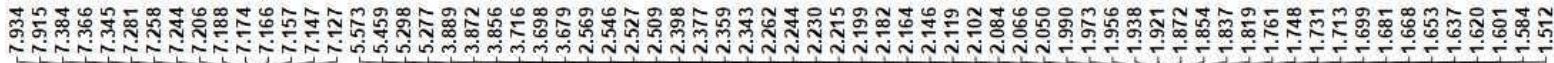

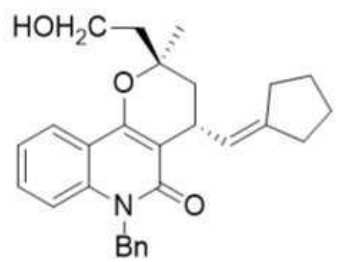

$4 \mathrm{vv}$

$400 \mathrm{MHz} \mathrm{CDCl}_{3}$

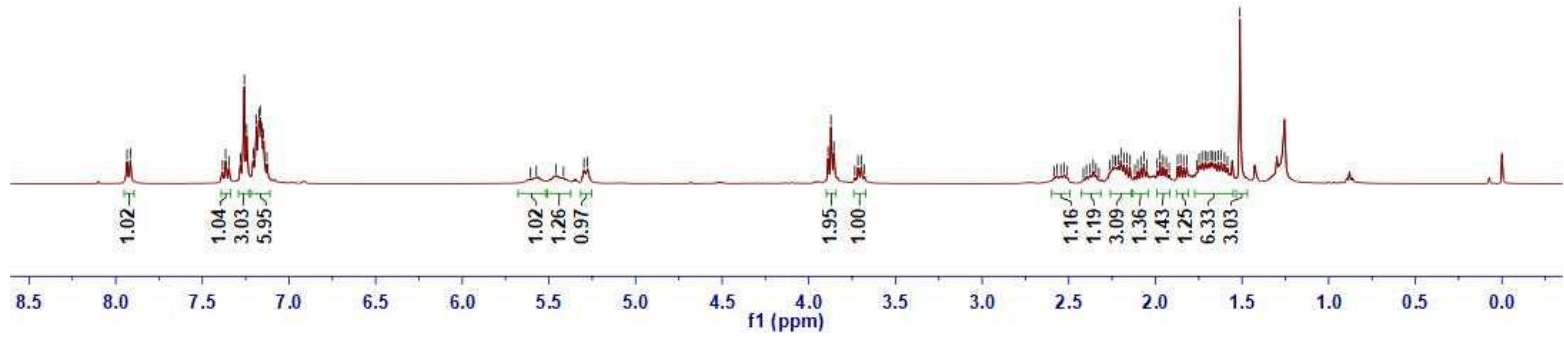

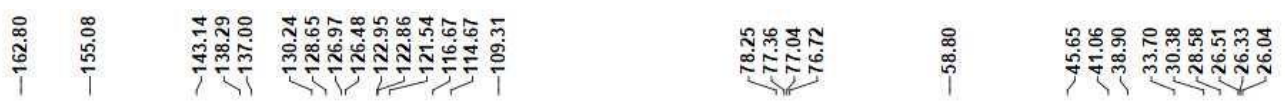

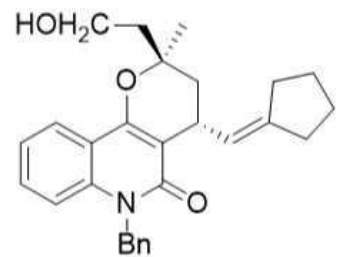

$4 \mathrm{vv}$

$100 \mathrm{MHz} C D C l_{3}$

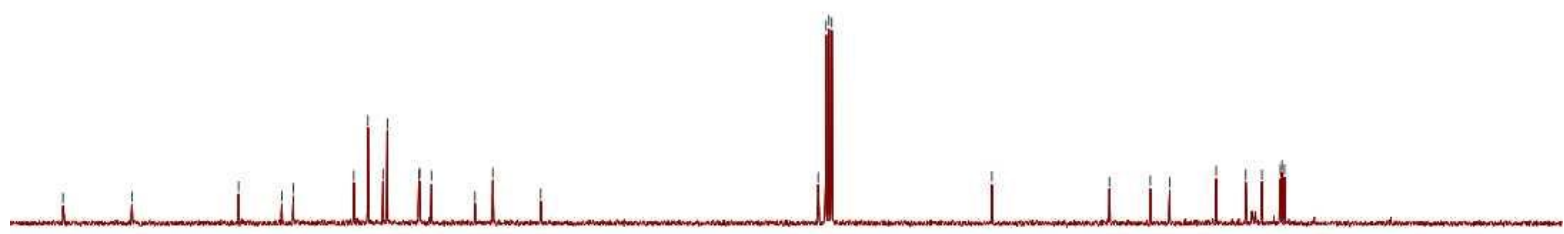

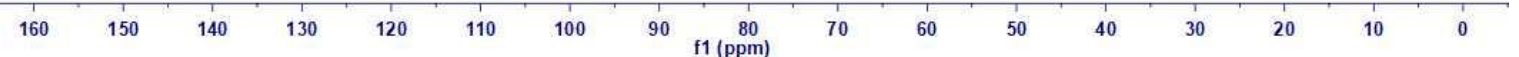


(8S,10R)-10-(2-hydroxyethyl)-10-methyl-8-(2-methylprop-1-en-1-yl)-4,5,9,10-tetrahydro-7H,8H-pyrano[3,2-c]pyrr olo[3,2,1-ij]quinolin-7-one (4w)
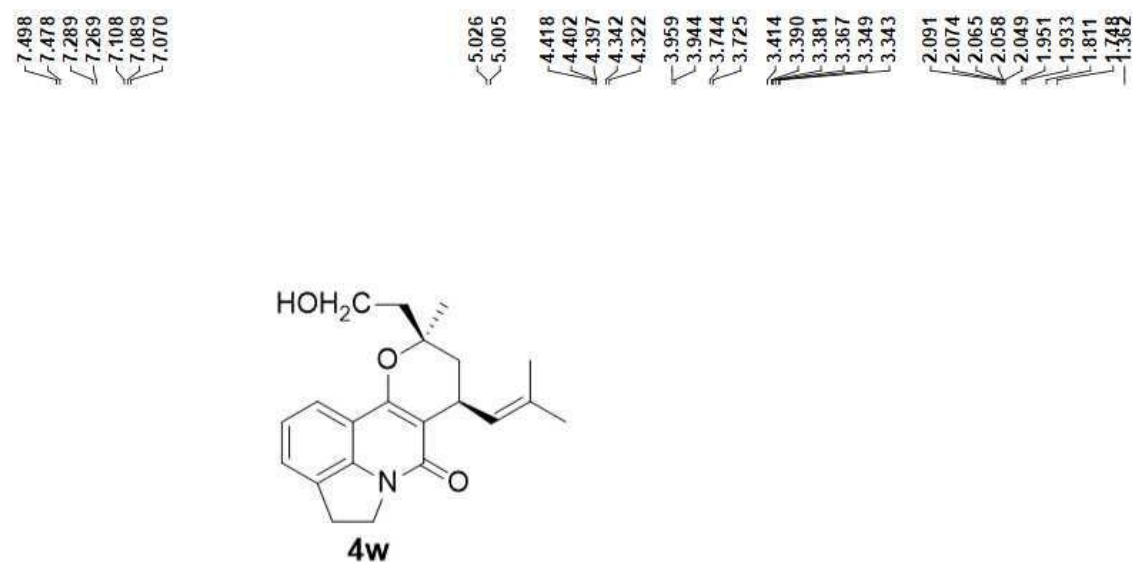

$400 \mathrm{MHz} \mathrm{CDCl}_{3}$

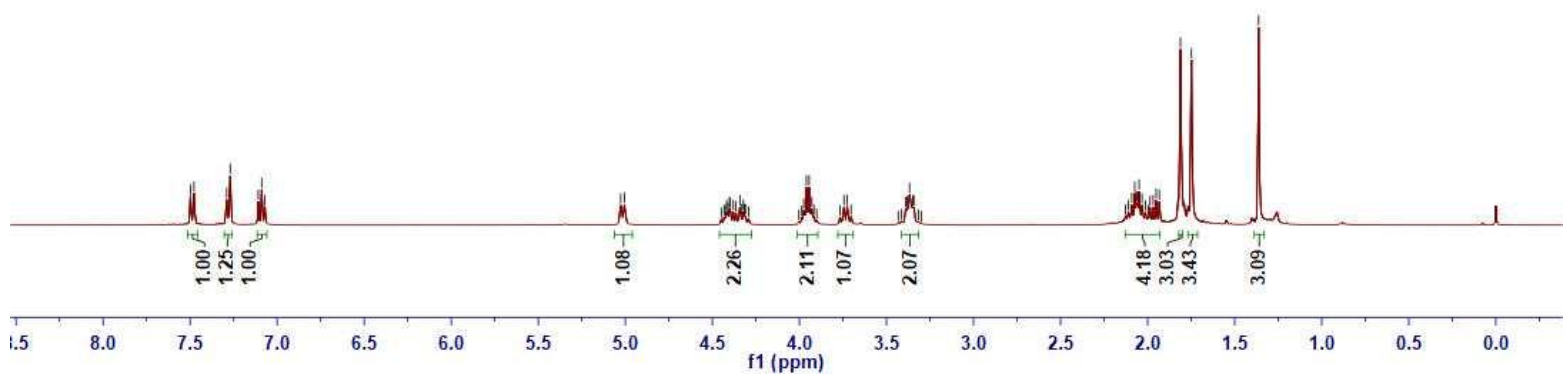

\begin{tabular}{|c|c|c|c|c|c|c|}
\hline 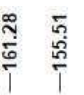 & $\underset{⿱ 亠 \pi}{\frac{\pi}{5}}$ & 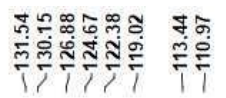 & 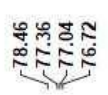 & $\stackrel{\substack{\infty \\
0}}{1}$ & 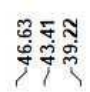 & 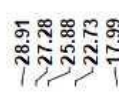 \\
\hline
\end{tabular}<smiles>CC(C)=CC1C[C@](C)(CCO)Oc2c1c(=O)n1c3c(cccc23)CC1</smiles>

$100 \mathrm{MHz} \mathrm{CDCl}_{3}$

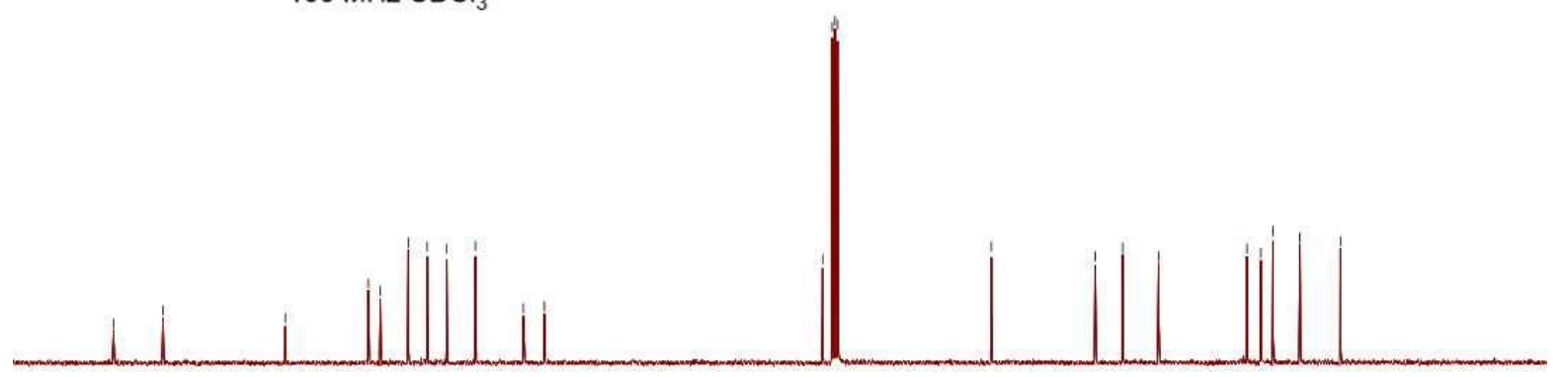

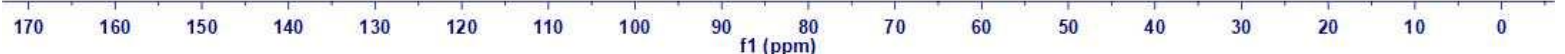


(8S,10S)-10-(2-hydroxyethyl)-10-methyl-8-(2-methylprop-1-en-1-yl)-4,5,9,10-tetrahydro-7H,8H-pyrano[3,2-c]pyrr olo[3,2,1-ij]quinolin-7-one (4ww)

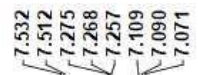

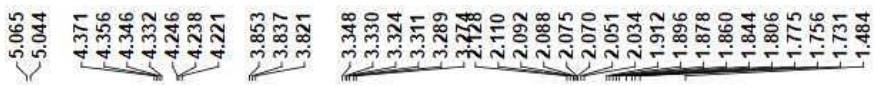

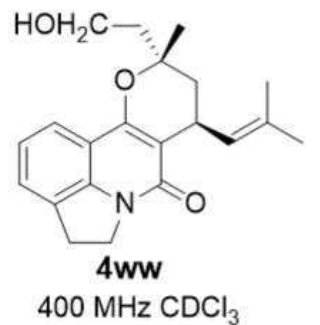

$400 \mathrm{MHz} \mathrm{CDCl}_{3}$
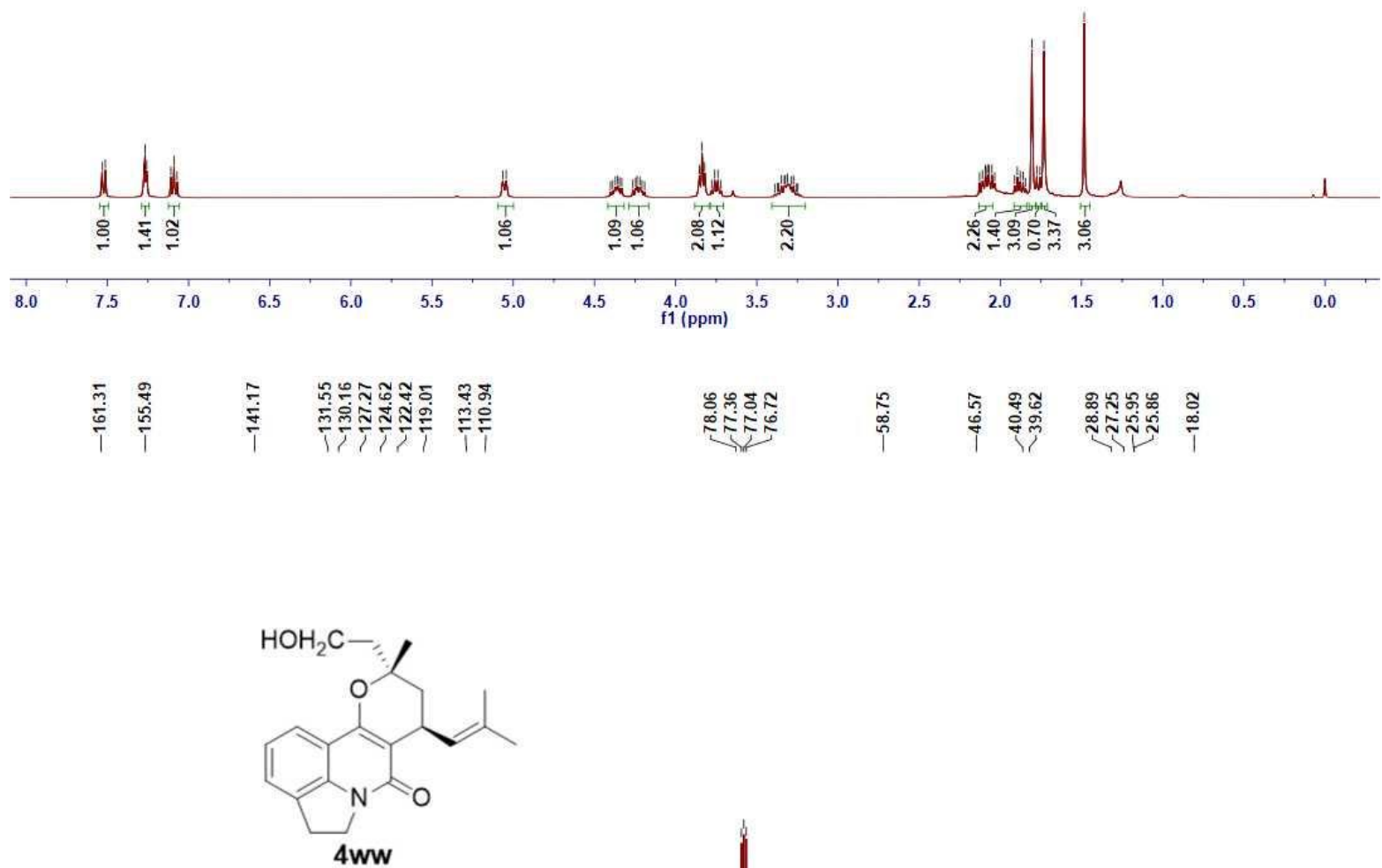

$100 \mathrm{MHz} \mathrm{CDCl}_{3}$

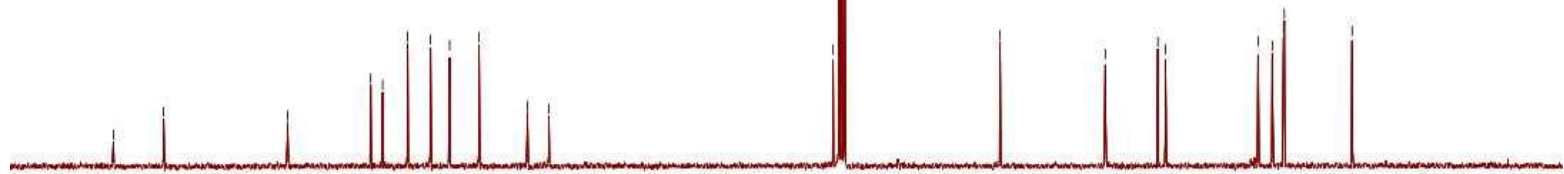

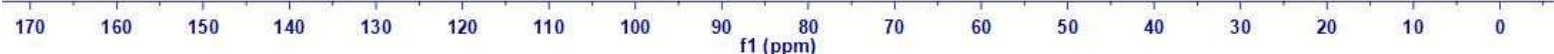


(9S,11R)-11-(2-hydroxyethyl)-11-methyl-9-(2-methylprop-1-en-1-yl)-5,6,10,11-tetrahydro-4H,8H,9H-pyrano[3,2-c] pyrido[3,2,1-ij]quinolin-8-one $(4 x)$
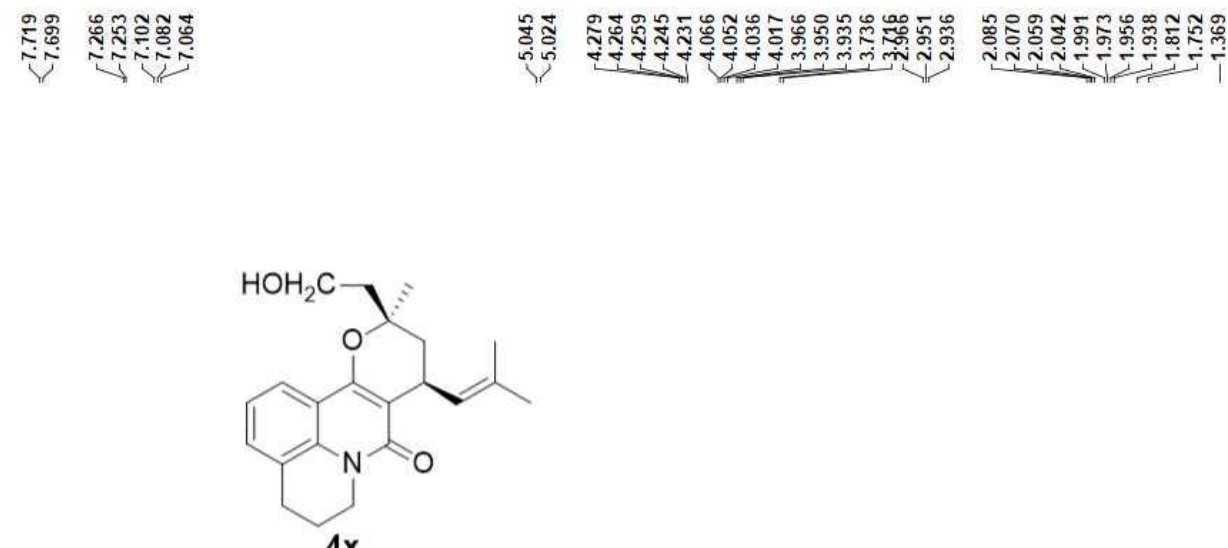

$400 \mathrm{MHz} \mathrm{CDCl}_{3}$

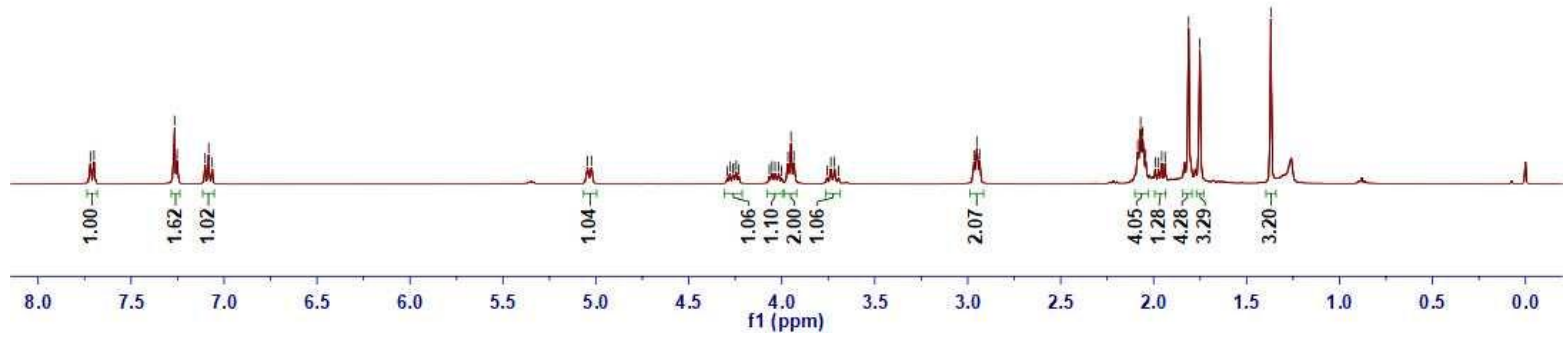

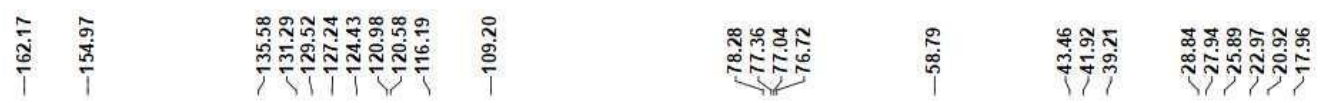

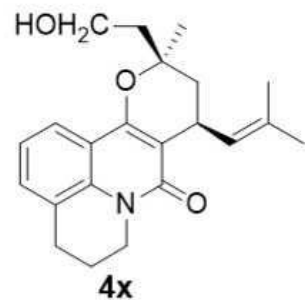

$100 \mathrm{MHz} \mathrm{CDCl}_{3}$

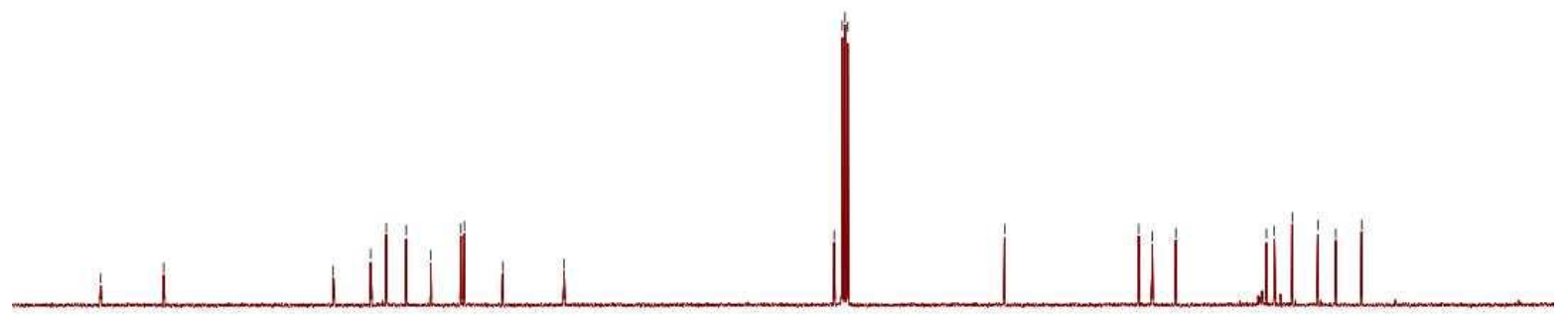

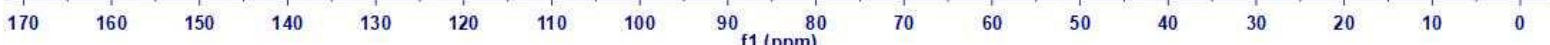


(9S,11S)-11-(2-hydroxyethyl)-11-methyl-9-(2-methylprop-1-en-1-yl)-5,6,10,11-tetrahydro-4H,8H,9H-pyrano[3,2c]pyrido[3,2,1-ij]quinolin-8-one (4xx)
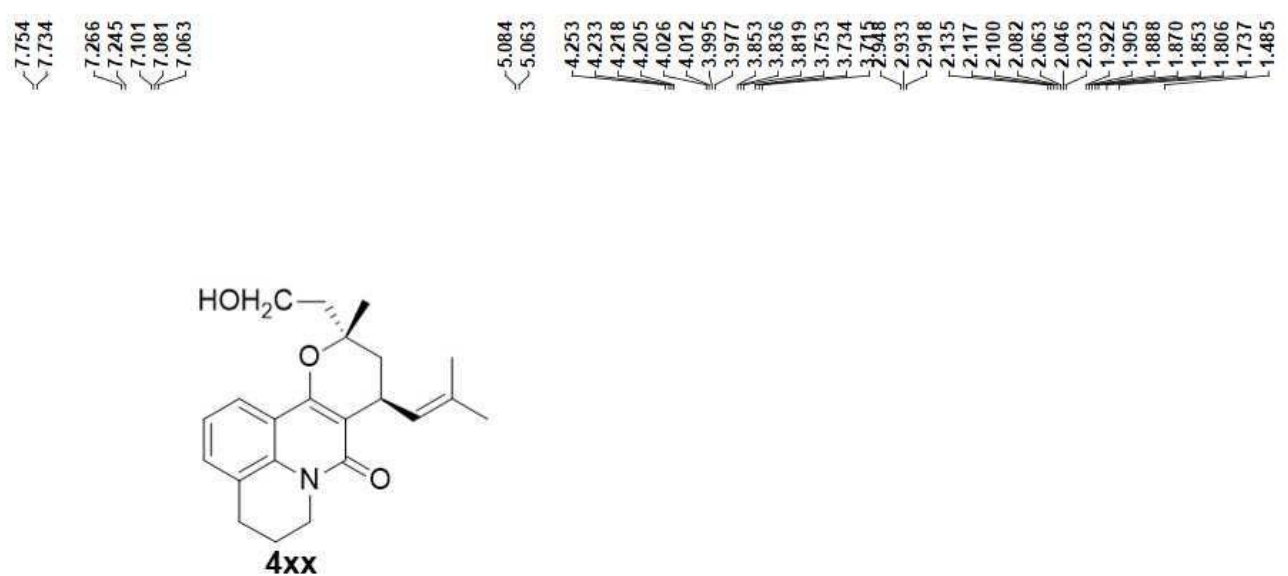

$400 \mathrm{MHz} \mathrm{CDCl}_{3}$
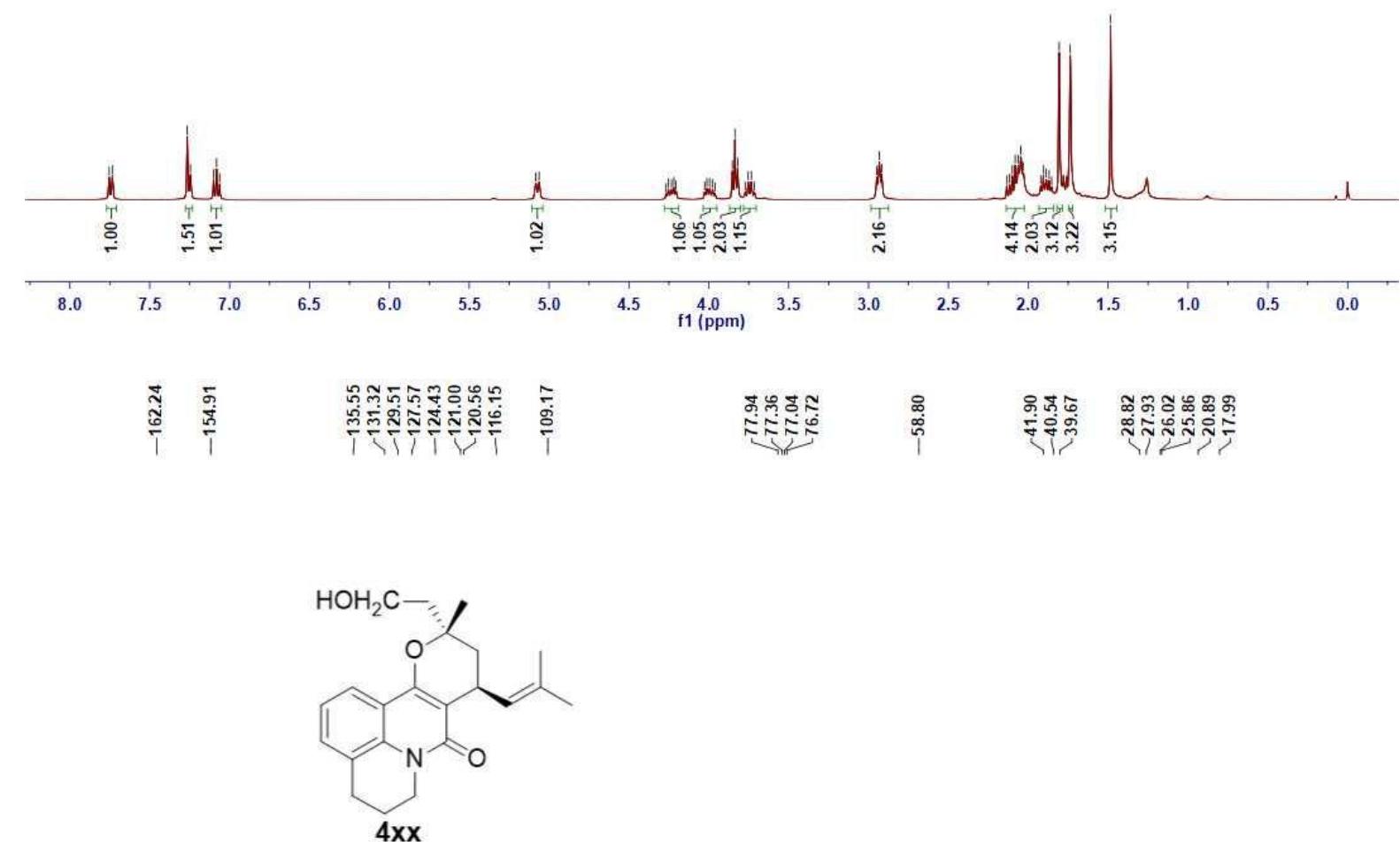

$100 \mathrm{MHz} \mathrm{CDCl}_{3}$

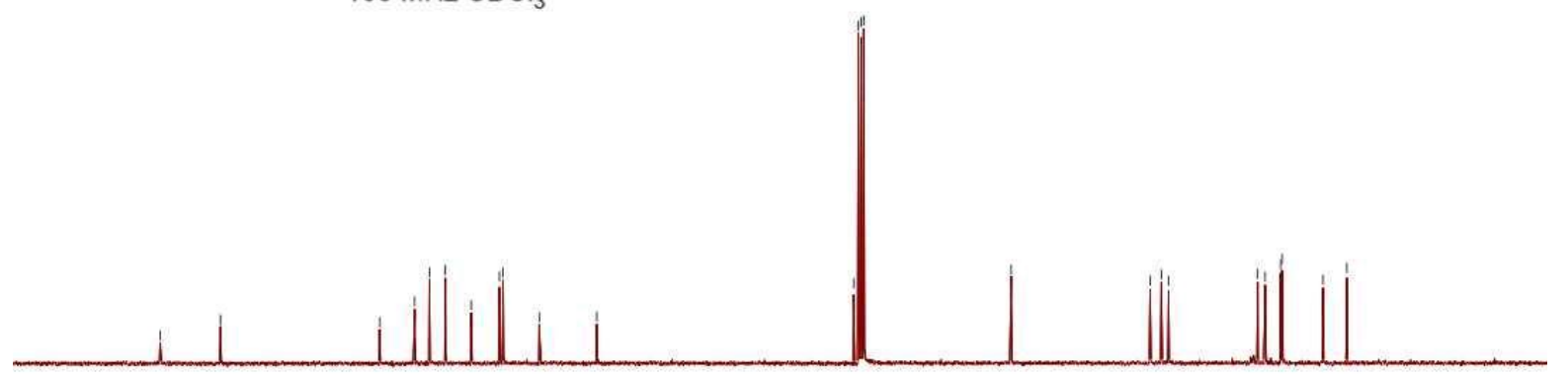

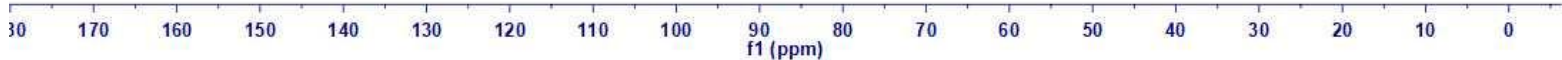


2-((2S,4S)-2,6-dimethyl-4-(2-methylprop-1-en-1-yl)-5-oxo-3,4,5,6-tetrahydro-2H-pyrano[3,2-c]quinolin-2-yl)acetic acid (9)
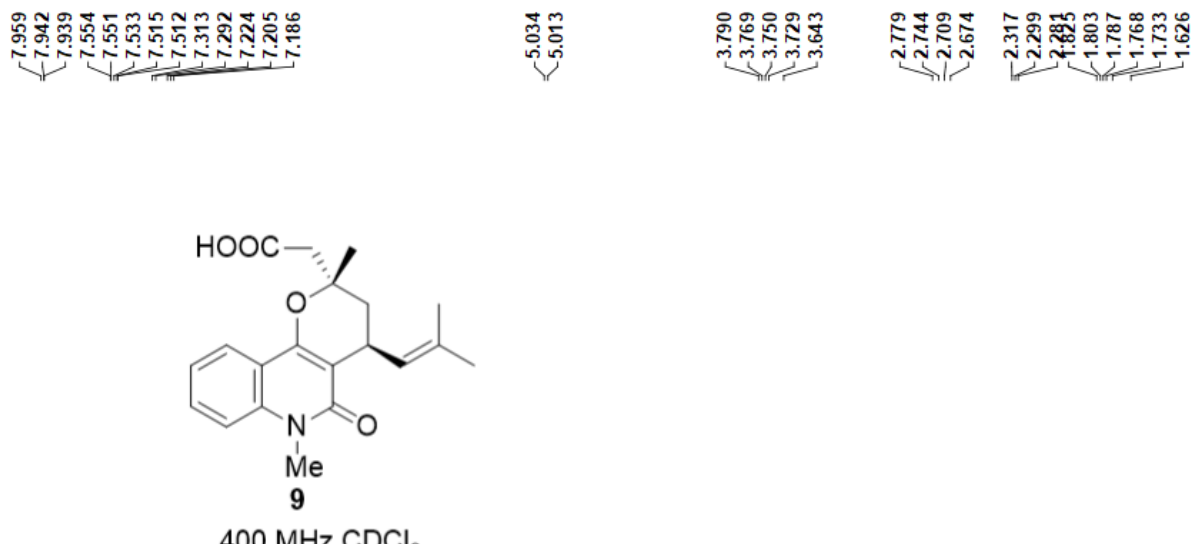

$400 \mathrm{MHz} \mathrm{CDCl}_{3}$
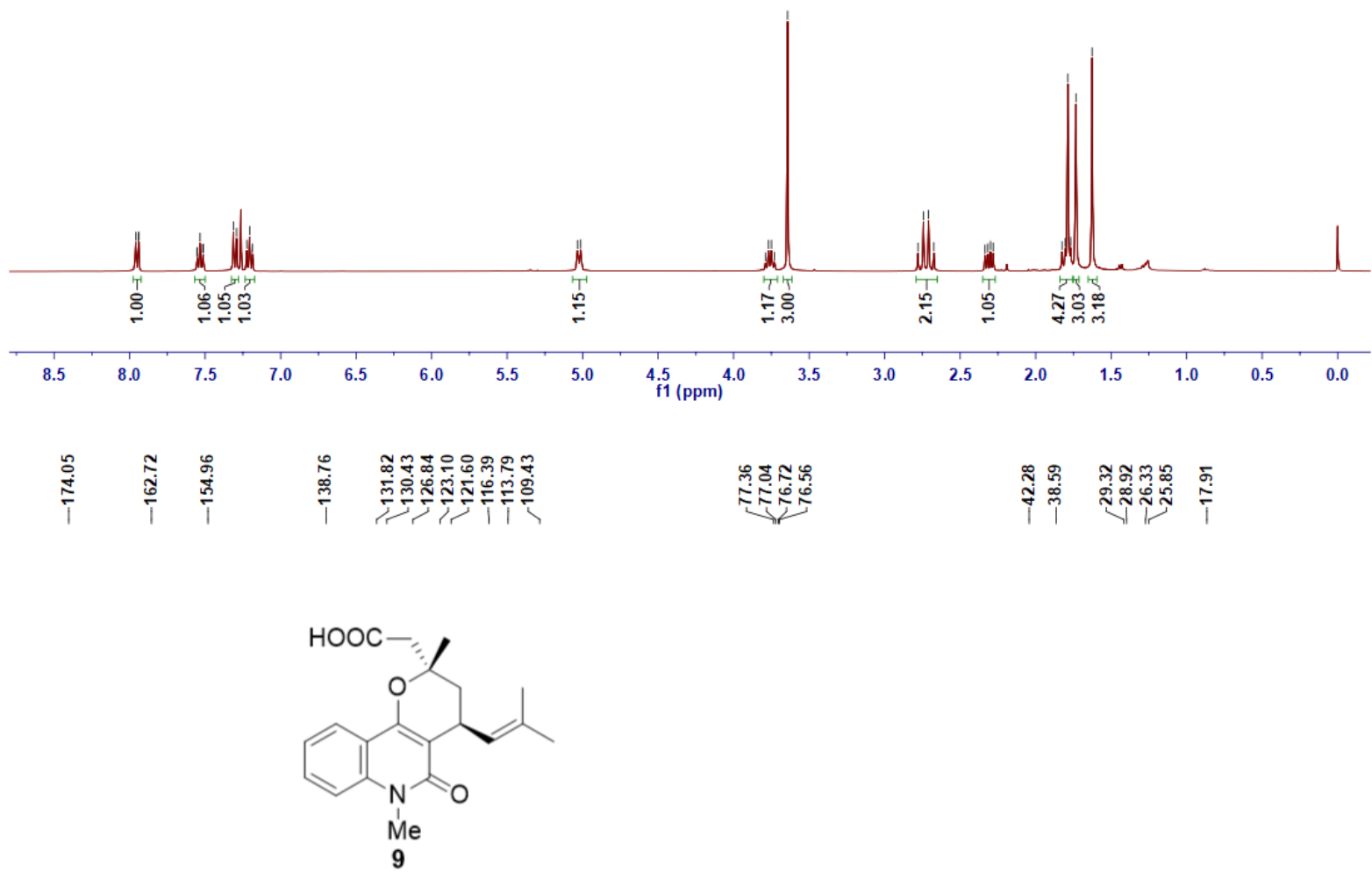

$100 \mathrm{MHz} \mathrm{CDCl}_{3}$

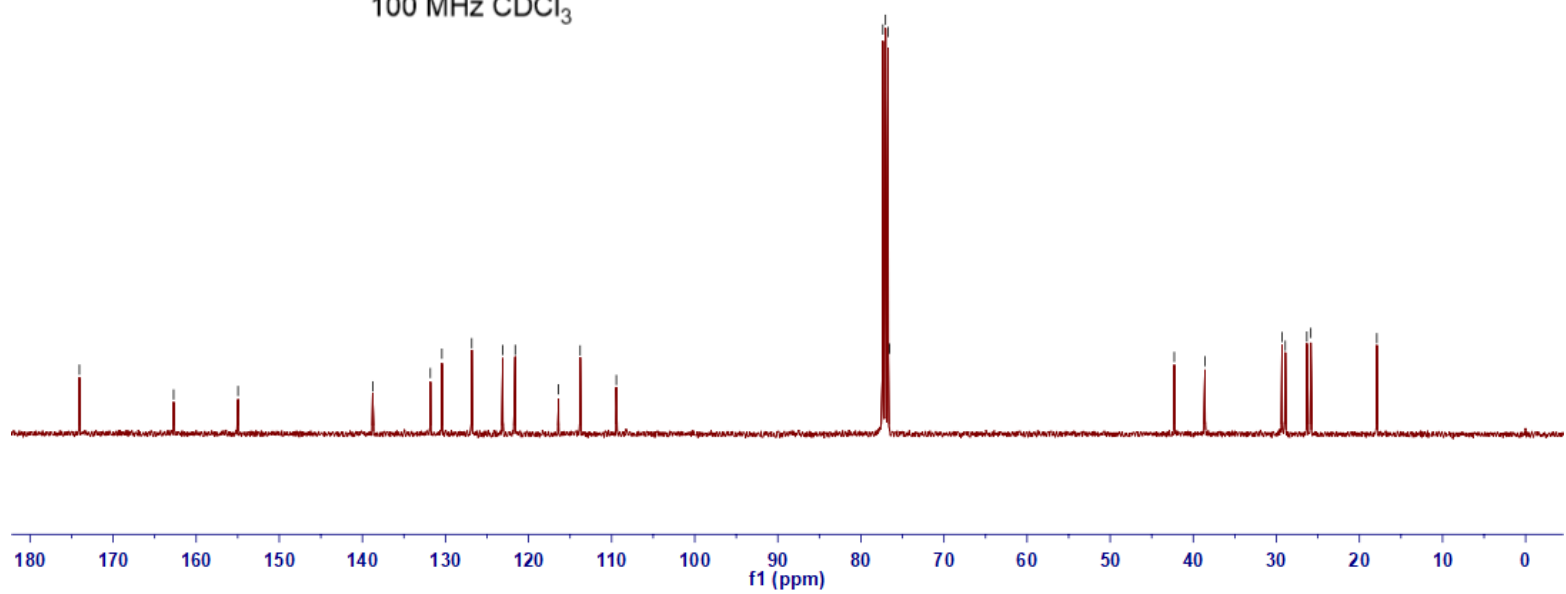


(2S,4R)-2,6-dimethyl-4-(2-methylprop-1-en-1-yl)-2-vinyl-2,3,4,6-tetrahydro-5H-pyrano[3,2-c]quinolin-5-one (3a)
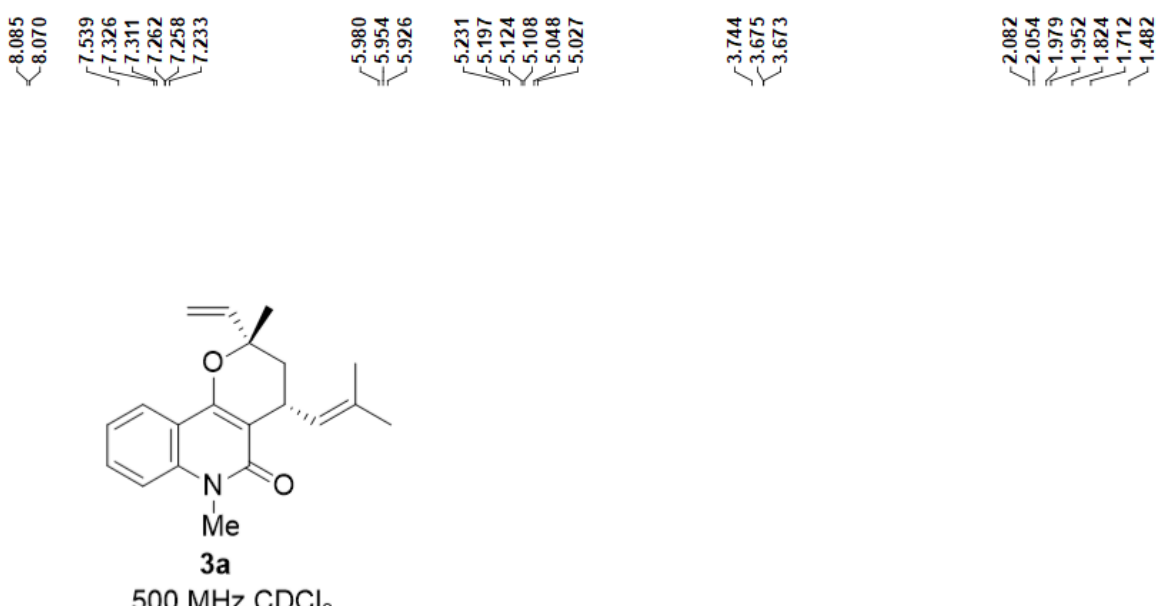

$500 \mathrm{MHz} \mathrm{CDCl}_{3}$

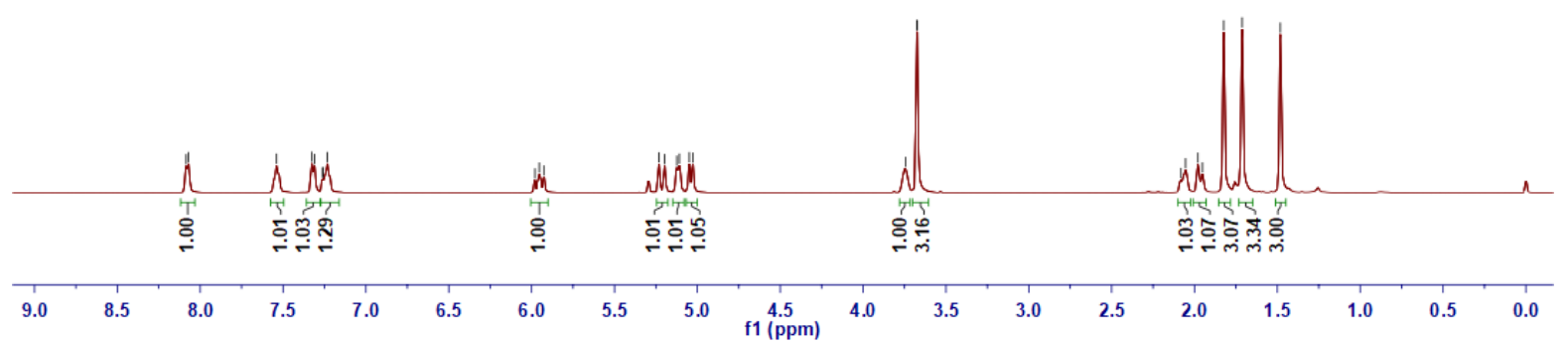

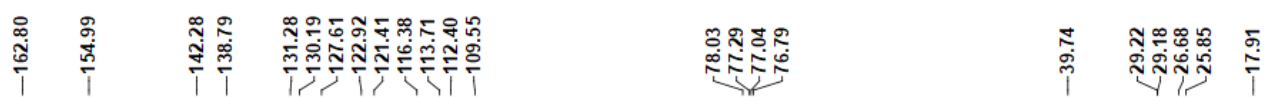

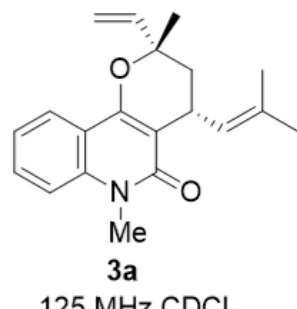

$125 \mathrm{MHz} \mathrm{CDCl}_{3}$

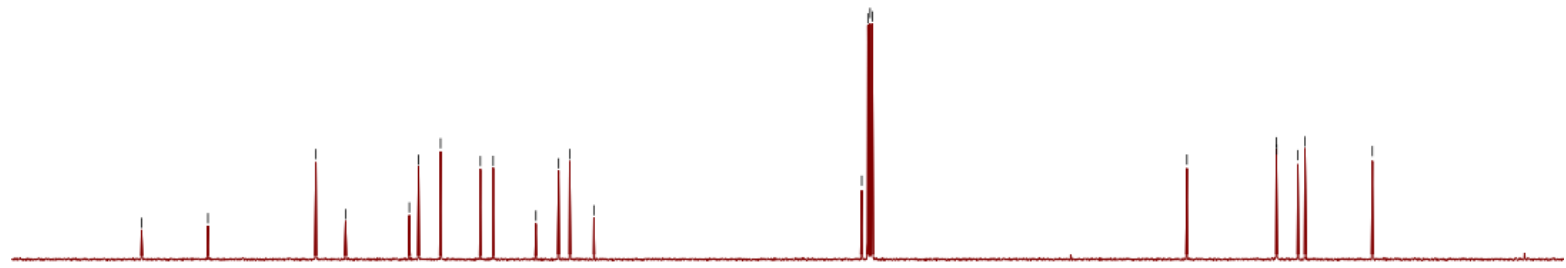

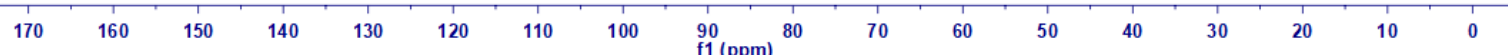


(2S,4R)-2-((E)-2-(5,7-dimethoxy-2-oxo-2H-chromen-8-yl)vinyl)-2,6-dimethyl-4-(2-methylprop-1-en-1-yl)-2,3,4,6-tet rahydro-5H-pyrano[3,2-c]quinolin-5-one (1a)
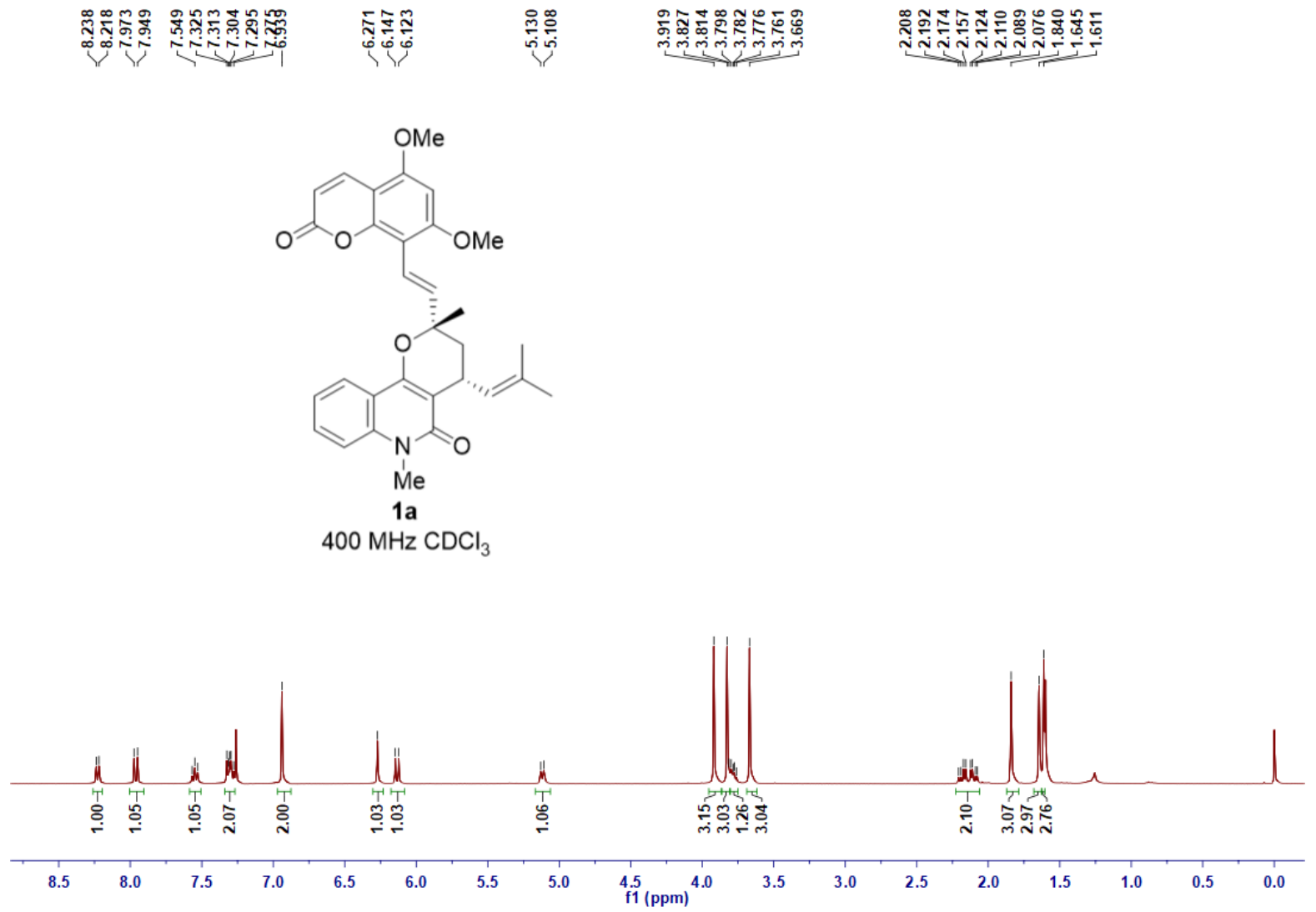

\begin{tabular}{|c|c|c|c|c|c|c|}
\hline 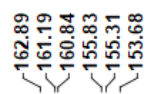 & 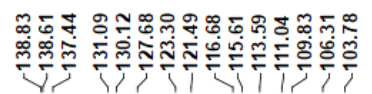 & స్ & 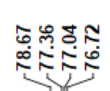 & ชัฐ & 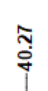 & 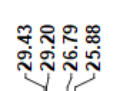 \\
\hline
\end{tabular}
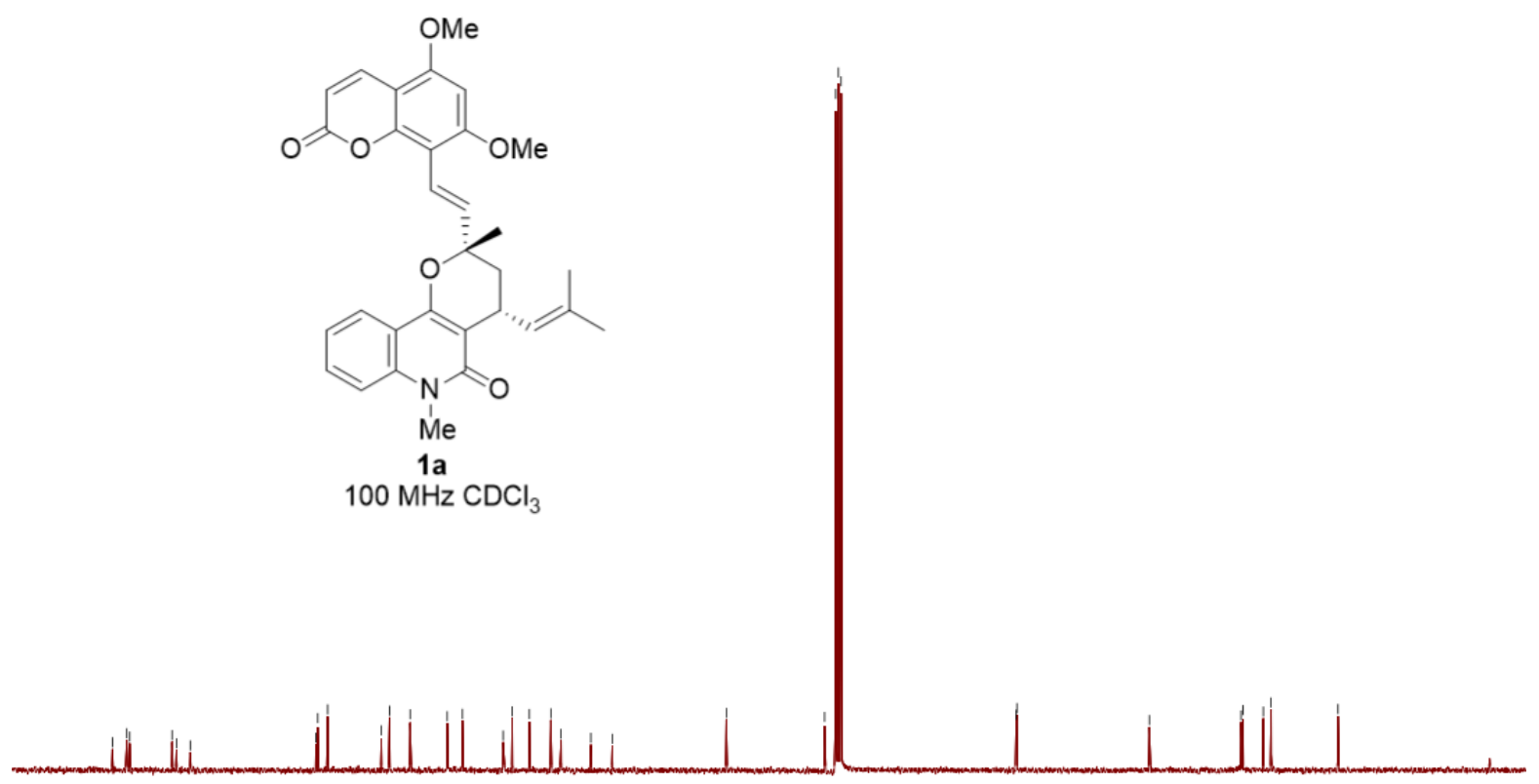

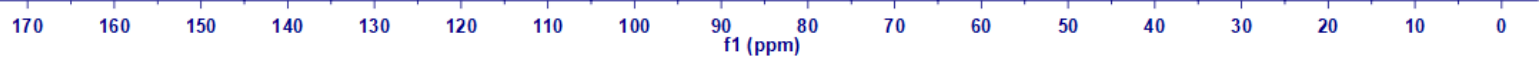


(2R,4R)-2,6-dimethyl-4-(2-methylprop-1-en-1-yl)-2-vinyl-2,3,4,6-tetrahydro-5H-pyrano[3,2-c]quinolin-5-one (3aa)

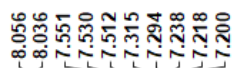

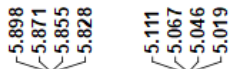

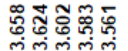

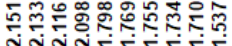
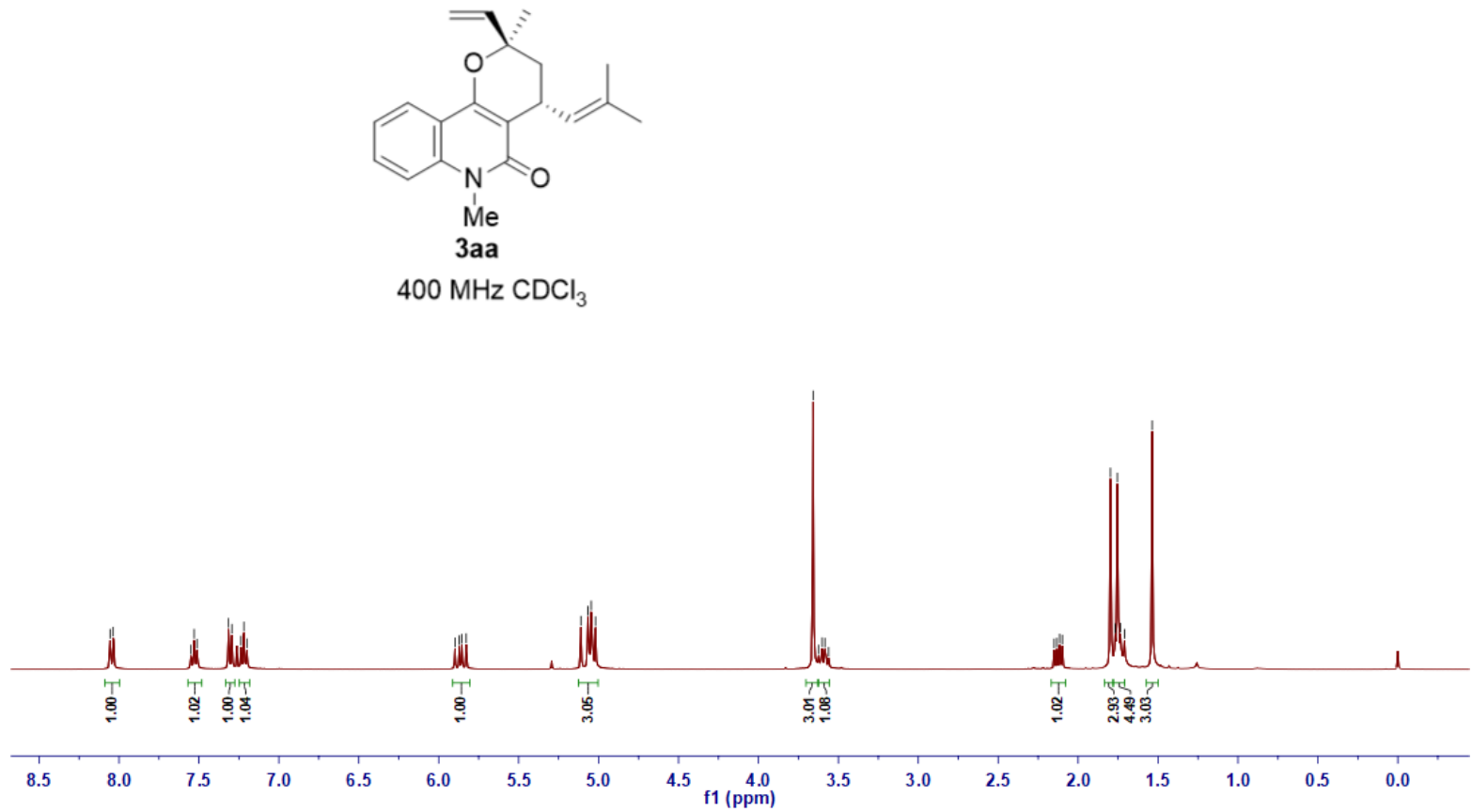

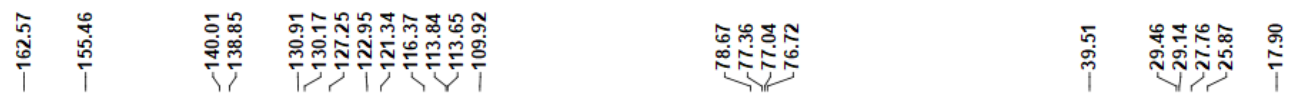
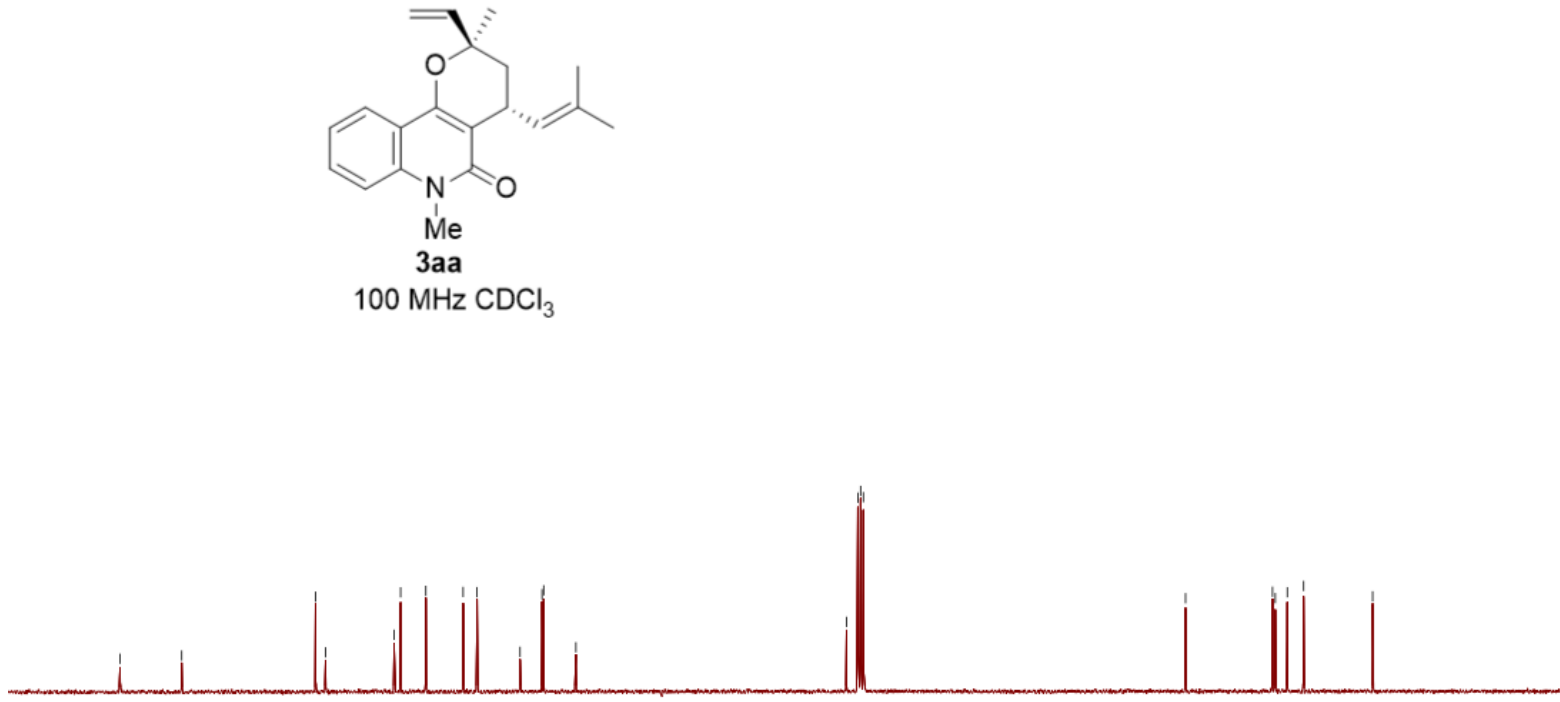

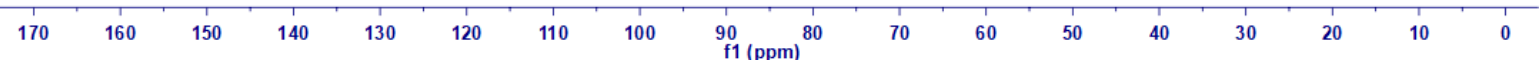


(2R,4R)-2-((E)-2-(5,7-dimethoxy-2-oxo-2H-chromen-8-yl)vinyl)-2,6-dimethyl-4-(2-methylprop-1-en-1-yl)-2,3,4,6-te trahydro-5H-pyrano[3,2-c]quinolin-5-one (1aa)

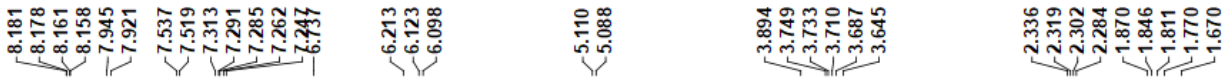
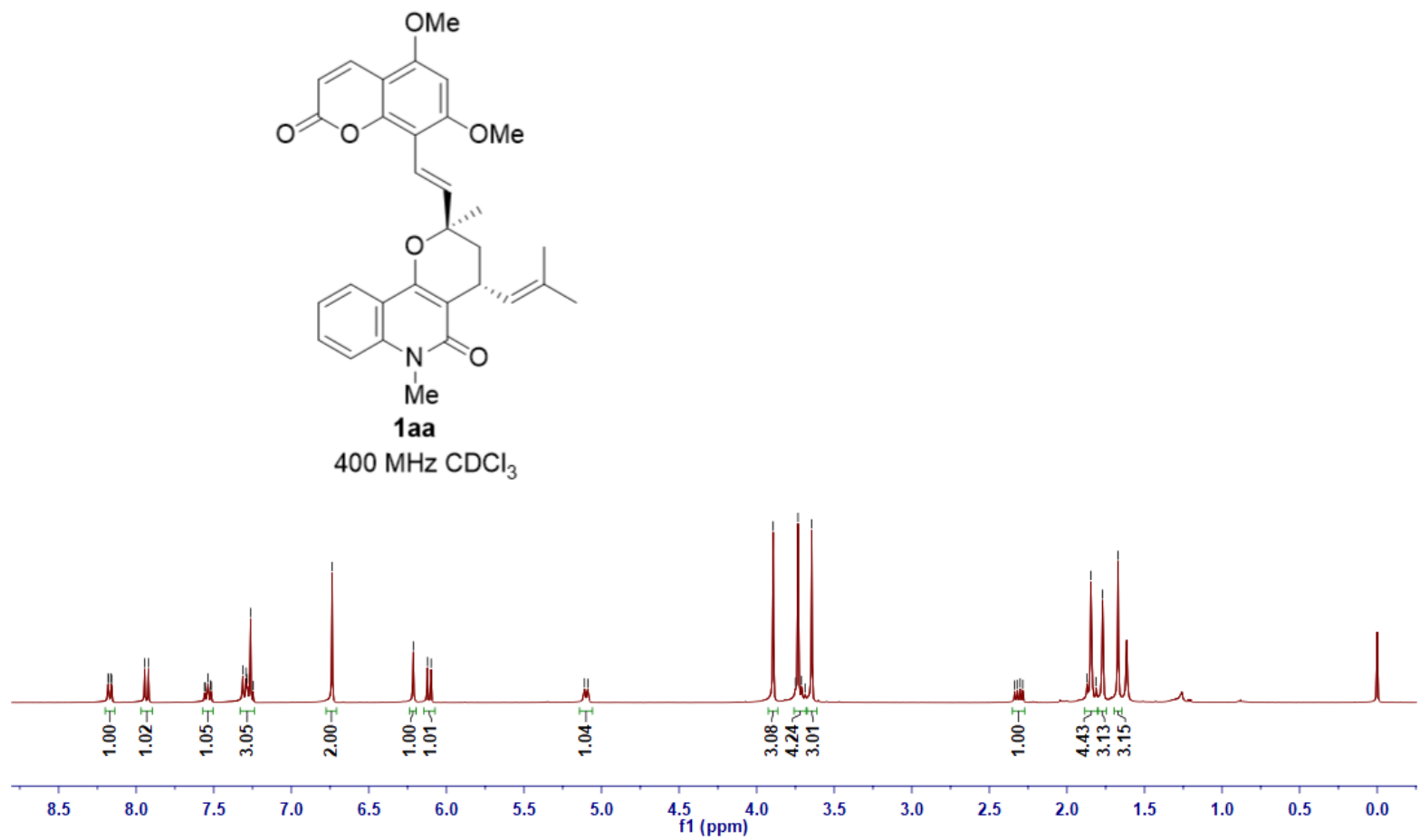

\begin{tabular}{|c|c|c|c|c|c|c|}
\hline 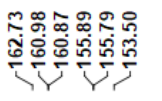 & 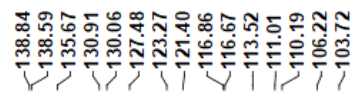 & ڤั̊ & 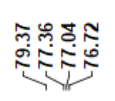 & 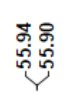 & \% & 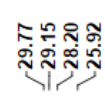 \\
\hline
\end{tabular}

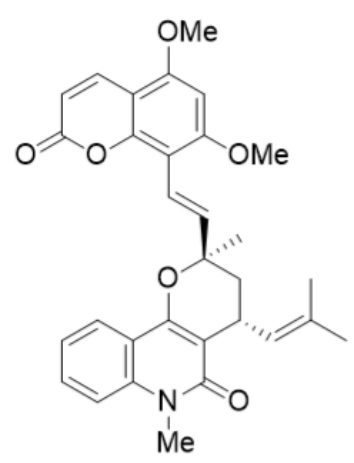

1aa

$100 \mathrm{MHz} \mathrm{CDCl}_{3}$

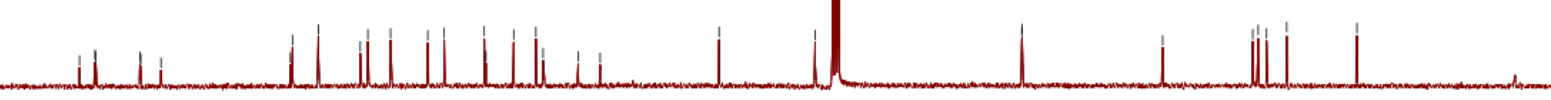

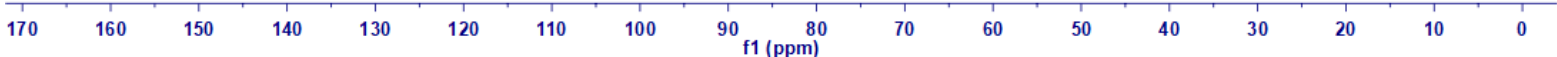

